\title{
The Ursa Major cluster of galaxies
}

\section{HI synthesis observations}

\author{
M. A. W. Verheijen ${ }^{1,2}$ and R. Sancisi ${ }^{2,3}$ \\ 1 NRAO, PO Box O, Socorro, NM 87801, USA \\ 2 Kapteyn Institute, Postbus 800, 9700 AV Groningen, The Netherlands \\ 3 Osservatorio Astronomico di Bologna, Via Ranzani 1, 40127 Bologna, Italy
}

Received 15 November 2000 / Accepted 1 December 2000

\begin{abstract}
In this data paper we present the results of an extensive $21 \mathrm{~cm}$-line synthesis imaging survey of 43 spiral galaxies in the nearby Ursa Major cluster using the Westerbork Synthesis Radio Telescope. Detailed kinematic information in the form of position-velocity diagrams and rotation curves is presented in an atlas together with HI channel maps, $21 \mathrm{~cm}$ continuum maps, global HI profiles, radial HI surface density profiles, integrated HI column density maps, and HI velocity fields. The relation between the corrected global HI linewidth and the rotational velocities $V_{\max }$ and $V_{\text {flat }}$ as derived from the rotation curves is investigated. Inclination angles obtained from the optical axis ratios are compared to those derived from the inclined HI disks and the HI velocity fields. The galaxies were not selected on the basis of their HI content but solely on the basis of their cluster membership and inclination which should be suitable for a kinematic analysis. The observed galaxies provide a well-defined, volume limited and equidistant sample, useful to investigate in detail the statistical properties of the Tully-Fisher relation and the dark matter halos around them.
\end{abstract}

Key words. galaxies: fundamental parameters - galaxies: kinematics and dynamics - galaxies: spiral - galaxies: structure

\section{Introduction}

The statistical properties of the Tully-Fisher relation (TFr), like its scatter and slope, and in more detail the characteristics of dark matter halos around galaxies, like their core densities and radii, are of great interest to those who study galaxy formation scenarios. The relevant observables for the TFr are total luminosities and rotational velocities while actual rotation curves and luminosity profiles are required to obtain constraints on the density profiles of dark matter halos. However, the available data sets from which these observables can be obtained are often suffering from incompleteness, distance uncertainties and inhomogeneous observing and data analysis techniques.

Furthermore, the interpretation of the observables is not always unambiguous. For instance, the rotational velocity of a galaxy is generally inferred from the width of its global HI profile and often this measured width is being related directly to the dark matter potential (e.g. Navarro \& Steinmetz 2000). However, the width of the HI profile is a complicated convolution of the 2-dimensional distribution of $\mathrm{HI}$ in a galaxy disk and the shape and extent of

Send offprint requests to: M. A. W. Verheijen, e-mail: mverheij@nrao.edu its rotation curve as sampled by the HI gas. Needless to say one has to exercise caution when relating the observed scatter and slope in the TFr to the outcome of numerical and semi-analytical simulations of galaxy formation. This is especially the case when the observed TFr was constructed to serve as an empirical distance estimator and the selection criteria and applied corrections to the raw observables were optimized to linearize the relation and minimize its scatter.

To overcome some of the observational issues, we initiated a program to obtain detailed multi-band photometric and kinematic information on individual galaxies in a welldefined, complete sample. The nearby Ursa Major cluster of galaxies provides a particularly suitable sample. In the first place, the galaxies in the cluster are all at roughly the same distance. Therefore, there is little doubt about their relative luminosities, sizes and masses. Furthermore, the Ursa Major cluster contains overwhelmingly gas-rich systems and the morphological mix of its galaxy members is close to that of the lower density field. A detailed discussion on the definition of the Ursa Major cluster is given by Tully et al. (1996) (Paper I). The characteristics of this cluster will be discussed in more detail in Sect. 2 . 
In this paper we present the results of an extensive $21 \mathrm{~cm}$-line synthesis imaging survey of individual galaxies in the Ursa Major cluster using the Westerbork Synthesis Radio Telescope (WSRT). The HI data are presented in the form of an atlas. Multi-band optical and near-infrared imaging photometry is presented in Paper I together with $B$-band and $K^{\prime}$-band images of all identified cluster members. Forthcoming papers in this series will use these data to investigate the TFr while the HI rotation curves will be supplemented in the inner regions with already obtained high resolution optical rotation curves to derive constraints on the structural properties of the dark matter halos and the mass-to-light ratios of the stellar populations.

This data paper is organized as follows. Section 2 describes the Ursa Major cluster in more detail and contains morphological and photometric information on the galaxies observed with the WSRT. Data acquisition and reduction procedures are explained in Sect. 3. Section 4 explains how the corrected linewidth can be matched to $V_{\max }$ and $V_{\text {flat }}$ from the rotation curves through an appropriate correction for turbulent motion. Inclinations are derived from the optical images, HI column density maps and HI velocity fields and Sect. 5 presents a comparison of these inclinations. The layout of the HI atlas is described in Sect. 6 with details about the various elements of the atlas pages. The HI properties of the Ursa Major cluster galaxies as a sample are presented in Sect. 7. Some concluding remarks are given in Sect. 8.

\section{The sample}

The nearby Ursa Major cluster as defined in Paper I has 79 identified members. It is located in the Supergalactic plane at an angular distance of 38 degrees from the core of the Virgo cluster. It has a recession velocity of $950 \mathrm{~km} \mathrm{~s}^{-1}$ and a velocity dispersion of only $\approx 150 \mathrm{~km} \mathrm{~s}^{-1}$. In Paper I and in Tully \& Verheijen (1997) (Paper II) a distance of 15.5 Mpc was adopted. However, new HST cepheid distances to local TFr calibrators (e.g. Sakai et al. 2000) and a new correction formalism for internal extinction (Tully et al. 1998) now place the Ursa Major cluster galaxies at a mean distance of $18.6 \mathrm{Mpc}$ (Tully \& Pierce 2000). At this distance, 1 arcmin corresponds to $5.4 \mathrm{kpc}$. The morphological mix of the cluster members is made up overwhelmingly by late type systems and only a dozen lenticulars are known members. Morphological and photometric properties in the optical and near-infrared of individual galaxies are described in detail in Paper I. The galaxy distribution shows no concentration toward any core and no X-ray emitting intra-cluster gas has been detected.

It should be noted that individual galaxies in the nearby Virgo (Warmels 1988a, 1988b; Cayatte et al. 1990), A1367 (Dickey \& Gavazzi 1991), Hercules (Dickey 1997) and Coma (Bravo-Alfaro et al. 2000) clusters have also been studied in detail and the effect of the dense environment on the properties of the HI disks in these clusters has been clearly demonstrated. The HI disks in the cores of these rich clusters are in general very small and often offset from the optical galaxy. On the other hand, the volume limited survey of the Hydra cluster (McMahon 1993) does not show such an HI deficiency although some interesting dynamical substructure has been revealed in this system. It should be stressed that the Ursa Major cluster is markedly different from these more massive and denser clusters. In selecting the Ursa Major sample, these environmental effects are carefully avoided as well as fore- and background contamination caused by high velocity dispersions and complex dynamical and spatial substructures.

Since the Ursa Major galaxies are all at the same distance, the effects of incompleteness and uncertain relative distances are minimized. A complete sample of 62 galaxies brighter than $M_{B} \approx-16.8$, i.e. roughly twice the luminosity of the Small Magellanic Cloud, was constructed and nearly all cluster members were observed with the WSRT. In this paper, however, only those 49 galaxies which are more inclined than 45 degrees, as derived from the optical axis ratio, will be considered for a detailed kinematic study.

Table 1 gives a summary of the positional and morphological properties of these 49 galaxies while photometrics are presented in Table 2, based on a $18.6 \mathrm{Mpc}$ distance. There are 3 additional galaxies in the tables which do not meet the luminosity $\left({ }^{f}\right)$ and inclination $\left({ }^{i}\right)$ criteria but happened to be in the same WSRT fields as galaxies from the complete sample. Of all those 52 galaxies, the HI synthesis data of 30 were fully analyzed. Thirteen systems were observed and detected but the HI data of these galaxies are presented in an abbreviated form comprising only the channel maps, global profiles and position-velocity diagrams. Two of the smaller galaxies were detected in HI but they are confused with the HI emission from their more massive companions. Finally, there are 7 galaxies in the complete sample which have not been observed or detected because of their low HI content known from single dish observations. These are in general S0 or Sa systems.

Table 1 presents the following positional and morphological information:

Column (1) gives the NGC or UGC numbers.

Columns (2) and (3) provide the equatorial coordinates (B1950) derived from the optical images.

Columns (4) and (5) give the Galactic coordinates.

Column (6) provides the morphological type.

Column (7) gives the observed major axis diameter of the 25th mag $\operatorname{arcsec}^{-2}$ blue isophote.

Column (8) contains the position angle of the receding side of the galaxy. For galaxies which are not observed or not detected in HI, this is the smallest position angle of the major axis measured eastward from the north.

Column (9) contains the observed ellipticity of the optical galaxy image.

Column (10) gives the inclination $i_{\text {opt }}$ as derived from the observed axis ratio $(b / a)$. See Sect. 5.1 for further details. Column (11) gives the adopted inclination angle as derived from several methods described in Sect. 5 .

Column (12) indicates whether a galaxy has a low (LSB) 
or high surface brightness (HSB) according to Paper II. Columns (13) and (14) provide the galactic extinction in the $B$-band according to Burstein \& Heiles (1984) (BH) and Schlegel et al. (1998) (SFD) as reported by the NASA Extragalactic Database.

Table 2 presents the following photometric information:

Column (1) gives the NGC or UGC numbers.

Columns (2)-(5) give the observed total magnitudes in the $B, R, I$ and $K^{\prime}$ passbands from Paper I.

Column (6) contains the corrected HI line widths at the $20 \%$ level, used to calculate the internal extinction as explained below.

Columns (7)-(10) present the calculated internal extinction corrections in thee $B, R, I$ and $K^{\prime}$ passbands toward face-on $A_{\lambda}^{i \rightarrow 0}$, calculated according to Tully et al. (1998):

$A_{\lambda}^{i \rightarrow 0}=\gamma_{\lambda} \log (a / b)$

where $a / b$ is the observed axis ratio of the galaxy as an indication of inclination while $\gamma_{\lambda}$ depends on the luminosity and is calculated according to

$\gamma_{B}=1.57+2.75\left(\log W_{R, I}^{i}-2.5\right)$

$\gamma_{R}=1.15+1.88\left(\log W_{R, I}^{i}-2.5\right)$

$\gamma_{I}=0.92+1.63\left(\log W_{R, I}^{i}-2.5\right)$

$\gamma_{K^{\prime}}=0.22+0.40\left(\log W_{R, I}^{i}-2.5\right)$

where $W_{R, I}^{i}$ is the distance independent HI line width corrected for instrumental resolution as described in Sect. 3.2, corrected for turbulent motion according to Tully \& Fouqué (1985) (TFq hereafter) with $W_{t, 20}=22 \mathrm{~km} \mathrm{~s}^{-1}$ as motivated in Sect. 4 and corrected for inclination using $i_{\text {adopt }}$ from Table 1 . For dwarf galaxies with $W_{R, I}^{i}<85 \mathrm{kms}^{-1}$ and for lenticulars with no dust features, the value of $\gamma_{\lambda}$ is set to zero at all passbands.

Columns (11)-(14) give the total absolute $B, R, I$ and $K^{\prime}$ magnitudes corrected for Galactic and internal extinction and a distance modulus of 31.35 corresponding to a distance to the Ursa Major cluster of $18.6 \mathrm{Mpc}$ :

$M_{\lambda}^{b, i}=m_{\lambda}^{\text {tot }}-A_{\lambda}^{b}-A_{\lambda}^{i \rightarrow 0}-31.35$

where the Galactic extinction $A_{B}^{b}$ is taken from SFD as listed in Table 1. Extinction corrections in the other passbands are made according to the Galactic reddening law given by Cardelli et al. (1989) as summarized by SFD under the Landolt filters in their Table 6 . It's given by $A_{R}^{b} / A_{B}^{b}=0.62, A_{I}^{b} / A_{B}^{b}=0.45$ and $A_{K^{\prime}}^{b} / A_{B}^{b}=0.08$.

Column (15) gives the diameter of the 25 th mag $\operatorname{arcsec}^{-2}$ blue isophote corrected for both galactic and internal extinction and projection according to TFq:

$\log \left(D_{25}^{b, i}\right)=\log \left(D_{25}\right)-0.22 \log \left(D_{25} / d_{25}\right)+0.09 A_{B}^{b}$

where $d_{25}$ is the minor axis diameter at the 25th mag $\operatorname{arcsec}^{-2}$ blue isophote and $A_{B}^{b}$ is taken from SFD.

\section{Data acquisition and reduction}

The HI data presented in this paper were obtained with the Westerbork Synthesis Radio Telescope (WSRT) between 1991 and 1996. The integration times varied between $1 \times 12^{\mathrm{h}}$ and $5 \times 12^{\mathrm{h}}$ depending on the required signal-to-noise. The angular resolution at the center of the cluster is $12^{\prime \prime} \times 16^{\prime \prime}$ or $1.08 \times 1.44 \mathrm{kpc}$ at the adopted distance of $18.6 \mathrm{Mpc}$. The $F W H M$ of the primary beam is 37.4 arcminutes or $202 \mathrm{kpc}$. As a result, often more than one galaxy was mapped in a single field of view. The observed bandwidth was either 2.5 or $5 \mathrm{MHz}$, depending on the width of the global profiles. The observations of the NGC 3992-group and the NGC 4111-group required a broad frequency band of $5 \mathrm{MHz}$ and at the same time also sufficient velocity resolution for the dwarf systems. To comply with the correlator restrictions, those two fields were observed only in one polarization (XX) which allowed for a velocity resolution of $10 \mathrm{~km} \mathrm{~s}^{-1}$ but resulted in less sensitivity. During the earlier measurements an on-line Hanning taper was applied but this tapering was abandoned later to obtain the highest possible velocity resolution. The various obtained velocity resolutions (dependent on the correlator restrictions) were 5, 8, 10, 20 or $33 \mathrm{~km} \mathrm{~s}^{-1}$, corresponding to typical rms-noise levels of respectively $3.1,1.9,2.9,1.6$ and 1.0 mJy beam $^{-1}$ for a single $12 \mathrm{~h}$ observation at the highest angular resolution. The data of NGC 4013 were kindly made available by $\mathrm{R}$. Bottema who studied this system in great detail (Bottema 1996 and references therein).

More details on the observational parameters for each field are tabulated in the atlas along with the data. What follows is a brief description of the reduction procedures.

The raw UV-data were calibrated, interactively flagged and Fast Fourier Transformed (FFT) using the NEWSTAR software developed at the NFRA in Dwingeloo. The UV points were weighted according to the local density of points in the UV plane and a Gaussian baseline taper was applied with a $F W H M$ of 2293 (m) which attenuates the longest baseline by $50 \%$. To deal with the frequency dependent antenna pattern, five antenna patterns were calculated for each data cube at a regular frequency separation thoughout the bandpass. Pixel sizes of 5 arcsec in RA and $\frac{5}{\sin (\delta)} \operatorname{arcsec}$ in declination ensure an adequate sampling of the synthesized beam, $12^{\prime \prime} \times 12^{\prime \prime} / \sin (\delta)$.

After the FFT, the datacubes and antenna patterns were further processed using the Groningen Image Processing SYstem (GIPSY). Several channels at the low and high velocity end of the bandpass were discarded because of their higher noise. As a result, there are 110 or 53 usable channels for a bandpass of 2.5 or $5 \mathrm{MHz}$ respectively, except for the N3992 and N4111 fields which had 110 channels across a $5 \mathrm{MHz}$ bandpass. All datacubes were smoothed to lower angular resolutions of $30^{\prime \prime} \times 30^{\prime \prime}$ and $60^{\prime \prime} \times 60^{\prime \prime}$. This facilitates the detection of extended low level HI emission and the identification of the "continuum" channels which are free from line emission. 
Table 1. All galaxies in the Ursa Major cluster brighter than $M^{b, i}(B)=-16.8$ and more inclined than 45 degrees

\begin{tabular}{|c|c|c|c|c|c|c|c|c|c|c|c|c|c|}
\hline \multirow{3}{*}{$\begin{array}{l}\text { Name } \\
(1)\end{array}$} & RA & Dec & \multicolumn{2}{|c|}{ Galactic } & \multirow{3}{*}{$\begin{array}{l}\text { Type } \\
(6)\end{array}$} & \multirow{3}{*}{$\begin{array}{c}D_{25}(\mathrm{~B}) \\
\left({ }^{\prime}\right) \\
(7)\end{array}$} & \multirow{3}{*}{$\begin{array}{l}\text { PA } \\
\left({ }^{\circ}\right) \\
(8)\end{array}$} & \multirow{3}{*}{$\begin{array}{c}1-b / a \\
(9)\end{array}$} & \multirow{3}{*}{$\begin{array}{c}i_{\mathrm{opt}} \\
\left({ }^{\circ}\right) \\
(10)\end{array}$} & \multirow{3}{*}{$\begin{array}{c}i_{\text {adopt }} \\
\left({ }^{\circ}\right) \\
(11)\end{array}$} & \multirow{3}{*}{$\begin{array}{l}\text { S.B. } \\
(12)\end{array}$} & \multirow{3}{*}{$\begin{array}{l}{[\mathrm{BH}]} \\
\mathrm{mag} \\
(13)\end{array}$} & \multirow{3}{*}{$\begin{array}{c}A_{B}^{\mathrm{b}}[\mathrm{SFD}] \\
\mathrm{mag} \\
(14)\end{array}$} \\
\hline & $(19$ & & Long. & Lat. & & & & & & & & & \\
\hline & $(2)$ & $(3)$ & (4) & (5) & & & & & & & & & \\
\hline \multicolumn{14}{|c|}{ Galaxies with fully analyzed HI data: } \\
\hline U6399 & 112035.9 & 511009 & 152.08 & 60.96 & $\mathrm{Sm}$ & 2.40 & 140 & 0.72 & 79 & $75 \pm 2$ & LSB & 0.00 & 0.07 \\
\hline U6446 & 112352.9 & 540121 & 147.56 & 59.14 & $\mathrm{Sd}$ & 2.27 & 200 & 0.38 & 54 & $51 \pm 3$ & LSB & 0.00 & 0.07 \\
\hline N3726 & 113038.7 & 471820 & 155.38 & 64.88 & $\mathrm{SBc}$ & 5.83 & 194 & 0.38 & 54 & $53 \pm 2$ & HSB & 0.01 & 0.07 \\
\hline N3769 & 113502.8 & 481010 & 152.72 & 64.75 & $\mathrm{SBb}$ & 2.97 & 150 & 0.69 & 76 & $70 \pm 2$ & HSB & 0.01 & 0.10 \\
\hline U6667 & 113945.3 & 515232 & 146.27 & 62.29 & Scd & 3.43 & 88 & 0.88 & 90 & $89 \pm 1$ & LSB & 0.00 & 0.07 \\
\hline N3877 & 114329.3 & 474621 & 150.72 & 65.96 & $\mathrm{Sc}$ & 5.40 & 36 & 0.78 & 84 & $76 \pm 1$ & HSB & 0.01 & 0.10 \\
\hline N3893 & 114600.2 & 485920 & 148.15 & 65.23 & $\mathrm{Sc}$ & 3.93 & 352 & 0.33 & 49 & $49 \pm 2$ & HSB & 0.02 & 0.09 \\
\hline N3917 & 114807.7 & 520609 & 143.65 & 62.79 & Scd & 4.67 & 257 & 0.76 & 82 & $79 \pm 2$ & LSB & 0.01 & 0.09 \\
\hline N3949 & 115105.5 & 480814 & 147.63 & 66.40 & $\mathrm{Sbc}$ & 2.90 & 297 & 0.38 & 54 & $55 \pm 2$ & HSB & 0.03 & 0.09 \\
\hline N3953 & 115112.4 & 523618 & 142.21 & 62.59 & $\mathrm{SBbc}$ & 6.10 & 13 & 0.50 & 62 & $62 \pm 1$ & HSB & 0.01 & 0.13 \\
\hline N3972 & 115309.0 & 553556 & 138.85 & 60.06 & Sbc & 3.43 & 298 & 0.72 & 79 & $77 \pm 1$ & HSB & 0.00 & 0.06 \\
\hline U6917 & 115353.1 & 504227 & 143.46 & 64.45 & SBd & 3.17 & 123 & 0.46 & 59 & $56 \pm 2$ & LSB & 0.03 & 0.12 \\
\hline U6923 & 115414.4 & 532619 & 140.51 & 62.06 & Sdm & 1.97 & 354 & 0.58 & 68 & $65 \pm 2$ & LSB & 0.00 & 0.12 \\
\hline $\mathrm{U} 6930^{i}$ & 115442.3 & 493341 & 144.54 & 65.51 & SBd & 3.00 & 39 & 0.14 & 32 & $31 \pm 3$ & LSB & 0.05 & 0.13 \\
\hline N3992 & 115500.9 & 533911 & 140.09 & 61.92 & $\mathrm{SBbc}$ & 6.93 & 248 & 0.44 & 58 & $56 \pm 2$ & HSB & 0.01 & 0.13 \\
\hline $\mathrm{U} 6940^{f}$ & 115512.4 & 533046 & 140.17 & 62.06 & Scd & 0.83 & 135 & 0.72 & 79 & $75 \pm 3$ & LSB & 0.00 & 0.12 \\
\hline $\mathrm{U} 6962^{i}$ & 115559.5 & 430044 & 154.08 & 71.05 & SBcd & 2.33 & 179 & 0.20 & 38 & $37 \pm 3$ & HSB & 0.00 & 0.09 \\
\hline N4010 & 115602.0 & 473216 & 146.68 & 67.36 & SBd & 4.63 & 65 & 0.88 & 90 & $89 \pm 1$ & LSB & 0.00 & 0.11 \\
\hline U6969 & 115612.9 & 534211 & 139.70 & 61.96 & $\mathrm{Sm}$ & 1.50 & 330 & 0.69 & 76 & $76 \pm 2$ & LSB & 0.01 & 0.13 \\
\hline U6973 & 115617.8 & 430003 & 153.97 & 71.10 & Sab & 2.67 & 40 & 0.61 & 70 & $71 \pm 3$ & HSB & 0.00 & 0.09 \\
\hline U6983 & 115634.9 & 525908 & 140.27 & 62.62 & SBcd & 3.20 & 270 & 0.34 & 50 & $49 \pm 1$ & LSB & 0.01 & 0.12 \\
\hline N4051 & 120036.4 & 444836 & 148.88 & 70.08 & $\mathrm{SBbc}$ & 5.90 & 311 & 0.34 & 50 & $49 \pm 3$ & HSB & 0.00 & 0.06 \\
\hline N4085 & 120250.4 & 503754 & 140.59 & 65.17 & $\mathrm{Sc}$ & 2.80 & 255 & 0.76 & 82 & $82 \pm 2$ & HSB & 0.01 & 0.08 \\
\hline N4088 & 120302.0 & 504903 & 140.33 & 65.01 & Sbc & 5.37 & 231 & 0.63 & 71 & $69 \pm 2$ & HSB & 0.01 & 0.09 \\
\hline N4100 & 120336.4 & 495141 & 141.11 & 65.92 & Sbc & 5.23 & 344 & 0.71 & 77 & $73 \pm 2$ & HSB & 0.03 & 0.10 \\
\hline N4102 & 120351.3 & 525922 & 138.08 & 63.07 & SBab & 3.00 & 38 & 0.44 & 58 & $56 \pm 2$ & HSB & 0.01 & 0.09 \\
\hline N4157 & 120834.2 & 504547 & 138.47 & 65.41 & $\mathrm{Sb}$ & 6.73 & 63 & 0.83 & 90 & $82 \pm 3$ & HSB & 0.02 & 0.09 \\
\hline N4183 & 121046.5 & 435833 & 145.39 & 71.73 & Scd & 4.77 & 346 & 0.86 & 90 & $82 \pm 2$ & LSB & 0.00 & 0.06 \\
\hline N4217 & 121321.6 & 472211 & 139.90 & 68.85 & $\mathrm{Sb}$ & 5.67 & 230 & 0.74 & 80 & $86 \pm 2$ & HSB & 0.00 & 0.08 \\
\hline N4389 & 122308.8 & 455741 & 136.73 & 70.74 & SBbc & 2.50 & 276 & 0.34 & 50 & $50 \pm 4$ & HSB & 0.00 & 0.06 \\
\hline \multicolumn{14}{|c|}{ Galaxies with partially analyzed HI data: } \\
\hline N3718 & 112949.9 & 532039 & 147.01 & 60.22 & $\mathrm{Sa}$ & 7.53 & 195 & 0.58 & 68 & $69 \pm 3$ & HSB & 0.00 & 0.06 \\
\hline N3729 & 113104.9 & 532408 & 146.64 & 60.28 & SBab & 2.80 & 164 & 0.32 & 48 & $49 \pm 3$ & HSB & 0.00 & 0.05 \\
\hline U6773 & 114522.1 & 500512 & 146.89 & 64.27 & $\mathrm{Sm}$ & 1.53 & 341 & 0.47 & 60 & $58 \pm 3$ & LSB & 0.00 & 0.07 \\
\hline U6818 & 114810.1 & 460509 & 151.76 & 67.78 & $\mathrm{Sd}$ & 2.20 & 77 & 0.72 & 79 & $75 \pm 3$ & LSB & 0.00 & 0.09 \\
\hline U6894 & 115247.3 & 545608 & 139.52 & 60.63 & Scd & 1.67 & 269 & 0.84 & 90 & $83 \pm 3$ & LSB & 0.00 & 0.06 \\
\hline N3985 & 115406.4 & 483648 & 145.94 & 66.27 & $\mathrm{Sm}$ & 1.40 & 70 & 0.37 & 53 & $51 \pm 3$ & HSB & 0.05 & 0.11 \\
\hline N4013 & 115556.8 & 441331 & 151.86 & 70.09 & $\mathrm{Sb}$ & 4.87 & 245 & 0.76 & 88 & $90 \pm 1$ & HSB & 0.00 & 0.07 \\
\hline U7089 & 120325.4 & 432518 & 149.90 & 71.52 & Sdm & 3.50 & 215 & 0.81 & 90 & $80 \pm 3$ & LSB & 0.00 & 0.07 \\
\hline U7094 & 120338.5 & 431405 & 150.14 & 71.70 & Sdm & 1.60 & 39 & 0.64 & 72 & $70 \pm 3$ & LSB & 0.00 & 0.06 \\
\hline N4117 & 120514.2 & 432417 & 149.07 & 71.72 & S0 & 1.53 & 21 & 0.56 & 67 & $68 \pm 3$ & LSB & 0.00 & 0.06 \\
\hline 138 & 120658.6 & 435749 & 147.29 & 71.40 & $\mathrm{Sa}$ & 2.43 & 151 & 0.37 & 53 & $53 \pm 3$ & HSB & 0.00 & 0.06 \\
\hline 218 & 121317.4 & 482436 & 138.88 & 67.88 & $\mathrm{Sm}$ & 1.17 & 316 & 0.40 & 55 & $53 \pm 3$ & HSB & 0.00 & 0.07 \\
\hline N4220 & 121342.8 & 480941 & 138.94 & 68.13 & $\mathrm{Sa}$ & 3.63 & 140 & 0.69 & 76 & $78 \pm 3$ & HSB & 0.00 & 0.08 \\
\hline Galaxies & $h$ confused & II data: & & & & & & & & & & & \\
\hline $1135+48$ & 113509.2 & 480931 & 152.71 & 64.77 & $\mathrm{Sm}$ & 1.23 & 114 & 0.69 & 76 & $73 \pm 3$ & LSB & 0.01 & 0.10 \\
\hline N3896 & 114618.6 & 485710 & 148.10 & 65.29 & $\mathrm{Sm}$ & 1.60 & 308 & 0.33 & 49 & $48 \pm 3$ & LSB & 0.02 & 0.09 \\
\hline Not obser & ed or too litt & le HI cont & ent: & & & & & & & & & & \\
\hline N3870 & 114317.5 & 502840 & 147.02 & 63.75 & S0a & 1.13 & 17 & 0.31 & 47 & $48 \pm 3$ & HSB & 0.00 & 0.07 \\
\hline N3990 & 115500.3 & 554413 & 138.25 & 60.04 & S0 & 1.47 & 40 & 0.50 & 62 & $63 \pm 3$ & HSB & 0.00 & 0.07 \\
\hline N4026 & 115650.7 & 511424 & 141.94 & 64.20 & S0 & 4.37 & 177 & 0.74 & 80 & $84 \pm 3$ & HSB & 0.04 & 0.10 \\
\hline N4111 & 120431.0 & 432040 & 149.53 & 71.69 & S0 & 4.47 & 150 & 0.78 & 84 & $90 \pm 3$ & HSB & 0.00 & 0.06 \\
\hline U7129 & 120623.6 & 420108 & 151.00 & 72.99 & $\mathrm{Sa}$ & 1.27 & 72 & 0.31 & 47 & $48 \pm 3$ & HSB & 0.00 & 0.06 \\
\hline N4143 & 120704.6 & 424844 & 149.18 & 72.40 & So & 2.60 & 143 & 0.46 & 59 & $60 \pm 3$ & HSB & 0.00 & 0.06 \\
\hline N4346 & 122101.2 & 471615 & 136.57 & 69.39 & So & 3.47 & 98 & 0.67 & 75 & $77 \pm 3$ & HSB & 0.00 & 0.06 \\
\hline
\end{tabular}


Table 2. Photometrics of all galaxies in the UMa cluster brighter than $M^{b, i}(B)=-16.8$ and more inclined than 45 degrees

\begin{tabular}{lrrrrrrrrrrrrrr}
\hline Name & $m_{B}^{\text {tot }}$ & $m_{R}^{\text {tot }}$ & $m_{I}^{\text {tot }}$ & $m_{K^{\prime}}^{\text {tot }}$ & $W_{R, I}^{i}$ & $A_{B}^{i}$ & $A_{R}^{i}$ & $A_{I}^{i}$ & $A_{K^{\prime}}^{i}$ & $M_{B}^{b, i}$ & $M_{R}^{b, i}$ & $M_{I}^{b, i}$ & $M_{K^{\prime}}^{b, i}$ & $D_{25}^{b, i}$ \\
& $\mathrm{mag}$ & $\mathrm{mag}$ & $\mathrm{mag}$ & $\mathrm{mag}$ & $\mathrm{km} \mathrm{s}^{-1}$ & $\operatorname{mag}$ & $\mathrm{mag}$ & $\mathrm{mag}$ & $\mathrm{mag}$ & $\mathrm{mag}$ & $\mathrm{mag}$ & $\mathrm{mag}$ & $\mathrm{mag}$ & $\left({ }^{\prime}\right)$ \\
$(1)$ & $(2)$ & $(3)$ & $(4)$ & $(5)$ & $(6)$ & $(7)$ & $(8)$ & $(9)$ & $(10)$ & $(11)$ & $(12)$ & $(13)$ & $(14)$ & $(15)$ \\
\hline \hline
\end{tabular}

Galaxies with fully analyzed HI data:

\begin{tabular}{|c|c|c|c|c|c|c|c|c|c|c|c|c|c|c|}
\hline \\
\hline 6399 & .33 & 13.31 & 2.88 & 11.09 & 172 & 0.47 & 0.36 & 0.27 & 0.06 & -17.56 & -18.44 & -18.77 & -20.33 & 1.84 \\
\hline U6446 & 13.52 & 12.81 & 12.58 & 11.50 & 174 & 0.18 & 0.14 & 0.10 & 0.02 & -18.08 & -18.72 & -18.90 & -19.88 & 2.07 \\
\hline N3726 & 11.00 & 9.97 & 9.51 & 7.96 & 331 & 0.34 & 0.25 & 0.20 & 0.05 & -20.76 & -21.67 & -22.07 & -23.45 & .32 \\
\hline N3769 & 12.80 & 11.56 & 10.99 & 9.10 & 256 & 0.67 & 0.50 & 0.39 & 0.09 & -19.32 & -20.35 & -20.80 & -22.35 & 2.34 \\
\hline U6667 & 14.33 & 13.11 & 12.63 & 10.81 & 167 & 0.74 & 0.58 & 0.43 & 0.10 & -17.83 & -18.87 & -19.18 & -20.65 & .18 \\
\hline N3877 & 11.91 & 10.46 & 9.72 & 7.75 & 335 & 1.06 & 0.78 & 0.62 & 0.15 & -20.60 & -21.73 & -22.29 & -23.76 & 3.95 \\
\hline N3893 & 11.20 & 10.19 & 9.71 & 7.84 & 382 & 0.31 & 0.23 & 0.18 & 0.04 & -20.55 & -21.45 & -21.86 & -23.56 & 3.67 \\
\hline N3917 & 12.66 & 11.42 & 10.85 & 9.08 & 276 & 0.87 & 0.64 & 0.51 & 0.12 & -19.65 & -20.63 & -21.05 & -22.40 & 3.48 \\
\hline N3949 & 11.55 & 10.69 & 10.28 & 8.43 & 321 & 0.33 & 0.24 & 0.20 & 0.05 & -20.22 & -20.96 & -21.31 & -22.98 & 2.66 \\
\hline N3953 & 11.03 & 9.66 & 9.02 & 7.03 & 446 & 0.60 & 0.43 & 0.35 & 0.08 & -21.05 & -22.20 & -22.74 & -24.41 & 5.38 \\
\hline N3972 & 13.09 & 11.90 & 11.34 & 9.39 & 264 & 0.76 & 0.56 & 0.44 & 0.11 & -19.08 & -20.05 & -20.48 & -22.08 & 2.62 \\
\hline U6917 & 13.15 & 12.16 & 11.74 & 10.30 & 224 & 0.31 & 0.23 & 0.18 & 0.04 & -18.63 & -19.49 & -19.84 & -21.10 & 2.84 \\
\hline U6923 & 13.91 & 12.97 & 12.36 & 11.04 & 160 & 0.28 & 0.22 & 0.16 & 0.04 & -17.84 & -18.67 & -19.20 & -20.36 & 1.67 \\
\hline $\mathrm{U} 6930^{i}$ & 12.70 & 11.71 & 11.39 & 10.33 & 231 & 0.08 & 0.06 & 0.05 & 0.01 & -18.86 & -19.78 & -20.07 & -21.04 & 2.98 \\
\hline N3992 & 10.86 & 9.55 & 8.94 & 7.23 & 547 & 0.56 & 0.40 & 0.33 & 0.08 & -21.18 & -22.28 & -22.80 & -24.21 & 6.27 \\
\hline $\mathrm{U} 6940^{f}$ & 16.45 & 15.65 & 15.44 & 13.99 & 50 & 0.00 & 0.00 & 0.00 & 0.00 & -15.02 & -15.77 & -15.96 & -17.37 & 0.64 \\
\hline $\mathrm{U} 6962^{i}$ & 12.88 & 11.88 & 11.42 & 10.11 & 327 & 0.16 & 0.11 & 0.09 & 0.02 & -18.72 & -19.64 & -20.06 & -21.27 & 2.26 \\
\hline N4010 & 13.36 & 12.14 & 11.55 & 9.22 & 254 & 1.20 & 0.89 & 0.70 & 0.17 & -19.30 & -20.17 & -20.55 & -22.31 & 2.97 \\
\hline U6969 & 15.12 & 14.32 & 14.04 & 12.58 & 117 & 0.20 & 0.17 & 0.11 & 0.02 & -16.56 & -17.28 & -17.48 & -18.80 & 1.19 \\
\hline U6973 & 12.94 & 11.26 & 10.53 & 8.23 & 364 & 0.71 & 0.52 & 0.42 & 0.10 & -19.21 & -20.67 & -21.28 & -23.23 & 2.21 \\
\hline U6983 & 13.10 & 12.27 & 11.91 & 10.52 & 221 & 0.21 & 0.16 & 0.12 & 0.03 & -18.58 & 9.31 & -19.61 & -20.87 & 2.99 \\
\hline N4051 & 10.98 & 9.88 & 9.37 & 7.86 & 308 & 0.28 & 0.21 & 0.17 & 0.04 & -20.71 & .72 & -22.18 & -23.54 & 5.45 \\
\hline N4085 & 13.09 & 11.87 & 11.28 & 9.20 & 247 & 0.78 & 0.58 & 0.46 & 0.11 & -19.12 & -20.11 & -20.57 & -22.27 & 2.08 \\
\hline N4088 & 11.23 & 10.00 & 9.37 & 7.46 & 362 & 0.74 & 0.54 & 0.43 & 0.10 & & .94 & -22.45 & -24.00 & 4.40 \\
\hline N4100 & 11.91 & 10.62 & 10.00 & 8.02 & 386 & 0.97 & 0.70 & 0.57 & 0.14 & & & -21.97 & -23.48 & 4.07 \\
\hline N4102 & 12.04 & 10.54 & 9.93 & 7.86 & 393 & 0.46 & 0.34 & 0.27 & 0.07 & & & -2 & -23.57 & 2.69 \\
\hline 157 & 12.12 & 10.60 & 9.88 & 7.52 & 399 & 1.40 & 1.02 & 0.82 & 0.20 & & & -2 & -24.04 & 4.64 \\
\hline 83 & & 11 & 11.51 & 9.76 & 228 & 1.01 & 0.76 & 0.59 & 14 & & & & 74 & .13 \\
\hline 217 & 1 & & 9.84 & 7.61 & 381 & 1. & 0.76 & 62 & 5 & & & & 90 & 29 \\
\hline N4389 & 12.56 & 11.33 & 10.87 & 9.12 & 212 & 0.20 & 0.15 & 0.12 & 0.03 & & & 63 & -22.27 & 2.31 \\
\hline \multicolumn{15}{|c|}{ Galaxies with partially analyzed HI data: } \\
\hline 718 & 28 & 95 & 9.29 & 7.47 & 476 & 0.77 & 0.55 & .45 & .11 & 90 & & -2 & -24.00 & 6.30 \\
\hline 729 & & 10.94 & 10.30 & 8.60 & 296 & 0.25 & 0.18 & 0.15 & .03 & & & 22 & -22.78 & 2.60 \\
\hline 73 & & 13.61 & 13.15 & 11.23 & 112 & 0.09 & 0.08 & 0.05 & 0.01 & 09 & & 28 & -20.14 & 1.35 \\
\hline & & $\$ 2$ & 13.15 & 70 & 151 & 0.39 & 0.31 & 0.22 & 0.05 & & & 46 & -19.71 & 1.69 \\
\hline & & & 1 & 40 & 124 & 0.37 & 0.31 & 0.21 & 55 & & & & 01 & 1.13 \\
\hline & & 26 & 11.81 & 10.19 & 180 & 0.18 & 0.14 & 0.10 & 0.02 & -1 & 30 & -1 & -2 & 1.29 \\
\hline 013 & 44 & 10.79 & 9.95 & 7.6 & 377 & 1.10 & 0.80 & 0.64 & 0.15 & -2 & 40 & -2 & -23.83 & 3.61 \\
\hline 89 & 73 & 12.77 & 12.36 & 11.11 & 138 & 0.42 & 0.34 & 0.24 & 0.05 & -1 & .96 & -19.26 & -20.30 & 2.46 \\
\hline 994 & .74 & 13.70 & 13.22 & 11.58 & 76 & 0.00 & 0.00 & 0.00 & 0.00 & -16.67 & -17.69 & -18.16 & -19.78 & 1.29 \\
\hline 117 & 14.05 & 12.47 & 11.81 & 9.98 & 285 & 0.00 & 0.00 & 0.00 & 0.00 & -17.36 & -18.92 & -19.57 & -21.38 & 1.29 \\
\hline 38 & 12.27 & 10.72 & 10.09 & 8.1 & 374 & 0.36 & 0.26 & 0.21 & 0.05 & -19.50 & -20.93 & -21.50 & -23.22 & 2.22 \\
\hline 18 & 3.69 & 12.83 & 12.41 & 10.8 & 50 & 0.15 & 0.12 & 0.09 & 12 & -1 & 68 & -19.06 & -20.55 & 1.06 \\
\hline N4220 & 12.34 & 10.79 & 10.03 & 8.36 & 399 & 0.94 & 0.68 & 0.55 & 0.13 & .03 & & & -23.13 & 2.85 \\
\hline \multicolumn{15}{|c|}{ Galaxies with confused HI data: } \\
\hline 1 & 05 & 05 & 13.61 & 11.9 & 111 & . & 5 & .09 & 2 & 7 & & & -1 & 0.97 \\
\hline N3896 & 13.75 & 12.96 & 12.47 & 11.35 & 83 & 0.0 & 0.00 & 0.00 & O & & & .92 & -20.01 & 1.49 \\
\hline \multicolumn{15}{|c|}{ Not observed or too little HI content: } \\
\hline & & 12.71 & 12.16 & 10.7 & 127 & 0.0 & 0.00 & .04 & 0.0 & -1 & & -1 & -2 & 1.06 \\
\hline 990 & 53 & 12.08 & 11.36 & 9.5 & & 0.00 & 0.00 & 0.00 & 0.00 & 89 & & -20.02 & -2 & 1.28 \\
\hline 026 & 11.71 & 10.25 & 9.57 & 7.65 & & 0.00 & 0.00 & 0.00 & 0.00 & -19.74 & -21.16 & -21.82 & -23.71 & 3.32 \\
\hline 111 & 11.40 & 9.95 & 9.25 & 7.60 & & 0.0 & 0.00 & 0.00 & 0.00 & -20.01 & -21.44 & -22.13 & -23.76 & 3.24 \\
\hline 129 & 14.13 & 12.80 & 12.19 & & $\cdots$ & 0.0 & 0. & 0.04 & 0. & -17.36 & -18.65 & -19.23 & & 1.19 \\
\hline & 12.06 & & 9.8 & 7.8 & $\cdots$ & $0 .($ & $0 .($ & 0.00 & & -19.35 & -20.83 & -21.54 & -23.50 & 2.30 \\
\hline N4346 & 12.14 & 10.69 & 9.96 & 8.21 & $\cdots$ & 0.00 & 0.00 & 0.00 & 0.00 & -19.27 & -20.70 & -21.42 & -23.15 & 2.75 \\
\hline
\end{tabular}


Table 3. A comparison of the widths and integrated fluxes from the present WSRT survey and from the literature

\begin{tabular}{|c|c|c|c|c|c|c|c|c|c|c|c|}
\hline \multirow[b]{2}{*}{$\begin{array}{c}\text { Name } \\
(1)\end{array}$} & \multicolumn{5}{|c|}{ This study } & \multicolumn{6}{|c|}{ Literature } \\
\hline & $\begin{array}{c}W_{20} \\
--- \\
(2)\end{array}$ & $\begin{array}{c} \pm \\
\mathrm{m} \mathrm{s}^{-1} \\
(3)\end{array}$ & $\begin{array}{c}\text { Res. } \\
--- \\
(4)\end{array}$ & $\begin{array}{l}\int S \mathrm{~d} v \\
-\mathrm{Jy} \mathrm{km} \\
(5)\end{array}$ & $\begin{array}{l} \pm \\
-1 \\
(6)\end{array}$ & $\begin{array}{c}W_{20} \\
--- \\
(7)\end{array}$ & $\begin{array}{c} \pm \\
\mathrm{m} \mathrm{s}^{-1} \\
(8)\end{array}$ & $\begin{array}{c}\text { Res. } \\
--- \\
(9)\end{array}$ & $\begin{array}{l}\int S \mathrm{~d} v \\
-\underset{\mathrm{Jy} \mathrm{km}}{(10)}\end{array}$ & $\begin{array}{c} \pm \\
\mathrm{s}^{-1}- \\
(11)\end{array}$ & $\begin{array}{l}\text { Ref. } \\
(12)\end{array}$ \\
\hline$\overline{~ U 6399}$ & 188.1 & 1.4 & 8.3 & 10.5 & 0.3 & $\overline{c 178}$ & 20 & 22 & 10.1 & 1.9 & 1 \\
\hline U6446 & 154.1 & 1.0 & 5.0 & 40.6 & 0.5 & 162 & 10 & 22 & 45.9 & 4.1 & 1 \\
\hline \multirow[t]{2}{*}{$\mathrm{N} 3718^{(c)}$} & 492.8 & 1.0 & 33.2 & 140.9 & 0.9 & 480 & 10 & 5.5 & 84.9 & 26.4 & 1 \\
\hline & & & & & & $508^{m}$ &.. & 33 & 120 & & $8^{\mathrm{WSRT}}$ \\
\hline N3726 & 286.5 & 1.6 & 5.0 & 89.8 & 0.8 & 290 & 10 & 5.5 & 83.9 & 10.8 & 1 \\
\hline N3729 noSD & 270.8 & 1.5 & 33.2 & 5.5 & 0.3 & $\begin{array}{c}\ldots \\
279^{m}\end{array}$ & $\begin{array}{l}. . \\
. .\end{array}$ & $\begin{array}{r}. . \\
33\end{array}$ & $\begin{array}{r}\ldots \\
25^{?}\end{array}$ & $\begin{array}{l}\cdots \\
\ldots\end{array}$ & $\ddot{8}^{\mathrm{WSRT}}$ \\
\hline $\mathrm{N} 3769^{i}$ & 265.3 & 6.7 & 8.3 & 62.3 & 0.6 & 276 & 20 & 7.4 & 44.1 & 4.2 & 2 \\
\hline U6667 & 187.5 & 1.4 & 5.0 & 11.0 & 0.4 & 210 & 20 & 22 & 11.6 & 2.2 & 1 \\
\hline N3877 & 373.4 & 5.0 & 33.2 & 19.5 & 0.6 & 352 & 10 & 22 & 24.8 & 5.6 & 1 \\
\hline U6773 & 110.4 & 2.3 & 8.3 & 5.6 & 0.4 & 118 & 8 & 22 & 5.6 & 0.7 & 6 \\
\hline $\mathrm{N} 3893^{(c)}$ & 310.9 & 1.0 & 5.0 & 69.9 & 0.5 & 313 & 8 & 22 & 85.3 & 5.1 & 1 \\
\hline N3917 & 294.5 & 1.9 & 8.3 & 24.9 & 0.6 & 284 & 10 & 22 & 21.9 & 4.7 & 1 \\
\hline U6818 & 166.9 & 2.3 & 8.3 & 13.9 & 0.2 & 168 & 15 & 22 & 14.8 & 2.1 & 1 \\
\hline N3949 & 286.5 & 1.4 & 8.3 & 44.8 & 0.4 & 289 & 10 & 22 & 42.7 & 5.4 & 1 \\
\hline $\mathrm{N} 3953^{l}$ & 441.9 & 2.4 & 33.1 & 39.3 & 0.8 & 423 & 10 & 22 & 41.0 & 3.9 & 1 \\
\hline U6894 & 141.8 & 1.1 & 8.3 & 5.8 & 0.2 & 159 & 20 & 7.4 & 5.1 & 1.7 & 2 \\
\hline N3972 & 281.2 & 1.4 & 8.3 & 16.6 & 0.4 & 270 & 15 & 22 & 14.0 & 2.6 & 1 \\
\hline U6917 & 208.9 & 3.2 & 8.3 & 26.2 & 0.3 & 211 & 10 & 22 & 31.5 & 4.1 & 1 \\
\hline N3985 & 160.2 & 3.7 & 8.3 & 15.7 & 0.6 & 168 & & 22 & 14.1 & 0.9 & 5 \\
\hline \multirow[t]{2}{*}{ U6923 } & 166.8 & 2.4 & 10.0 & 10.7 & 0.6 & 175 & 15 & 22 & 8.2 & 2.9 & 1 \\
\hline & & & & & & $189^{m}$ & 15 & 41.4 & 15.6 & & $12^{\mathrm{VLA}}$ \\
\hline U6930 & 136.5 & 0.5 & 8.3 & 42.7 & 0.3 & 145 & 8 & 22 & 38.2 & 3.5 & 1 \\
\hline \multirow[t]{2}{*}{$\mathrm{N} 3992^{l}$} & 478.5 & 1.4 & 10.0 & 74.6 & 1.5 & 480 & 10 & 22 & 81.2 & 5.3 & 1 \\
\hline & & & & & & $507^{m}$ & 15 & 41.4 & 79.9 & & $12^{\mathrm{VLA}}$ \\
\hline \multirow{2}{*}{ U6940 } & 59.3 & 3.8 & 10.0 & 2.1 & 0.3 & 226 & .. & 22 & 7.0 & 1.0 & 3 \\
\hline & & & & & & $121^{m}$ & 15 & 41.4 & 2.7 & & $12^{\mathrm{VLA}}$ \\
\hline N4013 & 425.0 & 0.9 & 33.0 & 41.5 & 0.2 & 403 & 10 & 22 & 33.8 & 3.7 & 1 \\
\hline \multirow[t]{2}{*}{$\mathrm{U} 6962^{(c)}$} & 220.3 & 6.6 & 8.3 & 10.0 & 0.3 & $\ldots$ & .. & 22 & 21.6 & 4.4 & 1 \\
\hline & & & & & & 235 & & 33 & 9.2 & 1.0 & $4^{\mathrm{WSRT}}$ \\
\hline N4010 & 277.7 & 1.0 & 8.3 & 38.2 & 0.3 & 281 & 10 & 22 & 38.1 & 3.4 & 1 \\
\hline \multirow[t]{2}{*}{$\mathrm{U} 6969^{c}$} & 132.1 & 6.4 & 10.0 & 6.1 & 0.5 & 146 &.. & 13.2 & 6.0 & 1.4 & 3 \\
\hline & & & & & & $159^{m}$ & 15 & 41.4 & 6.9 & $\ldots$ & $12^{\mathrm{VLA}}$ \\
\hline \multirow[t]{2}{*}{$\mathrm{U} 6973^{\text {noSD }}$} & 367.8 & 1.8 & 8.3 & 22.9 & 0.2 & $\ldots$ & .. & .. & $\ldots$ & $\ldots$ &.. \\
\hline & & & & & & 408 & & 33 & 18.3 & 1.2 & $4^{\mathrm{WSRT}}$ \\
\hline U6983 & 188.4 & 1.3 & 5.0 & 38.5 & 0.6 & 205 & 10 & 22 & 36.2 & 4.4 & 1 \\
\hline $\mathrm{N} 4051^{l}$ & 255.4 & 1.8 & 5.0 & 35.6 & 0.8 & 274 & 15 & 22 & 43.4 & 3.3 & 1 \\
\hline \multirow[t]{2}{*}{$\mathrm{N} 4085^{c}$} & 277.4 & 6.6 & 19.8 & 14.6 & 0.9 & 299 & 20 & 7.4 & 23.3 & 2.5 & 2 \\
\hline & & & & & & $311^{m}$ & .. & 33 & $24 !$ & $\ldots$ & $13^{\mathrm{WSRT}}$ \\
\hline \multirow[t]{2}{*}{$\mathrm{N} 4088^{(c)}$} & 371.4 & 1.7 & 19.8 & 102.9 & 1.1 & 381 & 8 & 22 & 109.2 & 6.4 & 1 \\
\hline & & & & & & $378^{m}$ & .. & 33 & $128^{!}$ & $\ldots$ & $13^{\mathrm{WSRT}}$ \\
\hline \multirow[t]{2}{*}{$\mathrm{U} 7089^{(c)}$} & 156.7 & 1.7 & 10.0 & 17.0 & 0.6 & 162 & 10 & 22 & 17.8 & 2.2 & 1 \\
\hline & & & & & & 176 & 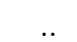 & 33.4 & 18.9 & & $11^{\mathrm{WSRT}}$ \\
\hline N4100 & 401.8 & 2.0 & 19.9 & 41.6 & 0.7 & 420 & 20 & 22 & 54.0 & 7.3 & 1 \\
\hline $\mathrm{U} 7094^{c}$ & 83.7 & 1.7 & 10.0 & 2.9 & 0.2 & 112 & 8 & 22 & 6.0 & 0.6 & 6 \\
\hline & & & & & & $153^{?}$ & & 33.4 & 2.5 & & $11^{\mathrm{WSRT}}$ \\
\hline N4102 & 349.8 & 2.0 & 8.3 & 8.0 & 0.2 & 327 & 20 & 7.4 & 10.3 & 2.1 & 2 \\
\hline $\mathrm{N} 4117^{\mathrm{noSD}}$ & 289.4 & 7.5 & 10.0 & 6.9 & 1.1 & $\ldots$ & .. &.. & $\ldots$ & $\ldots$ & .. \\
\hline & & & & & & 314 & & 33.4 & 5.3 & $\ldots$ & $11^{\mathrm{WSRT}}$ \\
\hline N4138 & 331.6 & 4.5 & 19.9 & 19.2 & 0.7 & $354^{m}$ & 30 & 6.8 & 16 & $\ldots$ & 14 \\
\hline & & & & & & 340 & 5 & 5.2 & 20.6 & 0.3 & $7^{\mathrm{VLA}}$ \\
\hline $\mathrm{N} 4157^{(c), l}$ & 427.6 & 2.2 & 19.9 & 107.4 & 1.6 & 436 & 10 & 22 & 123.9 & 9.5 & 1 \\
\hline N4183 & 249.6 & 1.2 & 8.3 & 48.9 & 0.7 & 258 & 10 & 22 & 49.6 & 5.3 & 1 \\
\hline N4218 & 138.0 & 5.0 & 8.3 & 7.8 & 0.2 & 160 & 20 & 13 & 5.7 & 0.9 & 9 \\
\hline N4217 & 428.1 & 5.1 & 33.2 & 33.8 & 0.7 & 426 & 20 & 22 & 51.8 & 7.2 & 1 \\
\hline N4220 & 438.1 & 1.3 & 33.1 & 4.4 & 0.3 & $382^{m}$ & .. & 11 & 3.3 & $\ldots$ & 10 \\
\hline N4389 & 184.0 & 1.5 & 8.3 & 7.6 & 0.2 & 174 & 20 & 7.4 & 7.6 & 0.8 & 2 \\
\hline
\end{tabular}


Table 3. continued. Notes

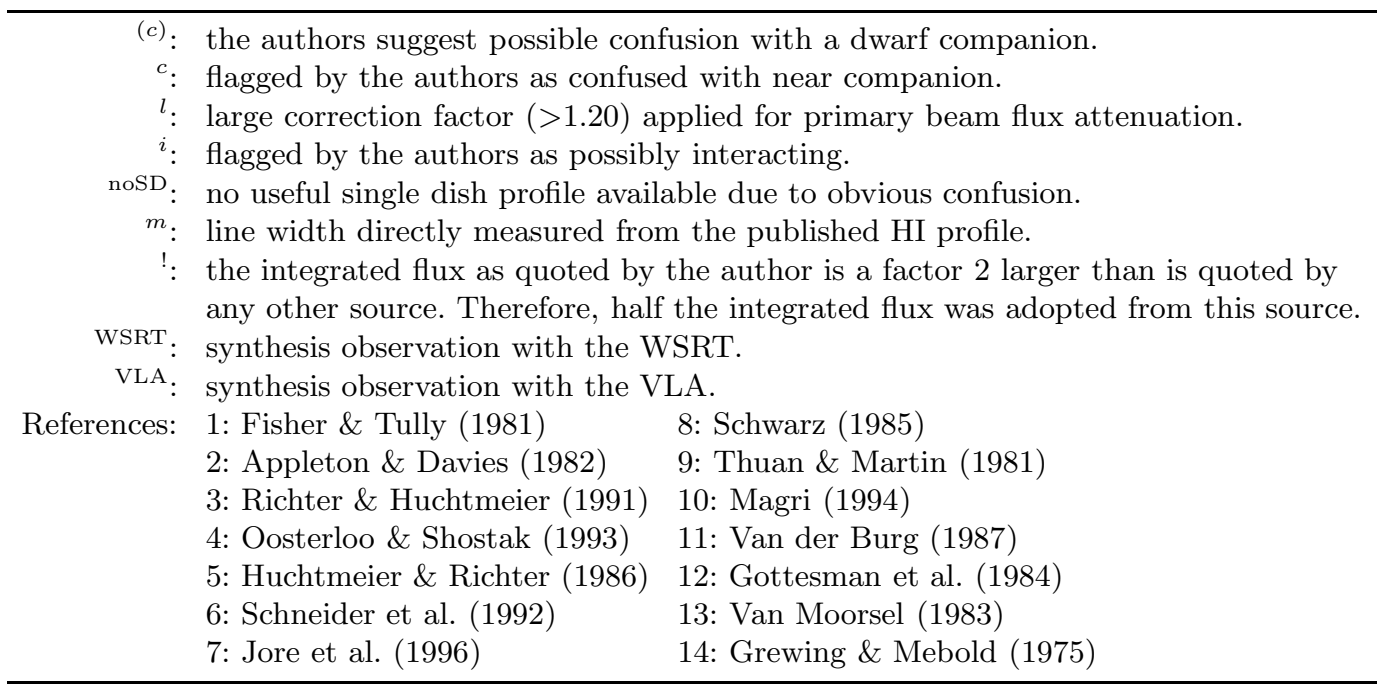

\subsection{The radio continuum emission}

The channels free from HI emission were averaged and the resulting continuum map was subtracted from all channels in the data cube. The residuals of the frequency dependent grating rings were only a minor fraction of the noise in the channels containing the line emission. The dirty continuum maps were cleaned (Högbom 1974) down to $0.3 \sigma$. The clean-components were restored with a Gaussian beam of similar $F W H M$ as the synthesized beam. When radio continuum emission from a galaxy was detected, its continuum flux was determined from the cleaned continuum map. In cases of no detection, an upper limit for extended emission was derived by calculating the rms scatter in the flux values obtained by integrating the noise in each of fifteen elliptical areas enclosed by the 25th mag blue isophote and positioned at various emission-free regions in the map.

\subsection{The HI channel maps and the global HI profiles}

At all three spatial resolutions, the regions of $\mathrm{HI}$ emission were defined by the areas enclosed by the $2 \sigma$ contours in the "dirty" 60 " resolution maps. Grating rings and noise peaks above this level were removed manually. The selected regions were enlarged by moving their boundaries 1 armin outwards to account for possible emission in the sidelobes. The resulting masks vary from channel to channel in shape, size and position due to the rotation of the HI disk. These masks defined the regions that were cleaned down to $0.3 \sigma$ at all three spatial resolutions.

The clean-components were restored with a Gaussian beam of similar $F W H M$ as the synthesized beam. The global HI profiles were derived by determining the primary beam corrected flux in each cleaned region. Since the size and shape of the clean masks vary as a function of velocity, the uncertainty in the flux densities at each velocity in the global HI profile varies as well. The noise on the global HI profile was determined by projecting each clean mask at nine different line-free positions in a channel map and integrating over each of them.

For further analysis, each profile was divided up in three equal velocity bins in which the peak fluxes $F_{\text {low }}^{\text {peak }}$, $F_{\text {mid }}^{\text {peak }}$ and $F_{\text {high }}^{\text {peak }}$ were determined for the low, middle and high velocity bin respectively. These three peak fluxes were then used to classify a global profile shape according to:

Double peaked: $\quad F_{\text {low }}^{\text {peak }}>F_{\text {mid }}^{\text {peak }}<F_{\text {high }}^{\text {peak }}$

Gaussian: $\quad F_{\text {low }}^{\text {peak }}<F_{\text {mid }}^{\text {peak }}>F_{\text {high }}^{\text {peak }}$

Distorted: $\quad F_{\text {low }}^{\text {peak }}<F_{\text {mid }}^{\text {peak }}<F_{\text {high }}^{\text {peak }}$

or $F_{\text {low }}^{\text {peak }}>F_{\text {mid }}^{\text {peak }}>F_{\text {high }}^{\text {peak }}$

Boxy: $\quad F_{\text {low }}^{\text {peak }} \approx F_{\text {mid }}^{\text {peak }} \approx F_{\text {high }}^{\text {peak }}$.

In case of a double peaked profile, the peak fluxes on both sides were considered separately when calculating the $20 \%$ and $50 \%$ levels. In all other cases, the overal peak flux was used. The four velocities $V_{\text {low }}^{20 \%}, V_{\text {low }}^{50 \%}, V_{\text {high }}^{50 \%}$ and $V_{\text {high }}^{20 \%}$ corresponding to these $20 \%$ and $50 \%$ levels were determined by linear interpolation between the data points, going from the center outward. In the few cases of nonmonotonically decreasing edges, this procedure tends to slightly underestimate the widths. The widths are calculated according to

$W_{20}=V_{\text {high }}^{20 \%}-V_{\text {low }}^{20 \%}$ and $W_{50}=V_{\text {high }}^{50 \%}-V_{\text {low }}^{50 \%}$.

The systemic velocity is calculated according to

$V_{\text {sys }}=0.25\left(V_{\text {low }}^{20 \%}+V_{\text {low }}^{50 \%}+V_{\text {high }}^{50 \%}+V_{\text {high }}^{20 \%}\right)$.

Because in interferometric measurements some flux may be lost due to the missing short baselines, it is useful to compare the widths and flux densities from the WSRT profiles with those from published single dish observations. However, a meaningful comparison requires that the 
profile widths are all corrected in the same way for instrumental broadening. In general, the widths that are published by various authors were corrected for instrumental broadening using nearly as many different methods. Therefore, the published line widths had to be decorrected first to ensure a uniformly applied correction. The de-corrected widths and integrated HI fluxes from the literature are compiled in Cols. (7)-(11) of Table 3 along with the results from this study in Cols. (2)-(6). Column (1) gives the NGC or UGC numbers.

Columns $(2,3)$ and $(7,8)$ give the widths of the global profiles at the $20 \%$ levels and the formal uncertainties.

Columns (4) and (9) give the velocity resolutions of the observations.

Columns $(5,6)$ and $(10,11)$ contain the integrated HI fluxes derived from the global profiles.

Column (12) provides the references to the literature sources.

In case the authors suggest that the single dish profile of a particular galaxy may be confused and synthesis data on that galaxy do exist, these synthesis data are included as well and used in the following comparison. However, first it will be explained how the observed linewidths are corrected for the different instrumental resolutions.

\subsubsection{Correcting $W_{20}$ for instrumental broadening}

The most widely used method to correct for broadening of the global HI profiles due to a finite instrumental velocity resolution was provided by Bottinelli et al. (1990). For the widths at the $20 \%$ and $50 \%$ levels of the peak flux they advocate the following linear relations:

$W_{20, R}=W_{20}-\delta W_{20}=W_{20}-0.55 R$

$W_{50, R}=W_{50}-\delta W_{50}=W_{50}-0.13 R$

where $W_{20}$ is the observed linewidth and $W_{20, R}$ is the linewidth corrected for the instrumental velocity resolution $R$ in $\mathrm{km} \mathrm{s}^{-1}$. This empirical prescription is based on comparing linewidths obtained at different resolutions.

However, the correction method applied here deviates from Bottinelli et al.'s method and is based on more analytic considerations. It is easy to imagine that both edges of an intrinsic global profile, when chopped off at their peaks and glued together, approximate a Gaussian with dispersion $\sigma_{0}$. The width at the $20 \%$ level of this "true" Gaussian is then given by

$W_{20, R}=\sigma_{0} \sqrt{8 \ln (5)}$

A spectral Hanning smoothing was applied to most of the WSRT observations presented in this paper. This smoothing function can also be approximated by a Gaussian with a $F W H M$ equal to the instrumental velocity resolution $R$ and has a dispersion $\sigma_{R}$

$$
\sigma_{R}=\frac{R}{\sqrt{8 \ln (2)}}
$$

The dispersion $\sigma_{\mathrm{c}}$ of the convolved observed Gaussian is then given by

$$
\sigma_{\mathrm{c}}=\sqrt{\sigma_{0}^{2}+\sigma_{R}^{2}}
$$

and the $20 \%$ line width of this convolved or observed Gaussian is given by

$$
\begin{aligned}
W_{20} & =\sigma_{\mathrm{c}} \sqrt{8 \ln (5)} \\
& =\sqrt{8 \ln (5)} \cdot \sqrt{\sigma_{0}^{2}+\sigma_{R}^{2}} \\
& =\sqrt{8 \ln (5)} \cdot \sqrt{\sigma_{0}^{2}+\frac{R^{2}}{8 \ln (2)}} .
\end{aligned}
$$

So, at the $20 \%$ level, the intrinsic width $W_{20, R}$ is broadened to $W_{20}$ by $\delta W$ given by

$$
\begin{aligned}
\delta W_{20} & =W_{20}-W_{20, R} \\
& =\sqrt{8 \ln (5)} \cdot \sqrt{\sigma_{0}^{2}+\frac{R^{2}}{8 \ln (2)}-\sigma_{0} \sqrt{8 \ln (5)}} \\
& =\sigma_{0} \sqrt{8 \ln (5)} \cdot\left[\sqrt{1+\frac{\left(R / \sigma_{0}\right)^{2}}{8 \ln (2)}}-1\right]
\end{aligned}
$$

The broadening $\delta W_{20}$ does not only depend on the instrumental resolution $R$ but also on the steepness of the slopes of the edges of the profile, expressed by $\sigma_{0}$. Fitting Gaussians to the edges of a profile yields $\sigma_{\mathrm{c}}$ from which $\sigma_{0}$ can be calculated given the known value of $\sigma_{R}$. The equation above can be rewritten using $\sigma_{\mathrm{c}}$ instead which results in

$$
\delta W_{20}=\sigma_{\mathrm{c}} \sqrt{8 \ln (2)}\left(\frac{\ln (5)}{\ln (2)}\right)^{2}\left[1-\sqrt{1-\frac{\left(R / \sigma_{\mathrm{c}}\right)^{2}}{8 \ln (2)}}\right] .
$$

However, no Gaussians were fitted to the edges of the new WSRT profiles. Instead it is assumed that the slopes of the edges of the profiles are more or less determined by the turbulent motion of the gas with a canonical velocity dispersion of $\sigma_{0}=10 \mathrm{~km} \mathrm{~s}^{-1}$. This results in

$$
\delta W_{20}=35.8 \cdot\left[\sqrt{1+\left(\frac{R}{23.5}\right)^{2}}-1\right]
$$

and similarly for the $50 \%$ level

$$
\delta W_{50}=23.5 \cdot\left[\sqrt{1+\left(\frac{R}{23.5}\right)^{2}}-1\right] .
$$

The differences between Bottinelli et al.'s linear presciption and our corrections $\left(\Delta \delta W=\delta \mathrm{W}^{B o t}-\delta W^{\text {our }}\right)$ are only minor and tabulated below for typical instrumental resolutions of the WSRT.

\begin{tabular}{r|rrrrr}
\hline level & \multicolumn{5}{|c}{$\Delta \delta W$} \\
\hline & $-----R\left(\mathrm{~km} \mathrm{~s}^{-1}\right)-----$ \\
& 5.0 & 8.3 & 16.5 & 19.9 & 33.1 \\
\hline $20 \%$ & 2.0 & 2.4 & 1.2 & 0.2 & -7.8 \\
$50 \%$ & 0.2 & -0.3 & -3.1 & -4.7 & -12.8 \\
\hline
\end{tabular}



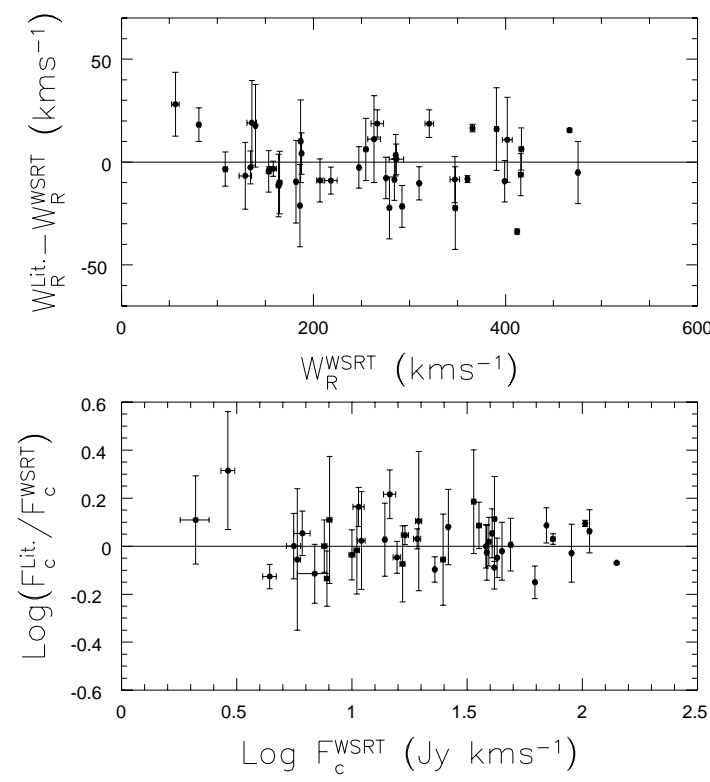

Fig. 1. A comparison of the present WSRT results with preexisting single dish and synthesis data from the literature

The larger differences occur for the poorest resolutions at which only the broadest profiles were observed. Consequently, the differences are a negligible fraction of the line widths.

Figure 1 shows the comparison of the widths and integrated fluxes derived from the new WSRT global profiles and those from the literature. There are no significant systematic differences. The unweighted average difference in widths is $-0.9 \pm 2.1 \mathrm{~km} \mathrm{~s}^{-1}$ with a rms scatter of $14 \mathrm{~km} \mathrm{~s}^{-1}$. The unweighted average difference in integrated flux is $4.7 \pm 3.5$ percent with a rms scatter of 25 percent. It can therefore be concluded that on average the WSRT results are in excellent agreement with the results from single dish observations.

\subsection{The total HI maps}

As a next step, the total integrated HI maps were constructed from the cleaned datacubes. The clean-masks were used to define the regions with $\mathrm{HI}$ emission. Outside these regions, the pixels were set to zero and all the channels containing a non-zero area were added to build up the integrated column density map. This was then corrected for attenuation by the primary beam. Although the advantage of this procedure is a higher signal-to-noise ratio at a certain pixel in the HI map, the disadvantage is that the noise is no longer uniform across the map. As a result, the $3 \sigma$-contour level in an integrated HI map is not defined. Signal-to-noise maps have been made, however, using the prescription outlined in Appendix A and the average pixel value of all pixels with $2.75<\left(\frac{S}{N}\right)<3.25$ was determined. This average value was adopted as the " $3 \sigma$ " level for the column density.

\subsection{The radial HI surface density profiles}

The integrated column density maps were used to derive the radial HI surface density profiles by azimuthally averaging in concentric ellipses. The orientations and widths of the ellipses were the same as those of the projected tilted rings fitted to the $\mathrm{HI}$ velocity field (see Sect. 3.6.1). In the case of a warp with overlapping ellipses, the flux in the overlapping regions was proportionally assigned to each ellipse. The azimuthal averaging was done separately for the receding and approaching halves of each tilted ring to reveal possible asymmetries. Pixels in the HI map without any measured signal were set to zero. Finally, the entire radial profile was scaled by the total HI mass as derived from the global HI profile. No attempt was made to correct the profiles for the effect of beam smearing.

This method for extracting the surface density profiles from integrated HI maps breaks down for nearly edge-on systems; the highly inclined annuli with large major axis diameters could still pick up some flux along the minor axis due to beam smearing. In such cases, Lucy's (1974) iterative deprojection scheme as adapted and developed by Warmels (1988b) might be preferable.

Due to the complex noise structure of the integrated HI map, no attempt was made to estimate the errors on the radial $\mathrm{HI}$ surface density profiles.

\subsection{The $H I$ velocity fields}

The HI data cubes were smoothed to velocity resolution of $\approx 19 \mathrm{~km} \mathrm{~s}^{-1}$ in order to obtain a good spectral signal-tonoise ratio. HI velocity fields were then constructed by fitting single Gaussians to the velocity profiles at each pixel. Initial estimates for the fits were given by the various moments of the profiles determined over the velocity range covered by the masks. Only those fits were accepted for which 1) the central velocity of the fitted Gaussian lies inside the masked volume, 2) the amplitude is larger than five times the rms noise in the profile and 3) the uncertainty in the central velocity is smaller than $\frac{1}{3}$ the velocity resolution.

Due to projection effects and beam smearing, the velocity profiles in highly inclined systems and in the central regions of galaxies may deviate strongly from a Gaussian shape. The exact shape depends on the spatial and kinematic distribution of the gas within a synthesized beam. Fitting single Gaussians to these usually skewed profiles results in an underestimate of the rotational velocity at that position. As a consequence, the gradients in the velocity field become shallower. There are several methods to correct for the effects of beam smearing. In the present cases, however, the signal-to-noise was in general too low to allow a useful application of these methods, and, since only a small number of systems were recognized as seriously affected, the HI velocity fields were not corrected for the effects of beam smearing. 


\subsection{Rotation curves}

The rotation curves were derived in two ways; 1) by fitting tilted-rings to the velocity fields (Begeman 1987) and 2) by estimating the rotational velocities by eye from the position-velocity diagrams.

\subsubsection{Using the velocity fields}

The determination of the rotation curves from the velocity fields was done in three steps by fitting tilted rings to the velocity field (see Begeman 1987, 1989). The widths of the rings were set at $\frac{2}{3}$ of the width of the synthesized beam (i.e. $10^{\prime \prime}, 20^{\prime \prime}$ or $40^{\prime \prime}$ ).

First, the systemic velocity and the dynamical center were determined. In this first step the inclination and position angles were the same for each ring and kept fixed at the values derived from the optical images. The systemic velocity, center and rotational velocity were fitted for each ring. All the points along the tilted ring were considered and weighted uniformly. In general, no significant trend as a function of radius could be detected for the systemic velocity and center. The adopted values were calculated as the average of all rings.

Second, the systemic velocity and center of rotation were kept fixed for each ring while the position angle, inclination and rotational velocity were fitted. All the points along the tilted ring were considered but weighted with $\cos (\theta)$ where $\theta$ is the angle in the plane of the galaxy measured from the receding side. Hence, points along the minor axis have zero weight. While the position angle can be determined accurately, the inclination and rotational velocity are rather strongly correlated for inclinations below 60 degrees and above 80 degrees (Begeman 1989). As a result, the fitted inclinations can vary by a large amount from one ring to another. However, a possible trend in the inclination with radius due to a central bar or a warp can be detected. A change in inclination angle often goes together with a change in the more accurately determined position angle.

Third, the rotational velocity was fitted again for each ring while keeping the systemic velocity, center of rotation, inclination and position angle fixed. Again, all the points along the tilted ring were considered but weighted with $\cos (\theta)$. The fixed values for the inclination and position angles were determined in the second step by averaging the solutions over all the rings or fixing a clear trend. For nearly edge-on galaxies, the inclinations determined in the second step were often overruled by higher values based on the clear presence of a dust lane (e.g. N4010, N4157, $\mathrm{N} 4217$ ) or the very thin distribution of gas in the column density maps (e.g. U6667). However, uncertainty in the inclinations of nearly edge-on systems does not significantly influence the amplitude of the rotational velocity.

The results of this 3-step procedure were used to construct a model velocity field. This model was subtracted from the actual observed velocity field to yield a map of the residual velocities. In some cases (e.g. N3769, N4051,
N4088) this residual map shows significant systematic residuals, indicative of non-circular motion or a bad model fit due to a noisy observed velocity field. As a further check, the derived rotation curve is projected onto the position-velocity maps along the major and minor axis.

The errors on the inclination and position angles and the rotational velocity are formal errors. They do not include possible systematic uncertainties due to, for instance, the beam smearing.

\subsubsection{Using the position-velocity diagrams}

It has already been remarked (see Sect. 3.5) that beam smearing affects the determination of the velocity fields, especially in the central regions of galaxies and in highly inclined disks. As a consequence, the rotation curves derived from such velocity fields are underestimated as one can see from their projection on the XV-maps. In order to overcome this problem, the rotation curves were derived directly from the major axis XV-maps in a manner similar to that used for edge-on systems (cf. Sancisi \& Allen 1979). This was done by two independent human neural networks trained to estimate the maximum rotational velocity from the asymmetric velocity profiles, taking into account the instrumental band- and beam-widths and the random gas motions. This was done for both the receding and approaching side of a galaxy. The rotation curves were then deprojected (also accounting for possible warps) by using the same position and inclination angles as fixed in the third step described in the previous section. In general, the average rotation curves derived from the XVdiagrams are in reasonable agreement with those obtained by the tilted ring fits. As expected, significant differences can only be noted for galaxies which are highly inclined or have a steeply rising rotation curve.

From the XV-diagrams it is clear that many galaxies have kinematic asymmetries in the sense that the rotation curve often rises more steeply on one side of a galaxy than on the other side (e.g. N3877, N3949). The rotation curves as derived from the velocity fields and XV-diagrams are tabulated in Table 4 for the approaching and receding parts separately. The adopted changes in inclination and position angles of N3718 and N4138 are motivated in the notes on the atlas pages of these galaxies. The uncertainties quoted in Table 4 are not 1-sigma Gaussian errors but rather reflect fiducial velocity ranges, based on the position-velocity diagrams.

\section{Matching $\mathrm{HI}$ linewidths to $V_{\max }$ and $V_{\text {flat }}$}

In this section, it will be investigated how the linewidth correction for turbulent motion can be used to match the finally corrected global HI linewidths to the actual rotational velocities measured from the rotation curves. 
Table 4. Rotation curves derived from velocity fields and XVdiagrams

\begin{tabular}{|c|c|c|c|c|c|c|c|c|c|}
\hline \multicolumn{2}{|c|}{$\begin{array}{c}\text { Rad. } V_{\text {rot }}^{\text {app }} \\
\left({ }^{\prime \prime}\right)--1\end{array}$} & $\mathrm{~km} \mathrm{~s}{ }^{ \pm}$ & & $\mathrm{km} \mathrm{s}^{ \pm}$ & & $V_{\text {rot }}^{\text {ave }}$ & \multicolumn{2}{|c|}{$\begin{array}{ll}i & \mathrm{PA} \\
\left.{ }^{\circ}\right) & \left({ }^{\circ}\right) \\
\end{array}$} \\
\hline \multicolumn{10}{|c|}{ U6399 } \\
\hline 10 & 25 & & 7 & & 10 & 7 & 25 & 75 & 141 \\
\hline 20 & & 10 & 7 & 49 & 7 & 7 & 46 & 75 & 141 \\
\hline 30 & & 12 & 7 & 61 & 7 & 5 & 61 & 75 & 141 \\
\hline 40 & 70 & 7 & 5 & 69 & 5 & 5 & 70 & 75 & 141 \\
\hline 50 & 77 & 7 & 5 & 78 & 3 & 5 & 78 & 75 & 141 \\
\hline 60 & 82 & 10 & 5 & 84 & 5 & 5 & 83 & 75 & 141 \\
\hline 70 & 84 & 5 & 5 & - & - & - & 84 & 75 & 141 \\
\hline 80 & 86 & 5 & 5 & - & - & - & 86 & 75 & 141 \\
\hline 90 & 88 & 5 & 5 & - & - & - & 88 & 75 & 141 \\
\hline \multicolumn{10}{|c|}{$U 6446$} \\
\hline 10 & 39 & 8 & 8 & & 10 & 10 & 31 & 51 & 188 \\
\hline 20 & 49 & 8 & 8 & & 8 & 10 & 55 & 51 & 188 \\
\hline 30 & 57 & 5 & 5 & 65 & 5 & 8 & 61 & 51 & 188 \\
\hline 40 & 63 & 5 & 5 & 65 & 5 & 8 & 64 & 51 & 188 \\
\hline 50 & 69 & 8 & 5 & 65 & 5 & 5 & 67 & 51 & 188 \\
\hline 60 & 71 & 5 & 5 & 70 & 5 & 5 & 70 & 51 & 188 \\
\hline 70 & 75 & 5 & 5 & 72 & 5 & 5 & 74 & 51 & 188 \\
\hline 80 & 79 & 8 & 5 & 77 & 5 & 5 & 78 & 51 & 188 \\
\hline 90 & 81 & 8 & 5 & 81 & 5 & 5 & 81 & 51 & 188 \\
\hline 100 & 81 & 5 & 5 & 80 & 5 & 5 & 81 & 51 & 188 \\
\hline 110 & 81 & 5 & 5 & 82 & 5 & 5 & 81 & 51 & 189 \\
\hline 120 & 82 & 8 & 5 & 83 & 5 & 8 & 82 & 51 & 191 \\
\hline 131 & 82 & 8 & 5 & 84 & 5 & 8 & 83 & 51 & 193 \\
\hline 142 & 83 & 8 & 8 & 86 & 8 & 8 & 85 & 51 & 195 \\
\hline 153 & 83 & 8 & 8 & 86 & 8 & 8 & 84 & 51 & 197 \\
\hline 164 & 82 & 11 & 11 & 85 & 8 & 11 & 83 & 51 & 199 \\
\hline 176 & 80 & 11 & 11 & - & - & - & 80 & 51 & 201 \\
\hline \multicolumn{10}{|c|}{ N3726 } \\
\hline 40 & 112 & 10 & 7 & 92 & & 10 & 102 & 53 & 195 \\
\hline 60 & 131 & 7 & 7 & 119 & 10 & 10 & 125 & 53 & 195 \\
\hline 80 & 144 & 5 & 5 & 146 & 7 & 10 & 145 & 53 & 195 \\
\hline 100 & 156 & 5 & 5 & 172 & 5 & 7 & 164 & 53 & 195 \\
\hline 120 & 154 & 7 & 7 & 171 & 5 & 7 & 162 & 53 & 195 \\
\hline 140 & 155 & 7 & 5 & 166 & 7 & 7 & 160 & 53 & 195 \\
\hline 160 & 152 & 7 & 7 & 159 & 5 & 7 & 156 & 53 & 195 \\
\hline 183 & 145 & 10 & 7 & 148 & 5 & 5 & 147 & 57 & 188 \\
\hline 256 & 157 & 8 & 8 & 159 & 6 & 6 & 158 & 72 & 180 \\
\hline 316 & 169 & 9 & 12 & - & - & - & 169 & 75 & 179 \\
\hline 344 & 169 & 9 & 12 & - & - & - & 169 & 75 & 179 \\
\hline 373 & 167 & 15 & 15 & - & - & - & 167 & 75 & 179 \\
\hline \multicolumn{10}{|c|}{ N3769 } \\
\hline 20 & 89 & & 10 & 86 & & 10 & 88 & 70 & 149 \\
\hline 40 & 103 & 10 & 8 & 109 & 13 & 10 & 106 & 70 & 149 \\
\hline 60 & 112 & 8 & 8 & 119 & 8 & 8 & 116 & 70 & 149 \\
\hline 80 & 120 & 8 & 8 & 130 & 8 & 10 & 125 & 70 & 149 \\
\hline 100 & 123 & 5 & 8 & 129 & 5 & 8 & 126 & 70 & 149 \\
\hline 120 & 124 & 5 & 8 & 122 & 5 & 8 & 123 & 70 & 150 \\
\hline 141 & 120 & 5 & 5 & 115 & 8 & 8 & 118 & 70 & 152 \\
\hline 166 & 120 & 8 & 10 & 110 & 8 & 10 & 115 & 70 & 155 \\
\hline 196 & 122 & 14 & 17 & - & - & - & 122 & 70 & 158 \\
\hline 364 & 121 & 10 & 10 & - & - & - & 121 & 70 & 167 \\
\hline 396 & 118 & 10 & 10 & - & - & - & 118 & 70 & 167 \\
\hline 426 & 113 & 11 & 11 & - & - & - & 113 & 70 & 168 \\
\hline \multicolumn{10}{|c|}{$U 666^{7}$} \\
\hline 10 & 27 & 5 & 5 & 27 & 7 & 7 & 27 & 89 & 89 \\
\hline 20 & 43 & 2 & 2 & 47 & 5 & 5 & 45 & 89 & 89 \\
\hline 30 & 55 & 5 & 5 & 59 & 7 & 5 & 57 & 89 & 89 \\
\hline 40 & 64 & 2 & 5 & 74 & 5 & 5 & 69 & 89 & 89 \\
\hline
\end{tabular}

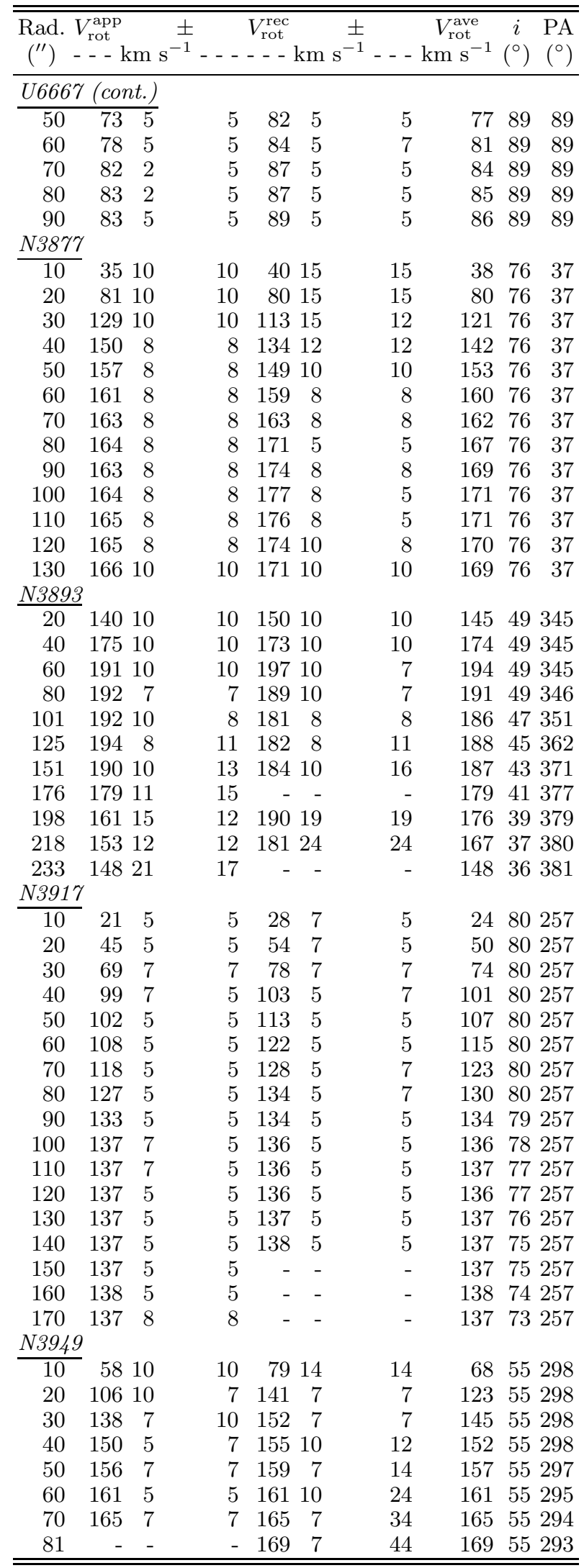


Table 4. continued

\begin{tabular}{|c|c|c|c|c|c|c|c|c|c|}
\hline \multirow{2}{*}{\multicolumn{3}{|c|}{$\begin{array}{l}\text { Rad. } V_{\text {rot }}^{\text {app }} \\
\left({ }^{\prime \prime}\right)---\mathrm{km} \mathrm{s}^{-1} \\
\end{array}$}} & \multicolumn{2}{|r|}{$\begin{array}{l}V_{\mathrm{rot}}^{\mathrm{rec}} \\
--.\end{array}$} & $\mathrm{km} \mathrm{s}^{-1}$ & & 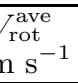 & \multicolumn{2}{|c|}{$\begin{array}{ll}i & \mathrm{PA} \\
\left.{ }^{\circ}\right) & \left({ }^{\circ}\right) \\
\end{array}$} \\
\hline & & & \\
\hline 40 & 178 & 7 & 10 & 184 & & 10 & 181 & 62 & 13 \\
\hline 60 & 200 & 10 & 10 & 207 & 10 & 7 & 203 & 62 & 13 \\
\hline 80 & 214 & 7 & 10 & 219 & 7 & 7 & 217 & 62 & 13 \\
\hline 100 & 218 & 7 & 10 & 227 & 10 & 7 & 223 & 62 & 13 \\
\hline 120 & 219 & 7 & 7 & 229 & 10 & 7 & 224 & 62 & 13 \\
\hline 140 & 222 & 7 & 7 & 224 & 10 & 10 & 223 & 62 & 13 \\
\hline 160 & 229 & 10 & 7 & 218 & 7 & 10 & 224 & 62 & 13 \\
\hline 175 & 234 & 10 & 7 & - & - & - & 234 & 62 & 13 \\
\hline 180 & - & - & - & 215 & & 10 & 215 & 62 & 13 \\
\hline \multicolumn{10}{|c|}{ N3972 } \\
\hline 10 & 24 & 5 & 5 & 55 & & 7 & 40 & 77 & 297 \\
\hline 20 & 68 & 7 & 7 & 78 & 7 & 7 & 73 & 77 & 7297 \\
\hline 30 & 86 & 12 & 10 & 93 & 7 & 7 & 89 & 77 & 7297 \\
\hline 40 & 101 & 10 & 7 & 103 & 5 & 7 & 102 & 77 & 7297 \\
\hline 50 & 111 & 7 & 5 & 110 & 5 & 7 & 110 & 77 & 7297 \\
\hline 60 & 117 & 7 & 5 & 116 & 5 & 7 & 116 & 77 & 7297 \\
\hline 70 & 122 & 5 & 5 & 124 & 7 & 7 & 123 & 77 & 7297 \\
\hline 80 & 129 & 5 & 5 & 131 & 5 & 7 & 130 & 77 & 7297 \\
\hline 86 & 131 & 7 & 7 & - & - & - & 131 & 77 & 7297 \\
\hline 90 & - & - & - & 134 & 5 & 7 & 134 & 77 & 7297 \\
\hline 100 & - & - & - & 134 & 5 & 5 & 134 & 77 & 7297 \\
\hline \multicolumn{10}{|c|}{ U6917 } \\
\hline 20 & 60 & 5 & 5 & 59 & 5 & 5 & 59 & 56 & 5123 \\
\hline 30 & 71 & 5 & 8 & 72 & 5 & 5 & 71 & 56 & 5123 \\
\hline 40 & 86 & 8 & 5 & 83 & 8 & 5 & 85 & 56 & 5123 \\
\hline 50 & 96 & 8 & 8 & 91 & 5 & 5 & 94 & 56 & 5123 \\
\hline 60 & 98 & 5 & 5 & 97 & 5 & 5 & 98 & 56 & 5123 \\
\hline 70 & 100 & 5 & 5 & 100 & 5 & 5 & 100 & 56 & 5123 \\
\hline 80 & 101 & 8 & 5 & 101 & 5 & 5 & 101 & 56 & 5123 \\
\hline 90 & 105 & 5 & 5 & 102 & 5 & 5 & 103 & 56 & 5123 \\
\hline 100 & 110 & 5 & 5 & 101 & 5 & 8 & 105 & 56 & 6123 \\
\hline 110 & 116 & 7 & 7 & 104 & 5 & 7 & 110 & 57 & 7123 \\
\hline 120 & - & - & - & 111 & 5 & 7 & 111 & 60 & 124 \\
\hline \multicolumn{10}{|c|}{$\underline{U 6923}$} \\
\hline 11 & 41 & 6 & 9 & - & - & - & 41 & 65 & 5341 \\
\hline 23 & 54 & 6 & 6 & - & - & - & 54 & 65 & 5341 \\
\hline 34 & 70 & 6 & 6 & 76 & 9 & 6 & 73 & 65 & 5341 \\
\hline 44 & 80 & 6 & 6 & 77 & 6 & 6 & 78 & 65 & 5344 \\
\hline 53 & - & - & - & 79 & 5 & 5 & 79 & 65 & 5347 \\
\hline 61 & - & - & - & 81 & 5 & 5 & 81 & 65 & 5350 \\
\hline \multicolumn{10}{|c|}{ U6930 } \\
\hline 20 & 58 & & 10 & 52 & & 12 & 55 & 32 & $2 \quad 39$ \\
\hline 40 & 88 & 10 & 7 & 83 & 12 & 10 & 85 & 32 & $2 \quad 39$ \\
\hline 60 & 94 & 7 & 7 & 94 & 10 & 10 & 94 & 32 & $2 \quad 39$ \\
\hline 80 & 98 & 7 & 7 & 100 & 7 & 7 & 99 & 32 & $\begin{array}{ll}2 & 39\end{array}$ \\
\hline 100 & 102 & 7 & 7 & 105 & 7 & 7 & 103 & 32 & $2 \quad 39$ \\
\hline 120 & 105 & 7 & 7 & 109 & 7 & 7 & 107 & 32 & $2 \quad 39$ \\
\hline 140 & 107 & 7 & 7 & 110 & 7 & 7 & 109 & 32 & $2 \quad 39$ \\
\hline 150 & - & - & - & 110 & 7 & 7 & 110 & 32 & $2 \quad 39$ \\
\hline 160 & 108 & 7 & 7 & - & - & - & 108 & 32 & $2 \quad 39$ \\
\hline 180 & 108 & 7 & 7 & - & - & - & 108 & 32 & $2 \quad 39$ \\
\hline 190 & 108 & 7 & 7 & - & - & - & 108 & 32 & $\begin{array}{ll}2 & 39\end{array}$ \\
\hline \multicolumn{10}{|c|}{ N3992 } \\
\hline 80 & 253 & 7 & 10 & 244 & & 12 & 249 & 56 & 6248 \\
\hline 120 & 264 & 7 & 10 & 265 & 7 & 12 & 264 & 56 & 6248 \\
\hline 160 & 273 & 7 & 10 & 272 & 7 & 7 & 272 & 56 & 6248 \\
\hline 200 & 274 & 7 & 7 & 268 & 7 & 10 & 271 & 56 & 6248 \\
\hline 240 & 273 & 7 & 7 & 256 & 7 & 7 & 264 & 56 & 6248 \\
\hline 280 & - & - & - & 242 & 7 & 7 & 242 & 56 & 6248 \\
\hline
\end{tabular}

Table 4. continued

\begin{tabular}{|c|c|c|c|c|c|c|c|c|c|}
\hline $\begin{array}{c}\text { Rad. } \\
\left({ }^{\prime \prime}\right)\end{array}$ & & $\mathrm{km} \mathrm{s}^{-}$ & & $\begin{array}{l}V_{\mathrm{ret}}^{\mathrm{rec}} \\
---. \\
\end{array}$ & $\mathrm{km} \mathrm{s}^{ \pm}$ & & $\begin{array}{l}\text { ave } \\
\text { rot } \\
\mathrm{n} \mathrm{s}^{-1} \\
\end{array}$ & $\begin{array}{c}i \\
\left(^{\circ}\right) \\
\end{array}$ & $\begin{array}{l}\text { PA } \\
\left({ }^{\circ}\right) \\
\end{array}$ \\
\hline N399 & $1 \mathrm{co}$ & nt.) & & & & & & & \\
\hline 320 & 247 & 7 & 7 & 242 & 7 & 7 & 244 & 56 & 248 \\
\hline 360 & 241 & 7 & 7 & 242 & 10 & 10 & 241 & 56 & 248 \\
\hline 400 & 237 & 7 & 10 & - & - & - & 237 & 56 & 248 \\
\hline U694 & & & & & & & & & \\
\hline 10 & 19 & 5 & 5 & 18 & 5 & 5 & 18 & 79 & 315 \\
\hline 20 & 41 & 5 & 5 & 34 & 5 & 8 & 37 & 79 & 315 \\
\hline U696 & & & & & & & & & \\
\hline 10 & 50 & & 15 & 75 & 12 & 10 & 62 & 37 & 359 \\
\hline 20 & 107 & & 7 & 106 & 10 & 7 & 106 & 37 & 359 \\
\hline 30 & 129 & 7 & 7 & 126 & 7 & 7 & 128 & & 359 \\
\hline 40 & 142 & 7 & 7 & 145 & 10 & 7 & 144 & 37 & 359 \\
\hline 50 & 155 & 7 & 7 & 163 & 10 & 10 & 159 & 37 & 359 \\
\hline 60 & 171 & 7 & 7 & - & - & - & 171 & 37 & 359 \\
\hline$N_{4} 01$ & & & & & & & & & \\
\hline$\overline{0}$ & 34 & & 15 & -34 & & 15 & 0 & 90 & 66 \\
\hline 10 & 59 & 12 & 10 & 20 & 7 & 7 & 39 & 90 & 66 \\
\hline 20 & 66 & 7 & 10 & 43 & 7 & 7 & 55 & 90 & 66 \\
\hline 30 & 69 & 5 & 10 & 62 & 12 & 10 & 66 & 90 & 66 \\
\hline 40 & 80 & 5 & 5 & 88 & 10 & 7 & 84 & 90 & 66 \\
\hline 50 & 84 & 5 & 5 & 104 & 12 & 10 & 94 & 90 & 66 \\
\hline 60 & 96 & 5 & 5 & 113 & 10 & 10 & 104 & 90 & 66 \\
\hline 70 & 108 & 7 & 5 & 122 & 7 & 7 & 115 & 90 & 66 \\
\hline 80 & 125 & 7 & 5 & 129 & 7 & 7 & 127 & 90 & 66 \\
\hline 90 & 128 & 7 & 5 & 131 & 7 & 7 & 129 & 90 & 66 \\
\hline 100 & 123 & 7 & 5 & 131 & 7 & 7 & 127 & 90 & 66 \\
\hline 110 & 119 & 7 & 5 & 129 & 7 & 7 & 124 & 90 & 66 \\
\hline 120 & 119 & 5 & 5 & 125 & 5 & 7 & 122 & 90 & 66 \\
\hline U696 & & & & & & & & & \\
\hline 10 & - & - & - & 26 & 5 & 7 & 26 & 76 & 330 \\
\hline 20 & 34 & 5 & 7 & 44 & 7 & 7 & 39 & 76 & 330 \\
\hline 31 & 46 & 5 & 5 & 58 & 5 & 7 & 52 & 76 & 330 \\
\hline 41 & 60 & 5 & 5 & 69 & 5 & 7 & 65 & 76 & 330 \\
\hline 51 & - & - & - & 79 & 5 & 5 & 79 & 76 & 330 \\
\hline U697. & & & & & & & & & \\
\hline 20 & 162 & 5 & 10 & 179 & 5 & 7 & 170 & 71 & 41 \\
\hline 30 & 174 & 5 & 7 & 174 & 5 & 7 & 174 & 71 & 41 \\
\hline 40 & 170 & 5 & 7 & 170 & 5 & 7 & 170 & 71 & 42 \\
\hline 50 & 170 & 5 & 7 & 170 & 5 & 7 & 170 & 71 & 44 \\
\hline 61 & 171 & 5 & 7 & 172 & 7 & 7 & 171 & 71 & 45 \\
\hline 72 & 174 & 5 & 8 & 174 & 8 & 8 & 174 & 71 & 46 \\
\hline 78 & - & - & - & 177 & 10 & 10 & 177 & 71 & 47 \\
\hline 84 & 178 & 5 & 8 & - & - & - & 178 & 71 & 47 \\
\hline 90 & 180 & 5 & 10 & - & - & - & 180 & 71 & 48 \\
\hline U698. & & & & & & & & & \\
\hline 20 & 58 & & 10 & 56 & 10 & 7 & 57 & 49 & 270 \\
\hline 30 & 93 & 7 & 5 & 82 & 7 & 7 & 87 & 49 & 270 \\
\hline 40 & 87 & 7 & 7 & 97 & 10 & 7 & 92 & 49 & 270 \\
\hline 50 & 84 & 7 & 7 & 103 & 7 & 7 & 94 & 49 & 270 \\
\hline 60 & 93 & 5 & 5 & 103 & 7 & 7 & 98 & 49 & 270 \\
\hline 70 & 94 & 5 & 5 & 105 & 7 & 7 & 100 & 49 & 270 \\
\hline 80 & 95 & 5 & 5 & 108 & 5 & 5 & 102 & 49 & 270 \\
\hline 90 & 100 & 7 & 7 & 113 & 5 & 5 & 107 & 49 & 270 \\
\hline 100 & 105 & 7 & 7 & 112 & 5 & 5 & 108 & 49 & 270 \\
\hline 110 & 111 & 7 & 7 & 110 & 5 & 5 & 111 & 49 & 270 \\
\hline 120 & 113 & 7 & 7 & 112 & 5 & 5 & 113 & 49 & 270 \\
\hline 130 & 111 & 7 & 7 & 110 & 7 & 7 & 111 & 49 & 270 \\
\hline 140 & 107 & 7 & 7 & 109 & 7 & 10 & 108 & 49 & 270 \\
\hline 145 & 102 & 10 & 10 & - & - & - & 102 & 49 & 270 \\
\hline 150 & - & - & - & 109 & 7 & 10 & 109 & 49 & 270 \\
\hline
\end{tabular}


Table 4. continued

\begin{tabular}{|c|c|c|c|c|c|c|c|c|c|}
\hline \multirow{2}{*}{\multicolumn{3}{|c|}{$\begin{array}{l}\text { Rad. } V_{\text {rot }}^{\text {app }} \\
\left({ }^{\prime \prime}\right)---\mathrm{km} \mathrm{s}^{-1} \\
\text { U6983 (cont.) }\end{array}$}} & \multicolumn{2}{|r|}{$\begin{array}{l}V_{\text {rot }}^{\mathrm{rec}} \\
--.\end{array}$} & $\mathrm{km} \mathrm{s}^{ \pm}$ & \multicolumn{2}{|c|}{$\begin{array}{c}V_{\text {rot }}^{\text {ave }} \\
\mathrm{km} \mathrm{s}^{-1}\end{array}$} & \multicolumn{2}{|c|}{$\begin{array}{ll}i & \text { PA } \\
\circ\end{array}$} \\
\hline & & & & & & & & & \\
\hline 160 & - & & - & 108 & 10 & 10 & 108 & & 270 \\
\hline 170 & - & & - & 108 & 10 & 10 & 108 & 49 & 270 \\
\hline 180 & - & - & - & 109 & 12 & 12 & 109 & 49 & 270 \\
\hline N405 & & & & & & & & & \\
\hline 20 & - & - & - & 121 & 15 & 15 & 121 & 49 & 310 \\
\hline 25 & 123 & 15 & 15 & - & - & - & 123 & & 310 \\
\hline 40 & 119 & 12 & 10 & 114 & & 10 & 116 & & 310 \\
\hline 60 & 146 & 10 & 10 & 133 & 10 & 10 & 140 & & 310 \\
\hline 80 & 163 & 7 & 10 & 156 & 10 & 10 & 160 & & 310 \\
\hline 100 & 158 & 7 & 7 & 165 & 7 & 7 & 162 & & 310 \\
\hline 115 & - & - & - & 170 & 7 & 7 & 170 & 49 & 310 \\
\hline 120 & 154 & 7 & 7 & - & - & - & 154 & 49 & 310 \\
\hline 140 & 153 & 10 & 10 & - & - & - & 153 & 49 & 310 \\
\hline$N 408$ & & & & & & & & & \\
\hline 10 & 35 & 10 & 5 & 50 & & 10 & 42 & 82 & 256 \\
\hline 20 & 71 & 7 & 5 & 89 & 10 & 10 & 80 & 82 & 256 \\
\hline 31 & 110 & 12 & 7 & 113 & 7 & 7 & 112 & 82 & 256 \\
\hline 41 & 126 & 7 & 5 & 127 & 7 & 7 & 127 & 82 & 256 \\
\hline 51 & 131 & 7 & 7 & 130 & 7 & 5 & 130 & 82 & 256 \\
\hline 61 & 134 & 7 & 7 & 133 & 5 & 5 & 133 & 82 & 256 \\
\hline 71 & 136 & 7 & 7 & - & - & - & 136 & 82 & 256 \\
\hline$N_{4} 08$ & & & & & & & & & \\
\hline 20 & 92 & 15 & 15 & 78 & & 15 & 85 & 69 & 230 \\
\hline 40 & 138 & 10 & 15 & 135 & & 10 & 136 & 69 & 230 \\
\hline 60 & 156 & 7 & 12 & 168 & 15 & 10 & 162 & 69 & 230 \\
\hline 80 & 167 & 7 & 10 & 191 & 10 & 10 & 179 & 69 & 230 \\
\hline 100 & 177 & 7 & 10 & 187 & 10 & 7 & 182 & 69 & 230 \\
\hline 120 & 185 & 7 & 12 & 174 & & 10 & 179 & 69 & 230 \\
\hline 140 & 187 & 7 & 12 & 162 & 7 & 7 & 174 & 69 & 230 \\
\hline 160 & 185 & 12 & 12 & 158 & 7 & 7 & 171 & 69 & 230 \\
\hline 180 & 175 & 10 & 10 & 161 & 7 & 7 & 168 & 69 & 230 \\
\hline 200 & 171 & 7 & 7 & 160 & 10 & 7 & 165 & 69 & 230 \\
\hline 210 & - & - & - & 156 & 10 & 7 & 156 & 69 & 229 \\
\hline 221 & 171 & 10 & 7 & - & - & - & 171 & 69 & 227 \\
\hline 246 & 174 & 8 & 8 & - & - & - & 174 & 69 & 224 \\
\hline$N_{4} 100$ & & & & & & & & & \\
\hline 20 & 67 & 15 & 15 & - & - & - & 67 & & 345 \\
\hline 30 & 102 & 15 & 20 & 139 & 15 & 7 & 121 & & 345 \\
\hline 40 & 138 & 12 & 12 & 159 & 10 & 7 & 148 & & 345 \\
\hline 50 & 164 & 7 & 10 & 173 & 10 & 7 & 168 & 73 & 345 \\
\hline 60 & 177 & 10 & 7 & 188 & 10 & 7 & 182 & 73 & 345 \\
\hline 70 & 188 & 7 & 7 & 193 & 7 & 7 & 191 & 73 & 345 \\
\hline 80 & 193 & 7 & 7 & 195 & 10 & 7 & 194 & 73 & 345 \\
\hline 90 & 195 & 10 & 7 & 195 & 10 & 7 & 195 & 73 & 345 \\
\hline 100 & 193 & 5 & 7 & 194 & 7 & 7 & 193 & 73 & 345 \\
\hline 110 & 192 & 5 & 5 & 192 & 7 & 5 & 192 & 73 & 345 \\
\hline 120 & 193 & 5 & 5 & 191 & 5 & 5 & 192 & 73 & 345 \\
\hline 130 & 192 & 5 & 5 & 190 & 7 & 7 & 191 & 73 & 345 \\
\hline 140 & 188 & 7 & 7 & 189 & 7 & 5 & 189 & 73 & 345 \\
\hline 150 & 183 & 7 & 10 & 187 & 7 & 5 & 185 & 73 & 345 \\
\hline 160 & 180 & 7 & 7 & 185 & 5 & 5 & 182 & 73 & 345 \\
\hline 170 & 175 & 10 & 10 & 183 & 10 & 7 & 179 & 72 & 346 \\
\hline 180 & 172 & 7 & 10 & 181 & & 7 & 177 & 71 & 346 \\
\hline 190 & 168 & 10 & 10 & 179 & & 8 & 174 & 71 & 346 \\
\hline 200 & - & - & - & 178 & 10 & 8 & 178 & 70 & 346 \\
\hline 210 & - & - & - & 177 & 8 & 5 & 177 & 70 & 347 \\
\hline 220 & 160 & 5 & 8 & 178 & 10 & 10 & 169 & 69 & 347 \\
\hline 230 & 158 & 5 & 8 & - & - & - & 158 & 69 & 347 \\
\hline 241 & 158 & 8 & 8 & - & - & - & 158 & 68 & 348 \\
\hline
\end{tabular}

Table 4. continued

\begin{tabular}{|c|c|c|c|c|c|c|c|c|c|}
\hline \multicolumn{3}{|c|}{$\begin{array}{l}\text { Rad. } V_{\text {rot }}^{\text {app }} \pm \\
\left({ }^{\prime \prime}\right)---\mathrm{km} \mathrm{s}^{-1}\end{array}$} & \multicolumn{2}{|r|}{$V_{\text {rot }}^{\text {rec }}$} & $\mathrm{km} \mathrm{s}^{\stackrel{ \pm}{-1}}$ & \multicolumn{2}{|c|}{$V_{\text {rot }}^{\text {ave }}$} & \multicolumn{2}{|c|}{$\begin{array}{ll}i & \text { PA } \\
\left.{ }^{\circ}\right) & \left({ }^{\circ}\right)\end{array}$} \\
\hline \\
\hline 251 & 158 & 8 & 10 & - & - & - & 158 & 68 & 348 \\
\hline 261 & 159 & 10 & 8 & - & - & - & 159 & 67 & 348 \\
\hline \multicolumn{10}{|c|}{$N_{4} 102$} \\
\hline 20 & 179 & 12 & 12 & 184 & & 12 & 181 & 56 & 39 \\
\hline 30 & 177 & & 10 & 181 & 10 & 12 & 179 & 56 & 39 \\
\hline 40 & 174 & 12 & 10 & 178 & 10 & 10 & 176 & 56 & 39 \\
\hline 50 & - & - & - & 178 & 15 & 10 & 178 & 56 & 39 \\
\hline \multicolumn{10}{|c|}{$N 4157$} \\
\hline 20 & 66 & 18 & 14 & 127 & 23 & 14 & 96 & 82 & 63 \\
\hline 40 & 142 & & 14 & 173 & 14 & 14 & 157 & 82 & 63 \\
\hline 60 & 192 & 9 & 14 & 191 & 11 & 14 & 192 & 82 & 63 \\
\hline 80 & 202 & 7 & 9 & 201 & 11 & 11 & 201 & 82 & 63 \\
\hline 100 & 198 & 11 & 11 & 204 & 9 & 9 & 201 & 82 & 63 \\
\hline 120 & 192 & 9 & 9 & 197 & 9 & 9 & 195 & 82 & 63 \\
\hline 140 & 191 & 9 & 9 & 188 & 9 & 7 & 190 & 82 & 63 \\
\hline 160 & 191 & 9 & 9 & 181 & 9 & 9 & 186 & 82 & 63 \\
\hline 180 & 192 & 9 & 9 & 176 & 9 & 9 & 184 & 82 & 63 \\
\hline 200 & 191 & 9 & 9 & 173 & 11 & 11 & 182 & 82 & 63 \\
\hline 220 & 190 & 7 & 7 & 173 & 14 & 9 & 181 & 82 & 63 \\
\hline 240 & 189 & 9 & 7 & 177 & 11 & 9 & 183 & 82 & 63 \\
\hline 260 & 189 & 11 & 7 & 181 & 9 & 9 & 185 & 82 & 63 \\
\hline 280 & 186 & 11 & 7 & - & - & - & 186 & 82 & 63 \\
\hline 300 & 186 & 11 & 11 & - & - & - & 186 & 82 & 63 \\
\hline 320 & 185 & 14 & 14 & - & - & - & 185 & 82 & 63 \\
\hline 340 & 185 & 14 & 14 & - & - & - & 185 & 82 & 63 \\
\hline \multicolumn{10}{|c|}{$N_{4} 183$} \\
\hline 10 & 56 & & 10 & 38 & 15 & 10 & 47 & 82 & 346 \\
\hline 20 & 71 & 7 & 7 & 61 & 12 & 10 & 66 & 82 & 346 \\
\hline 30 & 78 & 7 & 7 & 74 & 10 & 7 & 76 & 82 & 346 \\
\hline 40 & 88 & 7 & 7 & 84 & 10 & 7 & 86 & 82 & 346 \\
\hline 50 & 97 & 7 & 7 & 97 & 7 & 7 & 97 & 82 & 346 \\
\hline 60 & 100 & 7 & 7 & 99 & 7 & 7 & 99 & 82 & 346 \\
\hline 70 & 103 & 7 & 7 & 103 & 7 & 7 & 103 & 82 & 346 \\
\hline 80 & 106 & 7 & 7 & 107 & 7 & 7 & 107 & 82 & 346 \\
\hline 90 & 110 & 7 & 7 & 113 & 7 & 7 & 111 & 82 & 346 \\
\hline 100 & 112 & 7 & 7 & 117 & 10 & 10 & 114 & 82 & 346 \\
\hline 110 & 112 & 7 & 7 & 118 & 10 & 10 & 115 & 82 & 346 \\
\hline 120 & 108 & 7 & 7 & 114 & 10 & 7 & 111 & 82 & 346 \\
\hline 130 & 108 & 7 & 7 & 113 & 10 & 7 & 110 & 82 & 347 \\
\hline 141 & 111 & 7 & 7 & 112 & 7 & 7 & 111 & 82 & 347 \\
\hline 151 & 108 & 7 & 5 & 110 & 7 & 7 & 109 & 82 & 347 \\
\hline 161 & 106 & 5 & 5 & 109 & 7 & 7 & 108 & 82 & 347 \\
\hline 172 & 109 & 7 & 7 & 109 & 7 & 7 & 109 & 82 & 347 \\
\hline 183 & 112 & 7 & 7 & 110 & 7 & 7 & 111 & 82 & 348 \\
\hline 194 & 108 & 5 & 8 & 111 & 8 & 8 & 110 & 82 & 348 \\
\hline 205 & 106 & 5 & 8 & 111 & 8 & 8 & 109 & 82 & 348 \\
\hline 217 & 107 & 7 & 8 & 112 & 8 & 8 & 110 & 82 & 348 \\
\hline 229 & - & - & - & 112 & & 10 & 112 & 82 & 348 \\
\hline 241 & - & - & - & 113 & & 10 & 113 & 82 & 349 \\
\hline \multicolumn{10}{|c|}{ N4217 } \\
\hline 10 & 38 & & 10 & 57 & & 12 & 48 & 86 & 230 \\
\hline 20 & 82 & & 12 & 116 & & 10 & 99 & 86 & 230 \\
\hline 30 & 145 & & 10 & 148 & & 10 & 146 & 86 & 230 \\
\hline 40 & 162 & 7 & 10 & 165 & & 10 & 164 & 86 & 230 \\
\hline 50 & 176 & 7 & 10 & 172 & 7 & 10 & 174 & 86 & 230 \\
\hline 60 & 176 & 7 & 10 & 175 & & 7 & 175 & 86 & 230 \\
\hline 70 & 189 & 7 & 10 & 179 & 10 & 10 & 184 & 86 & 230 \\
\hline 80 & 188 & 7 & 10 & 182 & 10 & 10 & 185 & 86 & 230 \\
\hline 90 & 187 & 5 & 10 & 188 & 12 & 10 & 188 & 86 & 230 \\
\hline
\end{tabular}


Table 4. continued

\begin{tabular}{|c|c|c|c|c|c|c|c|c|}
\hline \multicolumn{2}{|c|}{$\begin{array}{c}\text { Rad. } V_{\text {rot }}^{\text {app }} \\
\left({ }^{\prime \prime}\right)---\mathrm{km} \mathrm{s}^{-} \\
\end{array}$} & \multicolumn{2}{|r|}{$\begin{array}{l}V_{\text {rot }}^{\mathrm{rec}} \\
----\end{array}$} & $\mathrm{km} \mathrm{s}^{ \pm}$ & \multicolumn{2}{|c|}{$\begin{array}{c}\begin{array}{c}V_{\text {rot }}^{\text {ave }} \\
\mathrm{km} \mathrm{s}^{-1}\end{array} \\
\end{array}$} & \multicolumn{2}{|c|}{$\begin{array}{ll}i & \mathrm{PA} \\
\left.{ }^{\circ}\right) & \left({ }^{\circ}\right) \\
\end{array}$} \\
\hline \multicolumn{9}{|c|}{ N4217 (cont.) } \\
\hline 100 & $188 \quad 7$ & 10 & 192 & 12 & 12 & 190 & & 230 \\
\hline 110 & 1917 & 10 & 191 & 10 & 10 & 191 & 86 & 230 \\
\hline 120 & 1927 & 7 & 189 & 10 & 7 & 191 & 86 & 230 \\
\hline 130 & 19110 & 7 & 187 & 10 & 7 & 189 & 86 & 230 \\
\hline 140 & $187 \quad 10$ & 7 & 185 & 12 & 10 & 186 & 86 & 230 \\
\hline 150 & 18310 & 7 & 183 & 10 & 10 & 183 & 86 & 230 \\
\hline 160 & 18012 & 7 & 178 & 10 & 10 & 179 & 86 & 230 \\
\hline 170 & $177 \quad 10$ & 10 & 177 & 10 & 12 & 177 & 86 & 230 \\
\hline 181 & 17812 & 12 & 177 & 10 & 14 & 177 & & 230 \\
\hline 191 & 17812 & 12 & - & - & - & 178 & 86 & 230 \\
\hline \multicolumn{9}{|c|}{ N4389 } \\
\hline 10 & 308 & 8 & & 10 & 8 & 27 & 50 & 277 \\
\hline 20 & 5610 & 8 & 50 & 13 & 8 & 53 & 50 & 277 \\
\hline 31 & 7013 & 8 & 69 & 10 & 8 & 69 & 50 & 277 \\
\hline 41 & 7913 & 8 & 88 & 8 & 8 & 84 & 50 & 277 \\
\hline 51 & 9210 & 10 & 99 & 8 & 8 & 96 & 50 & 277 \\
\hline 61 & $-\quad-$ & - & 110 & 8 & 8 & 110 & 50 & 277 \\
\hline
\end{tabular}

\begin{tabular}{|c|c|c|c|c|c|c|c|c|c|}
\hline \multicolumn{10}{|c|}{ Rotation curves derived from XV-diagrams only. } \\
\hline \multicolumn{10}{|c|}{$\overline{N 33718}$} \\
\hline 40 & 2281 & & 10 & 2281 & & 10 & 228 & 76 & 114 \\
\hline 80 & 2281 & & 10 & 2281 & & 10 & 228 & 80 & 130 \\
\hline 120 & 2281 & & 10 & 2281 & & 10 & 228 & 84 & 143 \\
\hline 160 & 2281 & & 10 & 2281 & 10 & 10 & 228 & 90 & 162 \\
\hline 200 & 2281 & & 10 & 2281 & 10 & 10 & 228 & 85 & 175 \\
\hline 240 & 2201 & & 10 & 2351 & 10 & 10 & 228 & 80 & 186 \\
\hline 280 & 2251 & & 10 & 2391 & 10 & 10 & 232 & 75 & 195 \\
\hline 320 & 2401 & & 10 & 2451 & 10 & 10 & 242 & 70 & 196 \\
\hline 360 & 2451 & & 10 & 2421 & & 10 & 244 & 65 & 196 \\
\hline 400 & 2351 & & 10 & 2401 & & 10 & 237 & 65 & 194 \\
\hline 420 & 2271 & & 10 & - & - & - & - & 65 & 194 \\
\hline N372: & & & & & & & & & \\
\hline 20 & 1183 & & 24 & 1381 & & 10 & 128 & 48 & 164 \\
\hline 40 & 1571 & & 12 & 1411 & & 12 & 149 & 48 & 164 \\
\hline 50 & - & - & - & 1441 & & 10 & 144 & 48 & 164 \\
\hline 60 & 1511 & & 10 & & - & - & 151 & 48 & 164 \\
\hline \multicolumn{10}{|c|}{ U6773 } \\
\hline 10 & 281 & & 7 & 341 & & 7 & 31 & 60 & 341 \\
\hline 20 & 38 & 7 & 7 & 48 & 7 & 5 & 43 & 60 & 341 \\
\hline 30 & 46 & & 5 & 44 & & 7 & 45 & 60 & 341 \\
\hline 40 & 47 & & 5 & 441 & & 7 & 45 & 60 & 341 \\
\hline \multicolumn{10}{|c|}{ U6818 } \\
\hline 10 & 27 & 7 & 7 & 201 & & 7 & 23 & 79 & 77 \\
\hline 20 & 281 & & 7 & 28 & 7 & 5 & 28 & 79 & 77 \\
\hline 30 & & 7 & 5 & 43 & 7 & 5 & 37 & 79 & 77 \\
\hline 40 & 43 & 7 & 5 & 53 & 7 & 5 & 48 & 79 & 77 \\
\hline 50 & 66 & 7 & 7 & 61 & 7 & 5 & 63 & 79 & 77 \\
\hline 60 & 77 & 7 & 10 & 66 & 7 & 5 & 71 & 79 & 77 \\
\hline 70 & 68 & 7 & 5 & - & - & - & 68 & 79 & 77 \\
\hline 80 & & 7 & 5 & & - & - & 74 & 79 & 7 \\
\hline \multicolumn{10}{|c|}{ N3985 } \\
\hline 0 & 81 & & 10 & -81 & & 10 & 0 & 53 & 70 \\
\hline 10 & 411 & & 7 & 371 & & 7 & 39 & 53 & 70 \\
\hline 20 & 60 & 7 & 10 & 891 & & 7 & 75 & 53 & 70 \\
\hline 25 & 681 & & 10 & - & - & - & 68 & 53 & 70 \\
\hline 30 & & - & - & 93 & 7 & 7 & 93 & 53 & 7 \\
\hline \multicolumn{10}{|c|}{ U6894 } \\
\hline 10 & 28 & 7 & 7 & 281 & & 10 & 28 & 89 & 269 \\
\hline 20 & 45 & 7 & 7 & 45 & 7 & 7 & 45 & 89 & 269 \\
\hline
\end{tabular}

Table 4. continued

\begin{tabular}{|c|c|c|c|c|c|c|c|c|c|}
\hline \multicolumn{3}{|c|}{$\begin{array}{ll}\text { Rad. } & V_{\text {rot }}^{\text {app }} \\
\left({ }^{\prime \prime}\right) & ---\mathrm{km} \mathrm{s}^{-1} \\
\end{array}$} & \multicolumn{2}{|c|}{$V_{\text {rot }}^{\text {rec }}$} & $\mathrm{km} \mathrm{s}^{\stackrel{ \pm}{-1}}$ & \multicolumn{2}{|c|}{$\begin{array}{c}V_{\text {rot }}^{\text {ave }} \\
-\mathrm{km} \mathrm{s}^{-1}\end{array}$} & \multicolumn{2}{|c|}{$\begin{array}{ll}i & \text { PA } \\
\circ & \left({ }^{\circ}\right) \\
\end{array}$} \\
\hline \multicolumn{10}{|c|}{ U6894 (cont.) } \\
\hline 30 & 56 & $\overline{7}$ & 5 & 56 & 5 & 5 & 56 & 89 & 269 \\
\hline 40 & 62 & 5 & 5 & 63 & 7 & 7 & 63 & 89 & 269 \\
\hline \multicolumn{10}{|c|}{ N4013 } \\
\hline 65 & - & - & - & 198 & 10 & 10 & 198 & 90 & 245 \\
\hline 73 & - & - & - & 195 & 5 & 5 & 195 & 90 & 245 \\
\hline 82 & 193 & 5 & 5 & 195 & 3 & 3 & 194 & 90 & 245 \\
\hline 91 & 195 & 4 & 4 & 195 & 3 & 3 & 195 & 90 & 245 \\
\hline 99 & 195 & 3 & 3 & 195 & 3 & 3 & 195 & 90 & 245 \\
\hline 108 & 195 & 3 & 3 & 193 & 4 & 4 & 195 & 90 & 245 \\
\hline 117 & 196 & 3 & 3 & 185 & 5 & 5 & 192 & 90 & 245 \\
\hline 125 & 195 & 3 & 3 & 178 & 5 & 5 & 188 & 90 & 245 \\
\hline 134 & 190 & 4 & 4 & 178 & 8 & 8 & 186 & 90 & 245 \\
\hline 143 & 190 & 4 & 4 & - & - & - & 186 & 90 & 245 \\
\hline 151 & 188 & 5 & 5 & - & - & - & 186 & 90 & 245 \\
\hline 160 & 187 & 6 & 6 & - & - & - & 185 & 90 & 245 \\
\hline 168 & 179 & 10 & 10 & - & - & - & 180 & 90 & 243 \\
\hline 177 & 163 & 8 & 8 & - & - & - & 163 & 90 & 240 \\
\hline 186 & 161 & 6 & 6 & - & - & - & 162 & 90 & 238 \\
\hline 194 & 162 & 5 & 5 & - & - & - & 164 & 90 & 236 \\
\hline 203 & 164 & 5 & 5 & 170 & 5 & 5 & 166 & 90 & 235 \\
\hline 212 & 164 & 6 & 6 & 168 & 5 & 5 & 166 & 90 & 233 \\
\hline 220 & 165 & 8 & 8 & 166 & 5 & 5 & 166 & 90 & 232 \\
\hline 229 & 165 & 7 & 7 & 166 & 5 & 5 & 166 & 90 & 230 \\
\hline 238 & 169 & 5 & 5 & 168 & 5 & 5 & 168 & 90 & 229 \\
\hline 246 & 173 & 5 & 5 & - & - & - & 172 & 90 & 228 \\
\hline 255 & 173 & 5 & 5 & - & - & - & 173 & 90 & 226 \\
\hline 264 & 172 & 5 & 5 & - & - & - & 171 & 90 & 225 \\
\hline 272 & 169 & 6 & 6 & 170 & 5 & 5 & 170 & 90 & 224 \\
\hline 281 & 162 & & 10 & 172 & 5 & 5 & 172 & 90 & 224 \\
\hline 289 & - & - & - & 174 & 5 & 5 & 173 & 90 & 223 \\
\hline 298 & - & - & - & 176 & 5 & 5 & 176 & 90 & 222 \\
\hline 307 & - & - & - & 178 & 5 & 5 & 178 & 90 & 221 \\
\hline 315 & - & - & - & 180 & 6 & 6 & 180 & 90 & 221 \\
\hline 324 & - & - & - & 180 & 8 & 8 & 180 & 90 & 220 \\
\hline 333 & - & - & - & 180 & 5 & 5 & 180 & 90 & 219 \\
\hline 341 & - & - & - & 180 & 5 & 5 & 180 & 90 & 219 \\
\hline 350 & - & - & - & 178 & 5 & 5 & 178 & 90 & 218 \\
\hline 359 & - & - & - & 174 & 5 & 5 & 174 & 90 & 218 \\
\hline 367 & - & - & - & 170 & 10 & 10 & 170 & 90 & 218 \\
\hline \multicolumn{10}{|c|}{ U7089 } \\
\hline 10 & 25 & 7 & 7 & 17 & 7 & 5 & 21 & 89 & 215 \\
\hline 20 & 38 & 5 & 5 & 35 & 7 & 7 & 36 & 89 & 215 \\
\hline 30 & 45 & 5 & 5 & 42 & 7 & 7 & 43 & 89 & 215 \\
\hline 40 & 51 & 7 & 7 & 51 & 5 & 5 & 51 & 89 & 215 \\
\hline 50 & 57 & 7 & 5 & 62 & 5 & 5 & 60 & 89 & 215 \\
\hline 60 & 63 & & 5 & 66 & 5 & 5 & 65 & 89 & 215 \\
\hline 70 & 66 & 7 & 5 & 69 & 5 & 5 & 68 & 89 & 215 \\
\hline 75 & - & - & - & 73 & 7 & 7 & 73 & 89 & 215 \\
\hline 80 & 70 & 7 & 5 & - & - & - & 70 & 89 & 215 \\
\hline \multicolumn{10}{|c|}{ U7089 (cont.) } \\
\hline 90 & 74 & $\overline{7}$ & 5 & - & - & - & 74 & 89 & 215 \\
\hline 100 & 78 & 7 & 5 & - & - & - & 78 & 89 & 215 \\
\hline 105 & 79 & 7 & 7 & - & - & - & 79 & 89 & 215 \\
\hline \multicolumn{10}{|c|}{ Uro94 } \\
\hline 20 & 32 & 5 & 5 & 32 & 5 & 5 & 32 & 72 & 39 \\
\hline 40 & 36 & 7 & 5 & 36 & 7 & 5 & 36 & 72 & 39 \\
\hline 60 & - & - & - & 35 & 7 & 5 & 35 & 72 & 39 \\
\hline
\end{tabular}


Table 4. continued

\begin{tabular}{|c|c|c|c|c|c|c|c|}
\hline \multicolumn{2}{|c|}{$\begin{array}{l}\text { Rad. } V_{\text {rot }}^{\text {app }} \\
\left(^{\prime \prime}\right)---\mathrm{km} \mathrm{s}^{-1} \\
\end{array}$} & & $\begin{array}{l}V_{\text {rot }}^{\text {rec }} \quad \pm \\
---\mathrm{km} \mathrm{s}^{-1}\end{array}$ & & $\begin{array}{l}\text { ave } \\
\text { rot } \\
\text { s }^{-1}\end{array}$ & $\begin{array}{c}i \\
\left(^{\circ}\right)\end{array}$ & $\begin{array}{l}\text { PA } \\
\left(^{\circ}\right)\end{array}$ \\
\hline \multicolumn{8}{|c|}{$N 4138$} \\
\hline 30 & 17819 & 4 & 18112 & 12 & 179 & & 15 \\
\hline 60 & 19110 & 10 & 20010 & & 5 & & 15 \\
\hline 90 & 18110 & 0 & 18115 & 20 & 181 & & 147 \\
\hline 122 & - & - & 16226 & 15 & 162 & & 1 \\
\hline 154 & 14514 & 14 & 14514 & 14 & 145 & & 14( \\
\hline 184 & - & - & 14718 & 18 & 147 & & 13 \\
\hline 213 & $-\quad-$ & - & 15021 & 21 & 150 & & 13 \\
\hline \multicolumn{8}{|c|}{ N4218 } \\
\hline 10 & 5110 & 7 & 7012 & & 60 & & 31 \\
\hline 20 & 8310 & 5 & $\begin{array}{ll}62 & 12\end{array}$ & 10 & 73 & & 31 \\
\hline
\end{tabular}

After the correction for instrumental resolution, the profile widths are generally corrected for broadening due to turbulent motions of the HI gas by applying TFq's formula

$$
\begin{aligned}
W_{R, l}^{2}= & W_{l}^{2}+W_{t, l}^{2}\left[1-2 \mathrm{e}^{-\left(\frac{W_{l}}{W_{c, l}}\right)^{2}}\right] \\
& -2 W_{l} W_{t, l}\left[1-\mathrm{e}^{-\left(\frac{W_{l}}{W_{c, l}}\right)^{2}}\right]
\end{aligned}
$$

where the subscript $l$ refers to the widths at the $l=20 \%$ or the $l=50 \%$ level of peak flux. This formula yields a linear subtraction of $W_{t, l}$ if $W_{l}>W_{c, l}$ and a quadratic subtraction if $W_{l}<W_{c, l}$. Values of $W_{t, l}$ and $W_{c, l}$ are different for line width corrections at the $20 \%$ and $50 \%$ levels. The values of $W_{c, l}$ indicate the profile widths where the transition from a boxy to a Gaussian shape occurs. The amount by which a global profile is broadened due to random motions is given by $W_{t, l}=2 k_{l} \sigma$ where, for a Gaussian velocity dispersion $\sigma, k_{20}=1.80$ and $k_{50}=1.18$.

The generally adopted values for $W_{c, l}$ are $W_{c, 20}=$ $120 \mathrm{~km} \mathrm{~s}^{-1}$ and $W_{c, 50}=100 \mathrm{~km} \mathrm{~s}^{-1}$. The more important values of $W_{t, l}$, however, have been subject of some debate among various authors. With our new HI synthesis data we can give a meaningful contribution to this debate.

Bottinelli et al. (1983) came up with an empirical approach, based on a minimization of the scatter in the TFrelation. They assumed an anisotropic velocity dispersion of the HI gas of $\sigma_{x}=\sigma_{y}=1.5 \sigma_{z}$ and a velocity dispersion perpendicular to the plane of $\sigma_{z}=10 \mathrm{~km} \mathrm{~s}^{-1}$. They determined the values of $k_{l}$ by minimizing the scatter in the TF-relation and found $k_{20}=1.89$ and $k_{50}=0.71$, indicating deviations from a Gaussian distribution (broader wings). Due to the assumed velocity anisotropy, $W_{t, l}$ has become a function of inclination angle and varies in the range $45<W_{t, 20}<57$ and $17<W_{t, 50}<21$ for inclinations ranging between $45^{\circ}<i<90^{\circ}$.

The same value of $k_{20}=1.89$ was adopted by $\mathrm{TFq}$ but they assumed an isotropic velocity dispersion of $\sigma_{x}=\sigma_{y}=\sigma_{z}=10 \mathrm{kms}^{-1}$ and consequently advocate $W_{t, 20}=2 \cdot 1.89 \cdot 10=38 \mathrm{~km} \mathrm{~s}^{-1}$, independent of inclination. They did not address the situation at the $50 \%$ level.

Fouqué et al. (1990) also assumed isotropy but adopted $\sigma=12 \mathrm{~km} \mathrm{~s}^{-1}$. They determined $k_{l}$ in a more direct way by comparing the corrected line width to the observed maximum rotational velocity $V_{\max }$ as derived from HI velocity fields. They found $k_{20}=1.96$ and $k_{50}=1.13$, indicating a near-Gaussian distribution, contrary to the findings of Bottinelli et al. Consequently, Fouqué et al. advocate the much larger values of $W_{t, 20}=47 \mathrm{~km} \mathrm{~s}^{-1}$ and $W_{t, 50}=27 \mathrm{~km} \mathrm{~s}^{-1}$ respectively.

A similar procedure was followed by Broeils (1992) using a sample of 21 galaxies with well defined HI velocity fields. Broeils made no a priori assumptions about the intrinsic velocity dispersion and did not decouple $k_{l}$ and $\sigma$. He did, however, recognize that $V_{\text {max }}$ may exceed $V_{\text {flat }}$ and he determined for each galaxy the values of $W_{t, l}^{\max }$ and $W_{t, l}^{\text {flat }}$ for which the differences

$\Delta W_{R, l}^{\max }=W_{R, l}-2 V_{\max } \sin (i)$

and

$\Delta W_{R, l}^{\mathrm{flat}}=W_{R, l}-2 V_{\text {flat }} \sin (i)$

become zero for each galaxy. He found mean values of

$W_{t, 20}^{\max }=21 \pm 2, \quad W_{t, 50}^{\max }=7 \pm 1$

$W_{t, 20}^{\text {flat }}=37 \pm 5, \quad W_{t, 50}^{\text {flat }}=25 \pm 4$.

(Note that he quoted the much larger scatters instead of the errors in the mean quoted above.) He rejected his results, probably discouraged by the large scatters, and adopted the values $W_{t, 20}=38$ and $W_{t, 50}=14 \mathrm{kms}^{-1}$ which he erroneously identifies with Bottinelli et al.'s results.

Finally, Rhee (1996a) performed the same investigation using 28 galaxies, most of them in common with Broeils' (1992) sample. Not surprisingly, he found

$$
\begin{aligned}
& W_{t, 20}^{\max }=20 \pm 2, \quad W_{t, 50}^{\max }=8 \pm 2 \\
& W_{t, 20}^{\text {flat }}=30 \pm 3, \quad W_{t, 50}^{\text {flat }}=18 \pm 3
\end{aligned}
$$

similar to Broeils' result.

Here, with our new and independent dataset, we follow the same strategy as Broeils and Rhee by investigating which values of $W_{t, l}$ allow an accurate retrieval of $V_{\max }$ and $V_{\text {flat }}$ from the broadened global profile. For this purpose we will only consider those 22 galaxies in our Ursa Major sample that show a flat part in their rotation curves (with a significant amount of HI gas) and that are free from a major change in inclination angle. Of these 22 , there are 6 galaxies with $V_{\max }>V_{\text {flat }}$. Note that both Broeils and Rhee used Bottinelli et al.'s prescription to correct for instrumental broadening which we are forced to adopt here to ensure a valid comparison between their 


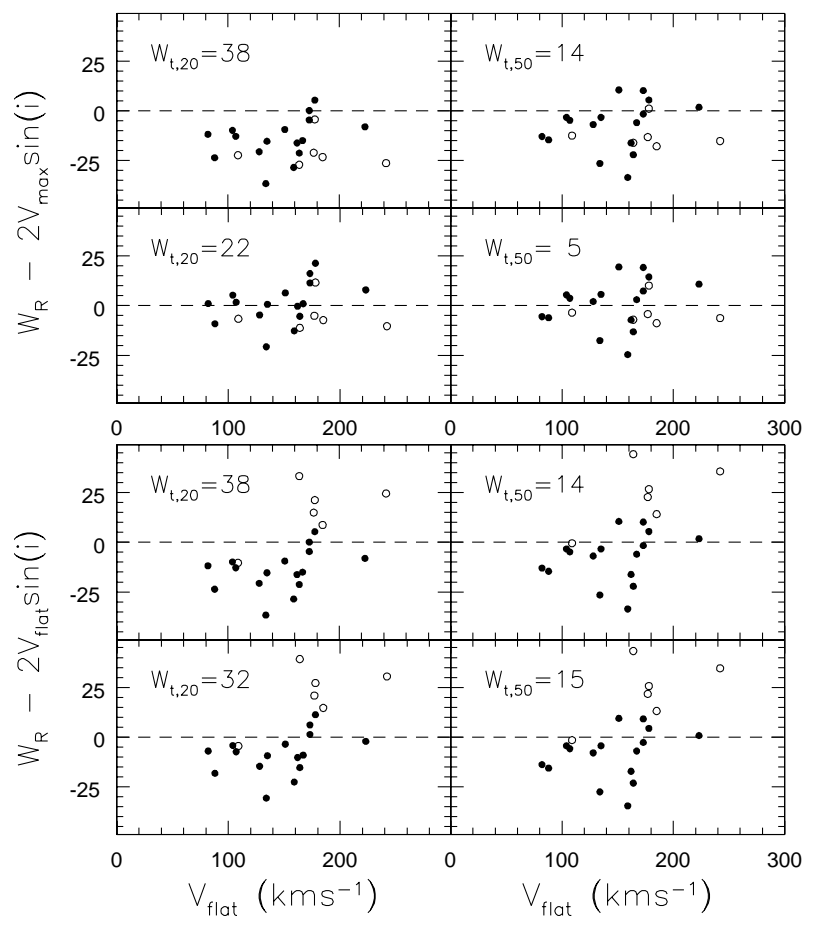

Fig. 2. Comparison of the global profile widths $W_{R, l}$, corrected for instrumental broadening and random motions, with $2 V_{\max } \sin (i)$ (upper panels) and with $2 V_{\text {flat }} \sin (i)$ (lower panels). The left panels consider $W_{R, 20}$ and the right panels $W_{R, 50}$. Different values of the random motion parameters $W_{t, l}$ are used. Open symbol indicate galaxies with declining rotation curves $\left(V_{\max }>V_{\text {flat }}\right)$ and filled symbols indicated galaxies without a declining part $\left(V_{\max }=V_{\text {flat }}\right)$. See Sect. 4 for further details

and our results. We calculated the values of $W_{t, l}^{\max }$ and $W_{t, l}^{\text {flat }}$ for which the average values

$$
\begin{aligned}
\overline{\Delta W_{R, l}^{\max }} & =\frac{1}{N} \sum\left(W_{R, l}-2 V_{\max } \sin (i)\right) \\
\overline{\Delta W_{R, l}^{\mathrm{flat}}} & =\frac{1}{N} \sum\left(W_{R, l}-2 V_{\text {flat }} \sin (i)\right)
\end{aligned}
$$

become zero. This is done for both the entire sample of $N=22$ galaxies and for the subsample of $N=16$ galaxies with $V_{\max }=V_{\text {flat }}$. For the entire sample we find

$$
\begin{aligned}
& W_{t, 20}^{\max }=22, \quad W_{t, 50}^{\max }=5 \\
& W_{t, 20}^{\text {flat }}=32, \quad W_{t, 50}^{\text {flat }}=15 .
\end{aligned}
$$

These values are in good agreement with the (rejected) results of Broeils and in excellent agreement with the results of Rhee. The values of $W_{t, l}^{\text {flat }}$ are larger than the values of $W_{t, l}^{\max }$ because the galaxies with $V_{\max }>V_{\text {flat }}$ in our sample have considerable amounts of HI gas at their peak velocity in the rotation curve. This gas, rotating at $V_{\max }$ broadens the global profile somewhat further. If we consider only the 16 galaxies for which $V_{\max }=V_{\text {flat }}$ we find

$$
W_{t, 20}^{\max }=W_{t, 20}^{\mathrm{flat}}=23, \quad W_{t, 50}^{\max }=W_{t, 50}^{\text {flat }}=6
$$

in agreement with the values of $W_{t, l}^{\max }$ we found when using all 22 galaxies.
Our results are illustrated in Fig. 2 where we show, for each of the 22 galaxies, the deviations $\Delta W_{R, l}^{\max }$ (upper panels) and $\Delta W_{R, l}^{\text {flat }}$ (lower panels) as a function of $V_{\text {flat }}$. Galaxies with $V_{\max }=V_{\text {flat }}$ are indicated by filled symbols, galaxies with $V_{\max }>V_{\text {flat }}$ are indicated by open symbols. The upper two panels in each block show the results one obtains when using Broeil's adopted values of $W_{t, 20}=38$ and $W_{t, 50}=14 \mathrm{~km} \mathrm{~s}^{-1}$.

From the upper panels in the upper block it is clear that the maximum rotational velocity as derived from the corrected global profiles is severely underestimated when using the values of $W_{t, l}$ derived by TFq and adopted by Broeils. This systematic underestimation disappears when $W_{t, 20}$ is decreased from 38 to $22 \mathrm{~km} \mathrm{~s}^{-1}$ and $W_{t, 50}$ is decreased from 14 to $5 \mathrm{~km} \mathrm{~s}^{-1}$. The upper two panels in the lower block show that if one is interested in the amplitude of the flat part, which is smaller than the maximum rotational velocity for galaxies with a declining rotation curve (open symbols), the average offset becomes less significant simply because the open symbols scatter upward. In this case, to obtain an average zero offset, we find similar values for $W_{t, l}$ as those adopted by Broeils. However, we find the curious situation that the corrected width of the global profile systematically overestimates $V_{\text {flat }}$ for galaxies with a declining rotation curve (open symbols) and systematically underestimates $V_{\text {flat }}$ for galaxies with a purely flat rotation curve (filled symbols).

From this we can conclude that, in a statistical sense, the maximum rotational velocity of a galaxy can be reasonably well retrieved from the width of the global profile when using $W_{t, 20}=22$ or $W_{t, 50}=5 \mathrm{~km} \mathrm{~s}^{-1}$. The amplitude of the flat part can not be retrieved consistently for a mixed sample containing galaxies with declining rotation curves. Note that we have explored only a restricted range of rotational velocities: $8-200 \mathrm{~km} \mathrm{~s}^{-1}$.

Our results also indicate a non-Gaussian distribution of random velocities in the sense that $W_{t, 20} / W_{t, 50} \neq$ $1.80 / 1.18$. Interpreting $W_{t, 20}$ and $W_{t, 50}$ in terms of velocity dispersions it follows that

$$
\begin{aligned}
& \sigma_{20}=W_{t, 20} / 2 k_{20}=6.1 \mathrm{kms}^{-1} \\
& \sigma_{50}=W_{t, 50} / 2 k_{50}=2.1 \mathrm{kms}
\end{aligned}
$$

where $k_{20}=1.80$ and $k_{50}=1.18$ for a Gaussian distribution. Recall, however, that we advocate a different correction for instrumental broadening than Bottinelli et al.'s scheme used by Broeils and Rhee. With our correction method for instrumental broadening we find the somewhat smaller values of:

$W_{t, 20}=22, \quad W_{t, 50}=2$.

These smaller values of $W_{t, l}$ allow to retrieve $V_{\text {flat }}$ from the global profiles of galaxies with purely flat rotation curves and $V_{\max }$ for galaxies with declining rotation curves. Applying our correction method for instrumental resolution and the above-mentioned value of $W_{t, 20}=22 \mathrm{~km} \mathrm{~s}^{-1}$ we find an rms scatter in $\Delta W_{20}=0.5 W_{R}^{i}-V_{\max }$ of $6.8 \mathrm{~km} \mathrm{~s}^{-1}$. 


\section{A comparison of inclinations}

Present day instrumentation allows accurate measurements of the luminosities and global HI profiles of galaxies. In general, the observed scatter in the TF-relation is larger than can be explained by the observational uncertainties in these measured parameters alone. However, the uncertainty in corrections sensitive to inclination contribute significantly to the observed scatter. For a sample of randomly oriented galaxies more inclined than 45 degrees, an uncertainty of 1,3 or 5 degrees in the inclination angle contributes respectively $0.04,0.12$ or 0.19 magnitudes to the scatter due to the uncertainty in line widths alone, assuming a slope in the TF-relation of -10 . Therefore, it is important to determine the inclination angle of a galaxy as accurate as possible and this issue deserves some special attention.

From the photometric and HI synthesis data available, three independent measurements of the inclination angle of a galaxy can in principle be obtained; $i_{\text {opt }}$ from the optical axis ratio, $i_{\mathrm{HI}}$ from the apparent ellipticity of the $\mathrm{HI}$ disk, and $i_{\mathrm{VF}}$ from fitting tilted rings to the HI velocity field. Each of these methods has its own systematic limitations which are important to recognize when estimating the actual inclination of a galaxy. In the following discussion we will briefly address those limitations and make an intercomparison of $i_{\mathrm{opt}}, i_{\mathrm{HI}}$, and $i_{\mathrm{VF}}$.

\section{1. $i_{\text {opt }}$ from optical axis ratios}

The most widely used formula to infer the inclination angle from the observed optical axis ratio $(b / a) \equiv q$ was provided by Hubble (1926):

$\cos ^{2}\left(i_{\mathrm{opt}}\right)=\frac{q^{2}-q_{0}^{2}}{1-q_{0}^{2}}$

where $q_{0}$ is the intrinsic thickness of an oblate stellar disk. Holmberg (1946) determined an average value of $q_{0}=0.20$ which is still commonly used although it is obvious from images of edge-on systems that large variations in $q_{0}$ exist. For instance, Fouqué et al. (1990) found $q_{0}$ to vary from 0.30 to 0.16 for spirals of morphological types Sa to $\mathrm{Sd}$ respectively and $q_{0}=0.42$ for galaxies of type Sdm-Im. Apart from the debate on the intrinsic thickness, the observed axis ratio $q$ itself has limited meaning since it is often defined at a certain isophote around which $q$ may still vary as a function of radius. From images of edgeon disks in the Ursa major cluster (see Paper I) it can often be observed that the axis ratio keeps increasing outward until the faintest isophotes. An extreme example is NGC 4389, dominated by a narrow bar and surrounded by an extended faint halo. The axis ratios presented in Table 1 were not determined at a fixed isophote but were chosen to represent the stellar disk instead of a bulge, lopsided structures or a faint halo.
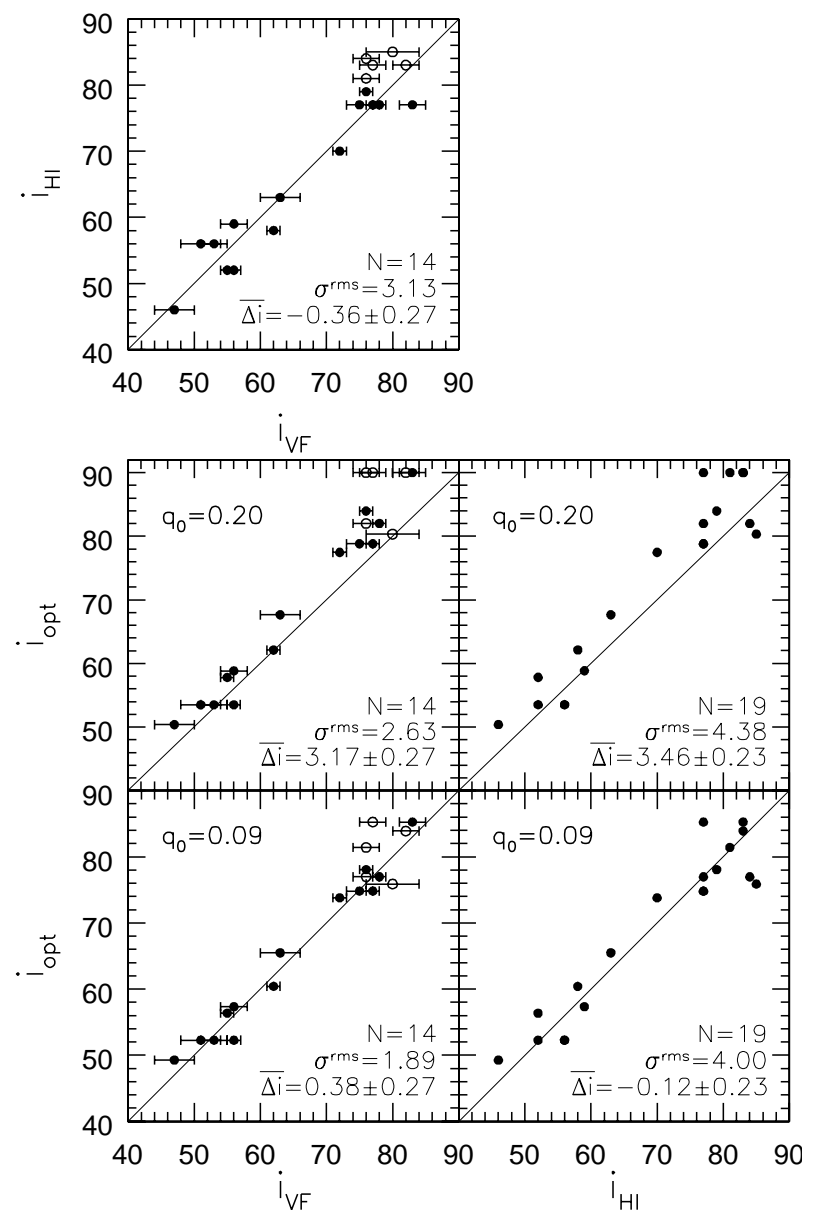

Fig. 3. Intercomparison of the three independently determined inclination angles $i_{\mathrm{opt}}, i_{\mathrm{HI}}$ and $i_{\mathrm{VF}}$. Only the filled symbols $\left(i_{\mathrm{HI}}<80^{\circ}\right.$ when $i_{\mathrm{VF}}$ is involved) are considered for the unweighted quantitative assessment

\section{2. $i_{H I}$ from the inclined $\mathrm{HI}$ disk}

Apart from the oblate stellar disk, the HI disk can also be used to determine the inclination. In general, the HI disk is much thinner than the stellar disk and its intrinsic thickness is of no concern. However, its patchiness, lopsidedness and the existence of warps and tidal tails may complicate the interpretation of the results from fitting ellipses to a certain HI isophote. Here, no correction for the intrinsic thickness of the HI layer was applied. However, the relatively large synthesized beams of imaging arrays at $21 \mathrm{~cm}$ may smear the observed HI disks to a rounder appearance. Therefore, a simple correction for beam smearing was applied to our measurements and the inclination of the HI disk was determined according to

$\cos ^{2}\left(i_{\mathrm{HI}}\right)=\frac{d_{\mathrm{HI}}^{2}-\Theta_{d}^{2}}{D_{\mathrm{HI}}^{2}-\Theta_{D}^{2}}$

where $D_{\mathrm{HI}}$ and $d_{\mathrm{HI}}$ are the observed major and minor axis diameters of the inclined HI disk obtained by fitting an ellipse to the outer column density levels. $\Theta_{D}$ and $\Theta_{d}$ are the sizes of the synthesized beam in the direction of the major and minor axis of the HI disk. 


\section{3. $i_{V F}$ from $\mathrm{HI}$ velocity fields}

The inclination angle of an HI disk can also be measured by fitting tilted-rings to its velocity field (Begeman 1989). However, the inclination angle and the rotational velocity are strongly coupled and reasonable results can only be obtained for inclination angles between roughly 50 and 75 degrees. This procedure requires accurate velocity fields with high signal-to-noise ratios as well as many independent points along a ring. The advantage that velocity fields offer is the possibility to identify warps and to check the kinematic regularity of the HI disk. For instance, the optical appearance of a galaxy may look very regular while the outer regions of the HI disk may be strongly warped toward edge-on (e.g. N3726). Such a warp would broaden the global profile and an inclination correction based on the optical axis ratio would lead to an overestimate of the rotational velocity when dividing the "warp-broadened" line width by $\sin \left(i_{\text {opt }}\right)$.

Note that the inclination measurement of a tilted ring may be affected by non-circular motions due to spiral arms, bars and lopsidedness.

\subsection{The comparison}

For the comparison between the three differently inferred inclination angles we considered only those 27 galaxies with fully reduced HI data for which the velocity fields and integrated HI maps are available. We excluded the interacting galaxies (N3769, N3893, U6973) because their outer isophotes (optical and $\mathrm{HI}$ ) are affected by tidal tails. We also excluded galaxies with perturbed or inadequately sampled velocity fields (N4088, U6969, N4389), galaxies with excessively patchy HI maps (N4102) and obviously lopsided galaxies (N4051). These eliminations leave us with 19 galaxies that have smooth outer isophotes, well filled HI disks and regular HI velocity fields.

Figure 3 presents the comparison between the three differently inferred inclination angles using two different values for $q_{0}$. When calculating mean differences and scatters using $i_{\mathrm{VF}}$, only galaxies with $i_{\mathrm{HI}}<80^{\circ}$ are considered because kinematic inclinations of highly inclined galaxies are systematically underestimated. The error bars on $i_{\mathrm{VF}}$ are based on the variations in $i_{\mathrm{VF}}$ between the various fitted rings but are not considered any further here.

The upper most panel compares $i_{\mathrm{VF}}$ with $i_{\mathrm{HI}}$. No significant offset is found for the 14 galaxies that meet the above-mentioned criteria. Assuming that $i_{\mathrm{VF}}$ and $i_{\mathrm{HI}}$ contribute equally to the scatter of 3.1 degrees implies that the inclination angle can be determined with an accuracy of 2.2 degrees from either the velocity fields or from the inclined HI disk. Note that the correlation turns up for $i_{\mathrm{HI}}>80^{\circ}$ due to the systematic underestimation of $i_{\mathrm{VF}}$ for highly inclined disks.

Comparing $i_{\mathrm{opt}}$ with $i_{\mathrm{VF}}$ and $i_{\mathrm{HI}}$ does show a significant offset of roughly 3 degrees when assuming $q_{0}=0.20$ (middle panels). This offset is biggest toward edge-on as would be expected in case of an overestimate of the intrin- sic thickness. Note that there are several galaxies with an observed optical axis ratio less than 0.20 which have been assigned an inclination angle of $90^{\circ}$.

This $3^{\circ}$ offset disappears when $q_{0}=0.09$ is used (lower panels) and the rms scatter is reduced to only 1.9 degrees for $i_{\mathrm{opt}}$ versus $i_{\mathrm{VF}}$ but is still 4.0 degrees in case of $i_{\mathrm{opt}}$ versus $i_{\mathrm{HI}}$. In the latter case, the scatter is caused by a few nearly edge-on systems for which the higher uncertainties have no influence on the deprojection of the rotational velocities.

The adopted inclinations and their errors, listed in Col. 11 of Table 1 are best estimates based on all the information available for a particular galaxy, including the morphology of dust lanes if present. For galaxies which lack fully reduced HI synthesis data, the inclination angles were inferred from the optical axis ratios using $q_{0}=0.09$ for galaxies of type Sc and later and $q_{0}=0.24$ for galaxies of type Sbc and earlier. The latter value of $q_{0}$ seemed justified by the observed axis ratios of the (nearly) edgeon systems N4013, N4026 and N4111 of types Sb, S0 and S0 respectively. Unfortunately, there are not enough suitable galaxies available to determine $q_{0}$ as a function of morphology.

\section{The atlas}

The atlas is presented in Appendix B and consists of two parts. The first part presents the data for the 30 galaxies which have been fully reduced and analyzed. The second part gives a less elaborate presentation of the remaining 13 galaxies which have been only partially reduced.

The reduction procedures described in Sect. 3 were applied to the data at all three angular resolutions. However, to facilitate the intercomparison of various results for a galaxy, it was decided to present the data of a particular galaxy at the same angular resolution as much as possible. The rotation curves are in some cases a combination of the rotation curves at various resolutions, the inner parts at the highest spatial resolution and the outer parts from data of lower resolution but higher signal-to-noise ratio. The channel maps are sometimes presented at a lower angular resolution than the other data.

Figure 4 shows the graphical layout for each of the 30 galaxies in the first part of the atlas. The data for each of the 13 galaxies in the second part of the atlas are presented on a single page which contains the tables and notes as well as the mosaic of channel maps, the global profile and the XV-diagram along the major axis. The contents of the various tables and panels are described below.

Tables and Notes - There are three tables presented for each galaxy. The upper left table contains information on the observations like date, integration time and correlator settings. The lower left table presents some of the quantities derived from the HI data like global profile widths, integrated HI flux, systemic velocity etc. The upper right table provides the noise and contour levels for the maps in the various panels. The contours in the 
First page

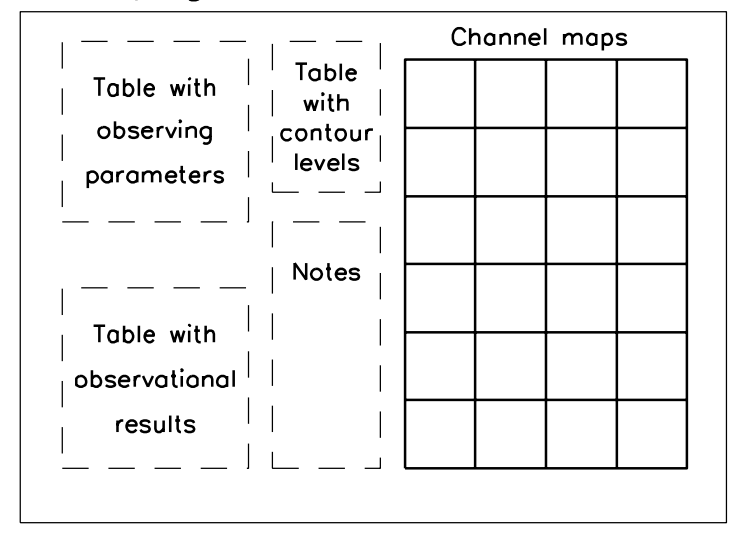

\section{Second page}

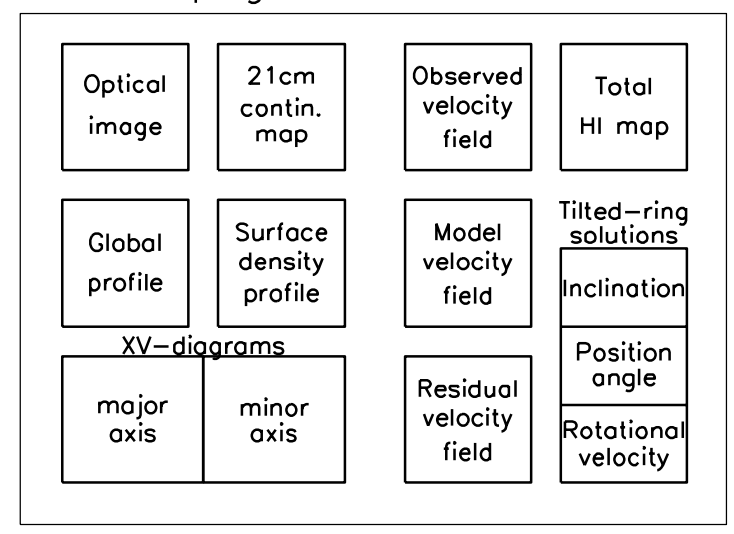

Fig. 4. Layout of the HI atlas pages for the 30 galaxies with fully reduced data. All the data for these galaxies are presented on two facing pages. Results for the 13 galaxies with partially reduced data are presented on a single page per galaxy and include only the channel maps, the global profile and the XVdiagram. The linear scale is $5.4 \mathrm{kpc}$ per arcminute

channel maps, $21 \mathrm{~cm}$ continuum maps and XV-diagrams are always drawn at levels which are related to the rms noise.

The notes contain information about specific aspects of a particular galaxy like optical and HI appearance.

Channel maps - The channel maps show how the HI emission behaves as a function of velocity. From these maps it is possible to recognize the presence of warps, non-circular motions and HI-bridges between interacting galaxies like $\mathrm{N} 3769 / 1135+48$ and $\mathrm{N} 3893 / \mathrm{N} 3896$. The contours are drawn at levels of $-3,-1.5$ (dashed), 1.5, 3, 4.5, 6, 9, $12,15, \ldots \sigma$. The value of the rms noise level $\sigma$ is given in the upper right table. In each panel, the cross indicates the adopted dynamical center of the galaxy. The ellipse in the upper left panel is centered on this position and the position angle is set at the kinematic major axis of the inner regions. The ellipticity represents the inclination as derived from the optical axis ratio and the major axis diameter is equal to $D_{25}^{b, i}$. The synthesized beam at half power is shown in the lower left corners of the left panels. The panel at the lower right shows the subtracted, "dirty" continuum map.

Optical image - The optical image of a galaxy was scanned from the blue POSS plates. In the upper left corner, the morphological type according to the RC3 is given. CCD images of far superior quality can be found in Paper I. An example is given for N3726 in which case the CCD image is pasted into the POSS image.

Radio continuum map - The cleaned $21 \mathrm{~cm}$ radio continuum map is plotted at the same scale as the optical image. The contours are drawn at levels of $-4,-2$ (dashed), 2, $4,8,16,32,64, \ldots \sigma$. The value of the rms noise $\sigma$ is given in the upper right table. The ellipse represents the optical image as in the upper left panel in the channels maps. The synthesized beam is plotted in the lower left corner and the small cross indicates the adopted position of the center of rotation.

Global profile - Each point in the global HI profile gives the primary-beam corrected, total HI flux density integrated over a single channel map. The errors are derived as explained in Sect. 3.2. The vertical arrow indicates the systemic velocity (lower left table on the first page) as derived from the $\mathrm{HI}$ velocity field and does not necessarily indicate the center of the global HI profile. A significant offset from the center could indicate non-circular motions or a strong lopsidedness. These anomalies can often be traced in the individual channel maps.

Surface density profile - The open and filled symbols indicate the azimuthally averaged radial surface densities for the approaching and receding sides. The solid line follows the average value. At the adopted distance of $18.6 \mathrm{Mpc}$, 1 arcmin corresponds to $5.4 \mathrm{kpc}$. The vertical arrow indicates $R_{25}^{b, i}$. The profile becomes unreliable for highly inclined systems since no correction for beam smearing was applied.

XV-diagrams - The position-velocity diagrams are shown for two orthogonal cuts through the adopted center of rotation along the kinematic major (left) and minor (right) axes. The position angles of these two axes are printed in the upper right corner of each panel. Note that the quoted position angles refer to the positive offset axes. Consequently, the position angle of the major axis refers to the receding side which also can be inferred from the channel maps. The vertical dashed line indicates the position of the center of rotation. The horizontal dashed line indicates the systemic velocity as derived from either the velocity field for galaxies with fully reduced data, or from the XV-diagram for galaxies in the second part of the atlas. The two vertical arrows show where the ellipse with major axis diameter $D_{25}^{b, i}$ intersects the XV-slice. The horizontal arrows in the left panel show the systemic velocity $V_{\text {sys }}^{\mathrm{GP}}$ derived from the global profile and $V_{\mathrm{sys}}^{\mathrm{GP}} \pm \frac{1}{2} W_{R, I}$ where $W_{R, I}$ is the width of the global profile at the $20 \%$ level of peak flux, corrected for instrumental broadening (see Sect. 3.2) and turbulent motions according to TFq.

Contours are at levels of $-3,-1.5$ (dashed), 1.5, 3, 4.5, $6,9,12,15, \ldots \sigma$. The value of the rms noise $\sigma$ is given in the upper right table. The cross in the lower left corners 
indicates the angular and velocity resolutions. An offset of 1 arcmin corresponds to a projected distance of $5.4 \mathrm{kpc}$ from the center.

The crosses give the projected rotational velocities as derived from the tilted rings fit. In some cases, at large radii, a cross can be found without any underlying signal in the XV-diagram. In such cases, the rotational velocity at that radius is defined by points in the velocity field away from the major axis. The open and filled circles indicate the projected rotational velocity estimated directly from these XV-diagrams. These points must be deprojected using the appropriate inclination and position angles to obtain the actual rotation curve for both halves of the galaxy.

Total HI map - All pixels in the total HI map with a positive signal have a greyscale value assigned. Because the signal-to-noise ratio along a contour is not constant, the " $3 \sigma$-contour" is not defined. Section 3.3 and the appendix explain why and how the noise varies across an integrated HI map.

The second contour in the total HI maps corresponds to the average value of all pixels with a signal-to-noise ratio between 2.75 and 3.25 and this contour can thus be considered as a pseudo $3 \sigma$-contour. Wherever a contour goes through an area with $\left(\frac{S}{N}\right)<3$, the contour is plotted much thinner. Consequently, the lowest contour, plotted at the " $1.5 \sigma$ " level, is plotted thin over most of its stretch. The various contour levels in atoms $\mathrm{cm}^{-2}$ are given in the upper right table. The size of the synthesized beam is plotted in the lower left corner. The beamwidths are the same as in the channel maps unless specified otherwise in the note. The small cross indicates the adopted position of the center of rotation ( 1 arcmin corresponds to $5.4 \mathrm{kpc}$ ).

Velocity fields - The greyscales indicate the pixels where a radial velocity was measured. Darker greyscales and white isovelocity contours indicate the receding side. The thick first black contour adjacent to the white ones indicates the adopted systemic velocity. In the ideal case of circular motion and no noise, this thick contour should be a straight line through the center and coinciding with the kinematic minor axis of the galaxy. The isovelocity contours are plotted with constant velocity intervals as given by the upper right table. The synthesized beam is plotted in the lower left corner.

The observed velocity field was modeled by fitting tilted rings to it. The orientation and rotational velocity of each ring were then used to construct the model velocity field. The model velocity field is plotted with the same orientation and on the same scale as the observed velocity field. The isovelocity contours are plotted at the same velocities in the observed as in the model velocity fields. For nearly edge-on systems, the model velocity field is only one or two pixels wide in which case no contours could be drawn.

The residual velocity field was made by subtracting the model from the observed velocity field. White contours indicate positive residuals, black contours indicate negative residuals. The contour levels are $\ldots,-15,-10,-5,5,10$, $15, \ldots \mathrm{km} \mathrm{s}^{-1}$.

Tilted-ring fits - The three combined panels show the results from the tilted-ring fits to the observed velocity field. The upper panel shows the inclination angle, the middle panel the position angle and the lower panel the rotational velocity.

The crosses with errorbars in the panels for inclination and position angle are the results from the second step of the fitting procedure as explained in Sect. 3.6.1. The dashed lines, mostly coinciding with the solid lines, in these upper two panels indicate the final values of the inclination and position angles kept fixed when the rotational velocity was fitted. The resulting rotation curve is shown by crosses with errorbars in the lower panel. The errorbars indicate the formal errors, as given by the least squares minimization algorithm.

The horizontal arrows in the upper two panels indicate the inclination and position angles as derived from the optical isophotes in the outer regions. The diamonds indicate the inclination and position angles as determined from the total HI maps. When the total HI maps are very patchy, these diamonds are very uncertain. The horizontal arrow in the lower panel indicates the rotational velocity as derived from the width of the global HI profile corrected for instrumental broadening, turbulent motion and inclination. The adopted inclination is representative for the outer parts. The vertical arrow in the lower panel indicates $R_{25}^{b, i}$

The solid lines in the upper and middle panels show the inclination and position angles that were adopted to deproject the radial velocities determined from the XVdiagrams. This deprojection results in the rotation curves plotted as open and filled circles in the lower panel (same symbols as in the XV-diagrams). Note that although the rotational velocities at a certain radius may be different for the approaching and receding sides, both sides were assumed to have the same inclination and position angles at that radius. The solid line in the lower panel shows the mean rotation curve derived from the XV-diagram. 1 arcmin on the horizontal axis corresponds to $5.4 \mathrm{kpc}$.

Tabulated data - The various parameters derived from the HI data presented here are summarized in Table 5. Column (1) gives the NGC or UGC numbers.

Columns (2)-(5) give the uncorrected widths with formal errors of the global profiles at $20 \%$ and $50 \%$ of the peak flux.

Column (6) gives the instrumental velocity resolution at which the global profiles were observed.

Columns (7) and (8) contain the heliocentric systemic velocities and their uncertainties as derived from the global profiles. 
Table 5. Results from the HI synthesis observations

\begin{tabular}{|c|c|c|c|c|c|c|c|c|c|c|c|c|c|c|c|c|c|c|c|c|c|}
\hline \multirow{2}{*}{$\begin{array}{c}\text { Name } \\
(1) \\
\end{array}$} & \multicolumn{2}{|c|}{$W_{20} \pm$} & \multicolumn{2}{|c|}{$\begin{array}{c}W_{50 \pm} \\
---\mathrm{km}\end{array}$} & Res. & \multicolumn{2}{|c|}{$V_{\text {hel }} \pm$} & \multicolumn{2}{|c|}{$\int_{\mathrm{Jy} \mathrm{km} \mathrm{s}^{-1}} S \mathrm{~d} v \pm$} & \multicolumn{2}{|c|}{$\begin{array}{c}F_{\nu \pm} \\
-\mathrm{mJy}-\end{array}$} & \multirow{2}{*}{$\begin{array}{r}R_{\mathrm{HI}} \\
\left({ }^{\prime}\right) \\
(13) \\
\end{array}$} & \multirow{2}{*}{$\begin{array}{r}R^{\operatorname{lmp}} \\
\left({ }^{\prime}\right) \\
(14) \\
\end{array}$} & \multicolumn{2}{|c|}{$\begin{array}{c}V_{\mathrm{rot}}^{\mathrm{Imp}} \pm \\
-\mathrm{km} \mathrm{s}^{-1}-\end{array}$} & \multicolumn{2}{|c|}{ shape } & \multicolumn{2}{|c|}{ 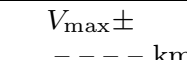 } & \multicolumn{2}{|c|}{$\begin{array}{r}V_{\text {flat }} \pm \\
----\end{array}$} \\
\hline & $(2)$ & $(3)$ & (4) & $(5)$ & $(6)$ & $(7)$ & $(8)$ & (9) & $(10)$ & (11) & $(12)$ & & & $(15)$ & $(16)$ & (17) & & $(18)$ & (19) & $(20)$ & $(21)$ \\
\hline \multicolumn{22}{|c|}{ Galaxies with fully reduced HI data: } \\
\hline U6399 & 188.1 & 1.4 & 172.5 & 2.9 & 8.3 & 791.5 & 0.6 & 10.5 & 0.3 & $<2.5$ & & 1.68 & 1.50 & 88 & 5 & $\mathrm{R} / \mathrm{F}$ & & 88 & 5 & 88 & 5 \\
\hline U6446 & 154.1 & 1.0 & 131.9 & 1.2 & 5.0 & 644.3 & 0.8 & 40.6 & 0.5 & $<7.2$ & & 2.96 & 2.93 & 80 & 11 & $\mathrm{~F}$ & $\mathrm{~L}$ & 82 & 4 & 82 & 4 \\
\hline N3726 & 286.5 & 1.6 & 260.6 & 1.8 & 5.0 & 865.6 & 0.9 & 89.8 & 0.8 & 49.7 & 5.0 & 4.24 & 6.22 & 167 & 15 & $\mathrm{~F} /(\mathrm{D})$ & & 162 & 9 & 162 & 9 \\
\hline N3769 & 265.3 & 6.7 & 230.5 & 3.6 & 8.3 & 737.3 & 1.8 & 62.3 & 0.6 & 12.1 & 2.9 & 4.31 & 7.10 & 113 & 11 & $\mathrm{~F} /(\mathrm{D})$ & & 122 & 8 & 122 & 8 \\
\hline U6667 & 187.5 & 1.4 & 178.1 & 1.9 & 5.0 & 973.2 & 1.2 & 11.0 & 0.4 & $<2.7$ & & 1.64 & 1.50 & 86 & 3 & $\mathrm{R}$ & $\mathrm{L}$ & 86 & 3 & 86 & 3 \\
\hline N3877 & 373.4 & 5.0 & 344.5 & 6.2 & 33.2 & 895.4 & 3.8 & 19.5 & 0.6 & 35.6 & 2.4 & 2.19 & 2.17 & 169 & 7 & $\mathrm{~F}$ & $\mathrm{~L}$ & 167 & 11 & 167 & 11 \\
\hline N3893 & 310.9 & 1.0 & 277.9 & 4.1 & 5.0 & 967.2 & 1.0 & 69.9 & 0.5 & 137.4 & 2.9 & 3.98 & 3.88 & 148 & 19 & $\mathrm{~F} /(\mathrm{D})$ & & 188 & 11 & 188 & 11 \\
\hline N3917 & 294.5 & 1.9 & 279.1 & 2.1 & 8.3 & 964.6 & 1.4 & 24.9 & 0.6 & $<7.2$ & & 2.69 & 2.83 & 137 & 8 & $\mathrm{~F}$ & & 135 & 3 & 135 & 3 \\
\hline N3949 & 286.5 & 1.4 & 258.3 & 1.7 & 8.3 & 800.2 & 1.2 & 44.8 & 0.4 & 134.1 & 3.6 & 2.62 & 1.35 & 169 & 8 & $\mathrm{~F}$ & $\mathrm{~L}$ & 164 & 7 & 164 & 7 \\
\hline N3953 & 441.9 & 2.4 & 413.9 & 3.2 & 33.1 & 1052.3 & 2.0 & 39.3 & 0.8 & 50.9 & 2.5 & 3.32 & 3.00 & 215 & 10 & $\mathrm{~F}$ & & 223 & 5 & 223 & 5 \\
\hline N3972 & 281.2 & 1.4 & 260.7 & 5.5 & 8.3 & 852.2 & 1.4 & 16.6 & 0.4 & $<5.8$ & & 1.92 & 1.67 & 134 & 5 & $\mathrm{R}$ & $\mathrm{L}$ & 134 & 5 & $\ldots$ & .. \\
\hline U6917 & 208.9 & 3.2 & 189.6 & 1.6 & 8.3 & 910.7 & 1.4 & 26.2 & 0.3 & $<4.4$ & & 2.42 & 2.00 & 111 & 7 & $\mathrm{R} / \mathrm{F}$ & & 104 & 4 & 104 & 4 \\
\hline U6923 & 166.8 & 2.4 & 147.1 & 4.5 & 10.0 & 1061.6 & 2.2 & 10.7 & 0.6 & $<2.6$ & & 1.29 & 1.02 & 81 & 5 & $\mathrm{R}$ & $\mathrm{L}$ & 81 & 5 & $\ldots$ & .. \\
\hline U6930 & 136.5 & 0.5 & 122.1 & 0.7 & 8.3 & 777.2 & 0.4 & 42.7 & 0.3 & $<5.8$ & & 3.20 & 3.17 & 108 & 7 & $\mathrm{R} / \mathrm{F}$ & & 107 & 4 & 107 & 4 \\
\hline N3992 & 478.5 & 1.4 & 461.4 & 2.4 & 10.0 & 1048.2 & 1.2 & 74.6 & 1.5 & 30.2 & 7.6 & 4.75 & 6.67 & 237 & 9 & $\mathrm{~F} / \mathrm{D}$ & & 272 & 6 & 242 & 5 \\
\hline U6940 & 59.3 & 3.8 & 40.6 & 7.8 & 10.0 & 1118.0 & 1.7 & 2.1 & 0.3 & $<1.3$ & & 0.61 & 0.33 & 37 & 4 & $\mathrm{R}$ & & 37 & 4 & $\ldots$ & .. \\
\hline U6962 & 220.3 & 6.6 & 182.4 & 3.7 & 8.3 & 807.4 & 3.2 & 10.0 & 0.3 & 13.4 & 1.7 & 1.38 & 1.00 & 171 & 7 & $\mathrm{R}$ & $\mathrm{L}$ & 171 & 7 & $\ldots$ & .. \\
\hline N4010 & 277.7 & 1.0 & 264.1 & 1.2 & 8.3 & 901.9 & 0.8 & 38.2 & 0.3 & 16.9 & 1.6 & 3.36 & 2.00 & 122 & 2 & $(\mathrm{R}) / \mathrm{F}$ & $\mathrm{L}$ & 128 & 9 & 128 & 9 \\
\hline U6969 & 132.1 & 6.4 & 123.5 & 2.9 & 10.0 & 1118.5 & 2.4 & 6.1 & 0.5 & $<3.8$ & & 0.95 & 0.85 & 79 & 5 & $\mathrm{R}$ & & 79 & 5 & $\ldots$ & \\
\hline U6973 & 367.8 & 1.8 & 350.4 & 1.2 & 8.3 & 700.5 & 1.0 & 22.9 & 0.2 & 127.5 & 2.1 & 2.21 & 1.50 & 180 & 8 & $\mathrm{~F} /(\mathrm{D})$ & & 173 & 10 & 173 & 10 \\
\hline U6983 & 188.4 & 1.3 & 173.0 & 1.1 & 5.0 & 1081.9 & 0.8 & 38.5 & 0.6 & $<5.4$ & & 3.07 & 3.00 & 109 & 12 & $\mathrm{~F}$ & & 107 & 7 & 107 & 7 \\
\hline N4051 & 255.4 & 1.8 & 224.6 & 1.5 & 5.0 & 700.3 & 1.2 & 35.6 & 0.8 & 26.5 & 2.6 & 2.89 & 2.33 & 153 & 10 & $\mathrm{R} / \mathrm{F}$ & $\mathrm{L}$ & 159 & 13 & 159 & 13 \\
\hline N4085 & 277.4 & 6.6 & 255.4 & 7.8 & 19.8 & 745.7 & 5.0 & 14.6 & 0.9 & 44.1 & 1.3 & 1.94 & 1.18 & 136 & 7 & $\mathrm{R} / \mathrm{F}$ & $\mathrm{L}$ & 134 & 6 & 134 & 6 \\
\hline N4088 & 371.4 & 1.7 & 342.1 & 1.9 & 19.8 & 756.7 & 1.2 & 102.9 & 1.1 & 222.3 & 1.9 & 4.25 & 4.10 & 174 & 8 & $F^{\prime} /(D)$ & $\mathrm{L}$ & 173 & 14 & 173 & 14 \\
\hline N4100 & 401.8 & 2.0 & 380.5 & 1.8 & 19.9 & 1074.4 & 1.3 & 41.6 & 0.7 & 54.3 & 1.7 & 3.45 & 4.35 & 159 & 9 & $\mathrm{~F} / \mathrm{D}$ & & 195 & 7 & 164 & 13 \\
\hline N4102 & 349.8 & 2.0 & 322.4 & 8.5 & 8.3 & 846.3 & 2.0 & 8.0 & 0.2 & 276.0 & 1.5 & 1.16 & 0.83 & 178 & 12 & $\mathrm{~F}$ & & 178 & 11 & 178 & 11 \\
\hline $\mathrm{N} 4157$ & 427.6 & 2.2 & 400.7 & 3.1 & 19.9 & 774.4 & 1.8 & 107.4 & 1.6 & 179.6 & 2.3 & 4.60 & 5.67 & 185 & 14 & $\mathrm{~F} / \mathrm{D}$ & & 201 & 7 & 185 & 10 \\
\hline N4183 & 249.6 & 1.2 & 232.5 & 1.5 & 8.3 & 930.1 & 1.0 & 48.9 & 0.7 & $<5.8$ & & 3.07 & 4.02 & 113 & 11 & $\mathrm{~F} / \mathrm{D}$ & $\mathrm{L}$ & 115 & 6 & 109 & 4 \\
\hline N4217 & 428.1 & 5.1 & 395.6 & 3.8 & 33.2 & 1027.0 & 3.0 & 33.8 & 0.7 & 115.6 & 2.2 & 3.19 & 3.17 & 178 & 12 & $\mathrm{~F} / \mathrm{D}$ & & 191 & 6 & 178 & 5 \\
\hline N4389 & 184.0 & 1.5 & 164.9 & 1.6 & 8.3 & 718.4 & 1.2 & 7.6 & 0.2 & 23.3 & 1.2 & 1.30 & 1.02 & 110 & 8 & $\mathrm{R}$ & & 110 & 8 & $\ldots$ & .. \\
\hline \multicolumn{22}{|c|}{ Galaxies with partially reduced HI data: } \\
\hline N3718 & 492.8 & 1.0 & 465.7 & 1.0 & 33.2 & 993.0 & 0.8 & 140.9 & 0.9 & 11.4 & 0.4 & & 6.67 & 223 & 12 & $\mathrm{~F}$ & & 232 & 11 & 232 & 11 \\
\hline N3729 & 270.8 & 1.5 & 253.2 & 3.9 & 33.2 & 1059.8 & 1.4 & 5.5 & 0.3 & 18.0 & 0.9 & & 1.00 & 151 & 11 & $\mathrm{~F}$ & & 151 & 11 & 151 & 11 \\
\hline U6773 & 110.4 & 2.3 & 91.1 & 2.2 & 8.3 & 923.6 & 1.6 & 5.6 & 0.4 & $<2.6$ & & & 0.67 & 45 & 5 & $\mathrm{R}$ & $\mathrm{L}$ & 45 & 5 & $\ldots$ & .. \\
\hline U6818 & 166.9 & 2.3 & 141.9 & 5.7 & 8.3 & 808.1 & 2.1 & 13.9 & 0.2 & 2.4 & 1.0 & & 1.33 & 74 & 7 & $\mathrm{R} /(\mathrm{F})$ & $\mathrm{L}$ & 73 & 5 & 73 & 5 \\
\hline U6894 & 141.8 & 1.1 & 132.2 & 1.5 & 8.3 & 848.6 & 1.8 & 5.8 & 0.2 & $<2.7$ & & & 0.67 & 63 & 5 & $\mathrm{R}$ & & 63 & 5 & $\ldots$ & .. \\
\hline N3985 & 160.2 & 3.7 & 88.0 & 2.4 & 8.3 & 948.2 & 2.0 & 15.7 & 0.6 & 9.7 & 1.4 & & 0.50 & 93 & 7 & $\mathrm{R}$ & & 93 & 7 & $\ldots$ & .. \\
\hline N4013 & 425.0 & 0.9 & 395.0 & 0.8 & 33.0 & 831.3 & 0.6 & 41.5 & 0.2 & 36.3 & 0.8 & & 6.12 & 170 & 10 & $\mathrm{~F} / \mathrm{D}$ & & 195 & 3 & 177 & 6 \\
\hline U7089 & 156.7 & 1.7 & 97.7 & 3.0 & 10.0 & 770.0 & 1.5 & 17.0 & 0.6 & $<3.4$ & & & 1.75 & 79 & 7 & $\mathrm{R}$ & $\mathrm{L}$ & 79 & 7 & $\ldots$ & .. \\
\hline U7094 & 83.7 & 1.7 & 71.9 & 5.5 & 10.0 & 779.6 & 1.6 & 2.9 & 0.2 & $<2.6$ & & & 1.00 & 35 & 6 & $\mathrm{R}$ & $\mathrm{L}$ & 35 & 6 & $\ldots$ & .. \\
\hline N4117 & 289.4 & 7.5 & 260.3 & 5.2 & 10.0 & 934.0 & 1.5 & 6.9 & 1.1 & 3.7 & 1.2 & & & & & $?$ & & & & & \\
\hline N4138 & 331.6 & 4.5 & 266.0 & 7.8 & 19.9 & 893.8 & 3.9 & 19.2 & 0.7 & 16.7 & 4.6 & & 3.55 & 150 & 21 & $\mathrm{~F} / \mathrm{D}$ & & 195 & 7 & 147 & 12 \\
\hline $\mathrm{N} 4218$ & 138.0 & 5.0 & 79.9 & 1.9 & 8.3 & 729.9 & 1.7 & 7.8 & 0.2 & 6.3 & 0.8 & & 0.33 & 73 & 7 & $\mathrm{R}$ & & 73 & 7 & $\ldots$ & . \\
\hline N4220 & 438.1 & 1.3 & 423.3 & 3.3 & 33.1 & 914.2 & 1.2 & 4.4 & 0.3 & $<4.9$ & & & & & & $?$ & & & & & \\
\hline
\end{tabular}




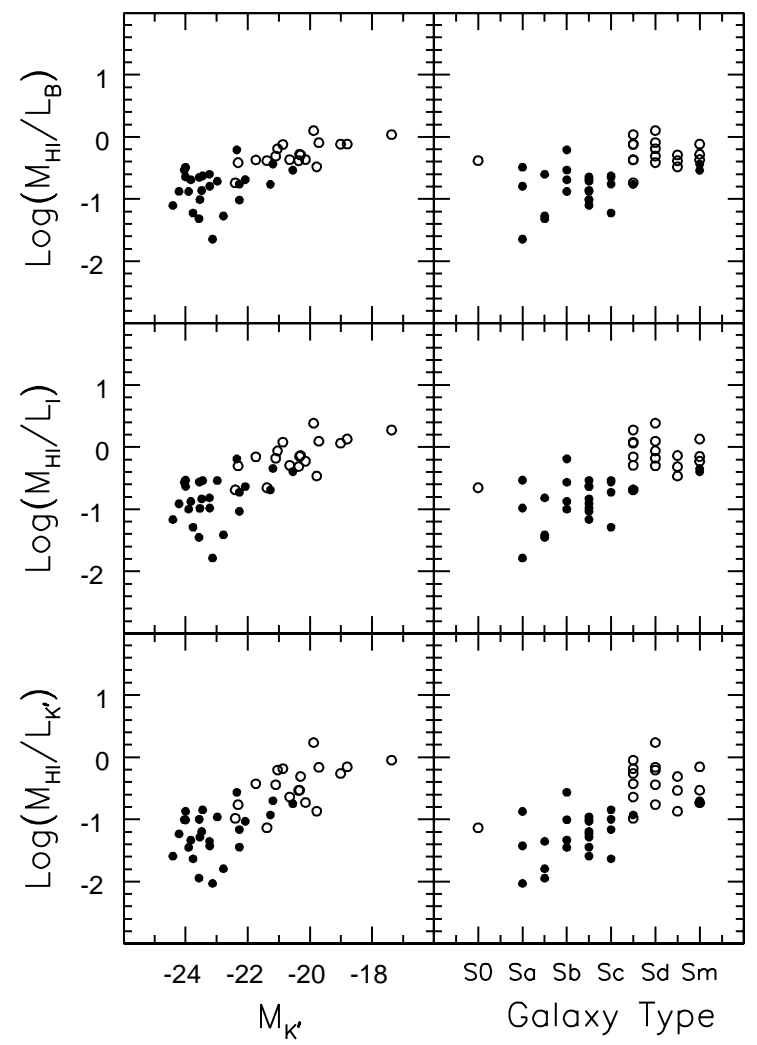

Fig. 5. Correlations between HI mass-to-light ratios and absolute $K^{\prime}$-band magnitudes (left column) and morphological type (right column). Solid symbols indicate HSB galaxies and open symbols denote galaxies of the LSB type

Columns (9) and (10) provide the integrated HI flux and the uncertainty in $\mathrm{Jy} \mathrm{km} \mathrm{s}^{-1}$.

Columns (11) and (12) contain the $21 \mathrm{~cm}$ continuum flux density and its uncertainty in mJy. In case no continuum flux was detected, a $3 \sigma$ upper limit for extended emission is given.

Column (13) gives the radius of the $\mathrm{HI}$ disk, $R_{\mathrm{HI}}$ in arcmin, at the azimuthally averaged surface density of $1 M_{\odot} \mathrm{pc}^{-2}$, measured from the radial surface density profiles.

Column (14) gives the radius $R^{\operatorname{lmp}}$ of the last measured point of the rotation curve in arcmin. The differences between $R^{\mathrm{lmp}}$ and $R_{\mathrm{HI}}$ depend on the sensitivity of the measurement and the distribution of the HI gas along the kinematic major axis.

Columns (15) and (16) give the rotational velocity of the last measured point $V^{\mathrm{lmp}}$ and its uncertainty.

Column (17) contains information on the overal shape of the rotation curve; $\mathrm{R}$ : rising rotation curve, $\mathrm{F}$ : the rotation curve shows a flat part, D: the rotation curve shows a declining part, L: lopsided.

Columns (18) and (19) give the maximum observed rotational velocity $V_{\max }$ and its uncertainty. For galaxies with a rising rotation curve (R) $V_{\max }=V^{\mathrm{lmp}}$.

Columns (20) and (21) give the average rotational velocity of the flat part of the rotation curve $V_{\text {flat }}$ and its uncertainty. For galaxies with a flat rotation curve (F)
$V_{\text {flat }}=V_{\text {max }}$ may deviate from $V^{\operatorname{lmp}}$ because $V_{\text {flat }}$ was averaged over the flat part of the rotation curve while $V^{\mathrm{lmp}}$ was measured at a single point.

\section{HI properties of spirals}

The HI survey of the Ursa Major cluster presented here provides not only the kinematical information necessary for the study of the Tully-Fisher relation and of the dark and luminous matter for a well defined sample of galaxies. It also serves to investigate the general HI properties of disks and to make a comparison with galaxies in the field and with galaxies in denser environments. The HI studies of the Virgo cluster galaxies by Warmels (1988a, 1988b) and especially by Cayatte et al. (1990) have shown that the spiral galaxies in the central parts of the cluster have smaller HI disks of lower surface density. In the Hydra Cluster McMahon (1993) did not find any such significant HI deficiency. She did find, however, a surprisingly large number of isolated HI-rich dwarf galaxies near the center of the cluster. Dickey (1997) surveyed the more distant Hercules SuperCluster and found a similar HI deficiency of spirals near the X-ray gas as in the case of Virgo.

The Ursa Major cluster differs from those just mentioned. It has no central concentration, no X-ray emitting gas and contains mainly spirals of late morphological types. In many respects its conditions are very similar to those of a field environment. For this reason it is useful to compare the properties of the Ursa Major spirals not only with those of galaxies in dense cluster environments but also with those of field galaxies as found in various recent studies (see e.g. Broeils 1992; Puche \& Carignan 1991; Rhee 1996a; Swaters 1999).

Here we give only a brief description of the global parameters and of the main properties of the $\mathrm{HI}$ disks of the Ursa Major galaxies. A more detailed discussion and a comparison with results from previous work is beyond the scope if this data paper.

\subsection{Global parameters}

Integral properties and global parameters of spiral galaxies have been derived for a large number of objects from single-dish observations (cf. Roberts \& Haynes 1994). In recent years also synthesis observations (Broeils 1992; Rhee 1996a) have been used to obtain similar information for smaller samples of galaxies.

The $M_{\mathrm{HI}} / L$ ratios obtained for the galaxies of the Ursa Major sample listed in Table 5 are shown here in Fig. 5 as a function of absolute magnitude and of morphological type. It is well known (see refs. above) that the $M_{\mathrm{HI}} / L$ ratio of galaxies depends on luminosity and morphological type. The present sample of galaxies shows a clear increase of the HI mass fraction with decreasing luminosity and from early to late morphological types. The correlation is clearly stronger for the $K^{\prime}$-band magnitudes which is a better tracer of the stellar mass. 


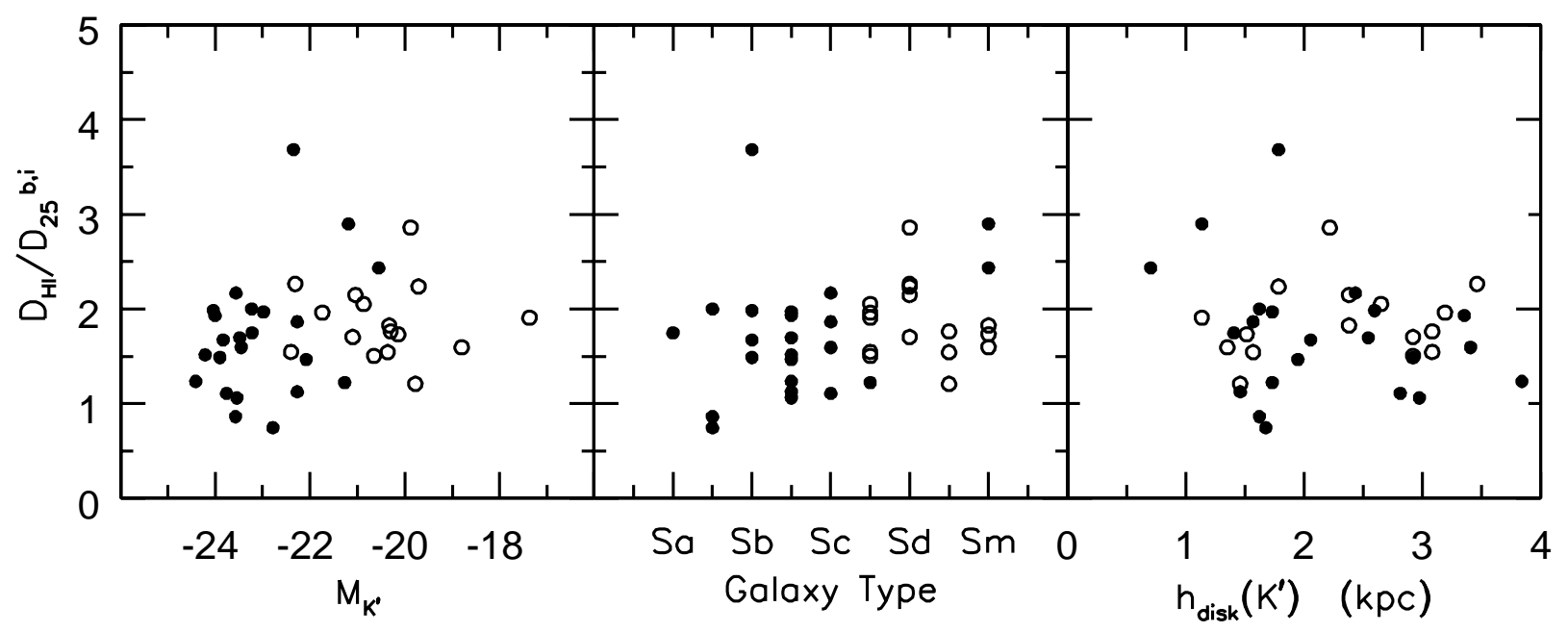

Fig. 6. Ratio of HI-to-optical diameter as a function of $K^{\prime}$ magnitudes, morphological type and disk scale length. The HI diameters were measured at the $1 M_{\odot} \mathrm{pc}^{-2}$ isodensity contour. Solid symbols indicate HSB galaxies and open symbols denote galaxies of the LSB type

\subsection{Sizes of $\mathrm{HI}$ disks and radial surface density profiles}

Detailed information on the sizes and radial distributions of HI disks has been obtained recently from synthesis observations of limited samples of field and cluster galaxies (Broeils \& Van Woerden 1994; Cayatte et al. 1994; Rhee $1996 \mathrm{a}$ and 1996b). Here we present only some of the main results on the comparison of $\mathrm{HI}$ and optical diameters, on the relation between HI mass and diameter and on the radial density profiles for the Ursa Major sample.

Figure 6 shows the ratio of the HI diameter $D_{\mathrm{HI}}$ (defined at an HI surface density of $1 M_{\odot} \mathrm{pc}^{-2}$ ) to the optical diameter $D_{25}^{b, i}$ as a function of luminosity, morphological type and disk scale-length. The diagrams do not indicate any clear trend or dependence of the diameter ratio on any of those quantities. The spread is large. There may be a hint of a slight increase of the ratio from early to later types and from more luminous to less luminous systems. For almost all galaxies $D_{\mathrm{HI}}$ is larger than $D_{25}^{b, i}$.

As shown in previous investigations (see refs. above), there is a tight correlation between HI mass and HI diameter as illustrated in Fig. 7. This implies a nearly constant mean HI surface density regardless of size. The HI mass correlates also with the optical diameter, but, as in previous work, with a much larger scatter.

\subsection{Warps, asymmetries and interactions}

The radial distributions of the HI surface densities are shown in Fig. 8. Only galaxies with fully reduced data, more inclined than 80 degrees and with $R_{\mathrm{HI}}<$ 1 arcmin are considered in order to avoid the most severe cases of beam smearing. There is clearly a considerable diversity of shapes and intensities. The upper row shows the profiles grouped for galaxies of similar morphological types. The dotted lines represent low surface brightness galaxies. No obvious trend with morphological type

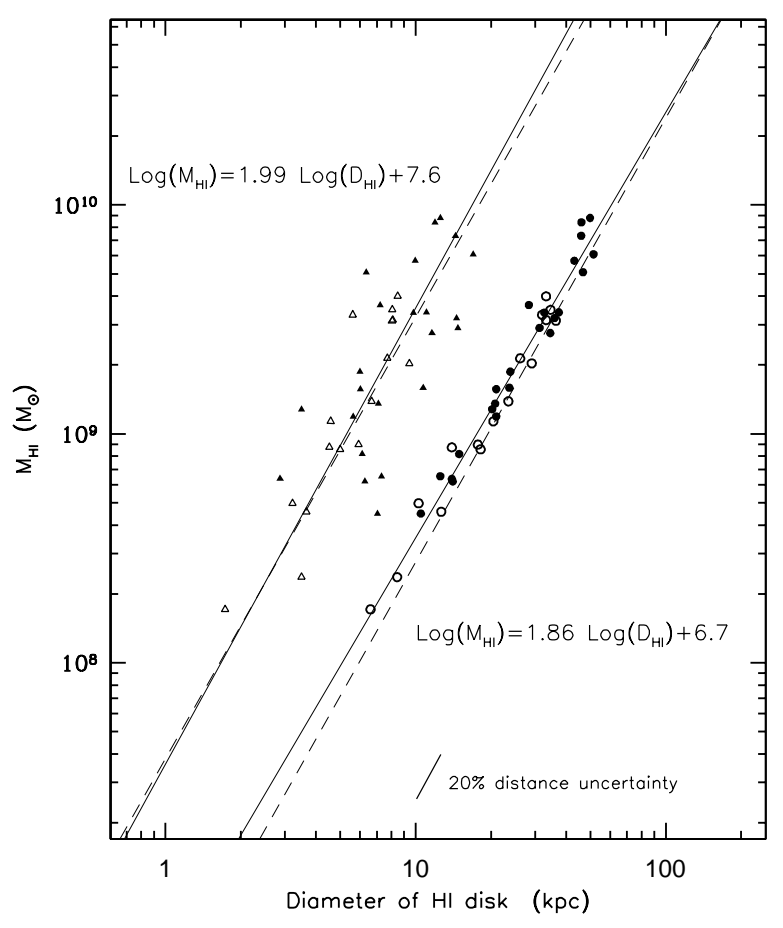

Fig. 7. Correlations between HI mass and the isophotal diameters of the stellar (triangles) and HI disks (circles). Solid lines indicate fits to the plotted data points while the dashed lines represent the fits found by Broeils (1992). Filled symbols indicate HSB galaxies and open symbols denote galaxies of the LSB type. Triangles are offset by 0.3 dex to the left

or surface brightness can be discerned. However, in the lower row, the profiles are grouped according to the galaxy properties as listed in Table 6 . In this case, a clear trend is visible in the sense that galaxies with high HI surface densities in their inner regions are either involved in interactions, are lopsided or display a warped HI disk. Especially in the case of interacting and strongly lopsided systems, no 
Table 6. Warps, asymmetries and interactions

\begin{tabular}{|c|c|c|c|c|c|}
\hline Name & Type & Warped & $\begin{array}{l}\text { Lops } \\
\text { HI distr. }\end{array}$ & $\begin{array}{l}\text { ded } \\
\text { HI kin. }\end{array}$ & $\begin{array}{l}\text { Inter- } \\
\text { acting }\end{array}$ \\
\hline U6399 & $\mathrm{Sm}$ & & x & & \\
\hline U6446 & $\mathrm{Sd}$ & & & $x$ & \\
\hline N3718 & $\mathrm{Sa}$ & $x$ & & & \\
\hline N3726 & $\mathrm{SBc}$ & $x$ & & & \\
\hline N3729 & SBab & & & & \\
\hline N3769 & $\overline{\mathrm{SBb}}$ & $x$ & & & $x$ \\
\hline U6667 & Scd & & & $x$ & \\
\hline N3877 & $\mathrm{Sc}$ & & & $x$ & \\
\hline U6773 & $\mathrm{Sm}$ & & & $\times$ & \\
\hline N3893 & $\mathrm{Sc}$ & & & & $x$ \\
\hline N3917 & Scd & & $x$ & & \\
\hline U6818 & $\mathrm{Sd}$ & & & $x$ & \\
\hline N3949 & Sbc & & $\times$ & $\times$ & \\
\hline N3953 & $\mathrm{SBbc}$ & & & & \\
\hline U6894 & Scd & & & & \\
\hline N3972 & $\mathrm{Sbc}$ & & & $x$ & \\
\hline U6917 & $\mathrm{SBd}$ & $x$ & & & \\
\hline N3985 & $\mathrm{Sm}$ & $\times$ & & & \\
\hline U6923 & $\mathrm{Sdm}$ & $x$ & & $x$ & \\
\hline U6930 & $\mathrm{SBd}$ & & $x$ & & \\
\hline N3992 & $\mathrm{SBbc}$ & & & & \\
\hline U6940 & Scd & & & & \\
\hline N4013 & $\mathrm{Sb}$ & $x$ & & & \\
\hline U6962 & SBcd & & & $x$ & $x$ \\
\hline N4010 & $\mathrm{SBd}$ & $\times$ & & $x$ & \\
\hline U6969 & $\mathrm{Sm}$ & & & & \\
\hline U6973 & Sab & $x$ & & & $x$ \\
\hline U6983 & SBcd & & & & \\
\hline N4051 & $\mathrm{SBbc}$ & & & $x$ & \\
\hline N4085 & $\mathrm{Sc}$ & & & $x$ & \\
\hline N4088 & $\mathrm{Sbc}$ & $x$ & $\bar{x}$ & $x$ & \\
\hline U7089 & $\mathrm{Sdm}$ & & $x$ & & \\
\hline N4100 & Sbc & $\times$ & & & \\
\hline U7094 & $\mathrm{Sdm}$ & & $x$ & & \\
\hline N4102 & $\mathrm{SBab}$ & & & & \\
\hline N4117 & S0 & & & & \\
\hline N4138 & $\mathrm{Sa}$ & $x$ & $x$ & & \\
\hline N4157 & $\mathrm{Sb}$ & $x$ & & & \\
\hline N4183 & Scd & $x$ & & & \\
\hline N4218 & $\mathrm{Sm}$ & & & & \\
\hline $\mathrm{N} 4217$ & $\mathrm{Sb}$ & & & & \\
\hline N4220 & $\mathrm{Sa}$ & & & & \\
\hline N4389 & $\mathrm{SBbc}$ & & & & \\
\hline
\end{tabular}

longlived stable gas orbits can be expected and evidently, the cold gas becomes concentrated toward the inner regions of the disk. Note that some galaxies appear in more than one panel.

Warps are thought to be a quite common feature of the outer HI layers of spiral galaxies. But, in spite of attempts made in recent years (Bosma 1991), a good statistics on their occurrence does not exist yet. The information on HI warps provided by the present survey of the Ursa Major sample is somewhat limited mainly because of the already noted small radial extent of the HI layers with respect to the optical. There are pronounced HI warps like the well-known ones found in NGC 3718 (Schwarz 1985) and NGC 4013 (Bottema 1995) and those seen in other galaxies (N3726, N3985 N4010, N4157, N4183). In most cases the warps are visible in the outer parts beyond the optical bright disk and are present in normal, regular, not interacting disks. But there are also systems, like NGC 4088, which are strongly distorted in their optical appearance and also in their kinematics. Similar distortions are found in clearly interacting systems like NGC 3769. In the sample of 43 galaxies (Table 6 ) there are at least 13 objects with clear indications of warping.

Also asymmetries are thought to occur frequently in field spirals (Richter \& Sancisi 1994). In the present sample we see a large number of objects (at least half, see Table 6) with a lopsided HI distribution and/or kinematics. The majority shows kinematical asymmetries: on one side of the disk the rotation curve rises more slowly and reaches its flat part at a larger radius than on the other side (see for example NGC 3877, 3949, 4051). Note that this occurs in the inner parts of non-interacting, regular, normal systems.

Finally, there are four galaxies in this sample of 43 which have close companions and show clear HI signs of tidal interactions. A few more objects (examples NGC 3718, 4088) show distortions or peculiar structures in their density and velocity maps.

\section{Concluding remarks}

This data paper has presented the results of an extensive HI synthesis imaging survey with the WSRT of a welldefined complete equidistant sample of spiral galaxies in the nearby Ursa Major cluster. Figures B.1 and B.2 show a compilation of all the available HI maps. Individual galaxies are at their proper position on the sky but they are individually four times enlarged. Some galaxies had to be shifted to avoid overlapping maps. Rotation curves have been derived for most galaxies as well as detailed information on the kinematical state of the galaxy disks as indicated by the presence of global perturbations, warps, interactions and lopsidedness. Since the galaxies were not selected on the basis of their HI size or content, the quality of the kinematical data varies widely from galaxy to galaxy. Nevertheless, these data will be useful for an analysis of the statistical properties of the Tully-Fisher relation and the rotation curves may be decomposed into contributions from the main dynamical constituents like the stellar and gaseous disks, the bulge and the dark matter halo. These issues will be addressed in forthcoming papers.

Those who would like to use the rotation curves for their own purposes are advised not to take the rotation curves at face value but to check their validity against the actual data and to take notice of the comments provided on the atlas pages. It should be realized that the rotation curves presented here are derived from the kinematics of the HI gas which is assumed to be a tracer of a galaxy's potential. There are some caveats to be aware of like the 

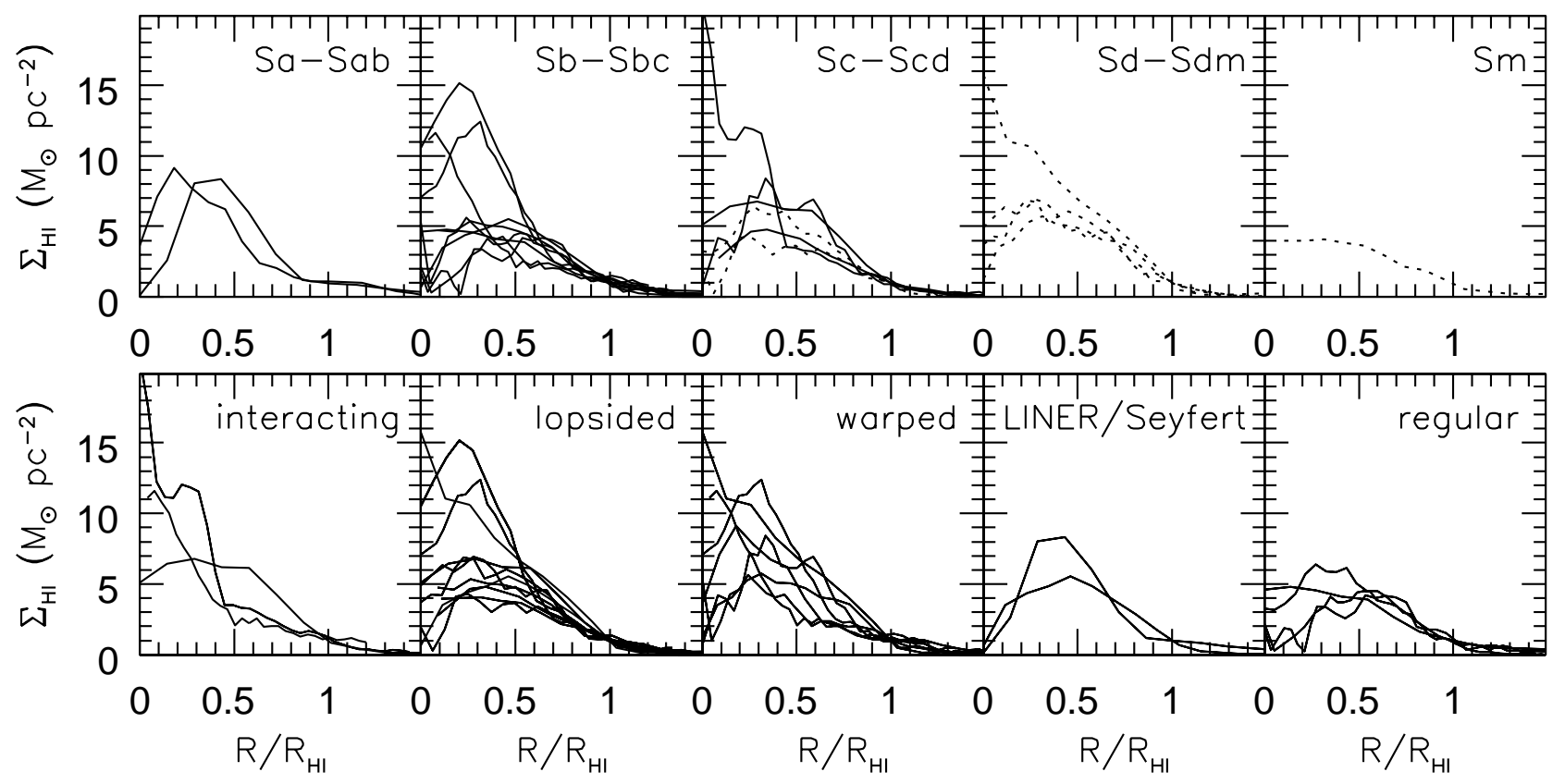

Fig. 8. Azimuthally averaged deprojected radial HI surface density profiles of galaxies with fully reduced data, less inclined than 80 degrees and with $R_{\mathrm{HI}}>1$ arcmin. The profiles were scaled in radius by the radius of the HI disk measured at the $1 M_{\odot} \mathrm{pc}^{-2}$ isodensity contour. Upper row: $\Sigma_{\mathrm{HI}}$ as a function of morphological type. The dashed lines indicate low surface brightness galaxies. Lower row: $\Sigma_{\mathrm{HI}}$ as a function of the kinematic state, according to Table 6, and nuclear activity

projection effects of streaming motions and partially filled HI disks of edge-on systems.

Finally, optical long-slit spectroscopy is already available for nearly all galaxies in the sample. These high resolution optical rotation curves will be used to supplement the HI rotation curves in the inner regions, allowing for better decompositions and maximum-disk constraints.

Acknowledgements. The Westerbork Synthesis Radio Telescope is operated by the Netherlands Foundation for Research in Astronomy (NFRA/ASTRON), with financial support by The Netherlands Organization for Scientific Research (NWO). This research has made use of the NASA/IPAC Extragalactic Database (NED) which is operated by the Jet Propulsion Laboratory, Caltech, under agreement with the National Aeronautics and Space Association. The results presented in this paper were obtained during MV's thesis research at the Kapteyn Institute of the University of Groningen, The Netherlands. This paper was finalized at the National Radio Astronomy Observatory which is a facility of the National Science Foundation operated under cooperative agreement by Associated Universities, Inc. This research has been supported by NATO Collaborative Research Grant 940271 .

\section{References}

Appleton, P. N., \& Davies, R. D. 1982, MNRAS, 201, 1073 Begeman, K. G. 1987, Thesis, University of Groningen Begeman, K. G. 1989, A\&A, 223, 47

Bosma, A. 1991, Warped and flaring HI disks, in Warped disks and inclined rings around galaxies, ed. S. Casertano, P. Sackett, \& F. Briggs (Cambridge University Press), 181

Bottema, R., 1995 A\&A, 295, 605

Bottema, R. 1996, A\&A, 306, 345
Bottinelli, L., Gouguenheim, L., Paturel, G., \& De Vaucouleurs, G. 1983, A\&A, 118, 4

Bottinelli, L., Gouguenheim, L., Fouqué, P., \& Paturel, G. 1990, A\&AS, 82, 391

Bravo-Alfaro, H., Cayatte, V., Van Gorkom, J. H., \& Balkowski, C. 2000, AJ, 119, 580

Broeils, A. H. 1992, Thesis, University of Groningen

Broeils, A. H., \& van Woerden, H. 1994, A\&AS, 107, 129

Burstein, D., \& Heiles, C. 1984, ApJS, 54, 33

Cardelli, J. A., Clayton, G. C., \& Mathis, J. S. 1989, ApJ, 345, 245

Cayatte, V., van Gorkom, J. H., Balkowski, C., \& Kotanyi, C. G. 1990, AJ, 100, 604

Cayatte, V., Kotanyi, C. G., Balkowski, C., \& van Gorkom, J. H. 1994, AJ, 107, 1003

Dickey, J. M., \& Gavazzi, G. 1991, ApJ, 373347

Dickey, J. M. 1997, AJ, 113, 1939

Fisher, J. R., \& Tully, R. B. 1981, ApJS, 47, 139

Fouqué, P., Bottinelli, L., Gouguenheim, L., \& Paturel, G. 1990, ApJ, 349, 1

Gottesman, S. T., Ball, R., \& Hunter Jr, J. H. 1984, ApJ, 286, 471

Grewing, M., \& Mebold, U. 1975, A\&A, 42, 119

Högbom, J. A. 1974, A\&AS, 15, 417

Holmberg, E. 1946, Medd. Lunds Astr. Obs., Ser. II, No. 117

Hubble, E. 1926, ApJ, 64, 321

Huchtmeier, W. K., \& Richter, O.-G. 1986, A\&AS, 63, 323

Jore, K. P., Broeils, A. H., \& Haynes, M. P. 1996, AJ, 112, 438

Lucy, L. B. 1974, AJ, 79, 745

Magri, C. 1994, AJ, 108, 896

McMahon, P. M. 1993, Thesis, University of Columbia

Navarro, J. F., \& Steinmetz, M. 2000, ApJ, 538, 477

Oosterloo, T., \& Shostak, S. 1993, A\&AS, 99, 379

Puche, D., \& Garignan, C. 1991, AJ, 378, 487

Rhee, M.-H. 1996a, Thesis, University of Groningen 
Rhee, M.-H. 1996b, A\&AS, 115, 407

Richter, O.-G., \& Huchtmeier, W. K. 1991, A\&AS, 87, 425

Richter, O.-G., \& Sancisi, R. 1994, A\&A, 295, 605

Roberts, M. S., \& Haynes, M. P. 1994, ARA\&A, 32, 115

Sakai, S., Mould, J. R., Hughes, S. M. G., et al. 2000, ApJ, 529,698

Sancisi, R., \& Allen, R. J. 1979, A\&A, 74, 73

Schlegel, D. J., Finkbeiner, D. P., \& Davis, M. 1998, ApJ, 500, 525

Schneider, S. E., Thuan, T. X., Mangum, J. G., \& Miller, J. 1992, ApJS, 81, 5

Schwarz, U. J. 1985, A\&A, 142, 273

Swater, R. A. 1999, Dark Matter in late type dwarf galaxies, Ph.D. Thesis, University of Groningen

Thuan, T. X., \& Martin, G. E. 1981, ApJ, 247, 823

Tully, R. B., \& Fouquè, P. 1985, ApJS, 58, 67 (TFq)

Tully, R. B., Verheijen, M. A. W., Pierce, M. J., Huang, J.-S., \& Wainscoat, R. J. 1996, AJ, 112, 2471 (Paper I)

Tully, R. B., \& Verheijen, M. A. W. 1997, ApJ, 484, 145 (Paper II)

Tully, R. B., Pierce, M. J., Huang, J.-S., et al. AJ, 115, 2264

Tully, R. B., \& Pierce, M. J. 2000, ApJ, 533, 744

Van der Burg, G. 1987, Ph.D. Thesis, University of Groningen

Van Moorsel, G. A. 1983, A\&AS, 54, 1

Warmels, R. H. 1988a, A\&AS, 72, 19

Warmels, R. H. 1988b, A\&AS, 72, 57

\section{Appendix A: The noise in an integrated $\mathrm{HI}$ map}

This Appendix explains how the noise in a total HI map can be calculated. A total HI map is usually constructed from a 3 dimensional datacube containing a number of so called channelmaps. Each channelmap shows an HI image of the galaxy at a certain velocity. A total HI map is made by adding those channelmaps which contain the HI signal. Before adding the channel maps the signal in each channelmap should be isolated. When the signal is not isolated one merely adds noise to the total HI map because the location of the signal in a channel map varies with velocity due to the galactic rotation. The signals can be isolated interactively by blotting away the surrounding noise or in a more objective way by taking a certain contour level in the smoothed maps as a mask. As a consequence of adding channel maps with isolated regions, the noise in the total HI map is not constant but varies from pixel to pixel. The noise at a certain pixel in the total HI map depends on the number $N$ of non-blank pixels at the same position in the individual channel maps that were added.

In case the data cube was obtained with an uniform taper during the observation, the noise $\sigma^{u}$ in two channelmaps will be independent. The noise equivalent bandwidth $B^{u}$ in a uniform tapered spectrum is equal to the channel separation b. When adding $N$ uniform tapered channelmaps at a certain pixel the noise $\sigma_{N}^{u}$ at the same pixel position in the total HI map will be increased by a factor $\sqrt{N}$ :

$\sigma_{N}^{u}=\sqrt{N} \sigma^{u}$.

Usually, the observations are made using a hanning taper in which case the noise in two adjacent channelmaps is no longer independent. A hanning taper effectively smooths the data in velocity by convolving the velocity profile at each pixel. If $U_{i}$ is the pixel value in the $i$ th uniform tapered channel map, the value $H_{i}$ in the $i$ th hanning tapered channelmap is given by

$H_{i}=\frac{1}{4} U_{i-1}+\frac{1}{2} U_{i}+\frac{1}{4} U_{i+1}$.

Since the $\sigma_{i}^{u}$ 's are independent and all equal to $\sigma^{u}$, the noise $\sigma_{i}^{h}$ in the $i$ th hanning tapered channelmap can be calculated according to

$$
\begin{aligned}
\sigma_{i}^{h} & =\left[\left(\frac{1}{4} \sigma_{i-1}^{u}\right)^{2}+\left(\frac{1}{2} \sigma_{i}^{u}\right)^{2}+\left(\frac{1}{4} \sigma_{i+1}^{u}\right)^{2}\right]^{\frac{1}{2}} \\
& =\left[\frac{1}{16}+\frac{1}{4}+\frac{1}{16}\right]^{\frac{1}{2}} \sigma^{u} \\
& =\frac{\sqrt{6}}{4} \sigma^{u}=0.61 \sigma^{u} .
\end{aligned}
$$

In this case the noise equivalent bandwidth $B^{h}$ for a hanning tapered spectrum is given by

$B^{h}=\frac{16}{6} B^{u}=2.67 B^{u}$.

As a consequence, the noise in two hanning tapered channelmaps may be correlated depending on their separation. Two channelmaps separated by one channelmap are correlated because both contain a quarter of the flux from the channel map between them. Only channel maps separated by more than one channel are independent. This will be shown in the following three cases in which two hanning tapered channel maps at different separations will be added.

1. Adding two adjacent hanning tapered channelmaps $i$ and $(i+1)$ gives a signal $H_{(i)+(i+1)}$ of

$$
\begin{aligned}
H_{(i)+(i+1)}= & H_{i}+H_{i+1} \\
= & \left(\frac{1}{4} U_{i-1}+\frac{1}{2} U_{i}+\frac{1}{4} U_{i+1}\right) \\
& +\left(\frac{1}{4} U_{i}+\frac{1}{2} U_{i+1}+\frac{1}{4} U_{i+2}\right) \\
= & \frac{1}{4} U_{i-1}+\frac{3}{4} U_{i}+\frac{3}{4} U_{i+1}+\frac{1}{4} U_{i+2}
\end{aligned}
$$

and the noise $\sigma_{(i)+(i+1)}^{h}$ in that map will be

$$
\begin{aligned}
\sigma_{(i)+(i+1)}^{h}= & {\left[\left(\frac{1}{4} \sigma_{i-1}^{u}\right)^{2}+\left(\frac{3}{4} \sigma_{i}^{u}\right)^{2}\right.} \\
& \left.+\left(\frac{3}{4} \sigma_{i+1}^{u}\right)^{2}+\left(\frac{1}{4} \sigma_{i+2}^{u}\right)^{2}\right]^{\frac{1}{2}} \\
= & \frac{\sqrt{20}}{4} \sigma^{u}=\frac{\sqrt{20}}{4} \frac{4}{\sqrt{6}} \sigma^{h} \\
= & \sqrt{3 \frac{1}{3}} \sigma^{h}=1.83 \sigma^{h} .
\end{aligned}
$$

2. Adding the hanning tapered channels $i$ and $(i+2)$ gives

$$
\begin{aligned}
H_{(i)+(i+2)}= & H_{i}+H_{i+2} \\
= & \left(\frac{1}{4} U_{i-1}+\frac{1}{2} U_{i}+\frac{1}{4} U_{i+1}\right) \\
& +\left(\frac{1}{4} U_{i+1}+\frac{1}{2} U_{i+2}+\frac{1}{4} U_{i+3}\right) \\
= & \frac{1}{4} U_{i-1}+\frac{1}{2} U_{i}+\frac{1}{2} U_{i+1} \\
& +\frac{1}{2} U_{i+2}+\frac{1}{4} U_{i+3}
\end{aligned}
$$


and the noise becomes

$$
\begin{aligned}
\sigma_{(i)+(i+2)}^{h}= & {\left[\left(\frac{1}{4} \sigma_{i-1}^{u}\right)^{2}+\left(\frac{1}{2} \sigma_{i}^{u}\right)^{2}+\left(\frac{1}{2} \sigma_{i+1}^{u}\right)^{2}\right.} \\
& \left.+\left(\frac{1}{2} \sigma_{i+2}^{u}\right)^{2}+\left(\frac{1}{4} \sigma_{i+3}^{u}\right)^{2}\right]^{\frac{1}{2}} \\
= & \frac{\sqrt{14}}{4} \sigma^{u}=\frac{\sqrt{14}}{4} \frac{4}{\sqrt{6}} \sigma^{h} \\
= & \sqrt{2 \frac{1}{3}} \sigma^{h}=1.53 \sigma^{h} .
\end{aligned}
$$

3. Adding the hanning tapered channels $i$ and $(i+3)$ gives

$$
\begin{aligned}
H_{(i)+(i+3)}= & H_{i}+H_{i+3} \\
= & \left(\frac{1}{4} U_{i-1}+\frac{1}{2} U_{i}+\frac{1}{4} U_{i+1}\right) \\
& +\left(\frac{1}{4} U_{i+2}+\frac{1}{2} U_{i+3}+\frac{1}{4} U_{i+4}\right)
\end{aligned}
$$

with a resulting noise of

$$
\begin{aligned}
\sigma_{(i)+(i+3)}^{h}= & {\left[\left(\frac{1}{4} \sigma_{i-1}^{u}\right)^{2}+\left(\frac{1}{2} \sigma_{i}^{u}\right)^{2}+\left(\frac{1}{4} \sigma_{i+1}^{u}\right)^{2}\right.} \\
& \left.+\left(\frac{1}{4} \sigma_{i+2}^{u}\right)^{2}+\left(\frac{1}{2} \sigma_{i+3}^{u}\right)^{2}+\left(\frac{1}{4} \sigma_{i+4}^{u}\right)^{2}\right]^{\frac{1}{2}} \\
= & \frac{\sqrt{12}}{4} \sigma^{u}=\frac{\sqrt{12}}{4} \frac{4}{\sqrt{6}} \sigma^{h} \\
= & \sqrt{2} \sigma^{h}=1.41 \sigma^{h} .
\end{aligned}
$$

So, channelmaps $i$ and $(i+3)$ are independent.

Because the noise is correlated, adding $N$ adjacent hanning tapered channelmaps does not give an increase of the noise with a factor $\sqrt{N}$ but with a factor $\sqrt{N-\frac{3}{4}} \cdot \frac{4}{\sqrt{6}}$ as is shown below. First the total signal $H_{N}$ is calculated.

\begin{tabular}{c|ccccccc}
\hline Channel & $U_{i-1}$ & $U_{i}$ & $U_{i+1}$ & $\cdots$ & $U_{i+N-2}$ & $U_{i+N-1}$ & $U_{i+N}$ \\
\hline$i$ & $1 / 4$ & $1 / 2$ & $1 / 4$ & & & & \\
$i+1$ & & $1 / 4$ & $1 / 2$ & $\cdots$ & & & \\
$i+2$ & & & $1 / 4$ & $\cdots$ & & & \\
$\cdots$ & & & & $\cdots$ & & & \\
$i+N-3$ & & & & $\cdots$ & $1 / 4$ & & \\
$i+N-2$ & & & & $\cdots$ & $1 / 2$ & $1 / 4$ & \\
$i+N-1$ & & & & & $1 / 4$ & $1 / 2$ & $1 / 4$ \\
\hline & $\frac{1}{4} U_{i-1} \frac{3}{4} U_{i}$ & $U_{i+1}$ & $\cdots$ & $U_{i+N-2}$ & $\frac{3}{4} U_{i+N-1}$ & $\frac{1}{4} U_{i+N}$
\end{tabular}

and thus

$$
\begin{aligned}
H_{N}= & \frac{1}{4} U_{i-1}+\frac{3}{4} U_{i}+U_{i+1}+\cdots \\
& +U_{i+N-2}+\frac{3}{4} U_{i+N-1}+\frac{1}{4} U_{i+N} .
\end{aligned}
$$

From this it follows that the noise $\sigma_{N}^{h}$ is given by

$$
\begin{aligned}
\sigma_{N}^{h} & =\left[\left(\frac{1}{4}\right)^{2}+\left(\frac{3}{4}\right)^{2}+(N-2) \cdot 1^{2}+\left(\frac{3}{4}\right)^{2}+\left(\frac{1}{4}\right)^{2}\right]^{\frac{1}{2}} \sigma^{u} \\
& =\left[N-\frac{3}{4}\right]^{\frac{1}{2}} \sigma^{u}=\left[N-\frac{3}{4}\right]^{\frac{1}{2}} \frac{4}{\sqrt{6}} \sigma^{h} \\
& =\sqrt{\left(N-\frac{3}{4}\right) B^{h}} \sigma^{h} .
\end{aligned}
$$

However, before the hanning tapered channelmaps are added to form a total HI map, the continuum must be subtracted. This operation introduces extra noise in the channelmaps which doesn't behave like a hanning tapered correlation. Here, it will be assumed that the average continuum map is formed by averaging $N_{1}$ line free channels at the low velocity end of the datacube and $N_{2}$ channels at the high velocity end which gives

$C_{\text {low }}=\frac{1}{N_{1}} \sum_{j=1}^{N_{1}} H_{j} \quad$ and $\quad C_{\text {high }}=\frac{1}{N_{2}} \sum_{j=1}^{N_{2}} H_{j}$.

Since all channels are hanning tapered the noise in these maps can be calculated according to

$\sigma_{C_{\text {low }}}=\frac{1}{N_{1}} \sqrt{\left(N_{1}-\frac{3}{4}\right)} \sigma^{u}$

and

$\sigma_{C_{\text {high }}}=\frac{1}{N_{2}} \sqrt{\left(N_{2}-\frac{3}{4}\right)} \sigma^{u}$.

The average continuum map to be subtracted is then formed by

$<C>=\frac{1}{2}\left(C_{\text {low }}+C_{\text {high }}\right)$.

Since $\sigma_{C_{\text {low }}}$ and $\sigma_{C_{\text {high }}}$ are independent it follows that the noise $\sigma_{<C>}$ in the finally averaged continuum map is given by

$$
\begin{aligned}
\sigma_{<C>} & =\frac{1}{2} \sqrt{\sigma_{C_{\text {low }}}^{2}+\sigma_{C_{\text {high }}}^{2}} \\
& =\sigma^{u} \sqrt{\left(\frac{N_{1}-\frac{3}{4}}{4 N_{1}^{2}}+\frac{N_{2}-\frac{3}{4}}{4 N_{2}^{2}}\right)} \\
& \equiv \sigma^{u} \mathcal{N} .
\end{aligned}
$$

After subtraction of the continuum the channelmaps only contain signal from the HI emission line. The signal in the channelmaps containing the line emission is now given by

$$
\begin{aligned}
L_{i} & =H_{i}-<C> \\
& =\frac{1}{4} U_{i-1}+\frac{1}{2} U_{i}+\frac{1}{4} U_{i+1}-<C>.
\end{aligned}
$$

Because $\sigma_{<C>}$ is independent from $\sigma_{i}^{u}$ in the velocity range which is not used to form the averaged continuum map, it can be written

$$
\begin{aligned}
\sigma_{i}^{l} & =\left[\left(\frac{1}{4} \sigma_{i-1}^{u}\right)^{2}+\left(\frac{1}{2} \sigma_{i}^{u}\right)^{2}+\left(\frac{1}{4} \sigma_{i+1}^{u}\right)^{2}+\sigma_{<C>}^{2}\right]^{\frac{1}{2}} \\
& =\left[\frac{1}{16}+\frac{4}{16}+\frac{1}{16}+\mathcal{N}^{2}\right]^{\frac{1}{2}} \sigma^{u} \\
& =\sqrt{\left(\frac{3}{8}+\mathcal{N}^{2}\right)} \sigma^{u} .
\end{aligned}
$$

When adding $N$ adjacent hanning tapered and continuum subtracted channelmaps containing the line emission, the signal $L_{N}$ will be

$$
\begin{aligned}
L_{N}= & \frac{1}{4} U_{i-1}+\frac{3}{4} U_{i}+U_{i+1}+\cdots \\
& \cdots+U_{i+N-2}+\frac{3}{4} U_{i+N-1}+\frac{1}{4} U_{i+N} \\
& -N \cdot\langle C>.
\end{aligned}
$$

The noise $\sigma_{N}^{l}$ at each pixel in the final map can be derived analogous to the calculation of $\sigma_{N}^{h}$ and is given by

$$
\begin{aligned}
\sigma_{N}^{l} & =\left[\left(N-\frac{3}{4}\right)+N^{2} \mathcal{N}^{2}\right]^{\frac{1}{2}} \sigma^{u} \\
& =B^{h} \sqrt{\left(\frac{\left(N-\frac{3}{4}\right)+N^{2} \mathcal{N}^{2}}{B^{h}}\right)} \sigma^{h} .
\end{aligned}
$$




\section{Appendix B: The $\mathrm{HI}$ atlas}

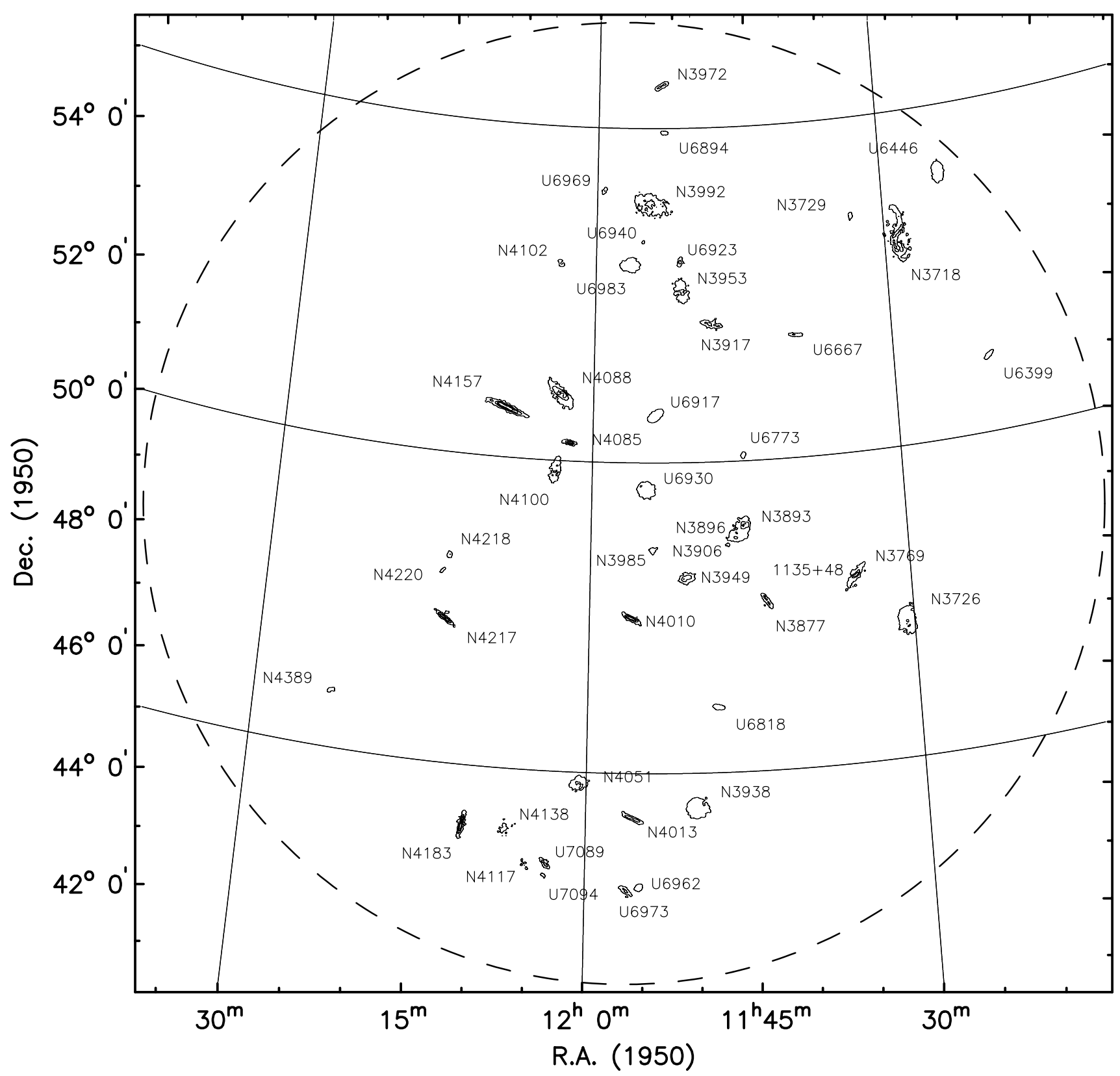

Fig. B.1. Integrated HI maps of all galaxies in the cluster more inclined than 45 degrees and brighter than $M(B)=-16.5$. The

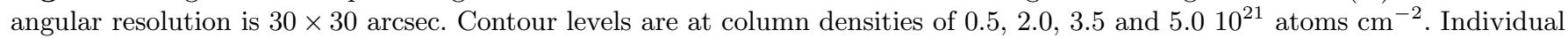
galaxies are four times enlarged. To avoid overlap, some galaxies are slightly displaced from their actual position. N3906, N3938, U6962 and U6930 are more face-on than 45 degrees. U6940 is fainter than the complete sample limit. The dashed circle indicates the adopted boundary of the cluster 


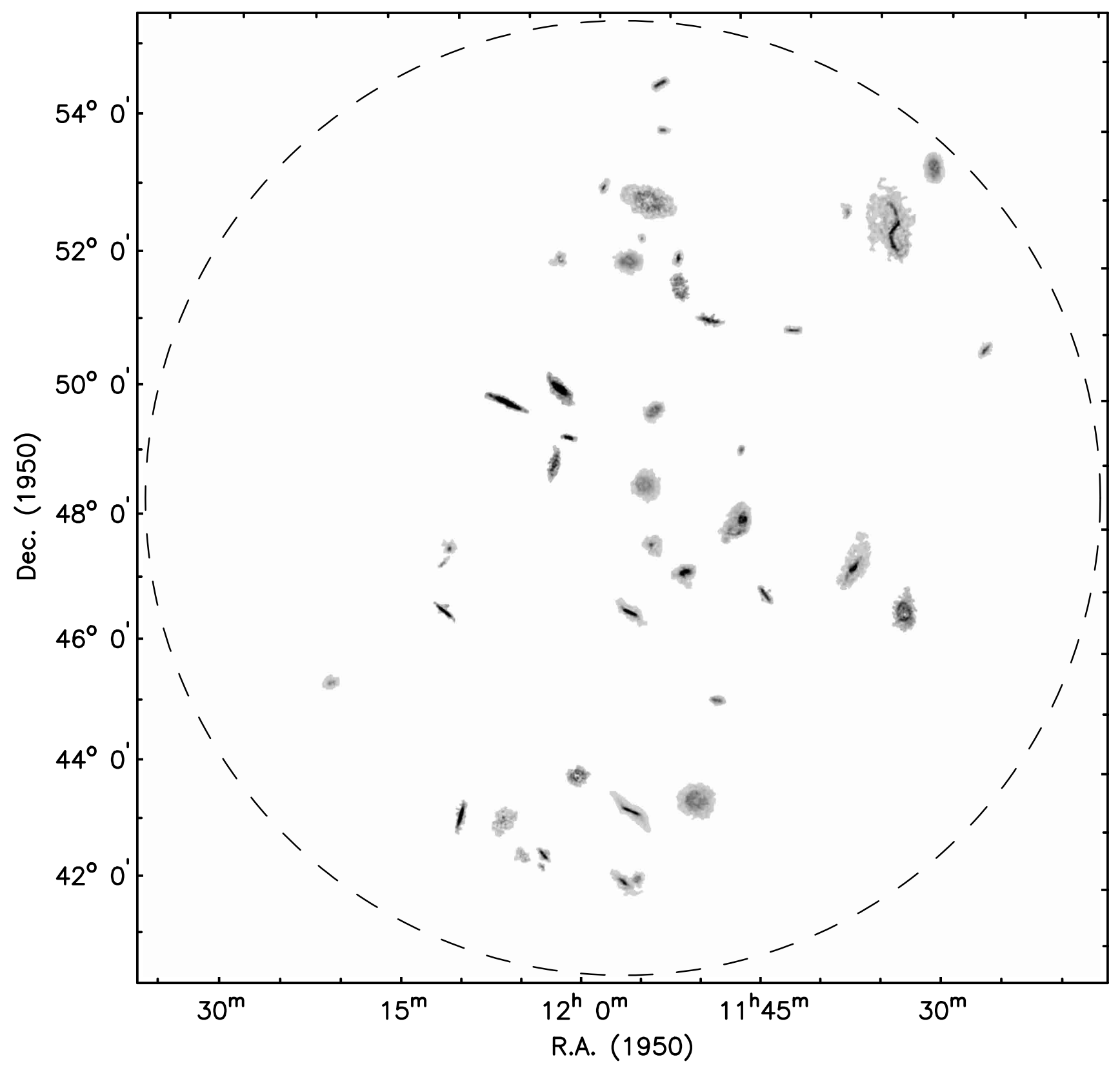

Fig. B.2. Same as Fig. B.1, displayed here in grayscale. Note the interacting pairs N3769/1135+48, N3893/N3896 and U6962/U6973. The galaxies N3718, N3726, N4010, N4013, N4088 and N4138 are strongly warped. It is clear that the integrated column density strongly depends on inclination. Many of the more face-on galaxies show a depletion of the HI gas in their inner regions 
Observing parameters for UGC 6399

\begin{tabular}{|c|c|}
\hline Length of observation & $1 \times 12$ \\
\hline Dates of observation & 6Aug90 \\
\hline Field center, $\alpha(1950)$ & $11: 20: 30$ \\
\hline$\delta(1950)$ & $51: 10: 00$ \\
\hline Central frequency & 1416.56 \\
\hline$V_{\text {hel }}$ of central channel & 810 \\
\hline Primary beam FWHM (arcmin) & 37.4 \\
\hline Nr. of interferometers & 40 \\
\hline Baselines (min-max-incr) & $36-2700-72$ \\
\hline Synthesized beam $(\alpha \times \delta)(\operatorname{arcsec})$ & $12.9 \times 16.6$ \\
\hline Bandwidth & 2.5 \\
\hline Number of channels & 127 \\
\hline Channel separation & 4.15 \\
\hline Velocity resolution & 8.29 \\
\hline rms noise in one channel $(\mathrm{K})$ & 5.54 \\
\hline $\begin{array}{l}\mathrm{K} \text {-mJy conversion, } \\
\text { equiv. of } 1 \mathrm{mJy} / \text { beam }\end{array}$ & 2.84 \\
\hline
\end{tabular}

Results from WSRT data

\begin{tabular}{|c|c|}
\hline \multicolumn{2}{|l|}{ From continuит map: } \\
\hline \multicolumn{2}{|l|}{ 21-cm flux density: } \\
\hline central point source $(\mathrm{mJy})$ & $<1.3(3 \sigma)$ \\
\hline extended source (mJy) & $<2.5(3 \sigma)$ \\
\hline \multicolumn{2}{|l|}{ From global profile: } \\
\hline$\overline{\text { Integrated HI-flux }}\left(\mathrm{Jy} \mathrm{km} \mathrm{s}^{-1}\right)$ & $10.5 \pm 0.3$ \\
\hline Hel. systemic velocity $\left(\mathrm{km} \mathrm{s}^{-1}\right)$ & $791.5 \pm 0.6$ \\
\hline HI profile width, $20 \%\left(\mathrm{~km} \mathrm{~s}^{-1}\right)$ & $188.1 \pm 1.4$ \\
\hline $50 \%\left(\mathrm{~km} \mathrm{~s}^{-1}\right)$ & $172.5 \pm 2.9$ \\
\hline \multicolumn{2}{|l|}{ From velocity field: } \\
\hline 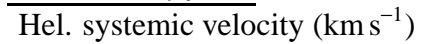 & $794.5 \pm 1.3$ \\
\hline Dynamical center, $\alpha(1950)$ & $11: 20: 35.8$ \\
\hline$\delta(1950)$ & $51: 09: 58$ \\
\hline \multicolumn{2}{|l|}{ From total HI map: } \\
\hline$\overline{\text { Geometric center, } \alpha(1950)}$ & $11: 20: 35.7$ \\
\hline$\delta(1950)$ & 51:10:01 \\
\hline Position angle & 139 \\
\hline Inclination angle & 77 \\
\hline Diameter of HI disk (arcmin) & 3.4 \\
\hline
\end{tabular}

Contour levels for U6399

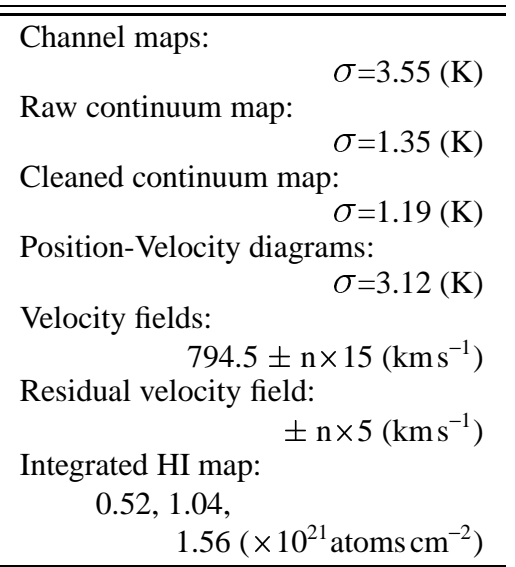

Note: This is the most isolated system in the cluster. The rotation curve does not show a flat part on the receding side and maybe just barely on the approaching side.

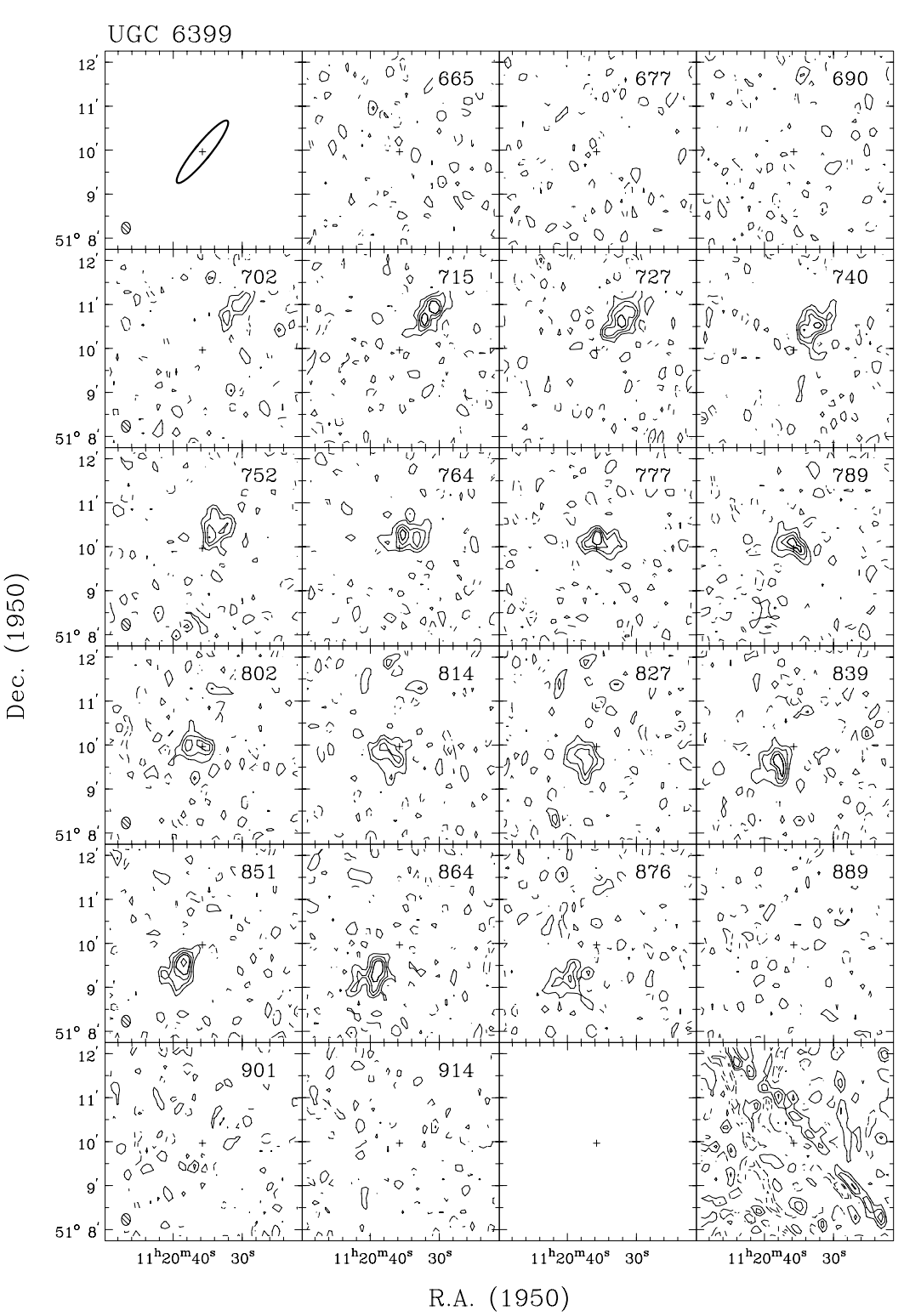

Channel maps at a resolution of $12^{\prime \prime} \times 16^{\prime \prime} \times 19 \mathrm{~km} \mathrm{~s}^{-1}$. Contour levels at $-3,-1.5$ (dashed), $1.5,3,4.5, \ldots \times \sigma$. 

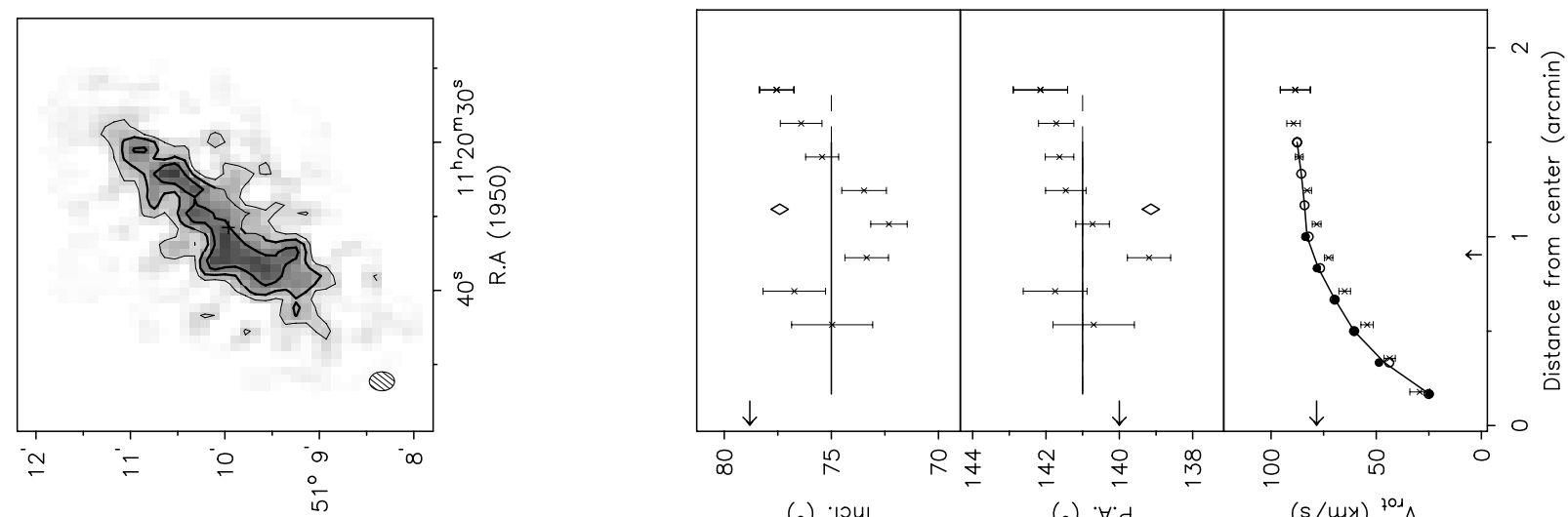
(o) $\cdot$ |ou|
(o) $\forall \cdot d$
$(s / m y){ }^{201} \wedge$

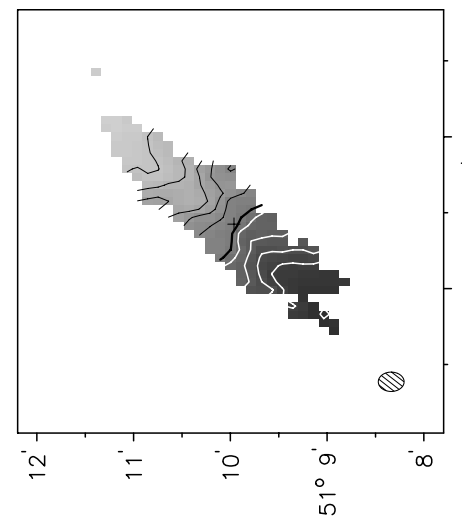

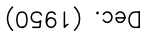
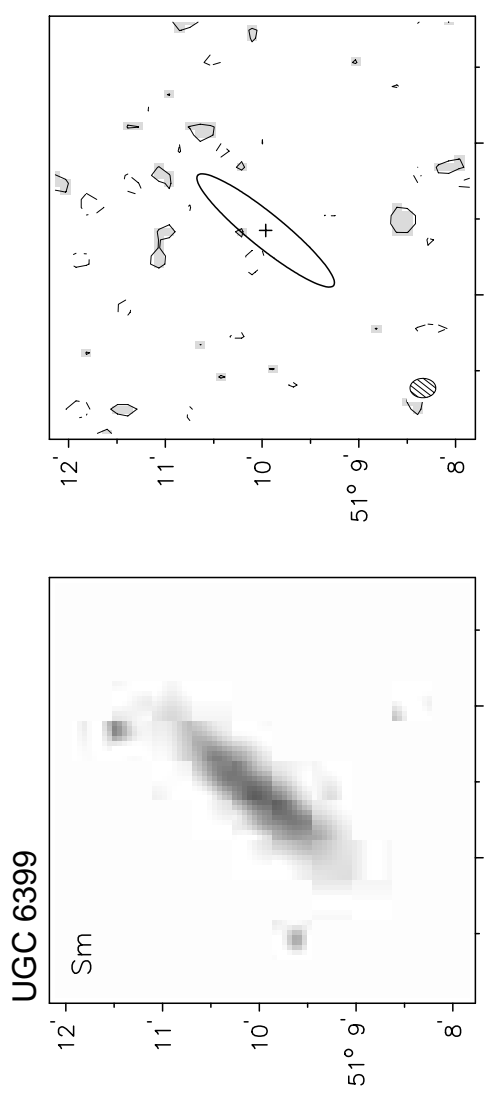

(0ร6l) $\cdot$ วә0

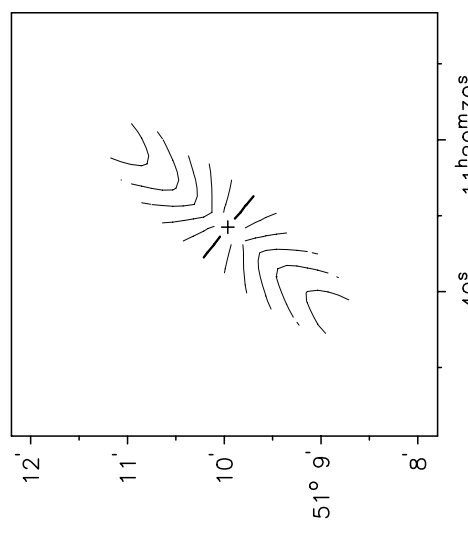

$(056 \mathrm{l}) \cdot 2 \cdot 0$
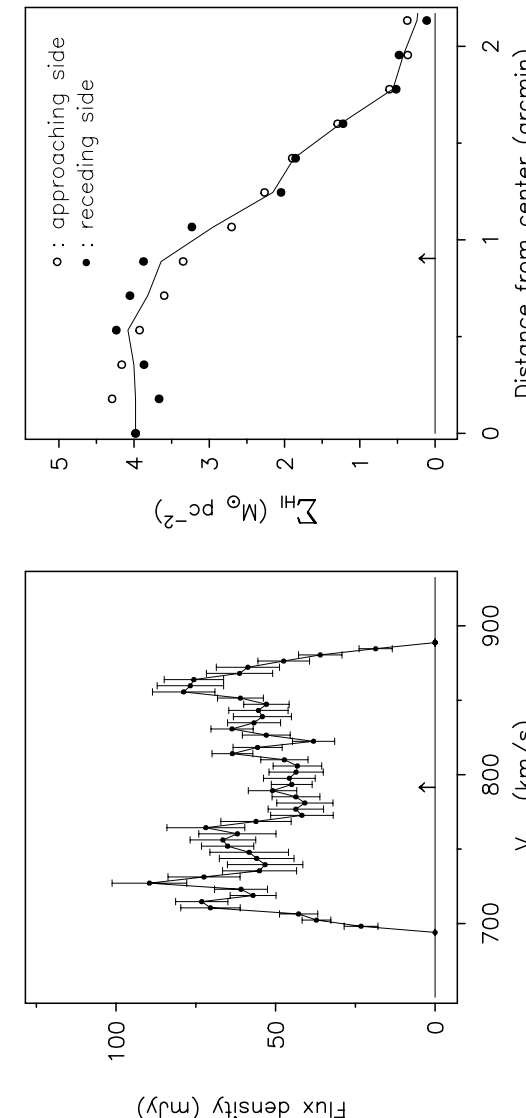

(Kৎщ) Kłısuәp xn!
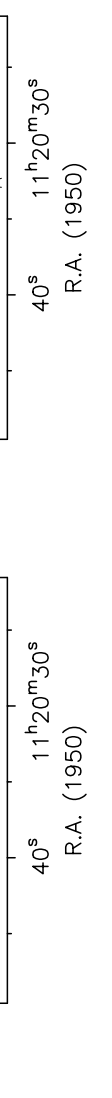

$(0 \mathrm{CL} \mathrm{l}) \cdot 200$

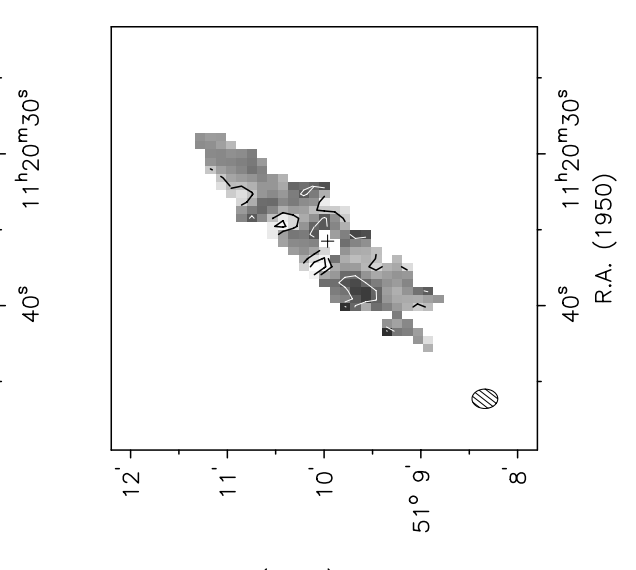


Observing parameters for UGC 6446

\begin{tabular}{|c|c|}
\hline Length of observation & $1 \times 12$ \\
\hline Date of observation & 07Jan94 \\
\hline Field center, $\alpha(1950)$ & $11: 23: 53$ \\
\hline$\delta(1950)$ & 54:01:28 \\
\hline Central frequency & 1417.40 \\
\hline$V_{\text {hel }}$ of central channel & 650 \\
\hline Primary beam FWHM (arcmin) & 37.4 \\
\hline Nr. of interferometers & 40 \\
\hline Baselines (min-max-incr) & $36-2700-72$ \\
\hline Synthesized beam $(\alpha \times \delta)$ (arcsec) & $12.1 \times 15.0$ \\
\hline Bandwidth & 2.5 \\
\hline Number of channels & 127 \\
\hline Channel separation & 4.14 \\
\hline Velocity resolution & 4.97 \\
\hline rms noise in one channel & 10.1 \\
\hline $\begin{array}{l}\text { K-mJy conversion, } \\
\text { equiv. of } 1 \mathrm{mJy} / \text { beam }\end{array}$ & 3.32 \\
\hline
\end{tabular}

Results from WSRT data

\begin{tabular}{|c|c|}
\hline rom continuит map: & \\
\hline 21-cm flux density: & \\
\hline ource (mJy) & $<1.2(3 \sigma)$ \\
\hline extended source & \\
\hline From global profile: & \\
\hline$\overline{\text { Integrated HI-flux }}\left(\mathrm{Jy} \mathrm{km} \mathrm{s}^{-1}\right)$ & $40.6 \pm 0.5$ \\
\hline Hel. systemic velocity $\left(\mathrm{km} \mathrm{s}^{-1}\right)$ & $644.3 \pm 0.8$ \\
\hline HI profile width, $20 \%\left(\mathrm{~km} \mathrm{~s}^{-1}\right)$ & $154.1 \pm 1.0$ \\
\hline $50 \%\left(\mathrm{~km} \mathrm{~s}^{-1}\right)$ & $131.9 \pm 1.2$ \\
\hline From velocity field: & \\
\hline 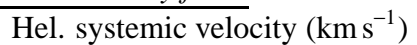 & \pm 0.4 \\
\hline Dynamical center, $\alpha(1$ & 3.0 \\
\hline$\delta(19$ & $54: 01: 23$ \\
\hline From total HI map: & \\
\hline$\overline{\text { Geometric center, } \alpha(1950)}$ & 52.9 \\
\hline$\delta(1950)$ & 54:01:29 \\
\hline Position angle & 188 \\
\hline Inclination angle & 55 \\
\hline Diameter of HI disk (arcmin) & 5.9 \\
\hline
\end{tabular}

Contour levels for U6446

\begin{tabular}{|c|c|}
\hline Channel maps: & $\sigma=3.98(\mathrm{~K})$ \\
\hline Raw continuum map: & $\sigma=1.44(\mathrm{~K})$ \\
\hline Cleaned continuum m & $\sigma=1.28(\mathrm{~K})$ \\
\hline Position-Velocity diag & $\begin{array}{l}\text { ams: } \\
\sigma=3.49(\mathrm{~K})\end{array}$ \\
\hline $\begin{array}{l}\text { Velocity fields: } \\
\quad 645.4 \pm \\
\text { Residual velocity field }\end{array}$ & $\times 15\left(\mathrm{~km} \mathrm{~s}^{-1}\right)$ \\
\hline $\begin{array}{c}\text { Integrated HI map: } \\
0.59,1.19, \\
1.78(\times 10\end{array}$ & $\begin{array}{l}\mathrm{n} \times 5\left(\mathrm{~km} \mathrm{~s}^{-1}\right) \\
{ }^{1} \text { atoms cm} \\
\end{array}$ \\
\hline
\end{tabular}

.

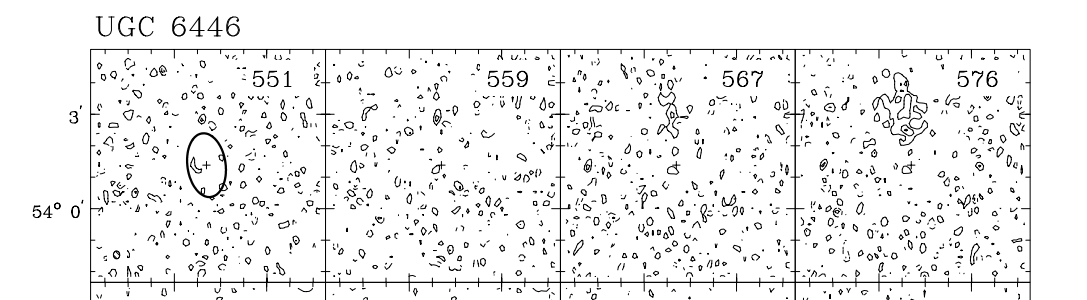

3

อิ

Note: This is a low surface brightness galaxy with possibly asymmetric rotation in the inner part; the rotation curve on the receding southern side seems to rise steeper than on the approaching northern side. The continuum map shows a grating ring from a source outside the mapped field of view. 

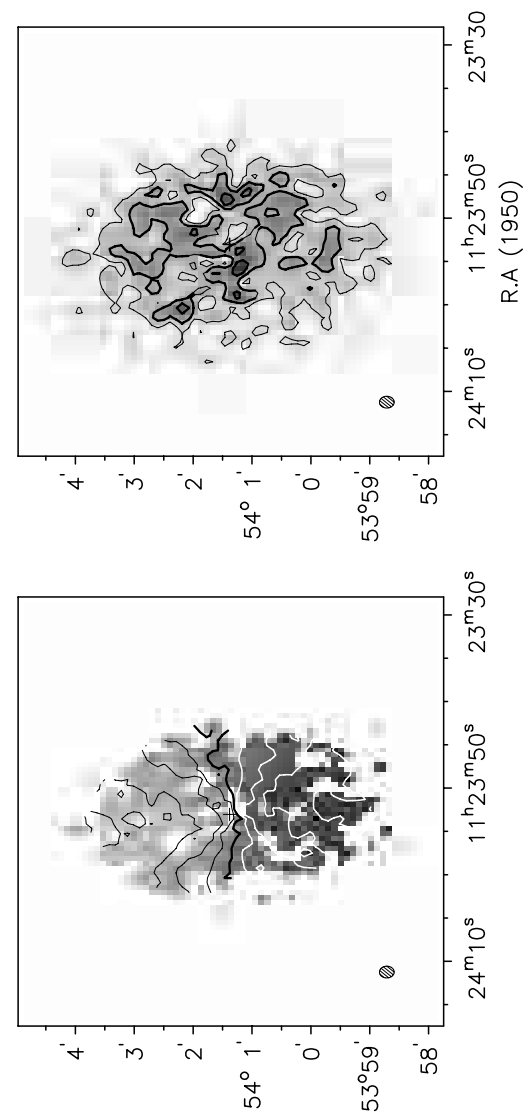

$(096 \mathrm{l}) \cdot 2$ ว
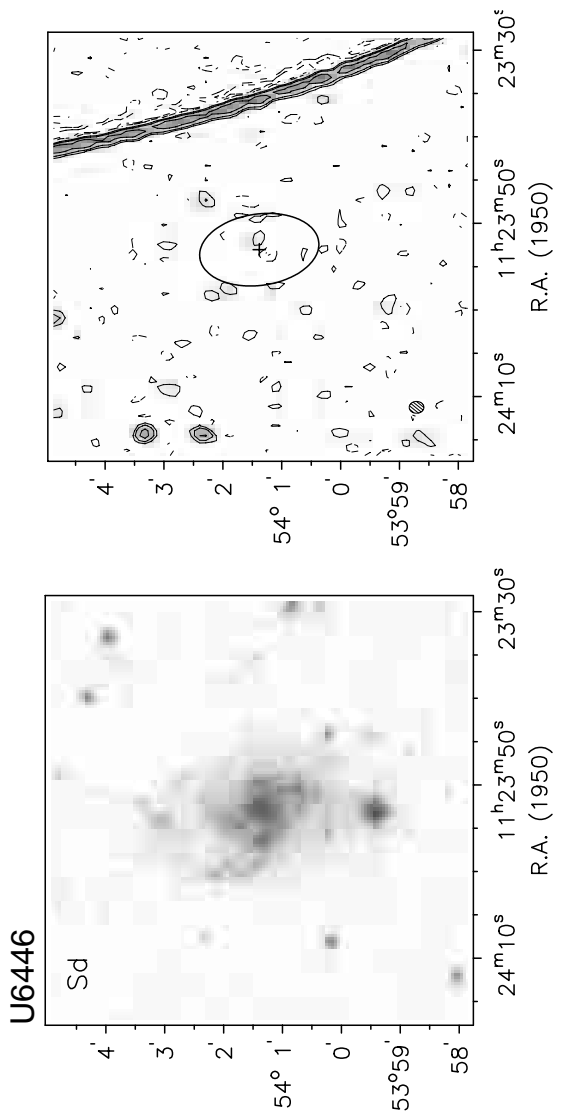

$(096 \mathrm{l}) \cdot 2$ ว

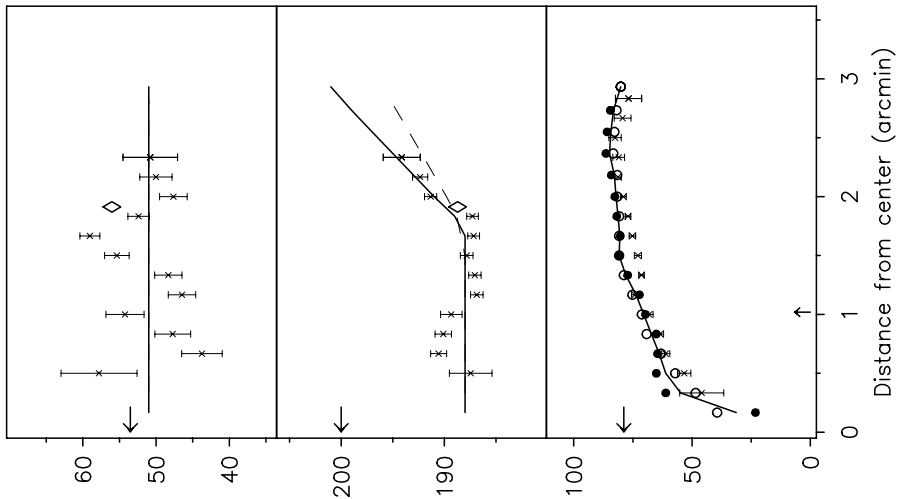

(०) · |оu|

(o) $\forall \cdot d$

$(s / m y)^{701} \wedge$

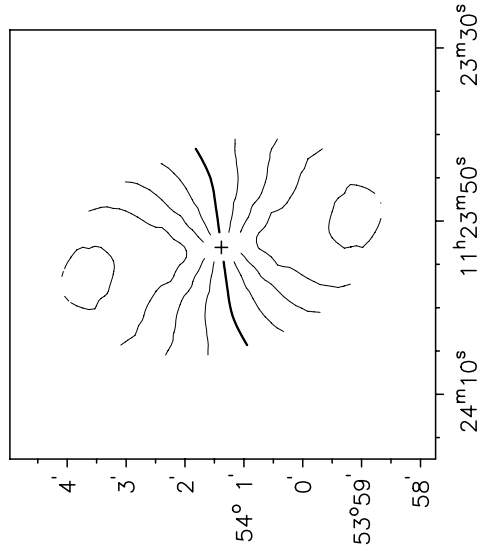

(056l) $\cdot 020$

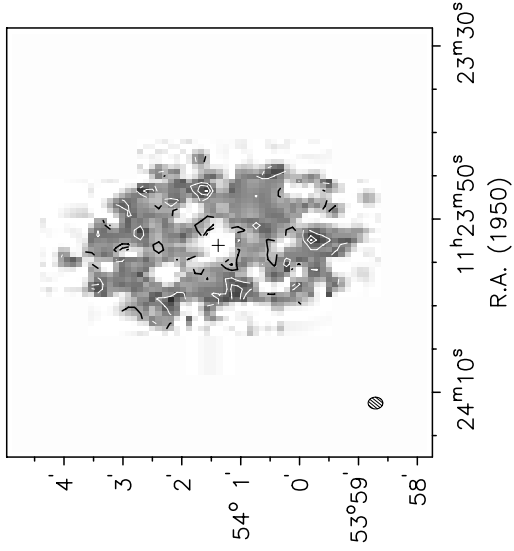

$(096 \mathrm{l}) \cdot 20^{2}$

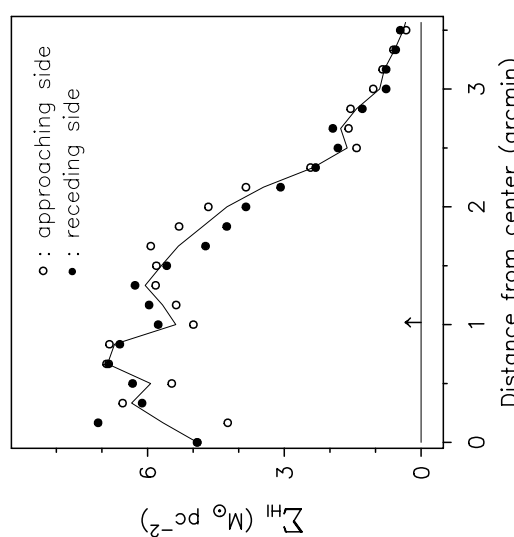

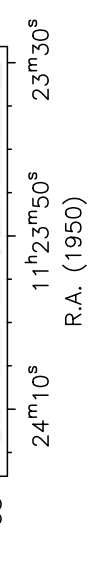

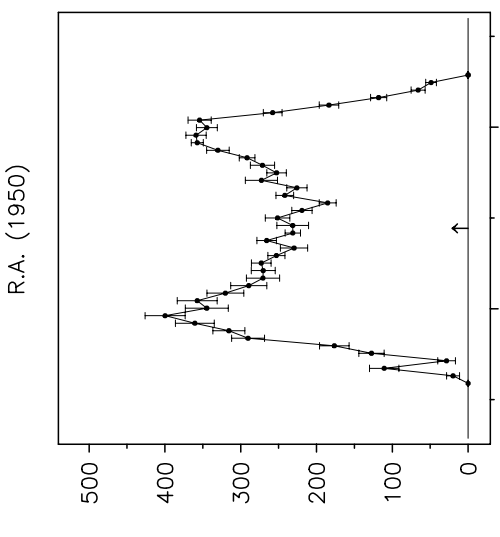

(Krw) Kł!suəp $\times$ nIJ

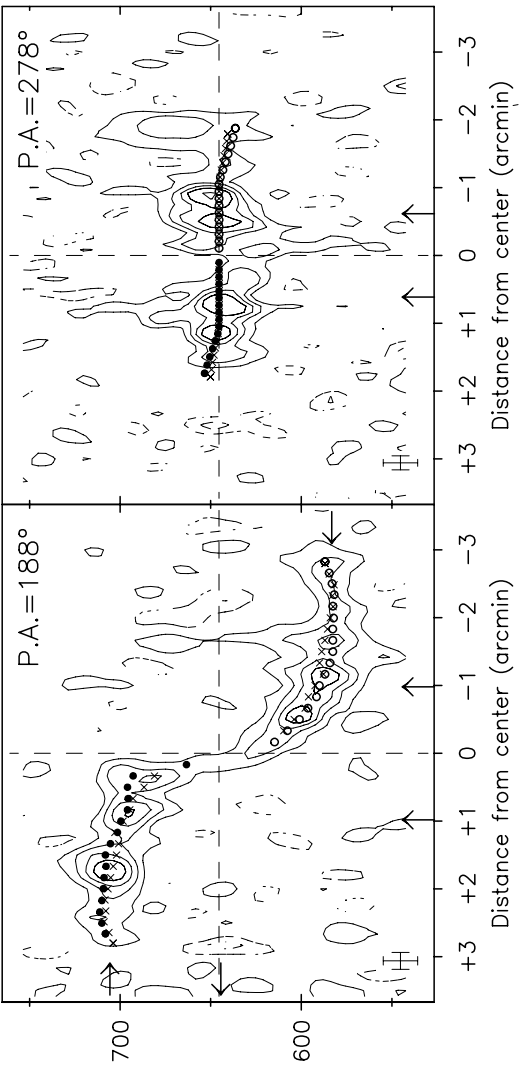

$(s / u y)^{124} \wedge$ 
Observing parameters for NGC 3726

\begin{tabular}{|c|c|}
\hline Length of observation & $1 \times 12$ \\
\hline Date of observation & 27Dec93 \\
\hline Field center, $\alpha(1950)$ & 11:30:38 \\
\hline$\delta(1950)$ & 47:18:13 \\
\hline Central frequency & 1416.39 \\
\hline$V_{\text {hel }}$ of central channel & 870 \\
\hline Primary beam FWHM (arcmin) & 37.4 \\
\hline Nr. of interferometers & 40 \\
\hline Baselines (min-max-incr) & $36-2700-72$ \\
\hline Synthesized beam $(\alpha \times \delta)(\operatorname{arcsec})$ & $12.2 \times 16.5$ \\
\hline Bandwidth & 2.5 \\
\hline Number of channels & 127 \\
\hline Channel separation & 4.13 \\
\hline Velocity resolution & 4.95 \\
\hline rms noise in one channel & 9.12 \\
\hline $\begin{array}{l}\text { K-mJy conversion, } \\
\text { equiv. of } 1 \mathrm{mJy} / \text { beam }\end{array}$ & 2.99 \\
\hline
\end{tabular}

equiv. of $1 \mathrm{mJy} / \mathrm{beam}$
Contour levels for N3726

Channel maps:
Raw continuum map: $\quad \sigma=0.36(\mathrm{~K})$
Cleaned continuum map: $\sigma=0.37(\mathrm{~K})$
$\sigma=0.37(\mathrm{~K})$
Position-Velocity diagrams:
$\quad \sigma=0.99(\mathrm{~K})$
Velocity fields: $\quad 862.6 \pm \mathrm{n} \times 20\left(\mathrm{~km} \mathrm{~s}^{-1}\right)$
Residual velocity field: $\quad \pm \mathrm{n} \times 5\left(\mathrm{~km} \mathrm{~s}^{-1}\right)$
Integrated HI map:
$0.41,0.82, \quad$
$1.23\left(\times 10^{21} \mathrm{atoms} \mathrm{cm}^{-2}\right)$

Note: This bright spiral is optically lopsided; the northern part has a higher surface brightnes than the southern part. However, the faintest isophotes are elliptical and centered on the bright nucleus.

The channel maps are shown at a resoThe channel maps are shown at a reso-
lution of $60 \times 60$ arcsec while the other lution of $60 \times 60$ arcsec while the other
data are presented at a spatial resolution data are presented at a spatial resolution
of $30 \times 30$ arcsec. The channel maps suggest that the HI disk is strongly warped toward edge-on beyond a radius of 3 arcmin. From the channel maps, the position angle of the outer disk is estimated at 179 degrees and the inclination angle at a minimum value of 75 nation angle at a minimum value of 75 degrees based upon the extent along the minor axis near the systemic velocity. The rotation curve in the inner region is asymmetric. The signature of the small

twisted velocity field.

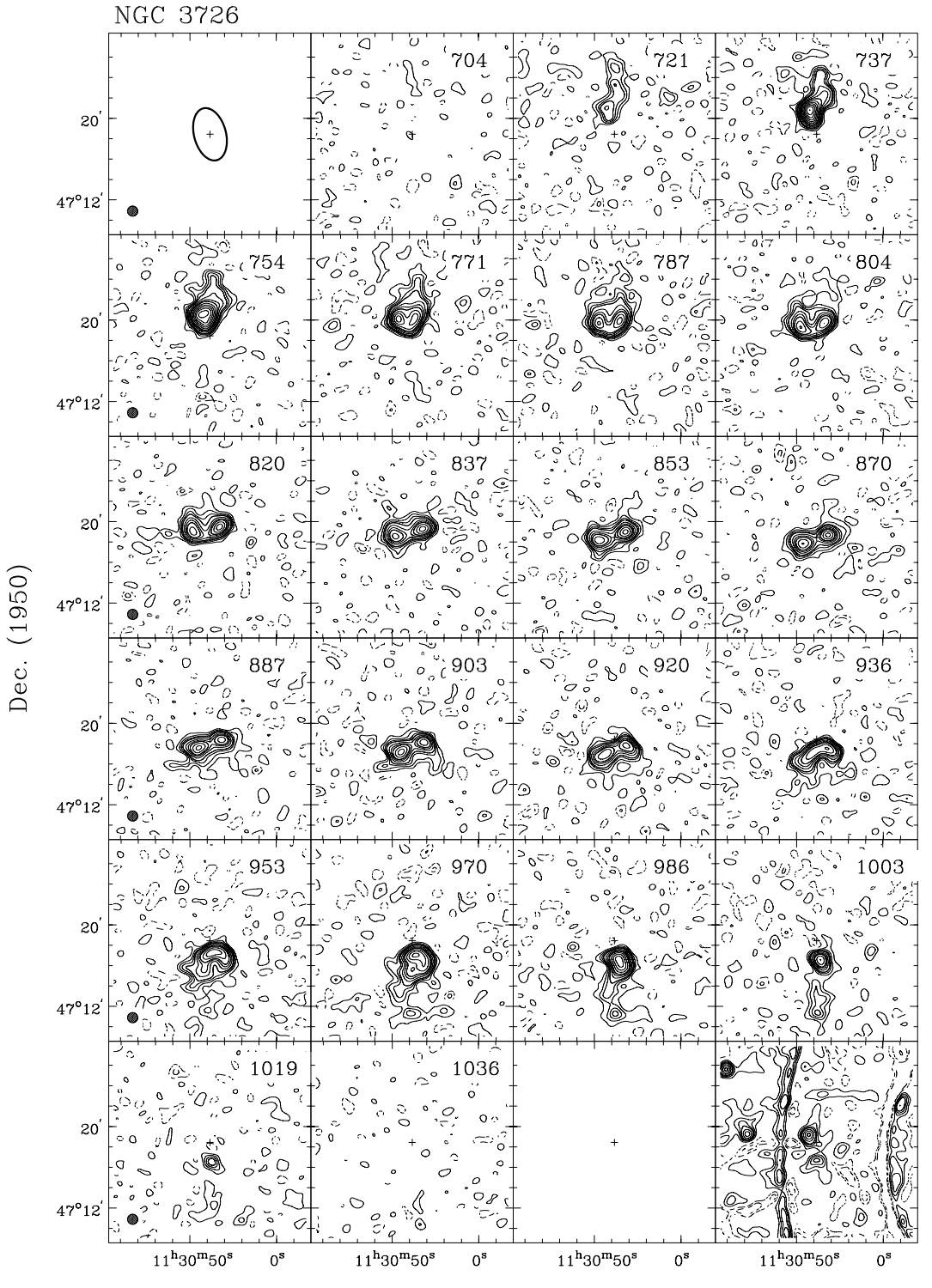

\section{From total HI map:}

Position angle $\delta(1950)$

Inclination angle (deg)

Diameter of HI disk (arcmin)

\begin{tabular}{|c|c|}
\hline \multicolumn{2}{|l|}{ Results from WSRT data } \\
\hline From continuum map: & \\
\hline$\overline{21-\mathrm{cm} \text { flux density }}$ & $49.7 \pm 5.0$ \\
\hline \multicolumn{2}{|l|}{ From global profile: } \\
\hline$\overline{\text { Integrated HI-flux }}\left(\mathrm{Jy} \mathrm{km} \mathrm{s}^{-1}\right)$ & $89.8 \pm 0.8$ \\
\hline Hel. systemic velocity $\left(\mathrm{km} \mathrm{s}^{-1}\right)$ & $865.6 \pm 0.9$ \\
\hline HI profile width, $20 \%\left(\mathrm{~km} \mathrm{~s}^{-1}\right)$ & $286.5 \pm 1.6$ \\
\hline $50 \%\left(\mathrm{~km} \mathrm{~s}^{-1}\right)$ & $260.6 \pm 1.8$ \\
\hline \multicolumn{2}{|l|}{ From velocity field: } \\
\hline 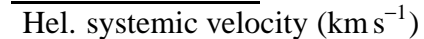 & $862.6 \pm 1.2$ \\
\hline Dynamical center, $\alpha(1950)$ & $11: 30: 38.3$ \\
\hline$\delta(1950)$ & $47: 18: 28$ \\
\hline \multicolumn{2}{|l|}{ From total HI map: } \\
\hline$\overline{\text { Geometric center, } \alpha} \alpha(1950)$ & $11: 30: 39.3$ \\
\hline$\delta(1950)$ & $47: 18: 34$ \\
\hline Position angle & 189 \\
\hline Inclination angle & 56 \\
\hline Diameter of HI disk (arcmin) & 8.5 \\
\hline
\end{tabular}



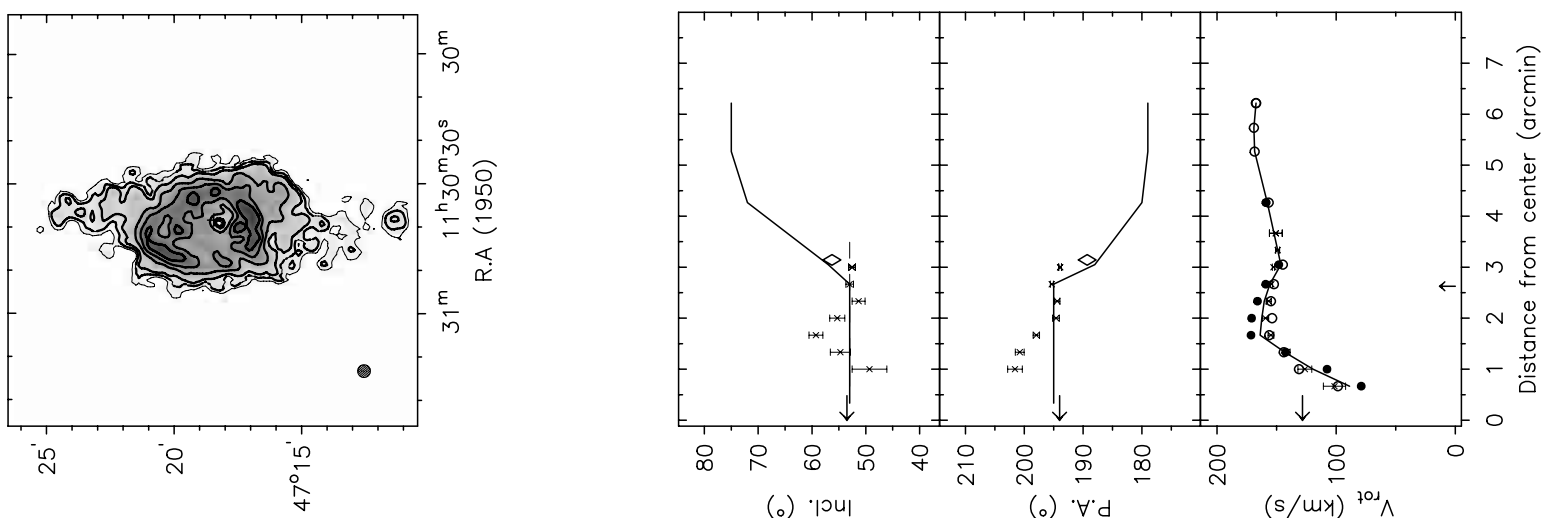

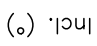

(o) $\cdot \forall \cdot d$

$(s / m y)^{701} \wedge$

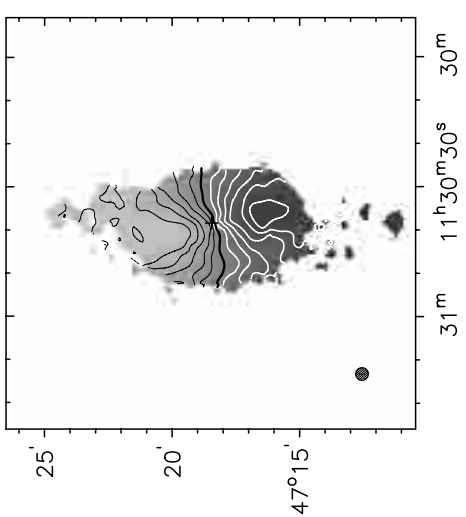

$(0 \mathrm{col}) \cdot 200$

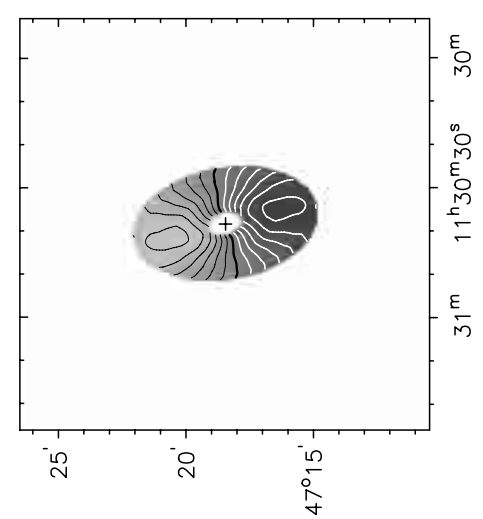

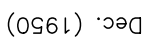

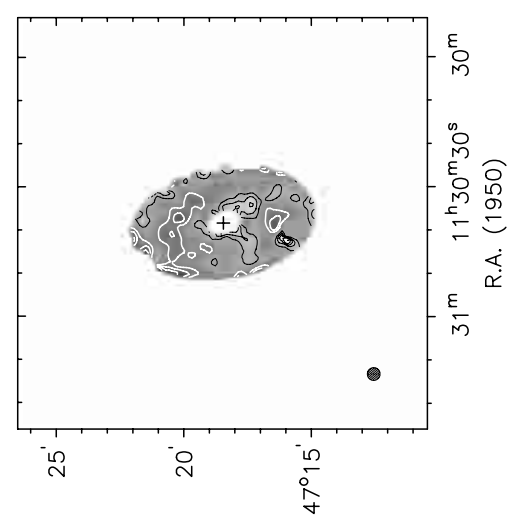

(0s6l) $\cdot 200$
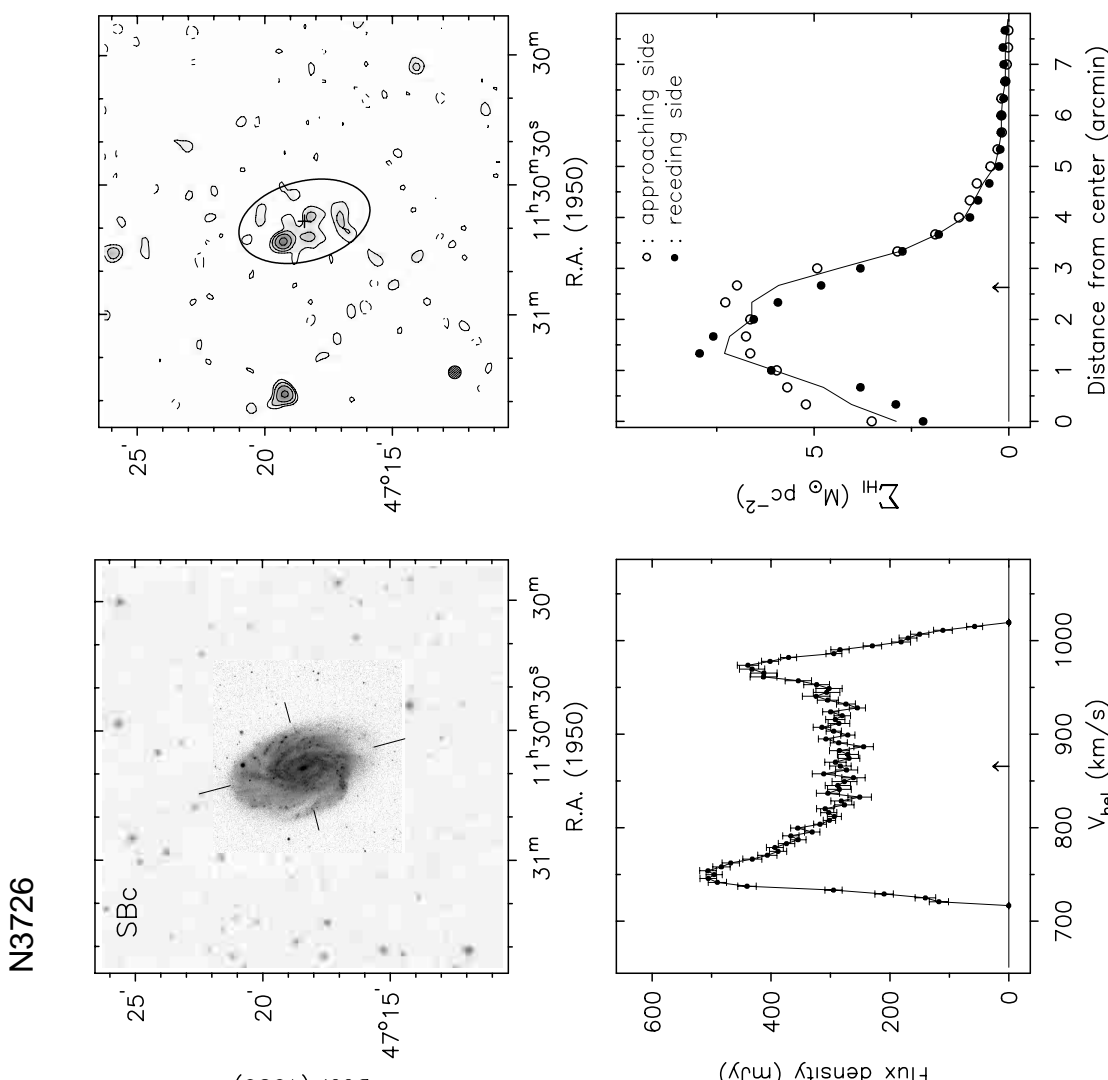

(096l) $\cdot 2$ م

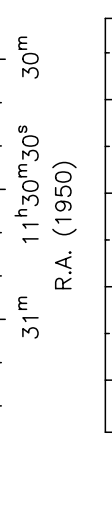

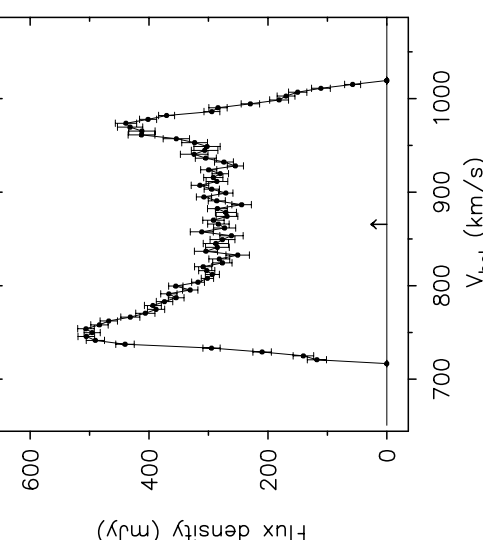

(Кгш) Kұ!suәp xn!

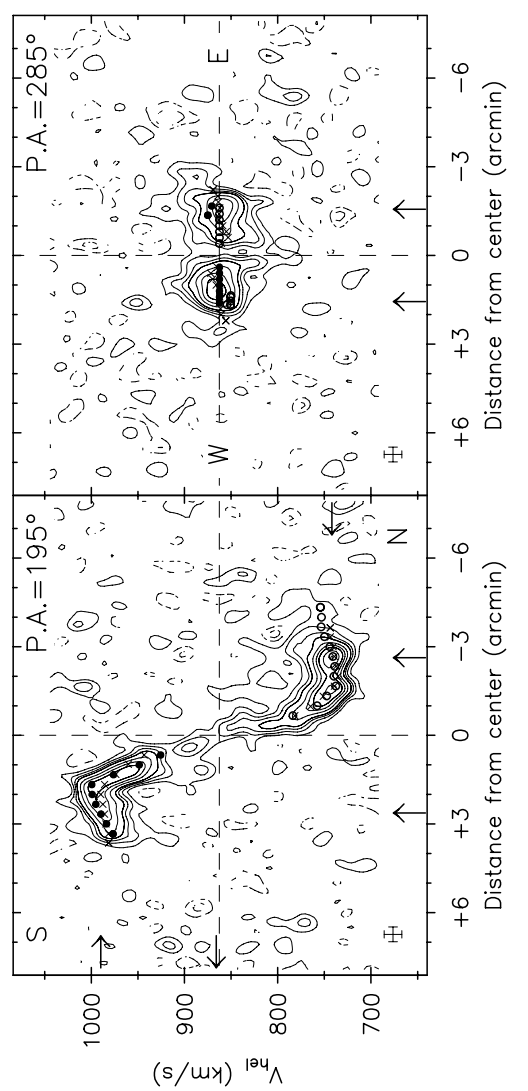


Observing parameters for NGC 3769

\begin{tabular}{|c|c|}
\hline Length of observation & $1 \times 12$ \\
\hline Date of observation & 2Aug90 \\
\hline Field center, $\alpha(1950)$ & 11:35:00 \\
\hline$\delta(1950)$ & 48:11:00 \\
\hline Central frequency & 1416.87 \\
\hline$V_{\text {hel }}$ of central channel & 740 \\
\hline Primary beam FWHM (arcmin) & 37.4 \\
\hline Nr. of interferometers & 40 \\
\hline Baselines (min-max-incr) & $36-2700-72$ \\
\hline Synthesized beam $(\alpha \times \delta)(\operatorname{arcsec})$ & $11.9 \times 15.9$ \\
\hline Bandwidth & 2.5 \\
\hline Number of channels & 127 \\
\hline Channel separation & 4.14 \\
\hline Velocity resolution & 8.29 \\
\hline rms noise in one channel & 6.53 \\
\hline $\begin{array}{l}\text { K-mJy conversion, } \\
\text { equiv. of } 1 \mathrm{mJy} / \text { beam }\end{array}$ & 3.18 \\
\hline
\end{tabular}

Results from WSRT data

\begin{tabular}{|c|c|}
\hline \multicolumn{2}{|l|}{ From continuum map: } \\
\hline From global profile: & $12.1 \pm 2.9$ \\
\hline$\overline{\text { Integrated HI-flux }}\left(\mathrm{Jy} \mathrm{km} \mathrm{s}^{-1}\right)$ & $62.3 \pm 0.6$ \\
\hline Hel. systemic velocity $\left(\mathrm{km} \mathrm{s}^{-1}\right)$ & $737.3 \pm 1.8$ \\
\hline HI profile width, $20 \%\left(\mathrm{~km} \mathrm{~s}^{-1}\right)$ & $265.3 \pm 6.7$ \\
\hline $50 \%\left(\mathrm{~km} \mathrm{~s}^{-1}\right)$ & $230.5 \pm 3.6$ \\
\hline \multicolumn{2}{|l|}{ From velocity field: } \\
\hline 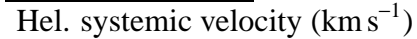 & $722.9 \pm 1.9$ \\
\hline $\begin{array}{r}\text { Dynamical center, } \alpha(1950) \\
\delta(1950)\end{array}$ & $\begin{array}{l}11: 35: 02.4 \\
48: 10: 12\end{array}$ \\
\hline \multicolumn{2}{|l|}{ From total HI map: } \\
\hline$\overline{\text { Geometric center, } \alpha(1950)}$ & $\begin{array}{l}11: 35: 03.9 \\
48 \cdot 10 \cdot 09\end{array}$ \\
\hline Position angle & 151 \\
\hline Inclination angle & 71 \\
\hline Diameter of HI disk (arcmin) & 8.6 \\
\hline
\end{tabular}

Contour levels for N3769

\begin{tabular}{|c|c|}
\hline \multicolumn{2}{|l|}{ Channel maps: } \\
\hline $\begin{array}{l}\text { Channel maps: } \\
\text { Raw continuum map: }\end{array}$ & $\sigma=0.72(\mathrm{~K})$ \\
\hline Cleaned continuum $\mathrm{m}$ & $\sigma=0.52(\mathrm{~K})$ \\
\hline Position-Velocity diag & $\begin{array}{l}\text { ams: } \\
\sigma=1.14(\mathrm{~K})\end{array}$ \\
\hline $\begin{array}{l}\text { Velocity fields: } \\
722.9 \pm \\
\text { Residual velocity fiel }\end{array}$ & $\times 20\left(\mathrm{~km} \mathrm{~s}^{-1}\right)$ \\
\hline $\begin{array}{c}\text { Integrated HI map: } \\
0.18,0.36,0 \\
1.07,1.24(\times 1\end{array}$ & $\begin{array}{l}\mathrm{n} \times 5\left(\mathrm{~km} \mathrm{~s}^{-1}\right) \\
\\
53,0.71,0.89 \\
\left.1 \text { atoms } \mathrm{cm}^{-2}\right)\end{array}$ \\
\hline
\end{tabular}

Note: N3769 is strongly interacting with its dwarf companion $1135+48$. This pair is also known as Arp 280. The HI disk is clearly warped and extends far beyond $D_{25}$. Confusion with its companion and with the tidal debris its companion and with the tidal debris
makes the velocity field in the southern part quite complex. The change in position angle is estimated from the channel maps around $645 \mathrm{~km} \mathrm{~s}^{-1}$ at 60 arcsec resolution. The rotational velocity is very uncertain beyond 2 arcmin where the warp sets in. Large scale noncircular motions are likely to occur in this system.

The channel maps are shown at a resolution of $60 \times 60$ arcsec while the other data are presented with a resolution of $30 \times 30$ arcsec.

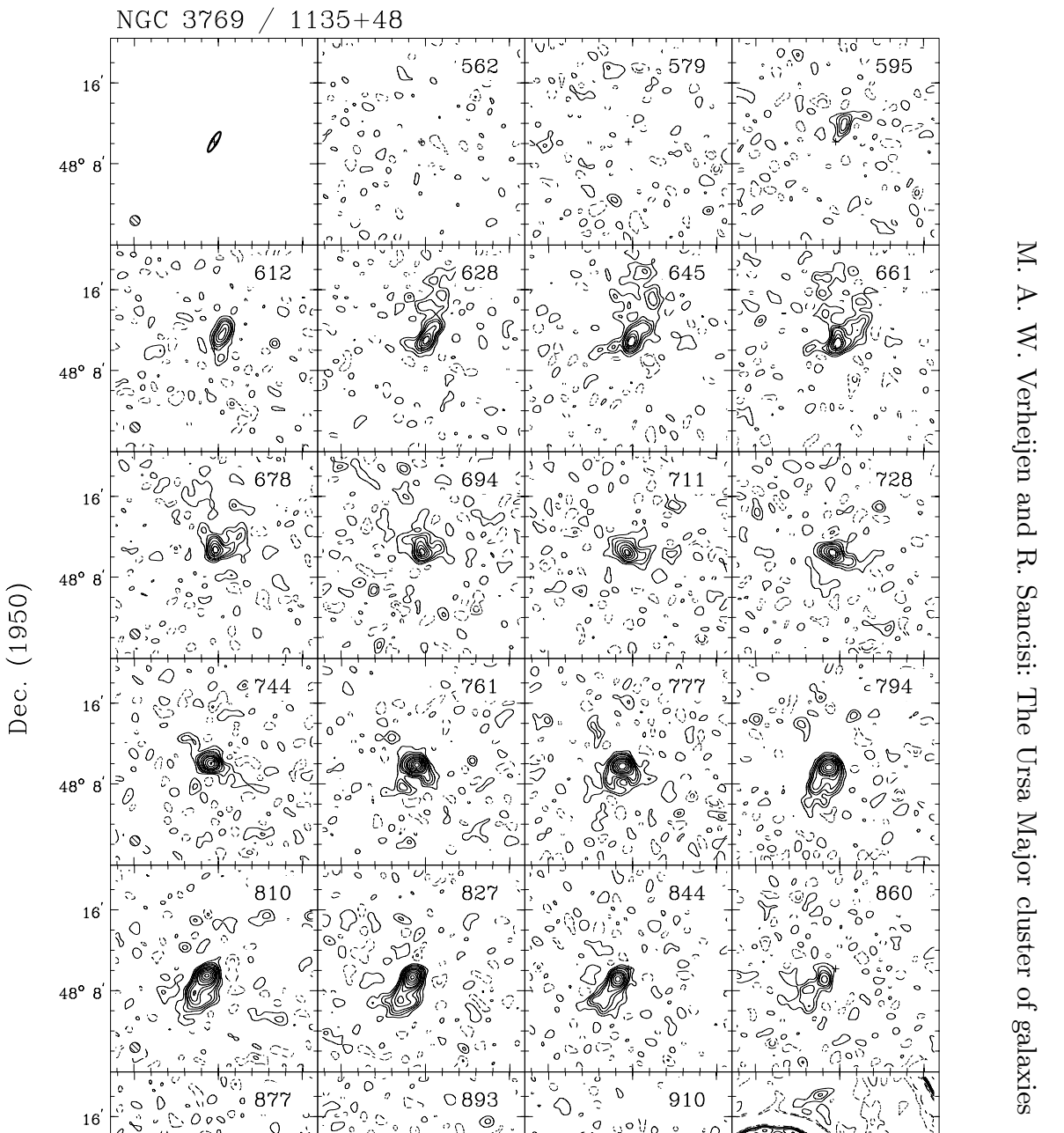

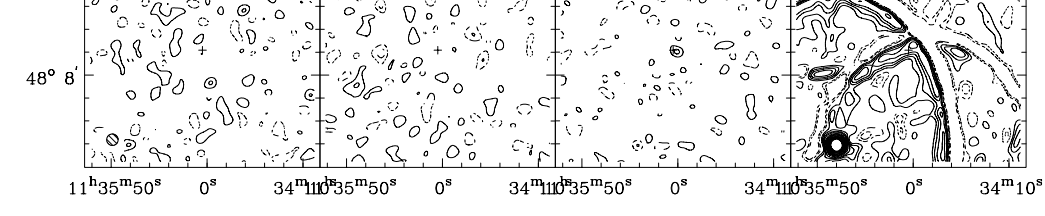

$$
\text { R.A. (1950) }
$$

Channel maps at a resolution of $60^{\prime \prime} \times 60^{\prime \prime} \times 19 \mathrm{~km} \mathrm{~s}^{-1}$. Contour levels at $-3,-1.5$ (dashed) $1.5,3,4.5, \ldots \times \sigma$. 

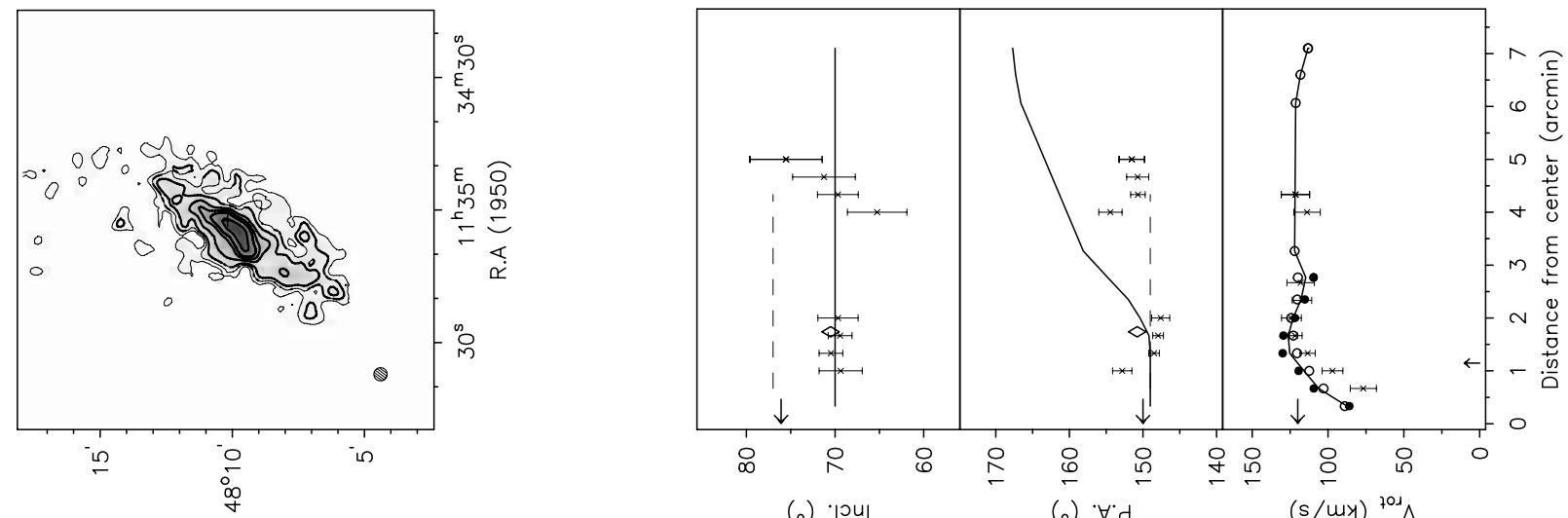
(o) '|ou|
(o) $\forall \cdot d$
$(s / m y){ }^{701} \wedge$

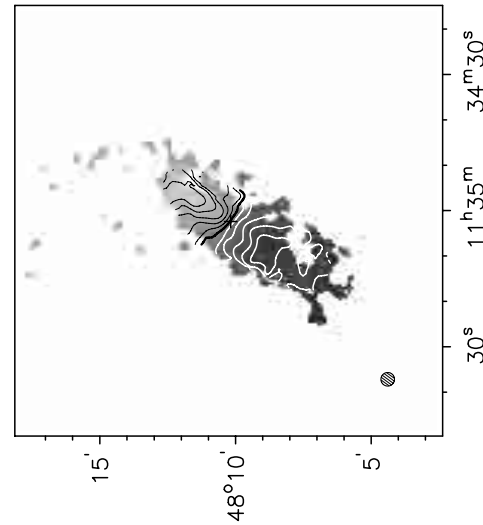

(096l) $\cdot 020$

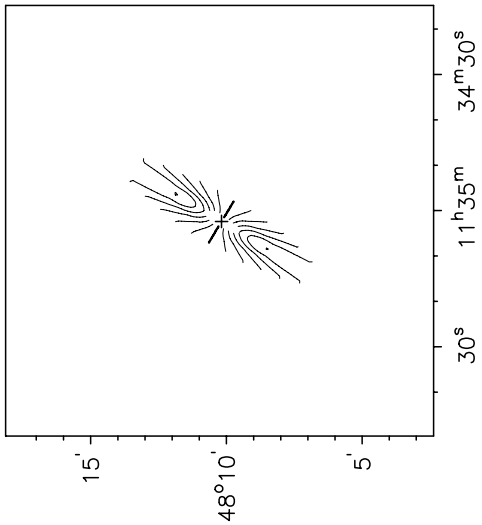

(096l) $\cdot 5$

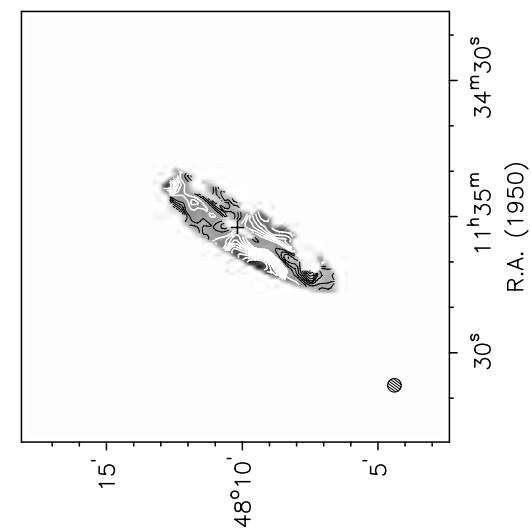

(0.56l)
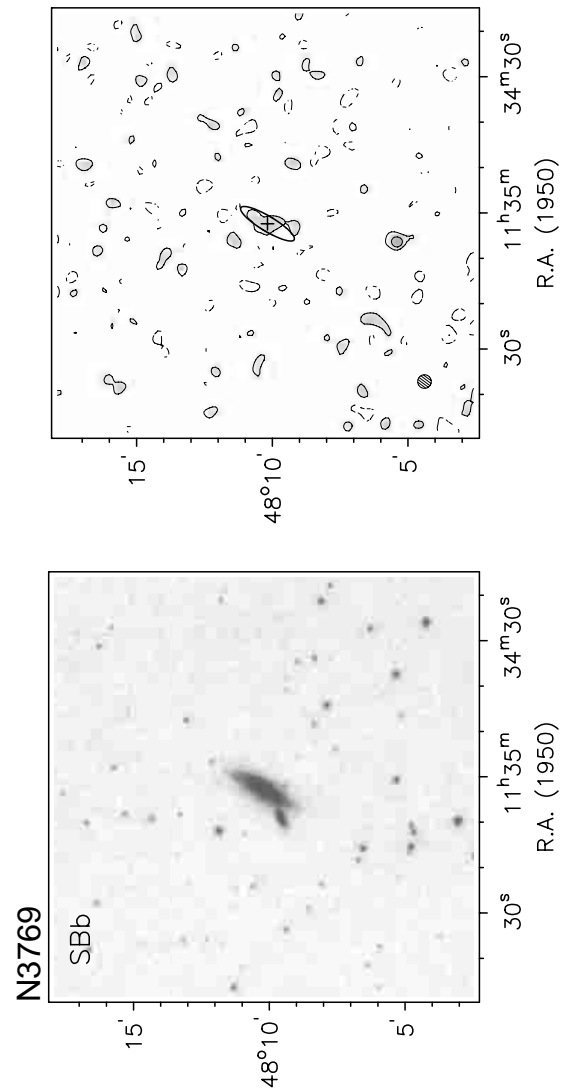

(0s6l) $\cdot 2$ م
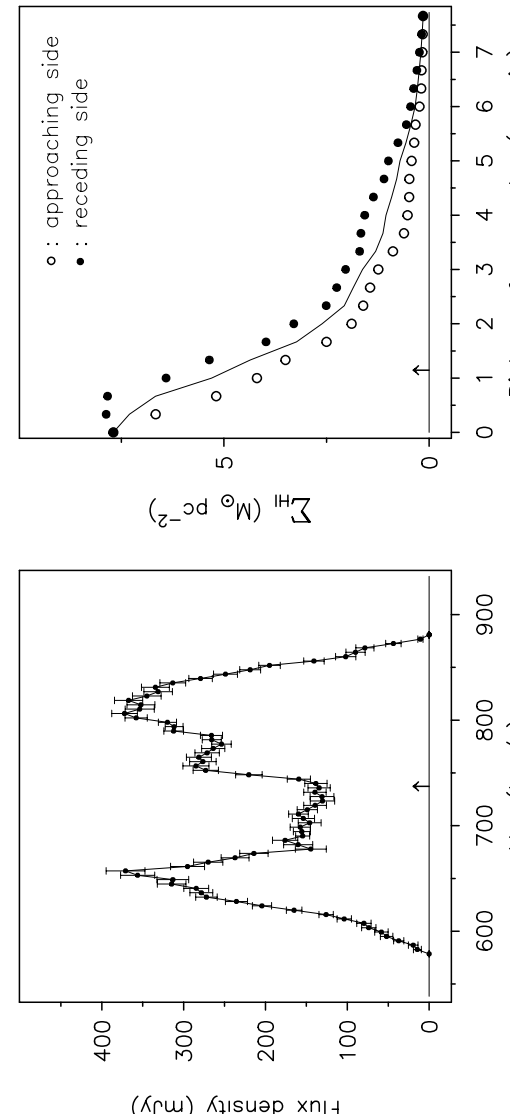

(Kৎm) K?!suəp xn!

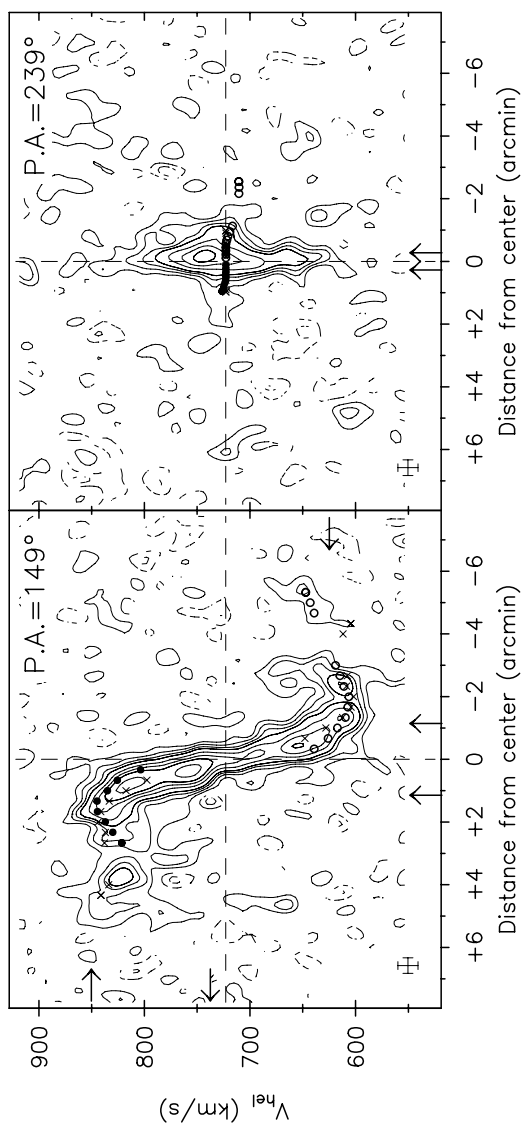


Observing parameters for UGC 6667

\begin{tabular}{|c|c|}
\hline Length of observation & $1 \times 12$ \\
\hline Date of observation & 16Dec94 \\
\hline Field center, $\alpha(1950)$ & $11: 39: 42$ \\
\hline$\delta(1950)$ & 51:50:00 \\
\hline Central frequency & 1415.78 \\
\hline$V_{\text {hel }}$ of central channel & 1000 \\
\hline Primary beam FWHM & 37.4 \\
\hline Nr. of interferometers & 40 \\
\hline Baselines (min-max-incr) & $36-2700-72$ \\
\hline Synthesized beam $(\alpha \times \delta)(\operatorname{arcsec})$ & $12.0 \times 15.4$ \\
\hline Bandwidth & 2.5 \\
\hline Number of channels & 127 \\
\hline Channel separation & 4.15 \\
\hline Velocity resolution & 4.98 \\
\hline rms noise in one channel & 10.4 \\
\hline $\begin{array}{l}\text { K-mJy conversion, } \\
\text { equiv. of } 1 \mathrm{mJy} / \text { beam }\end{array}$ & 3.26 \\
\hline
\end{tabular}

Results from WSRT data

\begin{tabular}{|c|c|}
\hline \multicolumn{2}{|l|}{ From continuит map: } \\
\hline \multicolumn{2}{|l|}{ 21-cm flux density: } \\
\hline central point source $(\mathrm{mJy})$ & $<1.3(3 \sigma)$ \\
\hline extended source (mJy) & $<2.7(3 \sigma)$ \\
\hline \multicolumn{2}{|l|}{ From global profile: } \\
\hline Integrated HI-flux $\left(\mathrm{Jy} \mathrm{km} \mathrm{s}^{-1}\right)$ & $11.0 \pm 0.4$ \\
\hline Hel. systemic velocity $\left(\mathrm{km} \mathrm{s}^{-1}\right)$ & $973.2 \pm 1.2$ \\
\hline HI profile width, $20 \%\left(\mathrm{~km} \mathrm{~s}^{-1}\right)$ & $187.5 \pm 1.4$ \\
\hline $50 \%\left(\mathrm{~km} \mathrm{~s}^{-1}\right)$ & $178.1 \pm 1.9$ \\
\hline \multicolumn{2}{|l|}{ From velocity field: } \\
\hline 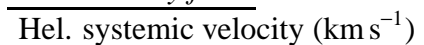 & $970.5 \pm 4.5$ \\
\hline Dynamical center, $\alpha(1950)$ & $11: 39: 44.9$ \\
\hline$\delta(1950)$ & $51: 52: 31$ \\
\hline \multicolumn{2}{|l|}{ From total HI map: } \\
\hline Geometric center, $\alpha(1950)$ & $11: 39: 45.0$ \\
\hline$\delta(1950)$ & $51: 52: 31$ \\
\hline Position angle & 88 \\
\hline Inclination angle & 48 \\
\hline Diameter of HI disk (arcmin) & 3.3 \\
\hline
\end{tabular}

Contour levels for U6667

\begin{tabular}{|c|c|}
\hline \\
\hline $\begin{array}{l}\text { Channel maps: } \\
\text { Raw continuum map: }\end{array}$ & $\sigma=1.55(\mathrm{~K})$ \\
\hline \multicolumn{2}{|c|}{ Cleaned continuum map: } \\
\hline Position-Velocity diag & $\begin{array}{l}\text { Ims: } \\
\sigma=3.58(\mathrm{~K})\end{array}$ \\
\hline $\begin{array}{l}\text { Velocity fields: } \\
\qquad 970.5 \pm \\
\text { Residual velocity field }\end{array}$ & $\times 15\left(\mathrm{~km} \mathrm{~s}^{-1}\right)$ \\
\hline $\begin{array}{c}\text { Integrated HI map: } \\
0.71,1.42, \\
2.13(\times 10\end{array}$ & $\begin{array}{l}\times 5\left(\mathrm{~km} \mathrm{~s}^{-1}\right) \\
\text { toms cm} \\
-2\end{array}$ \\
\hline
\end{tabular}

Note: The center of the integrated HI map corresponds to the optical center within a few arcseconds. The rotation curve at the receding side seems to be systematically higher than on the approaching side, which suggests that the adopted systemic velocity is a few $\mathrm{km} / \mathrm{s}$ too low or that the actual dynamical center is slightly offset from the geometrical center. Also notice the different shape of the rotation curve on the two sides.

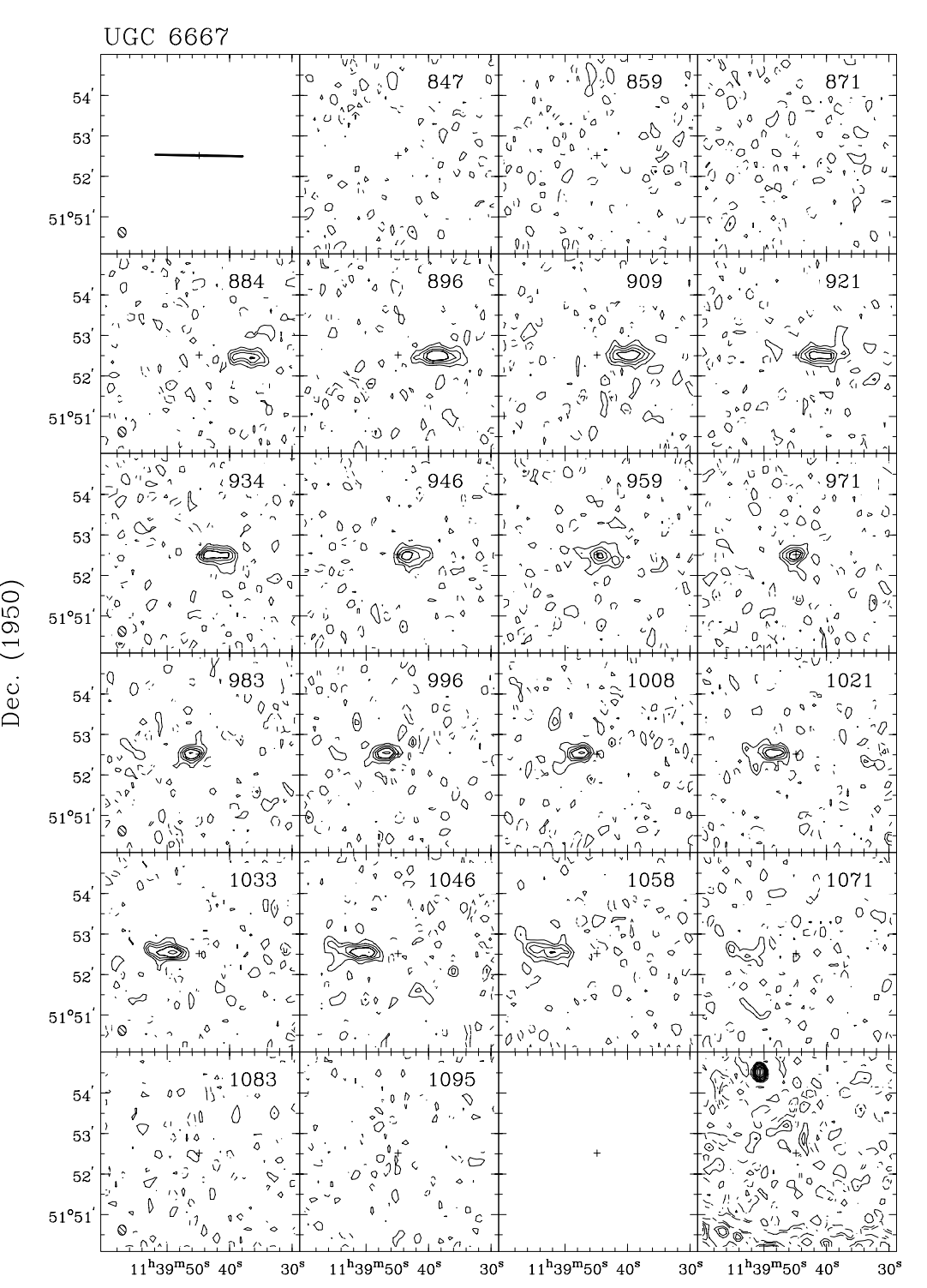

Channel maps at a resolution of $12^{\prime \prime} \times 15^{\prime \prime} \times 19 \mathrm{~km} \mathrm{~s}^{-1}$.

Contour levels at $-3,-1.5$ (dashed), $1.5,3,4.5, \ldots \times \sigma$. 

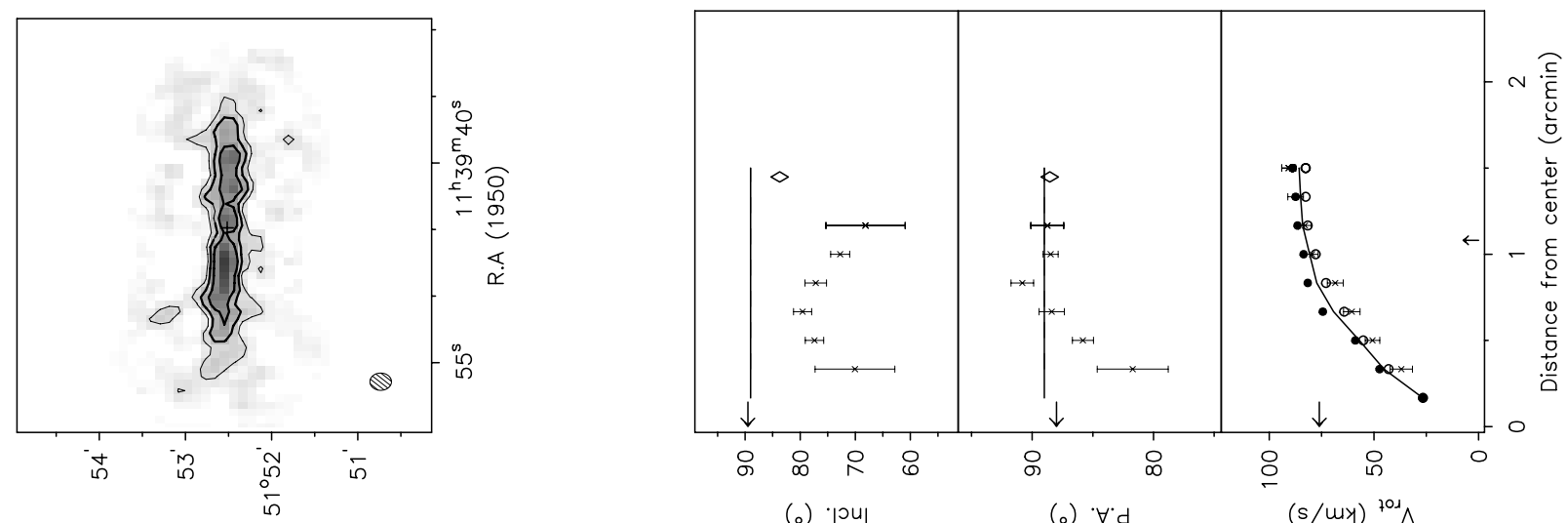
(o) '|⿰u|
(o) $\forall \cdot d$
$(s / m y){ }^{201} \wedge$

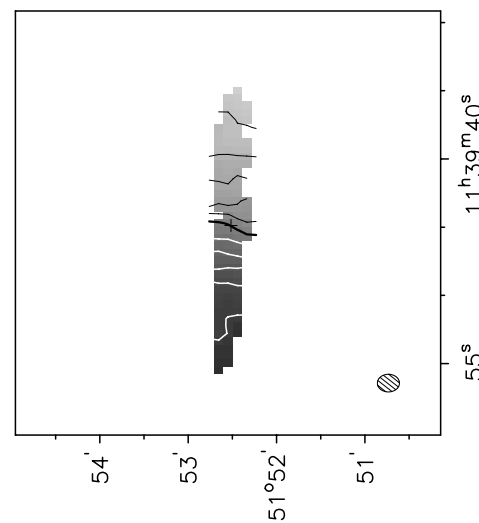

$(096 \mathrm{l}) \cdot 020$
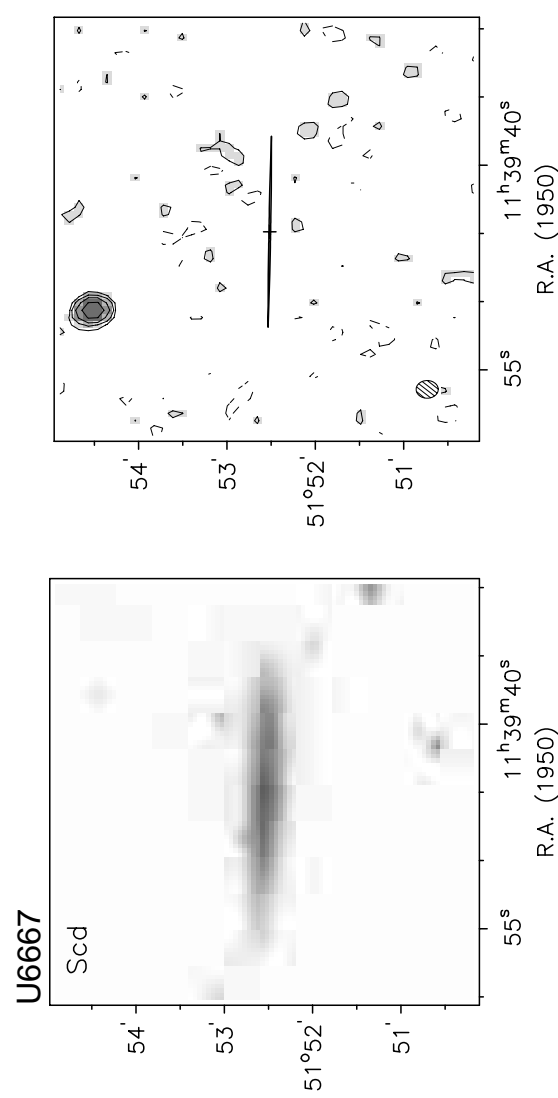

(0s6l) $\cdot 200$

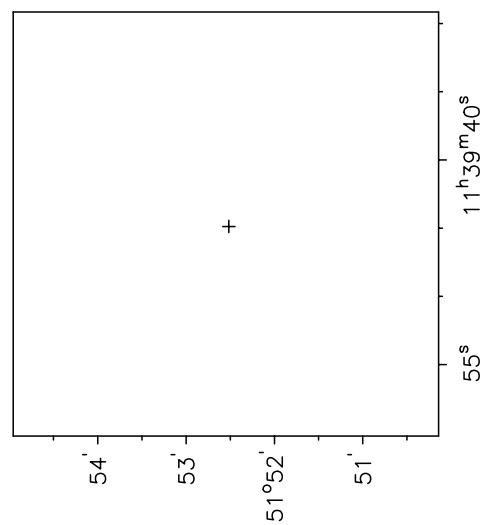

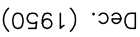

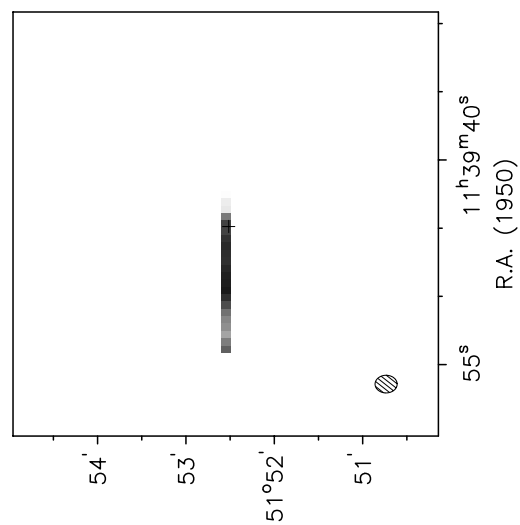

$(0961) \cdot 2.0$

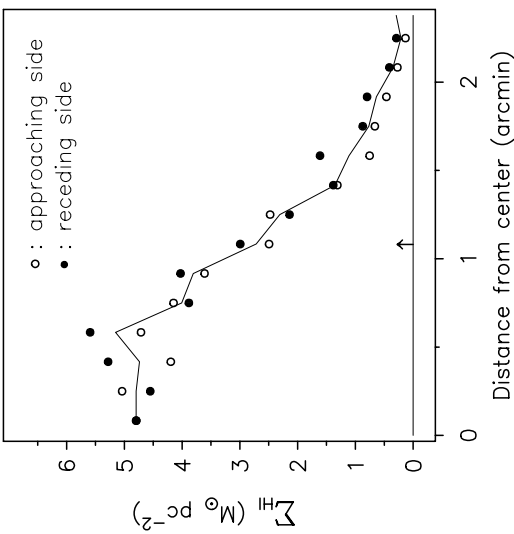

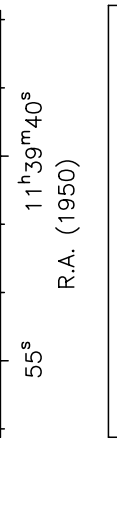

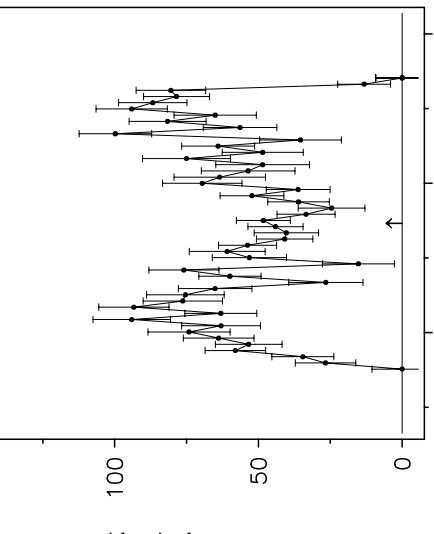

(Кгس) Kұ!suap xn!J

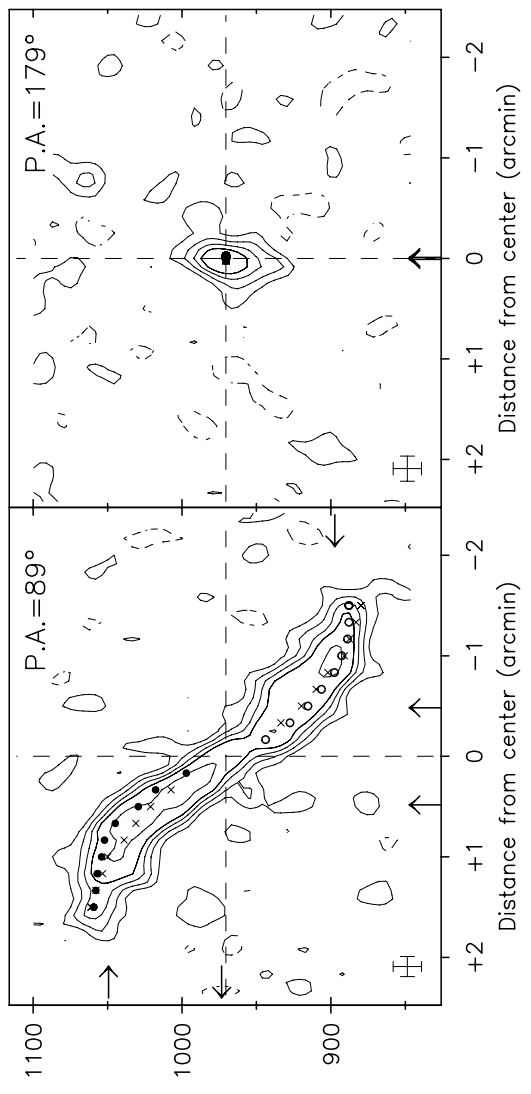

$(s / m y){ }^{124} \wedge$ 
Observing parameters for NGC 3877

\begin{tabular}{|c|c|}
\hline ength of observation & $2 \times 12$ \\
\hline Dates of observation & 3,9,14Aug90 \\
\hline Field center, $\alpha(1950)$ & $11: 43: 30$ \\
\hline$\delta(1950)$ & 47:45:00 \\
\hline Central frequency & 1416.12 \\
\hline$V_{\text {hel }}$ of central channel & 900 \\
\hline Primary beam FWHM & 37.4 \\
\hline $\mathrm{Nr}$ of interferometers & 40 \\
\hline Baselines (min-max-incr) & $36-2700-72$ \\
\hline Synthesized beam $(\alpha \times \delta)(\operatorname{arcsec})$ & $12.2 \times 17.6$ \\
\hline Bandwidth & 5.0 \\
\hline Number of channels & 63 \\
\hline Channel separation & 16.59 \\
\hline Velocity resolution & 33.18 \\
\hline rms noise in one channel & 1.96 \\
\hline $\begin{array}{l}\text { K-mJy conversion, } \\
\text { equiv. of } 1 \mathrm{mJy} / \text { beam }\end{array}$ & 2.81 \\
\hline
\end{tabular}

Results from WSRT data

\begin{tabular}{|c|c|}
\hline \multicolumn{2}{|l|}{ From continuum map: } \\
\hline 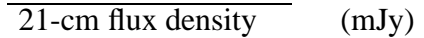 & $35.6 \pm 2.4$ \\
\hline \multicolumn{2}{|l|}{ Central point source position } \\
\hline$\alpha(1950)$ & $11: 43: 29.2$ \\
\hline$\delta(1950)$ & $47: 46: 21$ \\
\hline \multicolumn{2}{|l|}{ From global profile: } \\
\hline$\overline{\text { Integrated HI-flux }}\left(\mathrm{Jy} \mathrm{km} \mathrm{s}^{-1}\right)$ & $19.5 \pm 0.6$ \\
\hline Hel. systemic velocity $\left(\mathrm{km} \mathrm{s}^{-1}\right)$ & $895.4 \pm 3.8$ \\
\hline HI profile width, $20 \%\left(\mathrm{~km} \mathrm{~s}^{-1}\right)$ & $373.4 \pm 5.0$ \\
\hline $50 \%\left(\mathrm{~km} \mathrm{~s}^{-1}\right)$ & $344.5 \pm 6.2$ \\
\hline \multicolumn{2}{|l|}{ From velocity field: } \\
\hline 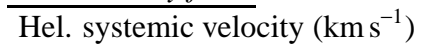 & $893.2 \pm 4.4$ \\
\hline Dynamical center, $\alpha(1950)$ & $11: 43: 28.9$ \\
\hline$\delta(1950)$ & $47: 46: 16$ \\
\hline \multicolumn{2}{|l|}{ From total HI map: } \\
\hline$\overline{\text { Geometric center, } \alpha(1950)}$ & $11: 43: 28.6$ \\
\hline$\delta(1950)$ & $47: 46: 15$ \\
\hline Position angle & 36 \\
\hline Inclination angle & 80 \\
\hline Diameter of HI disk (arcmin) & 4.4 \\
\hline
\end{tabular}

Contour levels for N3877

Channel maps: $\quad \sigma=1.96(\mathrm{~K})$

Raw continuum map:

Cleaned continuum map: $\sigma=0.67(\mathrm{~K})$

Position-Velocity diagrams:

Velocity fields:

$893.2 \pm \mathrm{n} \times 25\left(\mathrm{~km} \mathrm{~s}^{-1}\right)$ Residual velocity field: $\pm \mathrm{n} \times 5\left(\mathrm{~km} \mathrm{~s}^{-1}\right)$

Integrated HI map:

$0.80,1.61$,

$2.41\left(\times 10^{21}\right.$ atoms cm $\left.^{-2}\right)$

Note: This is a very regular system. The HI disk does not extend significantly beyond $D_{25}$. The peak intensity of the extended continuum emission coincides with a minimum in the HI distribution. The position of the weak central continuum point source coincides with the optical center but deviates some 5 arcsec (1 pixel) from the center of the HI map and the dynamical center. The coordinates of the dynamical center were used to make the position-velocity maps and the model velocity field.

The inclination angles of adjacent rings fitted to velocity field are quite consistent as a function of radius and is from the optical image and the HI map.

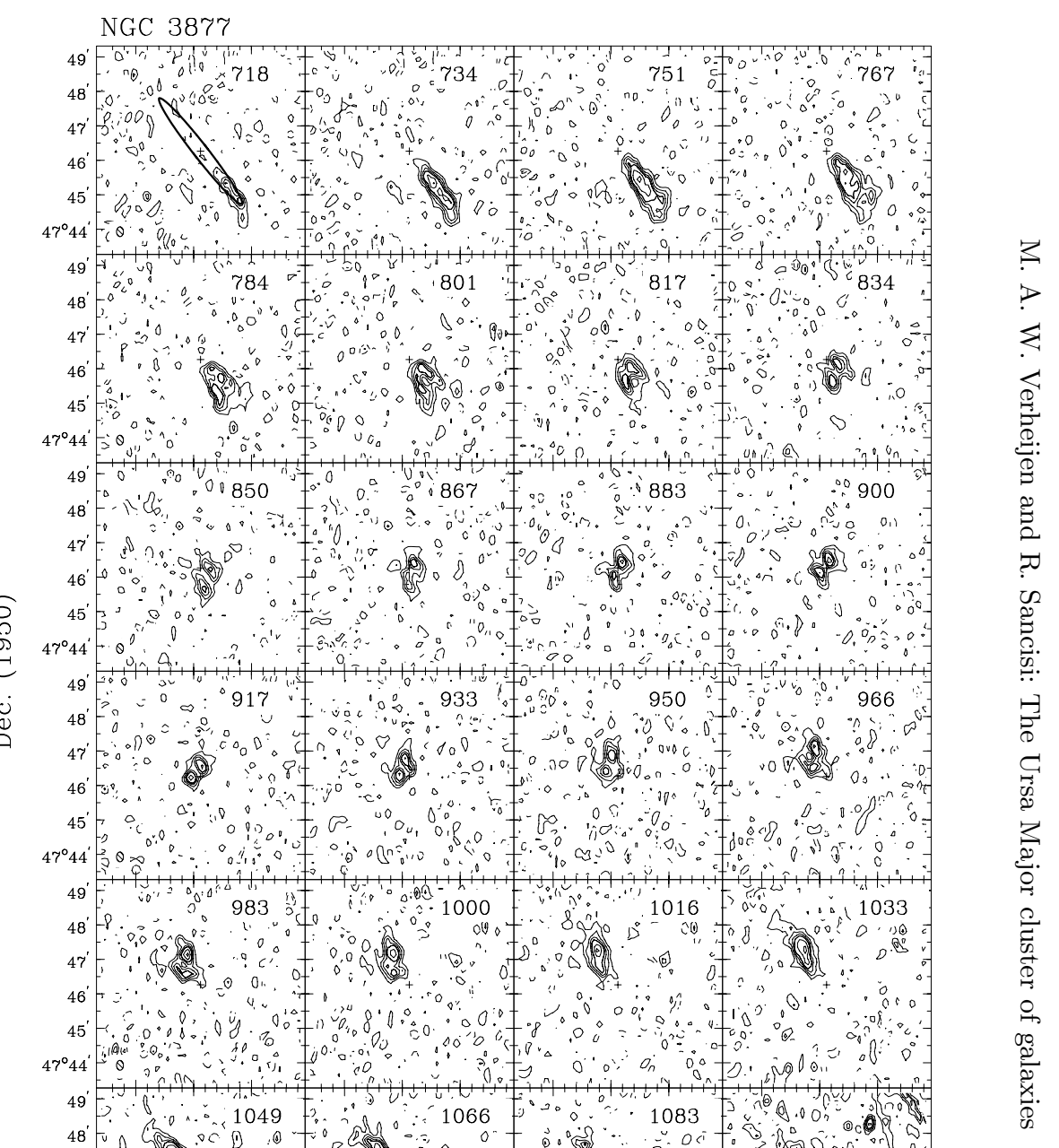

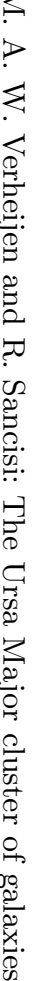



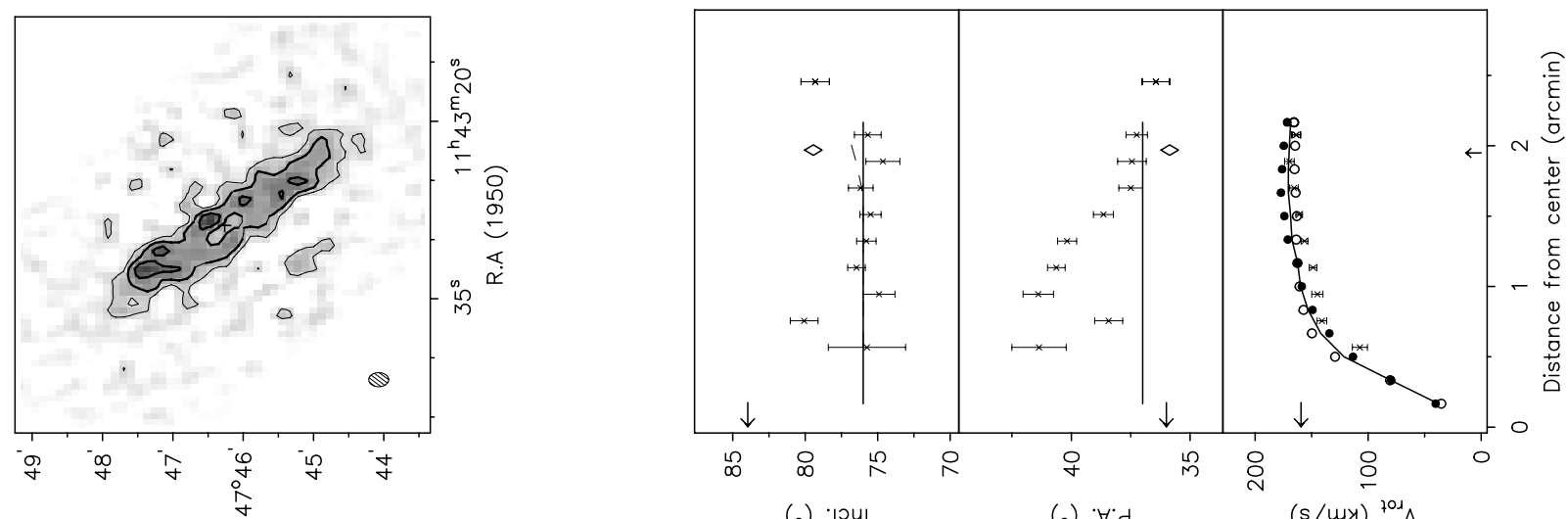
(o) '|⿰u|
(o) $\forall \cdot d$
$(s / m y)^{701} \wedge$

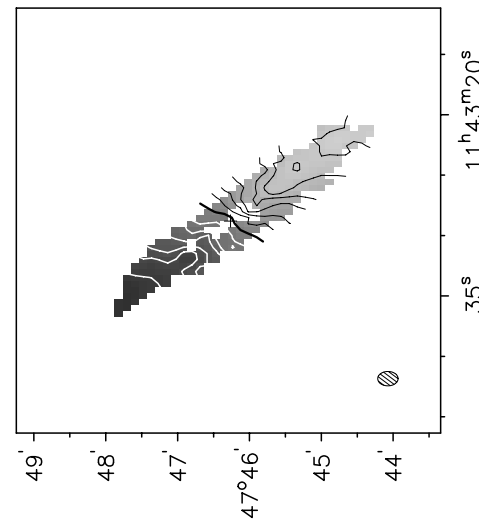

(096l) $\cdot 200$
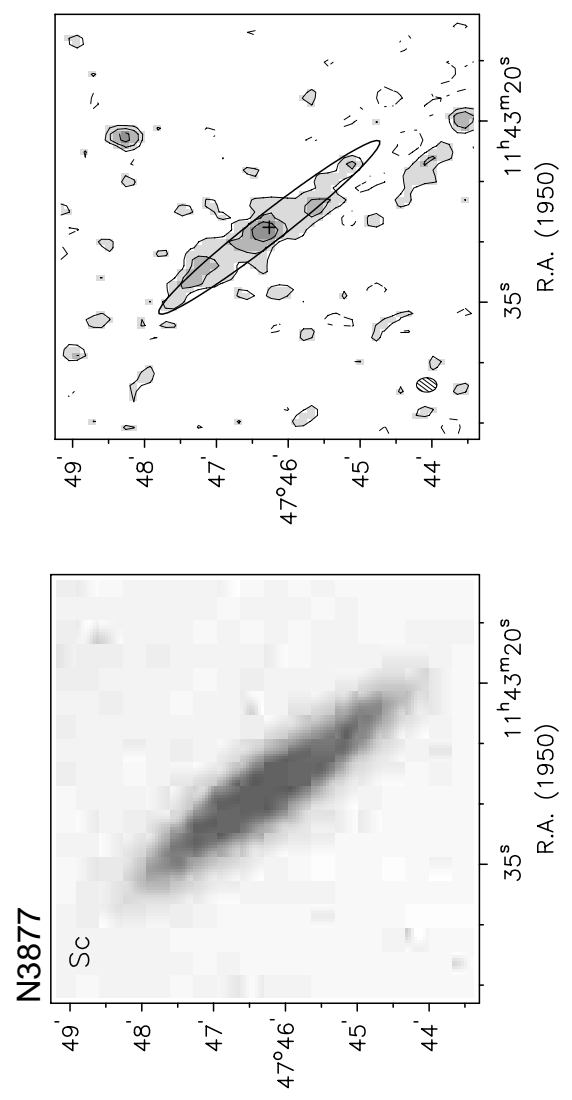

$(0961) \cdot 5 \circ 0$

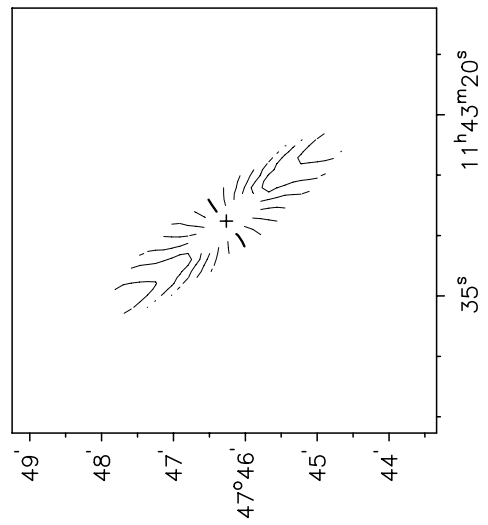

(056l) ' วә0

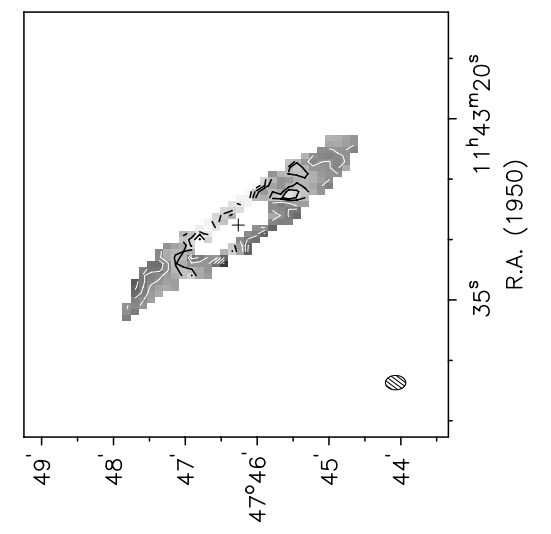

(0961) 'วә0
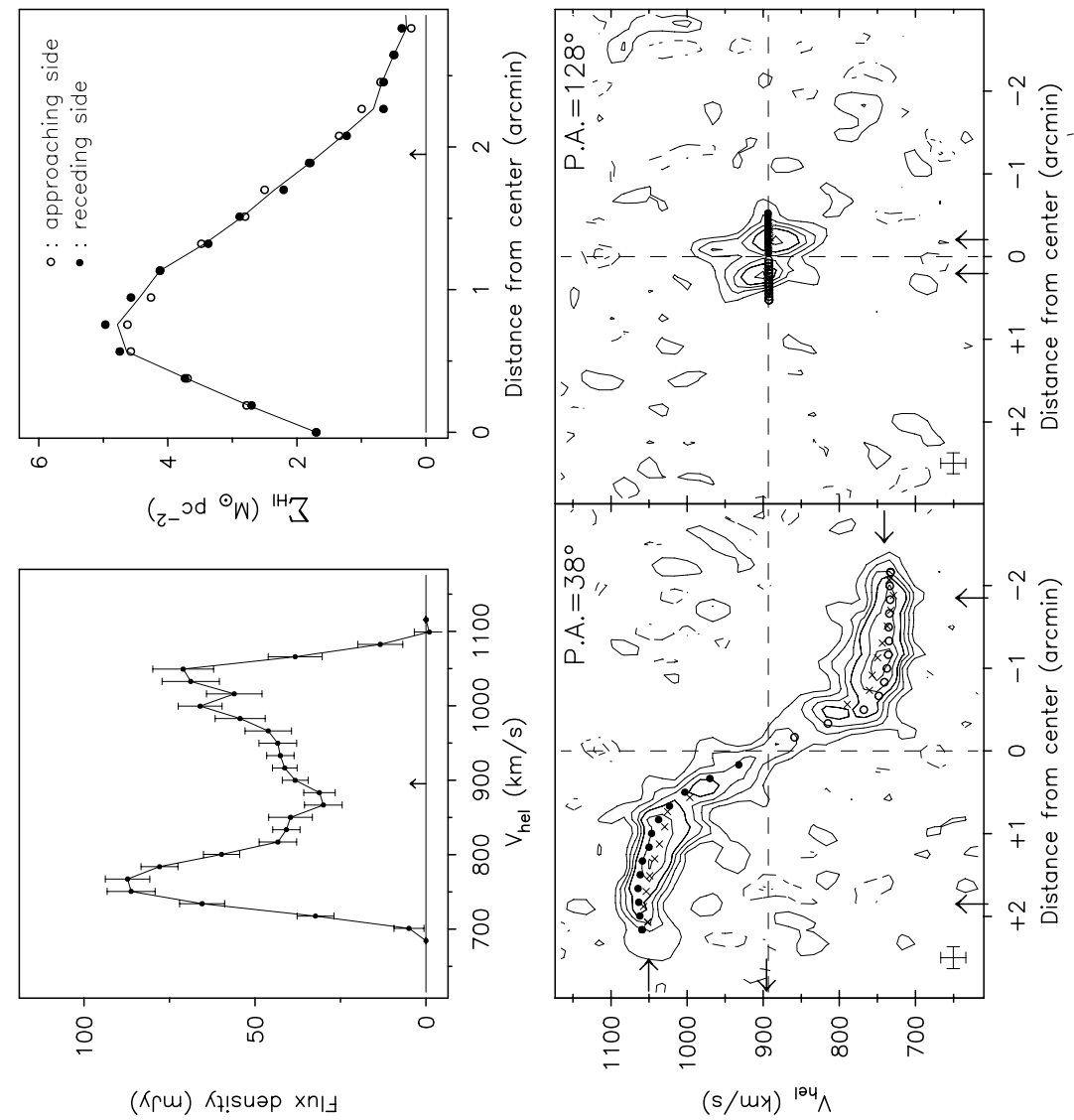


\begin{tabular}{|c|c|}
\hline Length of observation & $1 \times 12$ \\
\hline Date of observation & 31Dec93 \\
\hline Field center, $\alpha(1950)$ & 11:46:01 \\
\hline$\delta(1950)$ & 48:59:20 \\
\hline Central frequency & 1415.86 \\
\hline$V_{\text {hel }}$ of central channel & 980 \\
\hline Primary beam FWHM (arcmin) & 37.4 \\
\hline Nr. of interferometers & 40 \\
\hline Baselines (min-max-incr) & $36-2700-72$ \\
\hline Synthesized beam $(\alpha \times \delta)(\operatorname{arcsec})$ & $12.1 \times 16.1$ \\
\hline Bandwidth & 2.5 \\
\hline Number of channels & 127 \\
\hline Channel separation & 4.15 \\
\hline Velocity resolution & 4.98 \\
\hline rms noise in one channel (K) & 9.11 \\
\hline $\begin{array}{l}\text { K-mJy conversion, } \\
\text { equiv. of } 1 \mathrm{mJy} / \text { beam }\end{array}$ & 3.09 \\
\hline
\end{tabular}

Results from WSRT data

\begin{tabular}{|c|c|}
\hline \multicolumn{2}{|l|}{ From continuит map: } \\
\hline$\overline{21-\mathrm{cm} \text { flux density }}$ & $137.4 \pm 2.9$ \\
\hline \multicolumn{2}{|l|}{ Central point source position } \\
\hline$\alpha(1950)$ & $11: 45: 59.6$ \\
\hline$\delta(1950)$ & $48: 59: 23$ \\
\hline \multicolumn{2}{|l|}{ From global profile: } \\
\hline$\overline{\text { Integrated HI-flux }}\left(\mathrm{Jy} \mathrm{km} \mathrm{s}^{-1}\right)$ & $69.9 \pm 0.5$ \\
\hline Hel. systemic velocity $\left(\mathrm{km} \mathrm{s}^{-1}\right)$ & $967.2 \pm 1.0$ \\
\hline HI profile width, $20 \%\left(\mathrm{~km} \mathrm{~s}^{-1}\right)$ & $310.9 \pm 1.0$ \\
\hline $50 \%\left(\mathrm{~km} \mathrm{~s}^{-1}\right)$ & $277.9 \pm 4.1$ \\
\hline \multicolumn{2}{|l|}{ From velocity field: } \\
\hline 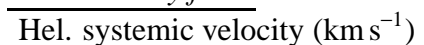 & $966.0 \pm 0.8$ \\
\hline Dynamical center, $\alpha(1950)$ & 11:46:00.2 \\
\hline$\delta(1950)$ & 48:59:21 \\
\hline \multicolumn{2}{|l|}{ From total HI map: } \\
\hline Geometric center, $\alpha(1950)$ & $11: 46: 01.3$ \\
\hline$\delta(1950)$ & $48: 59: 23$ \\
\hline Position angle & 51 \\
\hline Inclination angle & 49 \\
\hline Diameter of HI disk (arcmin) & 8.0 \\
\hline
\end{tabular}

Contour levels for N3893

Channel maps:
Raw continuum map: $\quad \sigma=1.04(\mathrm{~K})$
Cleaned continuum map:
$\quad \sigma=0.56(\mathrm{~K})$
Position-Velocity diagrams:
$\quad \sigma=0.95(\mathrm{~K})$
Velocity fields: $966.0 \pm \mathrm{n} \times 25\left(\mathrm{~km} \mathrm{~s}^{-1}\right)$
Residual velocity field:
$\quad \pm \mathrm{n} \times 5\left(\mathrm{~km} \mathrm{~s}^{-1}\right)$
Integrated HI map:
$0.20,0.40,0.60,0.80$
$1.00,1.20\left(\times 10^{21}\right.$ atoms cm $\left.{ }^{-2}\right)$

Note: The rotation curve beyond 2 arcmin is quite uncertain because of the tidal interaction with its companion N3896.

Compare this interacting pair with N3769/1135+48 which shows a similar optical configuration but a very different kinematical structure.

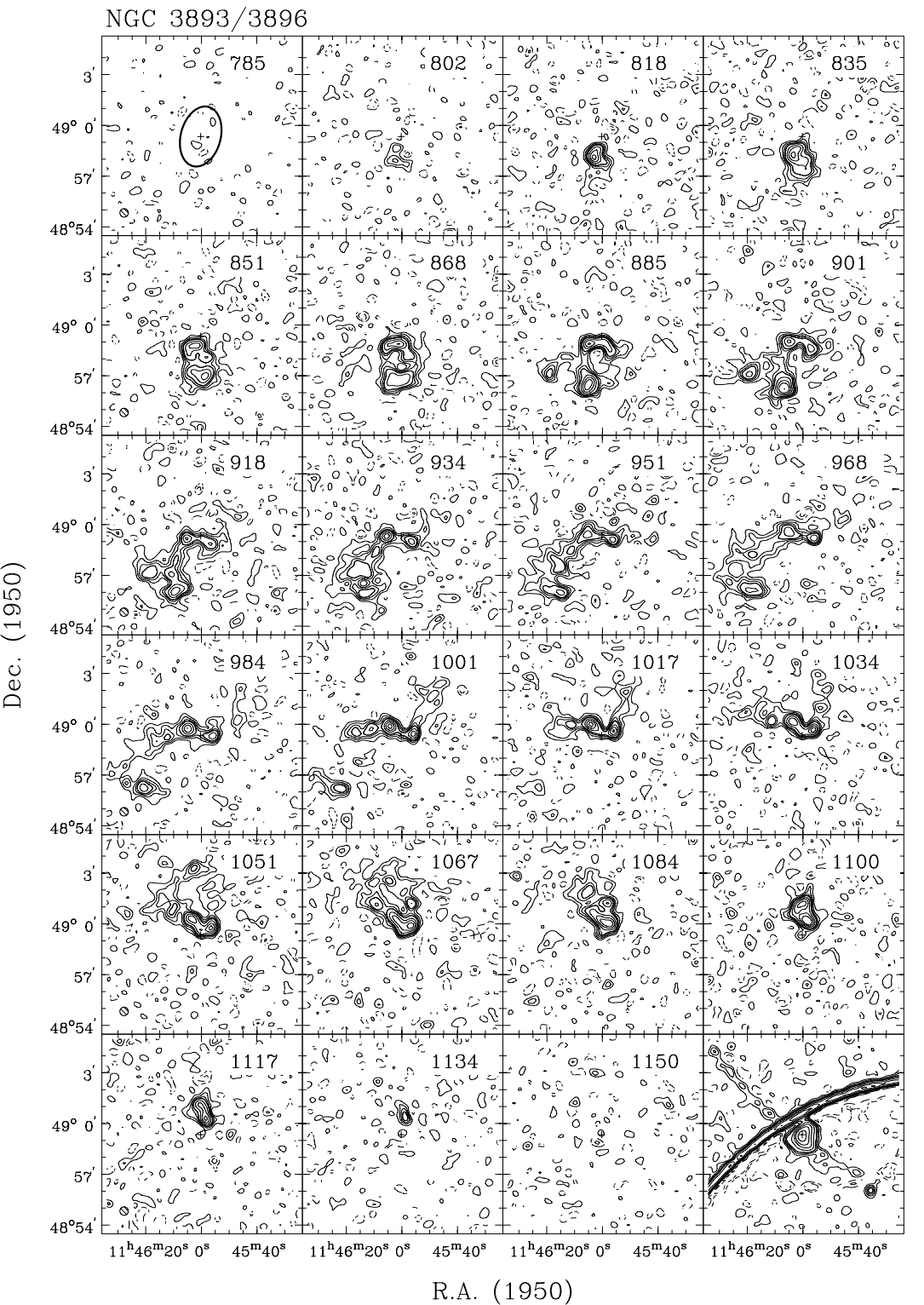

Channel maps at a resolution of $30^{\prime \prime} \times 30^{\prime \prime} \times 19 \mathrm{~km} \mathrm{~s}^{-1}$. Contour levels at $-3,-1.5$ (dashed), $1.5,3,4.5, \ldots \times \sigma$. 

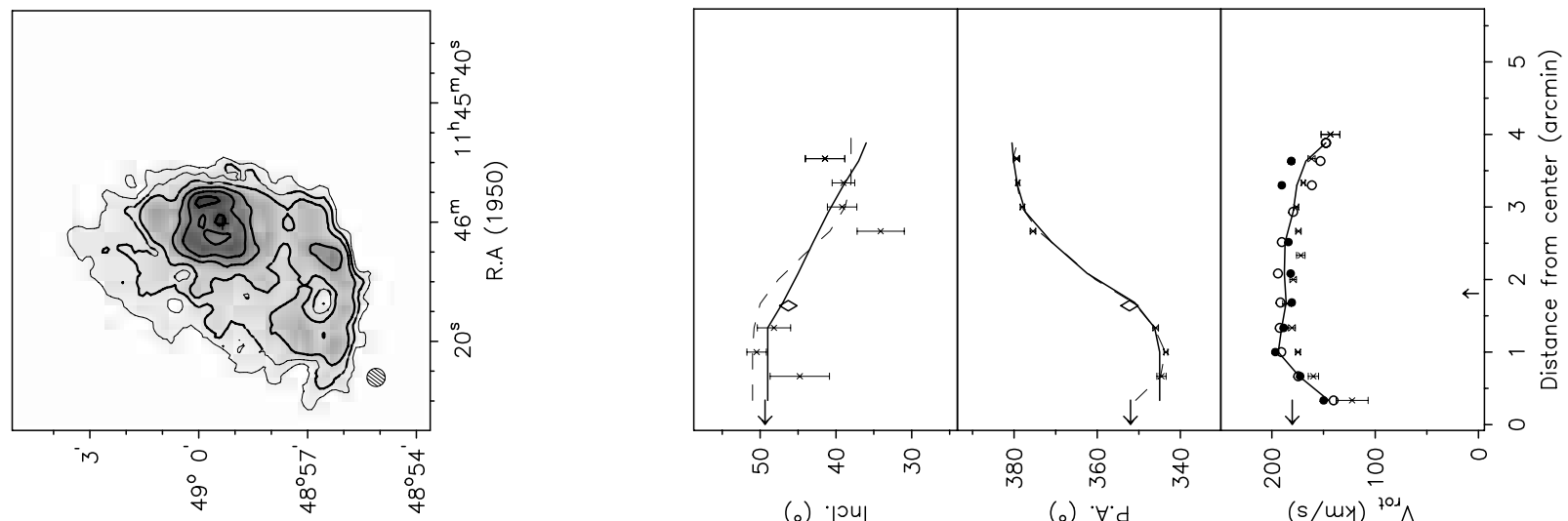

(०) '|गय|

(o) $\forall \cdot d$

$(s / m y){ }^{701} \wedge$

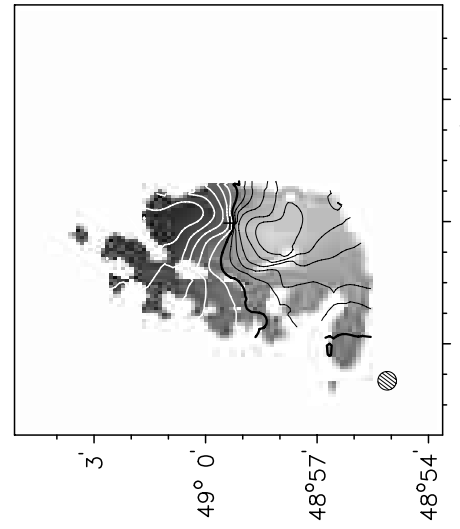

(096l) : गә0

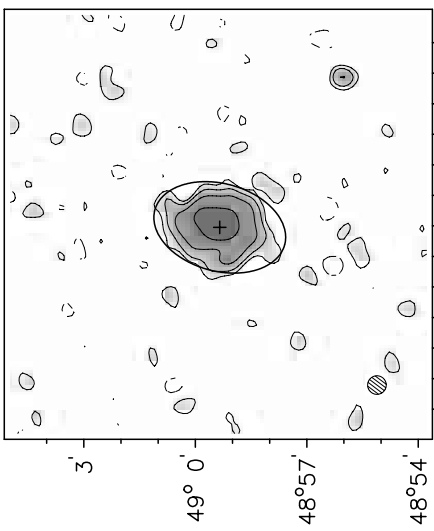

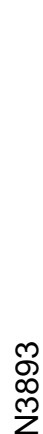

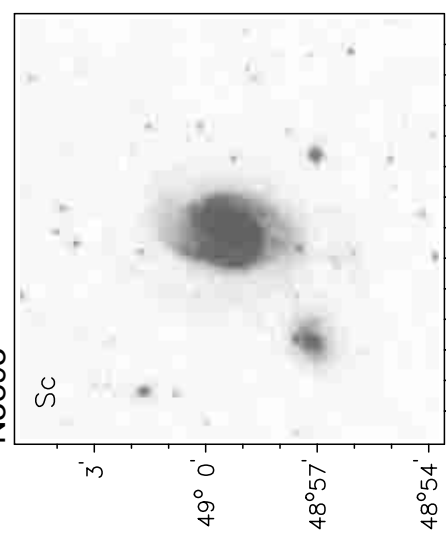

(096l) ' ०ә0

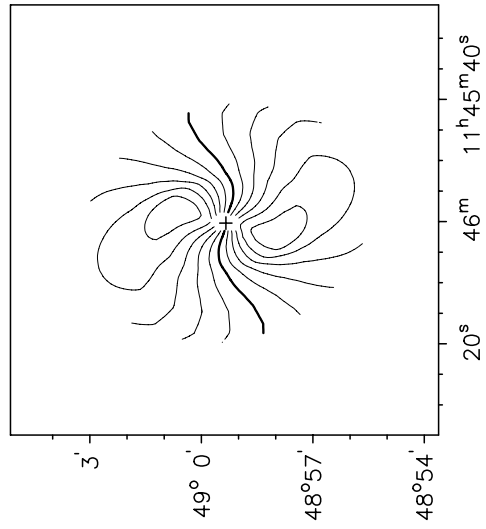

(096l) $\cdot 200$

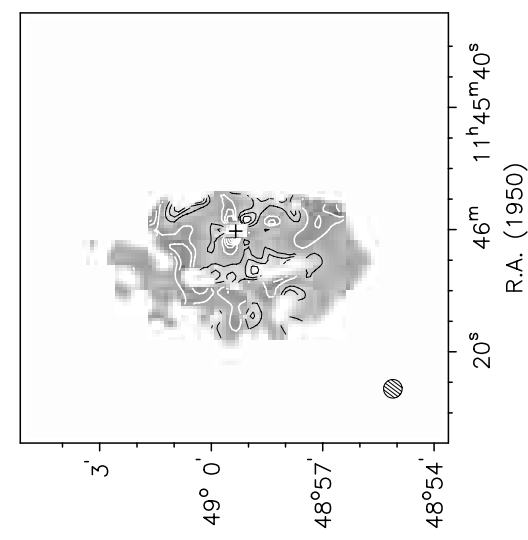

(056 L) :
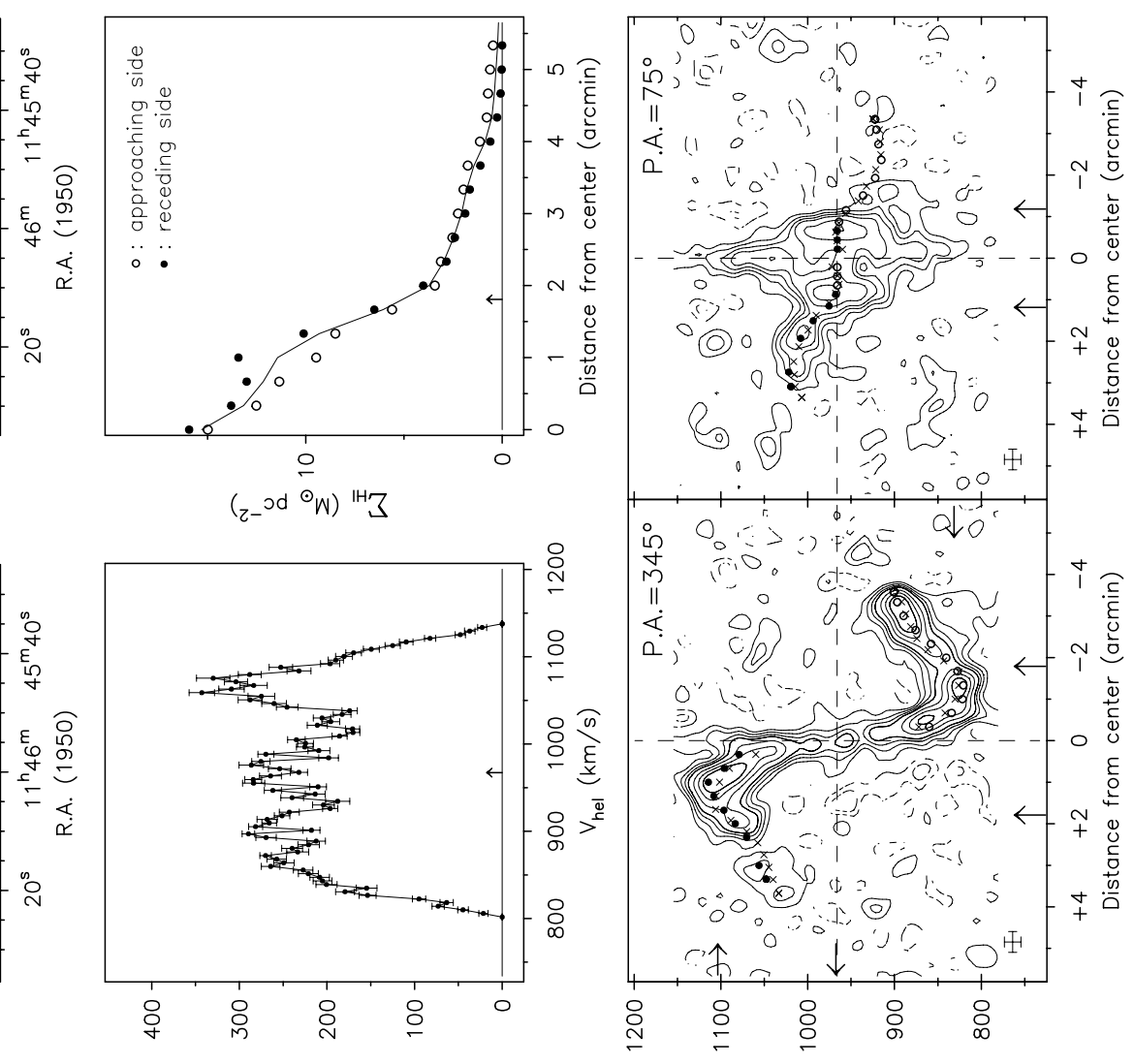

$(s / m y){ }^{104} \wedge$ 
Observing parameters for NGC 3917

\begin{tabular}{|c|c|}
\hline Length of observation & $1 \times 12$ \\
\hline Date of observation & 29Jul93 \\
\hline Field center, $\alpha(1950)$ & 11:48:08 \\
\hline$\delta(1950)$ & 52:06:14 \\
\hline Central frequency & 1415.78 \\
\hline$V_{\text {hel }}$ of central channel & 970 \\
\hline Primary beam FWHM (arcmin) & 37.4 \\
\hline Nr. of interferometers & 40 \\
\hline Baselines (min-max-incr) & $36-2700-72$ \\
\hline Synthesized beam $(\alpha \times \delta)(\operatorname{arcsec})$ & $11.7 \times 15.0$ \\
\hline Bandwidth & 2.5 \\
\hline Number of channels & 127 \\
\hline Channel separation & 4.15 \\
\hline Velocity resolution & 8.30 \\
\hline rms noise in one channel & 6.35 \\
\hline $\begin{array}{l}\text { K-mJy conversion, } \\
\text { equiv. of } 1 \mathrm{mJy} / \text { beam }\end{array}$ & 3.43 \\
\hline
\end{tabular}

Results from WSRT data

\begin{tabular}{|c|c|}
\hline \multicolumn{2}{|l|}{ From continuит map: } \\
\hline \multicolumn{2}{|l|}{ 21-cm flux density } \\
\hline central point source $(\mathrm{mJy})$ & $<1.6(3 \sigma)$ \\
\hline \multicolumn{2}{|l|}{ From global profile: } \\
\hline Integrated HI-flux $\left(\mathrm{Jy} \mathrm{km} \mathrm{s}^{-1}\right)$ & $24.9 \pm 0.6$ \\
\hline Hel. systemic velocity $\left(\mathrm{km} \mathrm{s}^{-1}\right)$ & $964.6 \pm 1.4$ \\
\hline HI profile width, $20 \%\left(\mathrm{~km} \mathrm{~s}^{-1}\right)$ & $294.5 \pm 1.9$ \\
\hline $50 \%\left(\mathrm{~km} \mathrm{~s}^{-1}\right)$ & $279.1 \pm 2.1$ \\
\hline \multicolumn{2}{|l|}{ From velocity field: } \\
\hline 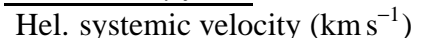 & $966.7 \pm 1.8$ \\
\hline Dynamical center, $\alpha(1950)$ & 11:48:07.7 \\
\hline$\delta(1950)$ & 52:06:07 \\
\hline \multicolumn{2}{|l|}{ From total HI map: } \\
\hline Geometric center, $\alpha(1950)$ & 11:48:08.9 \\
\hline$\delta(1950)$ & $52: 06: 10$ \\
\hline Position angle & 256 \\
\hline Inclination angle & 76 \\
\hline Diameter of HI disk (arcmin) & 5.4 \\
\hline
\end{tabular}

Contour levels for N3917

\begin{tabular}{l} 
Channel maps: \\
Raw continuum map: $\sigma=4.29(\mathrm{~K})$ \\
$\quad \sigma=1.89(\mathrm{~K})$ \\
Cleaned continuum map: $\sigma=1.87(\mathrm{~K})$ \\
Position-Velocity diagrams: \\
$\quad \sigma=3.60(\mathrm{~K})$ \\
Velocity fields: $966.7 \pm \mathrm{n} \times 20\left(\mathrm{~km} \mathrm{~s}^{-1}\right)$ \\
Residual velocity field: \\
Integrated HI map: \\
$1.39,2.78, \quad$ \\
$4.17\left(\times 10^{21}\right.$ atoms cm $\left.{ }^{-2}\right)$ \\
\hline
\end{tabular}

.

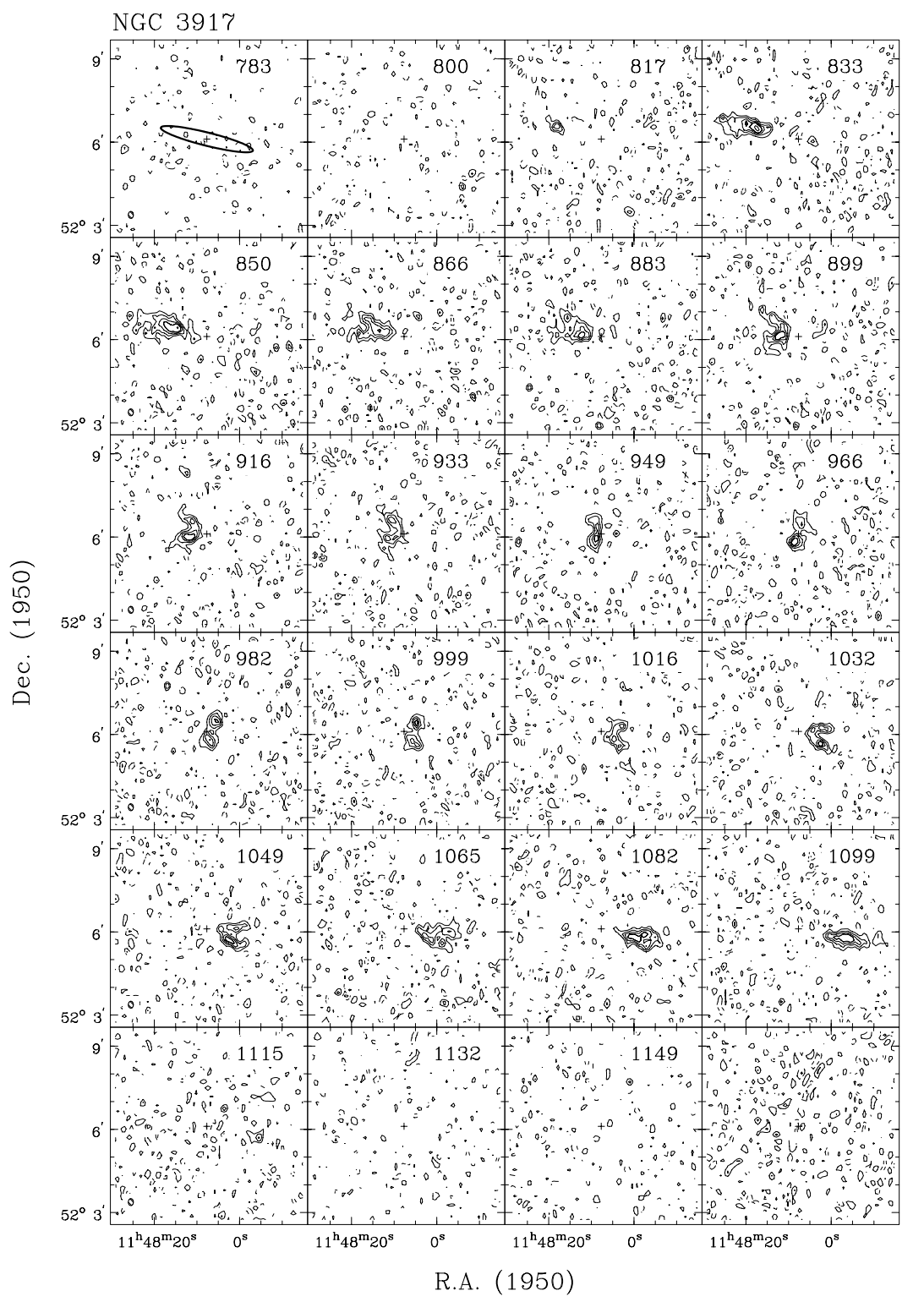

Note: This galaxy has a well defined symmetric rotation curve. The HI disk is a bit more extended at the approaching side. It has a dwarf companion (N3931) at 11 arcmin to the NNE.

Channel maps at a resolution of $11^{\prime \prime} \times 15^{\prime \prime} \times 19 \mathrm{~km} \mathrm{~s}^{-1}$.

Contour levels at $-3,-1.5$ (dashed), $1.5,3,4.5, \ldots \times \sigma$. 

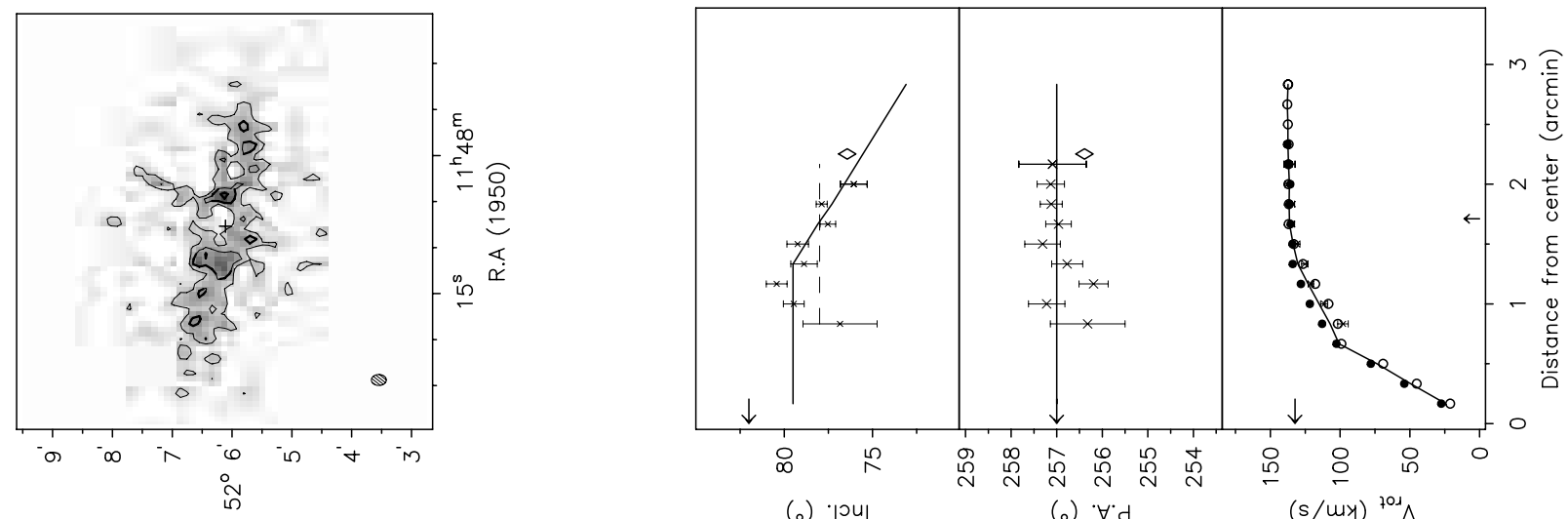
(०) '|गu|
(o) $\forall \cdot d$
$(s / m y){ }^{701} \wedge$

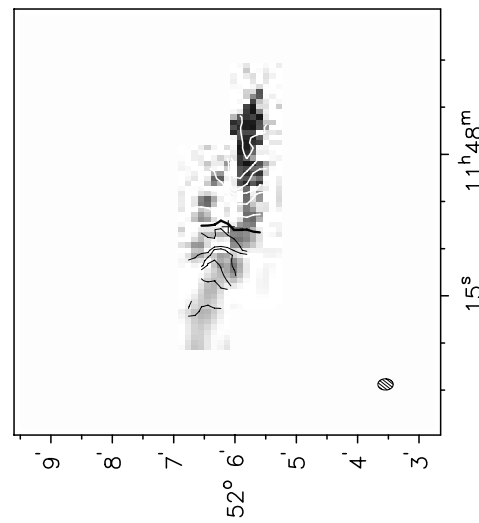

(096l) :
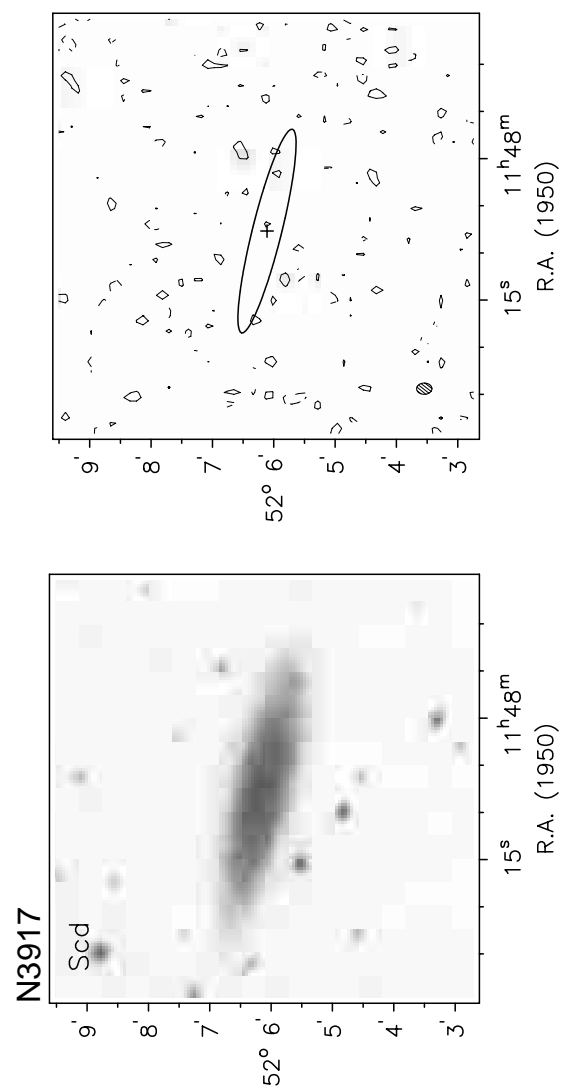

$(096 \mathrm{l}) \cdot 520$

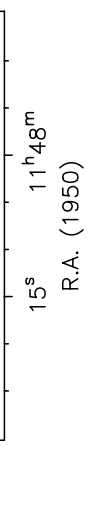

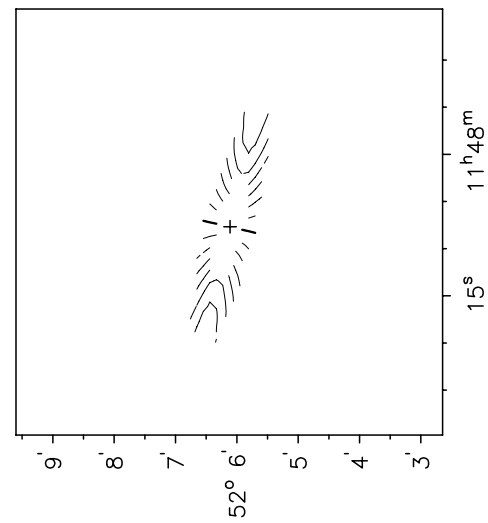

(096l) $\cdot 200$
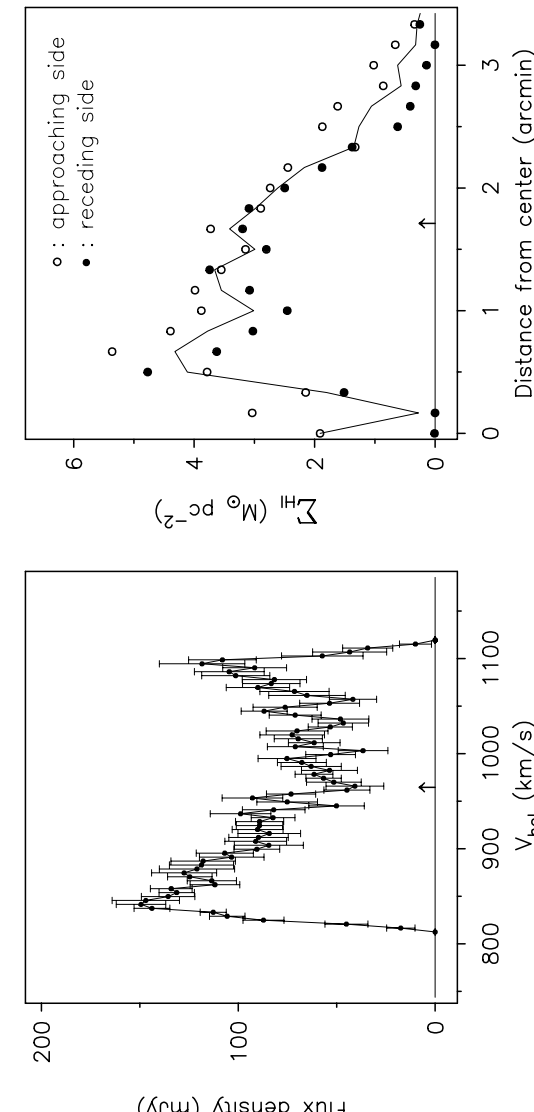

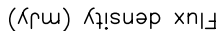

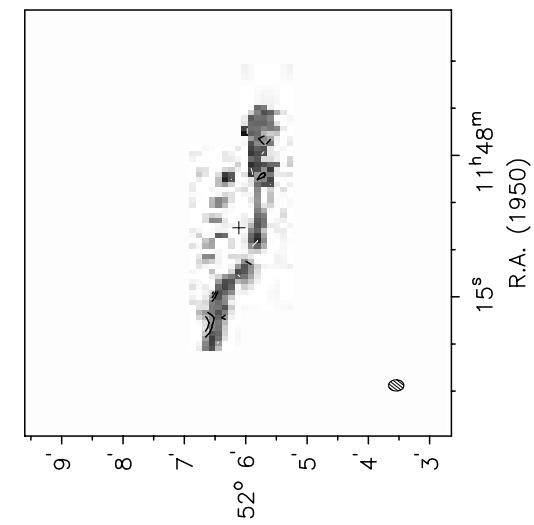

(096l) $\cdot 200$
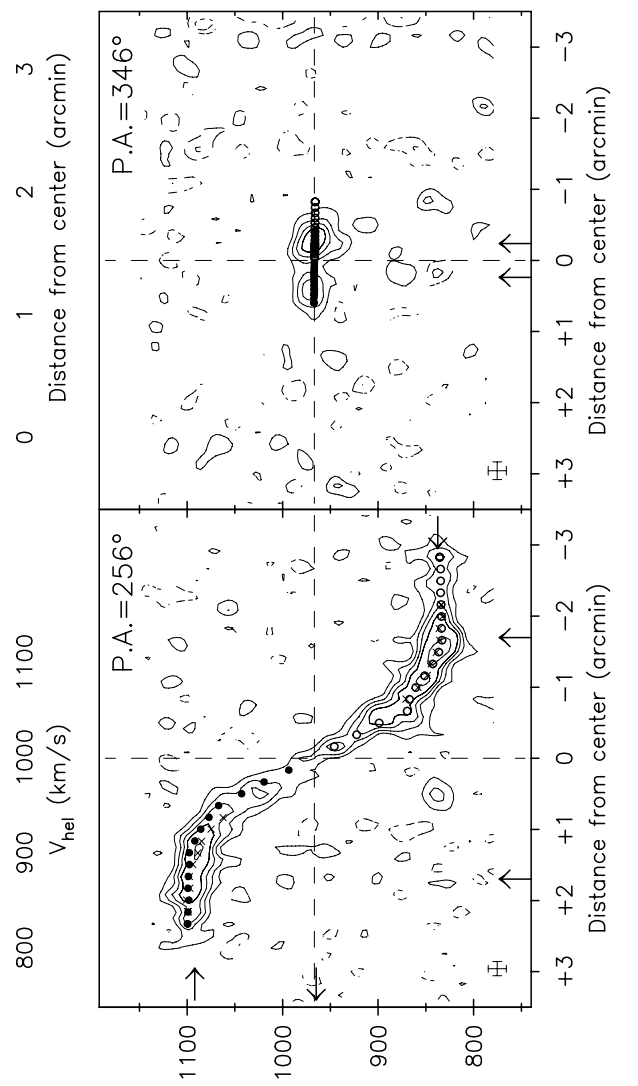

$(s / m y){ }^{124} \wedge$ 
Observing parameters for NGC 3949

\begin{tabular}{|c|c|}
\hline Length of observation & $1 \times 12$ \\
\hline Date of observation & 10Aug93 \\
\hline Field center, $\alpha(1950)$ & 11:51:05 \\
\hline$\delta(1950)$ & 48:08:16 \\
\hline Central frequency & 1416.61 \\
\hline$V_{\text {hel }}$ of central channel & 798 \\
\hline Primary beam FWHM & 37.4 \\
\hline Nr. of interferometers & 40 \\
\hline Baselines (min-max-incr) & $36-2700-72$ \\
\hline Synthesized beam $(\alpha \times \delta)(\operatorname{arcsec})$ & $11.9 \times 16.0$ \\
\hline Bandwidth & 2.5 \\
\hline Number of channels & 127 \\
\hline Channel separation & 4.14 \\
\hline Velocity resolution & 8.29 \\
\hline rms noise in one channel & 5.85 \\
\hline $\begin{array}{l}\text { K-mJy conversion, } \\
\text { equiv. of } 1 \mathrm{mJy} / \text { beam }\end{array}$ & 3.16 \\
\hline
\end{tabular}

Results from WSRT data

\begin{tabular}{|c|c|}
\hline From continuum map: & \\
\hline \multicolumn{2}{|l|}{ From global profile: } \\
\hline Integrated HI-flux $\left(\mathrm{Jy} \mathrm{km} \mathrm{s}^{-1}\right)$ & $44.8 \pm 0.4$ \\
\hline Hel. systemic velocity $\left(\mathrm{km} \mathrm{s}^{-1}\right)$ & $800.2 \pm 1.2$ \\
\hline HI profile width, $20 \%\left(\mathrm{~km} \mathrm{~s}^{-1}\right)$ & $286.5 \pm 1.4$ \\
\hline $50 \%\left(\mathrm{~km} \mathrm{~s}^{-1}\right)$ & $258.3 \pm 1.7$ \\
\hline \multicolumn{2}{|l|}{ From velocity field: } \\
\hline 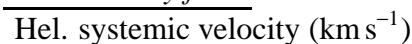 & $799.0 \pm 1.0$ \\
\hline $\begin{array}{l}\text { Dynamical center, } \alpha(1950) \\
\delta(1950)\end{array}$ & $\begin{array}{l}11: 51: 05.3 \\
48: 08: 14\end{array}$ \\
\hline \multicolumn{2}{|l|}{ From total HI map: } \\
\hline Geometric center, $\alpha(1950)$ & $11: 51: 04.5$ \\
\hline$\delta(1950)$ & 48:08:21 \\
\hline Position angle & 286 \\
\hline Inclination angle & 52 \\
\hline Diameter of HI disk (arcmin) & 5.4 \\
\hline
\end{tabular}

Contour levels for N3949

\begin{tabular}{|c|c|}
\hline \\
\hline \multirow{2}{*}{ Raw continuum map: } & $\sigma=3.80(\mathrm{~K})$ \\
\hline & $\sigma=1.69(\mathrm{~K})$ \\
\hline \multicolumn{2}{|c|}{ Cleaned continuum map: } \\
\hline Position-Velocity diag & $\sigma=3.48(\mathrm{~K})$ \\
\hline \multicolumn{2}{|l|}{ Velocity fields: } \\
\hline \multicolumn{2}{|c|}{$\begin{array}{l}\qquad 799.0 \pm \mathrm{n} \times 20\left(\mathrm{~km} \mathrm{~s}^{-1}\right) \\
\text { Residual velocity field: }\end{array}$} \\
\hline $\begin{array}{c}\text { Integrated HI map: } \\
0.85,1.71, \\
2.56(\times 10\end{array}$ & $\begin{array}{l}\mathrm{n} \times 5\left(\mathrm{~km} \mathrm{~s}^{-1}\right) \\
\left.{ }^{1} \text { atoms cm}{ }^{-2}\right)\end{array}$ \\
\hline
\end{tabular}

Note: The rotation curve rises more steeply on the receding side than on the approaching side. There is a possible companion about 1.5 arcmin to the north which may be interacting with N3949 and be responsible for the faint trace of HI gas on the west side of N3949 in the velocity range 868-918 $\mathrm{km} / \mathrm{s}$.

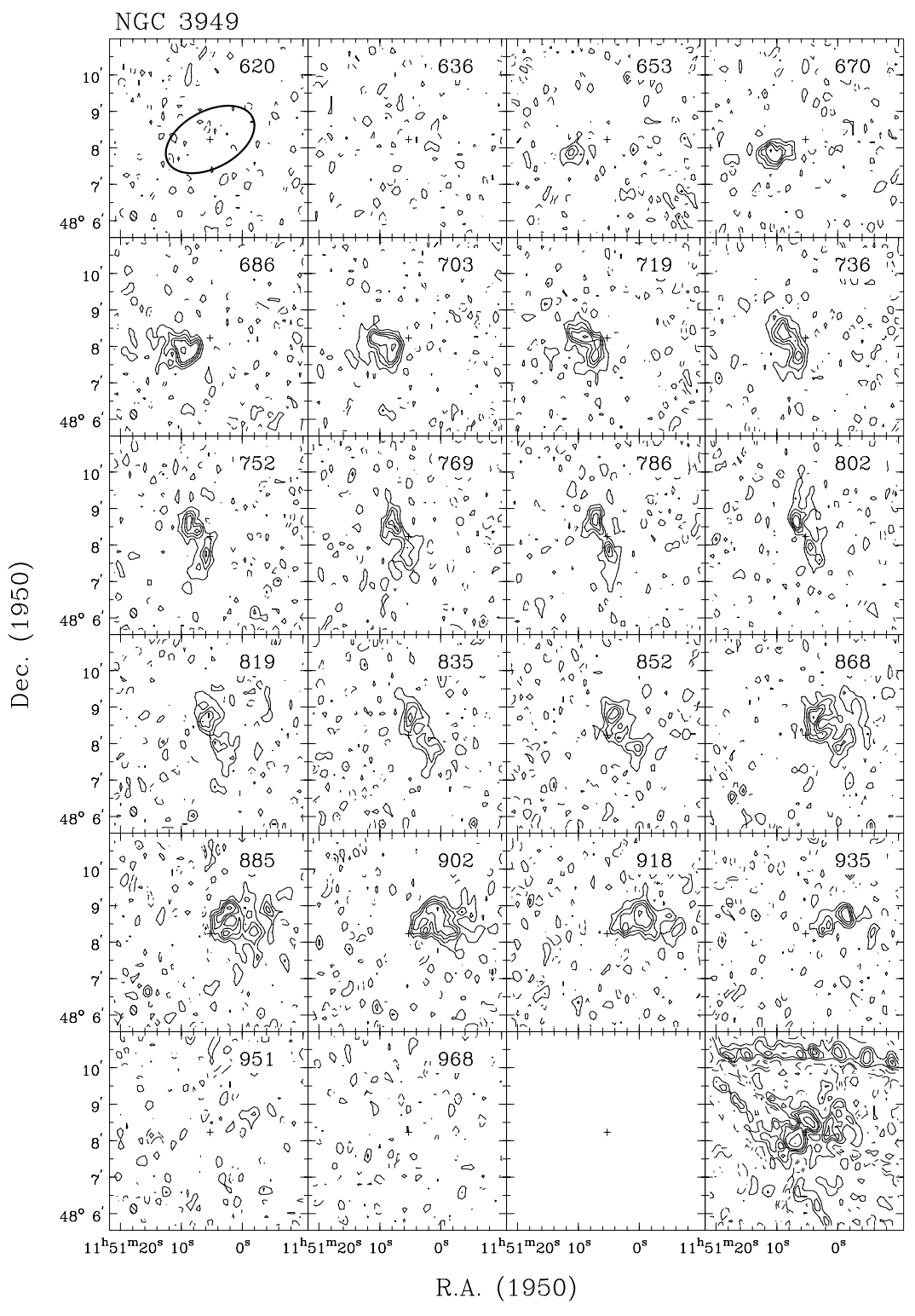

Channel maps at a resolution of $11^{\prime \prime} \times 16^{\prime \prime} \times 19 \mathrm{~km} \mathrm{~s}^{-1}$. Contour levels at $-3,-1.5$ (dashed) $1.5,3,4.5, \ldots \times \sigma$. 

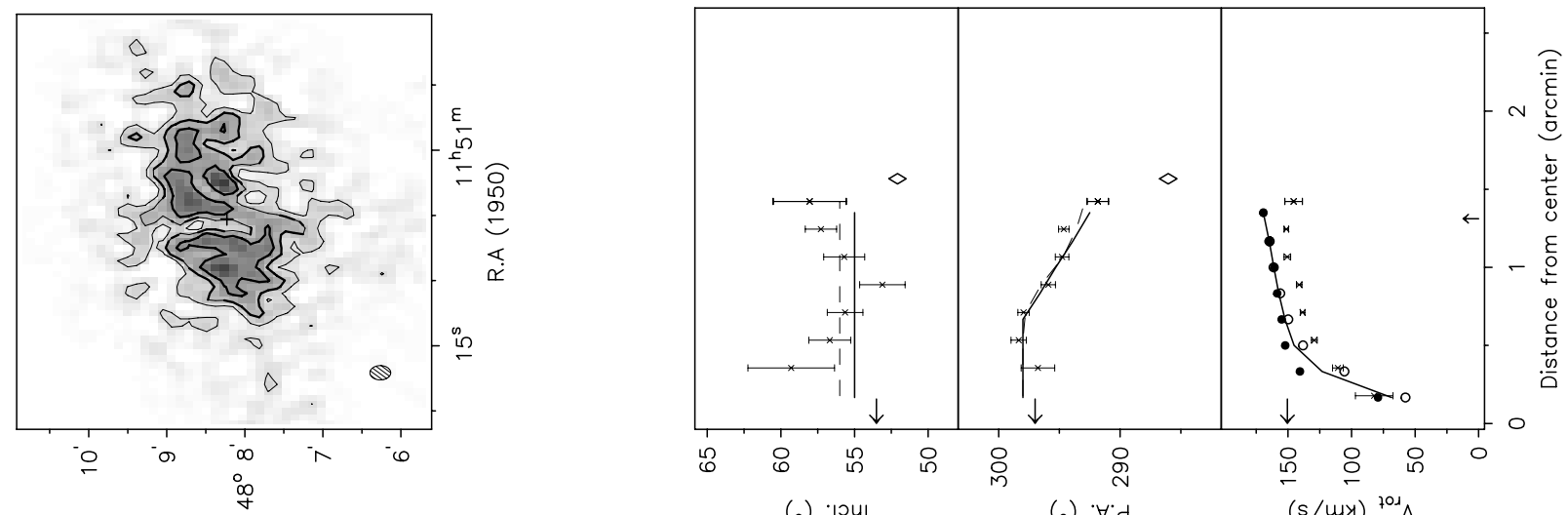
(o) '|⿰u|
(o) $\forall \cdot d$
$(s / m y){ }^{701} \wedge$

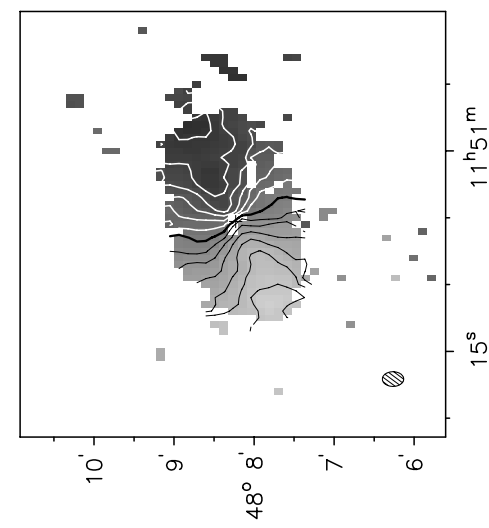

(096l) $\cdot 520$
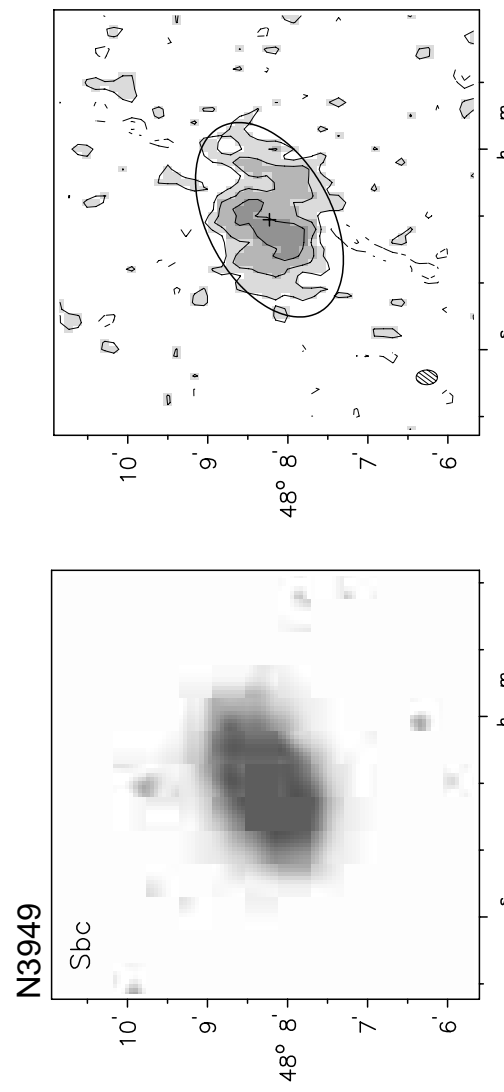

$(0 \mathrm{Og} \mathrm{L}) \cdot 2 \mathrm{0}$

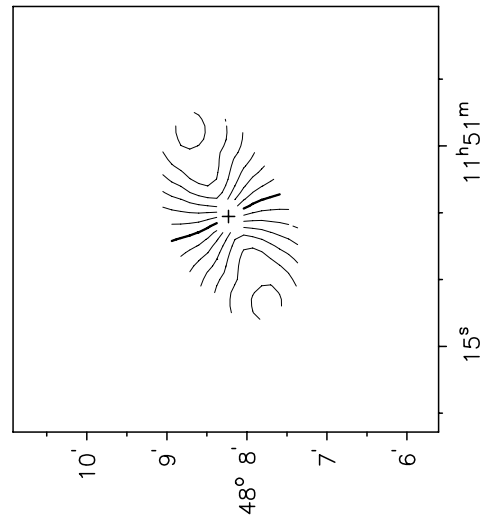

(096 L) · ว
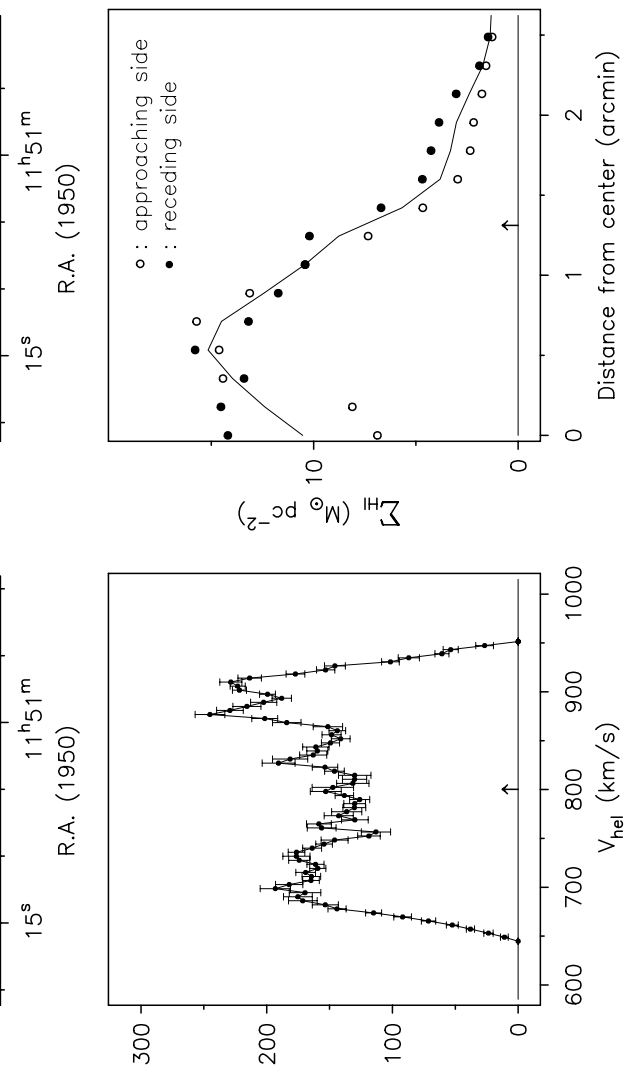

(Кгس) K?!suәp xn!

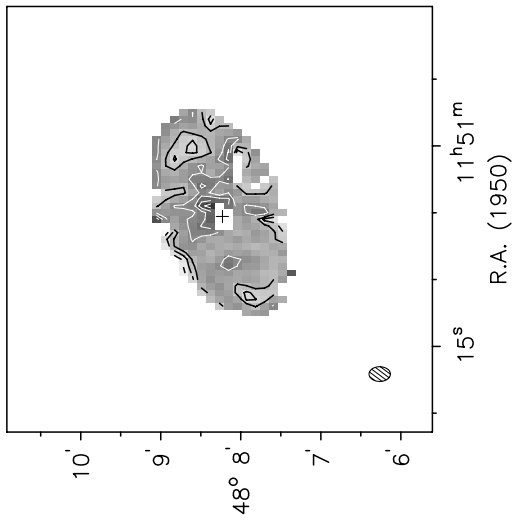

(0961) ·ә0

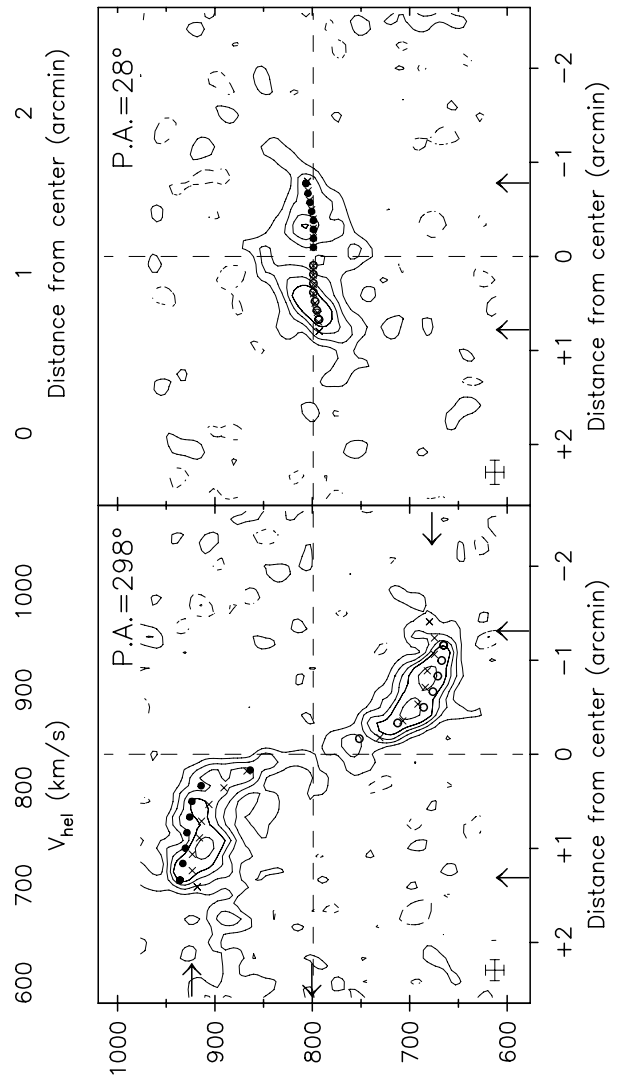

$(s / m y){ }^{194} \wedge$ 
Observing parameters for NGC 3953

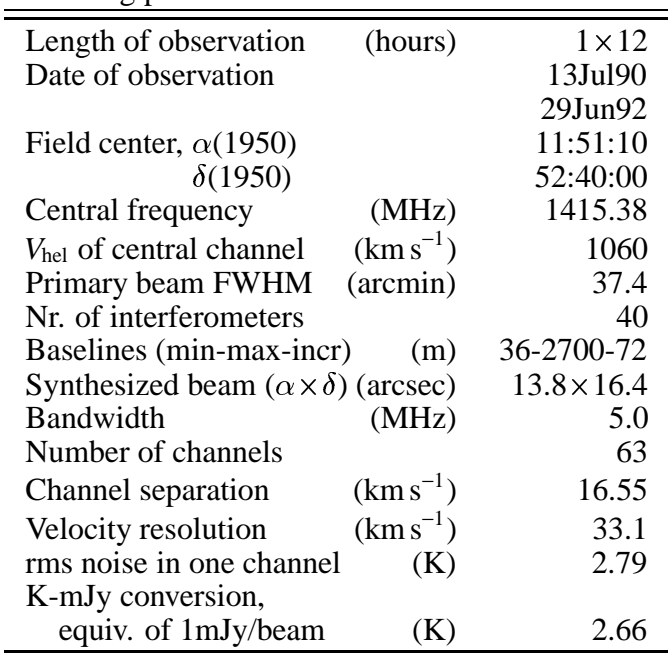

Results from WSRT data

\begin{tabular}{|c|c|}
\hline From continuum map: & \\
\hline $\begin{array}{l}21-\mathrm{cm} \text { flux density } \\
\text { From global profile: }\end{array}$ & $50.9 \pm 2.5$ \\
\hline Integrated HI-flux $\left(\mathrm{Jy} \mathrm{km} \mathrm{s}^{-1}\right)$ & $39.3 \pm 0.8$ \\
\hline Hel. systemic velocity $\left(\mathrm{km} \mathrm{s}^{-1}\right)$ & $1052.3 \pm 2.0$ \\
\hline HI profile width, $20 \%\left(\mathrm{~km} \mathrm{~s}^{-1}\right)$ & $441.9 \pm 2.4$ \\
\hline $50 \%\left(\mathrm{~km} \mathrm{~s}^{-1}\right)$ & $413.9 \pm 3.2$ \\
\hline \multicolumn{2}{|l|}{ From velocity field: } \\
\hline 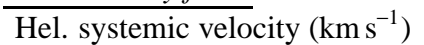 & $1051.4 \pm 0.8$ \\
\hline $\begin{array}{r}\text { Dynamical center, } \alpha(1950) \\
\delta(1950)\end{array}$ & $\begin{array}{l}11: 51: 12.4 \\
52: 36: 16\end{array}$ \\
\hline \multicolumn{2}{|l|}{ From total HI map: } \\
\hline$\overline{\text { Geometric center, } \alpha(1950)}$ & $11: 51: 12.5$ \\
\hline$\delta(1950)$ & $52: 36: 14$ \\
\hline Position angle & 11 \\
\hline Inclination angle & 58 \\
\hline Diameter of HI disk (arcmin) & 6.6 \\
\hline
\end{tabular}

Contour levels for N3953

Channel maps: $\quad \sigma=0.80(\mathrm{~K})$
Raw continuum map: $\quad \sigma=0.33(\mathrm{~K})$
Cleaned continuum map: $\sigma=0.30(\mathrm{~K})$
Position-Velocity diagrams:
$\quad \sigma=0.81(\mathrm{~K})$
Velocity fields: $\quad \mathrm{n} \times 30\left(\mathrm{~km} \mathrm{~s}^{-1}\right)$
Residual velocity field:
Integrated HI map: $\quad \pm \mathrm{n} \times 5\left(\mathrm{~km} \mathrm{~s}^{-1}\right)$
$0.33,0.66, \quad 0.99\left(\times 10^{21}\right.$ atoms cm

.

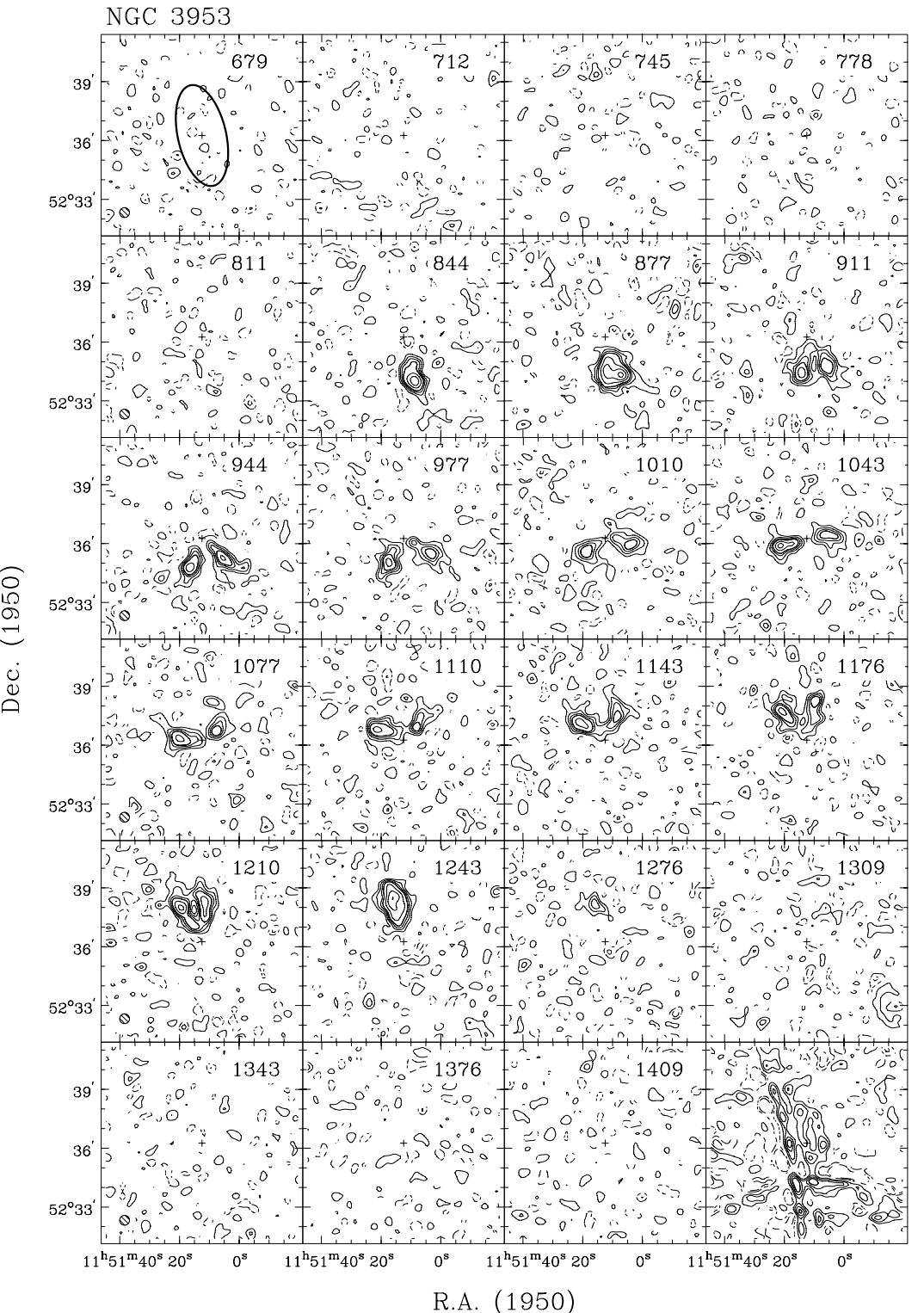

NGC 3953 is one of the most in the cluster. It is rather drops strongly near $\mathrm{D}_{25}$.
Channel maps at a resolution of $30^{\prime \prime} \times 30^{\prime \prime} \times 33 \mathrm{~km} \mathrm{~s}^{-1}$. Contour levels at $-3,-1.5$ (dashed), $1.5,3,4.5, \ldots \times \sigma$. 

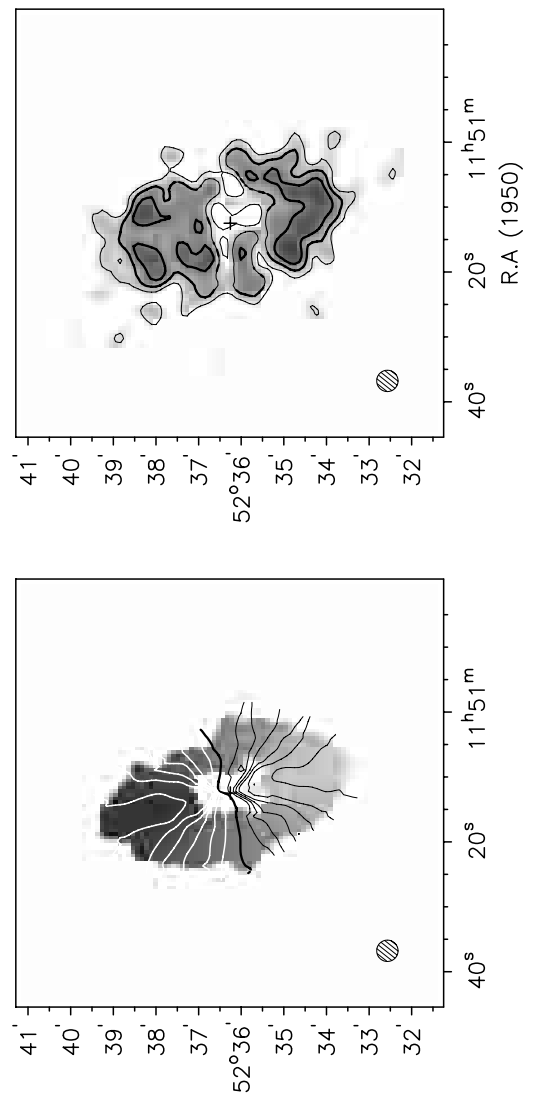

(096l) $\cdot 2 ә 0$

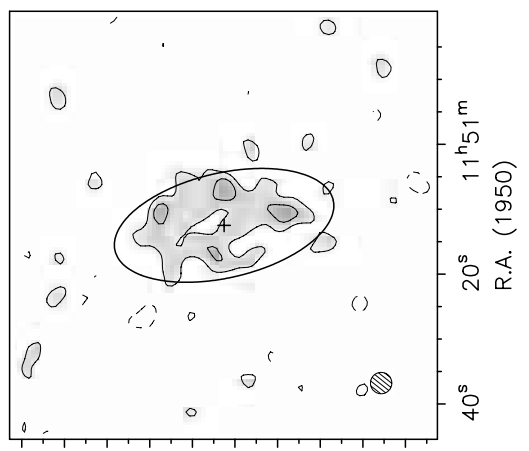

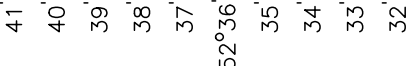

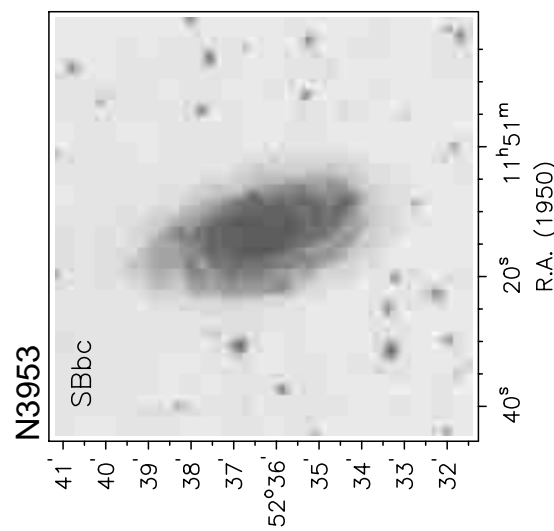

(096l) ' วә0

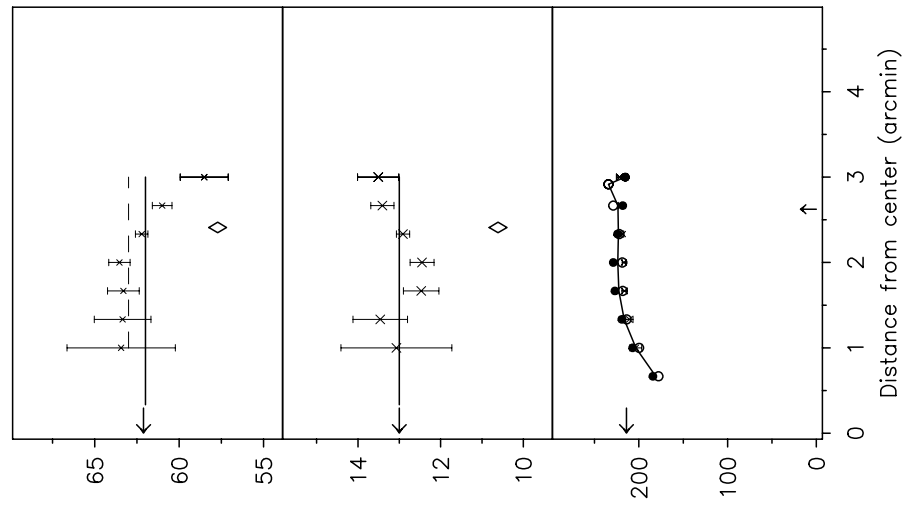

(०) '|ग0|

(o) $\forall \cdot d$

$(s / m x){ }^{701} \wedge$

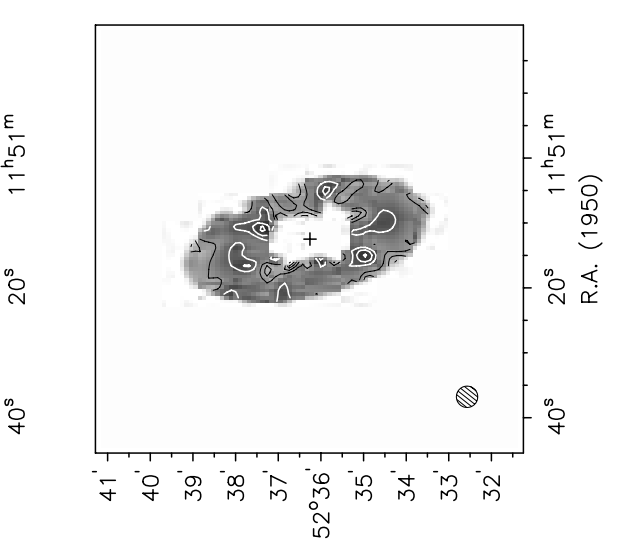

(0s6l) $\cdot 5 \partial 0$

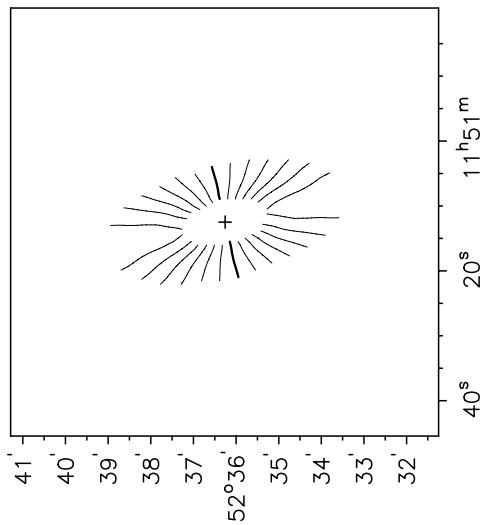

(096l) $\cdot 0 \curvearrowright 0$

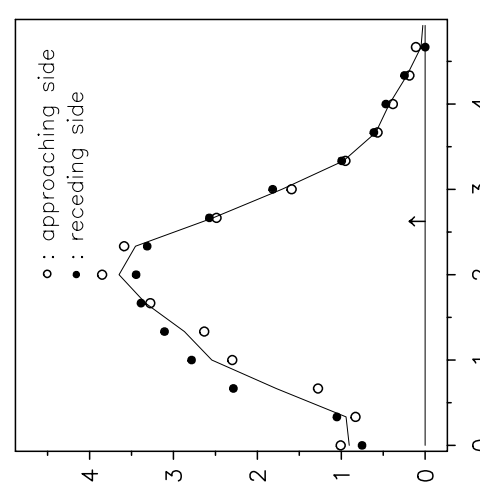

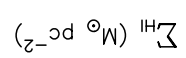

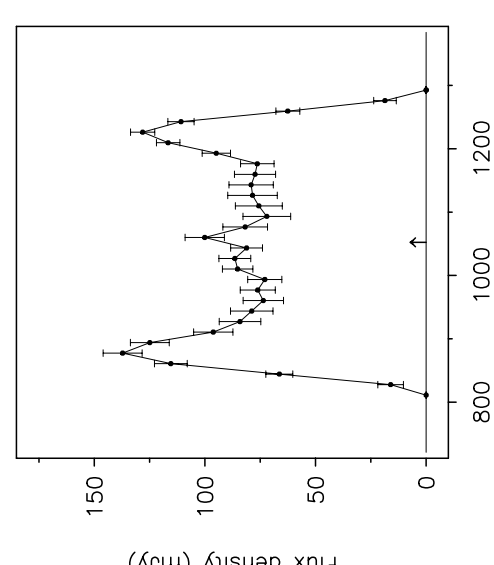

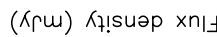
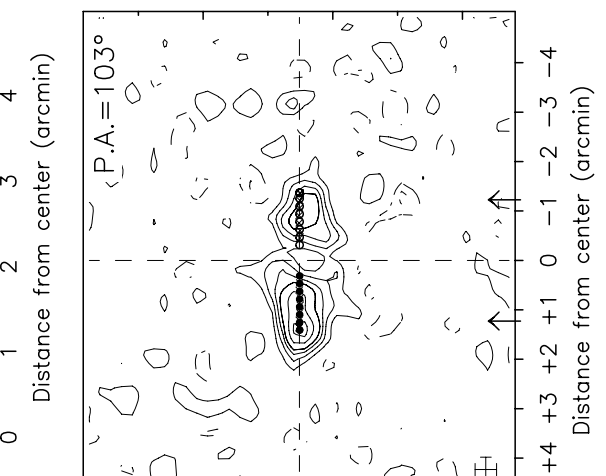

(1)

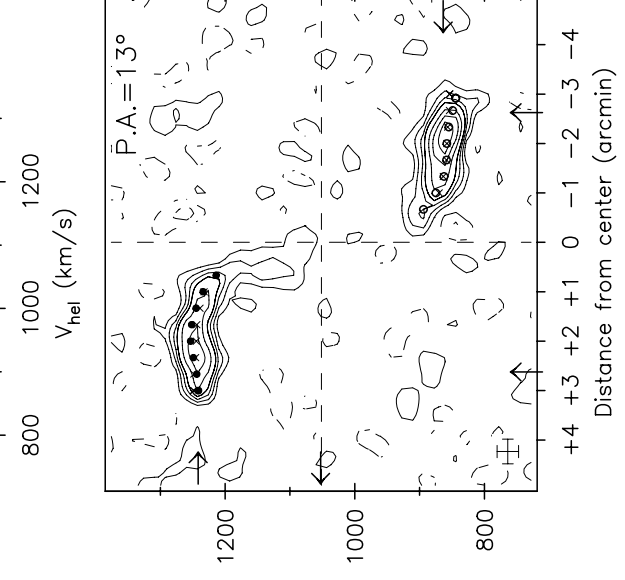

$(s / m y)^{\mid ⿰ 冫} \wedge$ 
Observing parameters for NGC 3972

\begin{tabular}{|c|c|}
\hline Length of observation & $1 \times 12$ \\
\hline Date of observation & 10Aug90 \\
\hline Field center, $\alpha(1950)$ & 11:53:05 \\
\hline$\delta(1950)$ & 55:33:00 \\
\hline Central frequency & 1416.37 \\
\hline$V_{\text {hel }}$ of central channel & 850 \\
\hline Primary beam FWHM & 37.4 \\
\hline Nr. of interferometers & 40 \\
\hline Baselines (min-max-incr) & $36-2700-72$ \\
\hline Synthesized beam $(\alpha \times \delta)(\operatorname{arcsec})$ & $11.7 \times 15.2$ \\
\hline Bandwidth & 2.5 \\
\hline Number of channels & 127 \\
\hline Channel separation & 4.15 \\
\hline Velocity resolution & 8.29 \\
\hline rms noise in one channel & 6.60 \\
\hline $\begin{array}{l}\text { K-mJy conversion, } \\
\text { equiv. of } 1 \mathrm{mJy} / \text { beam }\end{array}$ & 3.39 \\
\hline
\end{tabular}

Results from WSRT data

\begin{tabular}{|c|c|}
\hline \multicolumn{2}{|l|}{ From continuum map: } \\
\hline \multicolumn{2}{|l|}{ 21-cm flux density } \\
\hline central point source (mJy) & $<1.6(3 \sigma)$ \\
\hline extended source (mJy) & $<5.8(3 \sigma)$ \\
\hline \multicolumn{2}{|l|}{ From global profile: } \\
\hline$\overline{\text { Integrated HI-flux }}\left(\mathrm{Jy} \mathrm{km} \mathrm{s}^{-1}\right)$ & $16.6 \pm 0.4$ \\
\hline Hel. systemic velocity $\left(\mathrm{km} \mathrm{s}^{-1}\right)$ & $852.2 \pm 1.4$ \\
\hline HI profile width, $20 \%\left(\mathrm{~km} \mathrm{~s}^{-1}\right)$ & $281.2 \pm 1.4$ \\
\hline $50 \%\left(\mathrm{~km} \mathrm{~s}^{-1}\right)$ & $260.7 \pm 5.5$ \\
\hline \multicolumn{2}{|l|}{ From velocity field: } \\
\hline 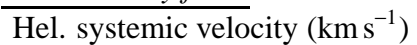 & $848.8 \pm 2.8$ \\
\hline Dynamical center, $\alpha(1950)$ & 11:53:08.5 \\
\hline$\delta(1950)$ & $55: 35: 54$ \\
\hline \multicolumn{2}{|l|}{ From total HI map: } \\
\hline$\overline{\text { Geometric center, } \alpha} \alpha(1950)$ & 11:53:08.1 \\
\hline$\delta(1950)$ & $55: 35: 54$ \\
\hline Position angle & 297 \\
\hline Inclination angle & 77 \\
\hline Diameter of HI disk (arcmin) & 3.8 \\
\hline
\end{tabular}

Contour levels for N3972

Channel maps: $\quad \sigma=4.24(\mathrm{~K})$

Raw continuum map: $\sigma=2.37(\mathrm{~K})$ Cleaned continuum map $\sigma=1.78(\mathrm{~K})$ Position-Velocity diagrams:

Velocity fields:

$$
\sigma=3.64(\mathrm{~K})
$$

$848.8 \pm \mathrm{n} \times 20\left(\mathrm{~km} \mathrm{~s}^{-1}\right)$

Residual velocity field: $\pm \mathrm{n} \times 5\left(\mathrm{~km} \mathrm{~s}^{-1}\right)$

Integrated HI map: $1.00,1.99$, $2.99\left(\times 10^{21}\right.$ atoms cm $\left.{ }^{-2}\right)$

Note: The HI disk does not extend much beyond $\mathrm{D}_{25}$ and the rotation curve is still somewhat rising at the last measured points.

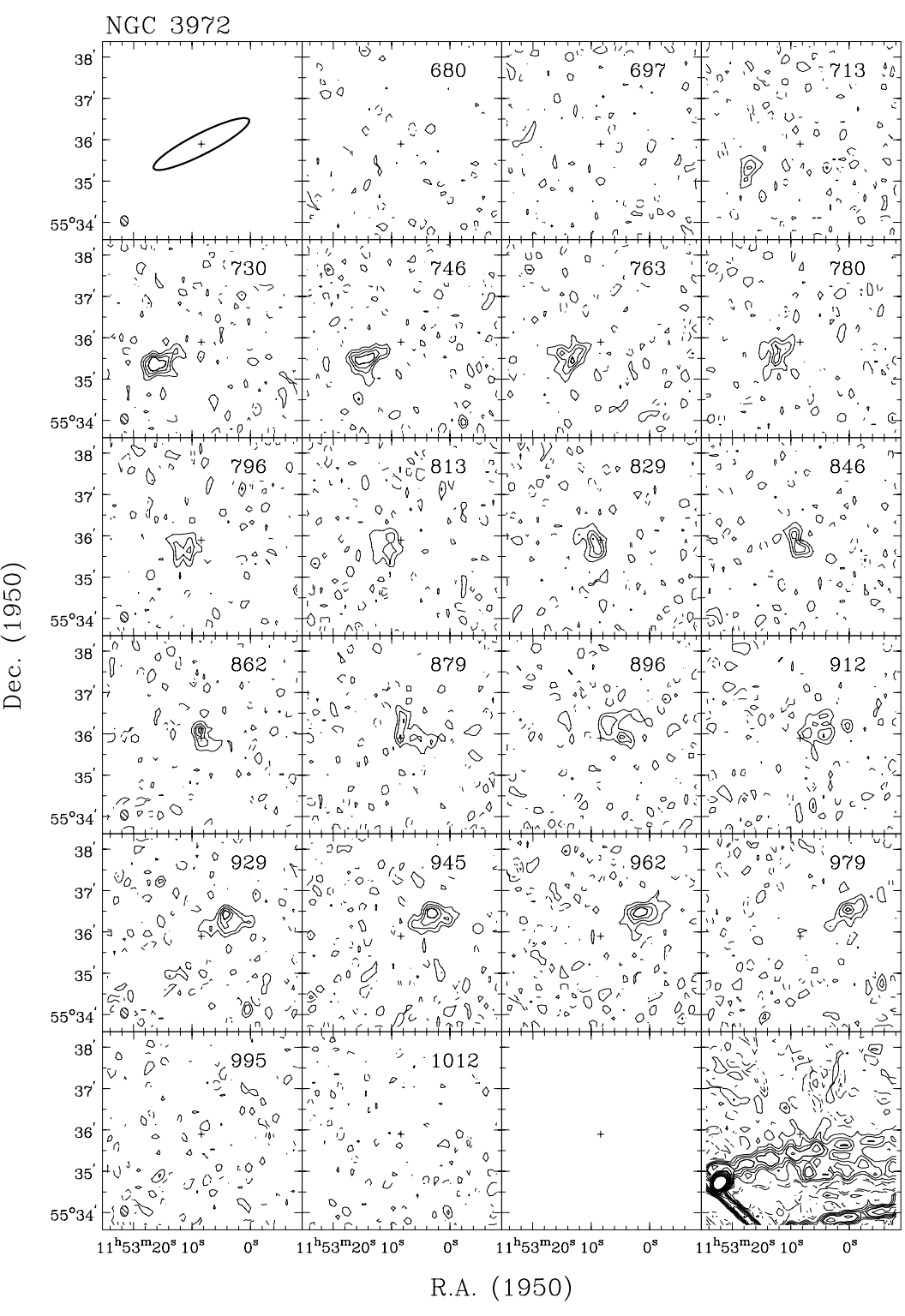

Channel maps at a resolution of $11^{\prime \prime} \times 15^{\prime \prime} \times 19 \mathrm{~km} \mathrm{~s}^{-1}$. Contour levels at $-3,-1.5$ (dashed) $1.5,3,4.5, \ldots \times \sigma$. 

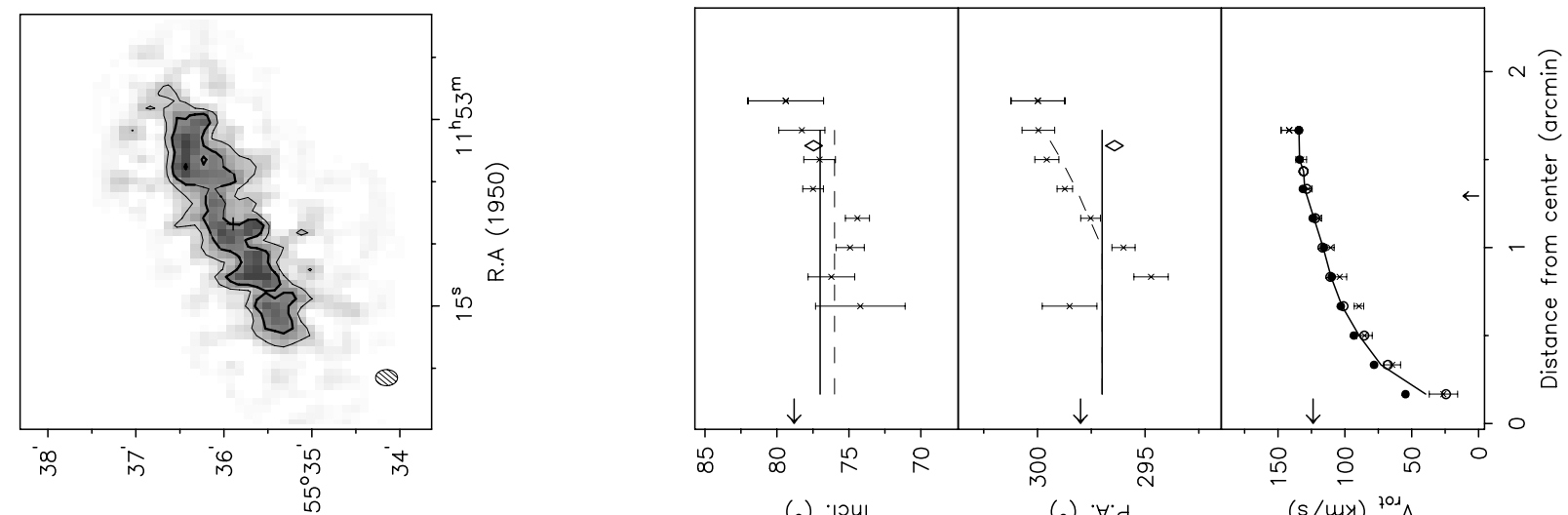
(.) '|गu|
(०) $\forall \cdot d$
$(s / m y){ }^{701} \wedge$

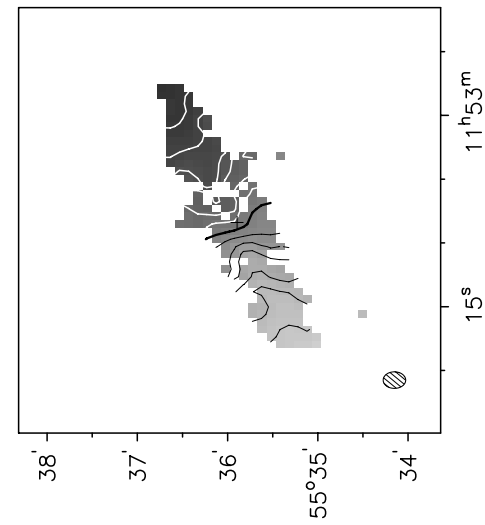

$(056 \mathrm{~L}) \cdot 200$
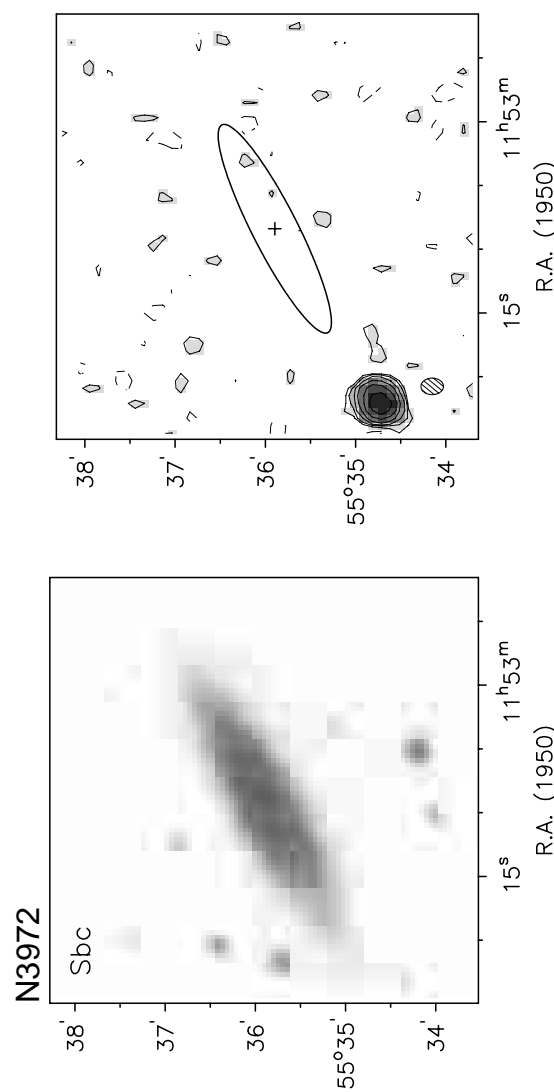

(096l) $\cdot 2 \partial 0$

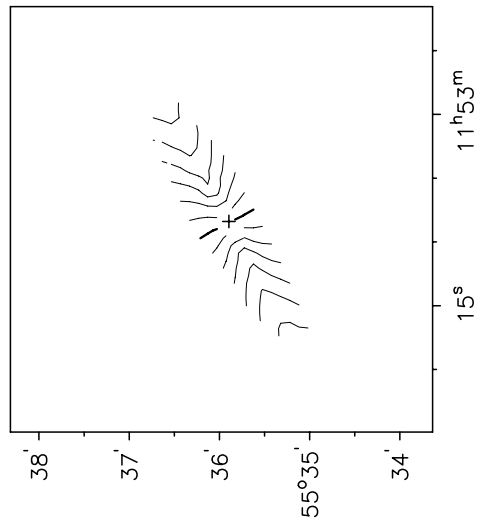

(0s6l) $\cdot 200$
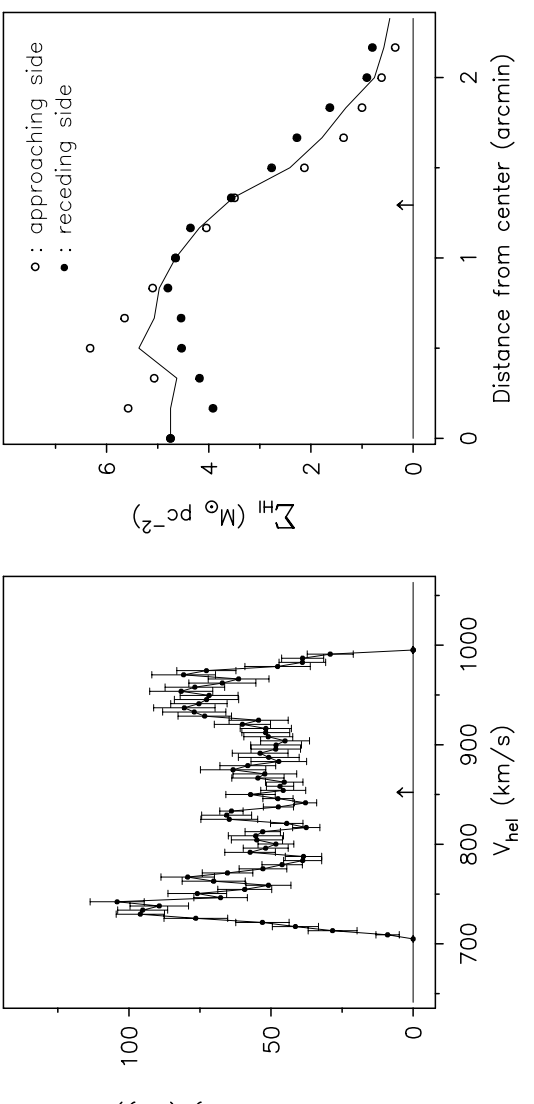

(Кгس) Kұ!suәp xn!」

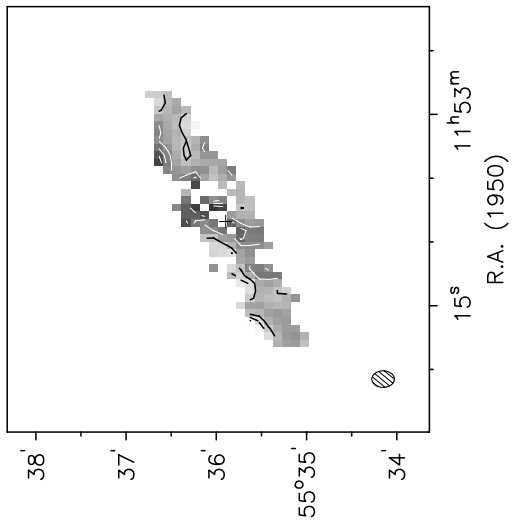

(096l) $\cdot 000$

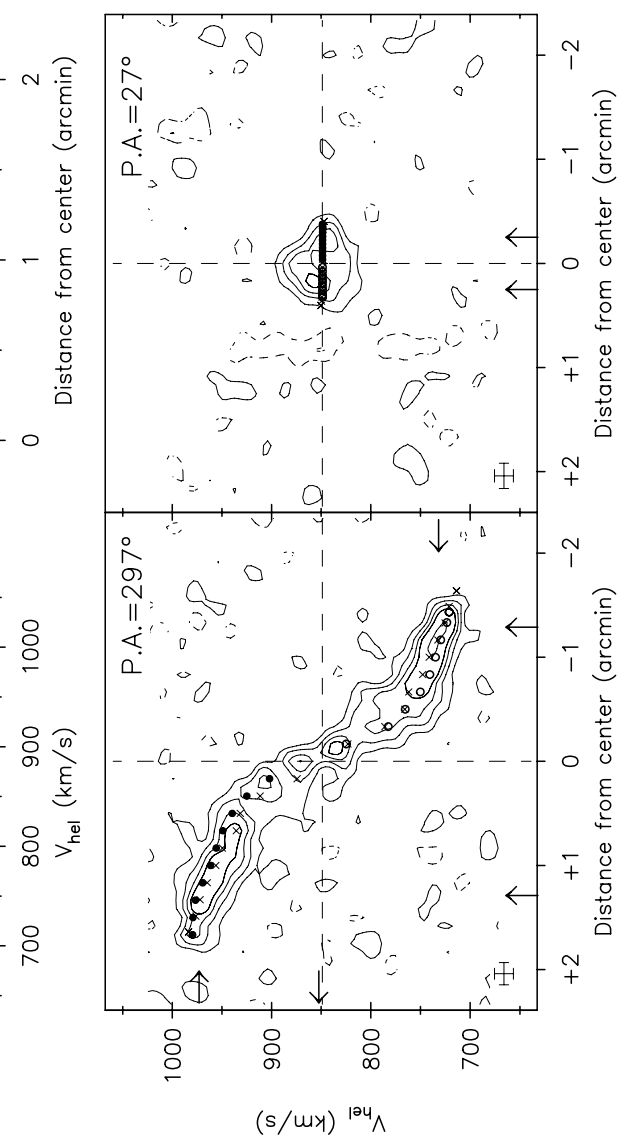


Observing parameters for UGC 6917

\begin{tabular}{|c|c|}
\hline Length of observation & $1 \times 12$ \\
\hline Date of observation & 19Apr91 \\
\hline Field center, $\alpha(1950)$ & 11:53:55 \\
\hline$\delta(1950)$ & 50:40:00 \\
\hline Central frequency & 1415.96 \\
\hline$V_{\text {hel }}$ of central channel & 925 \\
\hline Primary beam FWHM & 37.4 \\
\hline Nr. of interferometers & 40 \\
\hline Baselines (min-max-incr) & $36-2700-72$ \\
\hline Synthesized beam $(\alpha \times \delta)(\operatorname{arcsec})$ & $11.6 \times 17.2$ \\
\hline Bandwidth & 2.5 \\
\hline Number of channels & 127 \\
\hline Channel separation & 4.15 \\
\hline Velocity resolution & 8.30 \\
\hline rms noise in one channel & 5.28 \\
\hline $\begin{array}{l}\text { K-mJy conversion, } \\
\text { equiv. of } 1 \mathrm{mJy} / \text { beam }\end{array}$ & 3.02 \\
\hline
\end{tabular}

Results from WSRT data

\begin{tabular}{|c|c|}
\hline \multicolumn{2}{|l|}{ From continuит map: } \\
\hline \multicolumn{2}{|l|}{$\begin{array}{l}\text { 21-cm flux density } \\
\text {. }\end{array}$} \\
\hline central point source (mJy) & $<1.2(3 \sigma)$ \\
\hline extended source (mJy) & $<4.4(3 \sigma)$ \\
\hline \multicolumn{2}{|l|}{ From global profile: } \\
\hline$\overline{\text { Integrated HI-flux }}\left(\mathrm{Jy} \mathrm{km} \mathrm{s}^{-1}\right)$ & $26.2 \pm 0.3$ \\
\hline Hel. systemic velocity $\left(\mathrm{km} \mathrm{s}^{-1}\right)$ & $910.7 \pm 1.4$ \\
\hline HI profile width, $20 \%\left(\mathrm{~km} \mathrm{~s}^{-1}\right)$ & $208.9 \pm 3.2$ \\
\hline $50 \%\left(\mathrm{~km} \mathrm{~s}^{-1}\right)$ & $189.6 \pm 1.6$ \\
\hline \multicolumn{2}{|l|}{ From velocity field: } \\
\hline 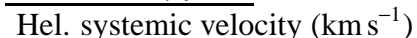 & $909.6 \pm 0.9$ \\
\hline Dynamical center, $\alpha(1950)$ & $11: 53: 53.1$ \\
\hline$\delta(1950)$ & $50: 42: 29$ \\
\hline \multicolumn{2}{|l|}{ From total HI map: } \\
\hline$\overline{\text { Geometric center, } \alpha} \alpha(1950)$ & $11: 53: 53.3$ \\
\hline$\delta(1950)$ & $50: 42: 25$ \\
\hline Position angle & 130 \\
\hline Inclination angle & 59 \\
\hline Diameter of HI disk (arcmin) & 4.8 \\
\hline
\end{tabular}
Diameter of Hil disk (arcmin)
Contour levels for U6917

Channel maps: $\quad \sigma=0.90(\mathrm{~K})$

Raw continuum map:

Cleaned continuum map

$\sigma=1.18(\mathrm{~K})$

Position-Velocity diagrams:

Velocity fields:

$909.6 \pm \mathrm{n} \times 20\left(\mathrm{~km} \mathrm{~s}^{-1}\right)$ Residual velocity field: $\pm \mathrm{n} \times 5\left(\mathrm{~km} \mathrm{~s}^{-1}\right)$

Integrated HI map:

$0.49,0.98$,

$1.47\left(\times 10^{21}\right.$ atoms cm $\left.^{-2}\right)$

Note: The apparent upturn of the rotation curve at the last measured points is not necessarily real. The disk seems to be warped toward more edge-on in the outer regions but the amplitude of this warp is difficult to measure. A flat rotation curve in the outer regions would mean that the inclination angle would mean that the inclination angle
increases by about 6 degrees which is well within the uncertainties. The channel maps are presented at a resolution of $30 \times 30$ arcsec while the other data are shown for the highest resolution of $11 \times 15$ arcsec.

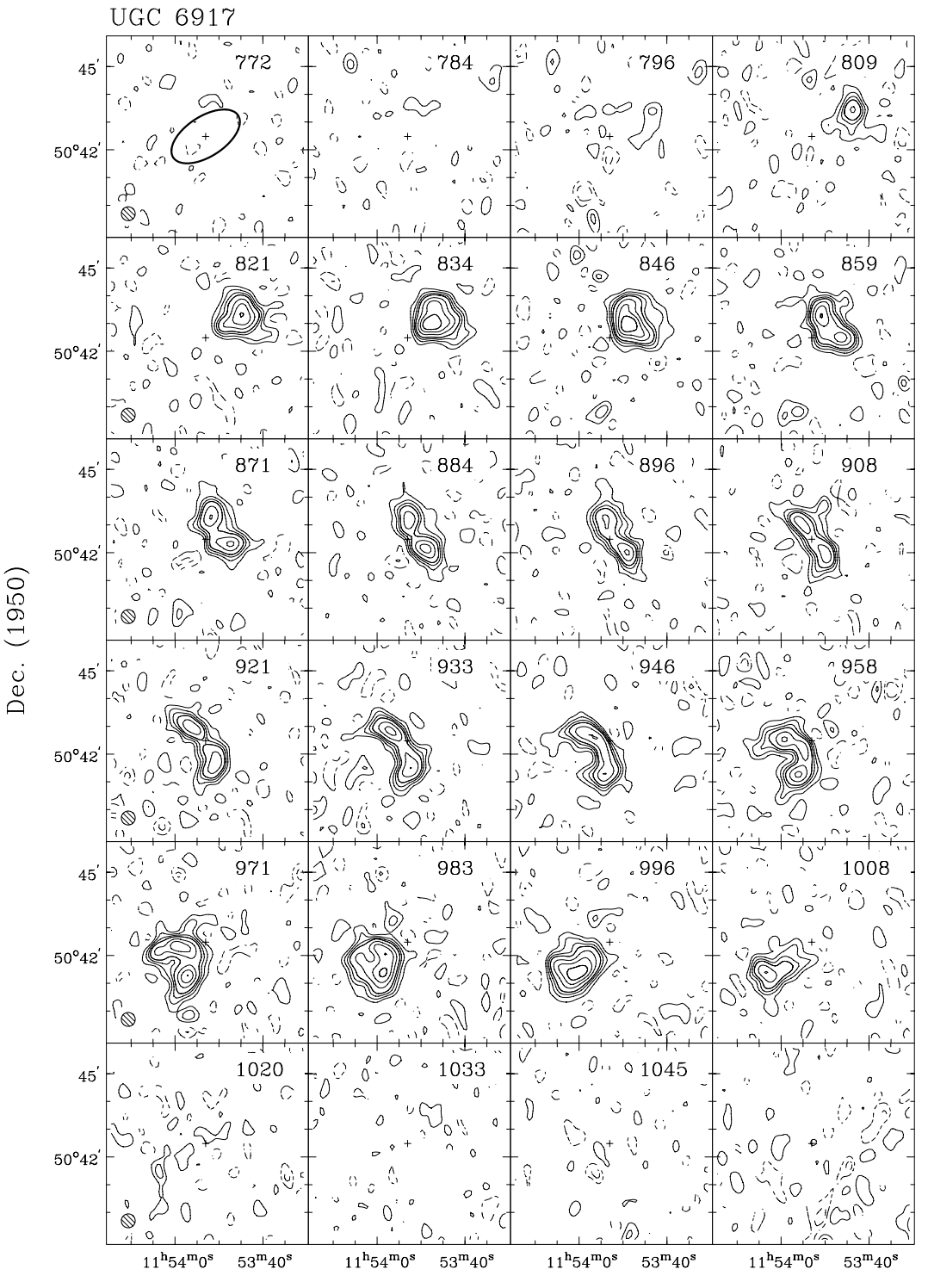



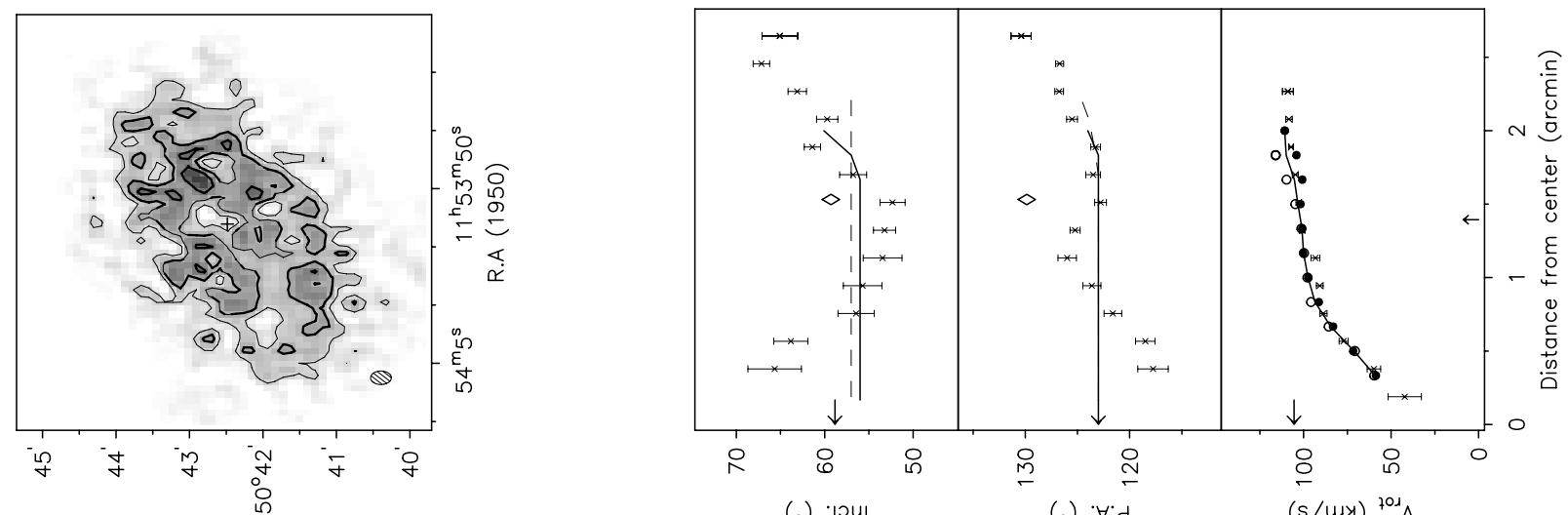

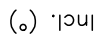

(o) $\forall \cdot d$

$(s / u x)^{701} \wedge$

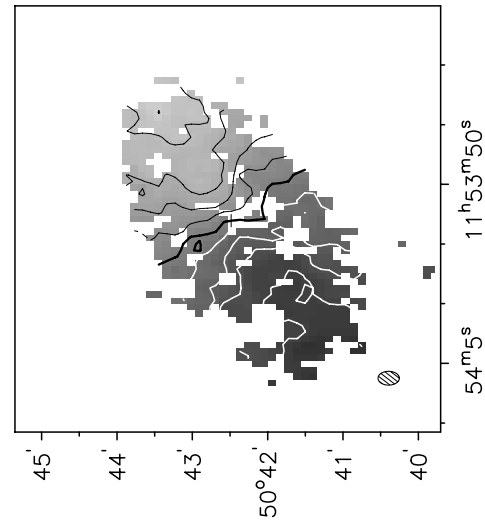

(0s6l) 'ว 0
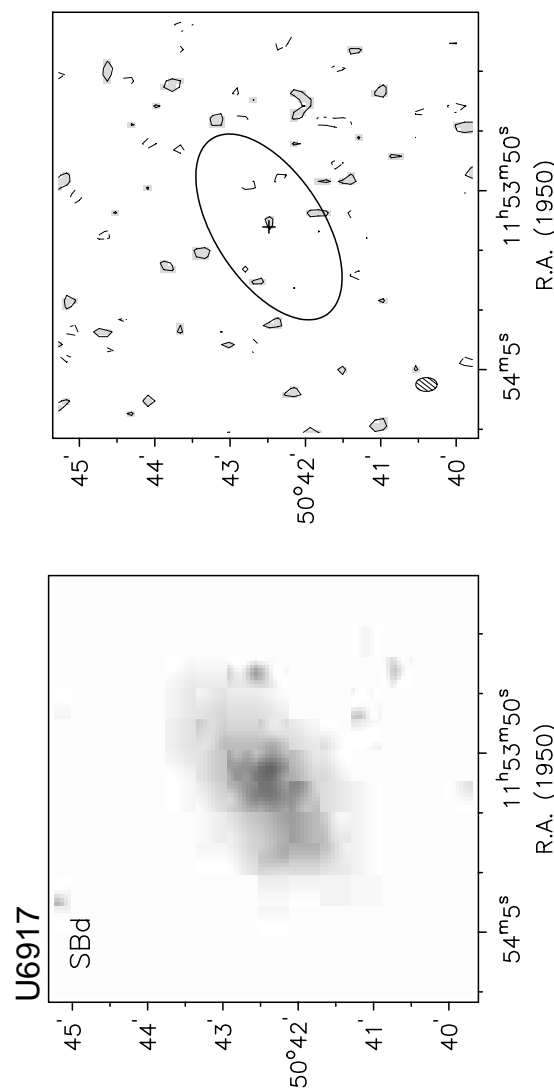

$(0 \mathrm{O} 6 \mathrm{l}) \cdot \mathrm{2} 0$

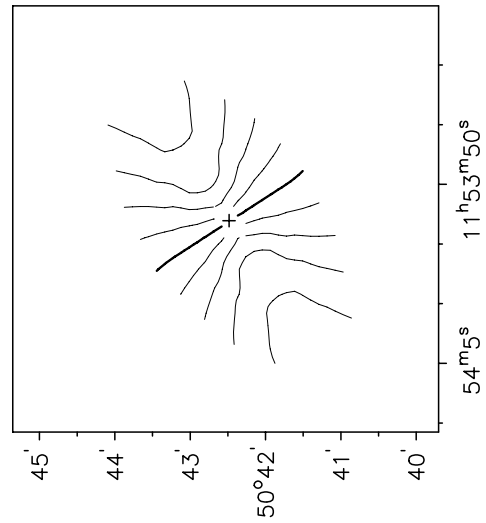

(0s6l) $\cdot 290$
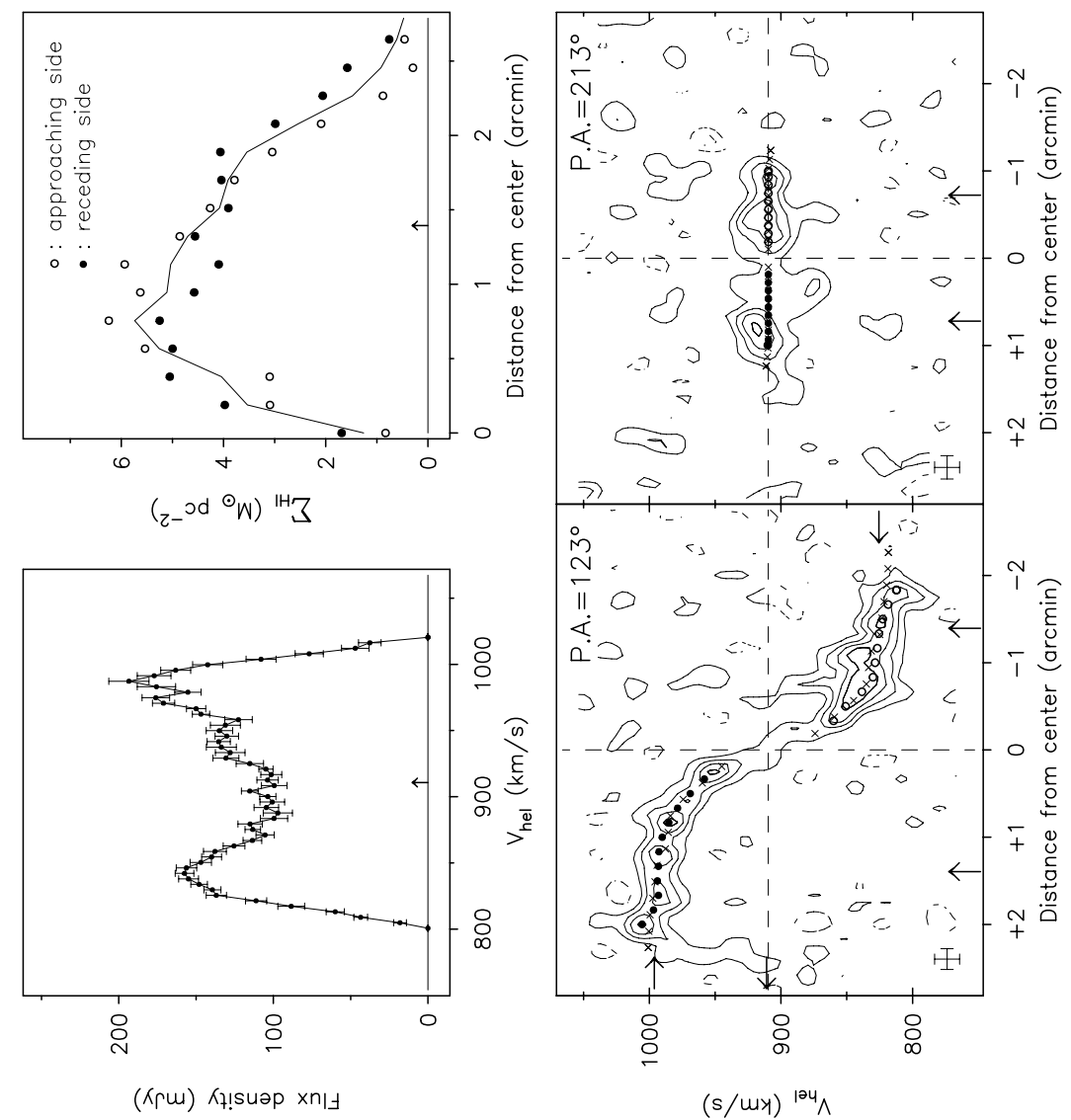

$(s / m y){ }^{104} \wedge$

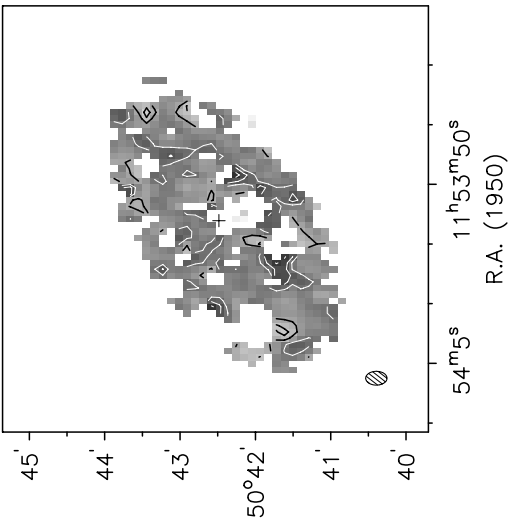

(0s6l) 
Observing parameters for UGC 6923

\begin{tabular}{|c|c|}
\hline Length of observation & $1 \times 12$ \\
\hline Date of observation & 23Dec94 \\
\hline Field center, $\alpha(1950)$ & 11:55:07 \\
\hline$\delta(1950)$ & 53:31:54 \\
\hline Central frequency & 1415.53 \\
\hline$V_{\text {hel }}$ of central channel & 1054 \\
\hline Primary beam FWHM & 37.4 \\
\hline Nr. of interferometers & 40 \\
\hline Baselines (min-max-incr) & $36-2700-72$ \\
\hline Synthesized beam $(\alpha \times \delta)(\operatorname{arcsec})$ & $12.1 \times 15.1$ \\
\hline Bandwidth & 5.0 \\
\hline Number of channels & 127 \\
\hline Channel separation & 8.31 \\
\hline Velocity resolution & 9.97 \\
\hline rms noise in one channel & 9.71 \\
\hline $\begin{array}{l}\text { K-mJy conversion, } \\
\text { equiv. of } 1 \mathrm{mJy} / \text { beam }\end{array}$ & 3.29 \\
\hline
\end{tabular}

Note: To achieve a sufficient velocity resolution over

a $5 \mathrm{MHz}$ bandwidth, only the $\mathrm{XX}$ dipoles could be

correlated due to limitations of the backend.

Results from WSRT data

\begin{tabular}{|c|c|}
\hline \multicolumn{2}{|l|}{ From continuит map: } \\
\hline \multicolumn{2}{|l|}{ 21-cm flux density } \\
\hline central point source (mJy) & $<1.3(3 \sigma)$ \\
\hline extended source (mJy) & $<2.6(3 \sigma)$ \\
\hline \multicolumn{2}{|l|}{ From global profile: } \\
\hline$\overline{\text { Integrated HI-flux }}\left(\mathrm{Jy} \mathrm{km} \mathrm{s}^{-1}\right)$ & $10.7 \pm 0.6$ \\
\hline Hel. systemic velocity $\left(\mathrm{km} \mathrm{s}^{-1}\right)$ & $1061.6 \pm 2.2$ \\
\hline HI profile width, $20 \%\left(\mathrm{~km} \mathrm{~s}^{-1}\right)$ & $166.8 \pm 2.4$ \\
\hline $50 \%\left(\mathrm{~km} \mathrm{~s}^{-1}\right)$ & $147.1 \pm 4.5$ \\
\hline \multicolumn{2}{|l|}{ From velocity field: } \\
\hline Hel. systemic velocity $\left(\mathrm{km} \mathrm{s}^{-1}\right)$ & $1066.0 \pm 1.7$ \\
\hline Dynamical center, $\alpha(1950)$ & $11: 54: 13.9$ \\
\hline$\delta(1950)$ & $53: 26: 22$ \\
\hline \multicolumn{2}{|l|}{ From total HI map: } \\
\hline$\overline{\text { Geometric center, } \alpha(1950)}$ & $11: 54: 14.4$ \\
\hline$\delta(1950)$ & $53: 26: 24$ \\
\hline Position angle & 346 \\
\hline Inclination angle & 64 \\
\hline Diameter of HI disk (arcmin) & 2.6 \\
\hline
\end{tabular}

Contour levels for U6923

Channel maps: $\quad \sigma=5.93(\mathrm{~K})$

Raw continuum map:

Cleaned continuum map $\sigma=1.42(\mathrm{~K})$

Position-Velocity diagrams:

Velocity fields:

$1066.0 \pm \mathrm{n} \times 15\left(\mathrm{~km} \mathrm{~s}^{-1}\right)$ Residual velocity field: $\pm \mathrm{n} \times 5\left(\mathrm{~km} \mathrm{~s}^{-1}\right)$

Integrated HI map: $1.19,2.39$ $3.58\left(\times 10^{21}\right.$ atoms cm $\left.^{-2}\right)$

)

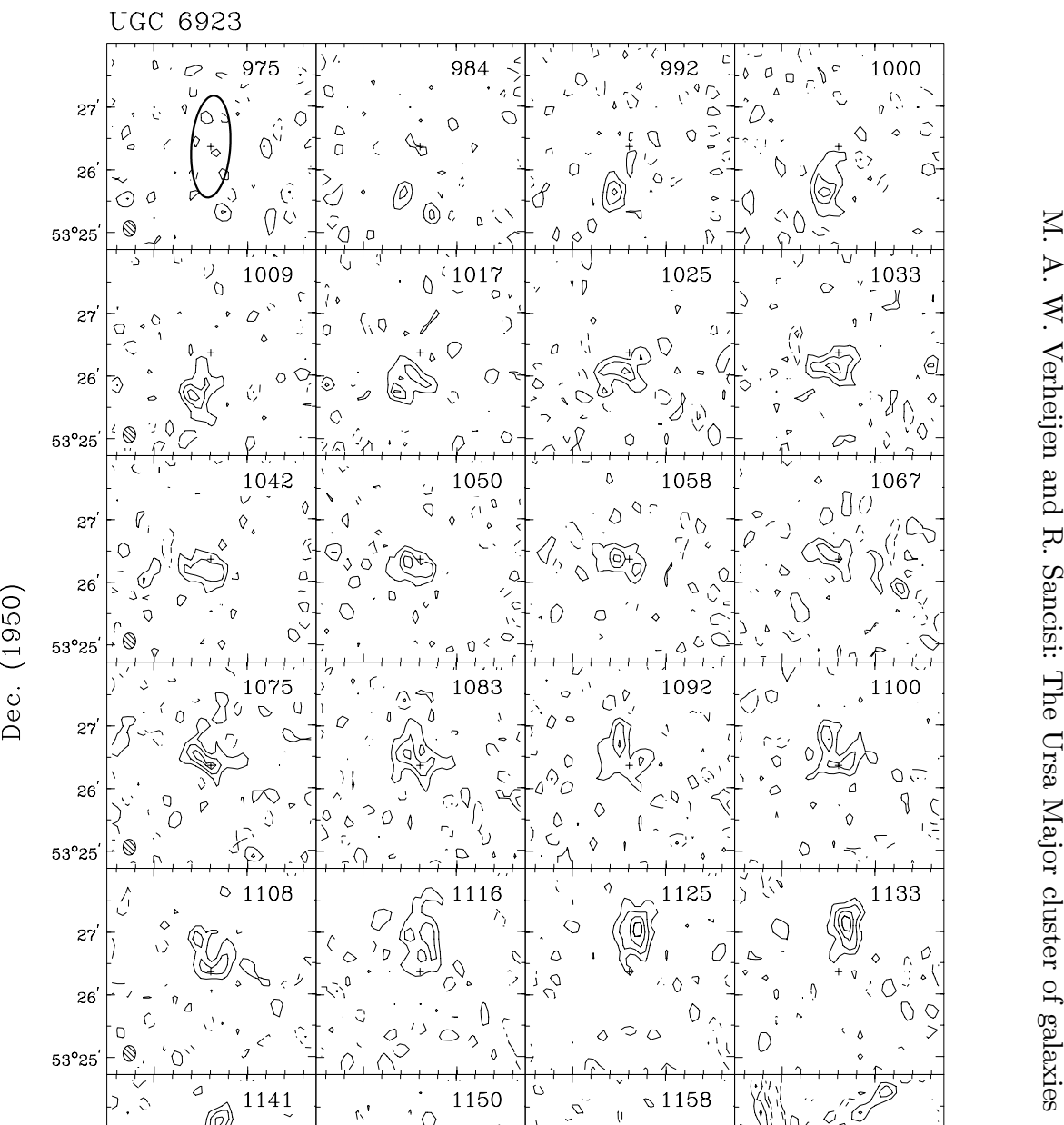

Note: This dwarf galaxy is a companion of NGC 3992. Optically, the disk seems to be warped which is also suggested by the HI velocity field. The HI surface density in this system is quite high and centrally concentrated.

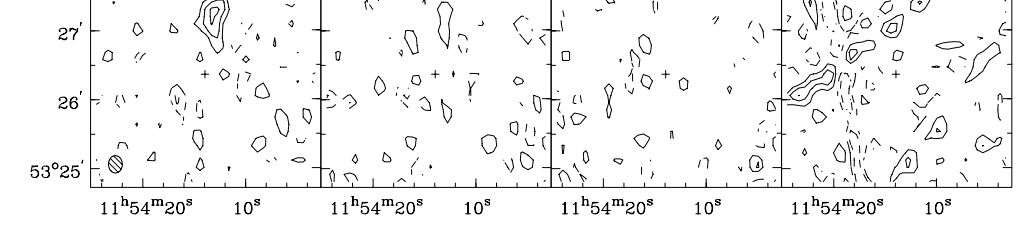

R.A. (1950)

Channel maps at a resolution of $12^{\prime \prime} \times 15^{\prime \prime} \times 17 \mathrm{~km} \mathrm{~s}^{-1}$. Contour levels at $-3,-1.5$ (dashed), $1.5,3,4.5, \ldots \times \sigma$. 

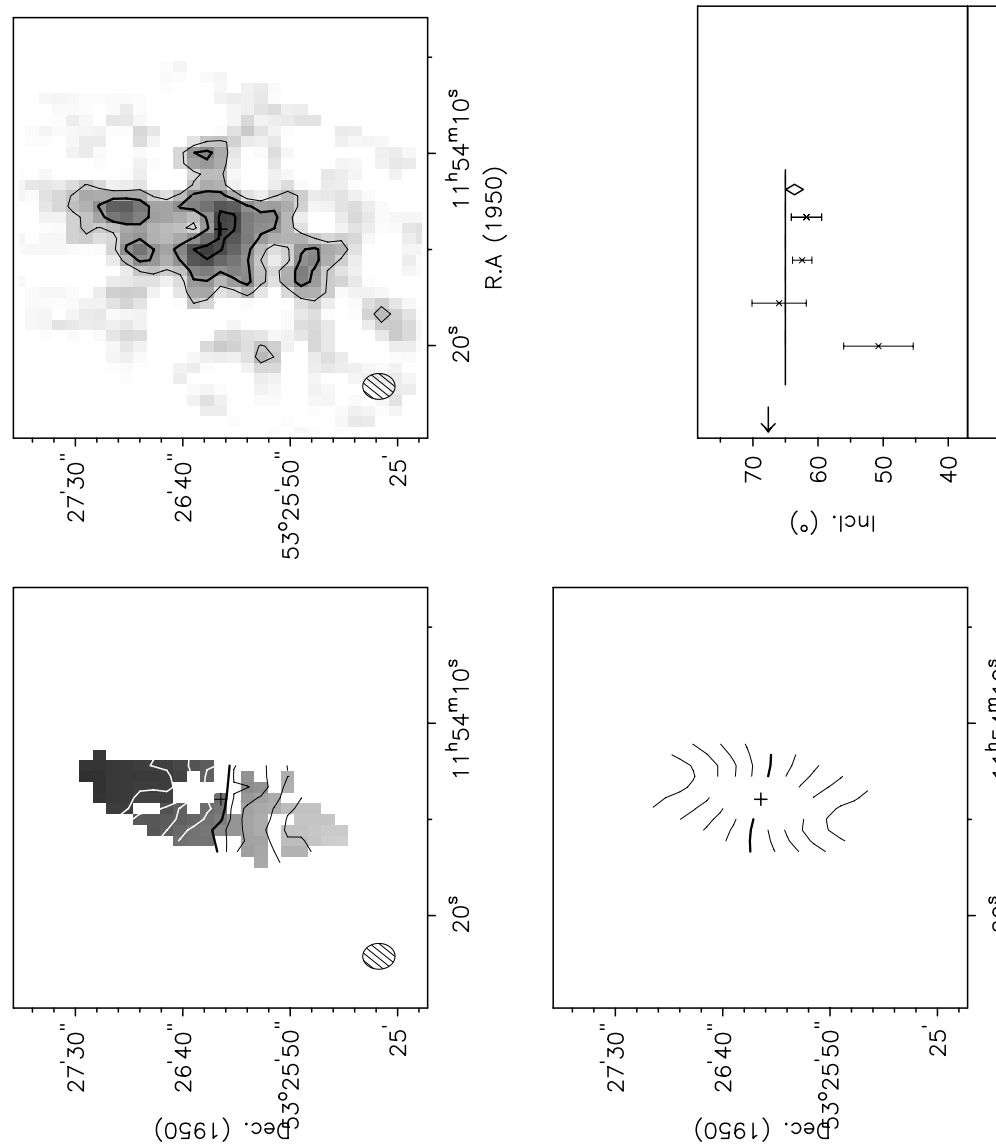

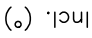

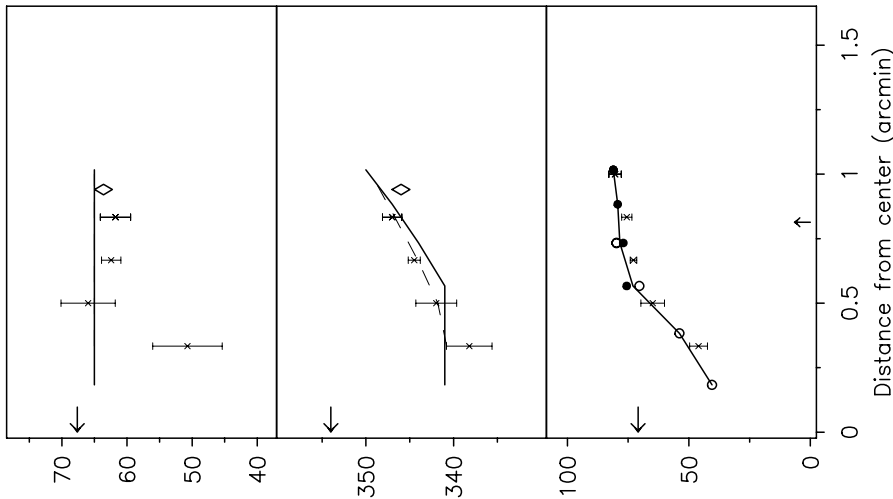

(o) $\forall \cdot d$

$(s / m y){ }^{701} \wedge$

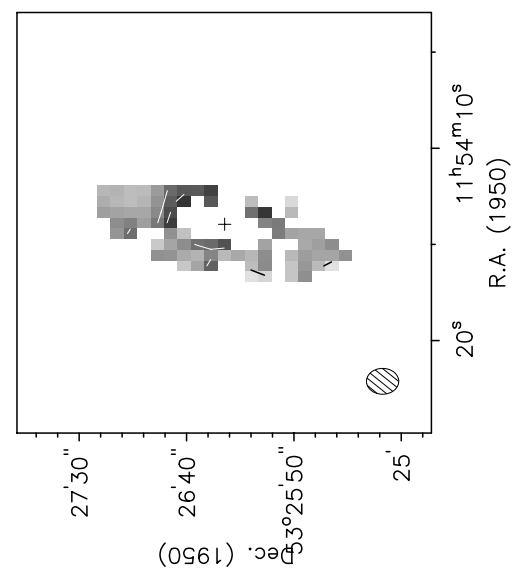

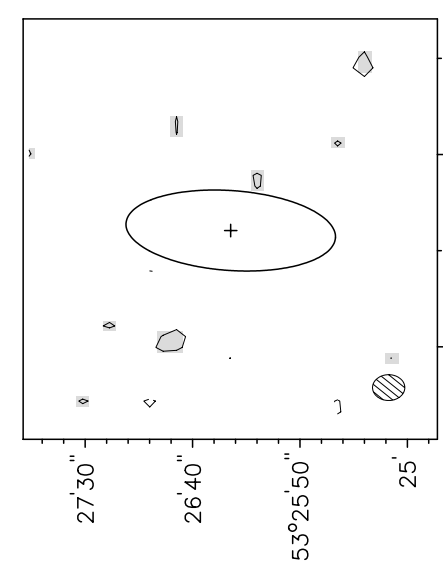

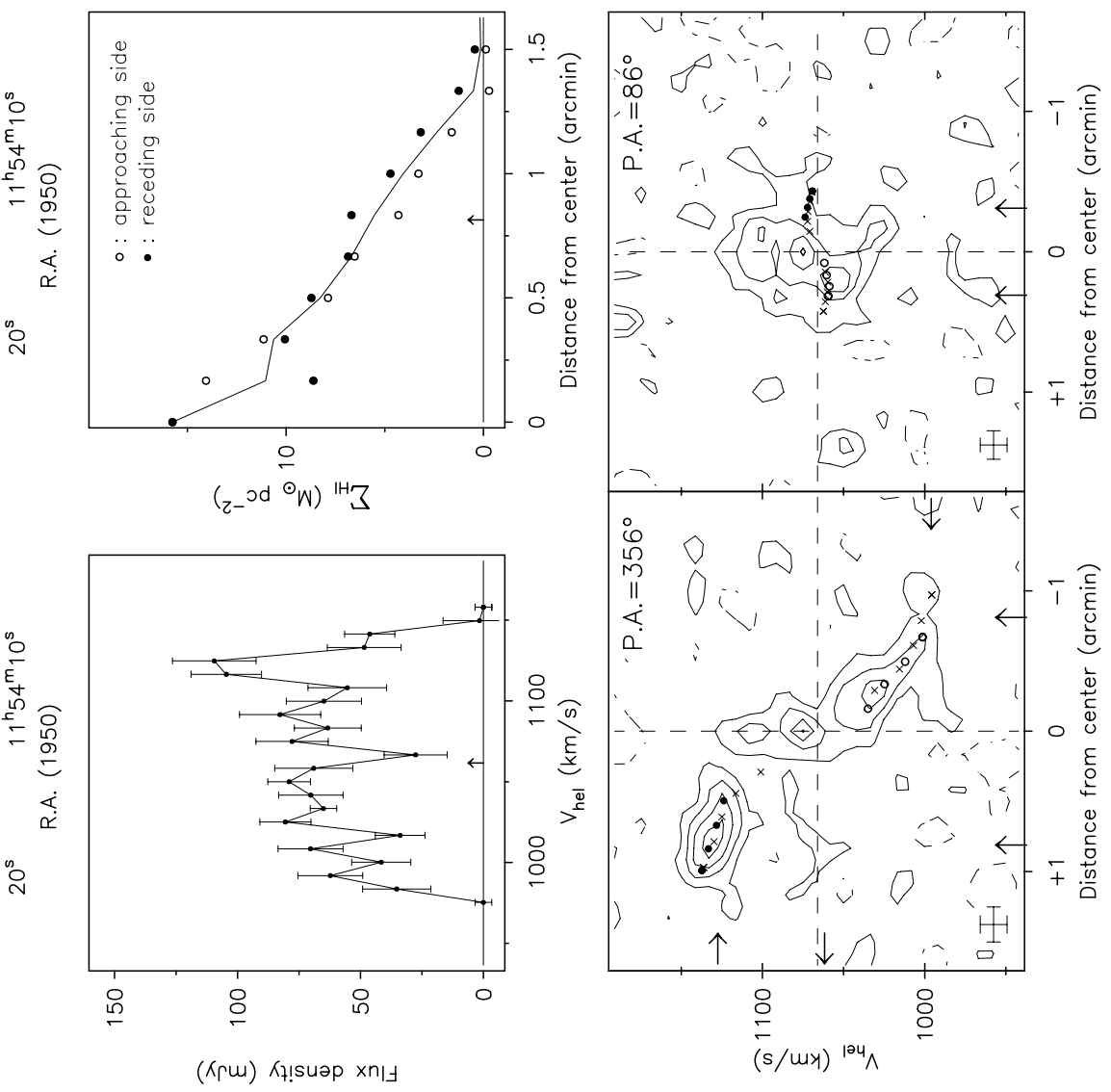


Observing parameters for UGC 6930

\begin{tabular}{|c|c|}
\hline Length of observation & $1 \times 12$ \\
\hline Date of observation & 20Aug93 \\
\hline Field center, $\alpha(1950)$ & 11:54:42 \\
\hline$\delta(1950)$ & 49:33:50 \\
\hline Central frequency & 1416.72 \\
\hline$V_{\text {hel }}$ of central channel & 778 \\
\hline Primary beam FWHM (arcmin) & 37.4 \\
\hline Nr. of interferometers & 40 \\
\hline Baselines (min-max-incr) & $36-2700-72$ \\
\hline Synthesized beam $(\alpha \times \delta)(\operatorname{arcsec})$ & $12.3 \times 16.8$ \\
\hline Bandwidth & 2.5 \\
\hline Number of channels & 127 \\
\hline Channel separation & 4.14 \\
\hline Velocity resolution & 8.29 \\
\hline rms noise in one channel $\quad(\mathrm{K})$ & 5.54 \\
\hline $\begin{array}{l}\text { K-mJy conversion, } \\
\text { equiv. of } 1 \mathrm{mJy} / \text { beam }\end{array}$ & 2.92 \\
\hline
\end{tabular}

Results from WSRT data

\begin{tabular}{|c|c|}
\hline \multicolumn{2}{|l|}{ From continuит map: } \\
\hline \multicolumn{2}{|l|}{ 21-cm flux density } \\
\hline central point source $(\mathrm{mJy})$ & $<1.2(3 \sigma)$ \\
\hline extended source (mJy) & $<5.8(3 \sigma)$ \\
\hline \multicolumn{2}{|l|}{ From global profile: } \\
\hline$\overline{\text { Integrated HI-flux }}\left(\mathrm{Jy} \mathrm{km} \mathrm{s}^{-1}\right)$ & $42.7 \pm 0.3$ \\
\hline Hel. systemic velocity $\left(\mathrm{km} \mathrm{s}^{-1}\right)$ & $777.2 \pm 0.4$ \\
\hline HI profile width, $20 \%\left(\mathrm{~km} \mathrm{~s}^{-1}\right)$ & $136.5 \pm 0.5$ \\
\hline $50 \%\left(\mathrm{~km} \mathrm{~s}^{-1}\right)$ & $122.1 \pm 0.7$ \\
\hline \multicolumn{2}{|l|}{ From velocity field: } \\
\hline 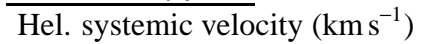 & $777.7 \pm 0.2$ \\
\hline Dynamical center, $\alpha(1950)$ & $11: 54: 41.9$ \\
\hline$\delta(1950)$ & 49:33:40 \\
\hline \multicolumn{2}{|l|}{ From total HI map: } \\
\hline$\overline{\text { Geometric center, } \alpha(1950)}$ & $11: 54: 43.1$ \\
\hline$\delta(1950)$ & 49:33:44 \\
\hline Position angle & 31 \\
\hline Inclination angle & 28 \\
\hline Diameter of HI disk (arcmin) & 6.4 \\
\hline
\end{tabular}
Diameter of HI disk (arcmin)
Contour levels for U6930

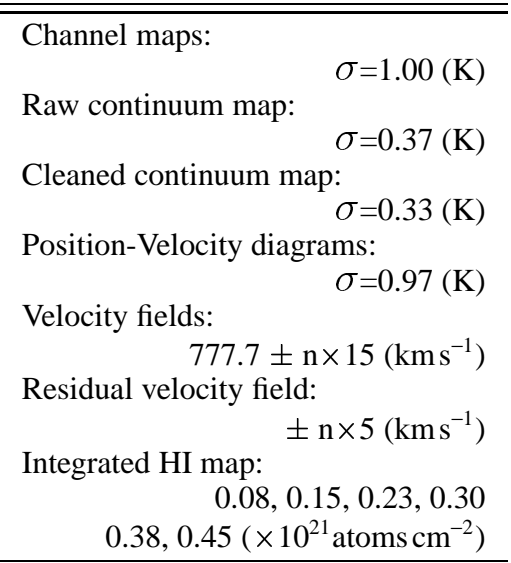

Note: This low surface brightness galaxy is located nearest to the adopted cluster center. It is more face-on than 45 degrees and therefore not fit for a study of the TF-relation. The HI disk is not symmetrical and is slightly more extended toward the south-east at the higher column density levels.

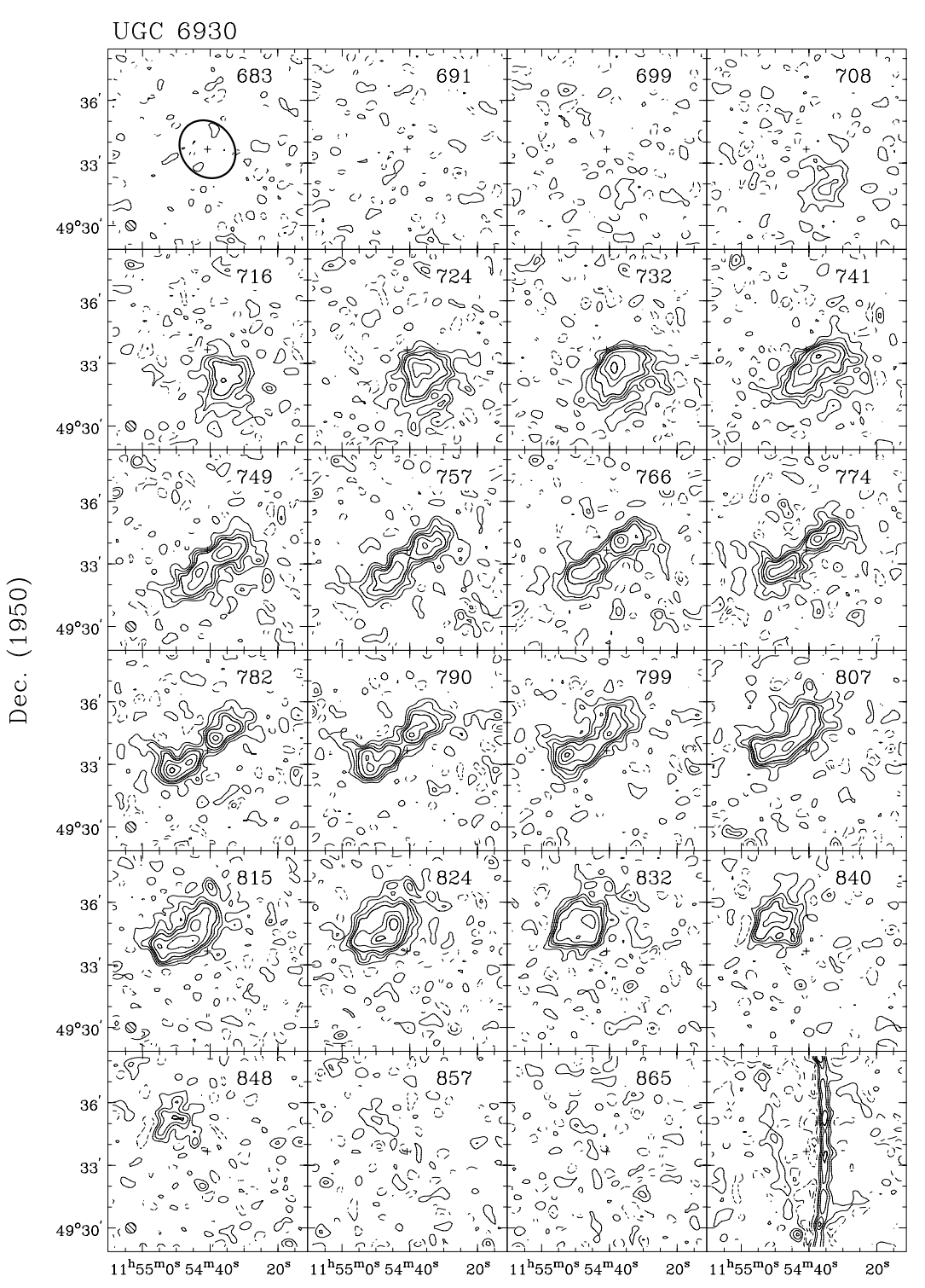




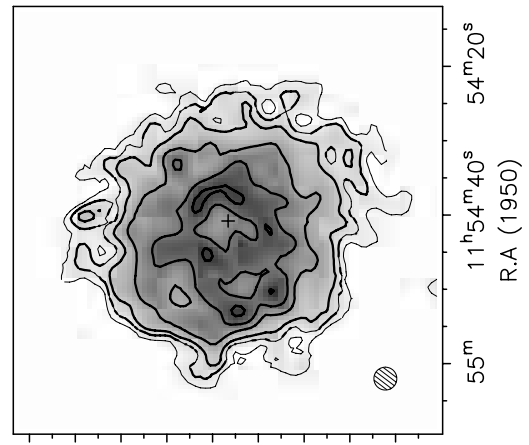

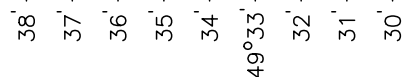

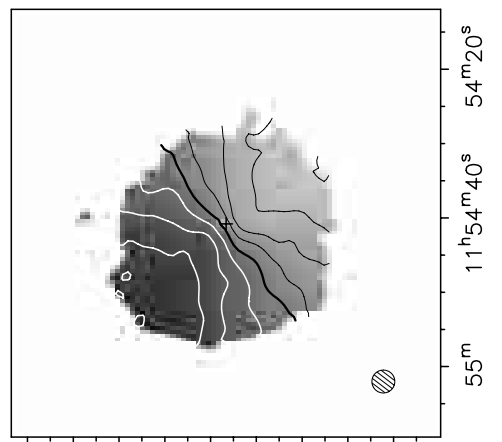

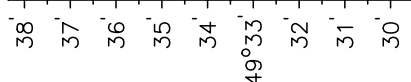

(0g6l) $\cdot 200$

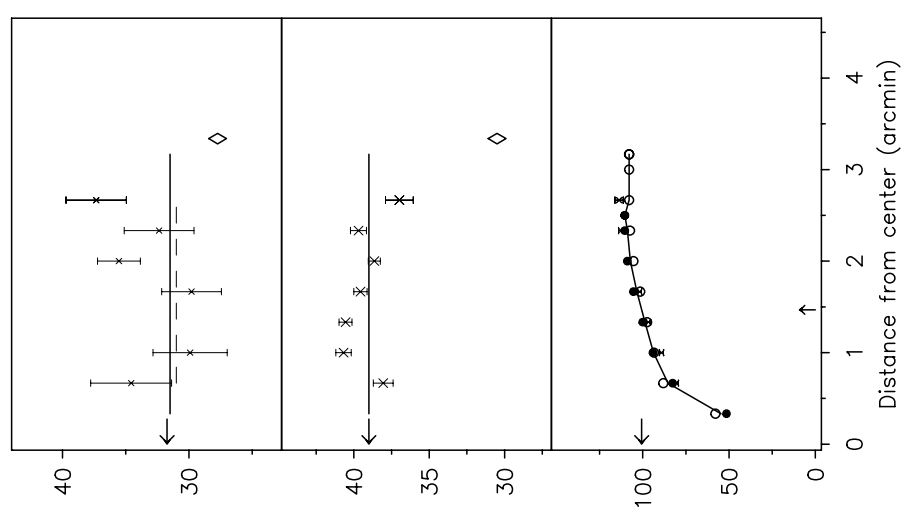

(०) $\cdot 10 \mathrm{ul}$

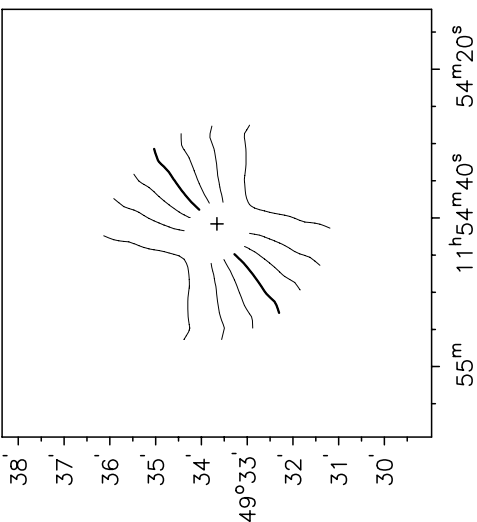

(096l) : गә0 (o) $\forall \cdot d$

$(s / m y){ }^{101} \wedge$

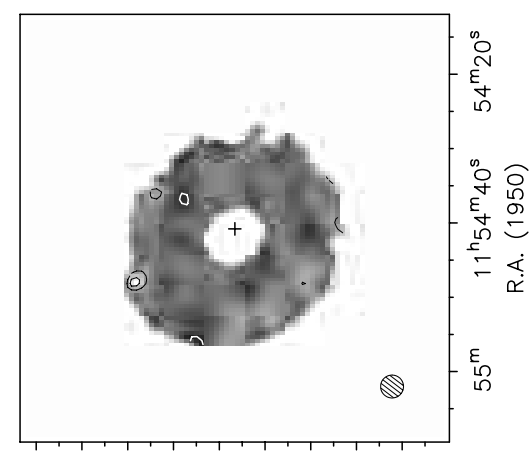

分

(096l) $\cdot 520$

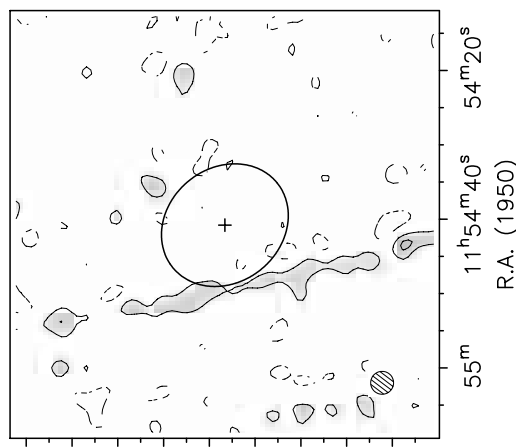

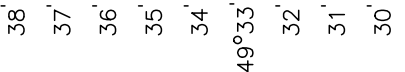

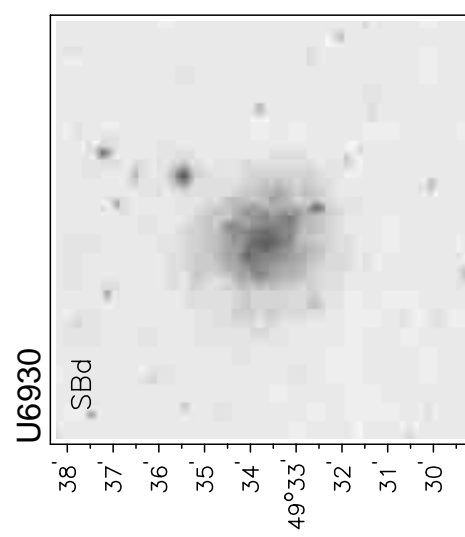

(096l) $\cdot 500$

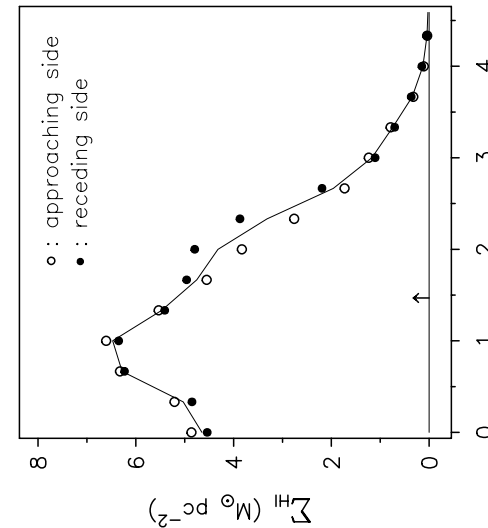

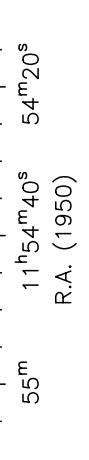

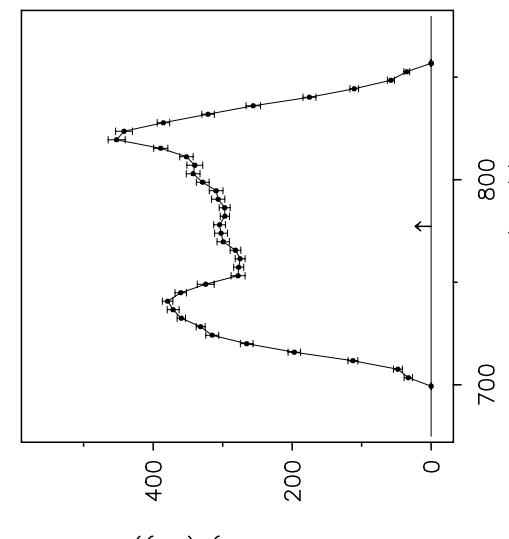

(Кгس) K?!suәp xn!

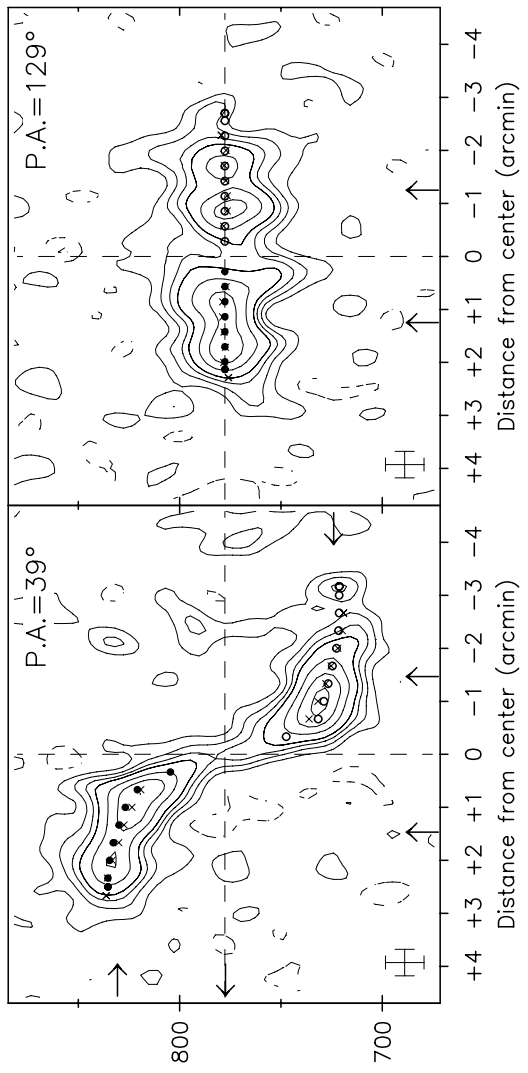

$(s / m y){ }^{124} \wedge$ 
Observing parameters for NGC 3992

\begin{tabular}{|c|c|}
\hline Length of observation & $1 \times 12$ \\
\hline Date of observation & 23Dec94 \\
\hline Field center, $\alpha(1950)$ & 11:55:07 \\
\hline$\delta(1950)$ & 53:31:54 \\
\hline Central frequency & 1415.53 \\
\hline$V_{\text {hel }}$ of central channel & 1054 \\
\hline Primary beam FWHM (arcmin) & 37.4 \\
\hline Nr. of interferometers & 40 \\
\hline Baselines (min-max-incr) & $36-2700-72$ \\
\hline Synthesized beam $(\alpha \times \delta)(\operatorname{arcsec})$ & $12.1 \times 15.1$ \\
\hline Bandwidth & 5.0 \\
\hline Number of channels & 127 \\
\hline Channel separation & 8.31 \\
\hline Velocity resolution & 9.97 \\
\hline rms noise in one channel & 9.71 \\
\hline $\begin{array}{l}\text { K-mJy conversion, } \\
\text { equiv. of } 1 \mathrm{mJy} / \text { beam }\end{array}$ & 3.29 \\
\hline
\end{tabular}

Note: To achieve a sufficient velocity resolution over

a $5 \mathrm{MHz}$ bandwidth, only the XX dipoles could be

correlated due to limitations of the backend.

\section{Results from WSRT data}

\begin{tabular}{|c|c|}
\hline \multicolumn{2}{|l|}{ From continuит map: } \\
\hline $\begin{array}{l}21-\mathrm{cm} \text { flux density } \\
\text { From global profile: }\end{array}$ & $30.2 \pm 7.6$ \\
\hline \multicolumn{2}{|l|}{ From global profile: } \\
\hline Integrated HI-flux $\quad\left(\mathrm{Jy} \mathrm{km} \mathrm{s}^{-1}\right)$ & $74.6 \pm 1.5$ \\
\hline Hel. systemic velocity $\left(\mathrm{km} \mathrm{s}^{-1}\right)$ & $1048.2 \pm 1.2$ \\
\hline HI profile width, $20 \%\left(\mathrm{~km} \mathrm{~s}^{-1}\right)$ & $478.5 \pm 1.4$ \\
\hline $50 \%\left(\mathrm{~km} \mathrm{~s}^{-1}\right)$ & $461.4 \pm 2.4$ \\
\hline \multicolumn{2}{|l|}{ From velocity field: } \\
\hline 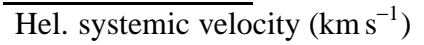 & $1049.5 \pm 0.6$ \\
\hline $\begin{array}{r}\text { Dynamical center, } \alpha(1950) \\
\delta(1950)\end{array}$ & $\begin{array}{l}\text { 11:55:00.7 } \\
53: 39: 09\end{array}$ \\
\hline \multicolumn{2}{|l|}{ From total HI map: } \\
\hline 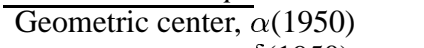 & $11: 54: 58.7$ \\
\hline$\delta(1950)$ & 53:39:03 \\
\hline Position angle & 251 \\
\hline Inclination angle & 52 \\
\hline Diameter of HI disk (arcmin) & 9.5 \\
\hline
\end{tabular}

Contour levels for N3992

Channel maps: $\quad \sigma=0.56(\mathrm{~K})$

Raw continuum map:

Cleaned continuum map:

$\sigma=0.15(\mathrm{~K})$

Position-Velocity diagrams:

Velocity fields:

$1049.5 \pm \mathrm{n} \times 30\left(\mathrm{~km} \mathrm{~s}^{-1}\right)$ Residual velocity field:

$$
\pm \mathrm{n} \times 5\left(\mathrm{~km} \mathrm{~s}^{-1}\right)
$$

Integrated HI map:

$0.45,0.90,1.36,1.81,2.26$

$2.71,3.16\left(\times 10^{20}\right.$ atoms $\left.\mathrm{cm}^{-2}\right)$

Note: NGC 3992 is the most massive spiral in the cluster. It has a prominent bar and well defined spiral arms. The rotation curve declines at 4 arcmin from the center by about $30 \mathrm{~km} \mathrm{~s}^{-1}$ and remains apparently flat in the outer parts. There are three dwarf companions detected in HI: U6923, U6940 and U6969.

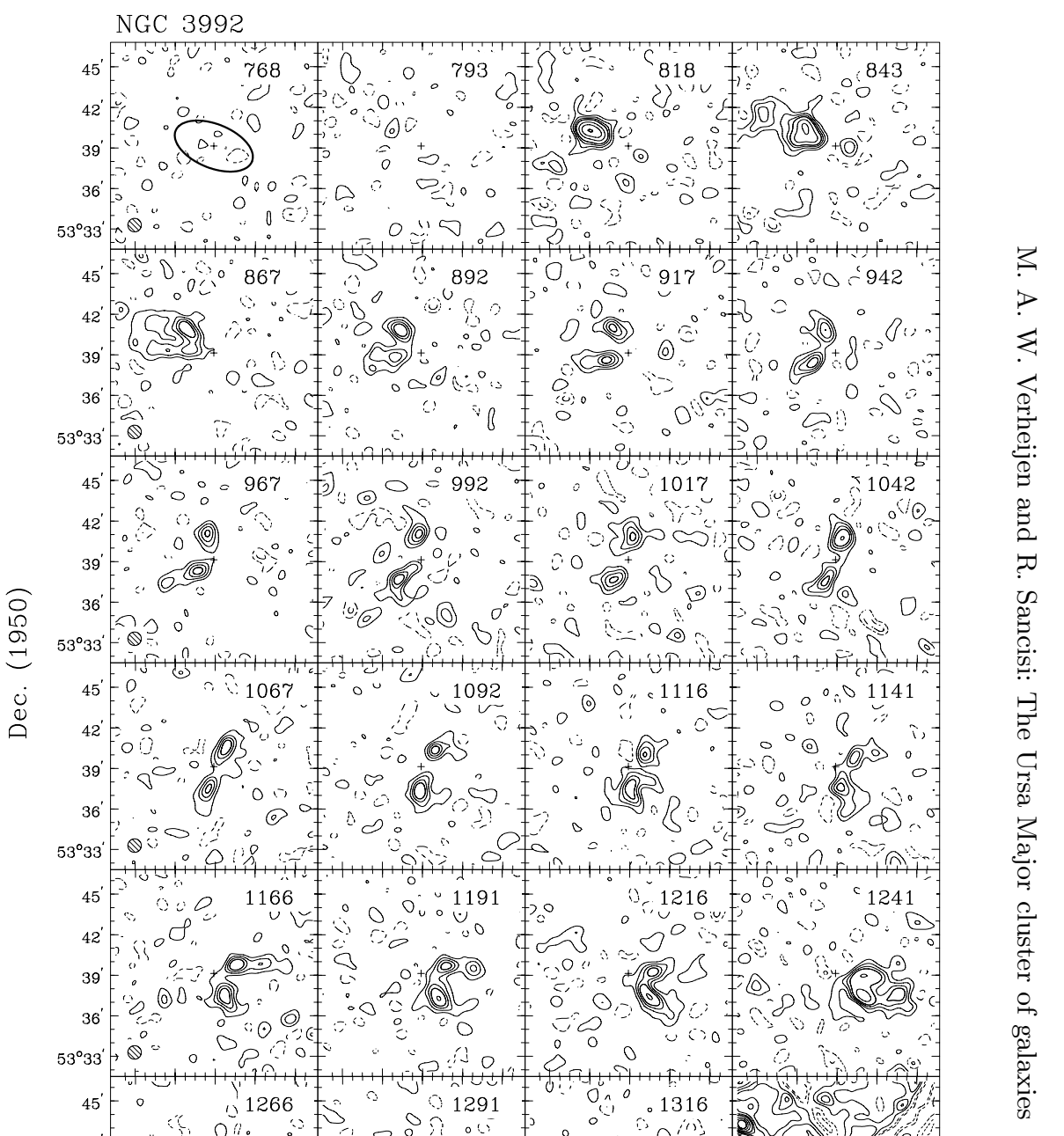

.

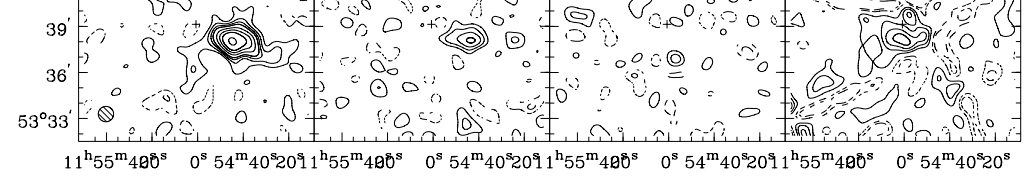

$$
\text { R.A. (1950) }
$$

Channel maps at a resolution of $60^{\prime \prime} \times 60^{\prime \prime} \times 17 \mathrm{~km} \mathrm{~s}^{-1}$. Contour levels at $-3,-1.5$ (dashed), $1.5,3,4.5, \ldots \times \sigma$. 

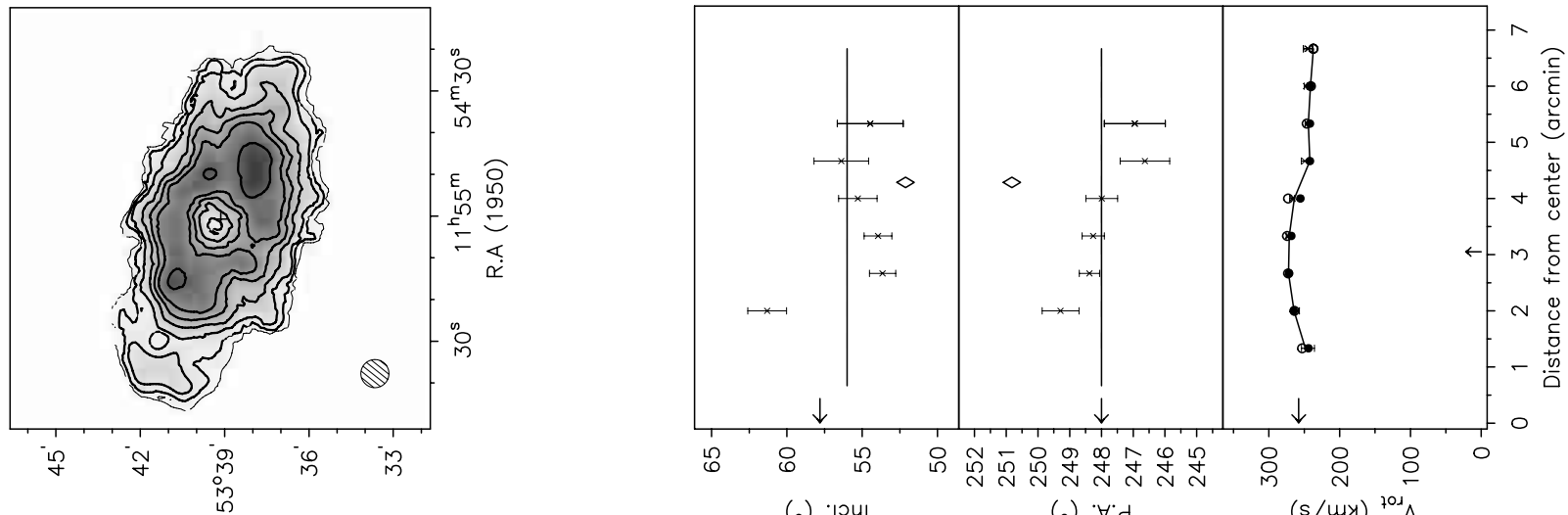

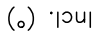

(o) $\forall \cdot d$

$(s / m x){ }^{701} \wedge$

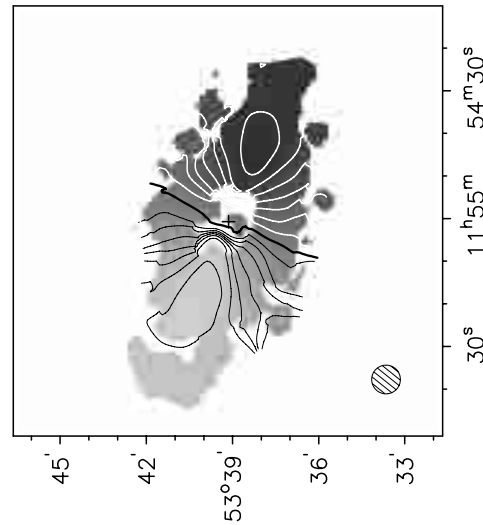

(0s6l) $\cdot 020$
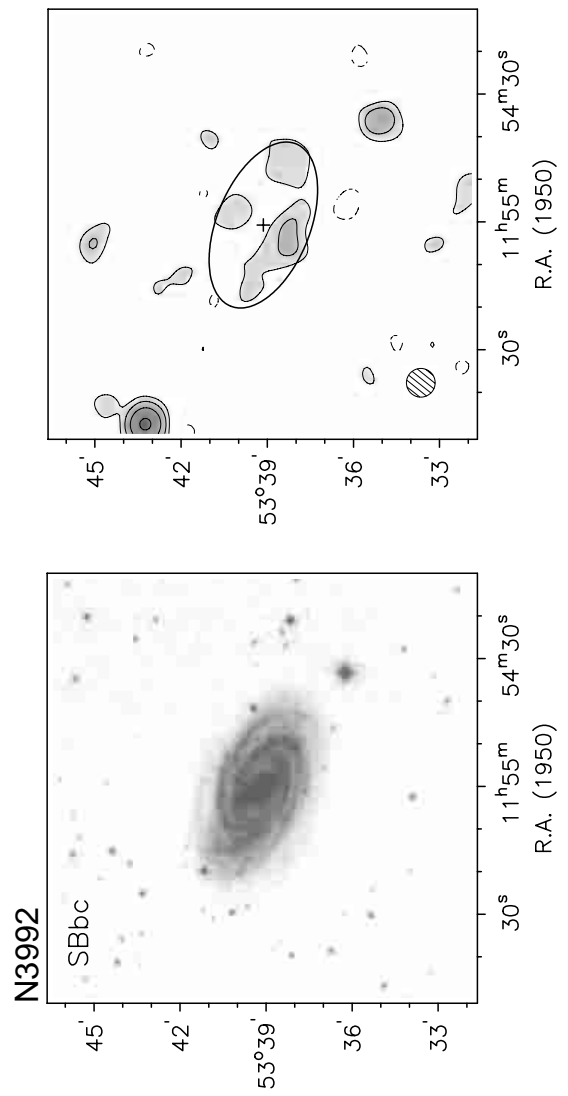

$(0961) \cdot 5 \circ 0$

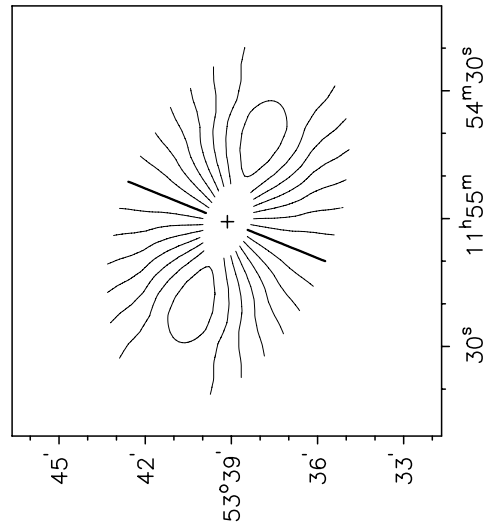

$(0 \mathrm{~s} 6 \mathrm{l}) \cdot \mathrm{0a0}$
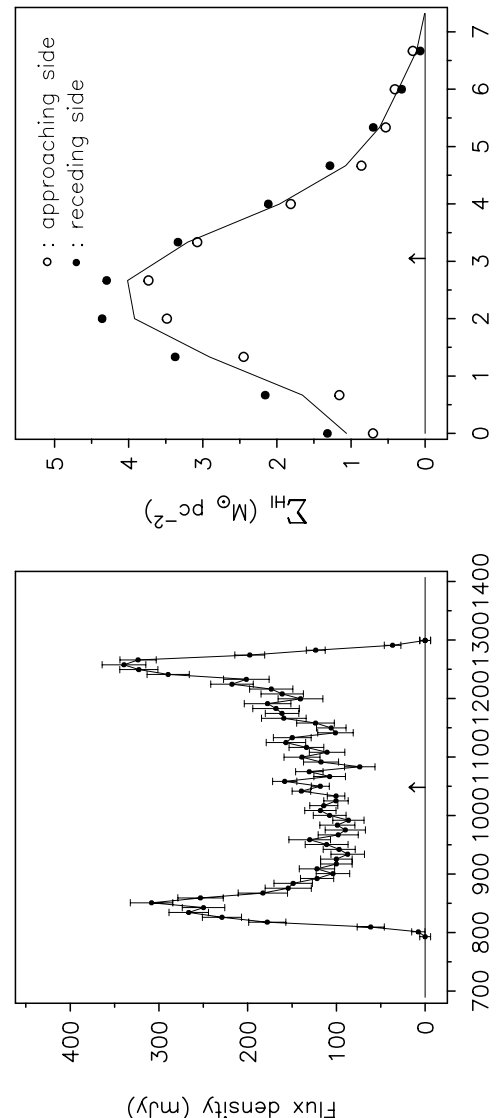

(КৎM) Kұ!!suәp xn!」

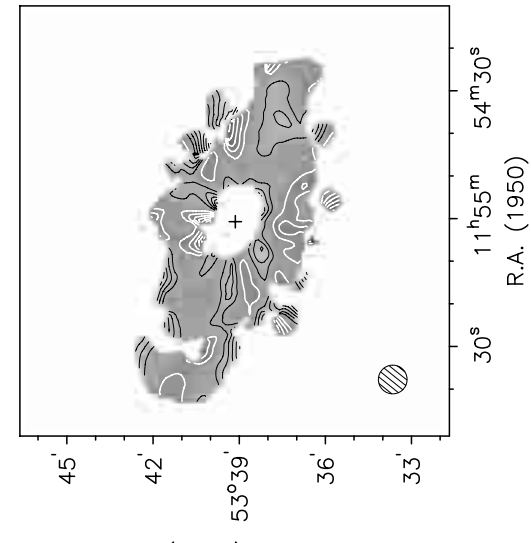

(0s6l) $\cdot 200$

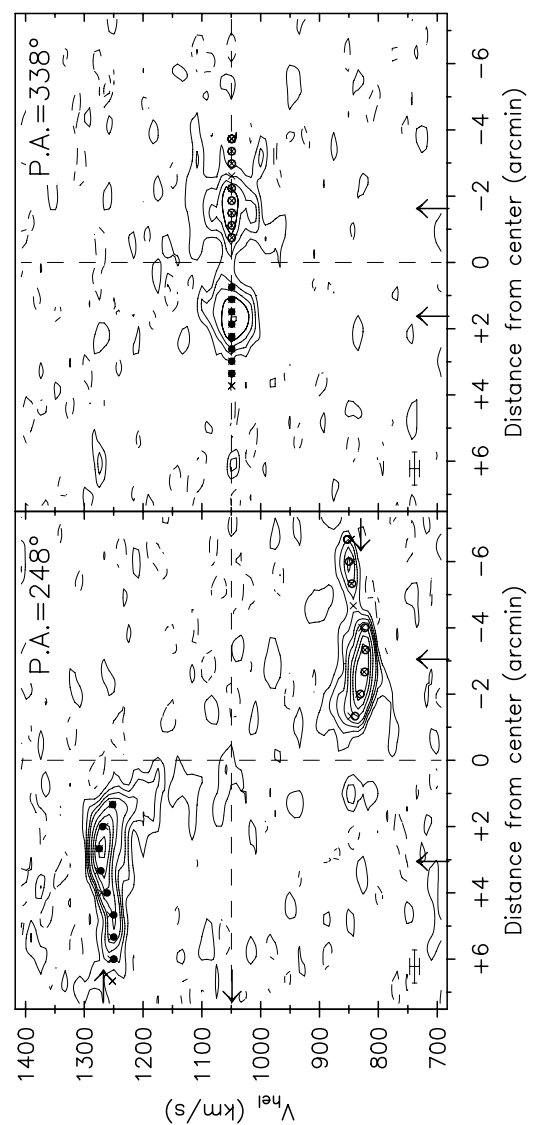


Observing parameters for UGC 6940

\begin{tabular}{|c|c|}
\hline Length of observation & $1 \times 12$ \\
\hline Date of observation & 23Dec94 \\
\hline Field center, $\alpha(1950)$ & 11:55:07 \\
\hline$\delta(1950)$ & 53:31:54 \\
\hline Central frequency & 1415.53 \\
\hline$V_{\text {hel }}$ of central channel & 1054 \\
\hline Primary beam FWHM & 37.4 \\
\hline Nr. of interferometers & 40 \\
\hline Baselines (min-max-incr) & $36-2700-72$ \\
\hline Synthesized beam $(\alpha \times \delta)(\operatorname{arcsec})$ & $12.1 \times 15.1$ \\
\hline Bandwidth & 5.0 \\
\hline Number of channels & 127 \\
\hline Channel separation & 8.31 \\
\hline Velocity resolution & 9.97 \\
\hline rms noise in one channel & 9.71 \\
\hline $\begin{array}{l}\text { K-mJy conversion, } \\
\text { equiv. of } 1 \mathrm{mJy} / \text { beam }\end{array}$ & 3.29 \\
\hline
\end{tabular}

Note: To achieve a sufficient velocity resolution over

a $5 \mathrm{MHz}$ bandwidth, only the XX dipoles could be

correlated due to limitations of the backend.

\section{Results from WSRT data}

\begin{tabular}{|c|c|}
\hline \\
\hline $\begin{array}{l}\text { 21-cm flux density } \\
\text { central point source (mJy) } \\
\text { extended source (mJy) }\end{array}$ & $\begin{array}{l}<1.3(3 \sigma) \\
<1.3(3 \sigma)\end{array}$ \\
\hline \multicolumn{2}{|l|}{ From global profile: } \\
\hline$\overline{\text { Integrated HI-flux }}\left(\mathrm{Jy} \mathrm{km} \mathrm{s}^{-1}\right)$ & $2.1 \pm 0.3$ \\
\hline Hel. systemic velocity $\left(\mathrm{km} \mathrm{s}^{-1}\right)$ & $1118.0 \pm 1.7$ \\
\hline HI profile width, $20 \%\left(\mathrm{~km} \mathrm{~s}^{-1}\right)$ & $59.3 \pm 3.8$ \\
\hline $50 \%\left(\mathrm{~km} \mathrm{~s}^{-1}\right)$ & $40.6 \pm 7.8$ \\
\hline \multicolumn{2}{|l|}{ From velocity field: } \\
\hline $\begin{array}{l}\text { Hel. systemic velocity }\left(\mathrm{km} \mathrm{s}^{-1}\right) \\
\text { From total HI map: }\end{array}$ & $1115.8 \pm 2.5$ \\
\hline Geometric center, $\alpha(1950)$ & $11: 55: 12.3$ \\
\hline$\delta(1950)$ & $53: 30: 56$ \\
\hline Position angle & 311 \\
\hline Inclination angle & 57 \\
\hline Diameter of HI disk (arcmin) & 1.2 \\
\hline
\end{tabular}

Contour levels for U6940

Channel maps: $\quad \sigma=5.93(\mathrm{~K})$

Raw continuum map:

Cleaned continuum map: $\sigma=1.45(\mathrm{~K})$ $\sigma=1.42(\mathrm{~K})$

Position-Velocity diagrams:

Velocity fields:

$$
\sigma=5.10(\mathrm{~K})
$$

$1115.8 \pm \mathrm{n} \times 10\left(\mathrm{~km} \mathrm{~s}^{-1}\right)$ Residual velocity field: $\pm \mathrm{n} \times 5\left(\mathrm{~km} \mathrm{~s}^{-1}\right)$

Integrated HI map: $0.82,1.64$ $2.46\left(\times 10^{21}\right.$ atoms cm $\left.^{-2}\right)$

Note: This dwarf system is among the faintest in the cluster. It spans just a few synthesized beams across and the HI rotation curve only shows the solid body part. In this case, the orientation of the disk is adopted from the optical image.

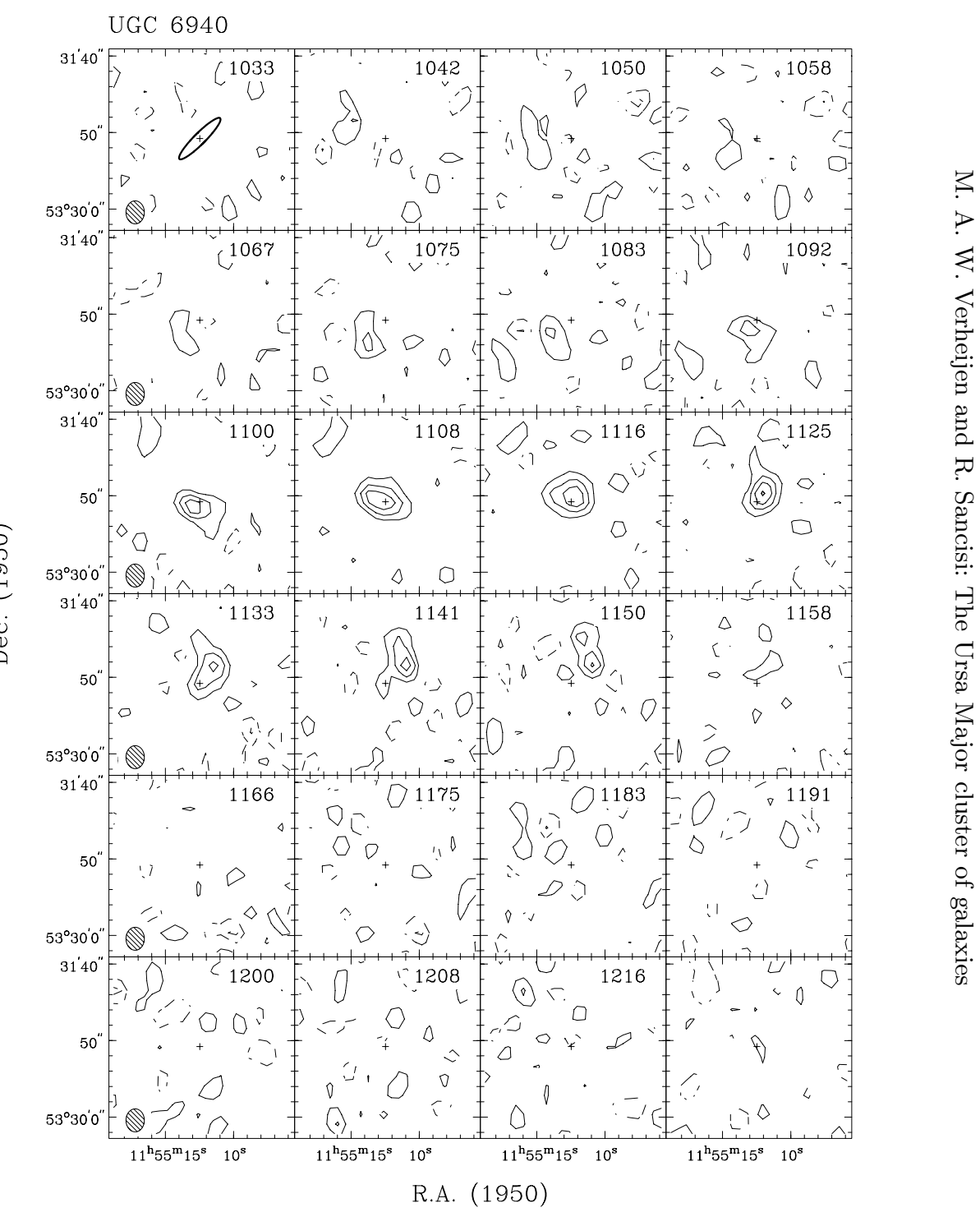

Channel maps at a resolution of $12^{\prime \prime} \times 15^{\prime \prime} \times 17 \mathrm{~km} \mathrm{~s}^{-1}$. Contour levels at $-3,-1.5$ (dashed), $1.5,3,4.5, \ldots \times \sigma$. 

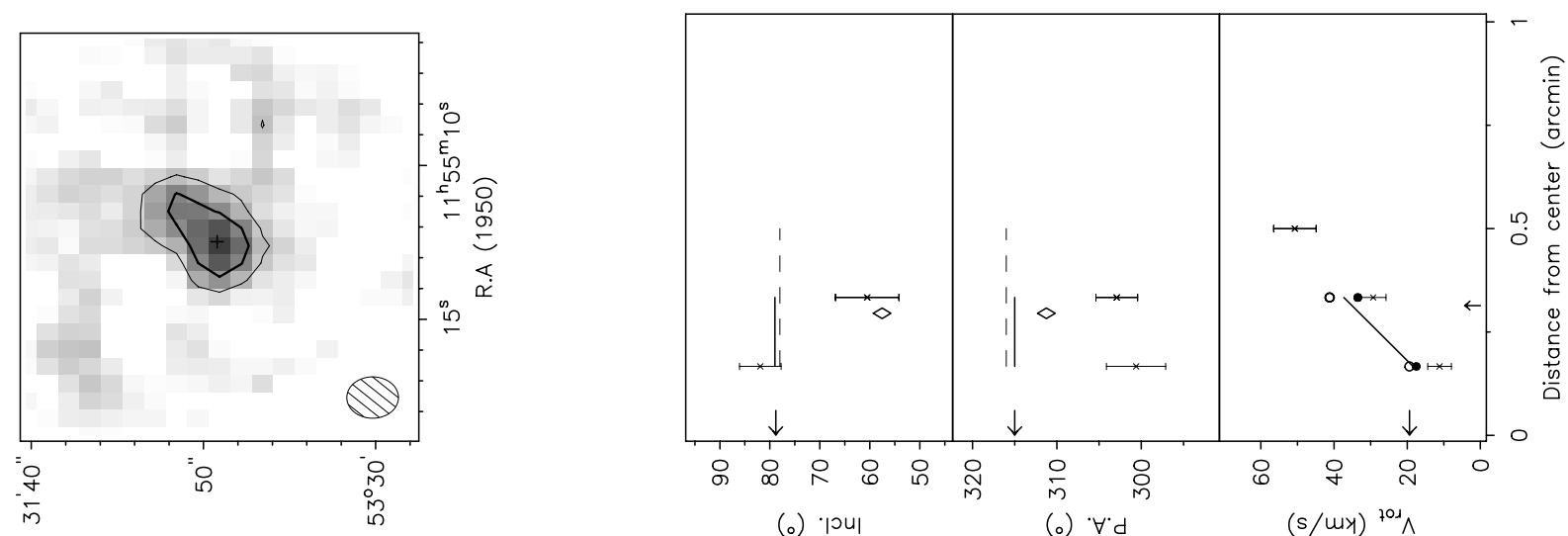
(०) '|⿰u|
(o) $\forall \cdot d$
$(s / m y){ }^{201} \wedge$

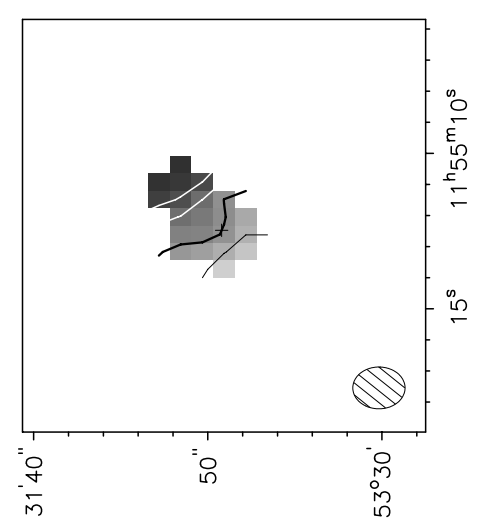

$(0 \mathrm{c} 6 \mathrm{l}) \cdot \mathrm{2a0}$
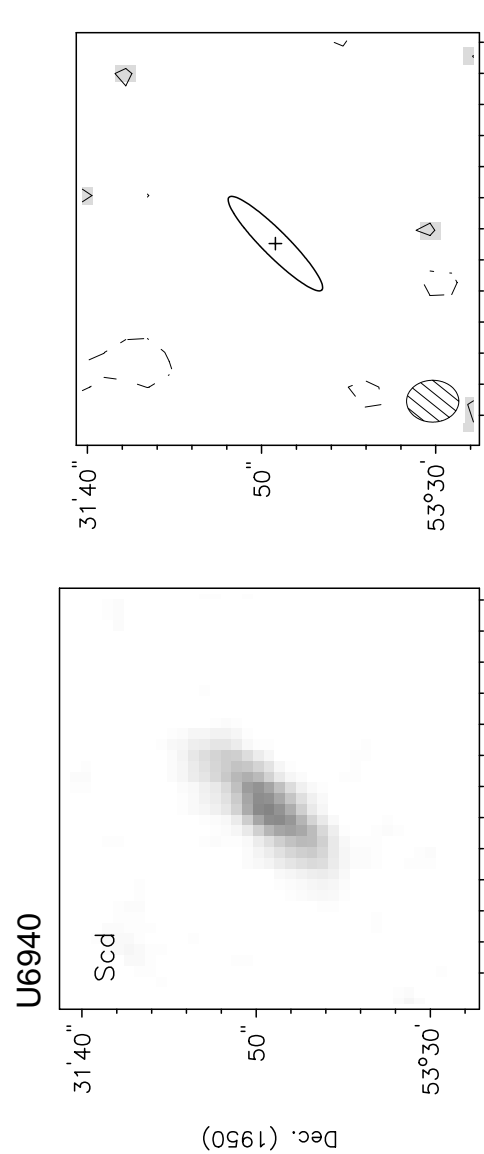

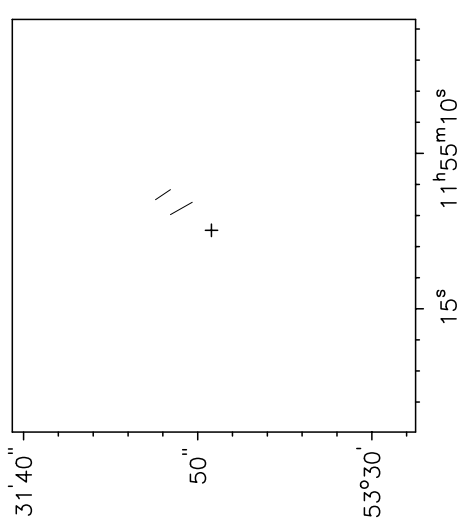

(0s6l)

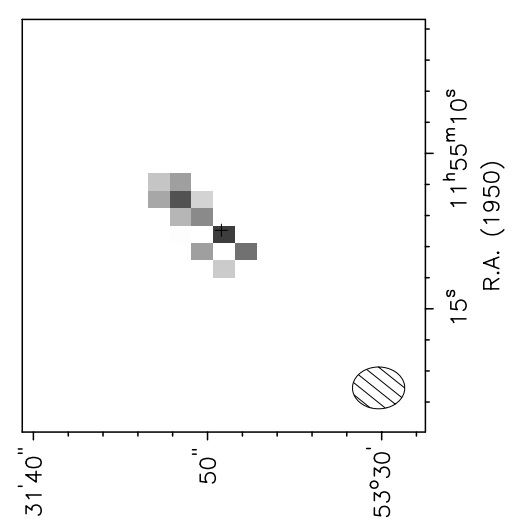

$(0 \mathrm{~g} 6 \mathrm{l}) \cdot \mathrm{0a}$
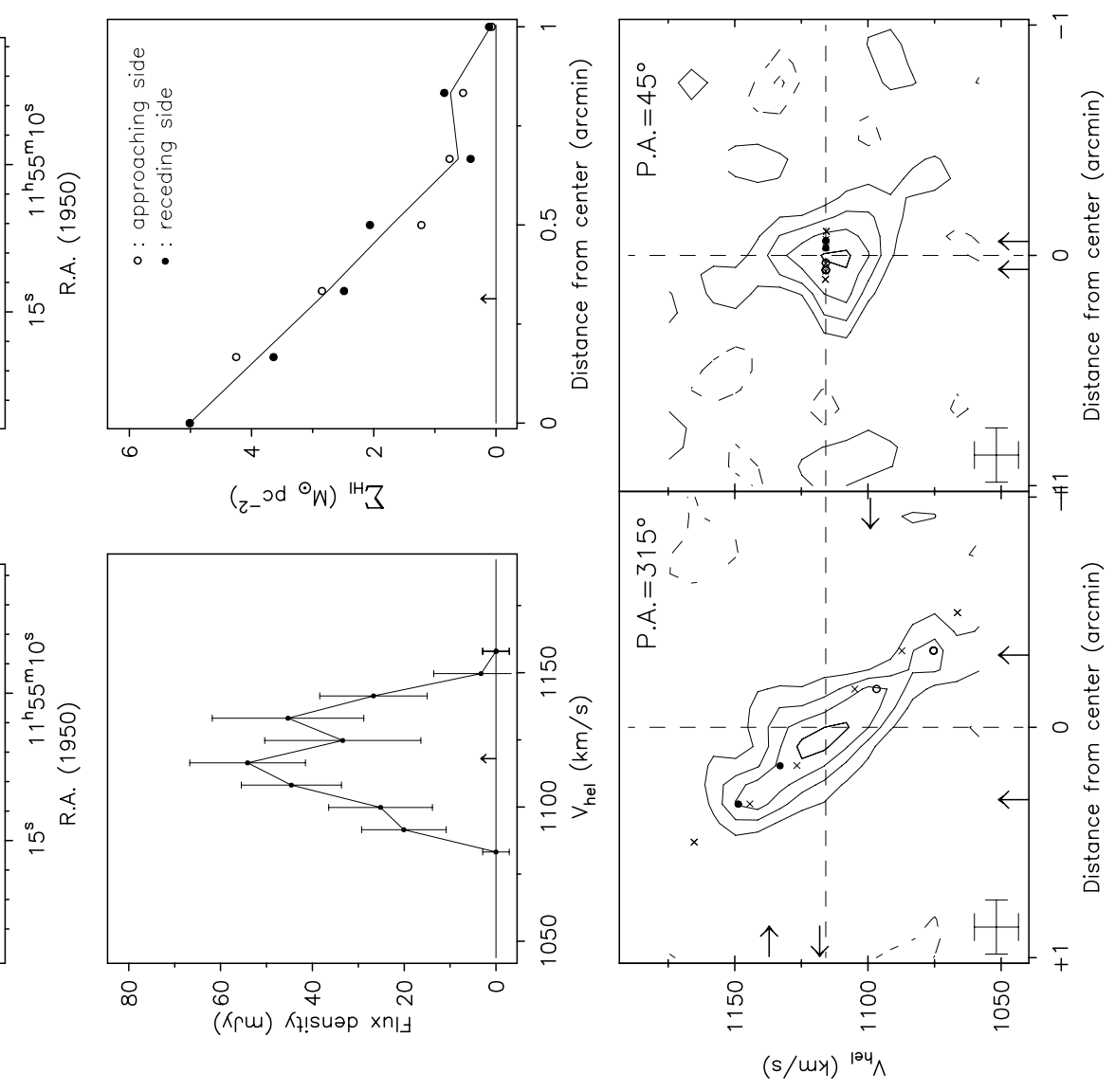
Observing parameters for UGC 6962

Length of observation (hours)

Dates of observation

Field center, $\alpha(1950)$

$$
\delta(1950)
$$

Central frequency

$V_{\text {hel }}$ of central channel

Primary beam FWHM

Baselines (min-max-incr)

Synthesized beam $(\alpha \times \delta)($ arcsec)

Bandwidth

Number of channels

Channel separation

Velocity resolution

rms noise in one channe

$\mathrm{K}$-mJy conversion,

equiv. of $1 \mathrm{mJy} / \mathrm{beam}$

\section{$(\mathrm{MHz})$}

$\left(\mathrm{km} \mathrm{s}^{-1}\right)$

$\left(\mathrm{km} \mathrm{s}^{-1}\right)$

(K)

2.70

\section{Results from WSRT data}

\begin{tabular}{|c|c|}
\hline \multicolumn{2}{|l|}{ From continuит map: } \\
\hline From global profile: & $13.4 \pm 1.7$ \\
\hline$\overline{\text { Integrated HI-flux }}\left(\mathrm{Jy} \mathrm{km} \mathrm{s}^{-1}\right)$ & $10.0 \pm 0 . ?$ \\
\hline velocity $\left(\mathrm{km} \mathrm{s}^{-1}\right)$ & 3.2 \\
\hline HI profile width, $20 \%\left(\mathrm{~km} \mathrm{~s}^{-1}\right)$ & 6.6 \\
\hline $50 \%\left(\mathrm{~km} \mathrm{~s}^{-1}\right)$ & \\
\hline \multicolumn{2}{|l|}{ From velocity field: } \\
\hline$\overline{\text { Hel. sy }}$ & \\
\hline Dynamic & \\
\hline \multicolumn{2}{|l|}{ From total HI map: } \\
\hline$\overline{\text { center, } \alpha} \alpha(1)$ & \\
\hline$\delta(10$ & $: 40$ \\
\hline Posi & 329 \\
\hline & 38 \\
\hline Diameter of HI disk (arcmin) & 2.8 \\
\hline
\end{tabular}
Diameter of HI disk (arcmin)
Contour levels for U6962

Channel maps: $\quad \sigma=2.03(\mathrm{~K})$

Raw continuum map:

Cleaned continuum map: $\sigma=1.11(\mathrm{~K})$

Position-Velocity diagrams:

Velocity fields:

$\sigma=1.69(\mathrm{~K})$

$810.1 \pm \mathrm{n} \times 20\left(\mathrm{~km} \mathrm{~s}^{-1}\right)$ Residual velocity field: $\pm \mathrm{n} \times 5\left(\mathrm{~km} \mathrm{~s}^{-1}\right)$

Integrated $\mathrm{HI}$ map: $0.44,0.88$,

$1.33\left(\times 10^{21}\right.$ atoms cm $\left.^{-2}\right)$

Note: This galaxy is slightly interacting with U6973, only 3.5 arcmin to the ESE. The kinematic major axis is at an angle of roughly 35 degrees with respect to the major axis of the optical image (see chapter 1) and the HI map. This could be a sign of ongoing tidal interaction with U6973 which may result in a severe warp. The derived 'rosult in a severe warp. The derived 'ro-
tation curve' for U6962 is most likely affected by strong non-circular motion and should therefore not be taken at face value.

The detected continuum emission comes from a low level extended region.

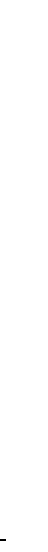

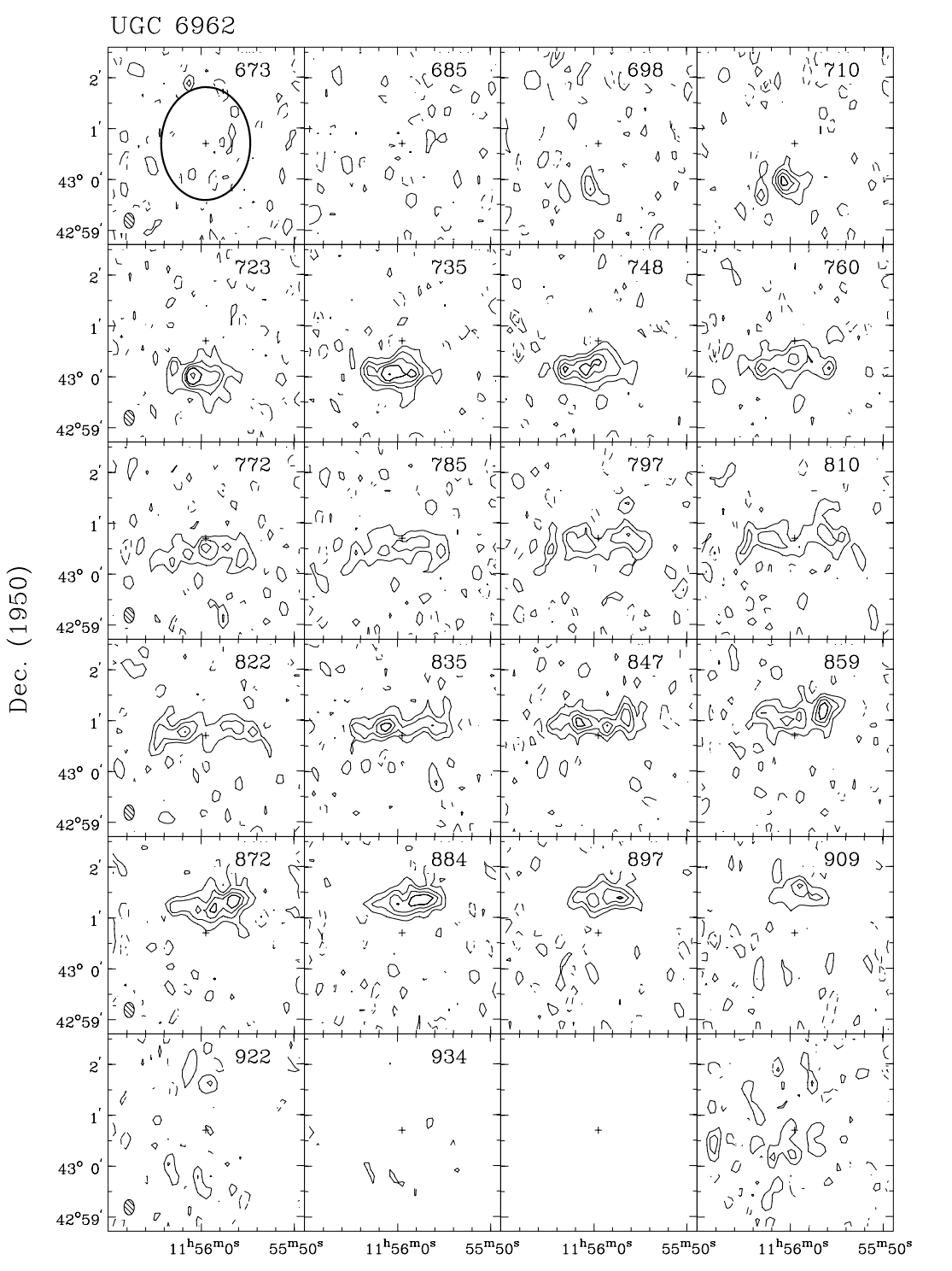

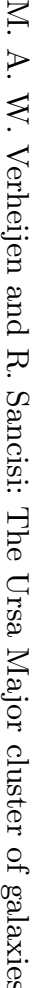




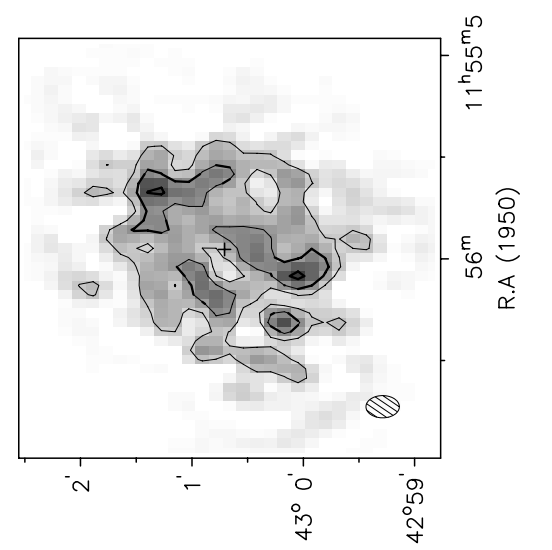

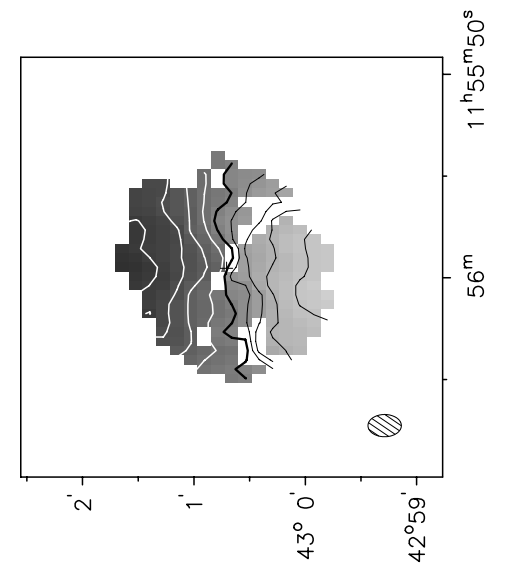

(0s6l) $\cdot 2 ә 0$
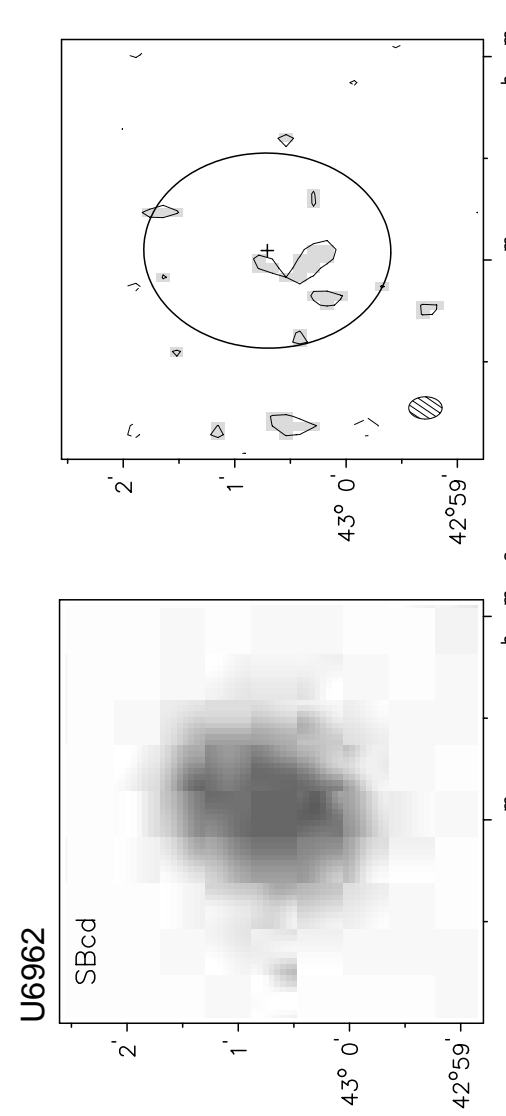

$(0 \mathrm{~s} 6 \mathrm{l}) \cdot 200$

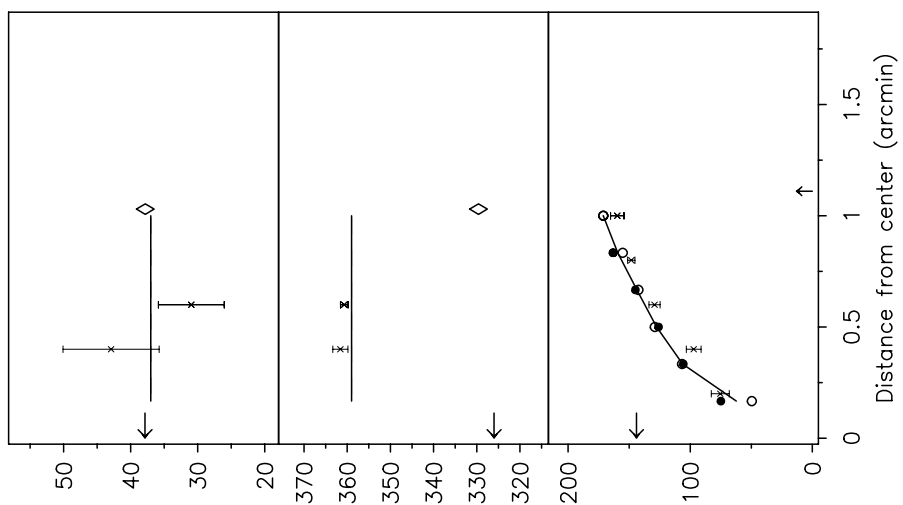

(०) '|गU|

(o) $\forall \cdot d$

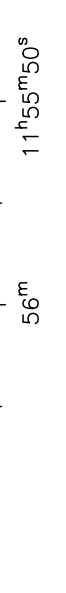

(0s6l) $\cdot 2 \circ 0$

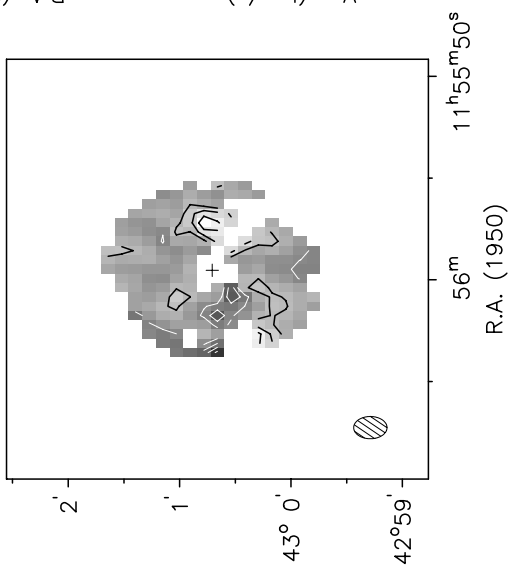

(0s6l) $\cdot{ }^{\circ} 0$

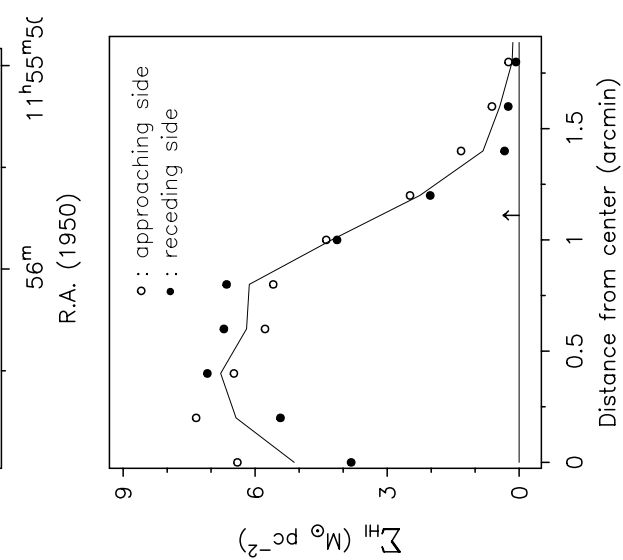

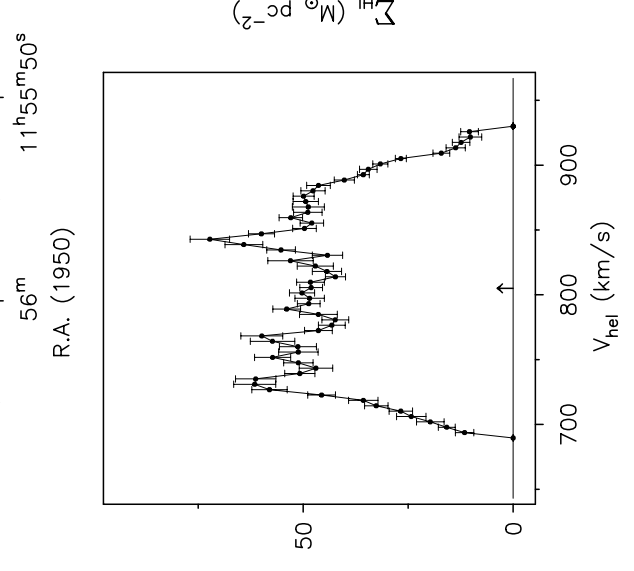

(кৎس) Kł!suәp xn!J

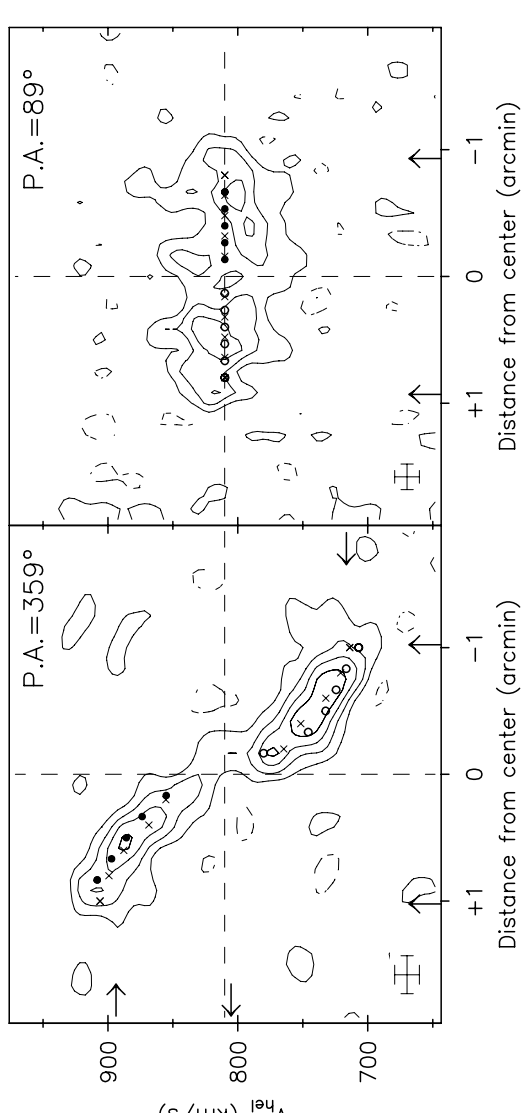

$(s / m y)^{194} \wedge$ 
Observing parameters for NGC 4010

\begin{tabular}{|c|c|}
\hline Length of observation & $1 \times 12$ \\
\hline Date of observation & 21Aug93 \\
\hline Field center, $\alpha(1950)$ & 11:56:03 \\
\hline$\delta(1950)$ & 47:32:20 \\
\hline Central frequency & 1416.11 \\
\hline$V_{\text {hel }}$ of central channel & 907 \\
\hline Primary beam FWHM (arcmin) & 37.4 \\
\hline Nr. of interferometers & 40 \\
\hline Baselines (min-max-incr) & $36-2700-72$ \\
\hline Synthesized beam $(\alpha \times \delta)(\operatorname{arcsec})$ & $12.5 \times 17.0$ \\
\hline Bandwidth & 2.5 \\
\hline Number of channels & 127 \\
\hline Channel separation & 4.15 \\
\hline Velocity resolution & 8.29 \\
\hline rms noise in one channel & 5.52 \\
\hline $\begin{array}{l}\text { K-mJy conversion, } \\
\text { equiv. of } 1 \mathrm{mJy} / \text { beam }\end{array}$ & 2.83 \\
\hline
\end{tabular}

Results from WSRT data

\begin{tabular}{|c|c|}
\hline \multicolumn{2}{|l|}{ From continuum map: } \\
\hline 21-cm flux density & 16.9. $x \pm 1.6$ \\
\hline Point source position, $\alpha(1950)$ & $11: 56: 01.6$ \\
\hline$\delta(1950)$ & $47: 32: 14$ \\
\hline \multicolumn{2}{|l|}{ From global profile: } \\
\hline Integrated HI-flux $\left(\mathrm{Jy} \mathrm{km} \mathrm{s}^{-1}\right)$ & $38.2 \pm 0.3$ \\
\hline Hel. systemic velocity $\left(\mathrm{km} \mathrm{s}^{-1}\right)$ & $901.9 \pm 0.8$ \\
\hline HI profile width, $20 \%\left(\mathrm{~km} \mathrm{~s}^{-1}\right)$ & $277.7 \pm 1.0$ \\
\hline $50 \%\left(\mathrm{~km} \mathrm{~s}^{-1}\right)$ & $264.1 \pm 1.2$ \\
\hline \multicolumn{2}{|l|}{ From velocity field: } \\
\hline 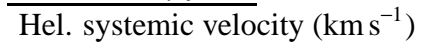 & $899.4 \pm 1.0$ \\
\hline Dynamical center, $\alpha(1950)$ & 11:56:02.1 \\
\hline$\delta(1950)$ & $47: 32: 16$ \\
\hline \multicolumn{2}{|l|}{ From total HI map: } \\
\hline$\overline{\text { Geometric center, } \alpha(1950)}$ & $11: 56: 02.1$ \\
\hline$\delta(1950)$ & $47: 32: 16$ \\
\hline Position angle & 62 \\
\hline Inclination angle & 77 \\
\hline Diameter of HI disk (arcmin) & 6.7 \\
\hline
\end{tabular}

Contour levels for N4010

Channel maps: $\quad \sigma=3.68(\mathrm{~K})$

Raw continuum map: $\sigma=1.52(\mathrm{~K})$ Cleaned continuum map: $\sigma=1.44(\mathrm{~K})$

Position-Velocity diagrams:

Velocity fields:

$899.4 \pm \mathrm{n} \times 20\left(\mathrm{~km} \mathrm{~s}^{-1}\right)$ Residual velocity field: $\pm \mathrm{n} \times 5\left(\mathrm{~km} \mathrm{~s}^{-1}\right)$

Integrated HI map:

$0.62,1.24,1.87,2.49$ $3.11\left(\times 10^{21}\right.$ atoms $\left.\mathrm{cm}^{-2}\right)$

Note: This optically lopsided edgeon system has a complicated velocity structure. A warp is clearly present as can be seen in the channel maps at the velocities 787 and $1019 \mathrm{~km} \mathrm{~s}^{-1}$.

The apparent decline of the (projected) rotation curve in the XV-diagram at the last measured points is due to the strong warp.

The asymmetry of the rotation curve in the inner parts may be due to streaming motions along a bar offset from the center and causing the optically lopsided appearance.

Because of the high inclination, the (face-on) surface density profile derived from the total HI map is quite uncertain. The dynamical center was chosen at the center of the HI map.

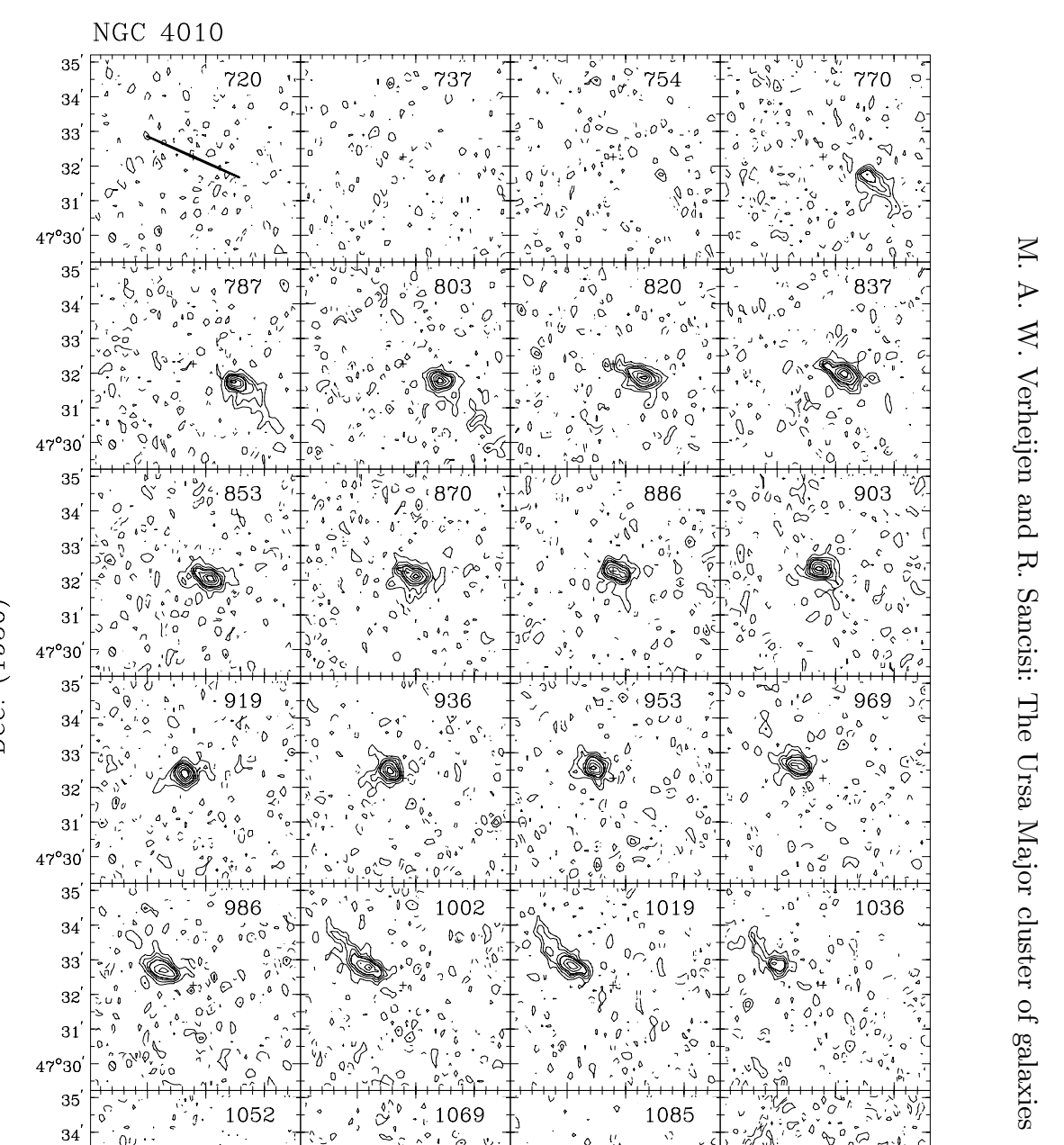

.

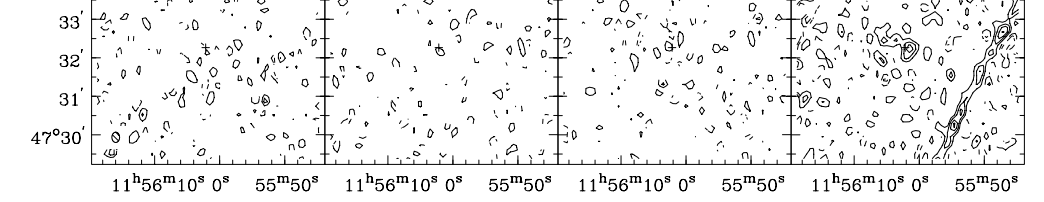

$$
\text { R.A. (1950) }
$$

Channel maps at a resolution of $12^{\prime \prime} \times 17^{\prime \prime} \times 19 \mathrm{~km} \mathrm{~s}^{-1}$.

Contour levels at $-3,-1.5$ (dashed), $1.5,3,4.5, \ldots \times \sigma$. 

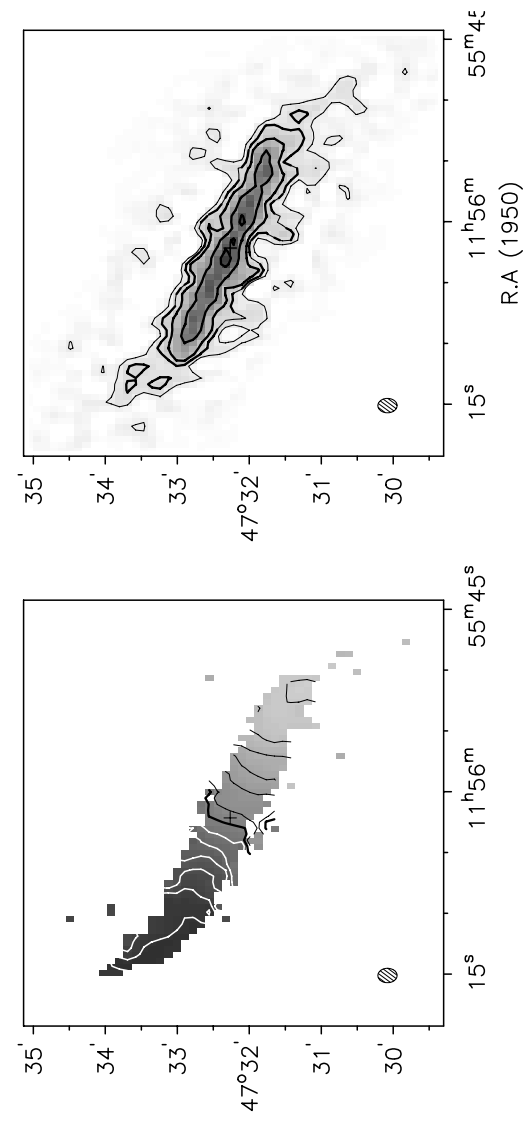

$(0 \mathrm{og} 6 \mathrm{l}) \cdot 020$

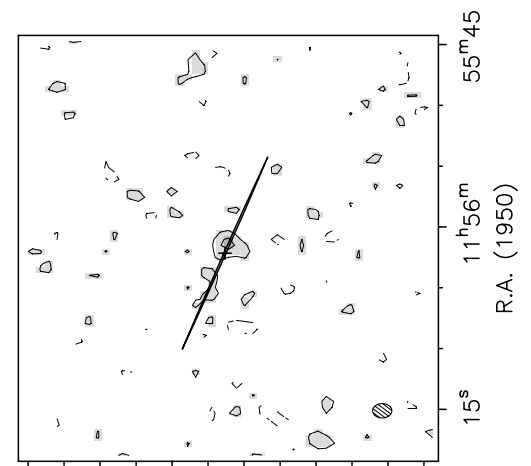

品

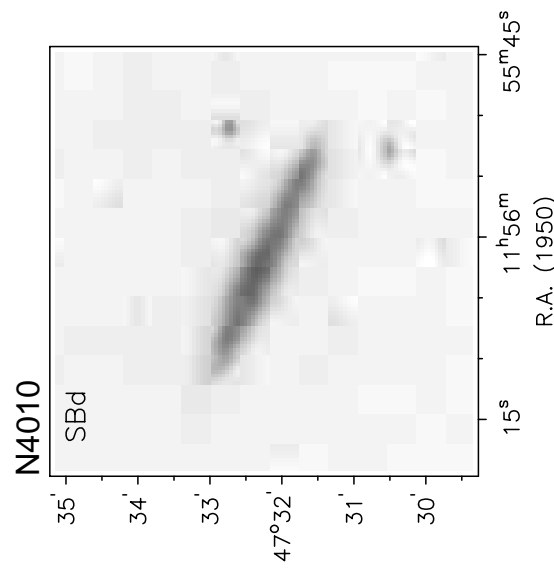

(0s6l) $\cdot 020$

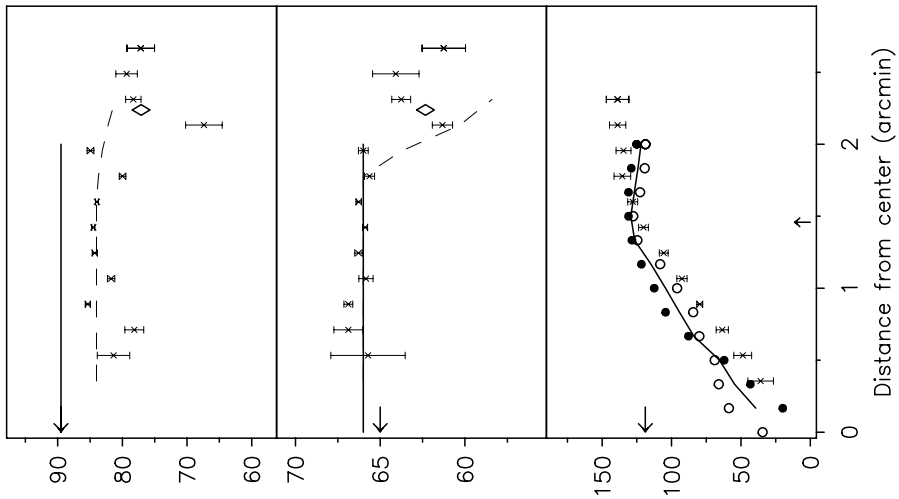

(०) '|⿰u|

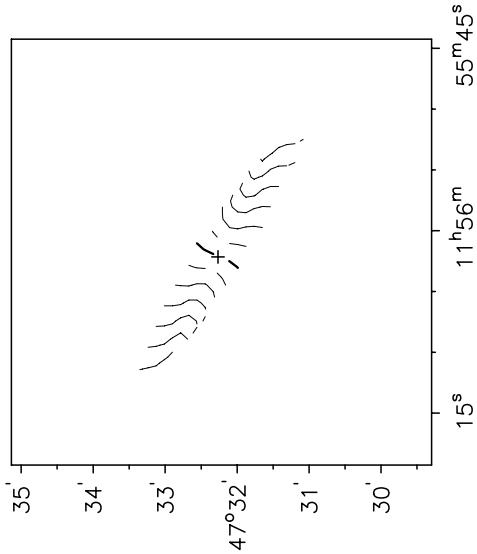

(096l) $\cdot 200$

(o) $\forall \cdot d$

$(s / m y){ }^{701} \wedge$

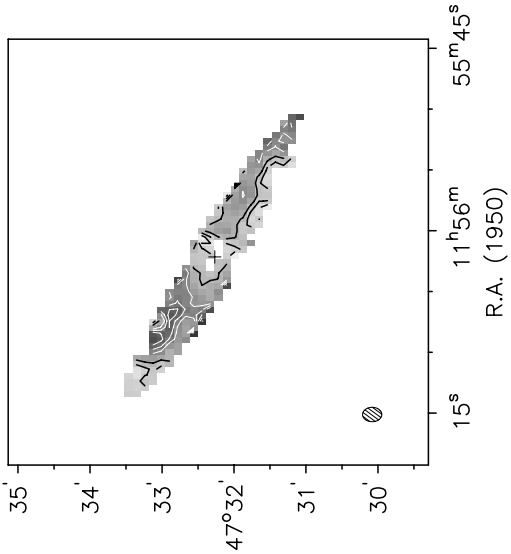

(0s6l) $\cdot 200$
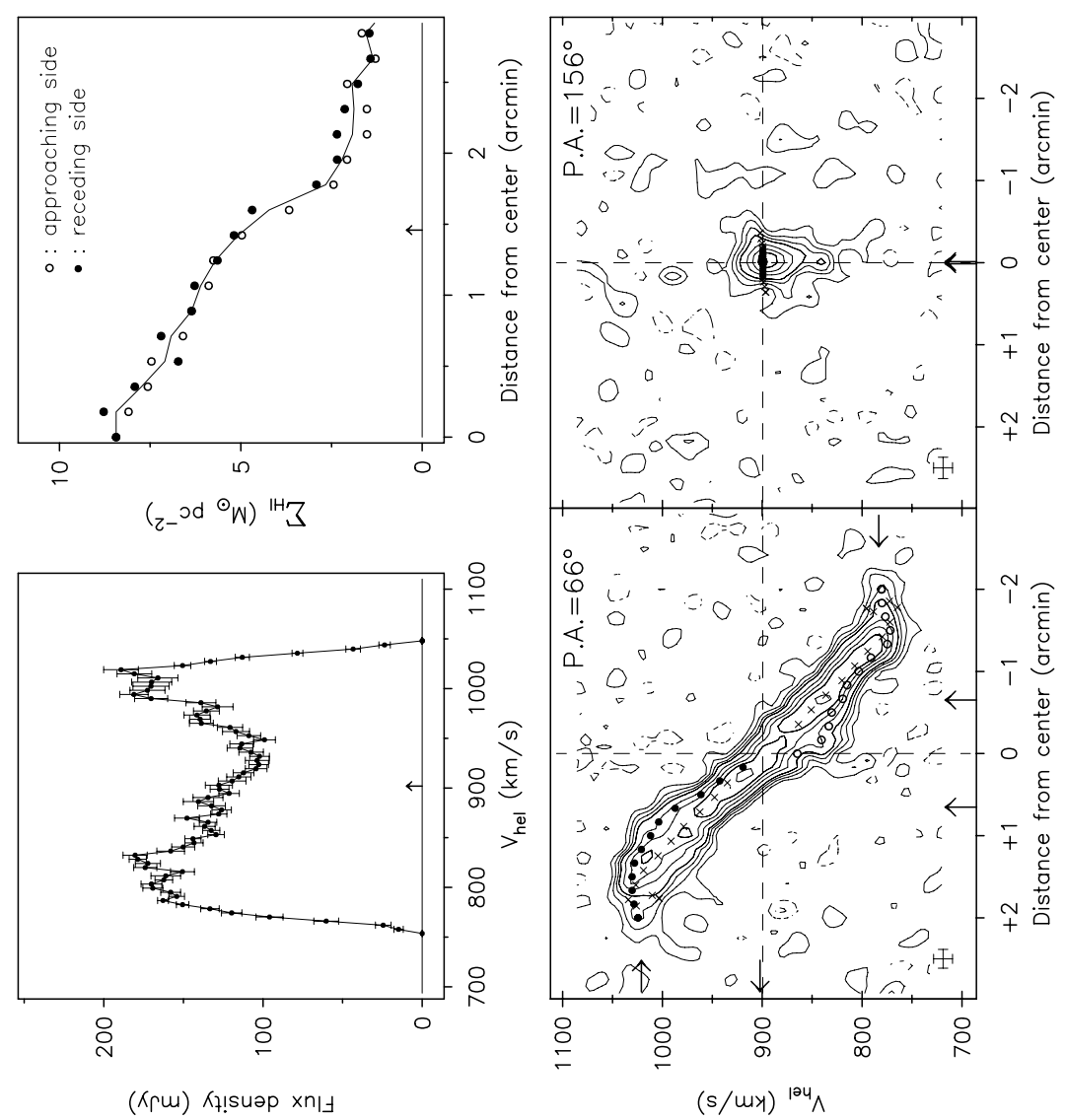


\begin{tabular}{|c|c|}
\hline Length of observation & $1 \times 12$ \\
\hline Date of observation & 23Dec94 \\
\hline Field center, $\alpha(1950)$ & 11:55:07 \\
\hline$\delta(1950)$ & $53: 31: 54$ \\
\hline Central frequency & 1415.53 \\
\hline$V_{\text {hel }}$ of central channel & 1054 \\
\hline Primary beam FWHM (arcmin) & 37.4 \\
\hline Nr. of interferometers & 40 \\
\hline Baselines (min-max-incr) & $36-2700-72$ \\
\hline Synthesized beam $(\alpha \times \delta)(\operatorname{arcsec})$ & $12.1 \times 15.1$ \\
\hline Bandwidth & 5.0 \\
\hline Number of channels & 127 \\
\hline Channel separation & 8.31 \\
\hline Velocity resolution & 9.97 \\
\hline rms noise in one channel $\quad(\mathrm{K})$ & 9.71 \\
\hline $\begin{array}{l}\text { K-mJy conversion, } \\
\text { equiv. of } 1 \mathrm{mJy} / \text { beam }\end{array}$ & 3.29 \\
\hline
\end{tabular}

Note: To achieve a sufficient velocity resolution over

a $5 \mathrm{MHz}$ bandwidth, only the XX dipoles could be

correlated due to limitations of the backend.

\section{Results from WSRT data}

\begin{tabular}{|c|c|}
\hline \multicolumn{2}{|l|}{ From continuum map: } \\
\hline \multicolumn{2}{|l|}{ 21-cm flux density } \\
\hline central point source $(\mathrm{mJy})$ & $<1.3(3 \sigma)$ \\
\hline extended source (mJy) & $<3.8(3 \sigma)$ \\
\hline \multicolumn{2}{|l|}{ From global profile: } \\
\hline$\overline{\text { Integrated HI-flux }}\left(\mathrm{Jy} \mathrm{km} \mathrm{s}^{-1}\right)$ & $6.1 \pm 0.5$ \\
\hline Hel. systemic velocity $\left(\mathrm{km} \mathrm{s}^{-1}\right)$ & $1118.5 \pm 2.4$ \\
\hline HI profile width, $20 \%\left(\mathrm{~km} \mathrm{~s}^{-1}\right)$ & $132.1 \pm 6.4$ \\
\hline $50 \%\left(\mathrm{~km} \mathrm{~s}^{-1}\right)$ & $123.5 \pm 2.9$ \\
\hline \multicolumn{2}{|l|}{ From velocity field: } \\
\hline 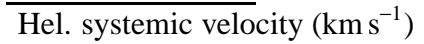 & $1117.6 \pm 1.2$ \\
\hline \multicolumn{2}{|l|}{ From total HI map: } \\
\hline$\overline{\text { Geometric center, }} \alpha(1950)$ & $11: 56: 12.7$ \\
\hline$\delta(1950)$ & $53: 42: 15$ \\
\hline Position angle & 327 \\
\hline Inclination angle & 76 \\
\hline Diameter of HI disk (arcmin) & 1.9 \\
\hline
\end{tabular}

Contour levels for U6969

Channel maps: $\quad \sigma=5.93(\mathrm{~K})$

Raw continuum map: $\sigma=1.45(\mathrm{~K})$

Cleaned continuum map: $\sigma=1.42(\mathrm{~K})$

Position-Velocity diagrams:

Velocity fields:

$1117.6 \pm \mathrm{n} \times 15\left(\mathrm{~km} \mathrm{~s}^{-1}\right)$ Residual velocity field: $\pm \mathrm{n} \times 5\left(\mathrm{~km} \mathrm{~s}^{-1}\right)$

Integrated HI map: $0.98,1.97$, $2.95\left(\times 10^{21}\right.$ atoms cm $\left.{ }^{-2}\right)$

Note: This dwarf galaxy is a companion of NGC 3992. The HI rotation curve shows only solid body rotation. Therefore, the dynamical center and inclination angle were adopted from the optical image.

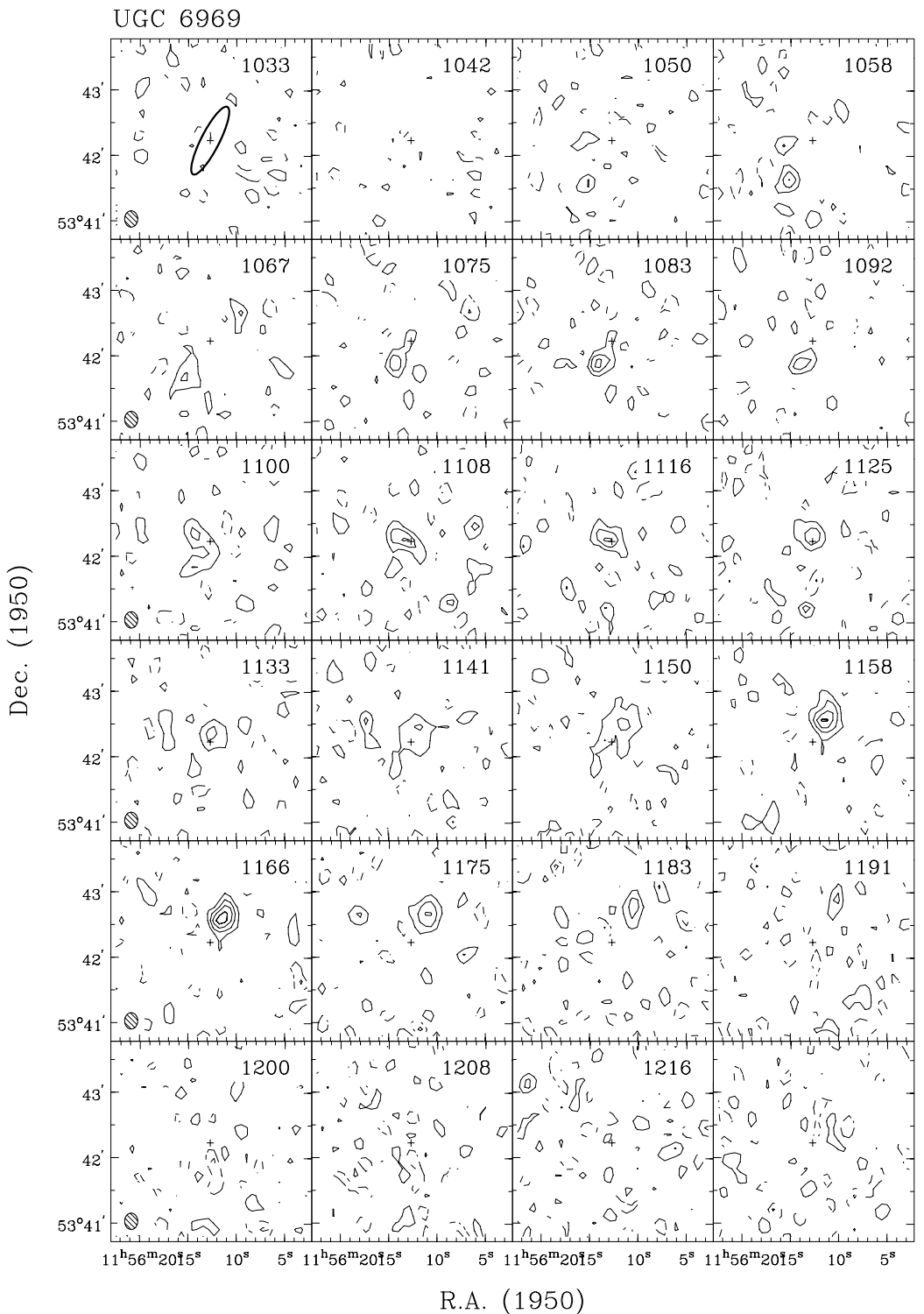

Channel maps at a resolution of $12^{\prime \prime} \times 15^{\prime \prime} \times 17 \mathrm{~km} \mathrm{~s}^{-1}$. Contour levels at $-3,-1.5$ (dashed), $1.5,3,4.5, \ldots \times \sigma$. 

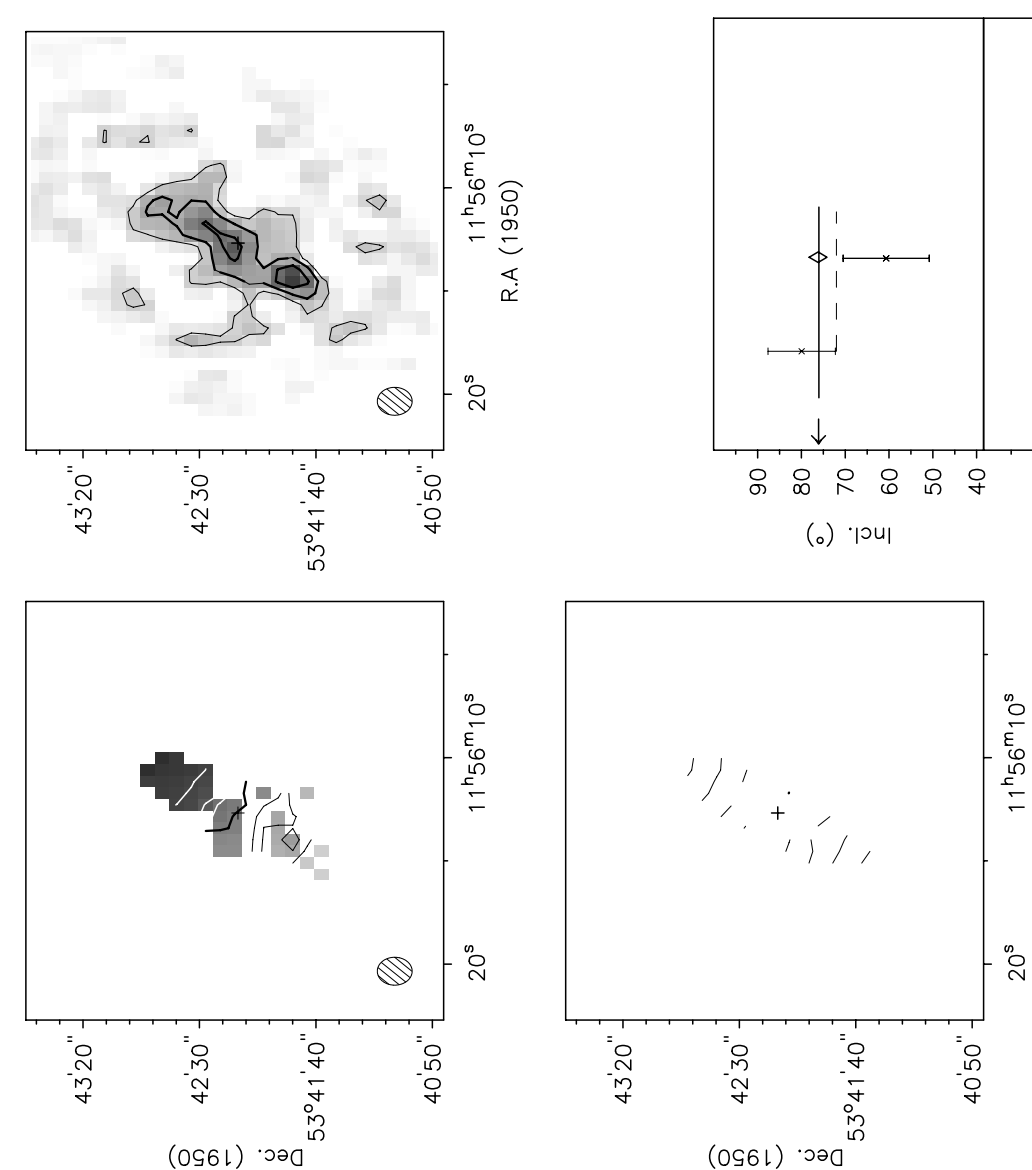

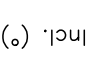

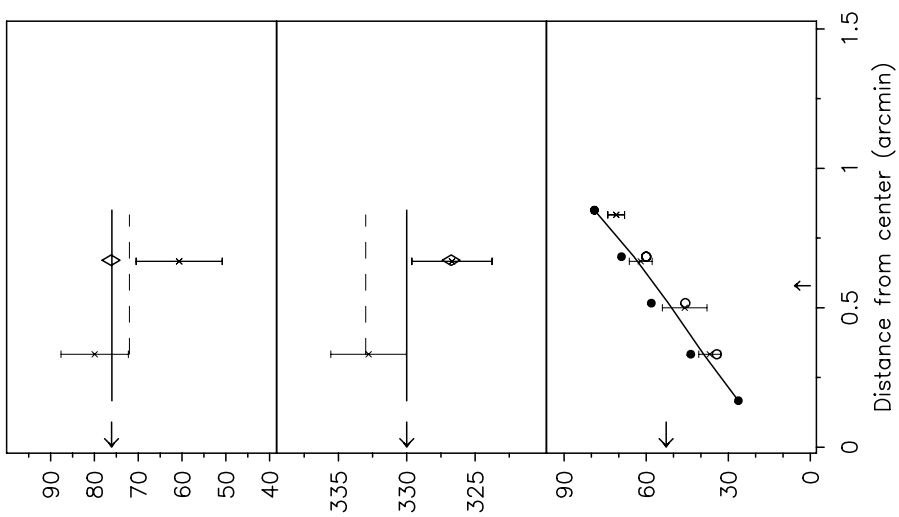

(o) $\forall \cdot d$

$(s / m y){ }^{701} \wedge$
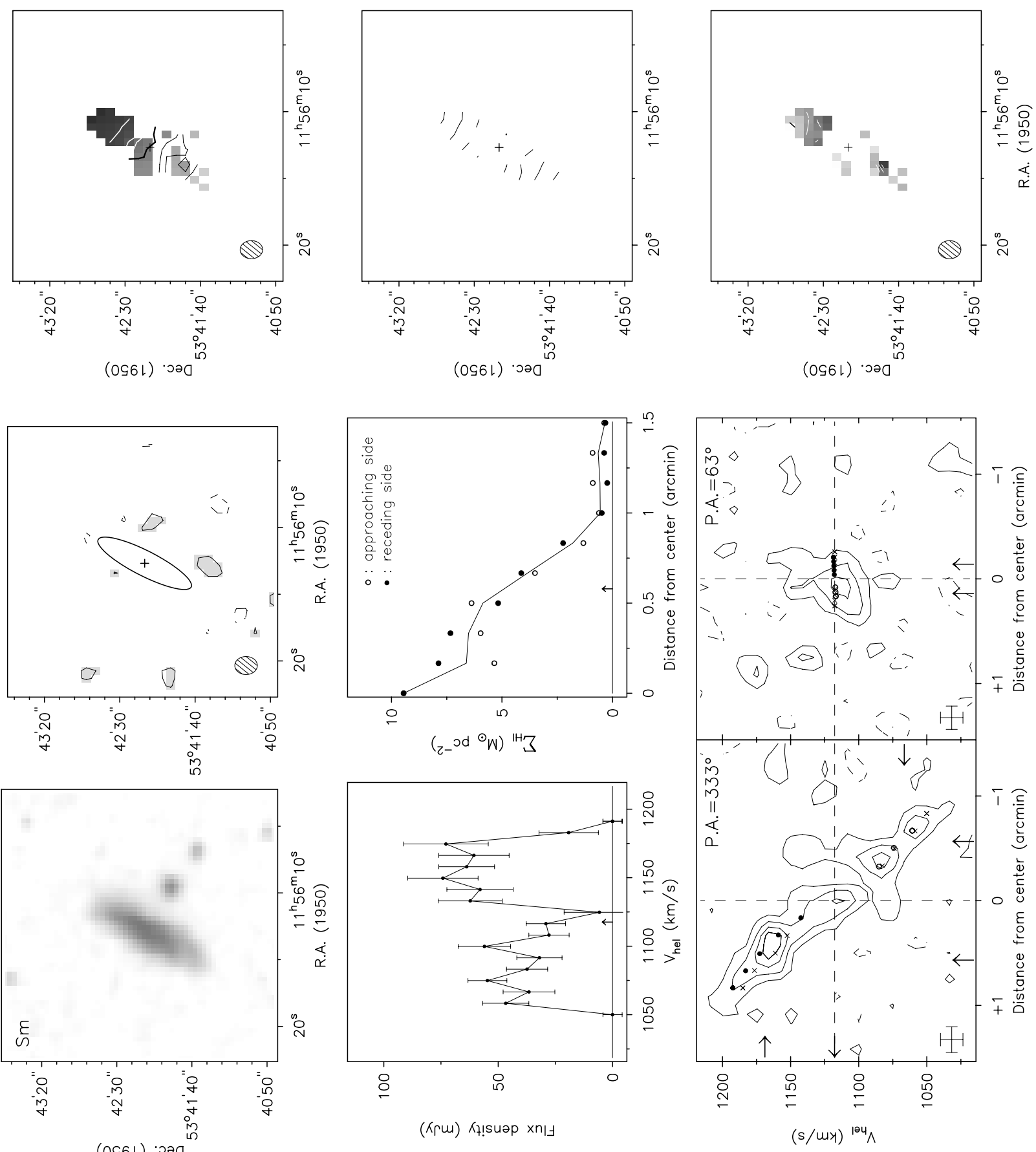

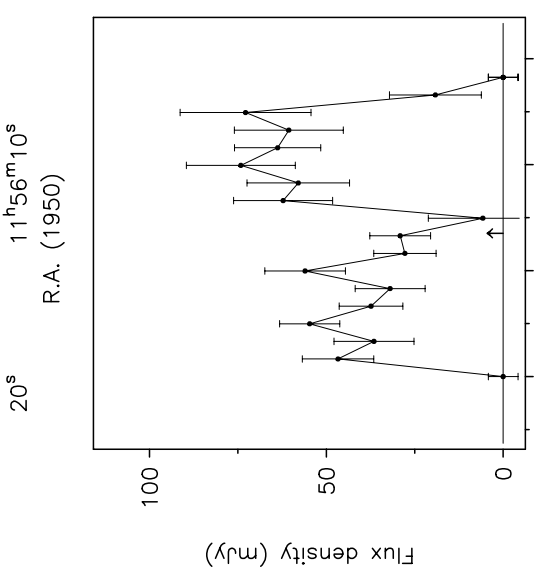

(Krui) Kt!suәp xn!z 
Observing parameters for UGC 6973

Length of observation (hours)

Dates of observation

Field center, $\alpha(1950)$

$$
\delta(1950)
$$

Central frequency

$V_{\text {hel }}$ of central channel

Primary beam FWHM

Synthesized beam $(\alpha \times \delta)($ arcsec)

Bandwidth

Number of channels

Channel separation

Velocity resolution

rms noise in one channe

$\mathrm{K}$-mJy conversion,

equiv. of $1 \mathrm{mJy} / \mathrm{beam}$

(MHz)

$\left(\mathrm{km} \mathrm{s}^{-1}\right)$

$\left(\mathrm{km} \mathrm{s}^{-1}\right)$

(K)

(K)
Baselines (min-max-incr)

Contour levels for U6973

Channel maps:

Raw continuum map:

$$
\sigma=2.03(\mathrm{~K})
$$

Cleaned continuum map

$\sigma$

Position-Velocity diagrams:

Velocity fields:

$701.4 \pm \mathrm{n} \times 25\left(\mathrm{~km} \mathrm{~s}^{-1}\right)$

Residual velocity field: $\pm \mathrm{n} \times 5\left(\mathrm{~km} \mathrm{~s}^{-1}\right)$

Integrated $\mathrm{HI}$ map: $0.83,1.67$,

$2.78\left(\times 10^{21}\right.$ atoms cm $\left.{ }^{-2}\right)$

\begin{tabular}{|c|c|}
\hline \multicolumn{2}{|l|}{ From continuит map: } \\
\hline 21-cm flux density & $127.5 \pm 2.1$ \\
\hline \multicolumn{2}{|l|}{ Central point source position } \\
\hline$\alpha(1950)$ & $11: 56: 17.7$ \\
\hline$\delta(1950)$ & 43:00:02 \\
\hline \multicolumn{2}{|l|}{ From global profile: } \\
\hline$\overline{\text { Integrated HI-flux }}\left(\mathrm{Jy} \mathrm{km} \mathrm{s}^{-1}\right)$ & $22.9 \pm 0.2$ \\
\hline Hel. systemic velocity $\left(\mathrm{km} \mathrm{s}^{-1}\right)$ & $700.5 \pm 1.0$ \\
\hline HI profile width, $20 \%\left(\mathrm{~km} \mathrm{~s}^{-1}\right)$ & $367.8 \pm 1.8$ \\
\hline $50 \%\left(\mathrm{~km} \mathrm{~s}^{-1}\right)$ & $350.4 \pm 1.2$ \\
\hline \multicolumn{2}{|l|}{ From velocity field: } \\
\hline 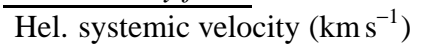 & $701.4 \pm 2.3$ \\
\hline Dynamical center, $\alpha(1950)$ & $11: 56: 17.8$ \\
\hline$\delta(1950)$ & 43:00:03 \\
\hline \multicolumn{2}{|l|}{ From total HI map: } \\
\hline$\overline{\text { Geometric center, } \alpha(1950)}$ & $11: 56: 17.2$ \\
\hline$\delta(1950)$ & 42:59:58 \\
\hline Position & 47 \\
\hline (deg) & 72 \\
\hline Diameter of HI disk & 4.4 \\
\hline
\end{tabular}

Results from WSRT data
Note: This galaxy is slightly interacting with U6962, only 3.5 arcmin to the WNW. The HI disk is somewhat warped and some very faint tidal debris can be seen in the channel maps. Due to severe beam smearing, the observed velocity field and the rotation curve derived from it are of limited use.

The optical image, showing a bright red and dusty inner part, and the strong extended continuum emission suggest vigorous star formation in the central regions.

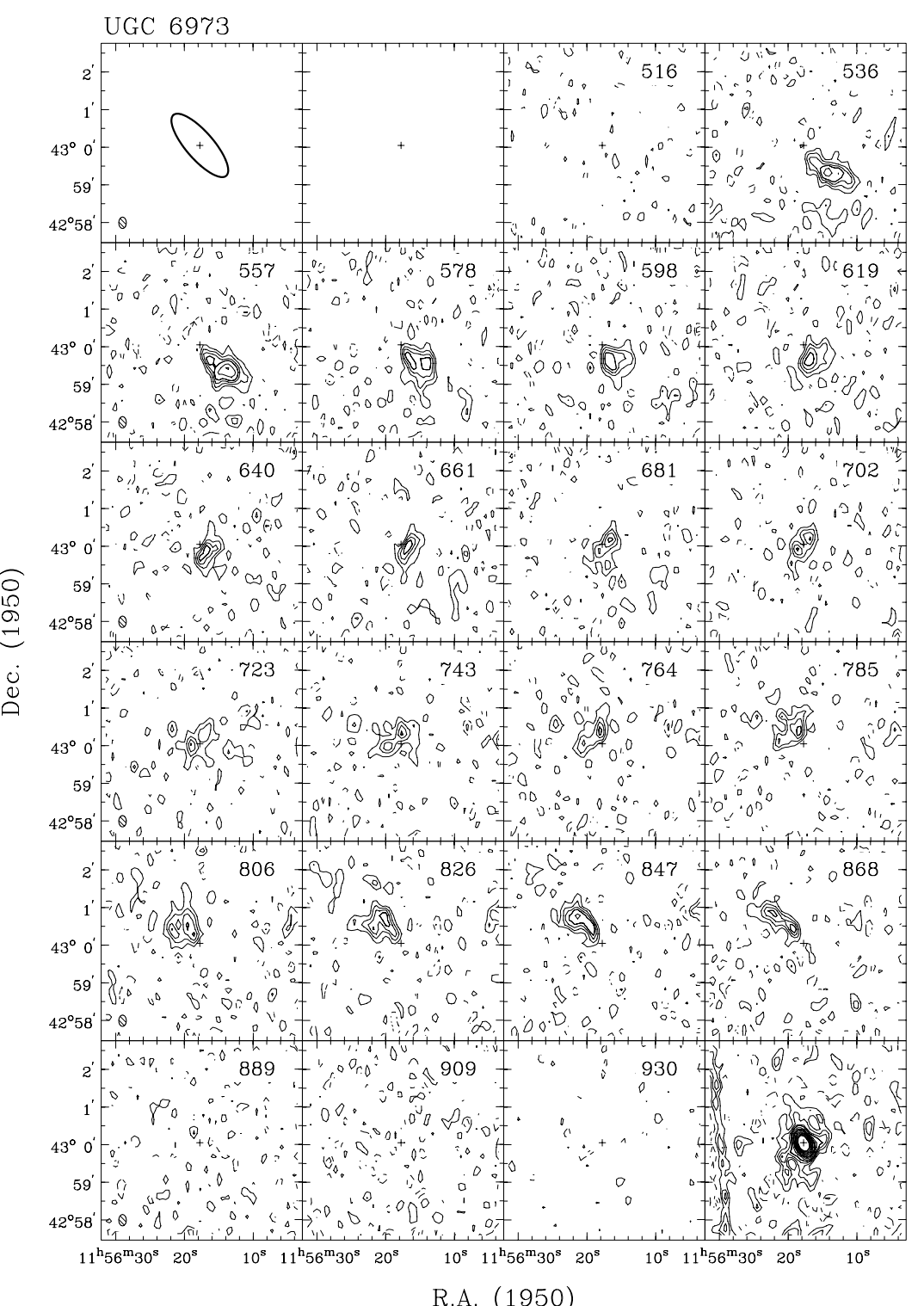

Channel maps at a resolution of $12^{\prime \prime} \times 18^{\prime \prime} \times 19 \mathrm{~km} \mathrm{~s}^{-1}$. Contour levels at $-3,-1.5$ (dashed), $1.5,3,4.5, \ldots \times \sigma$. 

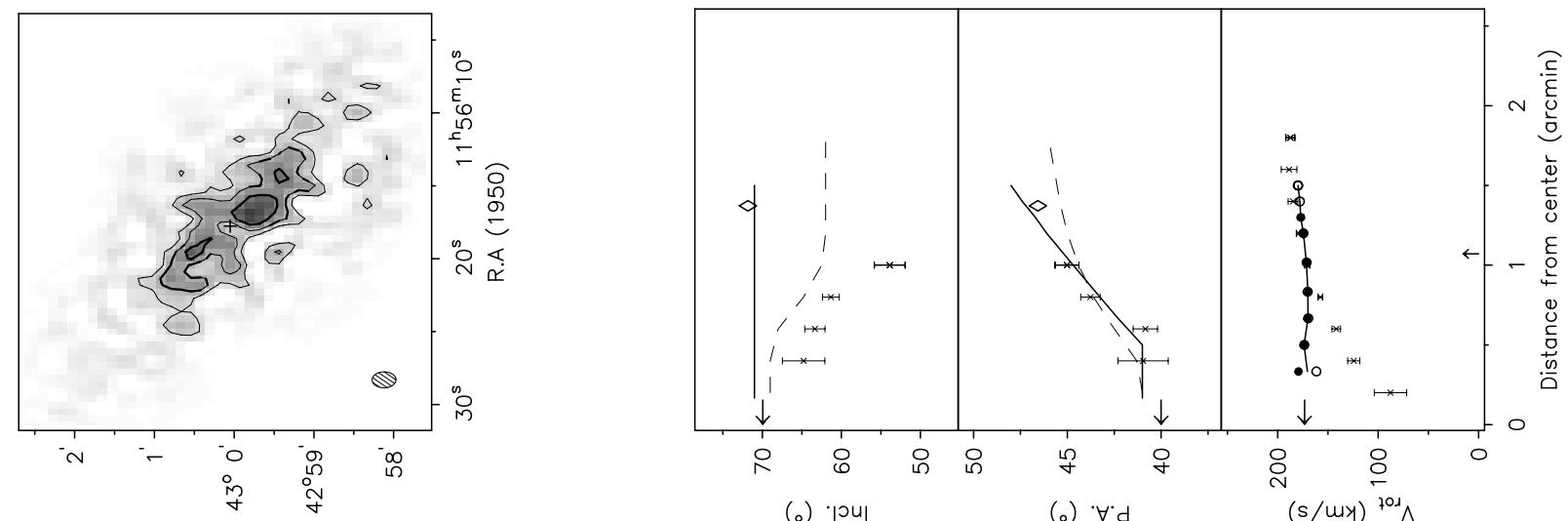

(o) '|0u|

(o) $\forall \cdot d$

$(s / m y){ }^{701} \wedge$

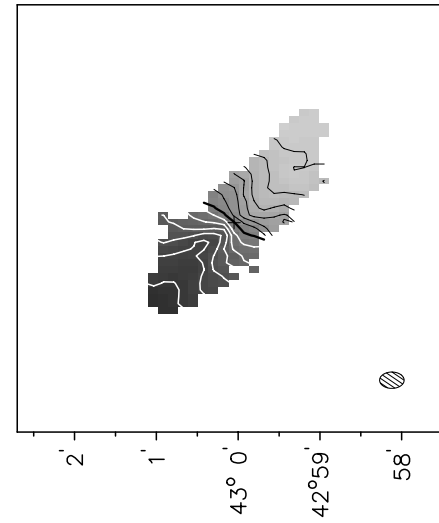

(096l) 'ग

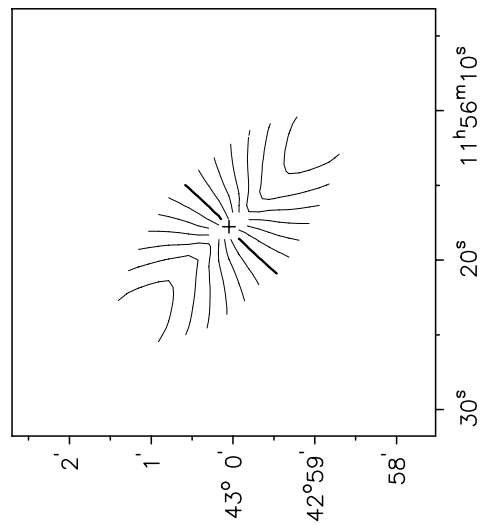

(096l)

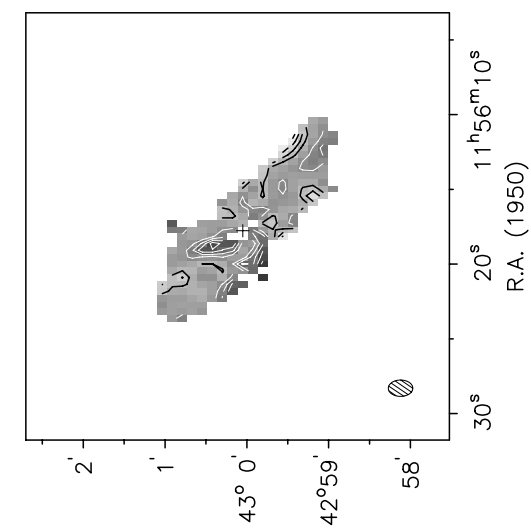

$(0,61) \cdot 200$
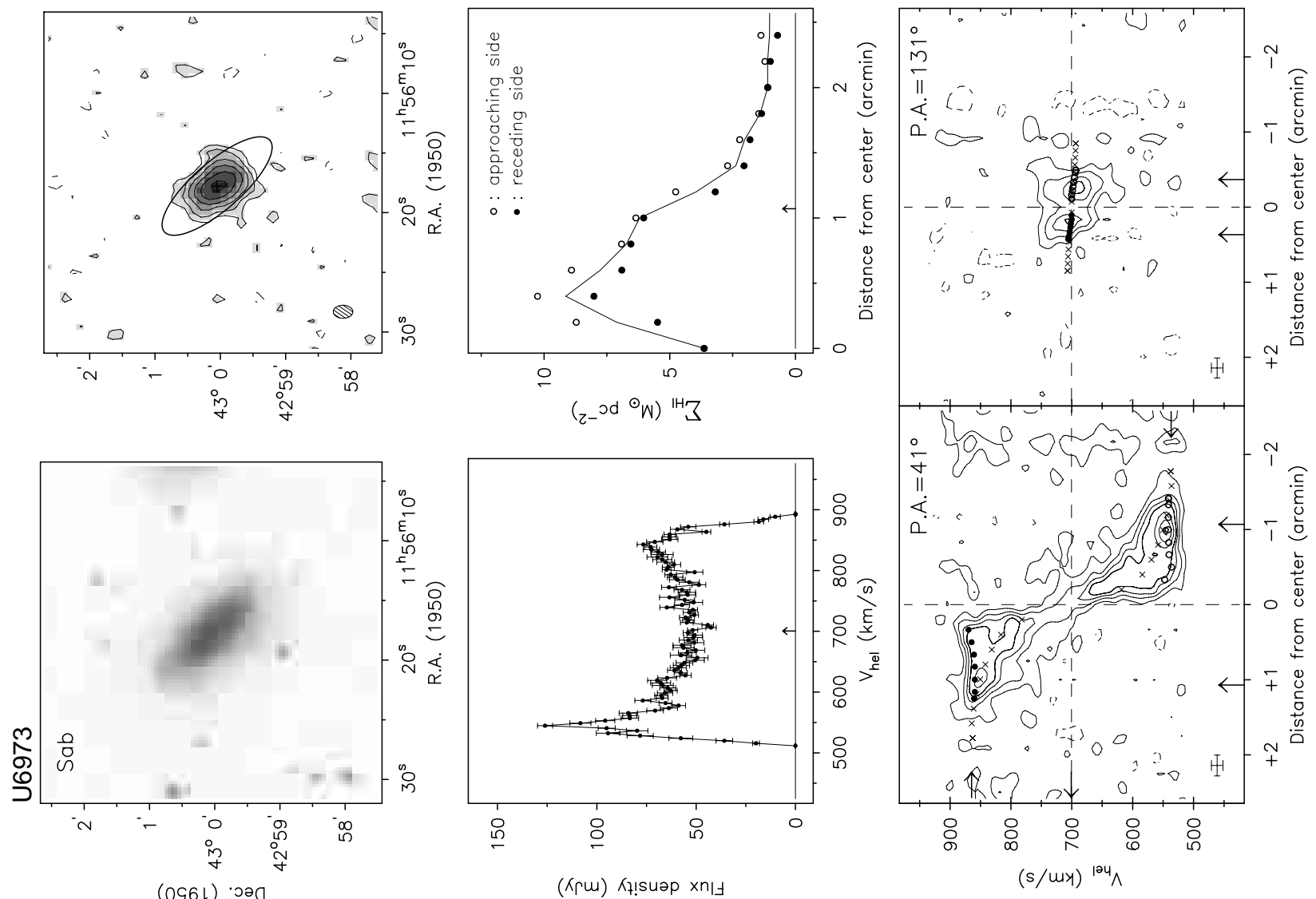
Observing parameters for UGC 6983

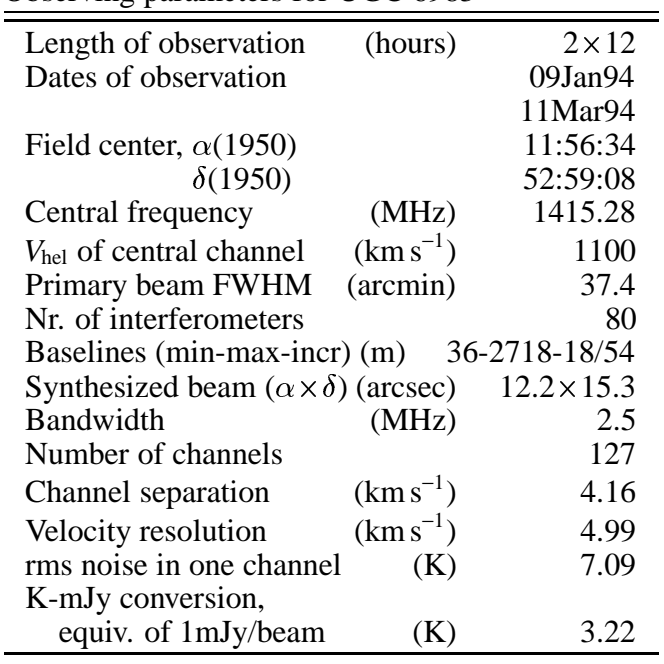

Results from WSRT data

\begin{tabular}{|c|c|}
\hline \multicolumn{2}{|l|}{ From continuит map: } \\
\hline \multicolumn{2}{|l|}{ 21-cm flux density } \\
\hline central point source (mJy) & $<0.9(3 \sigma)$ \\
\hline extended source (mJy) & $<5.4(3 \sigma)$ \\
\hline \multicolumn{2}{|l|}{ From global profile: } \\
\hline$\overline{\text { Integrated HI-flux }}\left(\mathrm{Jy} \mathrm{km} \mathrm{s}^{-1}\right)$ & $38.5 \pm 0.6$ \\
\hline Hel. systemic velocity $\left(\mathrm{km} \mathrm{s}^{-1}\right)$ & $1081.9 \pm 0.8$ \\
\hline HI profile width, $20 \%\left(\mathrm{~km} \mathrm{~s}^{-1}\right)$ & $188.4 \pm 1.3$ \\
\hline $50 \%\left(\mathrm{~km} \mathrm{~s}^{-1}\right)$ & $173.0 \pm 1.1$ \\
\hline \multicolumn{2}{|l|}{ From velocity field: } \\
\hline Hel. systemic velocity $\left(\mathrm{km} \mathrm{s}^{-1}\right)$ & $1082.1 \pm 0.4$ \\
\hline Dynamical center, $\alpha(1950)$ & $11: 56: 34.7$ \\
\hline$\delta(1950)$ & $52: 59: 10$ \\
\hline \multicolumn{2}{|l|}{ From total HI map: } \\
\hline$\overline{\text { Geometric center, } \alpha(1950)}$ & $11: 56: 35.4$ \\
\hline$\delta(1950)$ & 52:59:07 \\
\hline Position angle & 276 \\
\hline Inclination angle & 46 \\
\hline Diameter of HI disk (arcmin) & 6.1 \\
\hline
\end{tabular}

Contour levels for U6983

\begin{tabular}{|c|c|}
\hline \\
\hline $\begin{array}{l}\text { Channel maps: } \\
\text { Raw continuum map: }\end{array}$ & $\sigma=1.05(\mathrm{~K})$ \\
\hline \multicolumn{2}{|c|}{ Cleaned continuum map: } \\
\hline Position-Velocity diag & $\begin{array}{l}\text { Ims: } \\
\sigma=2.50(\mathrm{~K})\end{array}$ \\
\hline $\begin{array}{l}\text { Velocity fields: } \\
\qquad 1082.1 \pm \\
\text { Residual velocity field }\end{array}$ & $\times 15\left(\mathrm{~km} \mathrm{~s}^{-1}\right)$ \\
\hline $\begin{array}{c}\text { Integrated HI map: } \\
0.52,1.05, \\
1.57(\times 10\end{array}$ & $\begin{array}{l}\mathrm{n} \times 5\left(\mathrm{~km} \mathrm{~s}^{-1}\right) \\
\text { atoms cm } \\
-2\end{array}$ \\
\hline
\end{tabular}

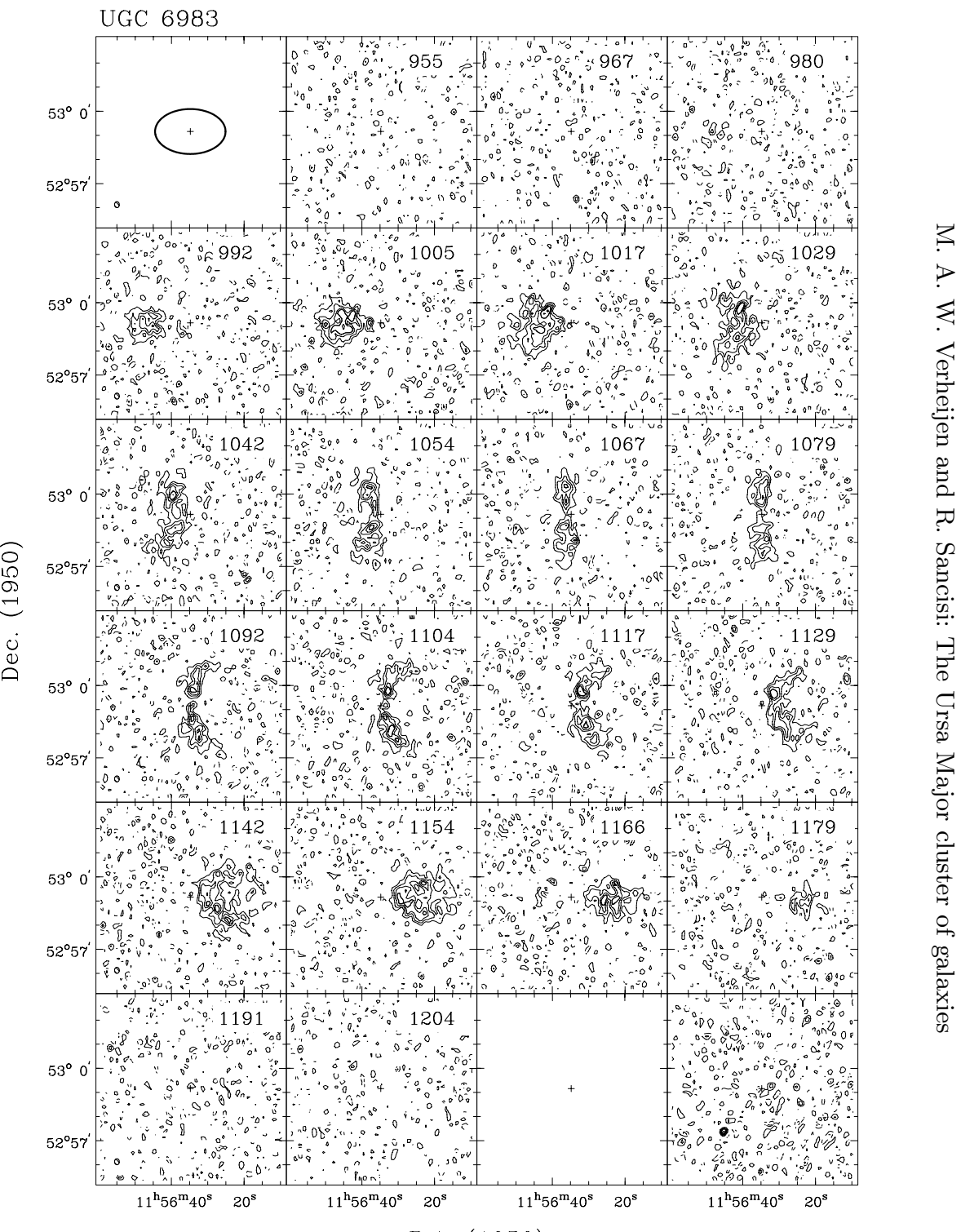

Note: UGC 6983 is a beautiful, regular,

low surface brightness galaxy with well defined spiral arms and a small bar.

Channel maps at a resolution of $12^{\prime \prime} \times 15^{\prime \prime} \times 19 \mathrm{~km} \mathrm{~s}^{-1}$. Contour levels at $-3,-1.5$ (dashed), $1.5,3,4.5, \ldots \times \sigma$. 

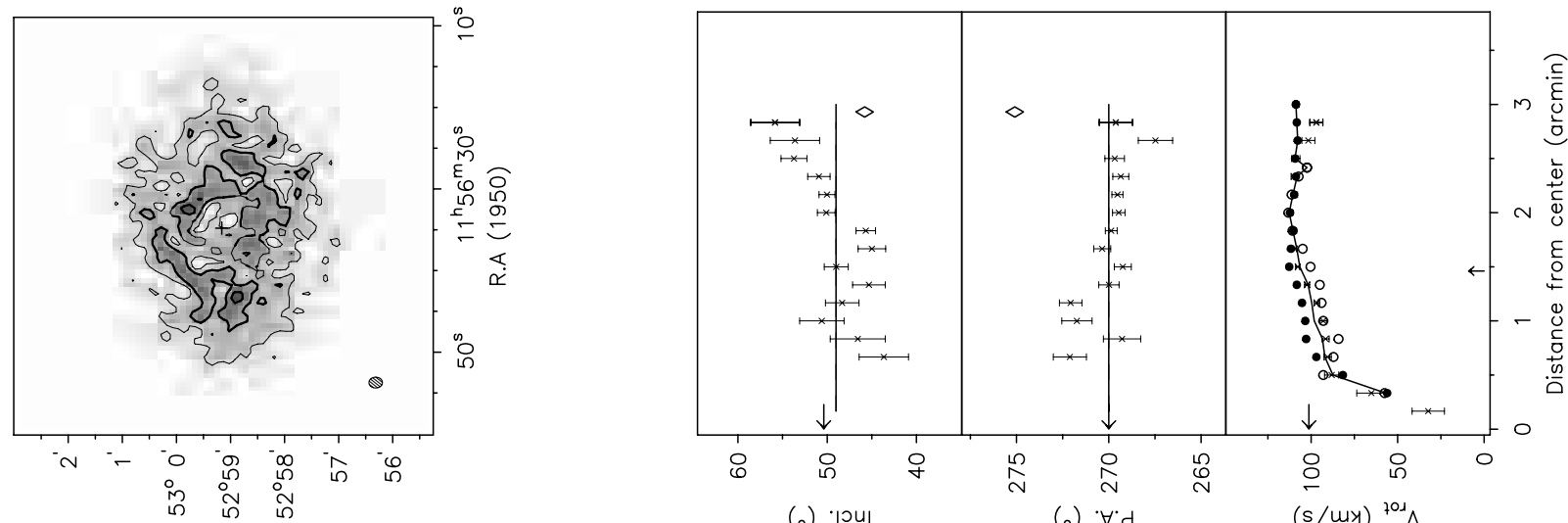

(.) ' pou

(o) $\forall \cdot d$

$(s / m y){ }^{701} \wedge$

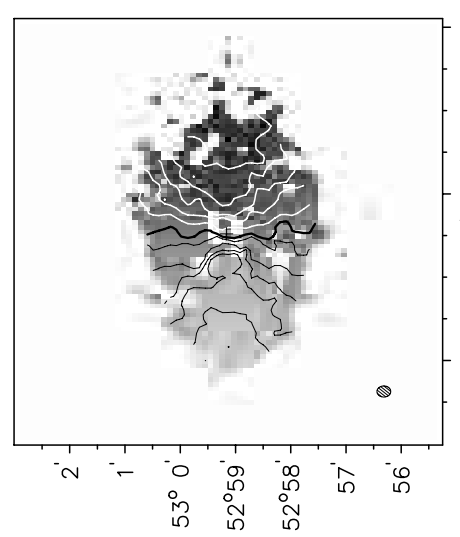

(096l) '
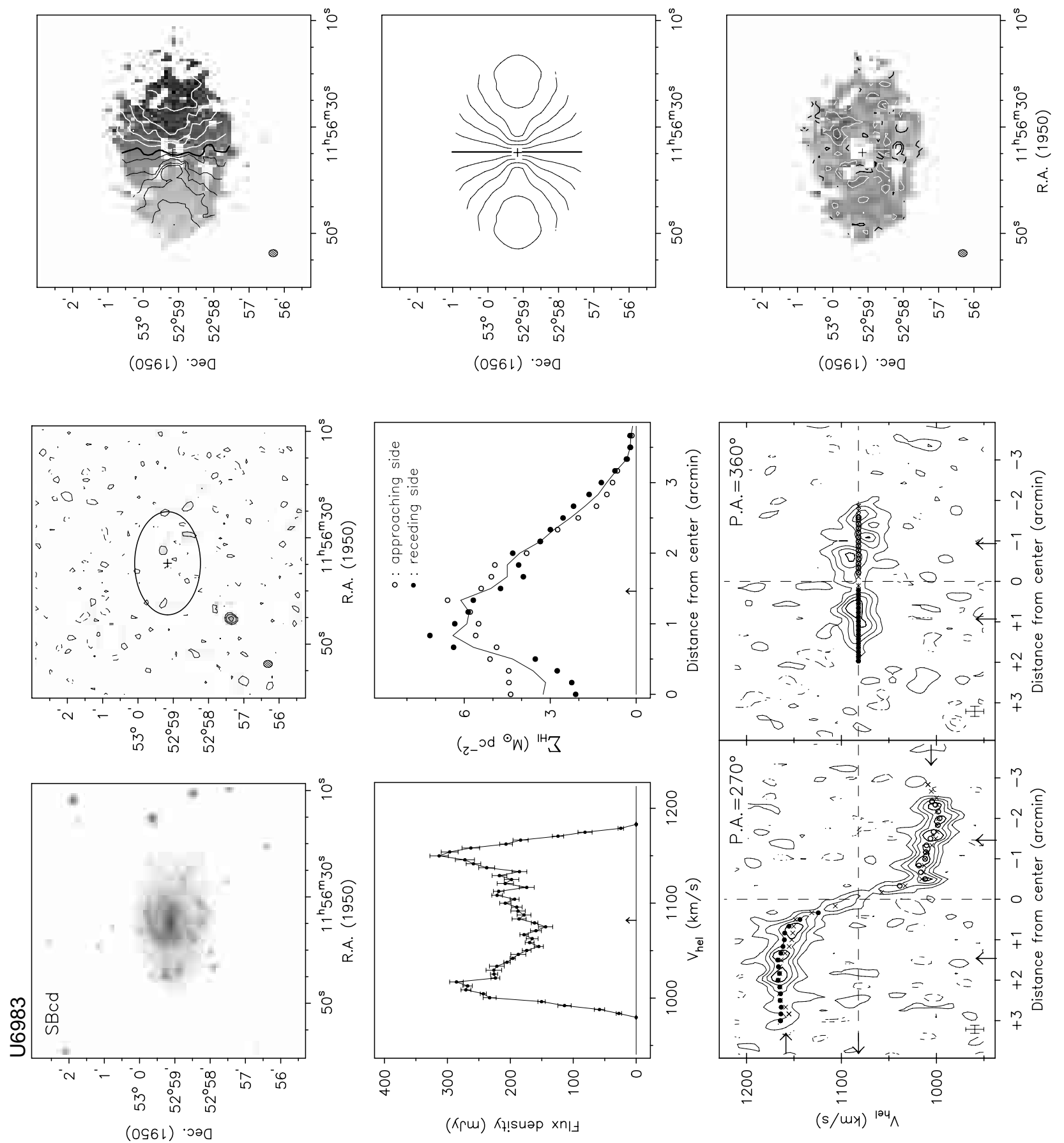
Observing parameters for NGC 4051

\begin{tabular}{|c|c|}
\hline Length of observation & $1 \times 12$ \\
\hline Date of observation & 01Feb94 \\
\hline Field center, $\alpha(1950)$ & 12:00:36 \\
\hline$\delta(1950)$ & 44:48:48 \\
\hline Central frequency & 1417.09 \\
\hline$V_{\text {hel }}$ of central channel & 720 \\
\hline Primary beam FWHM (arcmin) & 37.4 \\
\hline Nr. of interferometers & 40 \\
\hline Baselines (min-max-incr) & $36-2700-72$ \\
\hline Synthesized beam $(\alpha \times \delta)(\operatorname{arcsec})$ & $10.5 \times 15.1$ \\
\hline Bandwidth & 2.5 \\
\hline Number of channels & 127 \\
\hline Channel separation & 4.14 \\
\hline Velocity resolution & 4.96 \\
\hline rms noise in one channel & 13.3 \\
\hline $\begin{array}{l}\text { K-mJy conversion, } \\
\text { equiv. of } 1 \mathrm{mJy} / \text { beam }\end{array}$ & 3.80 \\
\hline
\end{tabular}

Results from WSRT data

\begin{tabular}{|c|c|}
\hline \multicolumn{2}{|l|}{ From continuum map: } \\
\hline 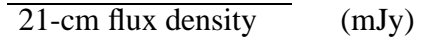 & $26.5 \pm 2.6$ \\
\hline \multicolumn{2}{|l|}{ Central point source position } \\
\hline$\alpha(1950)$ & $12: 00: 36.3$ \\
\hline$\delta(1950)$ & $44: 48: 35$ \\
\hline \multicolumn{2}{|l|}{ From global profile: } \\
\hline$\overline{\text { Integrated HI-flux }}\left(\mathrm{Jy} \mathrm{km} \mathrm{s}^{-1}\right)$ & $35.6 \pm 0.8$ \\
\hline Hel. systemic velocity $\left(\mathrm{km} \mathrm{s}^{-1}\right)$ & $700.3 \pm 1.2$ \\
\hline HI profile width, $20 \%\left(\mathrm{~km} \mathrm{~s}^{-1}\right)$ & $255.4 \pm 1.8$ \\
\hline $50 \%\left(\mathrm{~km} \mathrm{~s}^{-1}\right)$ & $224.6 \pm 1.5$ \\
\hline \multicolumn{2}{|l|}{ From velocity field: } \\
\hline 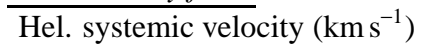 & $704.5 \pm 1.2$ \\
\hline Dynamical center, $\alpha(1950)$ & $12: 00: 36.6$ \\
\hline$\delta(1950)$ & $44: 48: 38$ \\
\hline \multicolumn{2}{|l|}{ From total HI map: } \\
\hline$\overline{\text { Geometric center, } \alpha(1950)}$ & $12: 00: 37.6$ \\
\hline$\delta(1950)$ & $44: 48: 42$ \\
\hline Position angle & 294 \\
\hline Inclination angle & 30 \\
\hline Diameter of HI disk (arcmin) & 5.8 \\
\hline
\end{tabular}

Contour levels for N4051

Channel maps: $\quad \sigma=1.37(\mathrm{~K})$

Raw continuum map: $\sigma=0.82(\mathrm{~K})$ Cleaned continuum map $\sigma=0.63(\mathrm{~K})$

Position-Velocity diagrams:

Velocity fields:

$704.5 \pm \mathrm{n} \times 20\left(\mathrm{~km} \mathrm{~s}^{-1}\right)$ Residual velocity field: $\pm \mathrm{n} \times 5\left(\mathrm{~km} \mathrm{~s}^{-1}\right)$

Integrated HI map:

$0.56,1.11$,

$1.67\left(\times 10^{21}\right.$ atoms cm $\left.{ }^{-2}\right)$

Note: This Seyfert galaxy is lopsided and has a central bar. The rotation curve is quite asymmetric as can be seen in the position-velocity diagrams. The position and inclinations angles derived from the velocity field are quite similar for the various rings and close to the values derived from the optical image. The upturn of the rotation curve in the inner part is uncertain but it is suggested by both the receding and approaching side.

The kinematic major axis seems to be somewhat bend and the iso-velocity contours in the observed velocity field are more separated in the north-eastern than in the south-western region. This suggests that non-circular motions are present in this system.

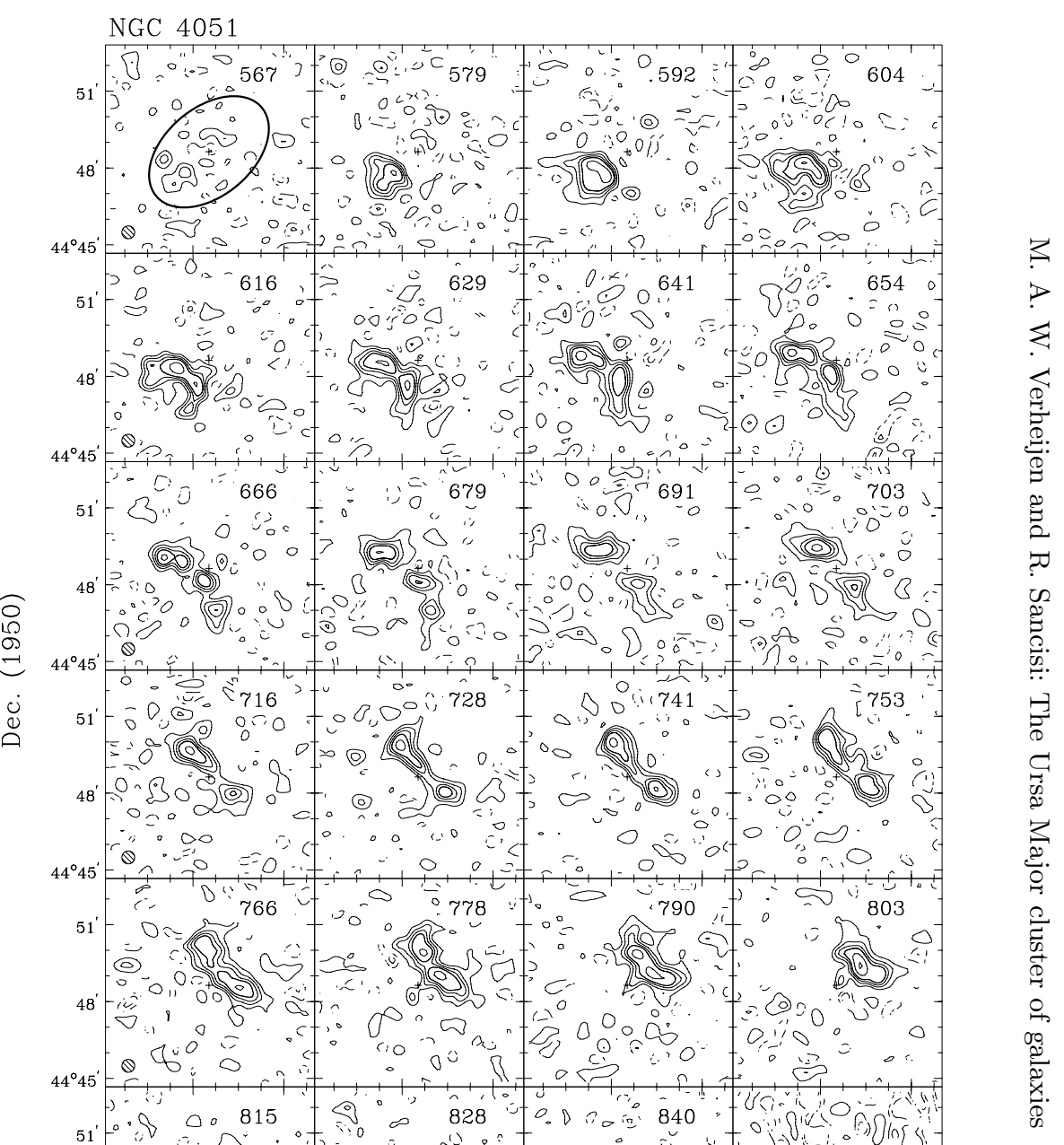

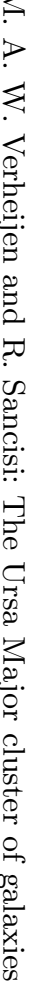

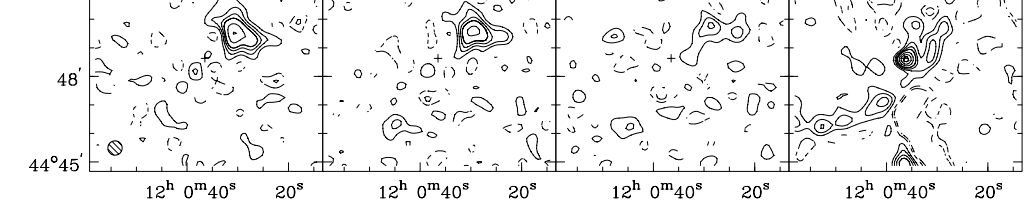

R.A. (1950)

Channel maps at a resolution of $30^{\prime \prime} \times 30^{\prime \prime} \times 19 \mathrm{~km} \mathrm{~s}^{-1}$. Contour levels at $-3,-1.5$ (dashed), $1.5,3,4.5, \ldots \times \sigma$. 

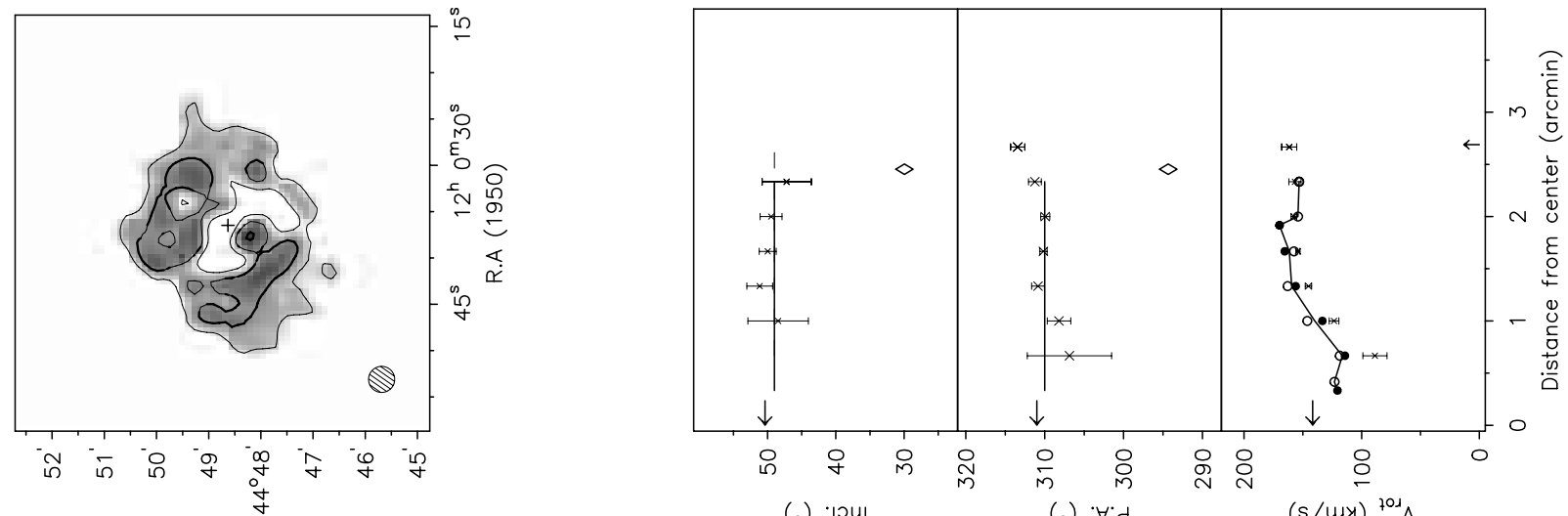
(o) 'pou
(o) $\forall \cdot d$
$(s / m y){ }^{701} \wedge$

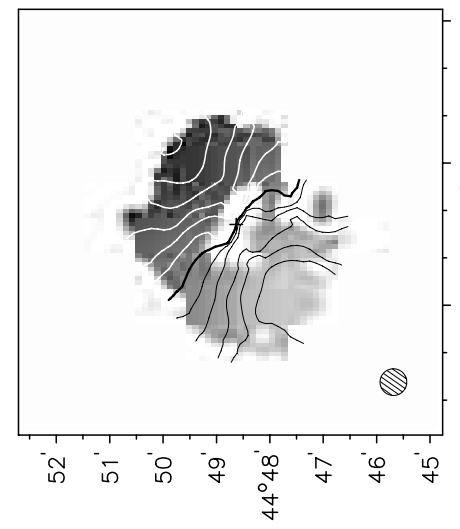

(0s6l) $\cdot 020$

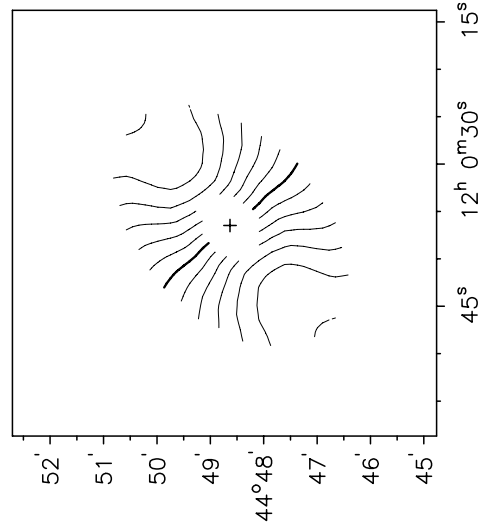

(0s6l)

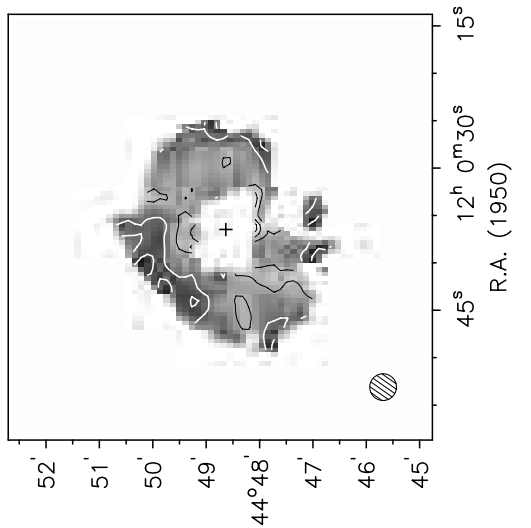

$(056 \mathrm{l}) \cdot$
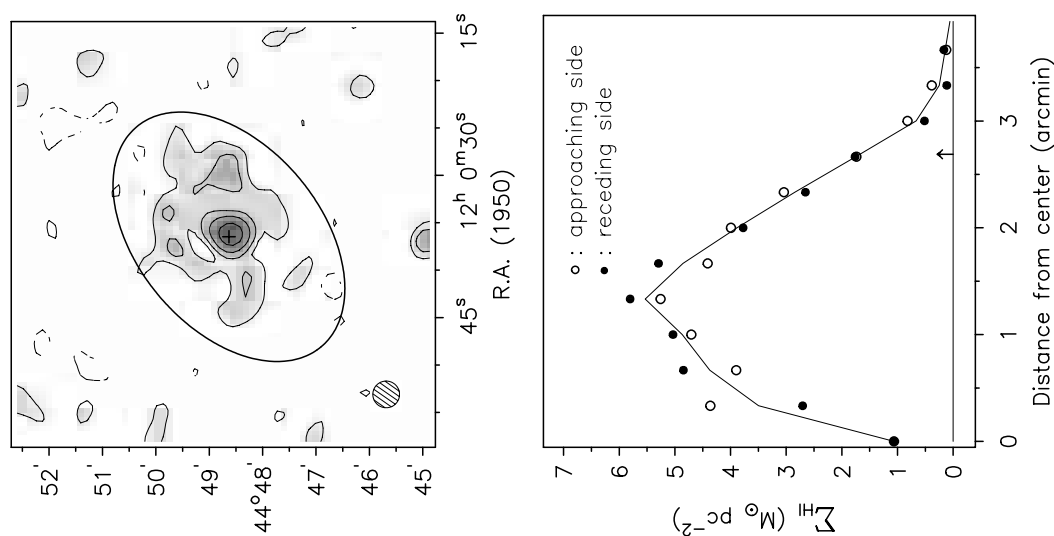

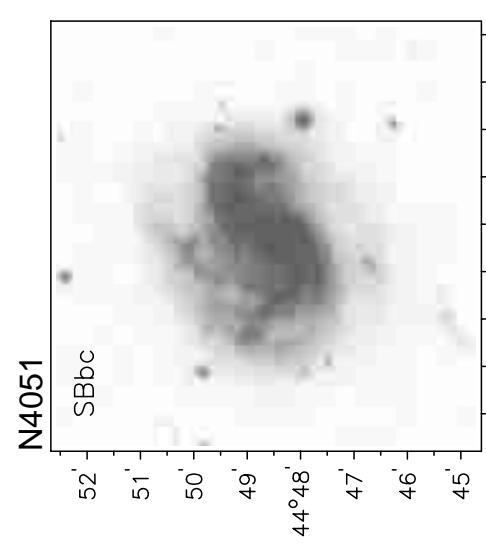

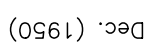

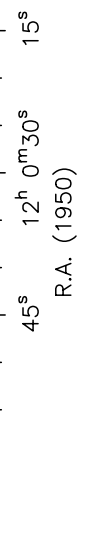

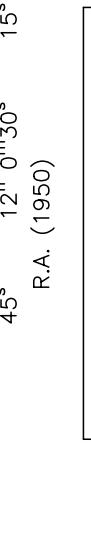

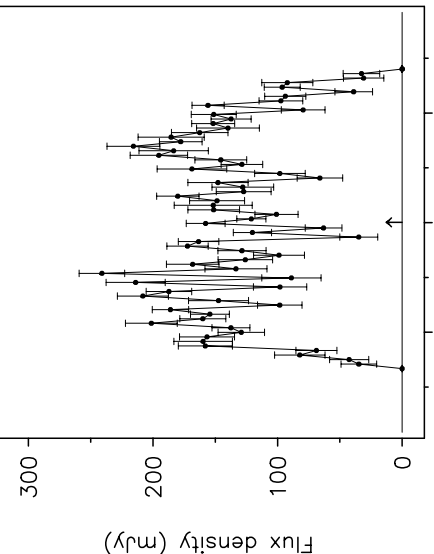

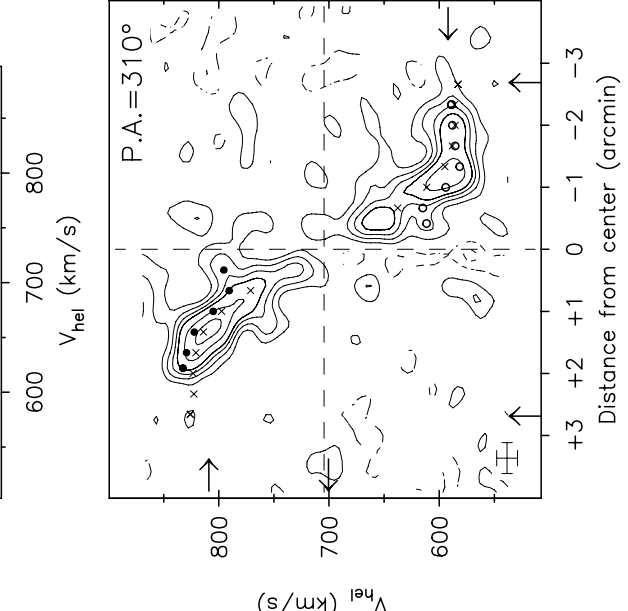


Observing parameters for NGC 4085

\begin{tabular}{|c|c|}
\hline Length of observation & $1 \times 12$ \\
\hline Date of observation & 13Jan94 \\
\hline Field center, $\alpha(1950)$ & 12:02:57 \\
\hline$\delta(1950)$ & $50: 43: 30$ \\
\hline Central frequency & 1416.69 \\
\hline$V_{\text {hel }}$ of central channel & 800 \\
\hline Primary beam FWHM (arcmin) & 37.4 \\
\hline Nr. of interferometers & 40 \\
\hline Baselines (min-max-incr) & $36-2700-72$ \\
\hline Synthesized beam $(\alpha \times \delta)(\operatorname{arcsec})$ & $12.0 \times 15.7$ \\
\hline Bandwidth & 5.0 \\
\hline Number of channels & 63 \\
\hline Channel separation & 16.5 \\
\hline Velocity resolution & 19.8 \\
\hline rms noise in one channel & 4.80 \\
\hline $\begin{array}{l}\text { K-mJy conversion, } \\
\text { equiv. of } 1 \mathrm{mJy} / \text { beam }\end{array}$ & 3.20 \\
\hline
\end{tabular}

Results from WSRT data

\begin{tabular}{|c|c|}
\hline \multicolumn{2}{|l|}{ From continuum map: } \\
\hline $21-\mathrm{cm}$ flux density & $44.1 \pm 1.3$ \\
\hline \multicolumn{2}{|l|}{ Central point source position } \\
\hline$\alpha(1950)$ & $12: 02: 50.2$ \\
\hline$\delta(1950)$ & $50: 37: 54$ \\
\hline \multicolumn{2}{|l|}{ From global profile: } \\
\hline$\overline{\text { Integrated HI-flux }}\left(\mathrm{Jy} \mathrm{km} \mathrm{s}^{-1}\right)$ & $14.6 \pm 0.9$ \\
\hline Hel. systemic velocity $\left(\mathrm{km} \mathrm{s}^{-1}\right)$ & $745.7 \pm 5.0$ \\
\hline HI profile width, $20 \%\left(\mathrm{~km} \mathrm{~s}^{-1}\right)$ & $277.4 \pm 6.6$ \\
\hline $50 \%\left(\mathrm{~km} \mathrm{~s}^{-1}\right)$ & $255.4 \pm 7.8$ \\
\hline \multicolumn{2}{|l|}{ From velocity field: } \\
\hline 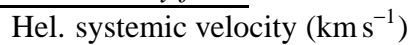 & 752 \\
\hline center, $\alpha(1950)$ & $12: 02: 50.3$ \\
\hline$\delta(1950)$ & $50: 37: 54$ \\
\hline \multicolumn{2}{|l|}{ From total HI map: } \\
\hline Geometric center, $\alpha(1950)$ & $12: 02: 50.8$ \\
\hline$\delta(1950)$ & $50: 37: 56$ \\
\hline (deg) & 256 \\
\hline Inclination angle & 83 \\
\hline Diameter of HI disk (arcmin) & 3.9 \\
\hline
\end{tabular}

Contour levels for N4085

Channel maps: $\quad \sigma=4.64(\mathrm{~K})$

Raw continuum map:

Cleaned continuum map: $\sigma=1.06(\mathrm{~K})$

Position-Velocity diagrams:

Velocity fields:

$$
\sigma=4.16(\mathrm{~K})
$$

$752.0 \pm \mathrm{n} \times 20\left(\mathrm{~km} \mathrm{~s}^{-1}\right)$ Residual velocity field: $\pm \mathrm{n} \times 5\left(\mathrm{~km} \mathrm{~s}^{-1}\right)$

Integrated HI map:

$1.77,3.54\left(\times 10^{21}\right.$ atoms cm $\left.^{-2}\right)$

Note: This galaxy is a companion of the strongly disturbed system NGC 4088 at 10 arcmin to the north. The HI disk does not extend much beyond $\mathrm{D}_{25}$. The extent of the radial HI density profile is due to the high inclination of the ellipses along which the column density is measured and the effect of beam smearing along the minor axis.

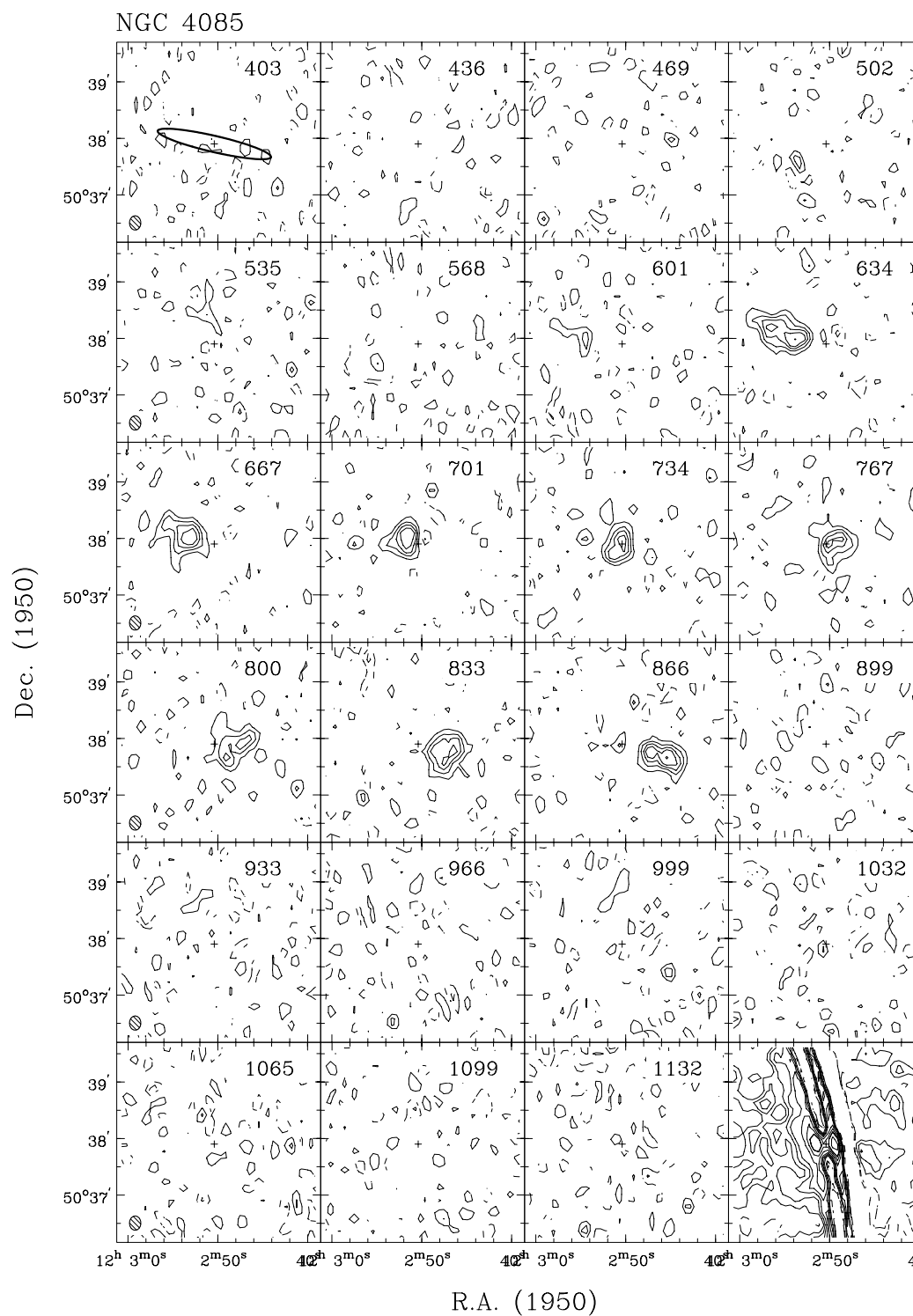

Channel maps at a resolution of $12^{\prime \prime} \times 15^{\prime \prime} \times 20 \mathrm{~km} \mathrm{~s}^{-1}$. Contour levels at $-3,-1.5$ (dashed), $1.5,3,4.5, \ldots \times \sigma$. 

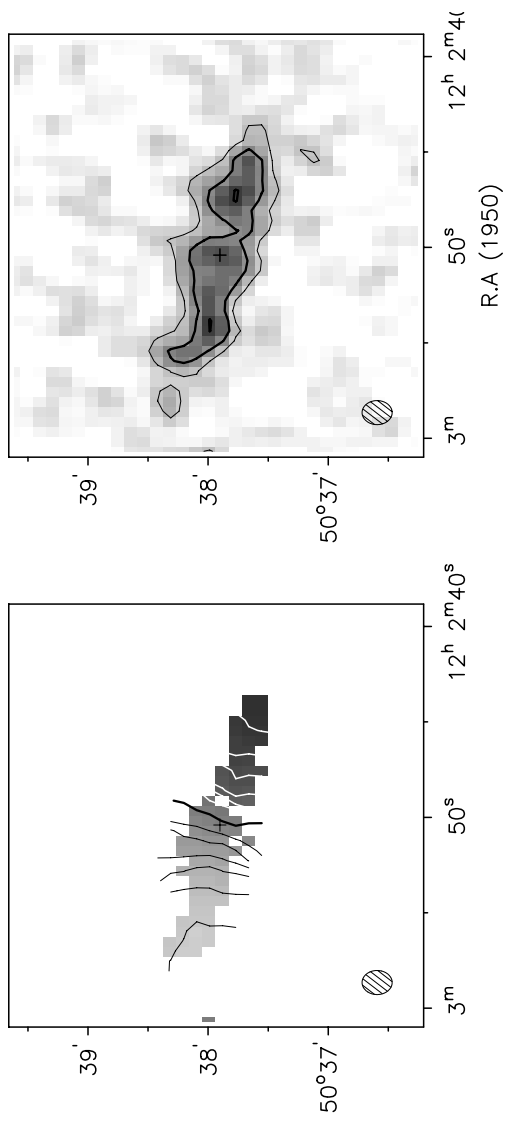

(096l) 'गә0
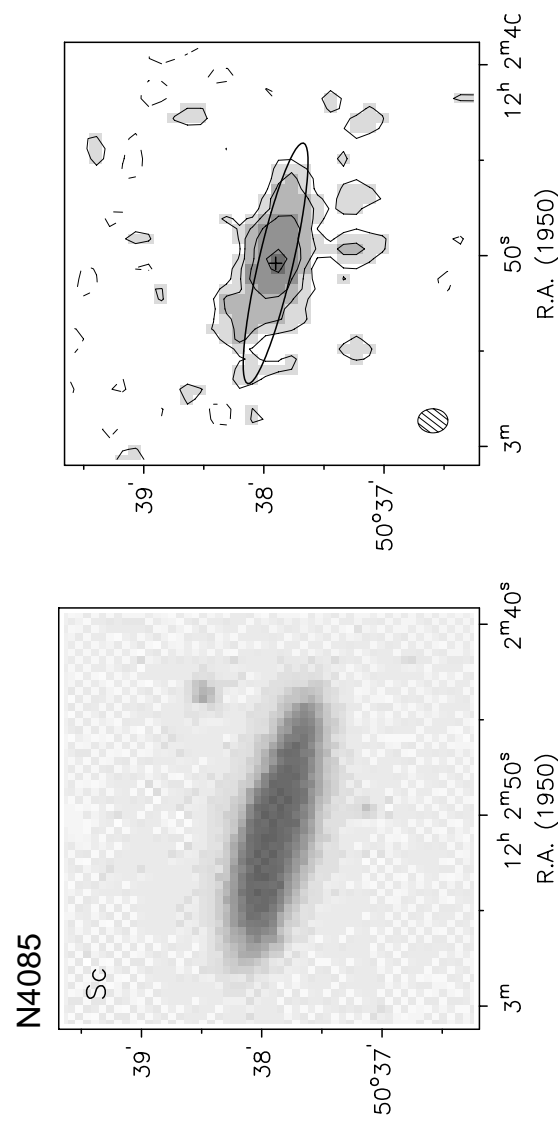

$(0961) \cdot 2 \partial 0$

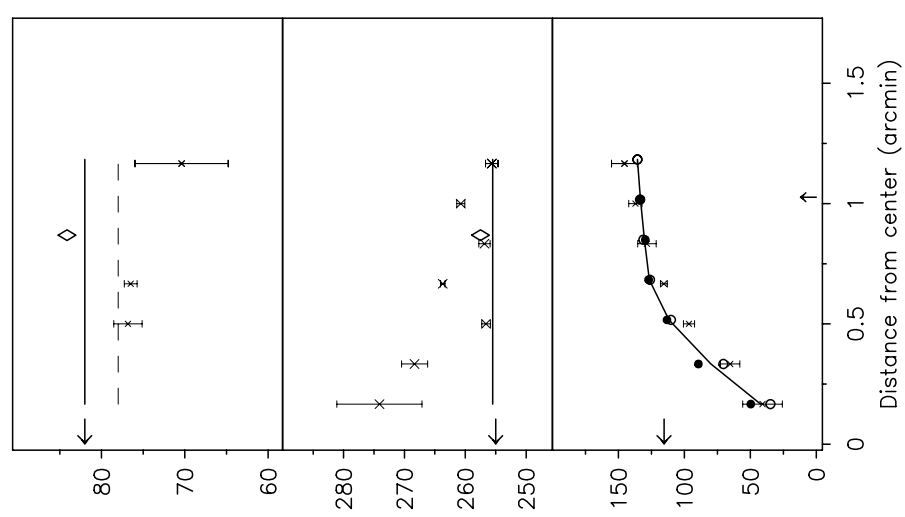

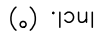

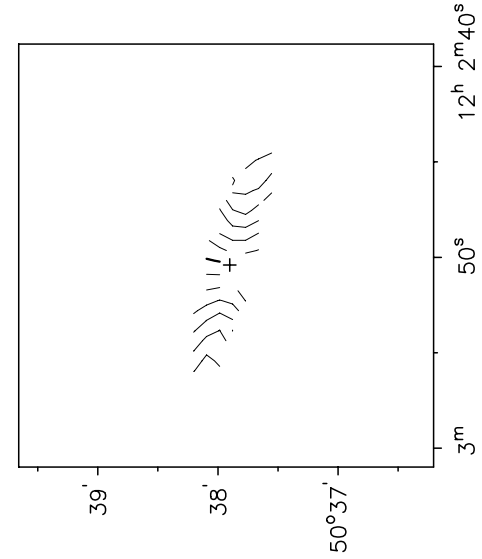

(096l) : .

(o) $\forall \cdot d$

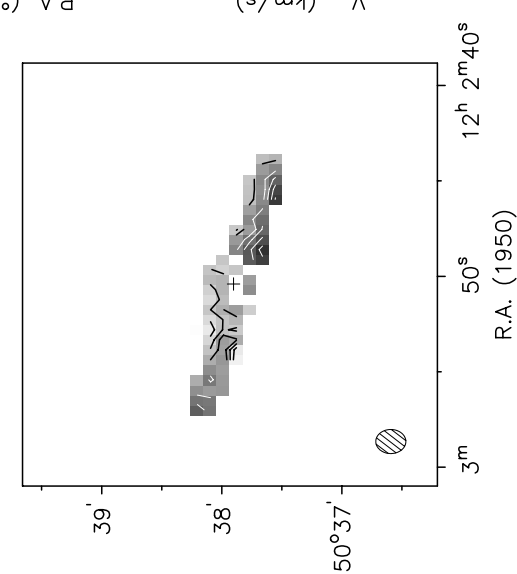

$(0961) \cdot$ วә0
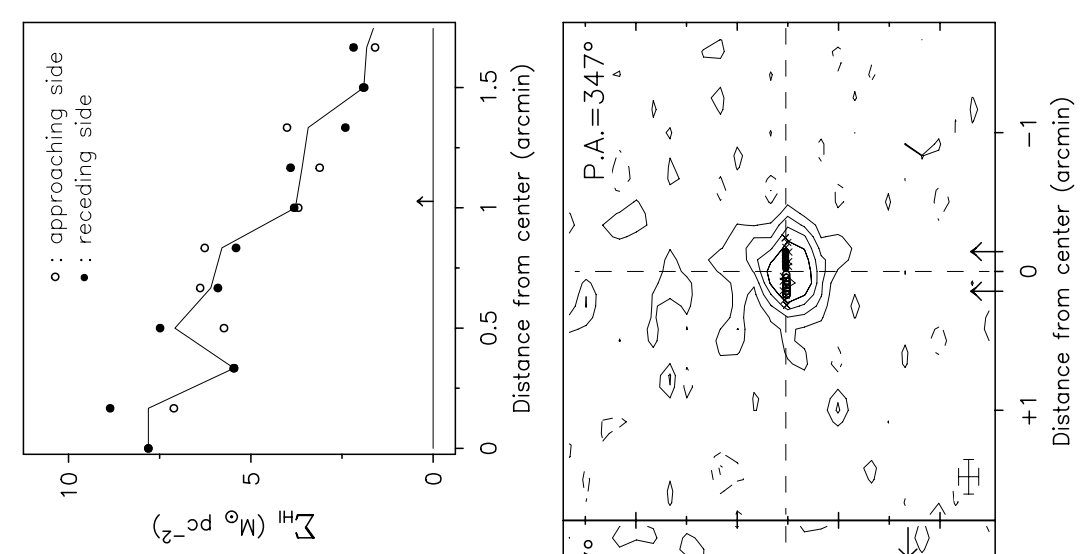
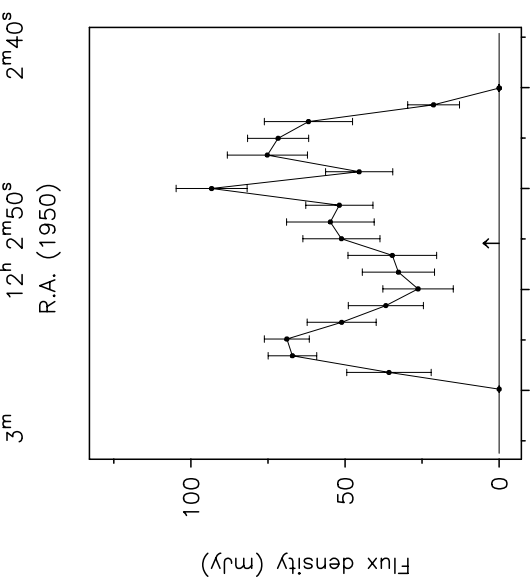

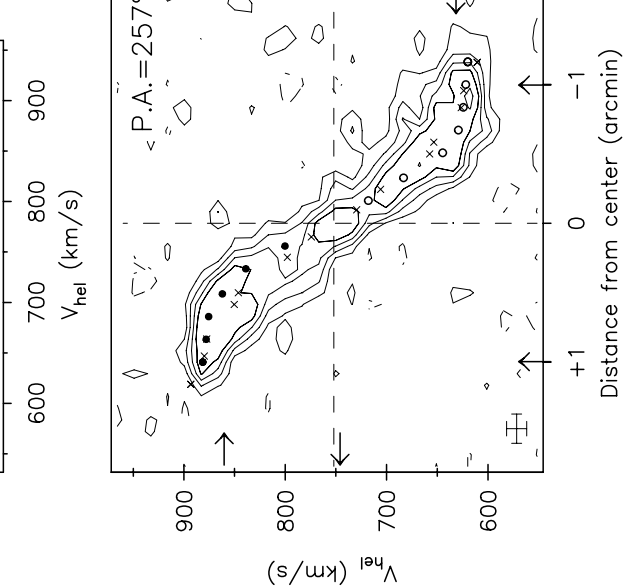


Observing parameters for NGC 4088

\begin{tabular}{|c|c|}
\hline Length of observation & $1 \times 12$ \\
\hline Date of observation & 13Jan94 \\
\hline Field center, $\alpha(1950)$ & 12:02:57 \\
\hline$\delta(1950)$ & $50: 43: 30$ \\
\hline Central frequency & 1416.69 \\
\hline$V_{\text {hel }}$ of central channel & 800 \\
\hline Primary beam FWHM (arcmin) & 37.4 \\
\hline Nr. of interferometers & 40 \\
\hline Baselines (min-max-incr) & $36-2700-72$ \\
\hline Synthesized beam $(\alpha \times \delta)(\operatorname{arcsec})$ & $12.0 \times 15.7$ \\
\hline Bandwidth & 5.0 \\
\hline Number of channels & 63 \\
\hline Channel separation & 16.5 \\
\hline Velocity resolution & 19.8 \\
\hline rms noise in one channel & 4.80 \\
\hline $\begin{array}{l}\text { K-mJy conversion, } \\
\text { equiv. of } 1 \mathrm{mJy} / \text { beam }\end{array}$ & 3.20 \\
\hline
\end{tabular}

Results from WSRT data

\begin{tabular}{|c|c|}
\hline \multicolumn{2}{|l|}{ From continuum map: } \\
\hline 21-cm flux density & $222.3 \pm 1.9$ \\
\hline \multicolumn{2}{|l|}{ Central point source position } \\
\hline$\alpha(1950)$ & 12:03:01.9 \\
\hline$\delta(1950)$ & 50:49:04 \\
\hline \multicolumn{2}{|l|}{ From global profile: } \\
\hline Integrated HI-flux $\left(\mathrm{Jy} \mathrm{km} \mathrm{s}^{-1}\right)$ & $102.9 \pm 1.1$ \\
\hline Hel. systemic velocity $\left(\mathrm{km} \mathrm{s}^{-1}\right)$ & $756.7 \pm 1.2$ \\
\hline HI profile width, $20 \%\left(\mathrm{~km} \mathrm{~s}^{-1}\right)$ & $371.4 \pm 1.7$ \\
\hline $50 \%\left(\mathrm{~km} \mathrm{~s}^{-1}\right)$ & $342.1 \pm 1.9$ \\
\hline \multicolumn{2}{|l|}{ From velocity field: } \\
\hline 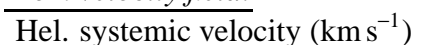 & $761.5 \pm 1.8$ \\
\hline Dynamical center, $\alpha(1950)$ & 12:03:02.4 \\
\hline$\delta(1950)$ & 50:49:06 \\
\hline \multicolumn{2}{|l|}{ From total HI map: } \\
\hline Geometric center $\alpha(1950)$ & 12:03:03.6 \\
\hline$\delta(1950)$ & $50: 49: 12$ \\
\hline Position angle & 231 \\
\hline Inclination angle & 69 \\
\hline Diameter of HI disk (arcmin) & 8.5 \\
\hline
\end{tabular}

Contour levels for N4088

\begin{tabular}{|c|c|}
\hline \\
\hline $\begin{array}{l}\text { Channel maps: } \\
\text { Raw continuum map: }\end{array}$ & $\sigma=0.33(\mathrm{~K})$ \\
\hline Cleaned continuum $\mathrm{m}$ & $\sigma=0.29(\mathrm{~K})$ \\
\hline Position-Velocity diag & $\begin{array}{l}\text { ams: } \\
\sigma=1.19(\mathrm{~K})\end{array}$ \\
\hline $\begin{array}{l}\text { Velocity fields: } \\
\quad 761.5 \pm \\
\text { Residual velocity fiel }\end{array}$ & $\times 25\left(\mathrm{~km} \mathrm{~s}^{-1}\right)$ \\
\hline $\begin{array}{r}\text { Integrated HI map: } \\
0.38,0 \\
1.89(\times 1\end{array}$ & $\begin{array}{l}\mathrm{n} \times 5\left(\mathrm{~km} \mathrm{~s}^{-1}\right) \\
75,1.13,1.51 \\
\left.1 \text { atoms } \mathrm{cm}^{-2}\right)\end{array}$ \\
\hline
\end{tabular}

Note: NGC 4088 is among the brightest systems in the cluster. It has a very high surface brightness and is very blue. The strong radio continuum emission and the $60 \mu \mathrm{m}$ flux suggest ongoing vigorous star formation. The disk is strongly distorted. The position-velocity diagram shows a strong asymmetry and comparison of the channel maps at 618 and $899 \mathrm{~km} \mathrm{~s}^{-1}$ shows that the warp is asymmetric as well. The position angle changes more in the southern than in the northern part. The extended radio continuum emission seems to originate even outside the optical regions. NGC 4085 is located 10 arcmin to the south.

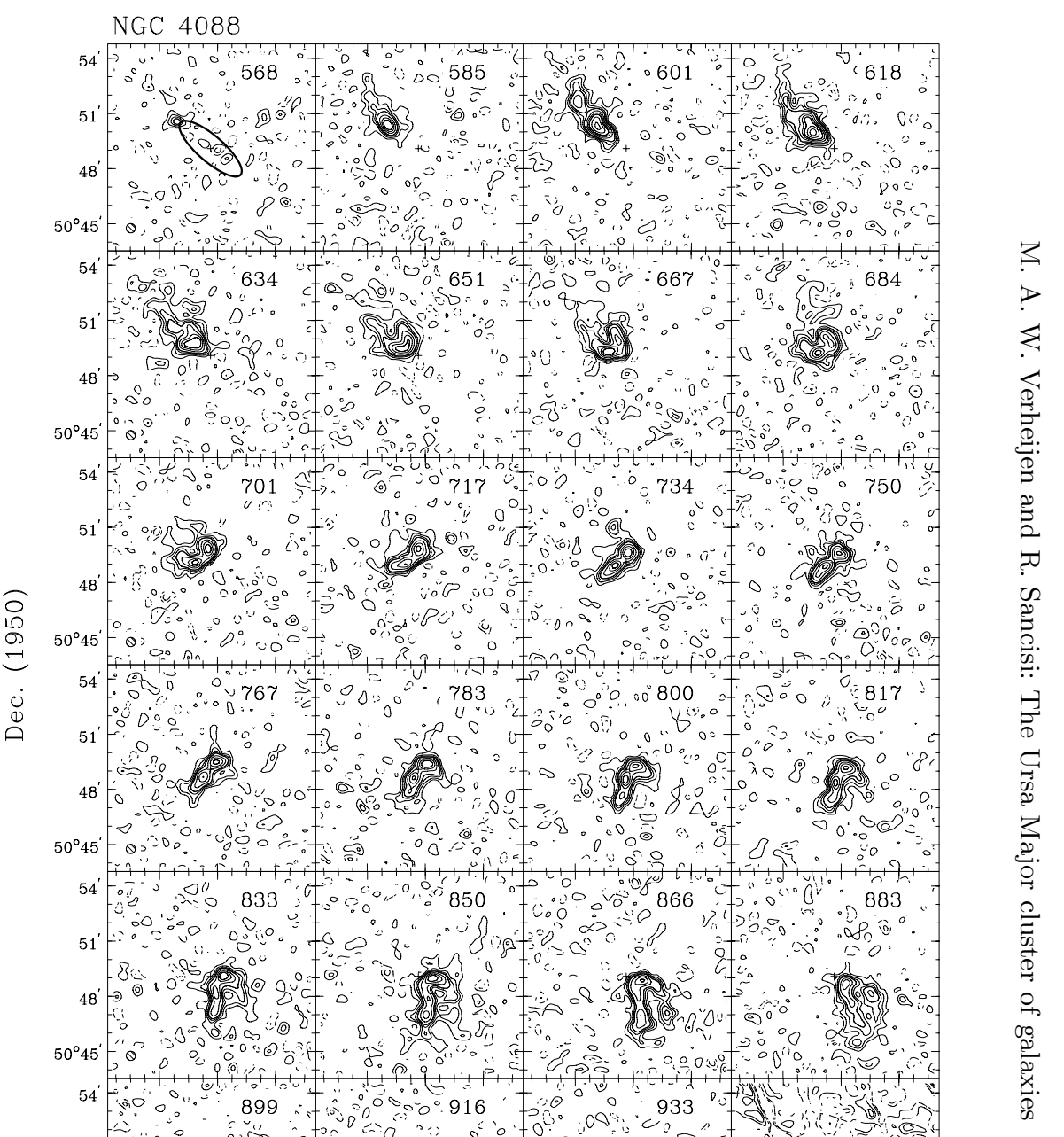

.

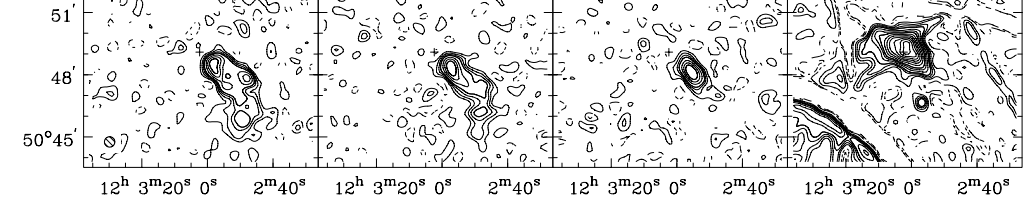

R.A. (1950)

Channel maps at a resolution of $30^{\prime \prime} \times 30^{\prime \prime} \times 20 \mathrm{~km} \mathrm{~s}^{-1}$. Contour levels at $-3,-1.5$ (dashed), $1.5,3,4.5, \ldots \times \sigma$. 

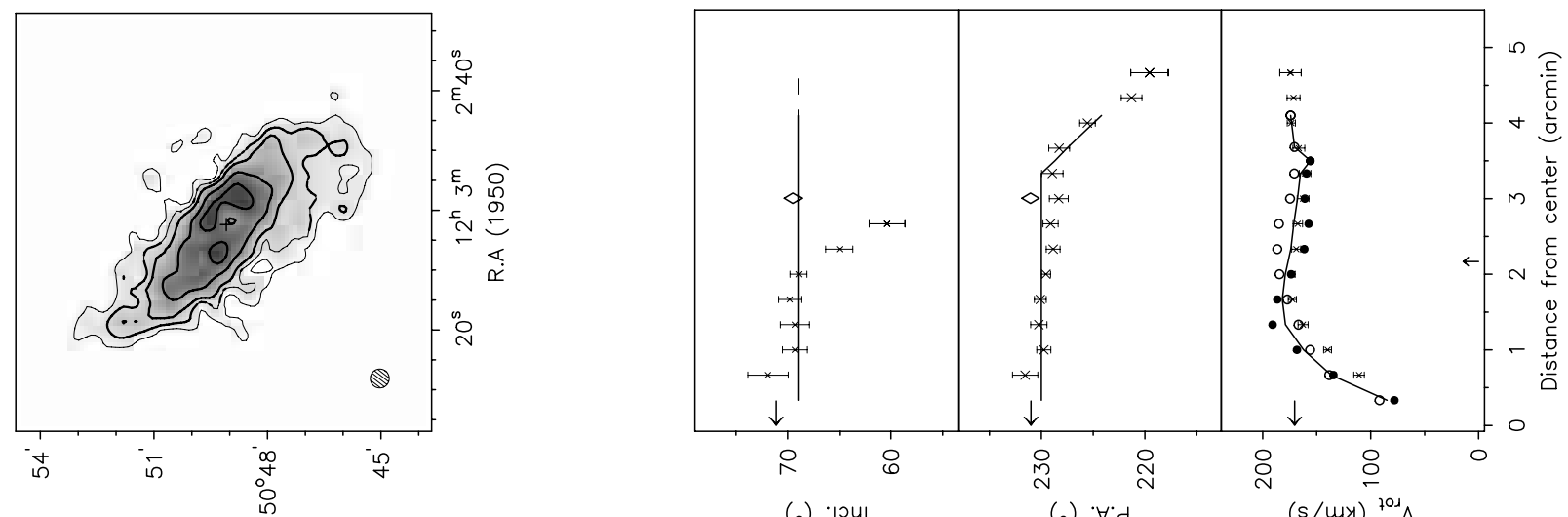

(०) '|गU|

(o) $\forall \cdot d$

$(s / m y){ }^{701} \wedge$

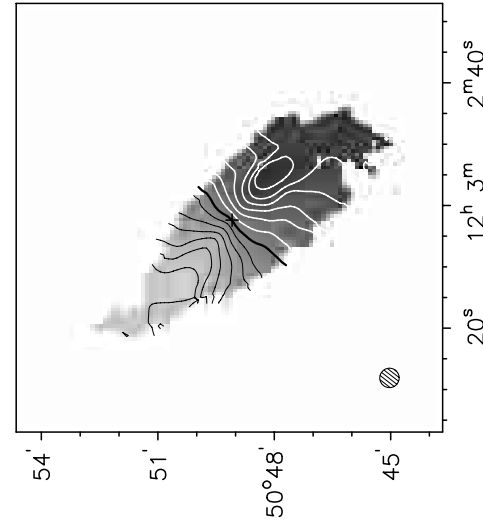

$(0 \mathrm{os} 6 \mathrm{l}) \cdot 020$

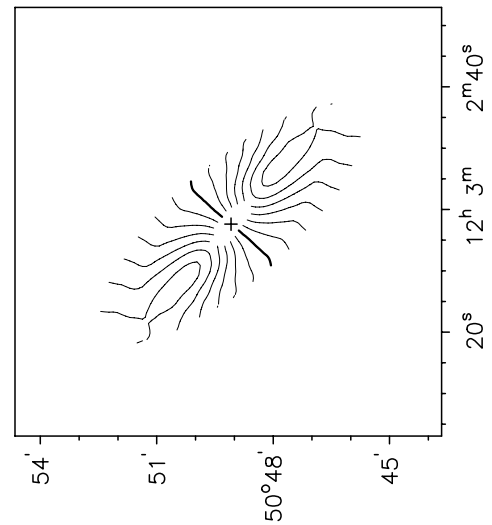

(0s6l) $\cdot 2$ م

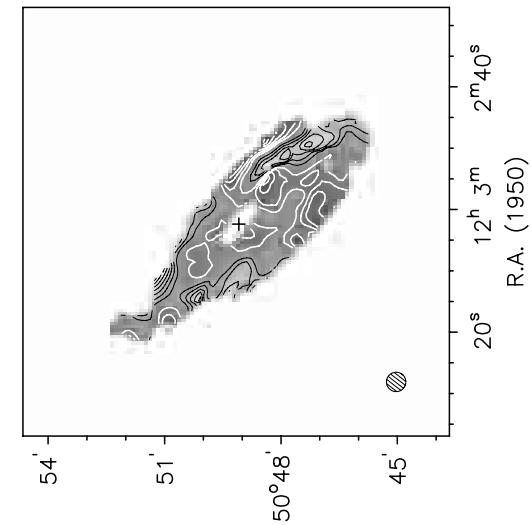

(0s6l) $\cdot 2$ ว
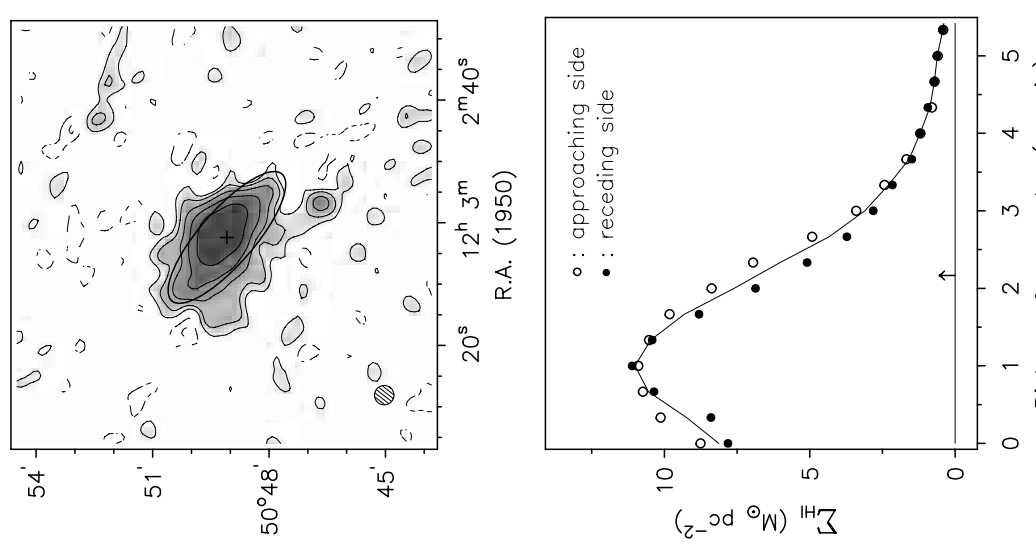

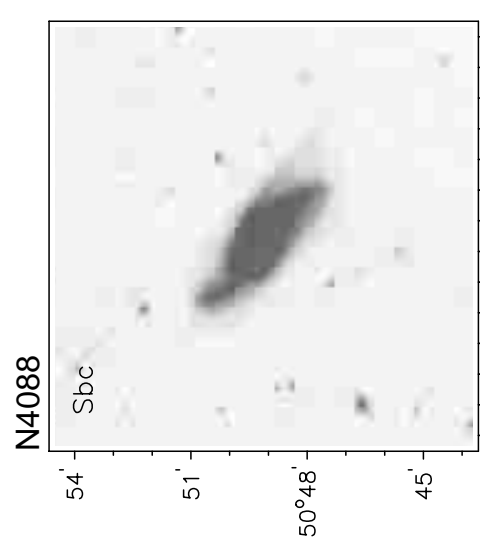

$(0961) \cdot 5 ə 0$

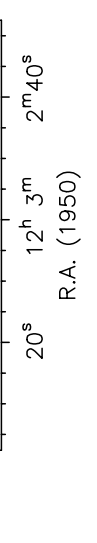

닐

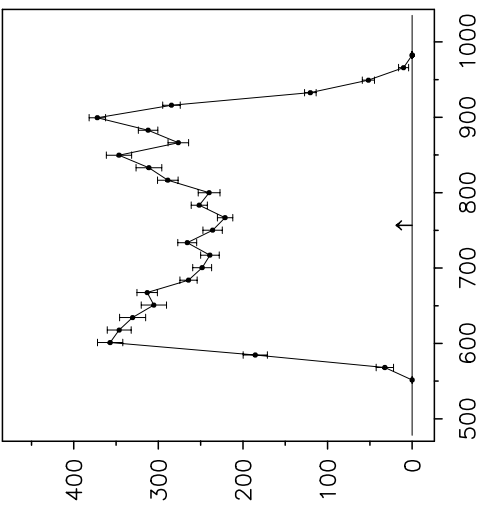

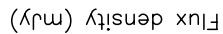

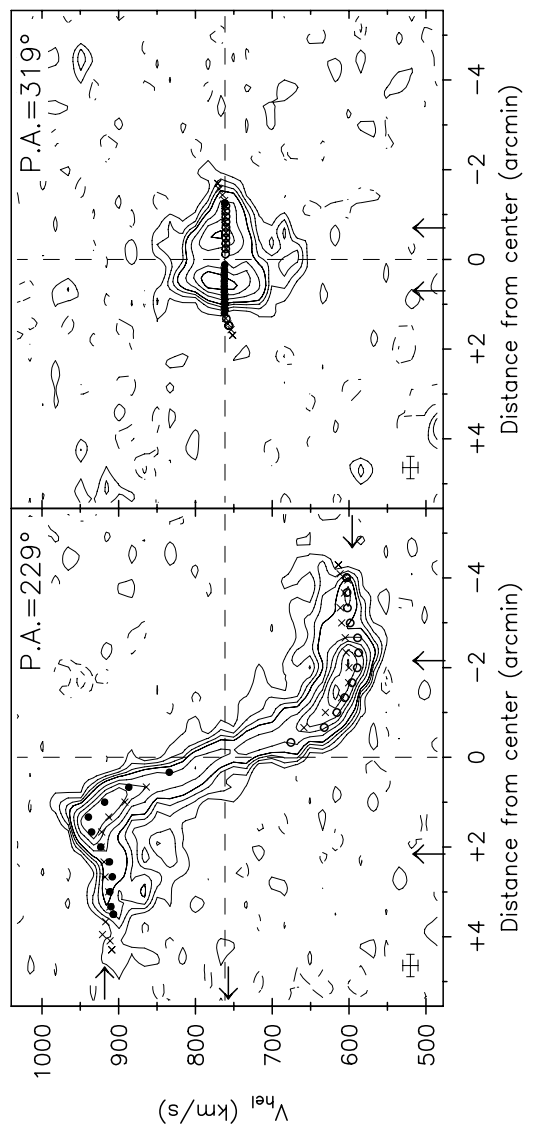


Observing parameters for NGC 4100

\begin{tabular}{|c|c|}
\hline Length of observation & $1 \times 12$ \\
\hline Date of observation & 06Jan94 \\
\hline Field center, $\alpha(1950)$ & 12:03:36 \\
\hline$\delta(1950)$ & 49:51:36 \\
\hline Central frequency & 1415.20 \\
\hline$V_{\text {hel }}$ of central channel & 1120 \\
\hline Primary beam FWHM (arcmin) & 37.4 \\
\hline Nr. of interferometers & 40 \\
\hline Baselines (min-max-incr) & $36-2700-72$ \\
\hline Synthesized beam $(\alpha \times \delta)(\operatorname{arcsec})$ & $12.1 \times 15.9$ \\
\hline Bandwidth & 5.0 \\
\hline Number of channels & 63 \\
\hline Channel separation & 16.61 \\
\hline Velocity resolution & 19.93 \\
\hline rms noise in one channel & 4.84 \\
\hline $\begin{array}{l}\text { K-mJy conversion, } \\
\text { equiv. of } 1 \mathrm{mJy} / \text { beam }\end{array}$ & 3.13 \\
\hline
\end{tabular}

Results from WSRT data

\begin{tabular}{|c|c|}
\hline \multicolumn{2}{|l|}{ From continuиm map: } \\
\hline $21-\mathrm{cm}$ flux density & $54.3 \pm 1.7$ \\
\hline \multicolumn{2}{|l|}{ Central point source position } \\
\hline$\alpha(1950)$ & $12: 03: 36.4$ \\
\hline$\delta(1950)$ & $49: 51: 40$ \\
\hline \multicolumn{2}{|l|}{ From global profile: } \\
\hline Integrated HI-flux $\left(\mathrm{Jy} \mathrm{km} \mathrm{s}^{-1}\right)$ & $41.6 \pm 0.7$ \\
\hline Hel. systemic velocity $\left(\mathrm{km} \mathrm{s}^{-1}\right)$ & $1074.4 \pm 1.3$ \\
\hline HI profile width, $20 \%\left(\mathrm{~km} \mathrm{~s}^{-1}\right)$ & $401.8 \pm 2.0$ \\
\hline $50 \%\left(\mathrm{~km} \mathrm{~s}^{-1}\right)$ & $380.5 \pm 1.8$ \\
\hline \multicolumn{2}{|l|}{ From velocity field: } \\
\hline 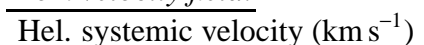 & $1071.2 \pm 0.9$ \\
\hline Dynamical center, $\alpha(1950)$ & $12: 03: 36.6$ \\
\hline$\delta(1950)$ & 49:51:40 \\
\hline \multicolumn{2}{|l|}{ From total HI map: } \\
\hline$\overline{\text { Geometric center, }} \alpha(1950)$ & $12: 03: 36.5$ \\
\hline$\delta(1950)$ & $49: 51: 40$ \\
\hline Position angle & 347 \\
\hline Inclination angle & 70 \\
\hline Diameter of HI disk (arcmin) & 6.9 \\
\hline
\end{tabular}

Contour levels for N4100

\begin{tabular}{|c|c|}
\hline \multicolumn{2}{|l|}{ Channel maps: } \\
\hline Raw continuum map: & $\sigma=0.37(\mathrm{~K})$ \\
\hline Cleaned continuum ma & $\sigma=0.30(\mathrm{~K})$ \\
\hline Position-Velocity diag & $\begin{array}{l}\text { Ims: } \\
\sigma=4.07(\mathrm{~K})\end{array}$ \\
\hline $\begin{array}{l}\text { Velocity fields: } \\
\qquad 1071.2 \pm \mathrm{r} \\
\text { Residual velocity field }\end{array}$ & $\times 30\left(\mathrm{~km} \mathrm{~s}^{-1}\right)$ \\
\hline $\begin{array}{c}\text { Integrated HI map: } \\
0.42,0.84, \\
1.26(\times 10\end{array}$ & $\begin{array}{l}\times 5\left(\mathrm{~km} \mathrm{~s}^{-1}\right) \\
\text { toms cm} \\
-2\end{array}$ \\
\hline
\end{tabular}

Note: The optical appearance of this spiral suggests a mild warp toward faceon or a strong widening of the pitchon or a strong widening of the pitch-
angle of the spiral arms in the outer regions. Also the HI distribution (see channel maps at 904 and $1236 \mathrm{~km} \mathrm{~s}^{-1}$ ) indicates a warp. The decline of the rotation curve indicated by the positionvelocity map is probably real. An unvelocity map is probably real. An un-
likely inclination of 49 degrees would likely inclination of 49 degrees would
be required instead of the adopted 67 degrees to give a flat curve.

The position-velocity diagrams are presented at the highest spatial resolution. The other data are shown for a resolution of $30 \times 30$ arcsec.

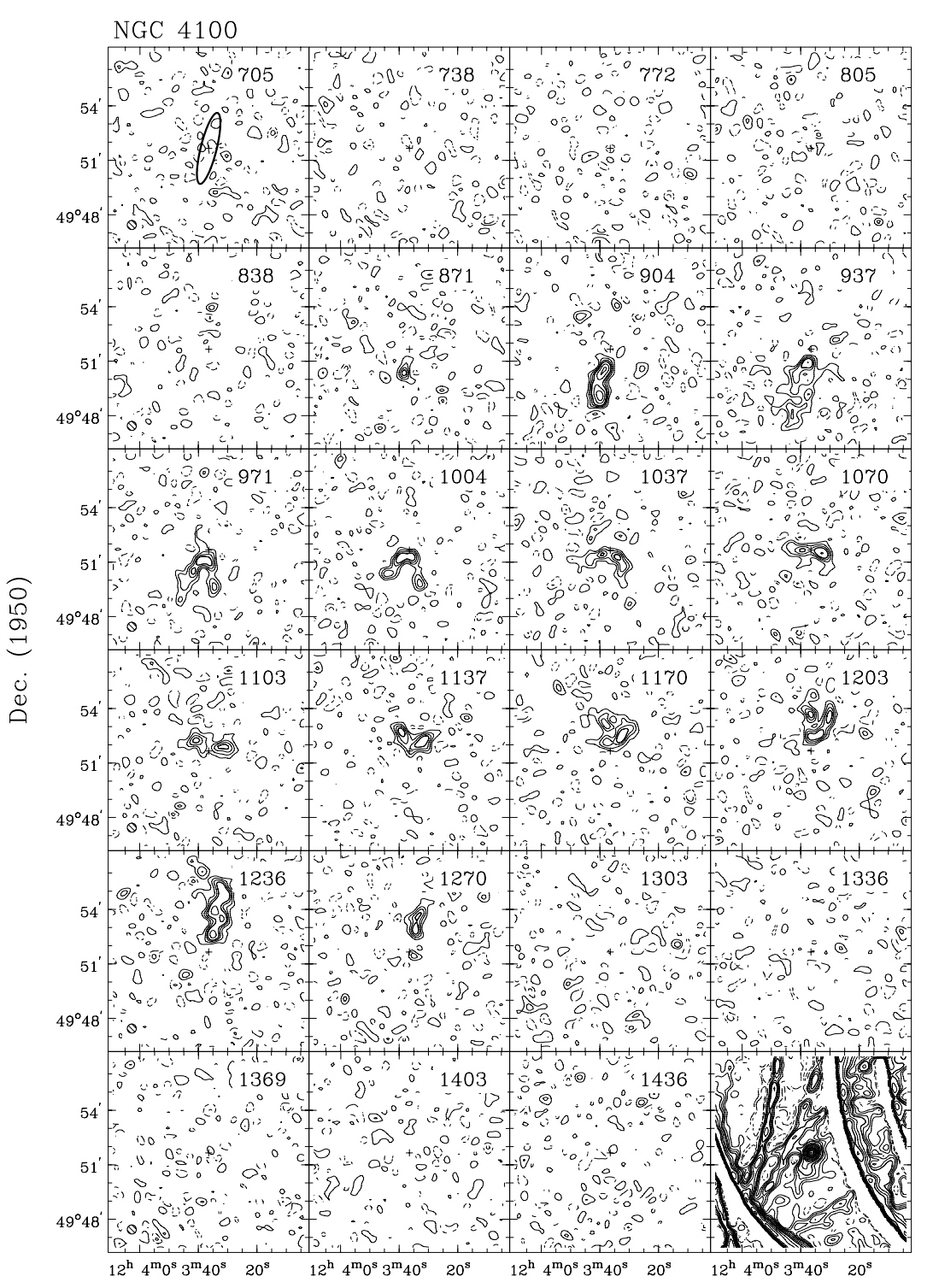

Channel maps at a resolution of $30^{\prime \prime} \times 30^{\prime \prime} \times 20 \mathrm{~km} \mathrm{~s}^{-1}$. Contour levels at $-3,-1.5$ (dashed), $1.5,3,4.5, \ldots \times \sigma$. 

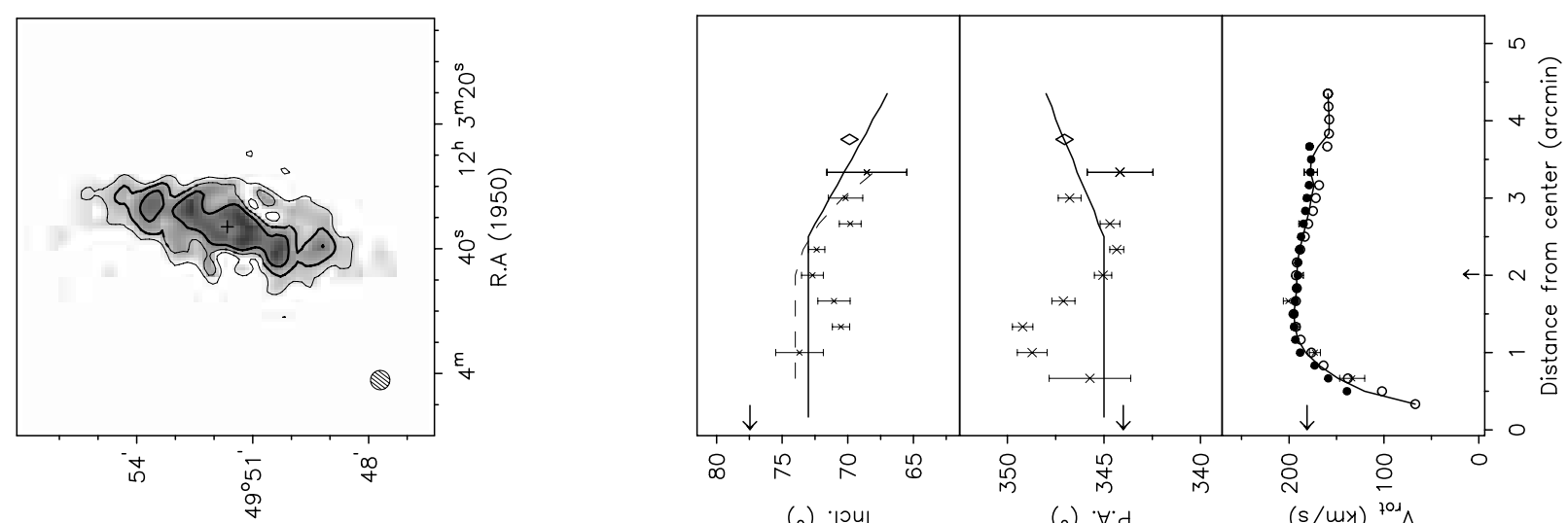

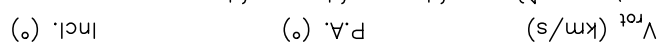

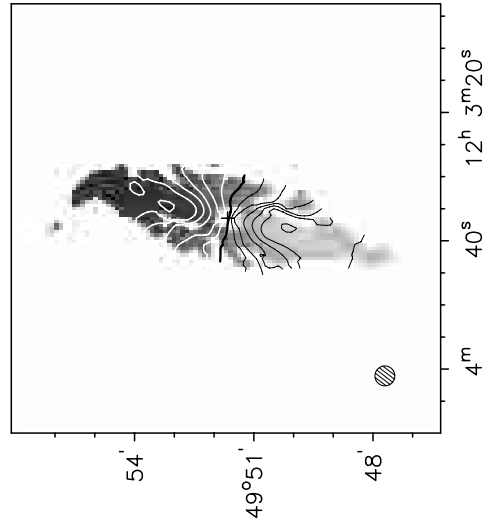

(096l) : วә0

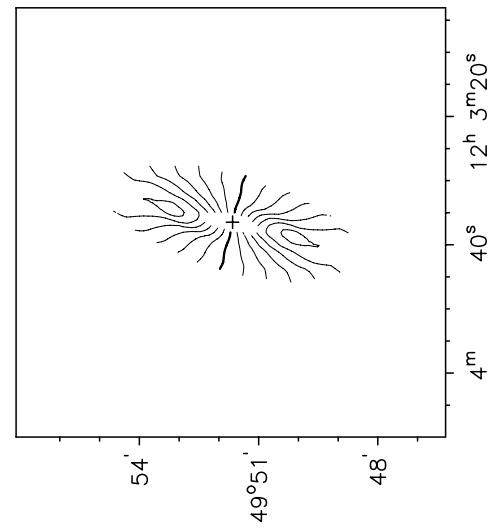

(096l) $\cdot 5 ว 0$

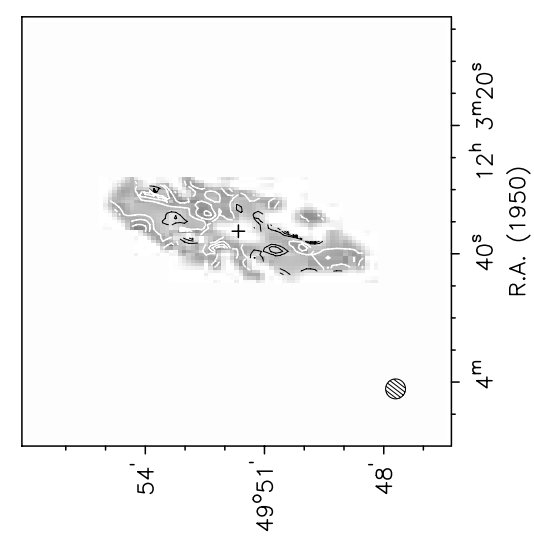

$(096 \mathrm{l}) \cdot 2 ә 0$
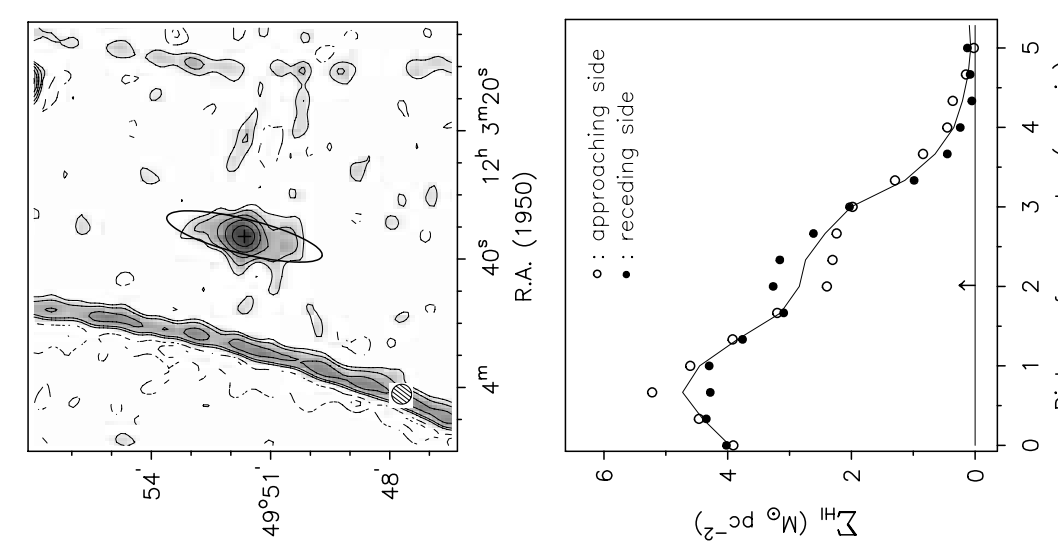

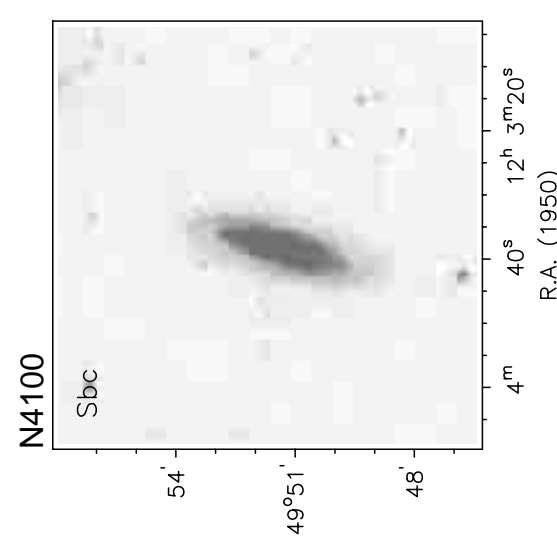

$(0961) \cdot 5 ə 0$

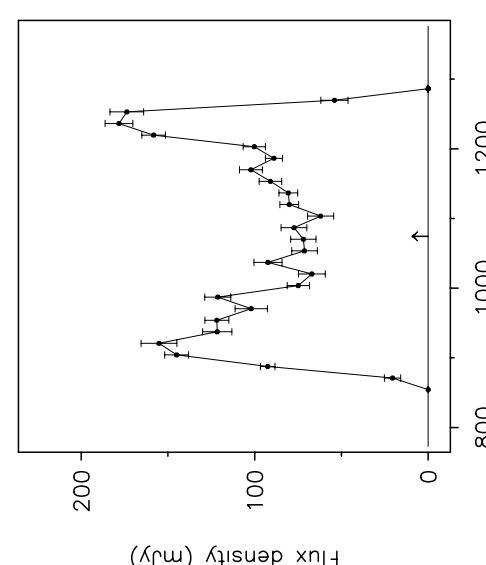

(кৎس) Kł!suap xn!J

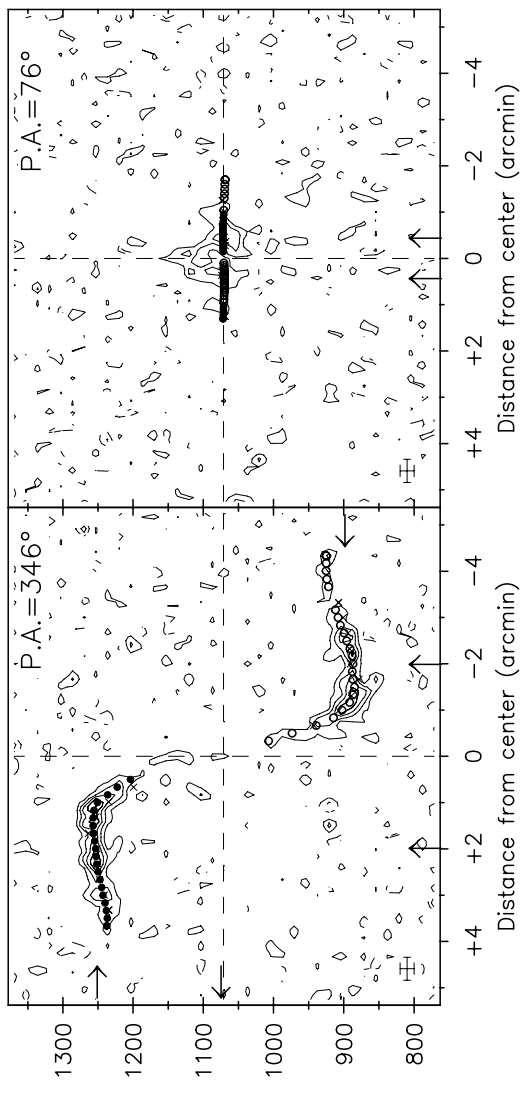

$(s / m y){ }^{194} \wedge$ 
Observing parameters for NGC 4102

\begin{tabular}{|c|c|}
\hline Length of observation & $4 \times 12$ \\
\hline Dates of observation & 11Aug90 \\
\hline Field center, $\alpha(1950)$ & 12:03:50 \\
\hline$\delta(1950)$ & 53:02:00 \\
\hline Central frequency & 1416.37 \\
\hline$V_{\text {hel }}$ of central channel & 850 \\
\hline Primary beam FWHM (arcmin) & 37.4 \\
\hline Nr. of interferometers & 80 \\
\hline Baselines (min-max-incr) & $36-2736-36$ \\
\hline Synthesized beam $(\alpha \times \delta)(\operatorname{arcsec})$ & $12.1 \times 15.1$ \\
\hline Bandwidth & 2.5 \\
\hline Number of channels & 127 \\
\hline Channel separation & 4.15 \\
\hline Velocity resolution & 8.29 \\
\hline rms noise in one channel $(\mathrm{K})$ & 3.30 \\
\hline $\begin{array}{l}\text { K-mJy conversion, } \\
\text { equiv. of } 1 \mathrm{mJy} / \text { beam }\end{array}$ & 3.30 \\
\hline
\end{tabular}

Results from WSRT data

\begin{tabular}{|c|c|}
\hline \multicolumn{2}{|l|}{ From continuum map: } \\
\hline 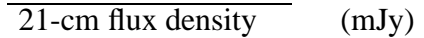 & $276.0 \pm 1.5$ \\
\hline \multicolumn{2}{|l|}{ Central point source position } \\
\hline$\alpha(1950)$ & $12: 03: 51.2$ \\
\hline$\delta(1950)$ & $52: 59: 21$ \\
\hline \multicolumn{2}{|l|}{ From global profile: } \\
\hline$\overline{\text { Integrated HI-flux }}\left(\mathrm{Jy} \mathrm{km} \mathrm{s}^{-1}\right)$ & $8.0 \pm 0.2$ \\
\hline Hel. systemic velocity $\left(\mathrm{km} \mathrm{s}^{-1}\right)$ & $846.3 \pm 2.0$ \\
\hline HI profile width, $20 \%\left(\mathrm{~km} \mathrm{~s}^{-1}\right)$ & $349.8 \pm 2.0$ \\
\hline $50 \%\left(\mathrm{~km} \mathrm{~s}^{-1}\right)$ & $322.4 \pm 8.5$ \\
\hline \multicolumn{2}{|l|}{ From velocity field: } \\
\hline 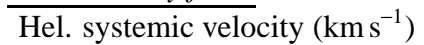 & $845.4 \pm 4.0$ \\
\hline Dynamical center, $\alpha(1950)$ & $12: 03: 51.2$ \\
\hline$\delta(1950)$ & $52: 59: 21$ \\
\hline \multicolumn{2}{|l|}{ From total HI map: } \\
\hline$\overline{\text { Geometric center, } \alpha(1950)}$ & $12: 03: 51.2$ \\
\hline$\delta(1950)$ & $52: 59: 22$ \\
\hline Position angle & 28 \\
\hline Inclination angle & 56 \\
\hline Diameter of HI disk (arcmin) & 2.3 \\
\hline
\end{tabular}

Contour levels for N4102

\begin{tabular}{|c|c|}
\hline \multicolumn{2}{|l|}{ Channel maps: } \\
\hline $\begin{array}{l}\text { Channel maps: } \\
\text { Raw continuum map: }\end{array}$ & $\sigma=1.25(\mathrm{~K})$ \\
\hline \multicolumn{2}{|c|}{$\begin{array}{l}\text { Cleaned continuum map: } \\
\qquad \sigma=1.09(\mathrm{~K})\end{array}$} \\
\hline \multicolumn{2}{|c|}{$\begin{array}{l}\text { Position-Velocity diagrams: } \\
\qquad \sigma=1 .\end{array}$} \\
\hline $\begin{array}{l}\text { Velocity fields: } \\
\qquad 845.4 \pm \\
\text { Residual velocity field }\end{array}$ & $25\left(\mathrm{~km} \mathrm{~s}^{-1}\right)$ \\
\hline $\begin{array}{c}\text { Integrated HI map: } \\
0.54,1.09 \\
1.63(\times 1\end{array}$ & $\begin{array}{l}\mathrm{n} \times 5\left(\mathrm{~km} \mathrm{~s}^{-1}\right) \\
{ }^{1} \text { atoms cm} \\
\end{array}$ \\
\hline
\end{tabular}

Note: This LINER galaxy shows HI in absorption against the central radio continuum source. The absorption is seen over the full velocity width of the global profile. The rotation curve reaches its maximum very close to the center.

The HI emission is concentrated in a ring-like structure that encompasses the bar. The position of the strong continuum source is taken as the dynamical center. The position angle derived from the total HI map is not meaningful due to the patchy-ness of the HI gas.

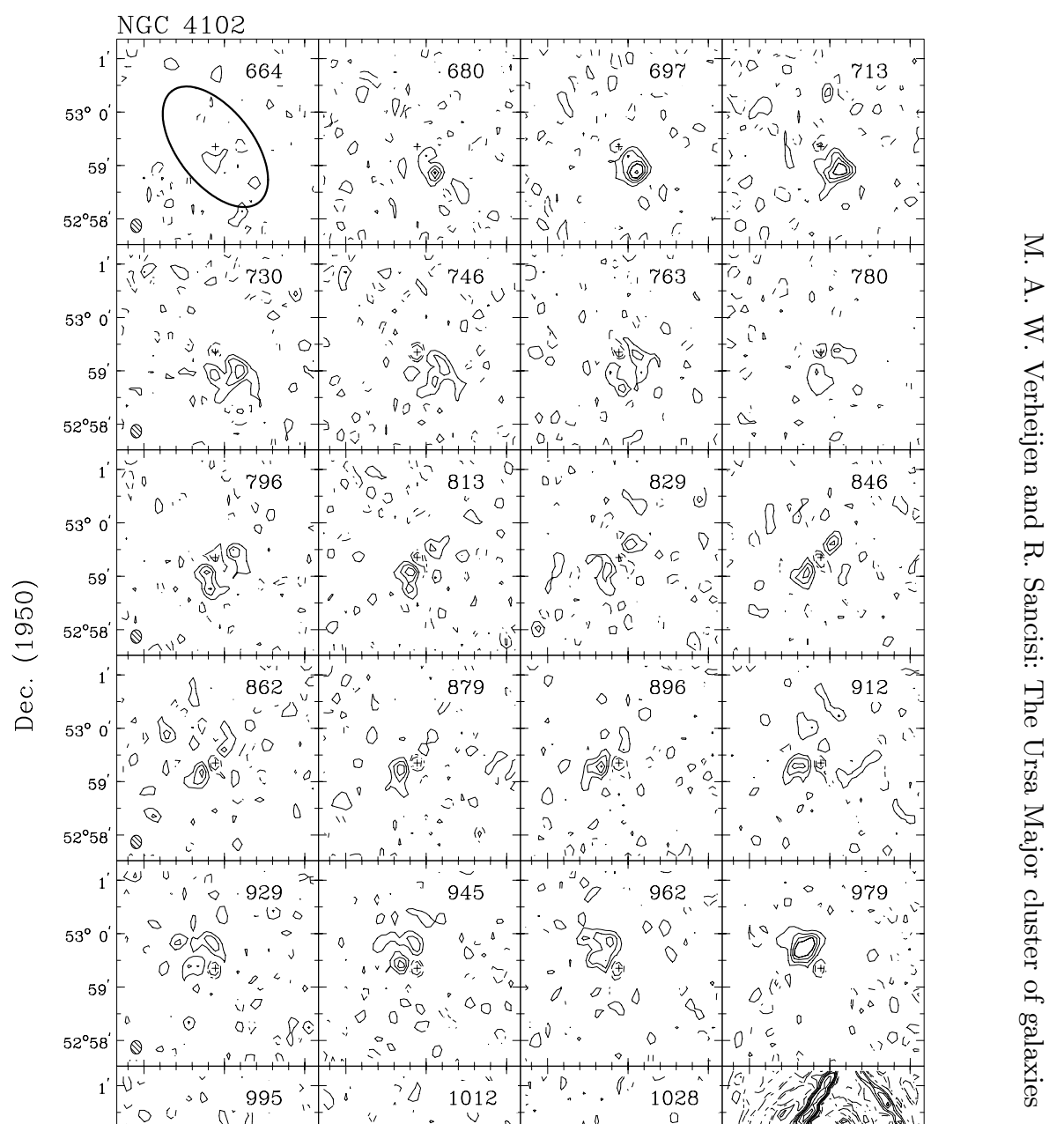

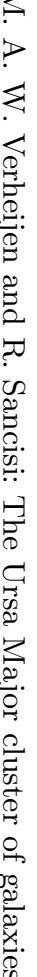



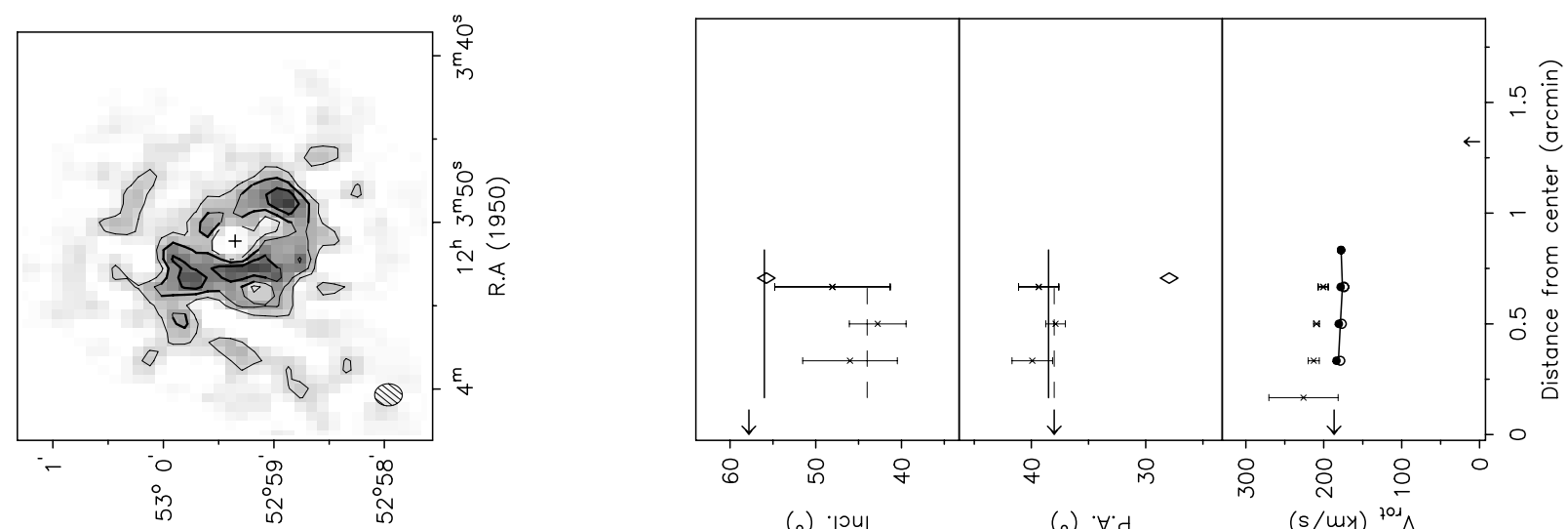

(०) $\cdot \mid \mathrm{ou}$

(o) $\forall \cdot d$

$(s / m y){ }^{701} \wedge$

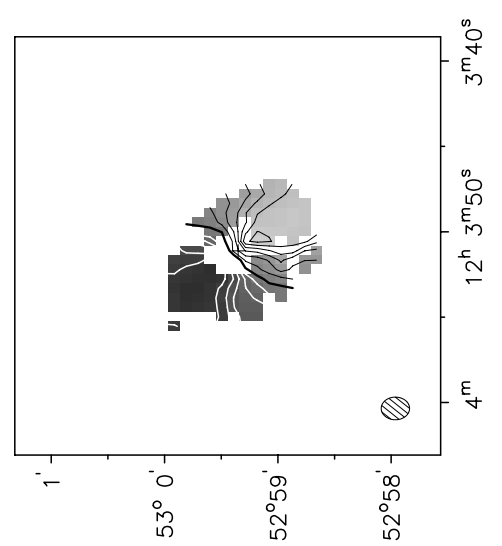

(0g6l) $\cdot 200$
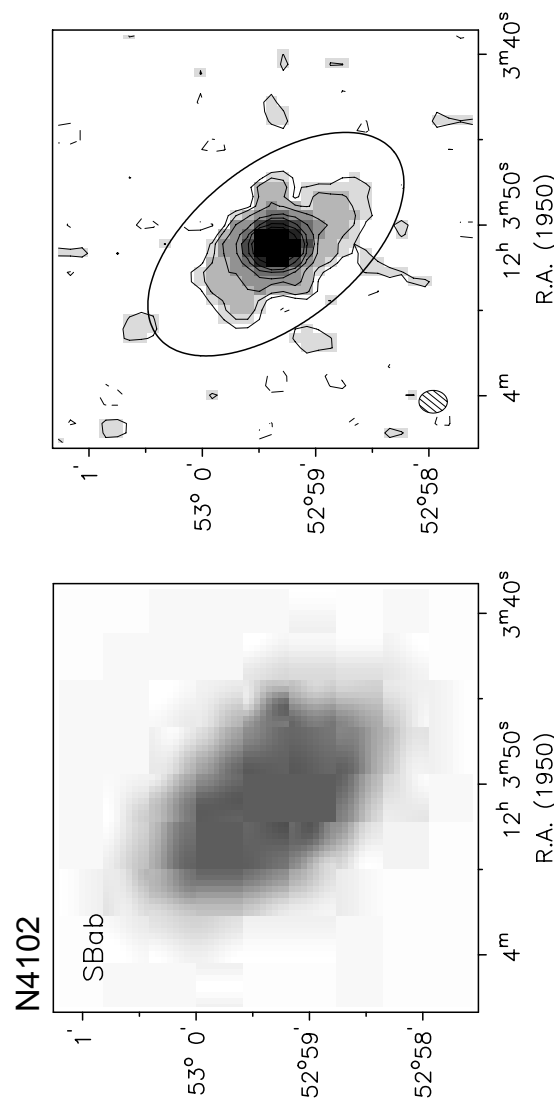

(0s6l) $\cdot 2 a 0$

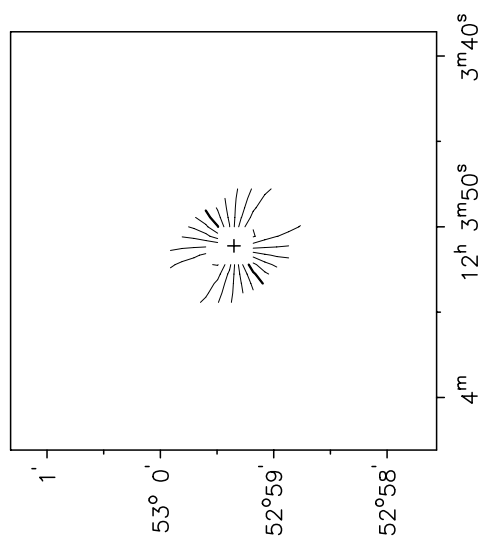

$(0 \mathrm{og} 6) \cdot 200$

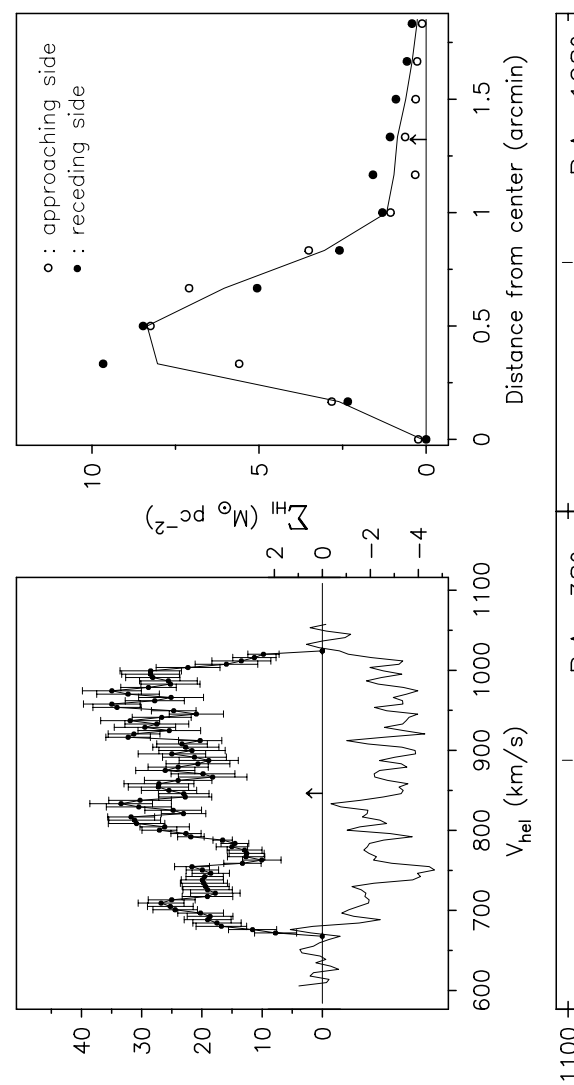

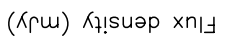
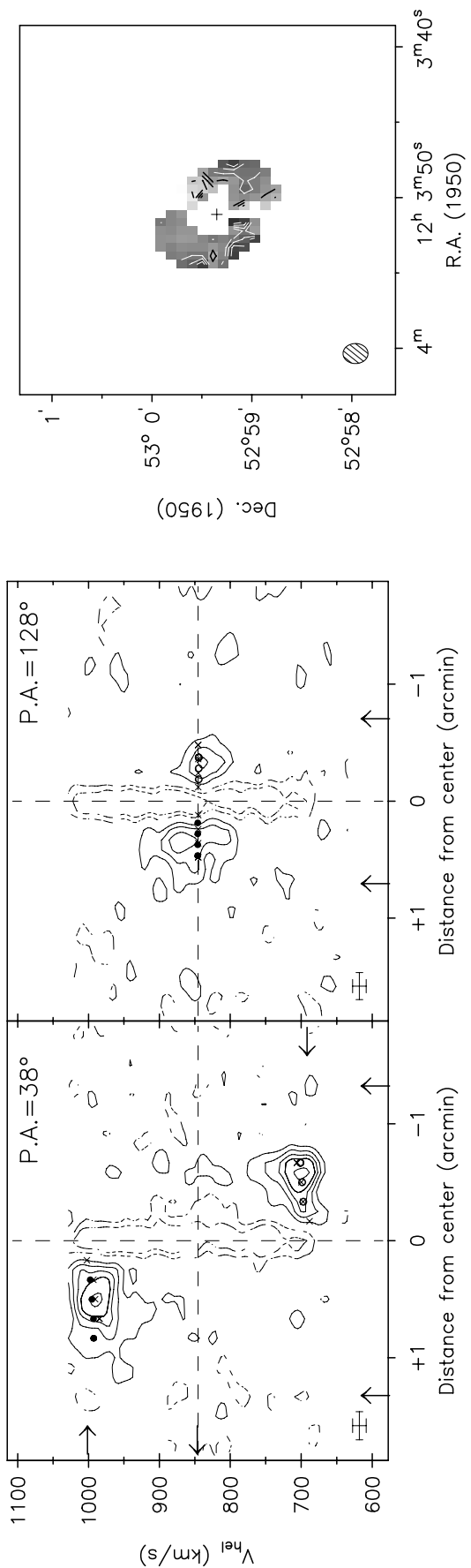
Observing parameters for NGC 4157

\begin{tabular}{|c|c|}
\hline Length of observation & $1 \times 12$ \\
\hline Date of observation & 29,31 Aug93 \\
\hline Field center, $\alpha(1950)$ & $12: 08: 35$ \\
\hline$\delta(1950)$ & $50: 45: 51$ \\
\hline Central frequency & 1416.75 \\
\hline$V_{\text {hel }}$ of central channel & 774 \\
\hline Primary beam FWHM (arcmin) & 37.4 \\
\hline Nr. of interferometers & 40 \\
\hline Baselines (min-max-incr) & $36-2700-72$ \\
\hline Synthesized beam $(\alpha \times \delta)(\operatorname{arcsec})$ & $11.8 \times 16.7$ \\
\hline Bandwidth & 5.0 \\
\hline Number of channels & 63 \\
\hline Channel separation & 16.57 \\
\hline Velocity resolution & 19.88 \\
\hline rms noise in one channel & 4.89 \\
\hline $\begin{array}{l}\text { K-mJy conversion, } \\
\text { equiv. of } 1 \mathrm{mJy} / \text { beam }\end{array}$ & 3.06 \\
\hline
\end{tabular}

Results from WSRT data

\begin{tabular}{|c|c|}
\hline \multicolumn{2}{|l|}{ From continuum map: } \\
\hline $\begin{array}{l}21-\mathrm{cm} \text { flux density } \\
\text { From global profile: }\end{array}$ & $179.6 \pm 2.3$ \\
\hline$\overline{\text { Integrated HI-flux }}\left(\mathrm{Jy} \mathrm{km} \mathrm{s}^{-1}\right)$ & $107.4 \pm 1.6$ \\
\hline Hel. systemic velocity $\left(\mathrm{km} \mathrm{s}^{-1}\right)$ & $774.4 \pm 1.8$ \\
\hline HI profile width, $20 \%\left(\mathrm{~km} \mathrm{~s}^{-1}\right)$ & $427.6 \pm 2.2$ \\
\hline $50 \%\left(\mathrm{~km} \mathrm{~s}^{-1}\right)$ & $400.7 \pm 3.1$ \\
\hline \multicolumn{2}{|l|}{ From velocity field: } \\
\hline 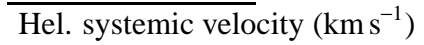 & $774.1 \pm 1.3$ \\
\hline $\begin{array}{r}\text { Dynamical center, } \alpha(1950) \\
\delta(1950)\end{array}$ & $\begin{array}{l}12: 08: 34.4 \\
50: 45: 50\end{array}$ \\
\hline \multicolumn{2}{|l|}{ From total HI map: } \\
\hline$\overline{\text { Geometric center, } \alpha(1950)}$ & $\begin{array}{l}12: 08: 32.7 \\
50 \cdot 45 \cdot 41\end{array}$ \\
\hline Position angle & 66 \\
\hline Inclination angle & 80 \\
\hline Diameter of HI disk (arcmin) & 9.2 \\
\hline
\end{tabular}

Contour levels for N4157

Channel maps: $\quad \sigma=1.34(\mathrm{~K})$

Raw continuum map:

Cleaned continuum map: $\sigma=0.33(\mathrm{~K})$

Position-Velocity diagrams:

Velocity fields:

$774.1 \pm \mathrm{n} \times 30\left(\mathrm{~km} \mathrm{~s}^{-1}\right)$ Residual velocity field: $\pm \mathrm{n} \times 5\left(\mathrm{~km} \mathrm{~s}^{-1}\right)$

Integrated HI map:

$0.39,0.78,1.16,1.55$ $1.94,2.33\left(\times 10^{21}\right.$ atoms cm $\left.^{-2}\right)$

Note: Because of the high inclination of NGC 4157 the velocity field derived here with the standard methods is not adequate for a detailed dynamical study. The formal results from the tilted-ring fits are merely shown for completeness and to give the global picture. The inclination of almost 90 degrees as derived from the optical axis ratio assuming an intrinsic thickness of 0.2 is definitely too high given the dust features in the optical image.

The rotation curve is declining by about $20 \mathrm{~km} \mathrm{~s}^{-s}$ at $\approx 2^{\prime}$ from the center and remains flat in the outer parts. To explain such a decline as due to inclination effects, the inclination angle would have to become 65 degrees in the outer parts. Such a low inclination is excluded by the axis ratio of the total HI map. Therefore, the rotation curve must map. Therefore, the rotation curve must cmin.

The extended radio continuum emission is asymmetric with respect to the center of the galaxy and originates partly in the halo.

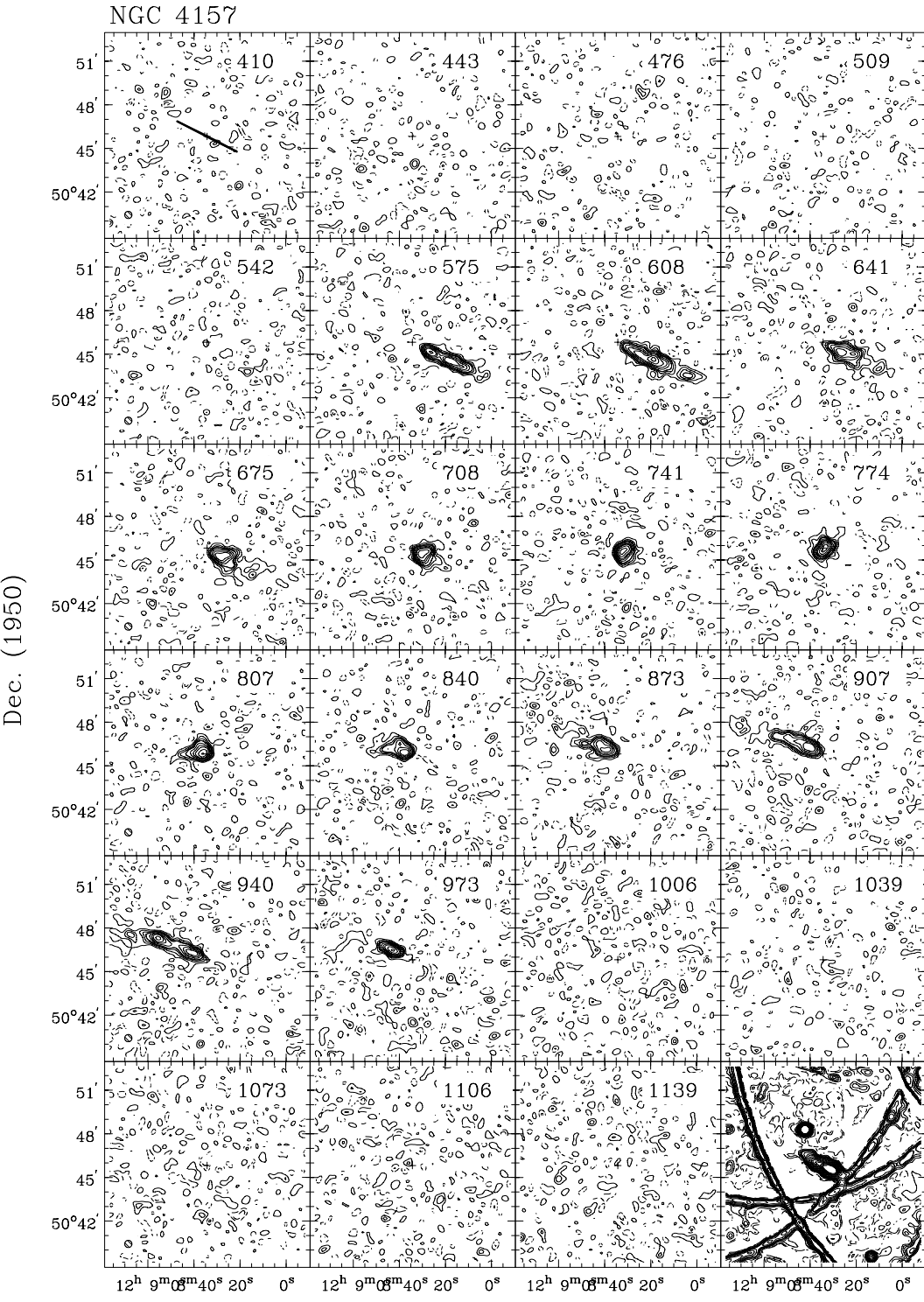

Channel maps at a resolution of $30^{\prime \prime} \times 30^{\prime \prime} \times 20 \mathrm{~km} \mathrm{~s}^{-1}$.

Contour levels at $-3,-1.5$ (dashed), $1.5,3,4.5, \ldots \times \sigma$. 

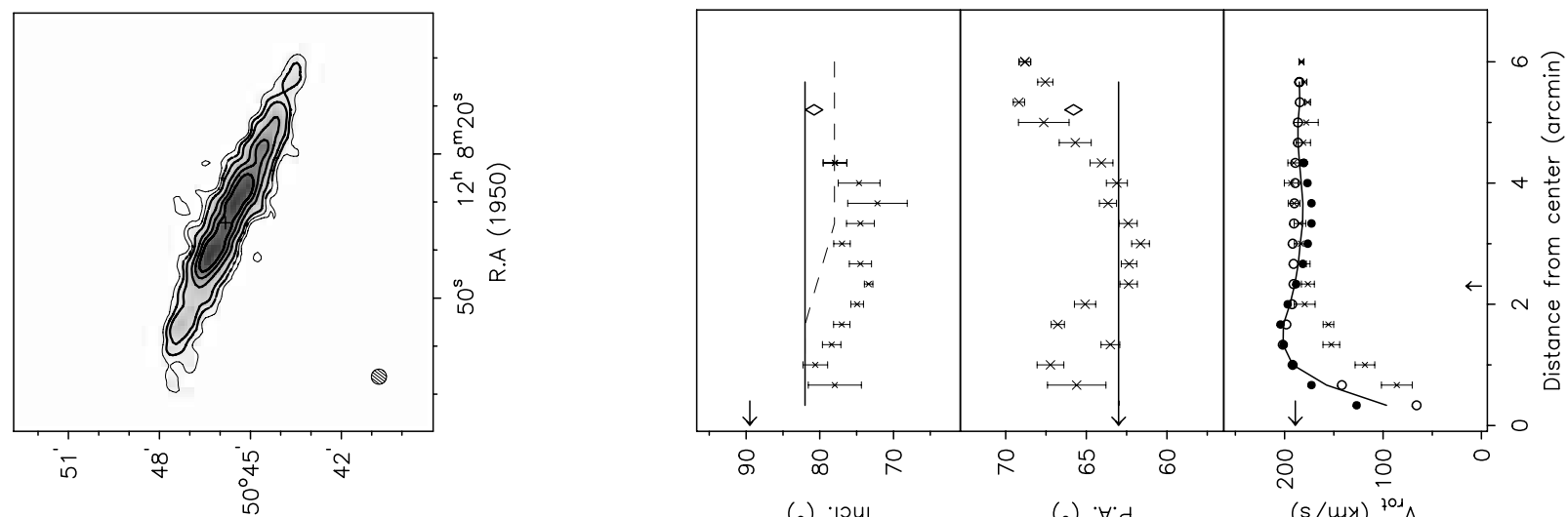

(०) '|⿰u|

(o) $\forall \cdot d$

$(s / m y){ }^{701} \wedge$

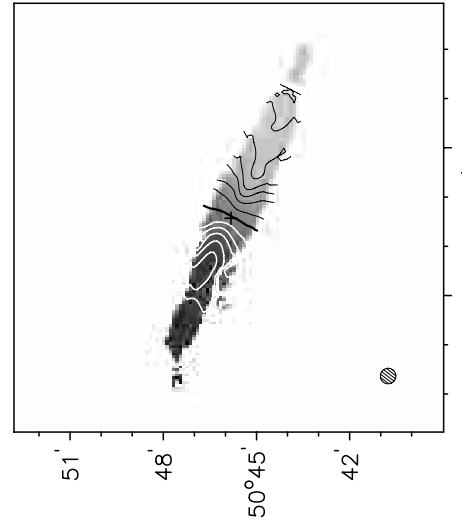

(096l) $\cdot 2$ م

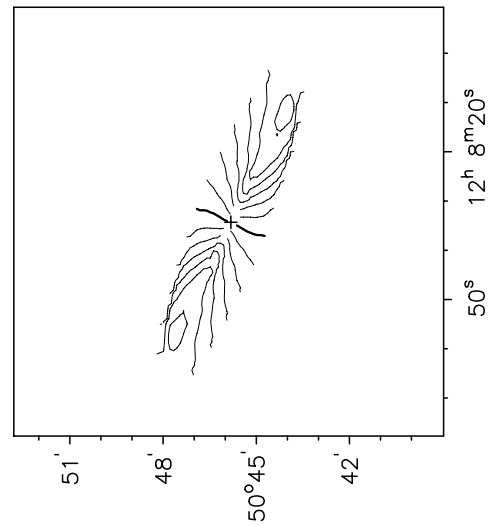

(0961) :

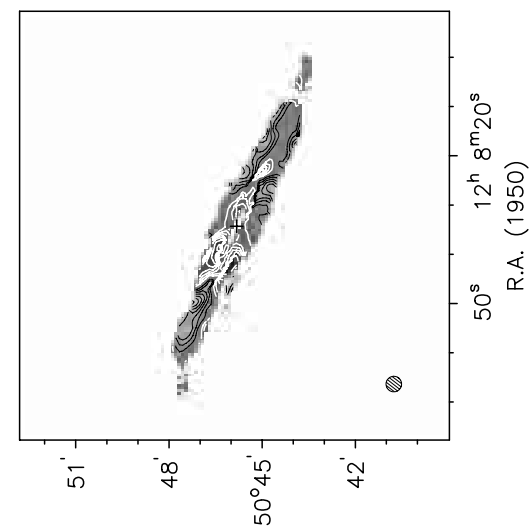

(0s6l) $\cdot 520$
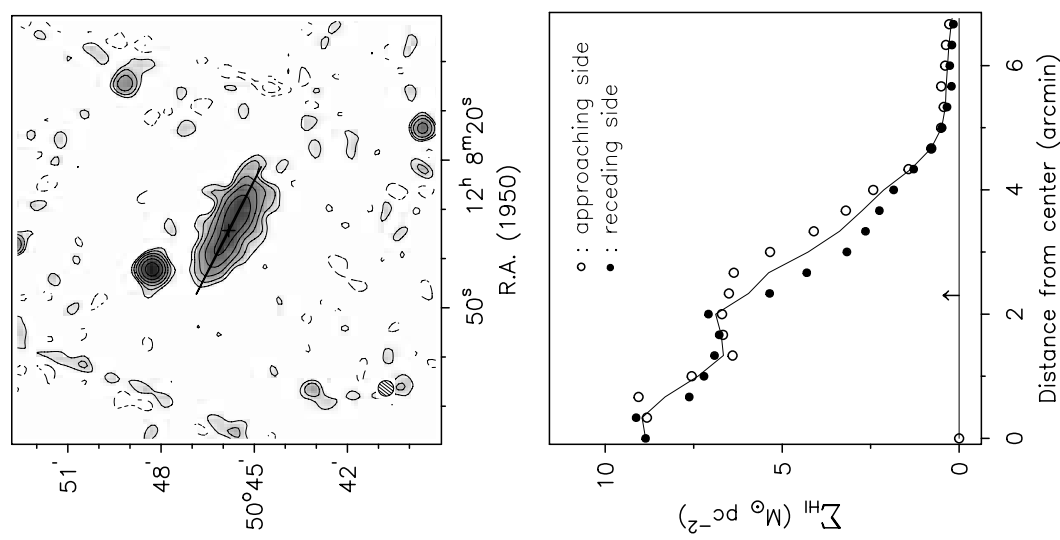

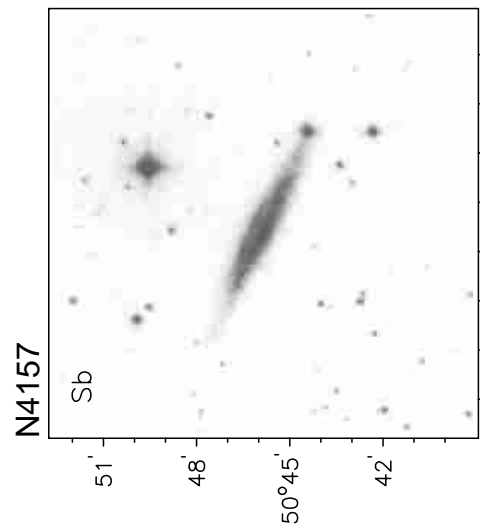

(096l) $\cdot 2 \circ 0$

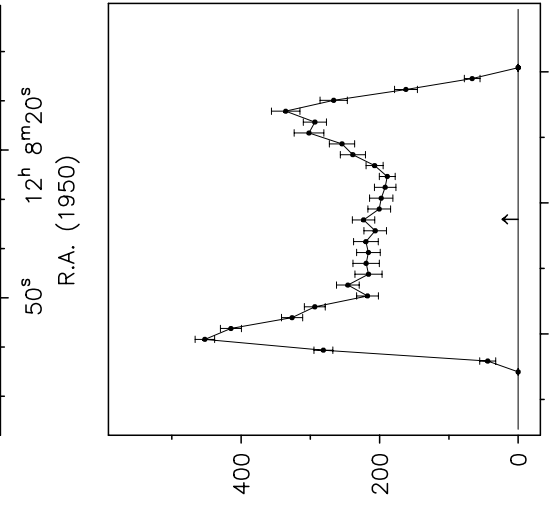

(кৎس) Kұ!suap xn!J

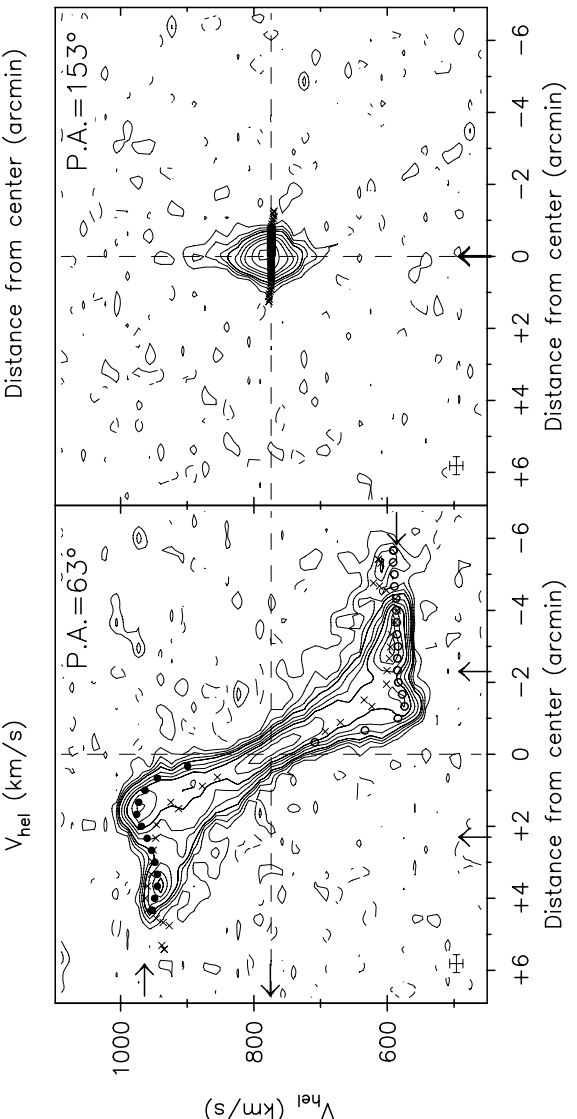


Observing parameters for NGC 4183

\begin{tabular}{|c|c|}
\hline Length of observation & $1 \times 12$ \\
\hline Date of observation & 02Aug93 \\
\hline Field center, $\alpha(1950)$ & $12: 10: 47$ \\
\hline$\delta(1950)$ & 43:58:35 \\
\hline Central frequency & 1415.95 \\
\hline$V_{\text {hel }}$ of central channel & 932 \\
\hline Primary beam FWHM & 37.4 \\
\hline Nr. of interferometers & 40 \\
\hline Baselines (min-max-incr) & $36-2700-72$ \\
\hline Synthesized beam $(\alpha \times \delta)(\operatorname{arcsec})$ & $11.8 \times 17.2$ \\
\hline Bandwidth & 2.5 \\
\hline Number of channels & 127 \\
\hline Channel separation & 4.15 \\
\hline Velocity resolution & 8.30 \\
\hline rms noise in one channel & 5.93 \\
\hline $\begin{array}{l}\text { K-mJy conversion, } \\
\text { equiv. of } 1 \mathrm{mJy} / \text { beam }\end{array}$ & 2.97 \\
\hline
\end{tabular}

Results from WSRT data

\begin{tabular}{|c|c|}
\hline \multicolumn{2}{|l|}{ From continuит map: } \\
\hline \multicolumn{2}{|l|}{ 21-cm flux density } \\
\hline central point source $(\mathrm{mJy})$ & $<1.5(3 \sigma)$ \\
\hline extended source (mJy) & $<5.8(3 \sigma)$ \\
\hline \multicolumn{2}{|l|}{ From global profile: } \\
\hline Integrated HI-flux $\left(\mathrm{Jy} \mathrm{km} \mathrm{s}^{-1}\right)$ & $48.9 \pm 0.7$ \\
\hline Hel. systemic velocity $\left(\mathrm{km} \mathrm{s}^{-1}\right)$ & $930.1 \pm 1.0$ \\
\hline HI profile width, $20 \%\left(\mathrm{~km} \mathrm{~s}^{-1}\right)$ & $249.6 \pm 1.2$ \\
\hline $50 \%\left(\mathrm{~km} \mathrm{~s}^{-1}\right)$ & $232.5 \pm 1.5$ \\
\hline \multicolumn{2}{|l|}{ From velocity field: } \\
\hline 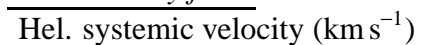 & $925.5 \pm 1.5$ \\
\hline Dynamical center, $\alpha(1950)$ & $12: 10: 46.2$ \\
\hline$\delta(1950)$ & 43:58:33 \\
\hline \multicolumn{2}{|l|}{ From total HI map: } \\
\hline Geometric center, $\alpha(1950)$ & $12: 10: 45.8$ \\
\hline$\delta(1950)$ & $43: 58: 41$ \\
\hline Position angle & 347 \\
\hline Inclination angle & 83 \\
\hline Diameter of HI disk (arcmin) & 6.1 \\
\hline
\end{tabular}

Contour levels for N4183

Channel maps: $\quad \sigma=3.86(\mathrm{~K})$

Raw continuum map:

Cleaned continuum map $\sigma=1.51(\mathrm{~K})$

Position-Velocity diagrams:

Velocity fields:

$$
\sigma=3.34(\mathrm{~K})
$$

$925.5 \pm \mathrm{n} \times 20\left(\mathrm{~km} \mathrm{~s}^{-1}\right)$

Residual velocity field: $\pm \mathrm{n} \times 5\left(\mathrm{~km} \mathrm{~s}^{-1}\right)$

Integrated HI map:

$1.06,2.13,3.19$

$4.26\left(\times 10^{21}\right.$ atoms cm $\left.{ }^{-2}\right)$

Note: This galaxy is slightly warped in the outer regions. The inclination derived from the optical axis ratio is too high due to the assumed intrinsic thickness of 0.2 which is probably too large.

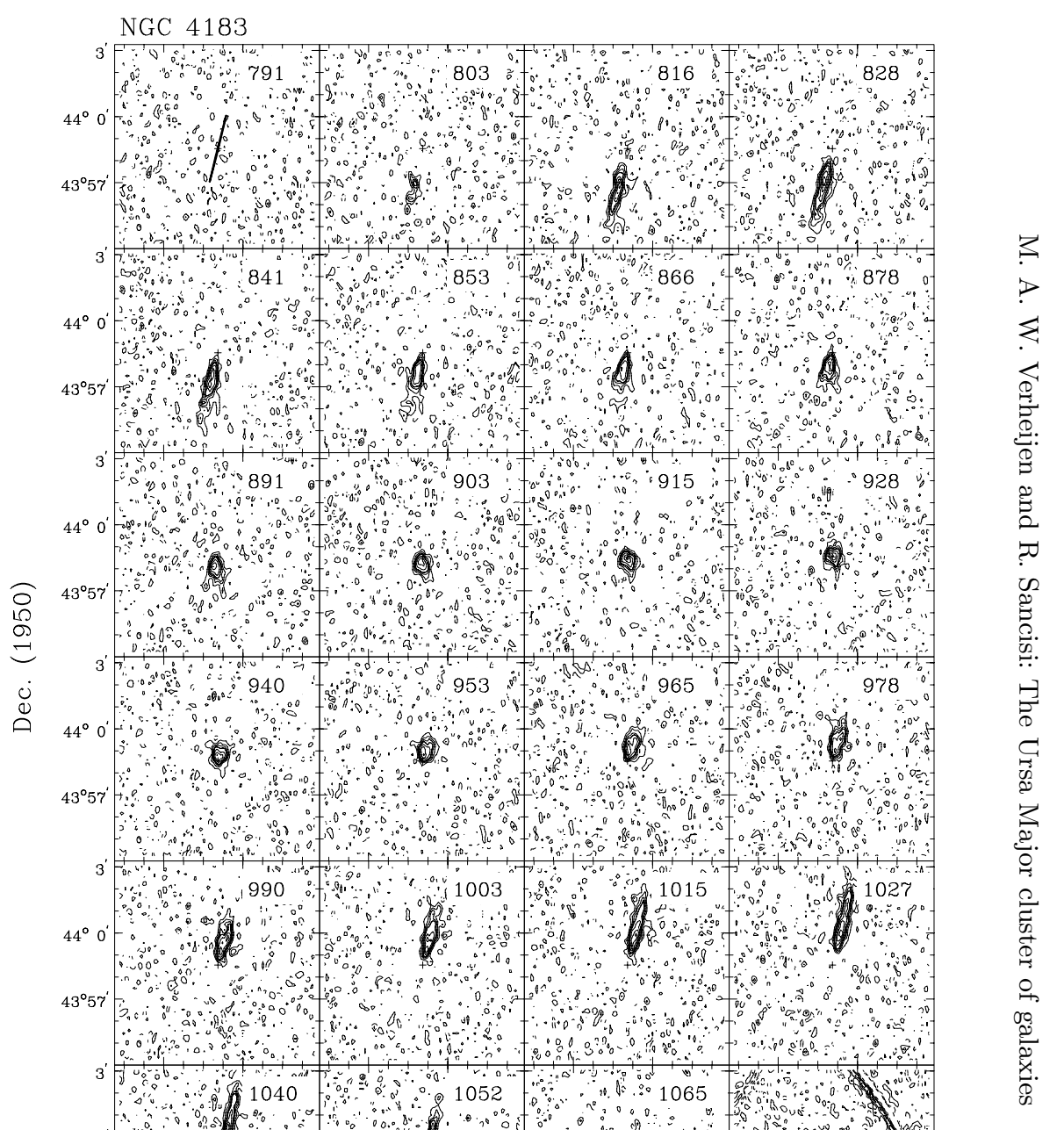

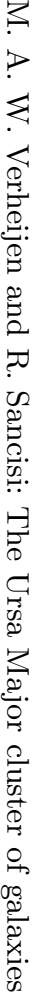



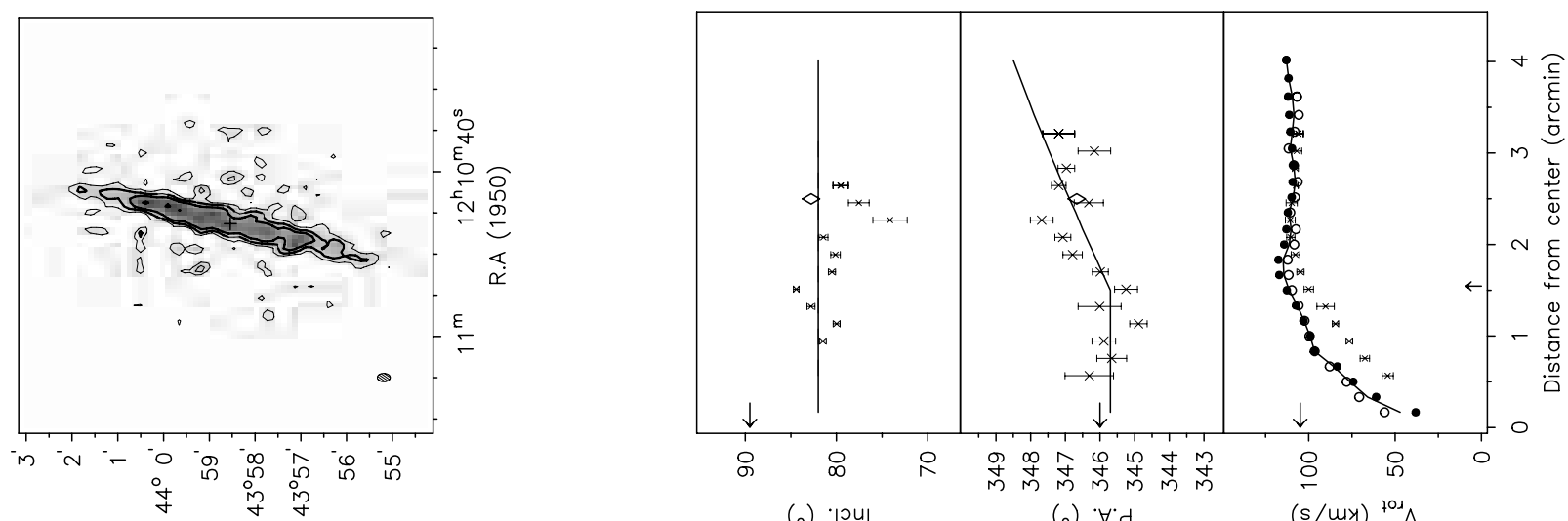
(०) '|⿰u|
(o) $\forall \cdot d$
$(s / m x){ }^{701} \wedge$

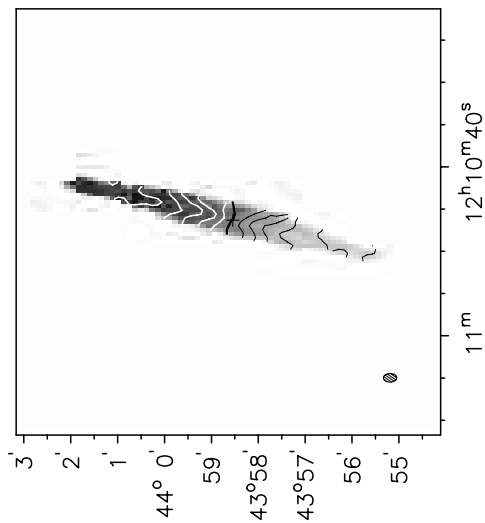

(056l) • गә0

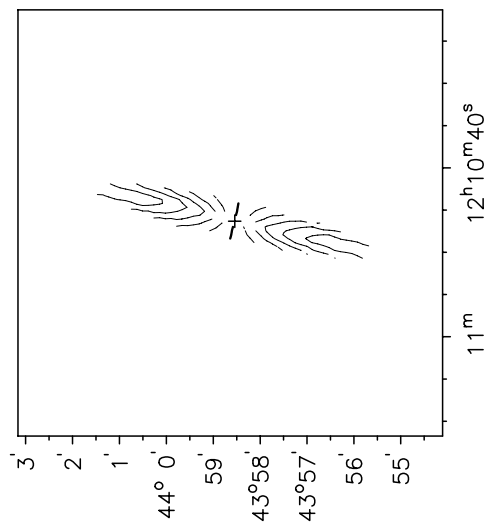

(056l) ग ग0

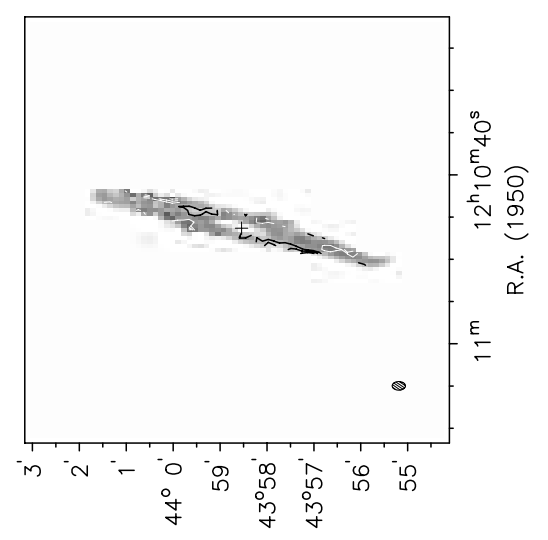

(096l)
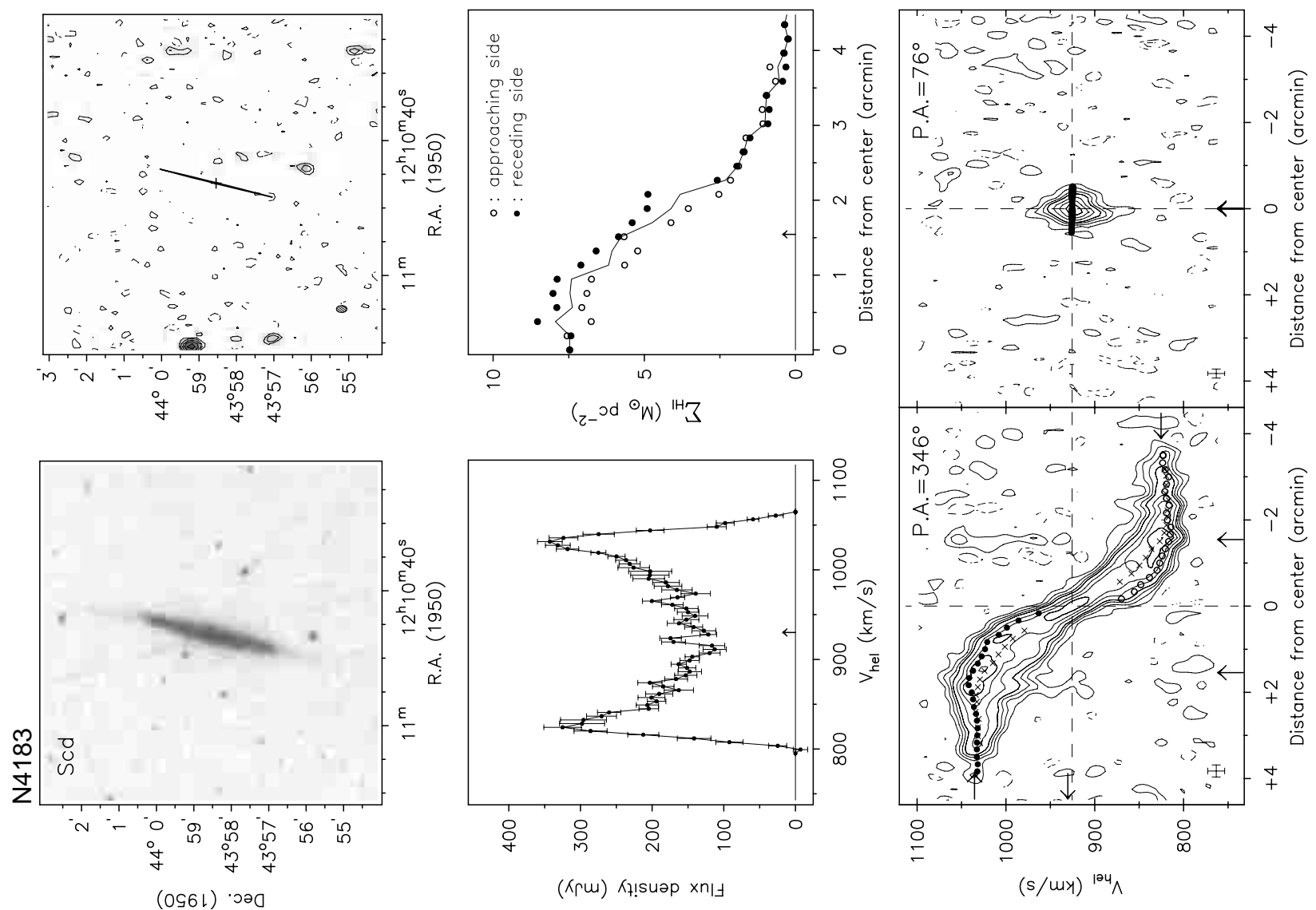
Observing parameters for NGC 4217

\begin{tabular}{|c|c|}
\hline Length of observation & $1 \times 12$ \\
\hline Date of observation & 16Aug90 \\
\hline Field center, $\alpha(1950)$ & $12: 13: 20$ \\
\hline$\delta(1950)$ & 47:20:00 \\
\hline Central frequency & 1415.52 \\
\hline$V_{\text {hel }}$ of central channel & 1030 \\
\hline Primary beam FWHM (arcmin) & 37.4 \\
\hline Nr. of interferometers & 40 \\
\hline Baselines (min-max-incr) & $36-2700-72$ \\
\hline Synthesized beam $(\alpha \times \delta)(\operatorname{arcsec})$ & $13.2 \times 18.6$ \\
\hline Bandwidth & 5.0 \\
\hline Number of channels & 63 \\
\hline Channel separation & 16.60 \\
\hline Velocity resolution & 33.20 \\
\hline rms noise in one channel & 2.45 \\
\hline $\begin{array}{l}\text { K-mJy conversion, } \\
\text { equiv. of } 1 \mathrm{mJy} / \text { beam }\end{array}$ & 2.45 \\
\hline
\end{tabular}

\section{Results from WSRT data}

\begin{tabular}{|c|c|}
\hline \multicolumn{2}{|l|}{ From continuum map: } \\
\hline $\begin{array}{l}21-\mathrm{cm} \text { flux density } \\
\text { From global profile: }\end{array}$ & $115.6 \pm 2.2$ \\
\hline Integrated HI-flux $\left(\mathrm{Jy} \mathrm{km} \mathrm{s}^{-1}\right)$ & $33.8 \pm 0.7$ \\
\hline Hel. systemic velocity $\left(\mathrm{km} \mathrm{s}^{-1}\right)$ & $1027.0 \pm 3.0$ \\
\hline HI profile width, $20 \%\left(\mathrm{~km} \mathrm{~s}^{-1}\right)$ & $428.1 \pm 5.1$ \\
\hline $50 \%\left(\mathrm{~km} \mathrm{~s}^{-1}\right)$ & $395.6 \pm 3.8$ \\
\hline From velocity field: & \\
\hline 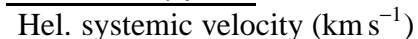 & $1028.8 \pm 2.7$ \\
\hline $\begin{array}{r}\text { Dynamical center, } \alpha(1950) \\
\delta(1950)\end{array}$ & $\begin{array}{l}12: 13: 21.6 \\
47: 22: 10\end{array}$ \\
\hline From total HI map: & \\
\hline $\begin{array}{r}\overline{\text { Geometric center, }} \alpha(1950) \\
\delta(1950)\end{array}$ & $\begin{array}{l}12: 13: 21.8 \\
47: 22: 11\end{array}$ \\
\hline Position angle & 230 \\
\hline Inclination angle & 85 \\
\hline Diameter of HI disk (arcmin) & 6.4 \\
\hline
\end{tabular}

Contour levels for N4217

\begin{tabular}{|c|c|}
\hline \\
\hline $\begin{array}{l}\text { Channel maps: } \\
\text { Raw continuum map: }\end{array}$ & $\sigma=0.93(\mathrm{~K})$ \\
\hline \multicolumn{2}{|c|}{ Cleaned continuum map: } \\
\hline Position-Velocity diag & $\begin{array}{l}\text { Ims: } \\
\sigma=2.21(\mathrm{~K})\end{array}$ \\
\hline $\begin{array}{l}\text { Velocity fields: } \\
\qquad 1028.8 \pm \mathrm{r} \\
\text { Residual velocity field }\end{array}$ & $\times 30\left(\mathrm{~km} \mathrm{~s}^{-1}\right)$ \\
\hline $\begin{array}{c}\text { Integrated HI map: } \\
0.91,1.82,2.74 \\
3.65(\times 10\end{array}$ & $\begin{array}{l}\times 5\left(\mathrm{~km} \mathrm{~s}^{-1}\right) \\
\text { atoms cm} \\
-2\end{array}$ \\
\hline
\end{tabular}

Note: This early type nearly edge-on spiral shows extended $21 \mathrm{~cm}$ continuum emission. Some HI line emission may be absorbed by the strong continuum source. The inclination derived from the optical axis ratio is probably an underestimate of the true inclination because the prominent bulge makes the galaxy look rounder than the assumed intrinsic thickness of 0.2 .

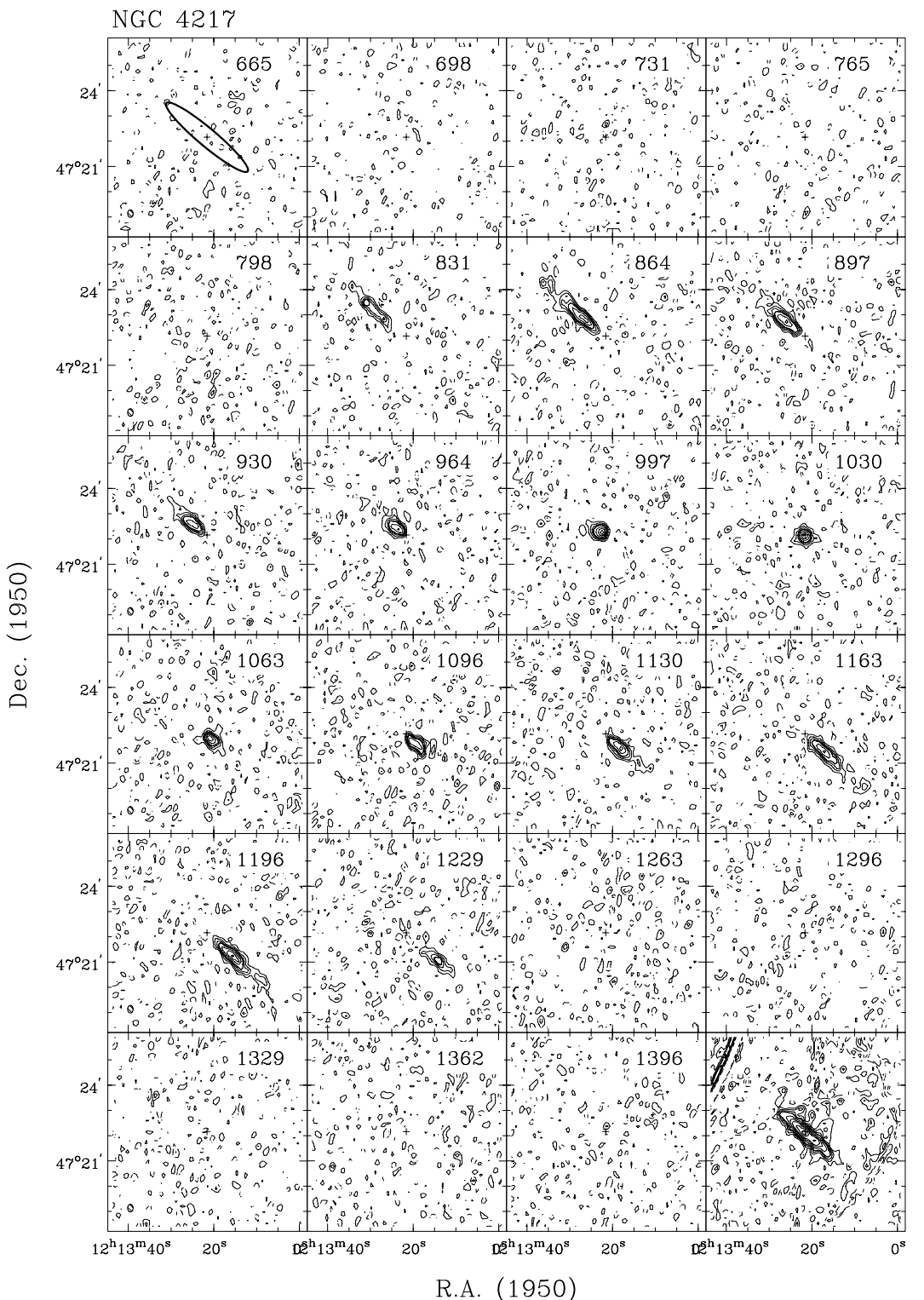

Channel maps at a resolution of $13^{\prime \prime} \times 18^{\prime \prime} \times 33 \mathrm{~km} \mathrm{~s}^{-1}$. Contour levels at $-3,-1.5$ (dashed), $1.5,3,4.5, \ldots \times \sigma$. 

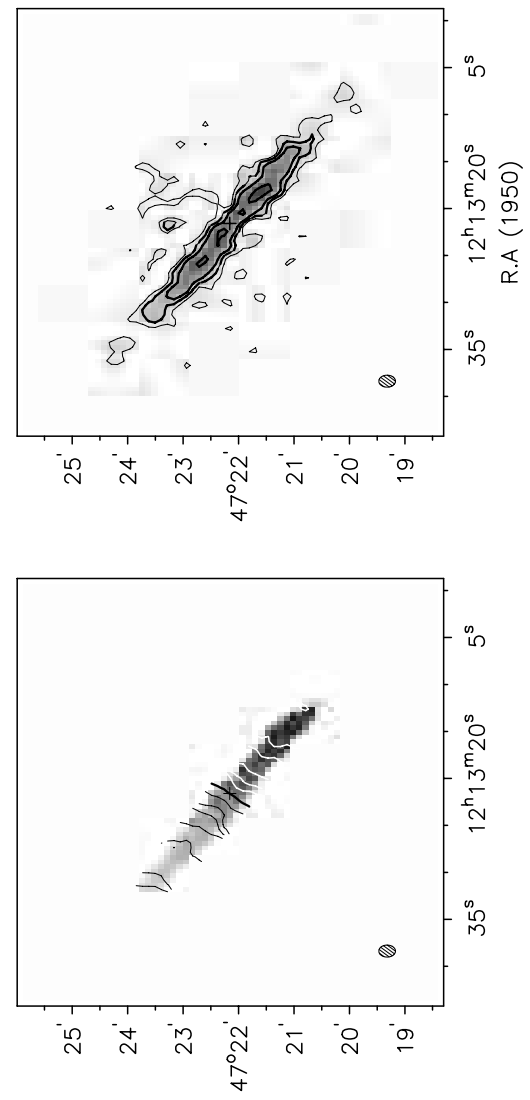

(096l) $\cdot 020$

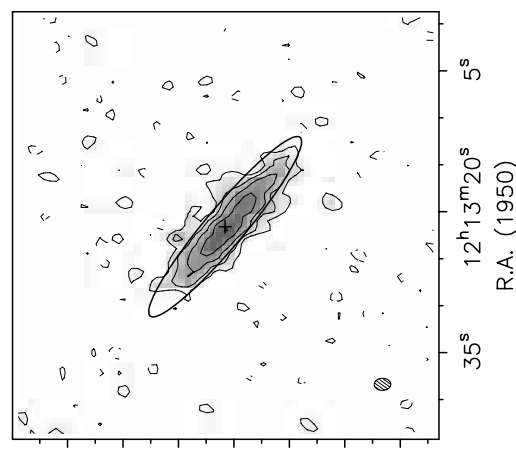

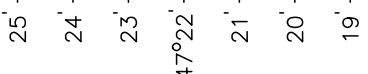

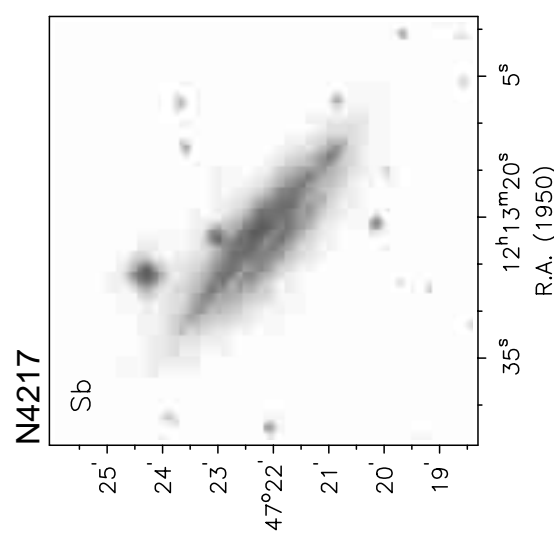

(096l) $\cdot 520$

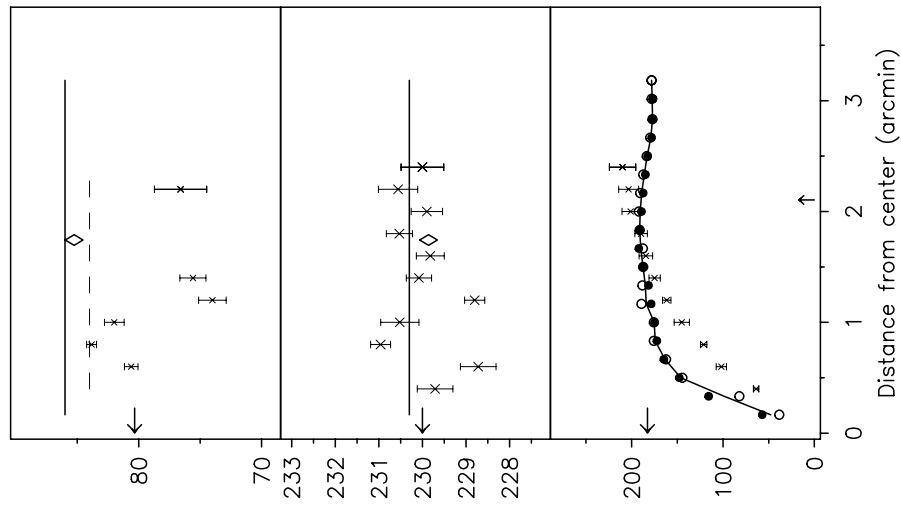

(०) '|⿰u|

(o) $\forall \cdot d$

$(s / m y){ }^{701} \wedge$

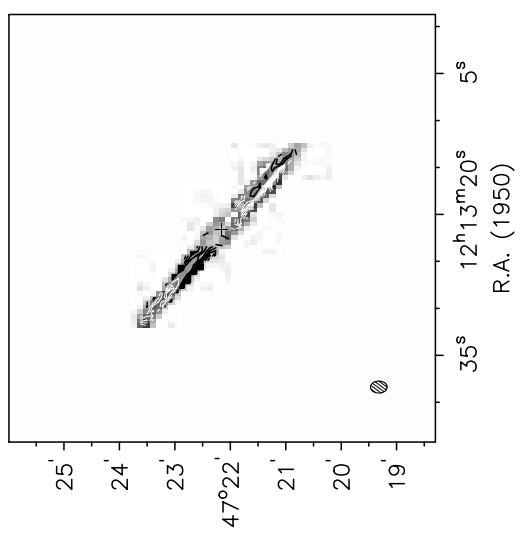

(0s6l) $\cdot 5$

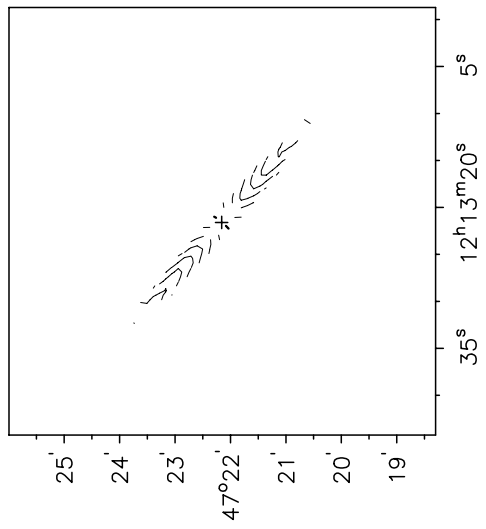

$(0961) \cdot 5 \circ 0$
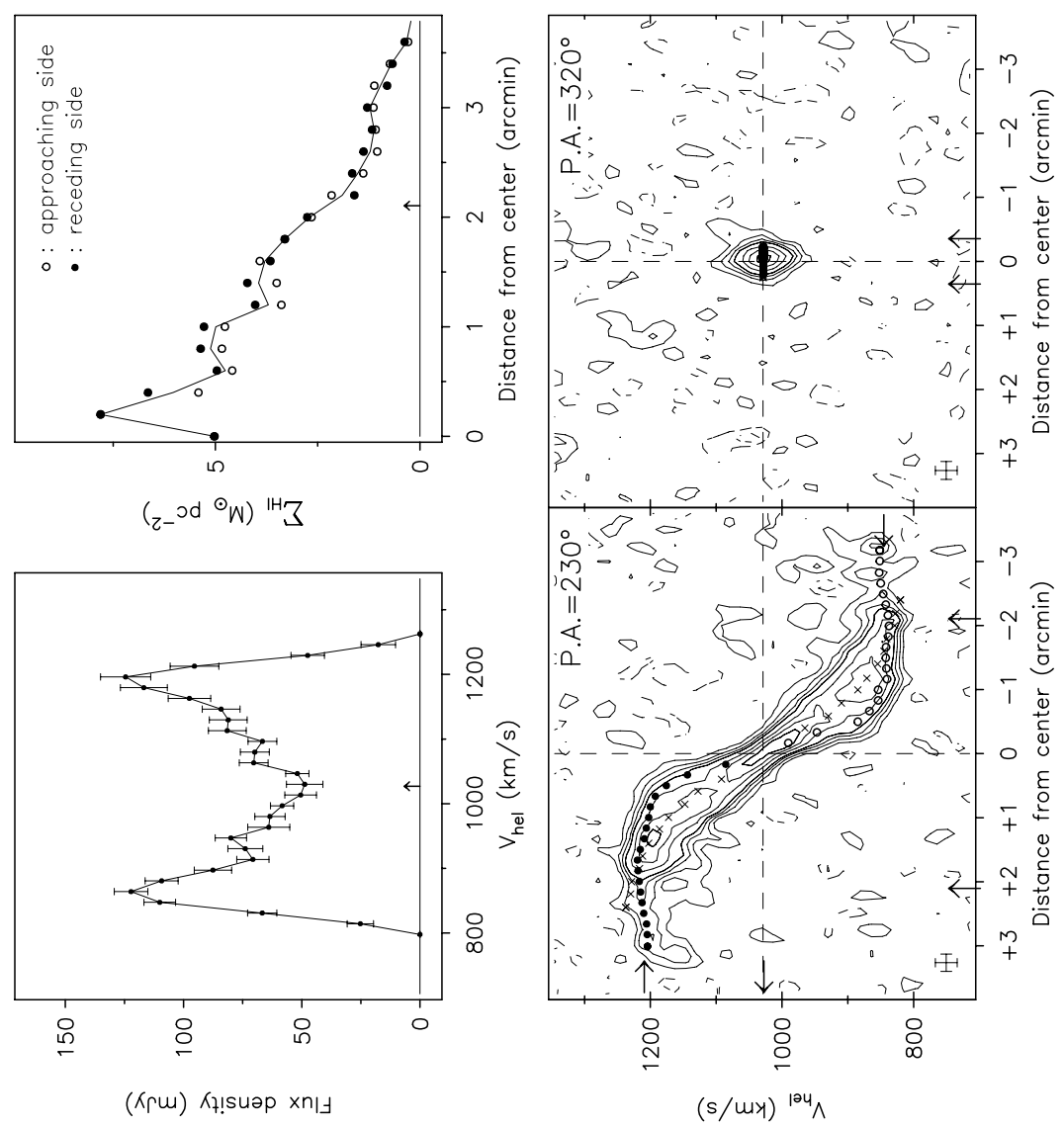
Observing parameters for NGC 4389

\begin{tabular}{|c|c|}
\hline Length of observation & $2 \times 12$ \\
\hline Date of observation & $\begin{array}{l}\text { 28Aug92 } \\
04 \mathrm{Dec} 93\end{array}$ \\
\hline Field center, $\alpha(1950)$ & $12: 23: 05$ \\
\hline$\delta(1950)$ & 46:00:00 \\
\hline Central frequency & 1416.90 \\
\hline$V_{\text {hel }}$ of central channel & 740 \\
\hline Primary beam FWHM (arcmin) & 37.4 \\
\hline Nr. of interferometers & 80 \\
\hline Baselines (min-max-incr) & $36-2736-36$ \\
\hline Synthesized beam $(\alpha \times \delta)(\operatorname{arcsec})$ & $12.0 \times 16.9$ \\
\hline Bandwidth & 2.5 \\
\hline Number of channels & 127 \\
\hline Channel separation & 4.14 \\
\hline Velocity resolution & 8.29 \\
\hline rms noise in one channel $(\mathrm{K})$ & 3.86 \\
\hline $\begin{array}{l}\text { K-mJy conversion, } \\
\text { equiv. of } 1 \mathrm{mJy} / \text { beam }\end{array}$ & 2.97 \\
\hline
\end{tabular}

Results from WSRT data

\begin{tabular}{|c|c|}
\hline \multicolumn{2}{|l|}{ From continuum map: } \\
\hline 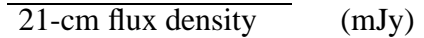 & $23.3 \pm 1.2$ \\
\hline \multicolumn{2}{|l|}{ Central point source position } \\
\hline$\alpha(1950)$ & 12:23:08.6 \\
\hline$\delta(1950)$ & $45: 57: 41$ \\
\hline \multicolumn{2}{|l|}{ From global profile: } \\
\hline$\overline{\text { Integrated HI-flux }}\left(\mathrm{Jy} \mathrm{km} \mathrm{s}^{-1}\right)$ & $7.6 \pm 0.2$ \\
\hline Hel. systemic velocity $\left(\mathrm{km} \mathrm{s}^{-1}\right)$ & $718.4 \pm 1.2$ \\
\hline HI profile width, $20 \%\left(\mathrm{~km} \mathrm{~s}^{-1}\right)$ & $184.0 \pm 1.5$ \\
\hline $50 \%\left(\mathrm{~km} \mathrm{~s}^{-1}\right)$ & $164.9 \pm 1.6$ \\
\hline \multicolumn{2}{|l|}{ From velocity field: } \\
\hline 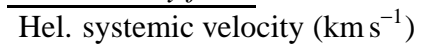 & $714.7 \pm 1.1$ \\
\hline Dynamical center, $\alpha(1950)$ & $12: 23: 08.6$ \\
\hline$\delta(1950)$ & $45: 57: 41$ \\
\hline \multicolumn{2}{|l|}{ From total HI map: } \\
\hline$\overline{\text { Geometric center, } \alpha(1950)}$ & $12: 23: 08.2$ \\
\hline$\delta(1950)$ & 45:57:44 \\
\hline Position angle & 281 \\
\hline Inclination angle & 40 \\
\hline Diameter of HI disk (arcmin) & 2.6 \\
\hline
\end{tabular}

Contour levels for N4389

Channel maps: $\quad \sigma=2.53(\mathrm{~K})$

Raw continuum map:

Cleaned continuum map $\sigma=0.83(\mathrm{~K})$

Position-Velocity diagrams:

Velocity fields:

$714.7 \pm \mathrm{n} \times 15\left(\mathrm{~km} \mathrm{~s}^{-1}\right)$ Residual velocity field: $\pm \mathrm{n} \times 5\left(\mathrm{~km} \mathrm{~s}^{-1}\right)$

Integrated HI map: $0.41,0.82$,

$1.23\left(\times 10^{21}\right.$ atoms cm $\left.{ }^{-2}\right)$

Note: The morphology and kinematics of this galaxy are completely dominated by a prominent bar. The position angle of the bar almost coincides with the position angle of the outer isophotes. The position of the central $21 \mathrm{~cm}$ continuum point source is the same as the center of the outer optical isophotes and is taken as the dynamical center. The HI gas is only found along the bar and the star forming regions at the end of the bar.

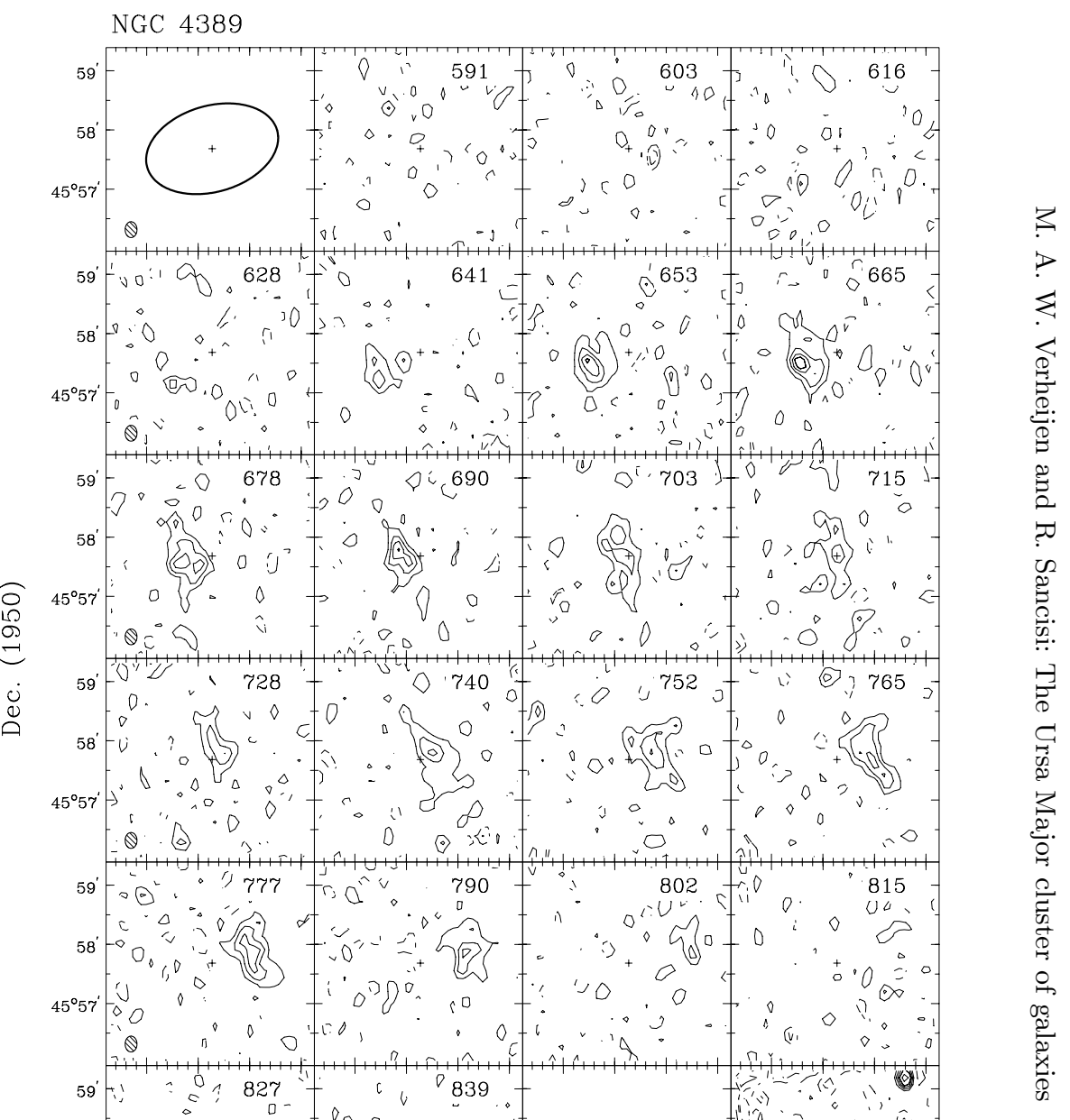

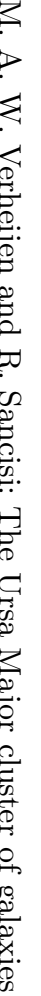

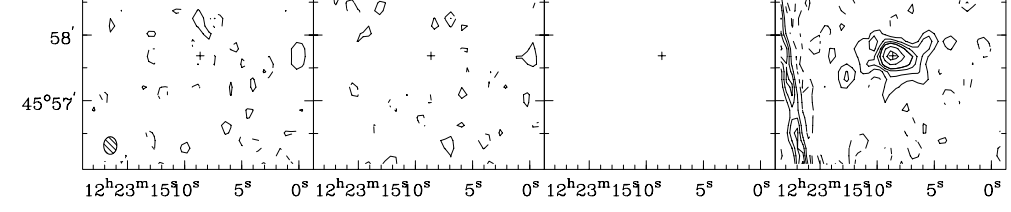

R.A. $(1950)$

Channel maps at a resolution of $12^{\prime \prime} \times 16^{\prime \prime} \times 19 \mathrm{~km} \mathrm{~s}^{-1}$. Contour levels at $-3,-1.5$ (dashed), $1.5,3,4.5, \ldots \times \sigma$. 

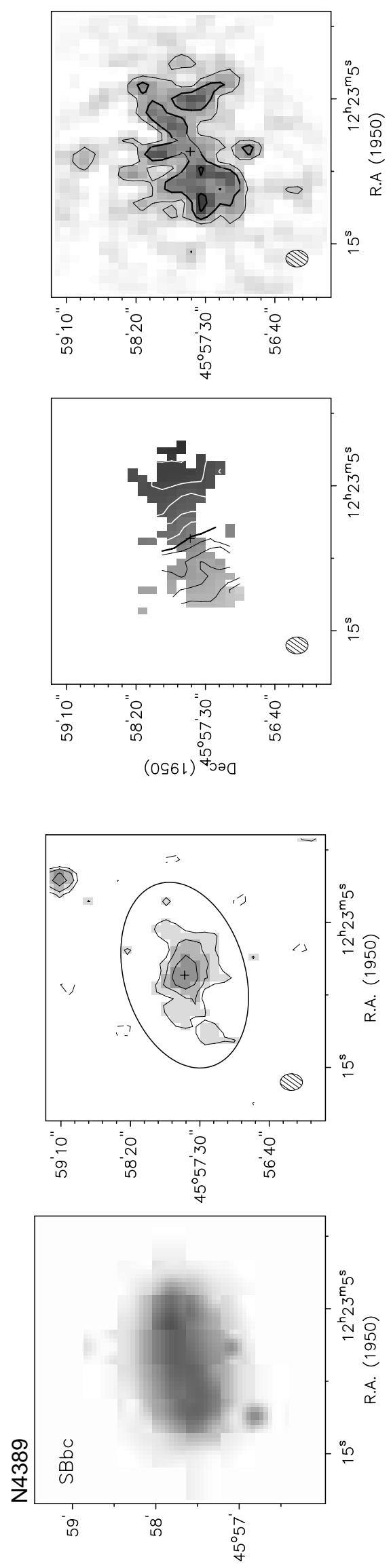

(056l) • วә0

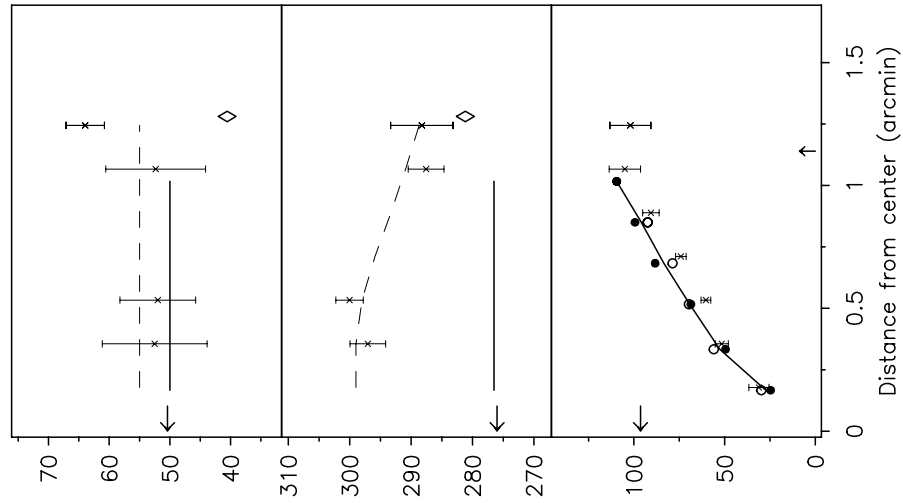

(o) $\cdot$ loul $\quad$ (o) $\forall \cdot d \quad(s / m y)^{701} \wedge$
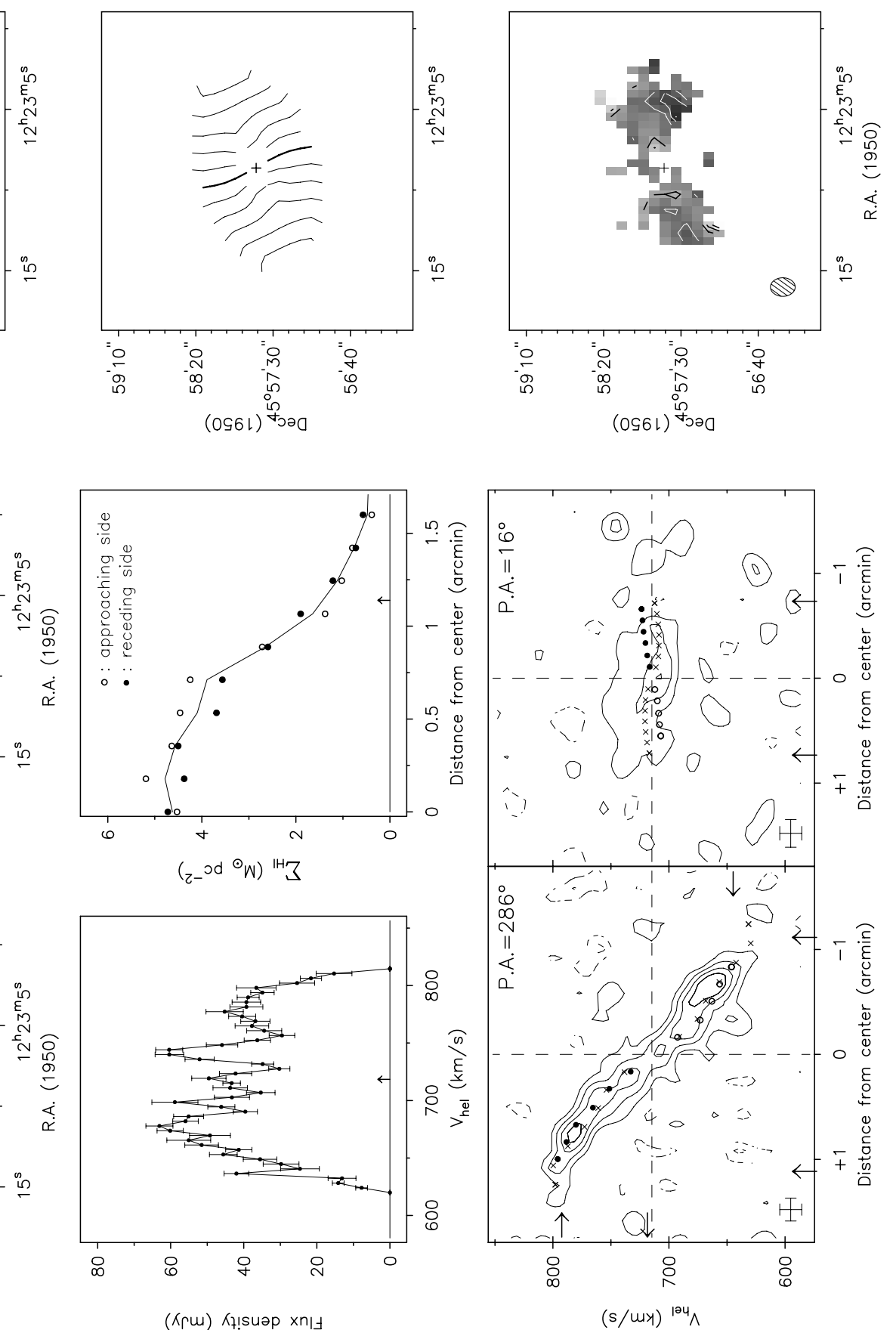


\begin{tabular}{|c|c|}
\hline Length of observation & $1 \times 12$ \\
\hline Date of observation & 15Apr91 \\
\hline Field center, $\alpha(1950)$ & 11:31:05 \\
\hline$\delta(1950)$ & $53: 23: 00$ \\
\hline Central frequency & 1415.27 \\
\hline$V_{\text {hel }}$ of central channel & 1070 \\
\hline Primary beam FWHM & 37.4 \\
\hline Nr. of interferometers & 40 \\
\hline Baselines (min-max-incr) & $36-2700-72$ \\
\hline Synthesized beam $(\alpha \times \delta)(\operatorname{arcsec})$ & $12.9 \times 17.2$ \\
\hline Bandwidth & 5.0 \\
\hline Number of channels & 53 \\
\hline Channel separation & 16.61 \\
\hline Velocity resolution & 33.22 \\
\hline rms noise in one channel & 2.85 \\
\hline $\begin{array}{l}\text { K-mJy conversion, } \\
\text { equiv. of } 1 \mathrm{mJy} / \text { beam }\end{array}$ & 2.71 \\
\hline
\end{tabular}

Contour levels for N3718

Channel maps:

Raw continuum maps:

$\sigma=0.27(\mathrm{~K})$

$\sigma=0.13(\mathrm{~K})$

Position-Velocity diagram:

$\sigma=0.26(\mathrm{~K})$ Note: NGC 3718 is the largest galaxy
in the cluster. It displays a symmetric but distorted morphology with extended spiral arms and a pronounced dust lane crossing its nucleus. The kinematics is very complex and shows an extremely warped HI disk. The inclination runs from roughly 75 degrees in the inner regions through edge-on to about gions through edge-on to about 65 degrees in the outer regions. The change in position angle is more than 95 degrees. This system has been studied in detail by Schwarz (1985). The rotation curve as projected onto the positionvelocity diagram is based on the results from his modelling.

NGC 3729 is located some 11 arcmin to the ENE.

From $X V$-diagram:

$$
\begin{array}{ll}
20 \%\left(\mathrm{~km} \mathrm{~s}^{-1}\right) & 492.8 \pm 1.0 \\
50 \%\left(\mathrm{~km} \mathrm{~s}^{-1}\right) & 465.7 \pm 1.0
\end{array}
$$

Hel. systemic velocity $\left(\mathrm{km} \mathrm{s}^{-1}\right) \quad 989.8$

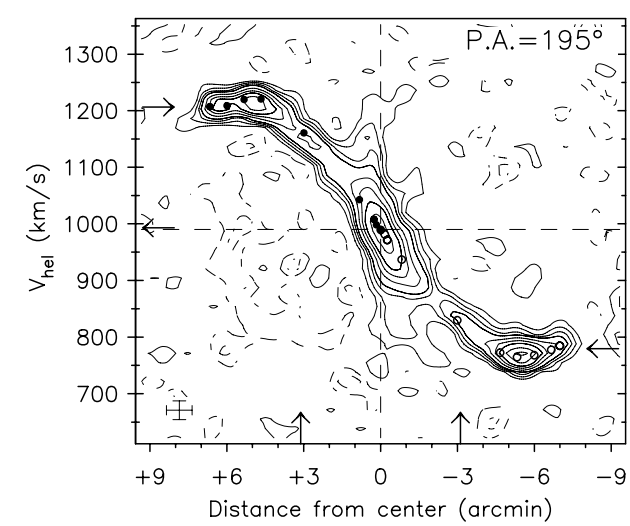

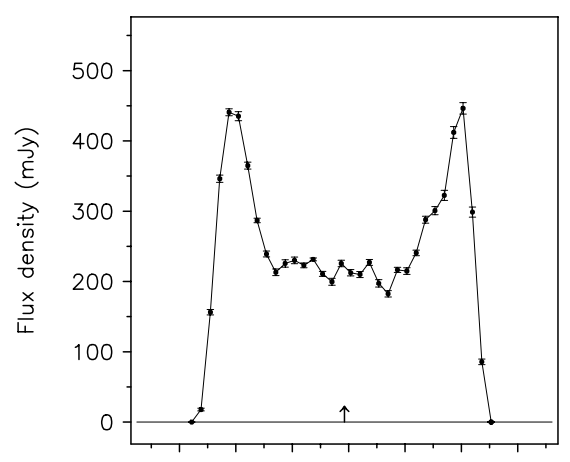

7008009001000110012001300 $V_{\text {hel }}(\mathrm{km} / \mathrm{s})$

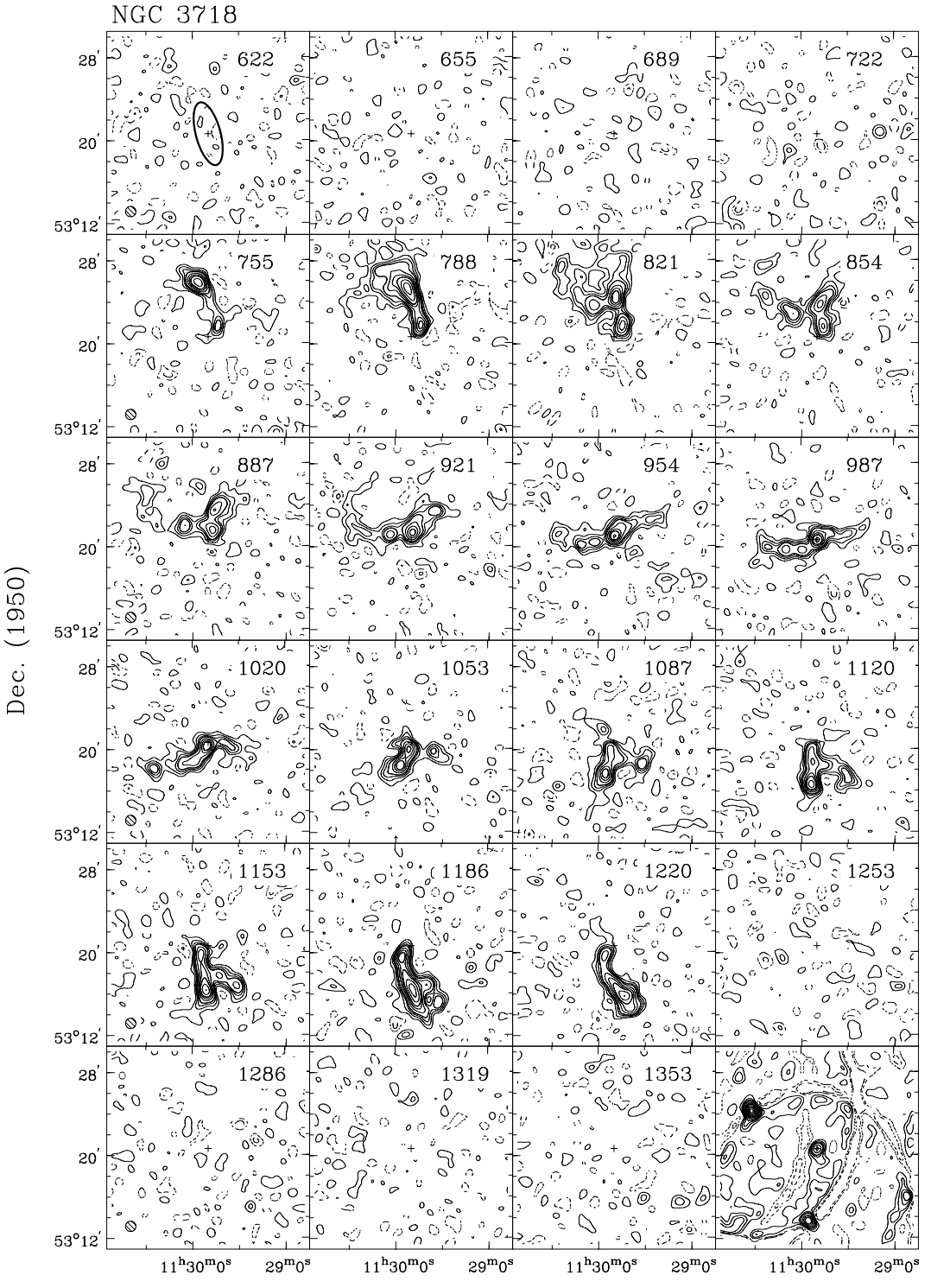

$$
\text { R.A. (1950) }
$$

Channel maps at a resolution of $60^{\prime \prime} \times 60^{\prime \prime} \times 33 \mathrm{~km} \mathrm{~s}^{-1}$.

Contour levels at $-3,-1.5$ (dashed), $1.5,3,4.5, \ldots \times \sigma$. 


\section{Observing parameters for NGC 3729}

\begin{tabular}{|c|c|}
\hline Length of observation & $1 \times 12$ \\
\hline Date of observation & 15Apr91 \\
\hline Field center, $\alpha(1950)$ & 11:31:05 \\
\hline$\delta(1950)$ & 53:23:00 \\
\hline Central frequency & 1415.27 \\
\hline$V_{\text {hel }}$ of central channel & 1070 \\
\hline Primary beam FWHM (arcmin) & 37.4 \\
\hline Nr. of interferometers & 40 \\
\hline Baselines (min-max-incr) & $36-2700-72$ \\
\hline Synthesized beam $(\alpha \times \delta)(\operatorname{arcsec})$ & $12.9 \times 17.2$ \\
\hline Bandwidth & 5.0 \\
\hline Number of channels & 53 \\
\hline Channel separation & 16.61 \\
\hline Velocity resolution & 33.22 \\
\hline rms noise in one channel & 2.85 \\
\hline $\begin{array}{l}\text { K-mJy conversion, } \\
\text { equiv. of } 1 \mathrm{mJy} / \text { beam }\end{array}$ & 2.71 \\
\hline
\end{tabular}

\section{Results from WSRT data}

\begin{tabular}{|c|c|}
\hline \multicolumn{2}{|l|}{ From continuum map: } \\
\hline 21-cm flux density & $18.0 \pm 0.9$ \\
\hline \multicolumn{2}{|l|}{ Central point source position } \\
\hline$\alpha(1950)$ & $11: 31: 04.8$ \\
\hline$\delta(1950)$ & $53: 24: 08$ \\
\hline \multicolumn{2}{|l|}{ From global profile: } \\
\hline$\overline{\text { Integrated HI-flux }}\left(\mathrm{Jy} \mathrm{km} \mathrm{s}^{-1}\right)$ & $5.5 \pm 0.3$ \\
\hline Hel. systemic velocity $\left(\mathrm{km} \mathrm{s}^{-1}\right)$ & $1059.8 \pm 1.4$ \\
\hline HI profile width, $20 \%\left(\mathrm{~km} \mathrm{~s}^{-1}\right)$ & $270.8 \pm 1.5$ \\
\hline $50 \%\left(\mathrm{~km} \mathrm{~s}^{-1}\right)$ & $253.2 \pm 3.9$ \\
\hline \multicolumn{2}{|l|}{ From XV-diagram: } \\
\hline 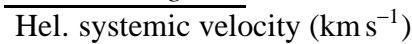 & 1062.8 \\
\hline
\end{tabular}

Note: NGC 3729 displays a bright ringlike structure which surrounds a diffuse bar and a double nucleus. On the northeastern side, just outside the ring, a diffuse blue patch can be seen. The HI gas is only detected in the bright ring with a faint extension toward the blue patch. The large disturbed galaxy N3718 is located about 11 armin to the WSW.
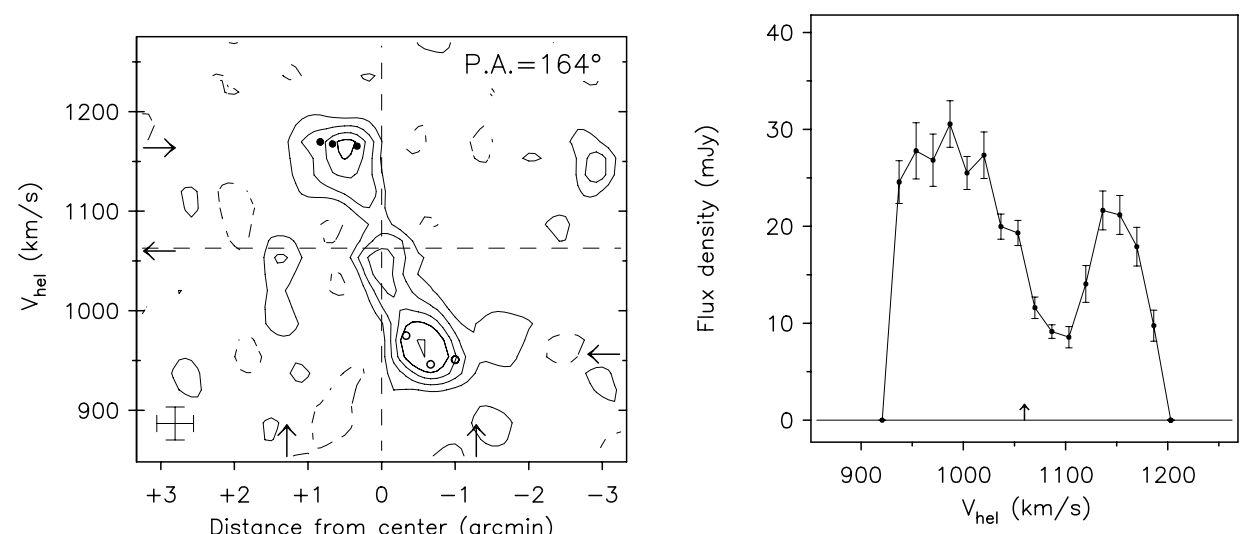

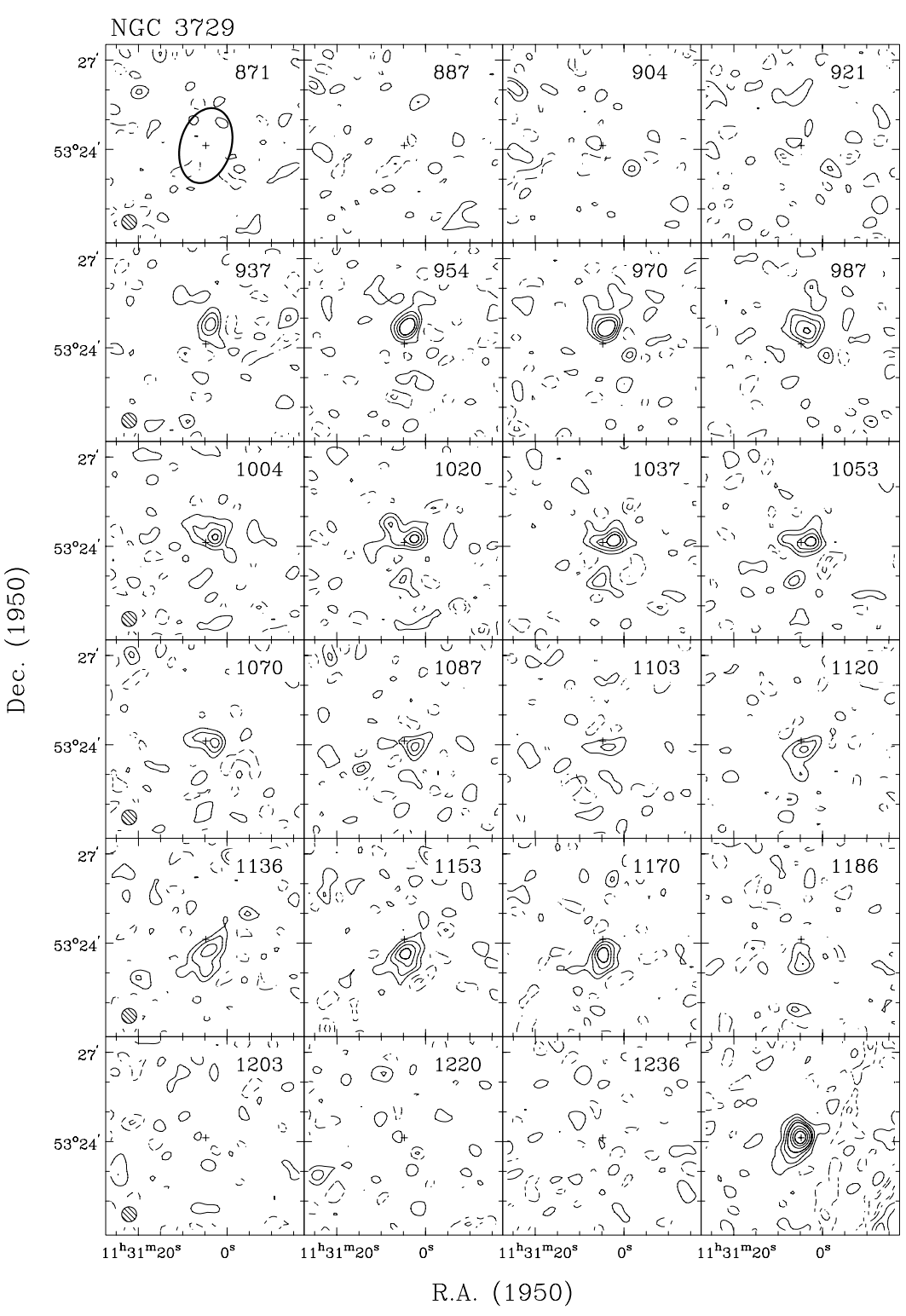

Channel maps at a resolution of $30^{\prime \prime} \times 30^{\prime \prime} \times 33 \mathrm{~km} \mathrm{~s}^{-1}$.

Contour levels at $-3,-1.5$ (dashed), 1.5, 3, 4.5, $\ldots \times \sigma$. 


\begin{tabular}{|c|c|}
\hline Length of observation & $1 \times 12$ \\
\hline Date of observation & 29Aug92 \\
\hline Field center, $\alpha(1950)$ & $11: 45: 24$ \\
\hline$\delta(1950)$ & 50:05:00 \\
\hline Central frequency & 1415.97 \\
\hline$V_{\text {hel }}$ of central channel & 940 \\
\hline Primary beam FWHM (arcmin) & 37.4 \\
\hline $\mathrm{Nr}$. of interferometers & 40 \\
\hline Baselines (min-max-incr) & $36-2700-72$ \\
\hline Synthesized beam $(\alpha \times \delta)(\operatorname{arcsec})$ & $12.6 \times 16.6$ \\
\hline Bandwidth & 2.5 \\
\hline Number of channels & 127 \\
\hline Channel separation & 4.15 \\
\hline Velocity resolution & 8.29 \\
\hline rms noise in one channel & 5.32 \\
\hline $\begin{array}{l}\text { K-mJy conversion, } \\
\text { equiv. of } 1 \mathrm{mJy} / \text { beam }\end{array}$ & 2.88 \\
\hline
\end{tabular}

\section{Results from WSRT data}

\section{From continuum map:}

\section{1-cm flux density}

central point source $(\mathrm{mJy}) \quad<1.2(3 \sigma)$

$$
\text { extended sour }
$$

From global profile:

Integrated HI-flux $\left(\mathrm{Jy} \mathrm{km} \mathrm{s}^{-1}\right) \quad 5.6 \pm 0.4$

Hel. systemic velocity $\left(\mathrm{km} \mathrm{s}^{-1}\right) \quad 923.6 \pm 1.6$

HI profile width, $20 \%\left(\mathrm{~km} \mathrm{~s}^{-1}\right) \quad 110.4 \pm 2.3$

\section{From $X V$-diagram:}

$$
50 \%\left(\mathrm{~km} \mathrm{~s}^{-1}\right) \quad 91.1 \pm 2.2
$$

Hel. systemic velocity $\left(\mathrm{km} \mathrm{s}^{-1}\right) \quad 922.3$

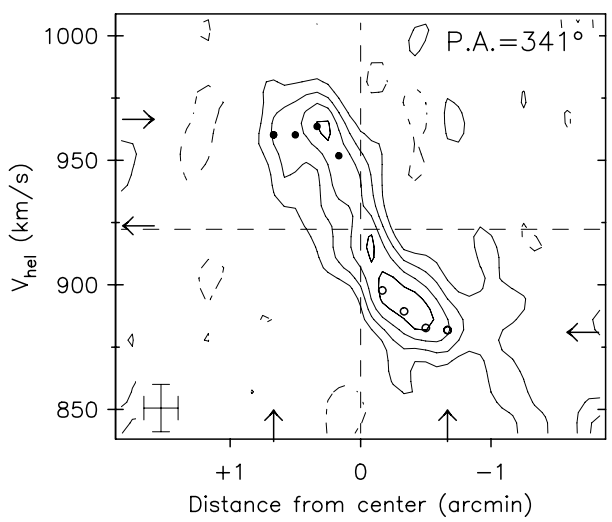

Contour levels for U6773

\begin{tabular}{ll}
\hline \hline Channel maps: & $\sigma=3.45(\mathrm{~K})$ \\
Raw continuum map: & $\sigma=1.47(\mathrm{~K})$ \\
Position-Velocity diagram: & \\
& $\sigma=2.88(\mathrm{~K})$ \\
\hline
\end{tabular}

Note: This dwarf galaxy shows an irregular morphology and the positionvelocity diagram suggests an asymmetric rotation curve. THe HI disk does not extend much beyond $\mathrm{D}_{25}$.

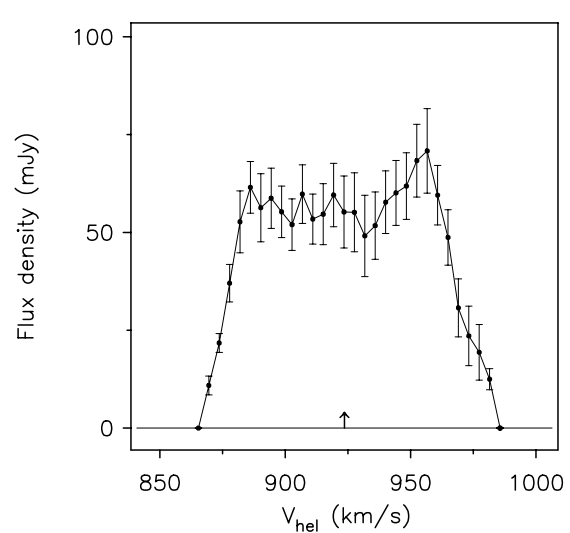

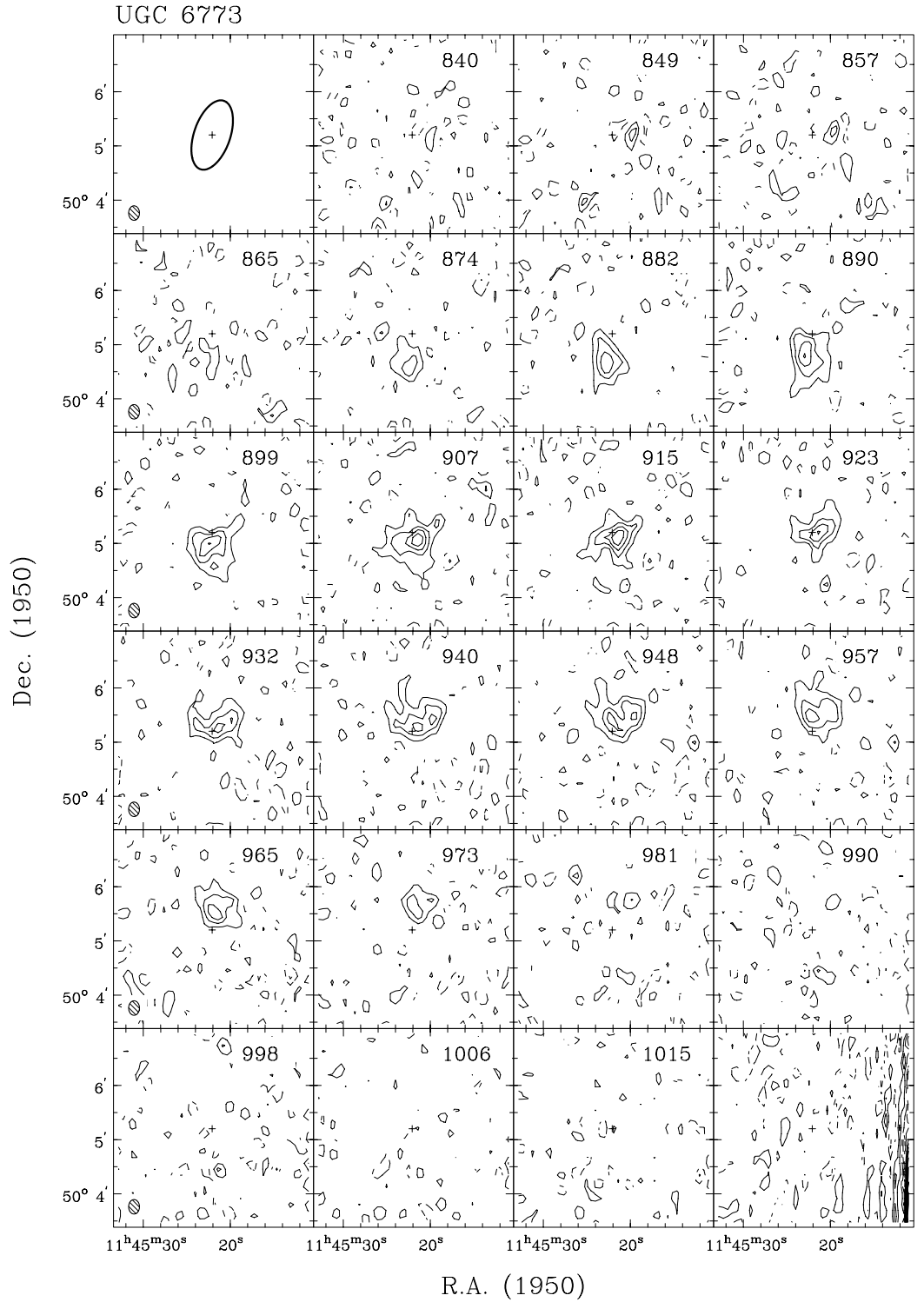

$6^{\prime \prime} \times 19 \mathrm{~km} \mathrm{~s}^{-1}$

Contour levels at $-3,-1.5$ (dashed), $1.5,3,4.5, \ldots \times \sigma$. 


\begin{tabular}{|c|c|}
\hline Length of observation & $1 \times 12$ \\
\hline Date of observation & 19Aug90 \\
\hline Field center, $\alpha(1950)$ & 11:48:10 \\
\hline$\delta(1950)$ & $46: 05: 00$ \\
\hline Central frequency & 1416.51 \\
\hline$V_{\text {hel }}$ of central channel & 820 \\
\hline Primary beam FWHM & 37.4 \\
\hline Nr. of interferometers & 40 \\
\hline Baselines (min-max-incr) & $36-2700-72$ \\
\hline Synthesized beam $(\alpha \times \delta)(\operatorname{arcsec})$ & $12.6 \times 17.6$ \\
\hline Bandwidth & 2.5 \\
\hline Number of channels & 127 \\
\hline Channel separation & 4.15 \\
\hline Velocity resolution & 8.29 \\
\hline rms noise in one channel & 5.03 \\
\hline $\begin{array}{l}\text { K-mJy conversion, } \\
\text { equiv. of } 1 \mathrm{mJy} / \text { beam }\end{array}$ & 2.72 \\
\hline
\end{tabular}

Results from WSRT data

\begin{tabular}{|c|c|}
\hline $\begin{array}{l}\text { From continuит map: } \\
21-\mathrm{cm} \text { flux density } \\
\text { From global profile: }\end{array}$ & $2.4 \pm 1.0$ \\
\hline$\overline{\text { Integrated HI-flux }}\left(\mathrm{Jy} \mathrm{km} \mathrm{s}^{-1}\right)$ & $13.9 \pm 0.2$ \\
\hline Hel. systemic velocity $\left(\mathrm{km} \mathrm{s}^{-1}\right)$ & $808.1 \pm 2.1$ \\
\hline HI profile width, $20 \%\left(\mathrm{~km} \mathrm{~s}^{-1}\right)$ & $166.9 \pm 2.3$ \\
\hline $50 \%\left(\mathrm{~km} \mathrm{~s}^{-1}\right)$ & $141.9 \pm 5.7$ \\
\hline
\end{tabular}

Hel. systemic velocity $\left(\mathrm{km} \mathrm{s}^{-1}\right) \quad 813.4$

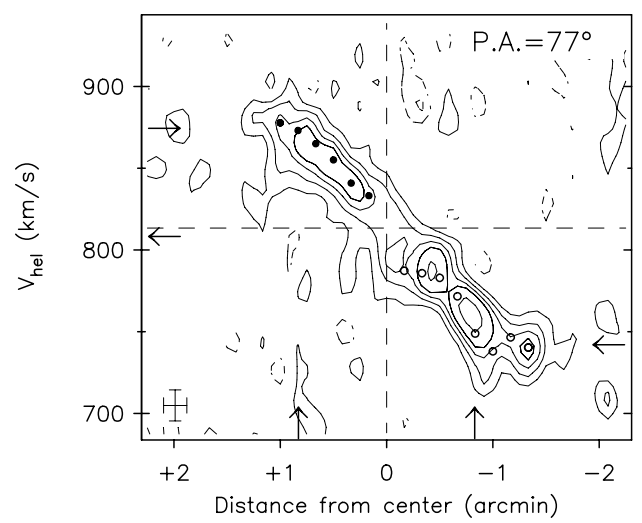

\section{Contour levels for U6818}

Channel maps:

Raw continuum map:

$\sigma=4.04(\mathrm{~K})$

$\sigma=2.07(\mathrm{~K})$

$$
\text { ms: }
$$

$\sigma=3.36(\mathrm{~K})$

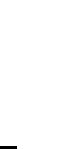

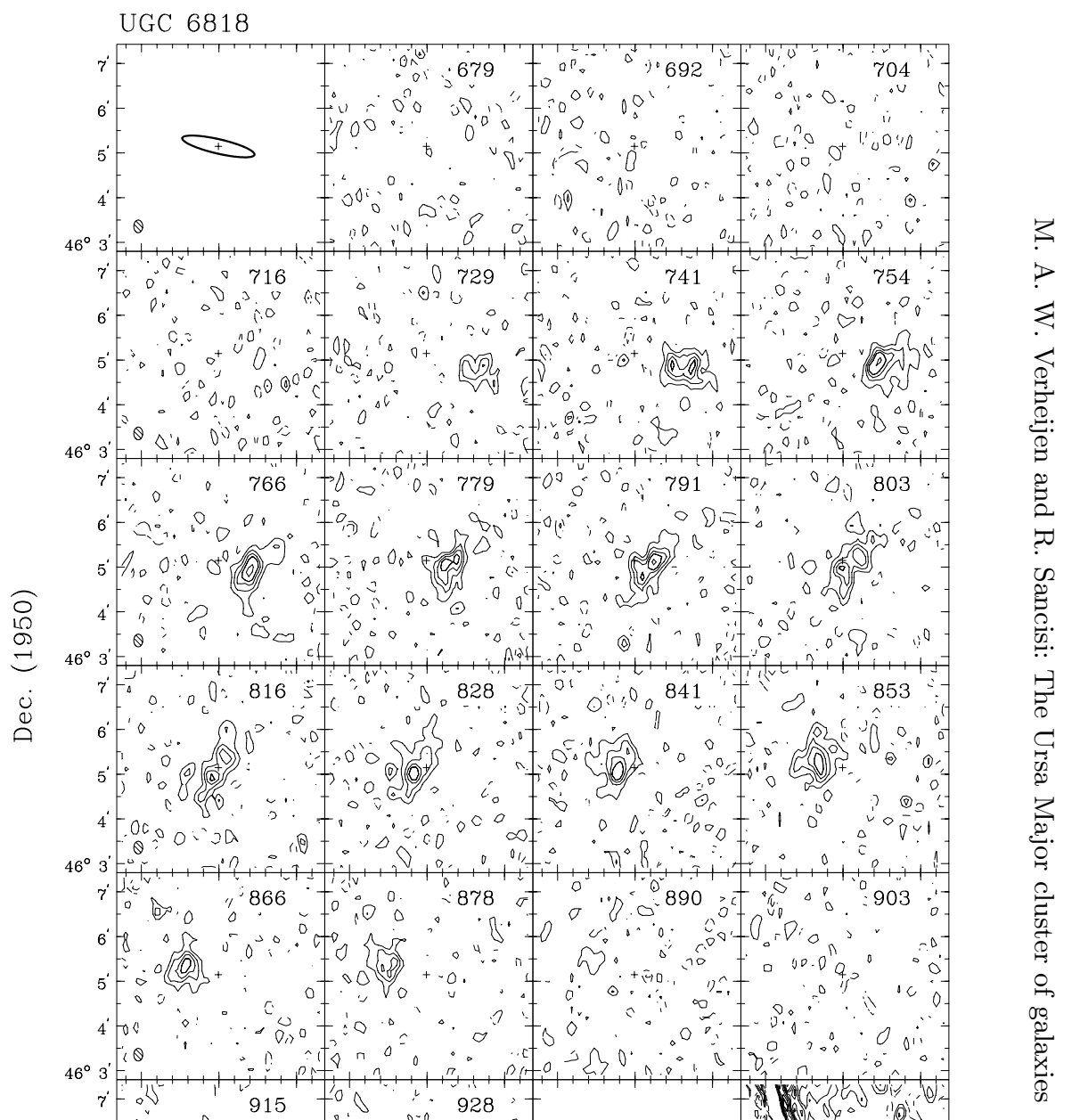
$\mathrm{m}=1$-like spiral arm toward the west and is probably tidally interacting with a faint companion at its north-western edge.

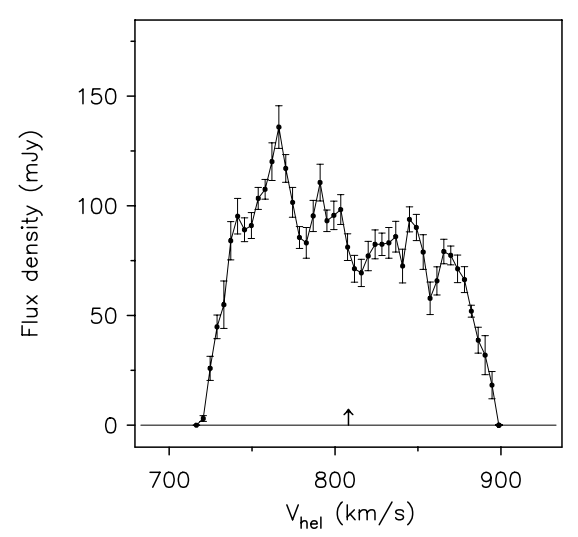

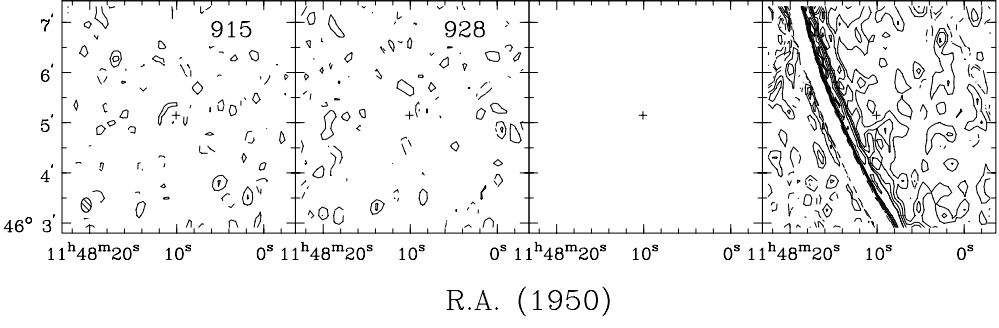

Channel maps at a resolution of $12^{\prime \prime} \times 17^{\prime \prime} \times 19 \mathrm{~km} \mathrm{~s}^{-1}$.

Contour levels at $-3,-1.5$ (dashed), $1.5,3,4.5, \ldots \times \sigma$. 


\begin{tabular}{|c|c|}
\hline Length of observation & $1 \times 12$ \\
\hline Date of observation & 19Aug93 \\
\hline Field center, $\alpha(1950)$ & 11:54:07 \\
\hline$\delta(1950)$ & $48: 36: 48$ \\
\hline Central frequency & 1415.87 \\
\hline$V_{\text {hel }}$ of central channel & 957 \\
\hline Primary beam FWHM (arcmin) & 37.4 \\
\hline Nr. of interferometers & 40 \\
\hline Baselines (min-max-incr) & $36-2700-72$ \\
\hline Synthesized beam $(\alpha \times \delta)(\operatorname{arcsec})$ & $12.3 \times 16.8$ \\
\hline Bandwidth & 2.5 \\
\hline Number of channels & 127 \\
\hline Channel separation & 4.15 \\
\hline Velocity resolution & 8.30 \\
\hline rms noise in one channel & 5.97 \\
\hline $\begin{array}{l}\text { K-mJy conversion, } \\
\text { equiv. of } 1 \mathrm{mJy} / \text { beam }\end{array}$ & 2.91 \\
\hline
\end{tabular}

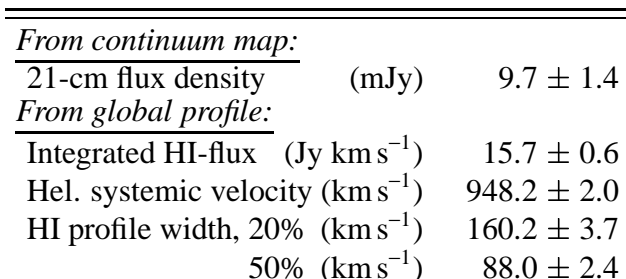

\section{From XV-diagram:}

\section{Results from WSRT data}

Contour levels for N3985

\begin{aligned} \hline Channel maps: & \\ $12 \times 16$ arcsec: & $\sigma=3.79(\mathrm{~K}) \\ 30 \times 30$ arcsec: & $\sigma=1.14(\mathrm{~K}) \\ 60 \times 60$ arcsec: & $\sigma=0.39(\mathrm{~K}) \\$ Raw continuum maps: & \\ $12 \times 16$ arcsec: & $\sigma=1.69(\mathrm{~K}) \\ 30 \times 30$ arcsec: & $\sigma=0.63(\mathrm{~K}) \\ 60 \times 60$ arcsec: & $\sigma=0.36(\mathrm{~K}) \\$ Position-Velocity diagram: & \end{aligned}

$\sigma=3.35(\mathrm{~K})$

Note: This small high surface brightness galaxy displays smooth outer elliptical isophotes but the inner regions are irregular.

The HI kinematics is quite patchy and chaotic as is shown by the channel maps at various angular resolutions. Two components can be identified; 1) a high surface density disk in the inner region and 2) a very extended low surface density disk with a kinematic major axis nearly perpendicular to the optical major axis.

Hel. systemic velocity $\left(\mathrm{km} \mathrm{s}^{-1}\right) \quad 949.8$
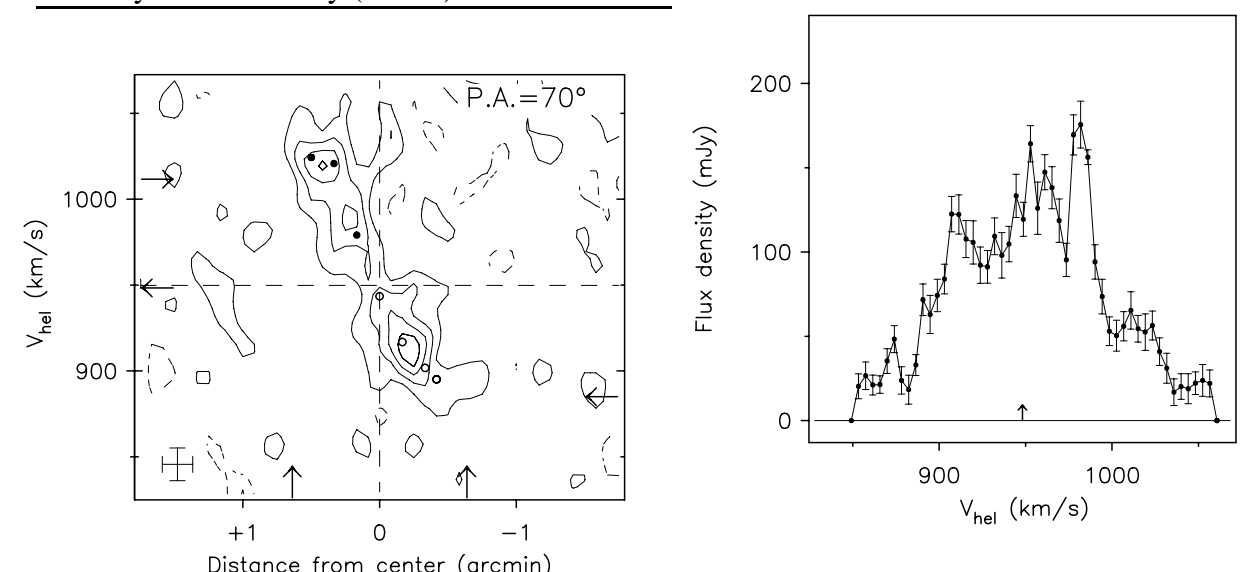

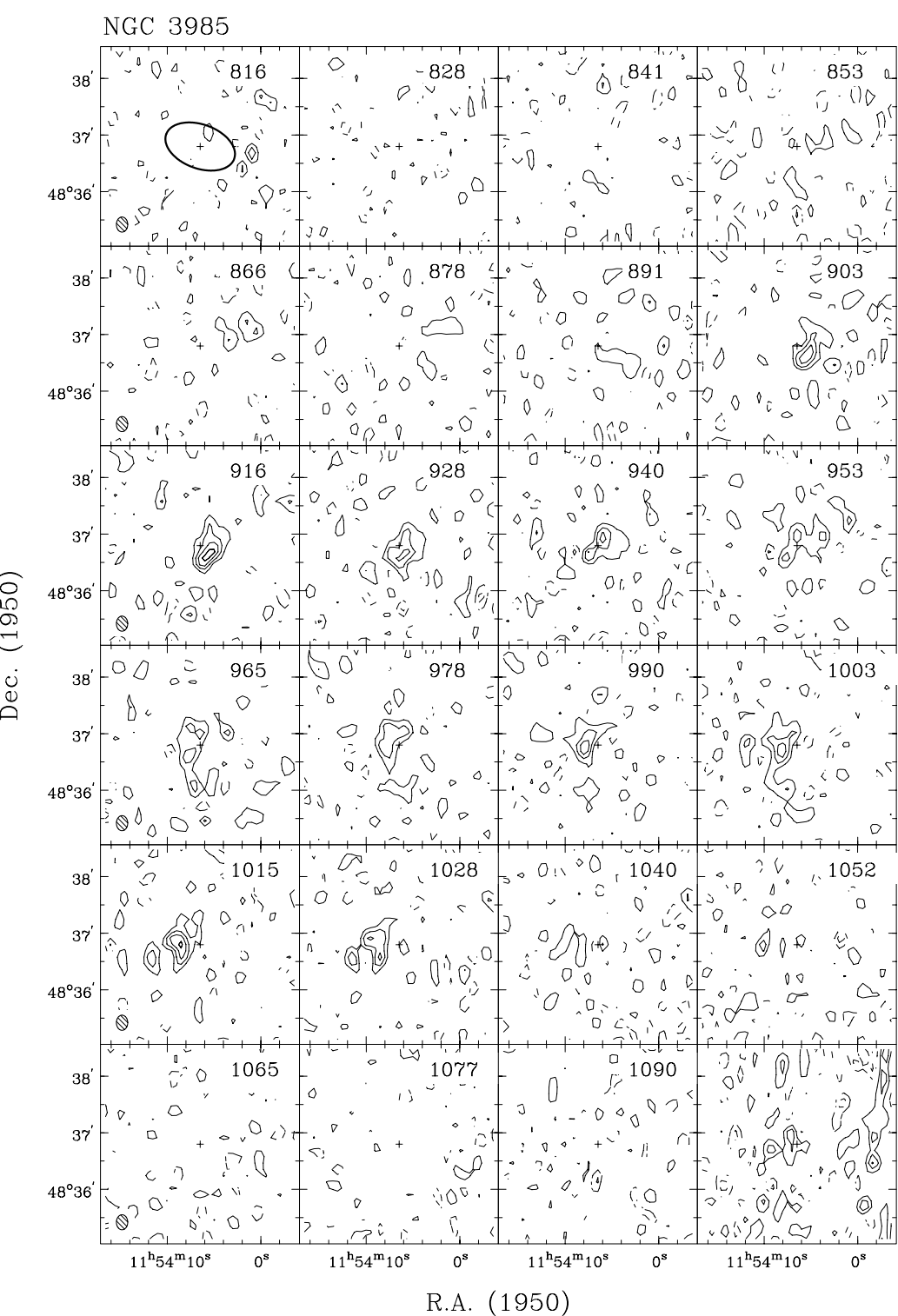

3
0
0
0
0
0
0
0
0
0
0
0
0
0
0
0
0
0
0
0
0
0
0
0
0
0
0
0
0
0
0
0

Channel maps at a resolution of $12^{\prime \prime} \times 16^{\prime \prime} \times 19 \mathrm{~km} \mathrm{~s}^{-1}$.

Contour levels at $-3,-1.5$ (dashed), $1.5,3,4.5, \ldots \times \sigma$. 


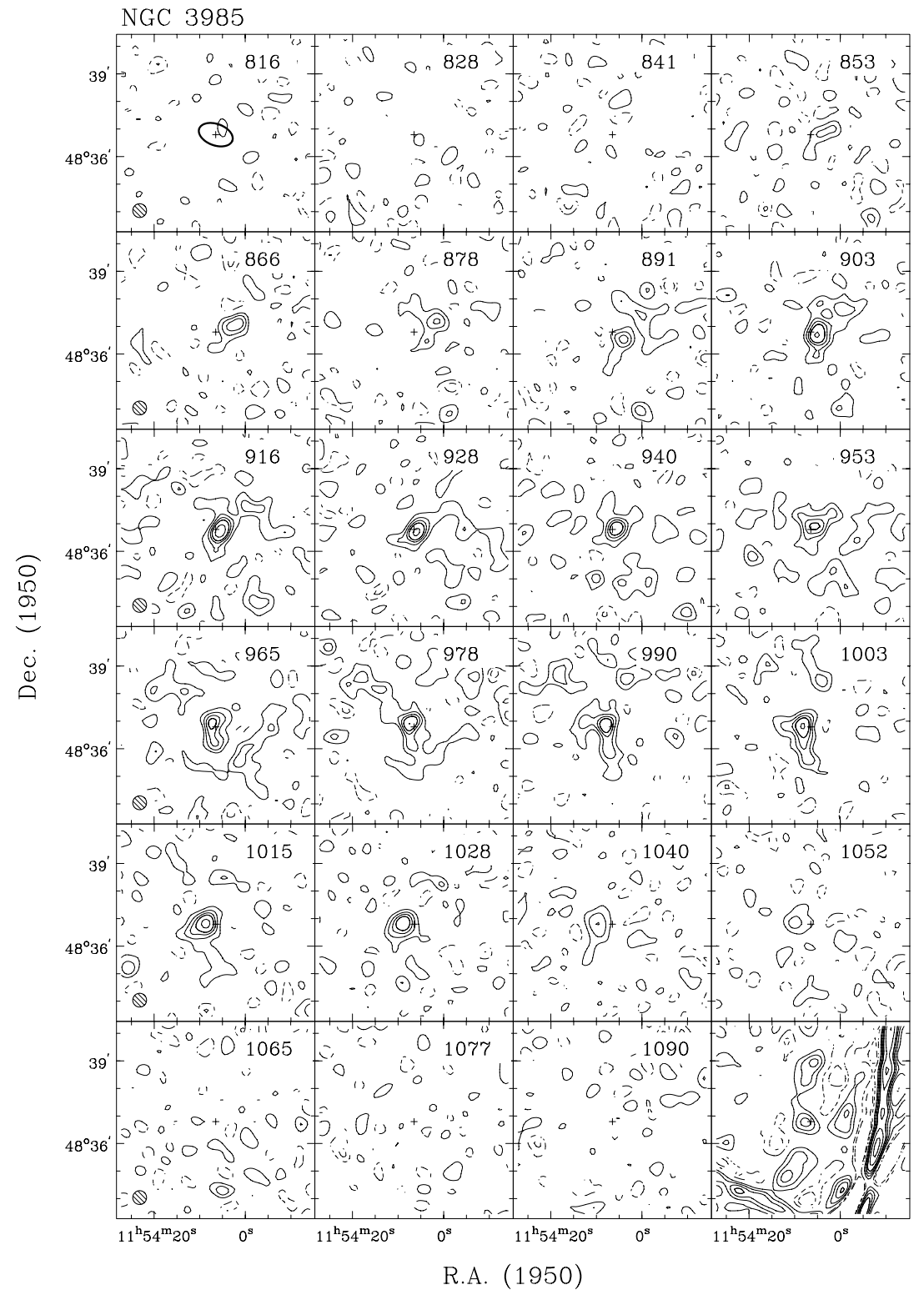

Channel maps at a resolution of $30^{\prime \prime} \times 30^{\prime \prime} \times 19 \mathrm{~km} \mathrm{~s}^{-1}$.

Contour levels at $-3,-1.5$ (dashed), $1.5,3,4.5, \ldots \times \sigma$.

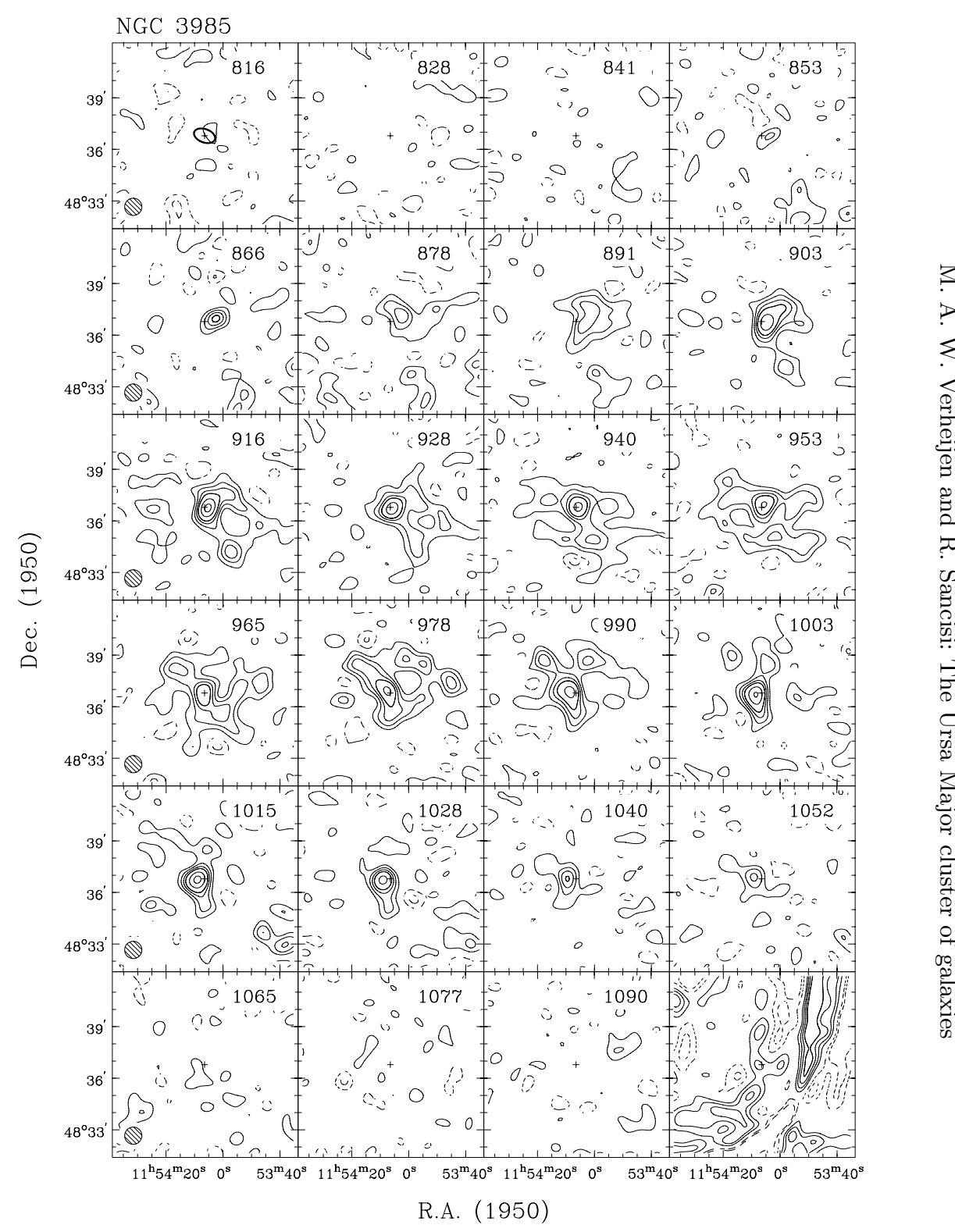

Channel maps at a resolution of $60^{\prime \prime} \times 60^{\prime \prime} \times 19 \mathrm{~km} \mathrm{~s}^{-1}$.

Contour levels at $-3,-1.5$ (dashed) $1.5,3,4.5, \ldots \times \sigma$. 


\begin{tabular}{|c|c|}
\hline Length of observation & $1 \times 12$ \\
\hline Date of observation & 18Apr91 \\
\hline Field center, $\alpha(1950)$ & $11: 52: 50$ \\
\hline$\delta(1950)$ & 54:55:00 \\
\hline Central frequency & 1416.69 \\
\hline$V_{\text {hel }}$ of central channel & 770 \\
\hline Primary beam FWHM & 37.4 \\
\hline Nr. of interferometers & 40 \\
\hline Baselines (min-max-incr) & $36-2700-72$ \\
\hline Synthesized beam $(\alpha \times \delta)(\operatorname{arcsec})$ & $12.5 \times 14.3$ \\
\hline Bandwidth & 2.5 \\
\hline Number of channels & 127 \\
\hline Channel separation & 4.14 \\
\hline Velocity resolution & 8.29 \\
\hline rms noise in one channel & 6.57 \\
\hline $\begin{array}{l}\text { K-mJy conversion, } \\
\text { equiv. of } 1 \mathrm{mJy} / \mathrm{beam}\end{array}$ & 3.37 \\
\hline
\end{tabular}

\section{Results from WSRT data}

\section{From continuum map:}

21-cm flux density

$$
\begin{aligned}
\text { central point source }(\mathrm{mJy}) & <1.2(3 \sigma) \\
\text { extended source }(\mathrm{mJy}) & <2.7(3 \sigma)
\end{aligned}
$$

From global profile:

Integrated HI-flux $\left(\mathrm{Jy} \mathrm{km} \mathrm{s}^{-1}\right) \quad 5.8 \pm 0.2$

Hel. systemic velocity $\left(\mathrm{km} \mathrm{s}^{-1}\right) \quad 848.6 \pm 1.8$

HI profile width, $20 \%\left(\mathrm{~km} \mathrm{~s}^{-1}\right) \quad 141.8 \pm 1.1$

$\begin{array}{ll}50 \%\left(\mathrm{~km} \mathrm{~s}^{-1}\right) & 132.2 \pm 1.5\end{array}$

\section{From XV-diagram:}

$$
50 \%\left(\mathrm{~km} \mathrm{~s}^{-1}\right) \quad 132.2 \pm 1.5
$$

Hel. systemic velocity $\left(\mathrm{km} \mathrm{s}^{-1}\right) \quad 848.3$

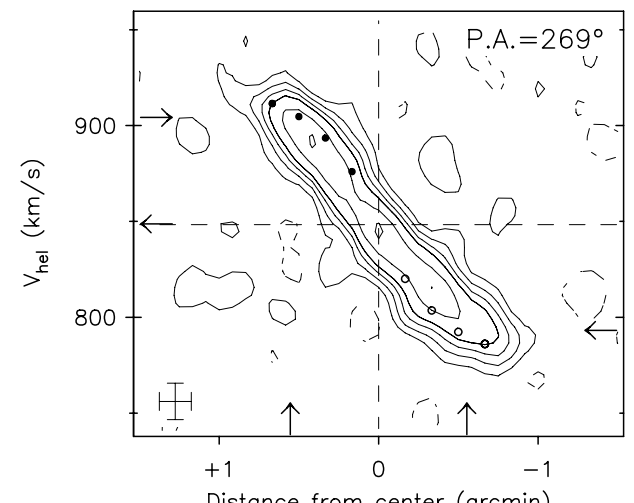

Distance from center (arcmin)
Contour levels for U6894

\begin{tabular}{l} 
Channel maps: \\
Raw continuum map: $\sigma=3.12(\mathrm{~K})$ \\
$\quad \sigma=1.06(\mathrm{~K})$ \\
Position-Velocity diagrams: \\
$\sigma=2.72(\mathrm{~K})$ \\
\hline
\end{tabular}

Note: The HI emission does not extend far beyond the optical image of this edge-on system. The rotation curve is still rising at the last measured point.

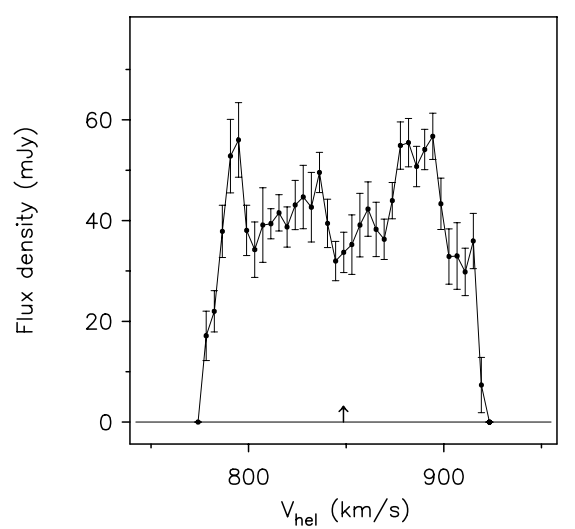

UGC 6894

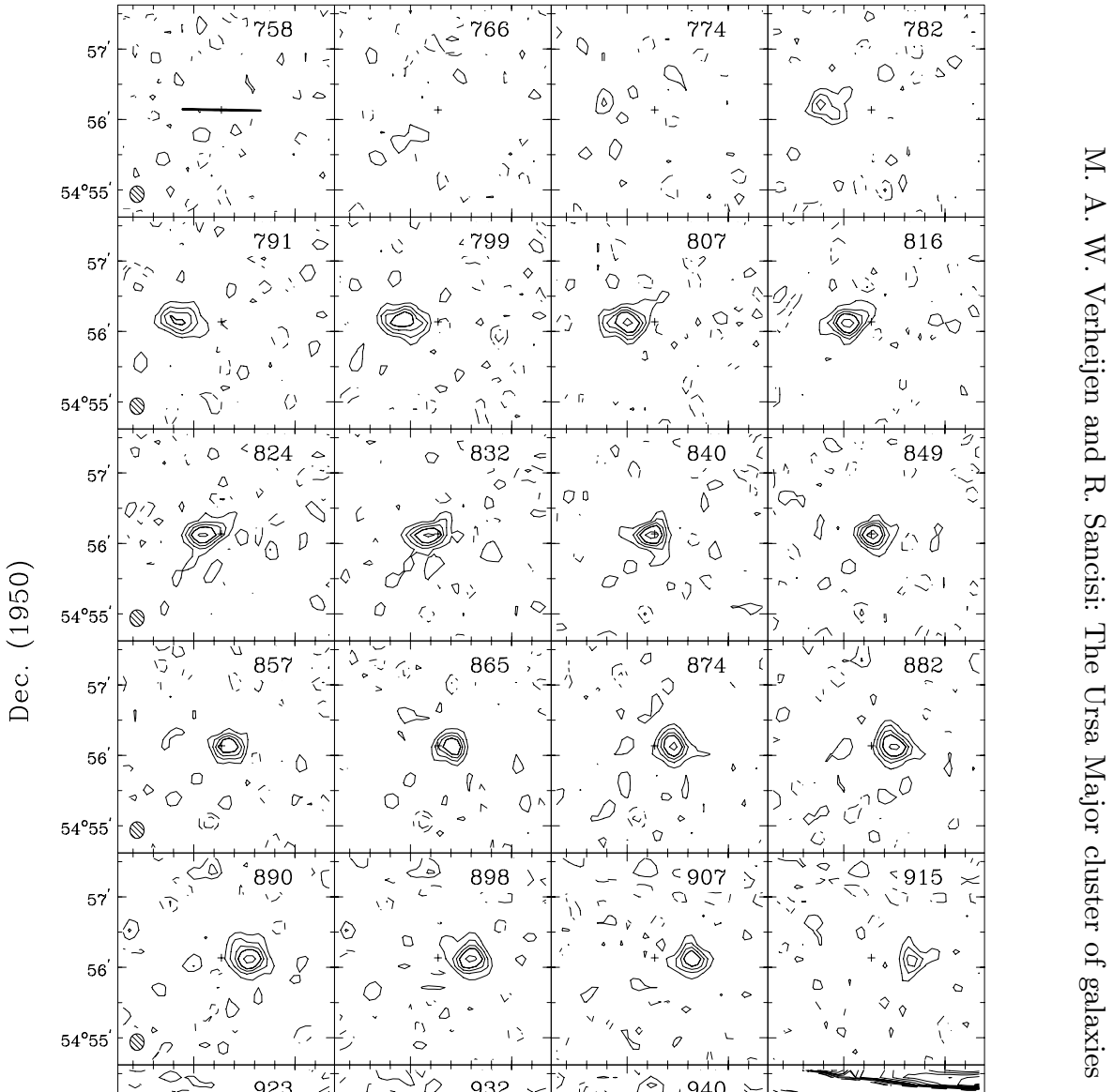

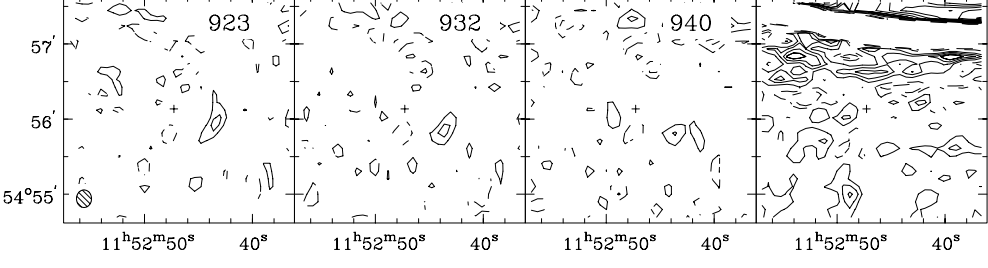

R.A. (1950)

Channel maps at a resolution of $12^{\prime \prime} \times 14^{\prime \prime} \times 19 \mathrm{~km} \mathrm{~s}^{-1}$.

Contour levels at $-3,-1.5$ (dashed), $1.5,3,4.5, \ldots \times \sigma$. 


\section{Observing parameters for NGC 4013}

\begin{tabular}{|c|c|}
\hline Length of observation & $8.3 \times 12$ \\
\hline Dates of observation & Dec86-Mar87 \\
\hline Field center, $\alpha(1950)$ & $11: 55: 40$ \\
\hline 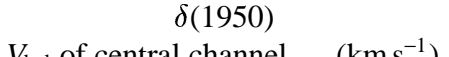 & 44:17:00 \\
\hline $\begin{array}{l}V_{\text {hel }} \text { of central channel } \\
\text { Primarv beam FWHM }\end{array}$ & $\begin{array}{r}820 \\
374\end{array}$ \\
\hline $\begin{array}{l}\text { Primary beam FWHM } \\
\text { Nr. of interferometers }\end{array}$ & $\begin{array}{r}37.4 \\
80\end{array}$ \\
\hline Baselines (min-max-incr) & $36-2736-36$ \\
\hline Synthesized beam $(\alpha \times \delta)(\operatorname{arcsec})$ & $12.3 \times 17.6$ \\
\hline Bandwidth & 5.0 \\
\hline Number of channels & 63 \\
\hline Channel separation & 16.49 \\
\hline Velocity resolution & 33.0 \\
\hline rms noise in one channel & 0.96 \\
\hline $\begin{array}{l}\text { K-mJy conversion, } \\
\text { equiv. of } 1 \mathrm{mJy} / \text { beam }\end{array}$ & 2.78 \\
\hline
\end{tabular}

Results from WSRT data

\begin{tabular}{|c|c|}
\hline \multicolumn{2}{|l|}{ From continuит map: } \\
\hline 21-cm flux density & $36.3 \pm 0$ \\
\hline \multicolumn{2}{|l|}{ Central point source position } \\
\hline & \\
\hline & \\
\hline \multicolumn{2}{|l|}{ From global profile: } \\
\hline$\overline{\mathrm{x}}(\mathrm{Jy})$ & 41. \\
\hline velocity $\left(\mathrm{km} \mathrm{s}^{-1}\right)$ & $831.3 \pm 0.6$ \\
\hline HI profile width, $20 \%\left(\mathrm{~km} \mathrm{~s}^{-1}\right)$ & $425.0 \pm 0.9$ \\
\hline $50 \%(\mathrm{~km}$ & $395.0 \pm 0.8$ \\
\hline
\end{tabular}

From XV-diagram: $50 \%\left(\mathrm{~km} \mathrm{~s}^{-1}\right) \quad 395.0 \pm 0.8$

Hel. systemic velocity $\left(\mathrm{km} \mathrm{s}^{-1}\right)$

$835 \pm 3$

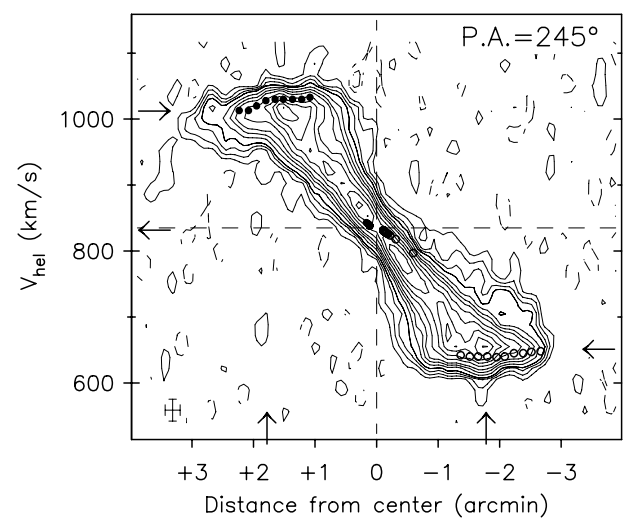

\section{Contour levels for N4013}

Channel maps:

Raw continuum maps:

$\sigma=0.96(\mathrm{~K})$

$\sigma=0.47(\mathrm{~K})$

Position-Velocity diagram

$\sigma=0.84(\mathrm{~K})$

Note: The data presented here was kindly provided by Bottema who extensively studied this warped, edge-on system. The rotation curve was adopted from the analysis by Bottema (1995).

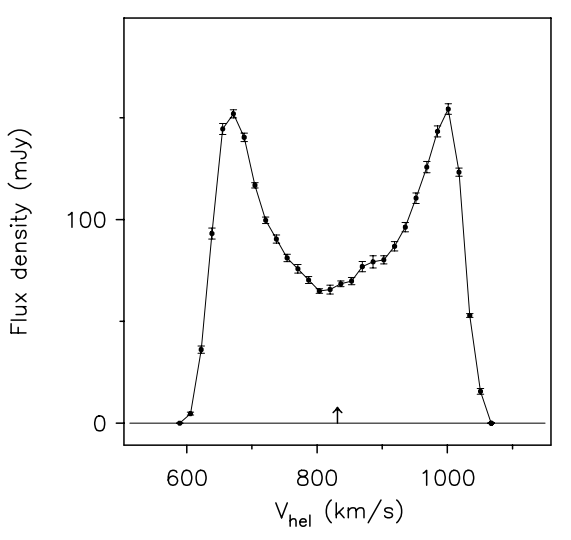

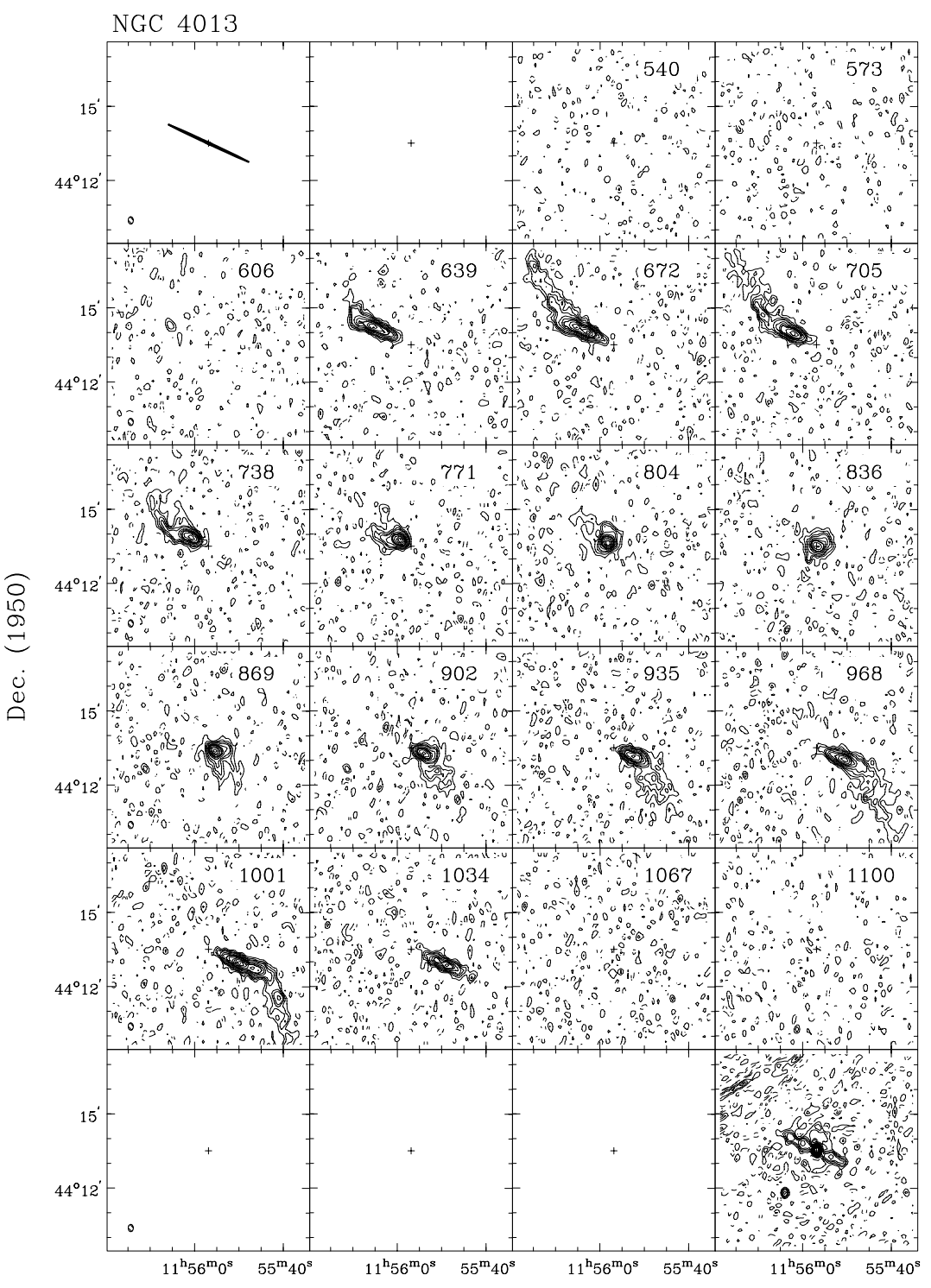

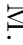

Channel maps at a resolution of $12^{\prime \prime} \times 17^{\prime \prime} \times 33 \mathrm{~km} \mathrm{~s}^{-1}$. Contour levels at $-3,-1.5$ (dashed), $1.5,3,4.5, \ldots \times \sigma$. 


\begin{tabular}{|c|c|}
\hline Length of observation & $1 \times 12$ \\
\hline Date of observation & 22Dec94 \\
\hline Field center, $\alpha(1950)$ & 12:04:32 \\
\hline$\delta(1950)$ & $43: 17: 41$ \\
\hline Central frequency & 1416.49 \\
\hline$V_{\text {hel }}$ of central channel & 850 \\
\hline Primary beam FWHM & 37.4 \\
\hline Nr. of interferometers & 40 \\
\hline Baselines (min-max-incr) & $36-2700-72$ \\
\hline Synthesized beam $(\alpha \times \delta)(\operatorname{arcsec})$ & $11.9 \times 17.9$ \\
\hline Bandwidth & 5.0 \\
\hline Number of channels & 127 \\
\hline Channel separation & 8.29 \\
\hline Velocity resolution & 9.95 \\
\hline rms noise in one channel & 8.34 \\
\hline $\begin{array}{l}\text { K-mJy conversion, } \\
\text { equiv. of } 1 \mathrm{mJy} / \mathrm{beam}\end{array}$ & 2.83 \\
\hline
\end{tabular}

Note: To achieve a sufficient velocity resolution over

a $5 \mathrm{MHz}$ badwidh only the XX dipoles could be

correlated due to limitations of the backen

Results from WSRT data

From continuum map:

21-cm flux density

$\begin{aligned} \text { central point source (mJy) } & <1.2(3 \sigma) \\ \text { extended source (mJy) } & <3.4(3 \sigma)\end{aligned}$

From global profile:

Integrated HI-flux $\left(\mathrm{Jy} \mathrm{km} \mathrm{s}^{-1}\right) \quad 17.0 \pm 0.6$

Hel. systemic velocity $\left(\mathrm{km} \mathrm{s}^{-1}\right) \quad 770.0 \pm 1.5$

HI profile width, $20 \%\left(\mathrm{~km} \mathrm{~s}^{-1}\right) \quad 156.7 \pm 1.7$ $50 \%\left(\mathrm{~km} \mathrm{~s}^{-1}\right) \quad 97.7 \pm 3.0$

From $X V$-diagram:

Hel. systemic velocity $\left(\mathrm{km} \mathrm{s}^{-1}\right) \quad 778.3$

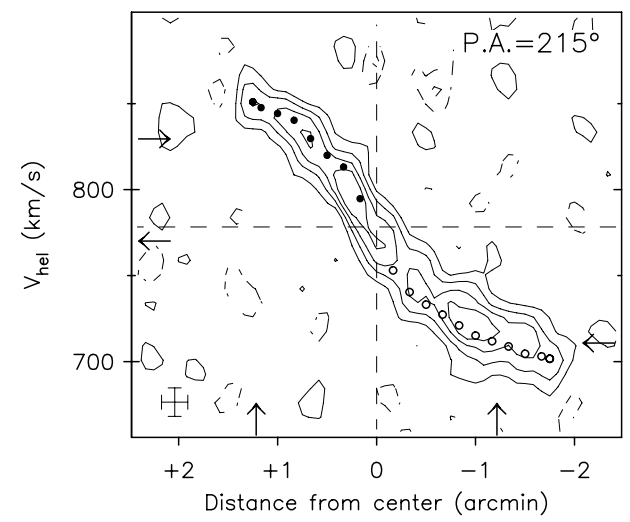

Contour levels for U7089

Channel maps:

$\sigma=5.09(\mathrm{~K})$

Raw continuum map: $\sigma=1.13(\mathrm{~K})$ Position-Velocity diagram:

$\sigma=4.52(\mathrm{~K})$

Note: This galaxy is a member of a rather tight group within the Ursa Major cluster. The group is dominated by the S0 system N4111 and several dwarf galaxies (U7094, N4117, N4118 and $1203+43)$. N4111 and N4118 were not detected in the HI line.

detected in the HI line. optically and in the distribution of the HI gas which is more extended on the approaching side. The rotation curve, however, is quite symmetric and does not reach the flat part on either side of not reach the galaxy.
the

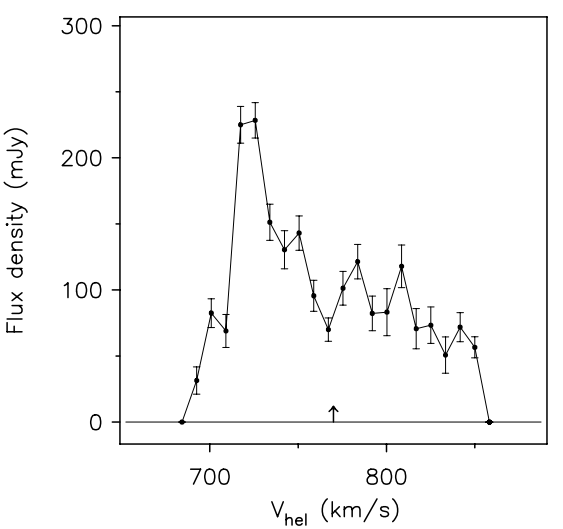

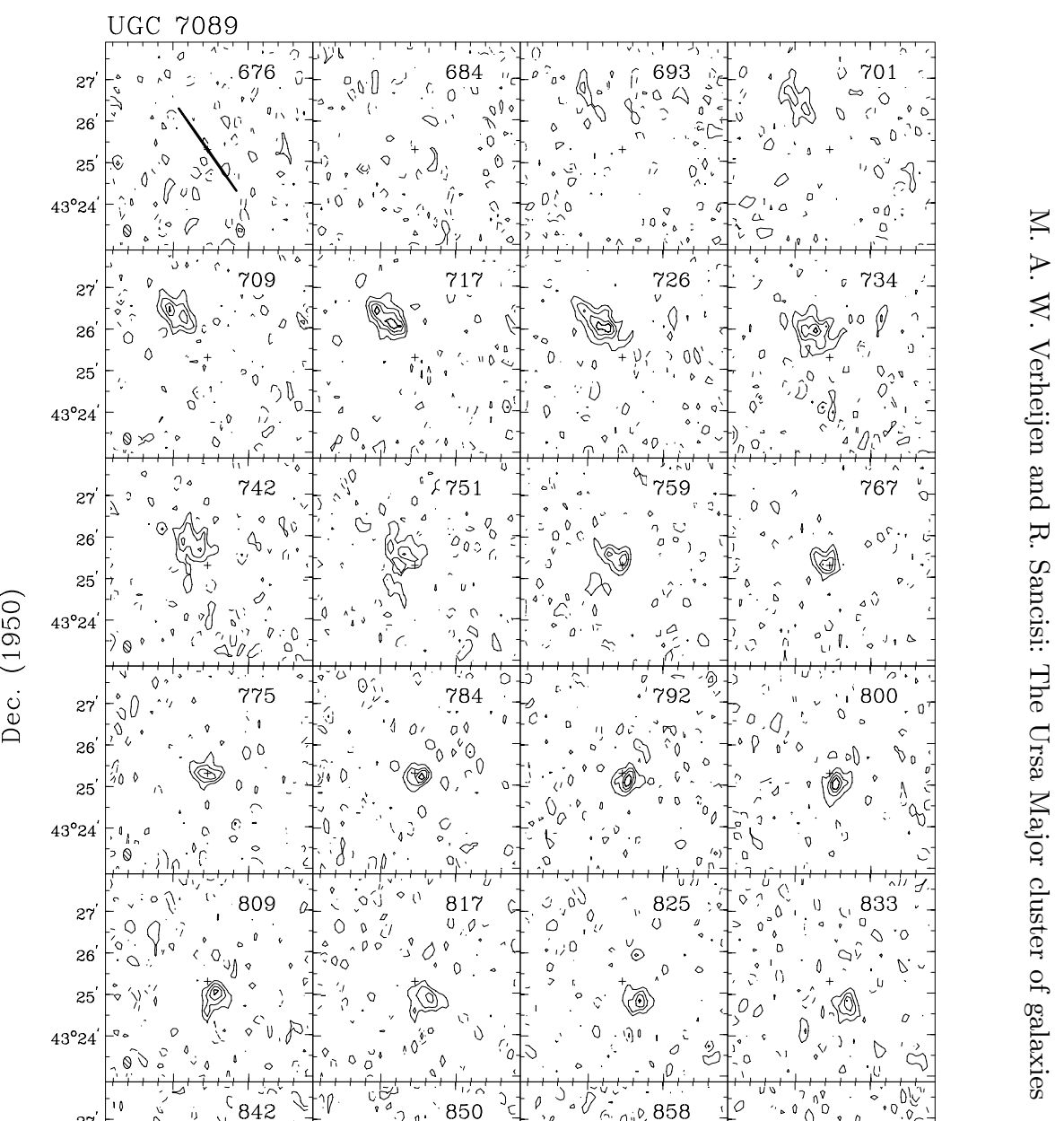

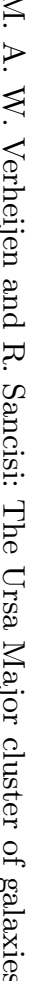

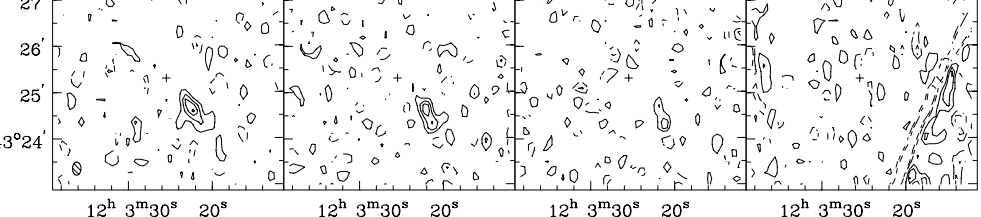

R.A. (1950)

Channel maps at a resolution of $11^{\prime \prime} \times 17^{\prime \prime} \times 17 \mathrm{~km} \mathrm{~s}^{-1}$.

Contour levels at $-3,-1.5$ (dashed), $1.5,3,4.5, \ldots \times \sigma$. 


\section{Observing parameters for UGC 7094}

\begin{tabular}{|c|c|}
\hline Length of observation & $1 \times 12$ \\
\hline Date of observation & 22Dec94 \\
\hline Field center, $\alpha(1950)$ & $12: 04: 32$ \\
\hline$\delta(1950)$ & 43:17:41 \\
\hline Central frequency & 1416.49 \\
\hline$V_{\text {hel }}$ of central channel & 850 \\
\hline Primary beam FWHM & 37.4 \\
\hline Nr. of interferometers & 40 \\
\hline Baselines (min-max-incr) & $36-2700-72$ \\
\hline Synthesized beam $(\alpha \times \delta)(\operatorname{arcsec})$ & $11.9 \times 17.9$ \\
\hline Bandwidth & 5.0 \\
\hline Number of channels & 127 \\
\hline Channel separation & 8.29 \\
\hline Velocity resolution & 9.95 \\
\hline rms noise in one channel & 8.34 \\
\hline $\begin{array}{l}\text { K-mJy conversion, } \\
\text { equiv. of } 1 \mathrm{mJy} / \text { beam }\end{array}$ & 2.83 \\
\hline
\end{tabular}

Note: To achieve a sufficient velocity resolution over

a $5 \mathrm{MHz}$ bandwidth, only the $\mathrm{XX}$ dipoles could be

correlated due to limitations of the backen

\section{Results from WSRT data}

\section{From continuum map: \\ 21-cm flux density}

central point source $(\mathrm{mJy}) \quad<1.2(3 \sigma)$ extended source $(\mathrm{mJy}) \quad<2.6(3 \sigma)$

From global profile:

Integrated HI-flux $\left(\mathrm{Jy} \mathrm{km} \mathrm{s}^{-1}\right) \quad 2.9 \pm 0.2$

Hel. systemic velocity $\left(\mathrm{km} \mathrm{s}^{-1}\right) \quad 779.6 \pm 1.6$

HI profile width, $20 \%\left(\mathrm{~km} \mathrm{~s}^{-1}\right) \quad 83.7 \pm 1.7$

$$
50 \%\left(\mathrm{~km} \mathrm{~s}^{-1}\right) \quad 71.9 \pm 5.5
$$

From $X V$-diagram:

Hel. systemic velocity $\left(\mathrm{km} \mathrm{s}^{-1}\right) \quad 776.9$

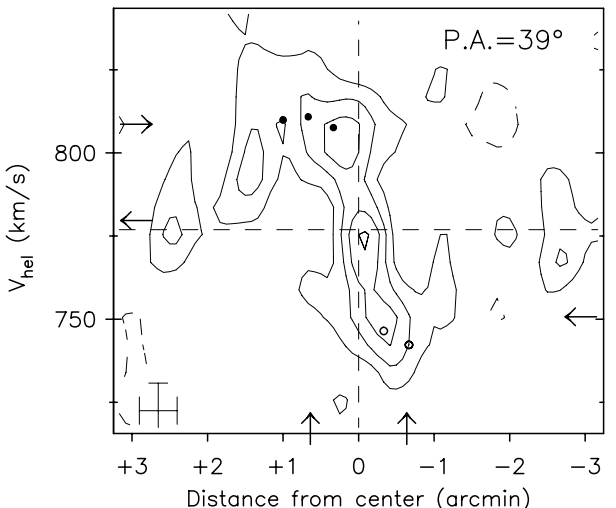

\section{Contour levels for U7094}

Channel maps:

\section{$\sigma=1.57(\mathrm{~K})$}

Raw continuum map:

$\sigma=0.36(\mathrm{~K})$

Position-Velocity diagram:

$\sigma=1.54(\mathrm{~K})$

Note: This edge-on galaxy is a member of a rather tight group within the Ursa Major cluster. The group is dominated by the S0 system N4111 and several dwarf galaxies (U7089, N4117, N4118 and 1203+43). N4111 and N4118 were not detected in the $\mathrm{HI}$ line.

The position-velocity diagram seems to suggest that the projected rotation curve of UGC 7094 reaches a maximum within 1 arcmin from the center and then declines to nearly zero at 2.5 arcmin from the center. This effect could be caused by a warp which reaches nearly face-on at the largest radii. However, the signal-to-noise level is too low to validate any model. $1203+43$ is located only 5 arcmin to the south-west while N4111 and U7089 lie 11 arcmin to the north and nort-east respectively.

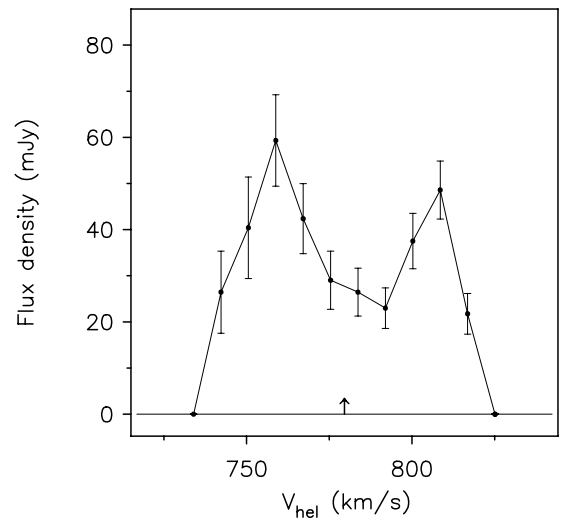

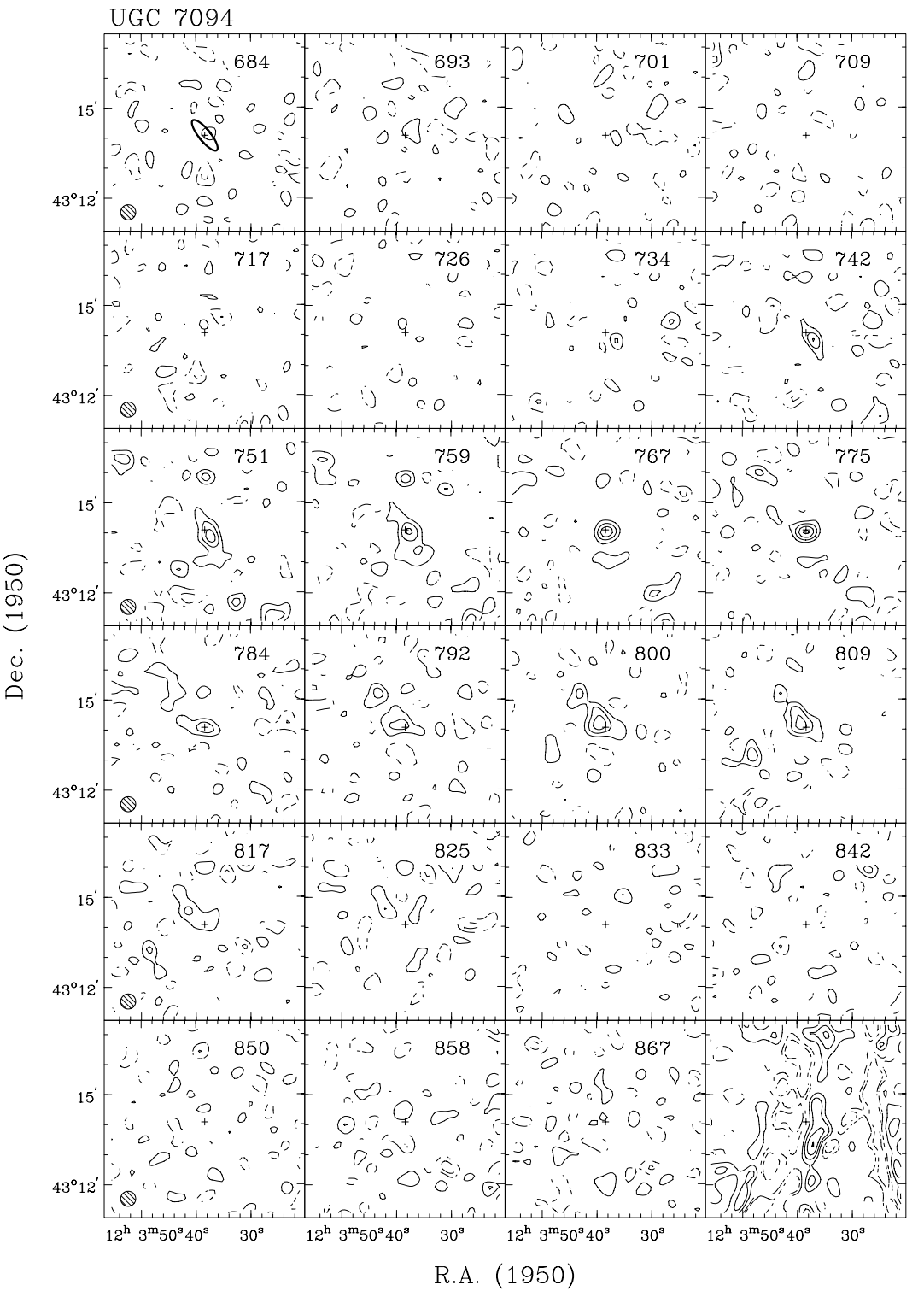

Channel maps at a resolution of $30^{\prime \prime} \times 30^{\prime \prime} \times 17 \mathrm{~km} \mathrm{~s}^{-1}$.

Contour levels at $-3,-1.5$ (dashed), $1.5,3,4.5, \ldots \times \sigma$. 


\begin{tabular}{|c|c|}
\hline Length of observation & $1 \times 12$ \\
\hline Date of observation & 22Dec94 \\
\hline Field center, $\alpha(1950)$ & 12:04:32 \\
\hline$\delta(1950)$ & 43:17:41 \\
\hline Central frequency & 1416.49 \\
\hline$V_{\text {hel }}$ of central channel & 850 \\
\hline Primary beam FWHM & 37.4 \\
\hline Nr. of interferometers & 40 \\
\hline Baselines (min-max-incr) & $36-2700-72$ \\
\hline Synthesized beam $(\alpha \times \delta)(\operatorname{arcsec})$ & $11.9 \times 17.9$ \\
\hline Bandwidth & 5.0 \\
\hline Number of channels & 127 \\
\hline Channel separation & 8.29 \\
\hline Velocity resolution & 9.95 \\
\hline rms noise in one channel & 8.34 \\
\hline $\begin{array}{l}\text { K-mJy conversion, } \\
\text { equiv. of } 1 \mathrm{mJy} / \text { beam }\end{array}$ & 2.83 \\
\hline
\end{tabular}

Note: To achieve a sufficient velocity resolution over

a $5 \mathrm{MHz}$ bandwidth,

correlated due to limitations of the backend.

Results from WSRT data

\begin{tabular}{|c|c|}
\hline \multicolumn{2}{|l|}{ From continuит map: } \\
\hline 21-cm flux density & $3.7 \pm 1.2$ \\
\hline \multicolumn{2}{|l|}{ Central point source position } \\
\hline$\alpha(1950)$ & $12: 05: 14.0$ \\
\hline$\delta(1950)$ & 43:24:17 \\
\hline \multicolumn{2}{|l|}{ From global profile: } \\
\hline$\overline{\text { Integrated HI-flux }}\left(\mathrm{Jy} \mathrm{km} \mathrm{s}^{-1}\right)$ & $6.9 \pm 1.1$ \\
\hline Hel. systemic velocity $\left(\mathrm{km} \mathrm{s}^{-1}\right)$ & $934.0 \pm 1.5$ \\
\hline HI profile width, $20 \%\left(\mathrm{~km} \mathrm{~s}^{-1}\right)$ & $289.4 \pm 7.5$ \\
\hline $50 \%\left(\mathrm{~km} \mathrm{~s}^{-1}\right)$ & $260.3 \pm 5.2$ \\
\hline
\end{tabular}

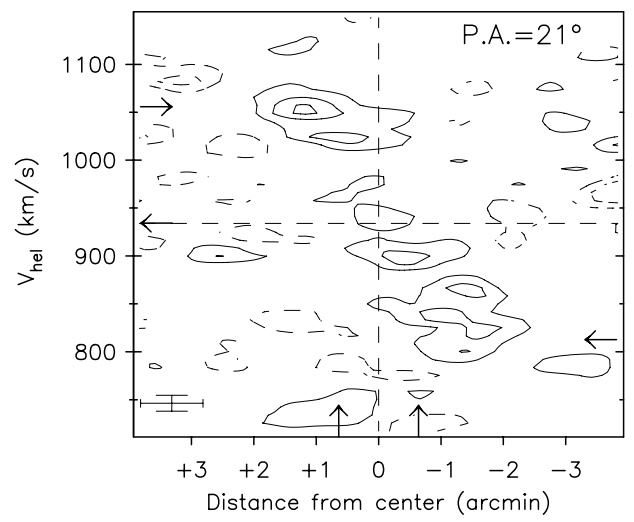

Contour levels for N4117

\begin{tabular}{ll}
\hline \hline Channel maps: & $\sigma=0.56(\mathrm{~K})$ \\
Raw continuum map: & $\sigma=0.12(\mathrm{~K})$ \\
Position-Velocity diagram: & $\sigma=0.55(\mathrm{~K})$ \\
\hline
\end{tabular}

Note: This galaxy is a member of a rather tight group within the Ursa Major cluster. The group is dominated by the S0 system N4111 and several dwarf galaxies (U7089, U7094, N4118 and galaxies (U7089, U7094, N4118 and
$1203+43)$. N4111 and N4118 were not detected in the HI line.

NGC 4117 is a dwarf S0 system with a faint $\mathrm{HI}$ ring just outside $\mathrm{R}_{25}$. The signal-to-noise is too low to derive a rotation curve from the positiontation curve from the position-velocity diagram. N4118 is situated 1.5 arcmin to the south-east.

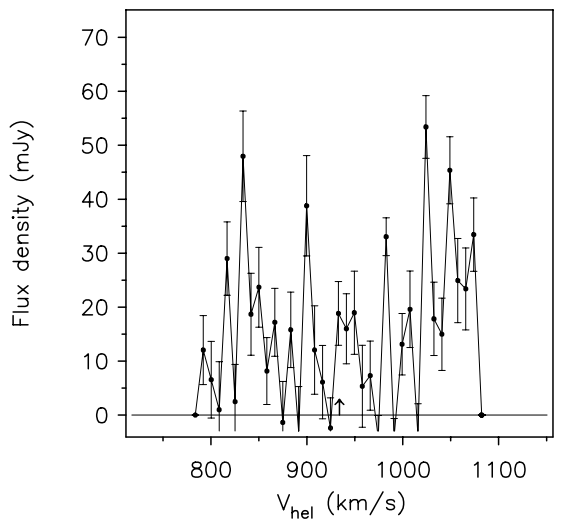

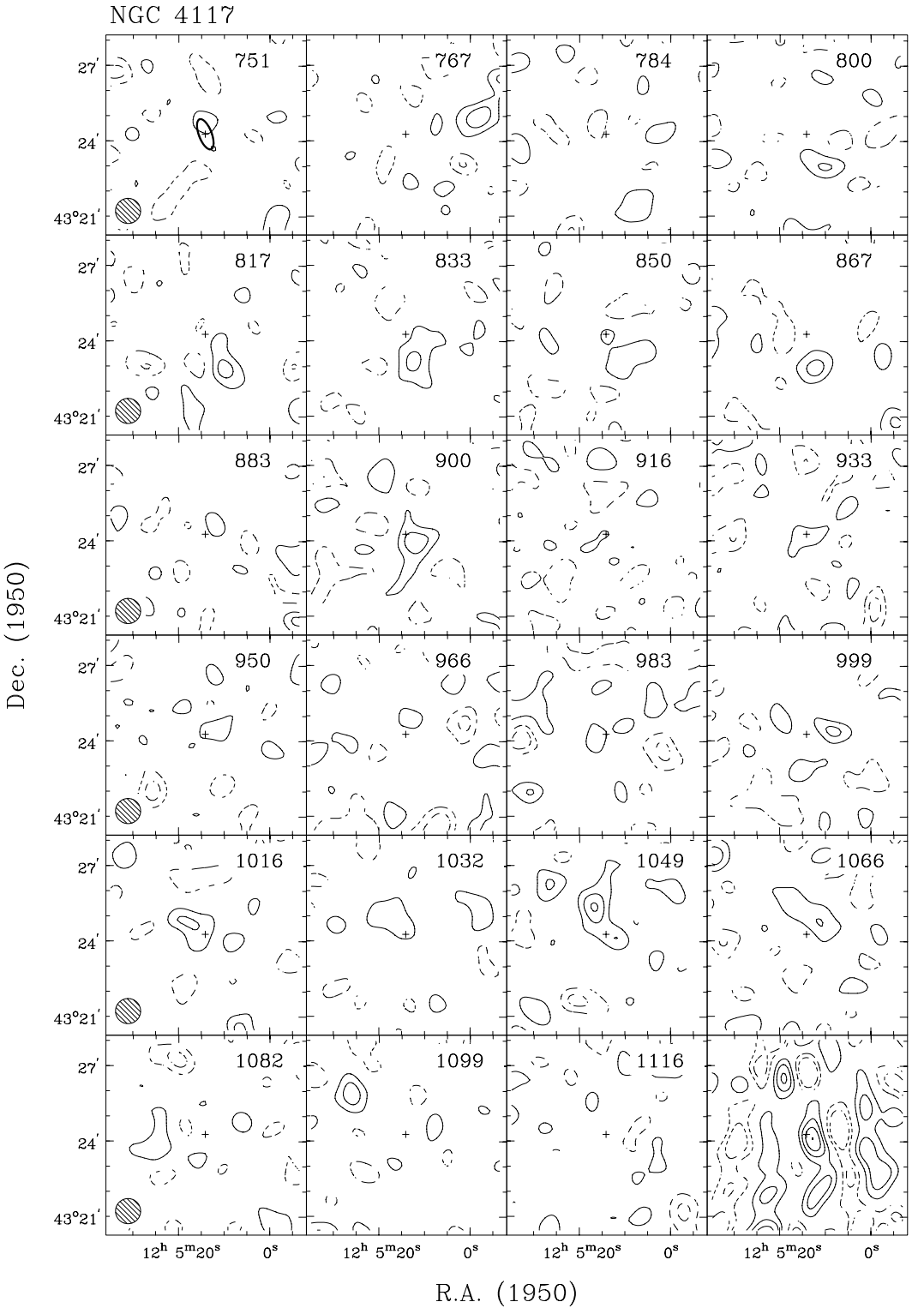

Channel maps at a resolution of $60^{\prime \prime} \times 60^{\prime \prime} \times 17 \mathrm{~km} \mathrm{~s}^{-1}$.

Contour levels at $-3,-1.5$ (dashed), $1.5,3,4.5, \ldots \times \sigma$. 


\begin{tabular}{|c|c|}
\hline Length of observation & $1 \times 12$ \\
\hline Date of observation & 19Dec94 \\
\hline Field center, $\alpha(1950)$ & 12:06:59 \\
\hline$\delta(1950)$ & $43: 54: 57$ \\
\hline Central frequency & 1416.26 \\
\hline$V_{\text {hel }}$ of central channel & 900 \\
\hline Primary beam FWHM (arcmin) & 37.4 \\
\hline Nr. of interferometers & 40 \\
\hline Baselines (min-max-incr) & $36-2700-72$ \\
\hline Synthesized beam $(\alpha \times \delta)(\operatorname{arcsec})$ & $12.1 \times 17.3$ \\
\hline Bandwidth & 5.0 \\
\hline Number of channels & 63 \\
\hline Channel separation & 16.59 \\
\hline Velocity resolution & 19.91 \\
\hline rms noise in one channel & 4.89 \\
\hline $\begin{array}{l}\text { K-mJy conversion, } \\
\text { equiv. of } 1 \mathrm{mJy} / \text { beam }\end{array}$ & 2.88 \\
\hline
\end{tabular}

Results from WSRT data

From continuum map:

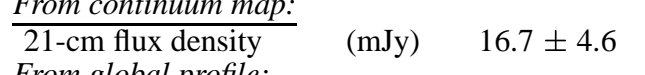

From global profile:

Integrated HI-flux $\left(\mathrm{Jy} \mathrm{km} \mathrm{s}^{-1}\right) \quad 19.2 \pm 0.7$

Hel. systemic velocity $\left(\mathrm{km} \mathrm{s}^{-1}\right) \quad 893.8 \pm 3.9$

HI profile width, $20 \%\left(\mathrm{~km} \mathrm{~s}^{-1}\right) \quad 331.6 \pm 4.5$

$$
50 \%\left(\mathrm{~km} \mathrm{~s}^{-1}\right) \quad 266.0 \pm 7.8
$$

\section{From XV-diagram:}

Hel. systemic velocity $\left(\mathrm{km} \mathrm{s}^{-1}\right) \quad 888.0$

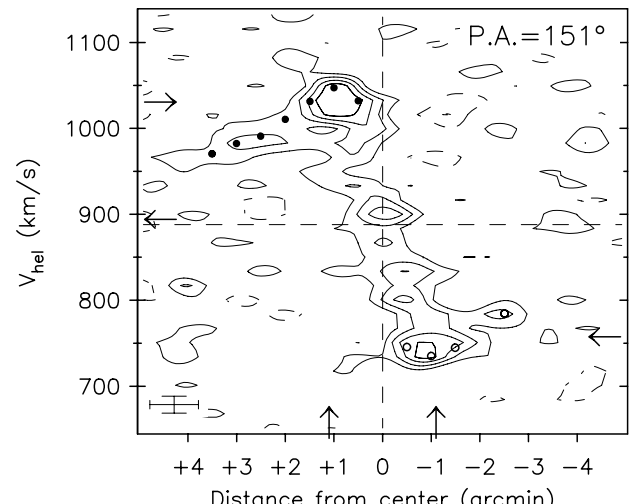

Distance from center (arcmin)

\section{Contour levels for N4138}

Channel maps:

\section{$\sigma=0.58(\mathrm{~K})$}

Raw continuum map:

$\sigma=0.29(\mathrm{~K})$

Position-Velocity diagrams

$\sigma=0.57(\mathrm{~K})$

Note: A large fraction (20\%) of the stars in this early type spiral is counter rotating. For that reason, N4138 has been studied extensively in the past. Jore et al (1996) have observed this galaxy at $21 \mathrm{~cm}$ with the VLA and obtained HI data with high S/N. For deprojection of the rotation curve derived from the position-velocity diagram of our WSRT data, the optical position angle and inclination were used for the inner regions. In the outer regions, the position angle and inclination from the velocity field of Jore et al were used. The HI disk is strongly warped toward faceon but not strong enough to account for the observed decline.

The strong point source at 3 arcmin to the $\mathrm{SW}$ is $3 \mathrm{C} 378.6$.

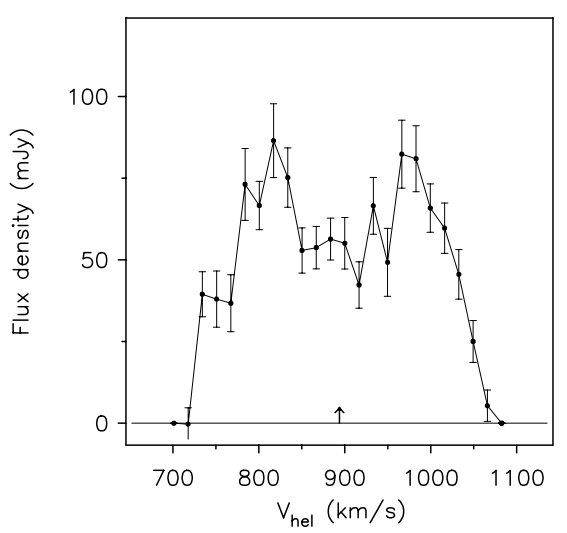

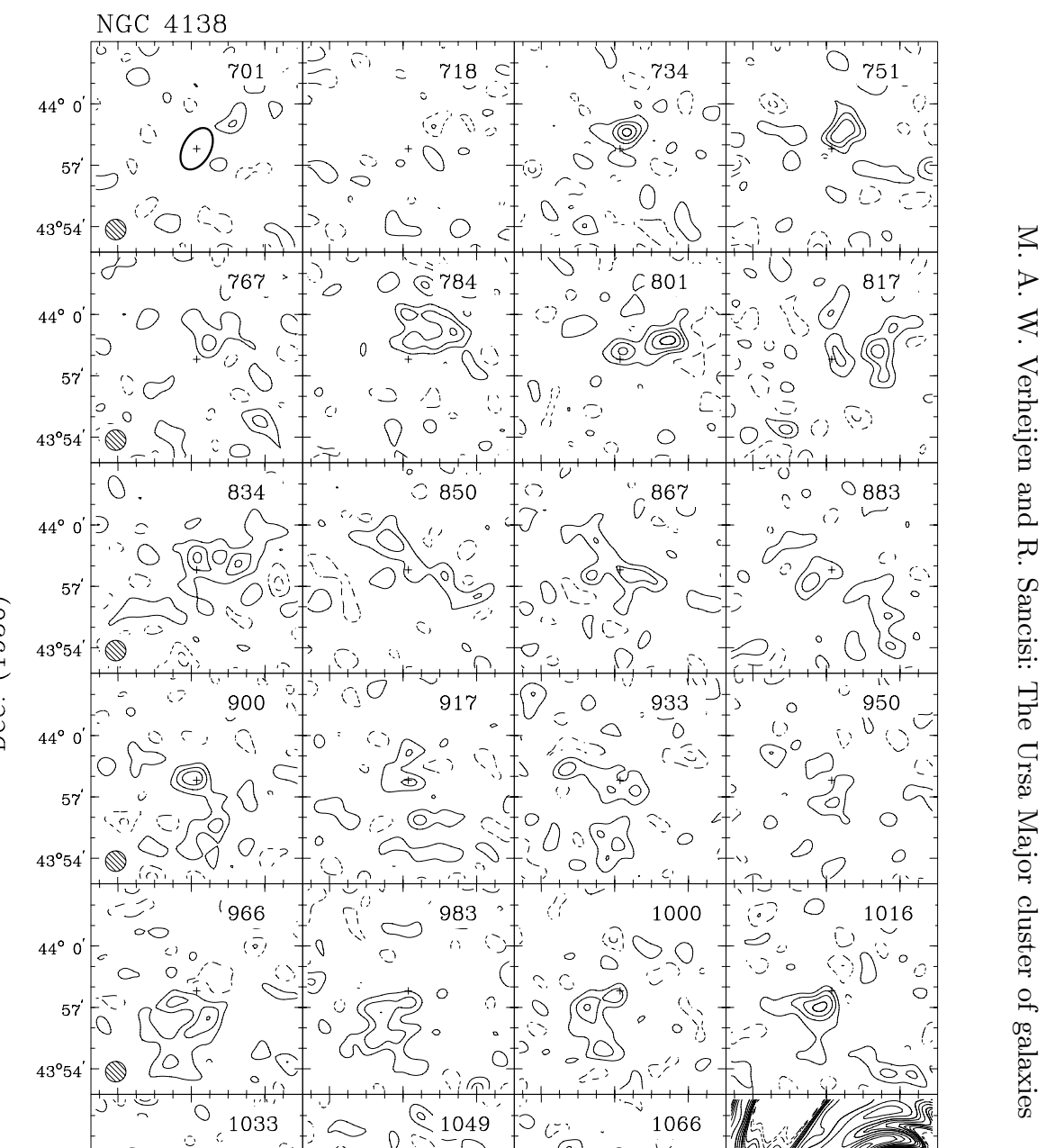

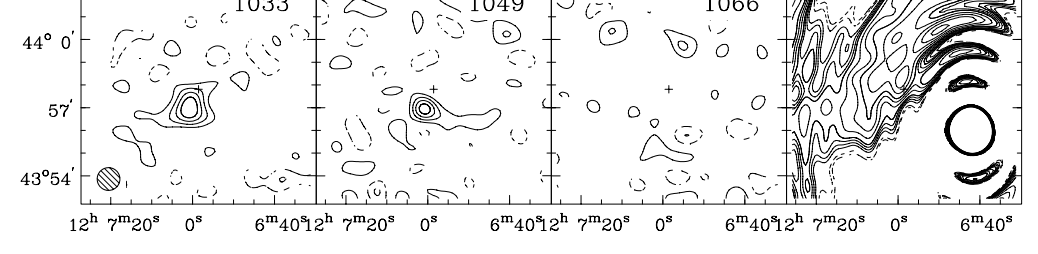

R.A. (1950)

Channel maps at a resolution of $60^{\prime \prime} \times 60^{\prime \prime} \times 20 \mathrm{~km} \mathrm{~s}^{-1}$.

Contour levels at $-3,-1.5$ (dashed), $1.5,3,4.5, \ldots \times \sigma$. 


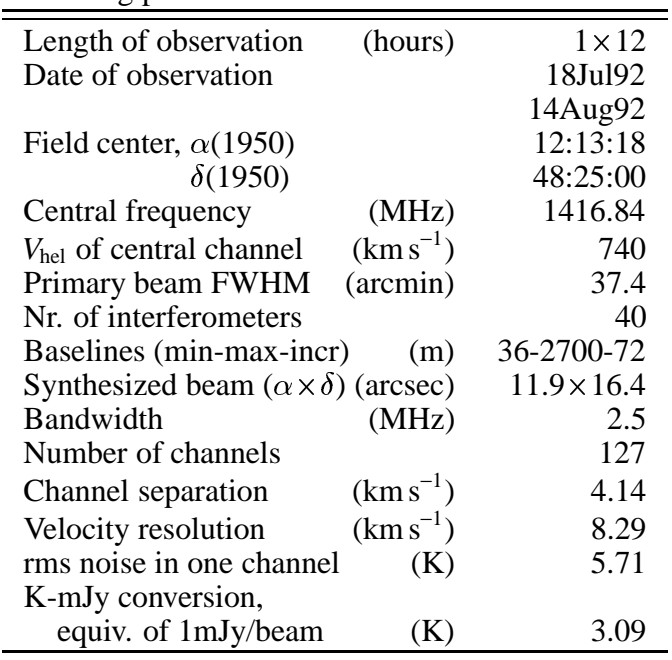

Results from WSRT data

\section{From continuum map:}

\section{1-cm flux density}

From global profile:

Integrated HI-flux $\left(\mathrm{Jy} \mathrm{km} \mathrm{s}^{-1}\right) \quad 7.8 \pm 0.2$

Hel. systemic velocity $\left(\mathrm{km} \mathrm{s}^{-1}\right) \quad 729.9 \pm 1.7$

HI profile width, $20 \%\left(\mathrm{~km} \mathrm{~s}^{-1}\right) \quad 138.0 \pm 5.0$

From $X V$-diagram: $50 \%\left(\mathrm{~km} \mathrm{~s}^{-1}\right) \quad 79.9 \pm 1.9$

Hel. systemic velocity $\left(\mathrm{km} \mathrm{s}^{-1}\right) \quad 723.7$

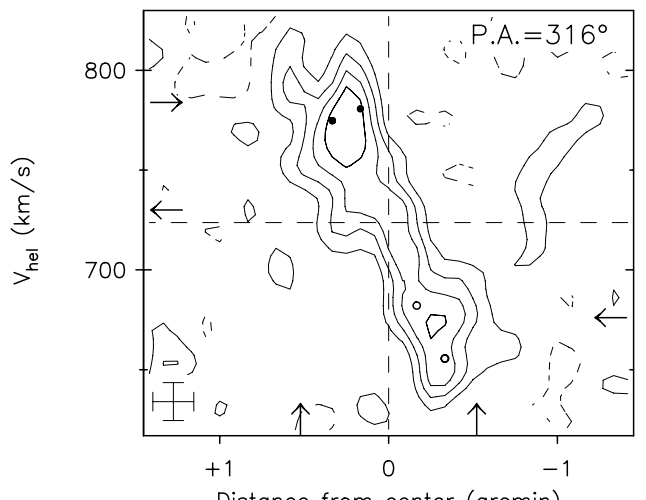

Distance from center (arcmin)
Contour levels for N4218

\begin{tabular}{ll}
\hline Channel maps: & $\sigma=3.71(\mathrm{~K})$ \\
Raw continuum maps: & $\sigma=1.28(\mathrm{~K})$ \\
Position-Velocity diagram: & $\sigma=3.24(\mathrm{~K})$ \\
&
\end{tabular}

Note: NGC 4218 is a Blue Compact Dwarf galaxy with a slightly irregular optical morphology. The HI distribution is a little bit lopsided. The HI disk does not extend significantly beyond $R_{25}$ and the rotation curve is ill deyond $\mathrm{R}_{25}$ and the rotation
fined in the inner regions.

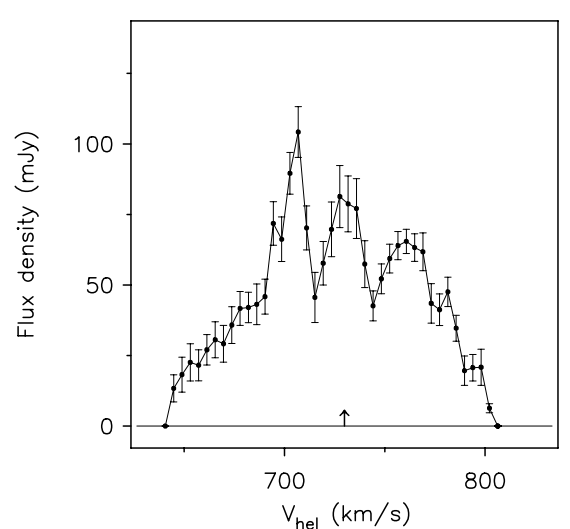

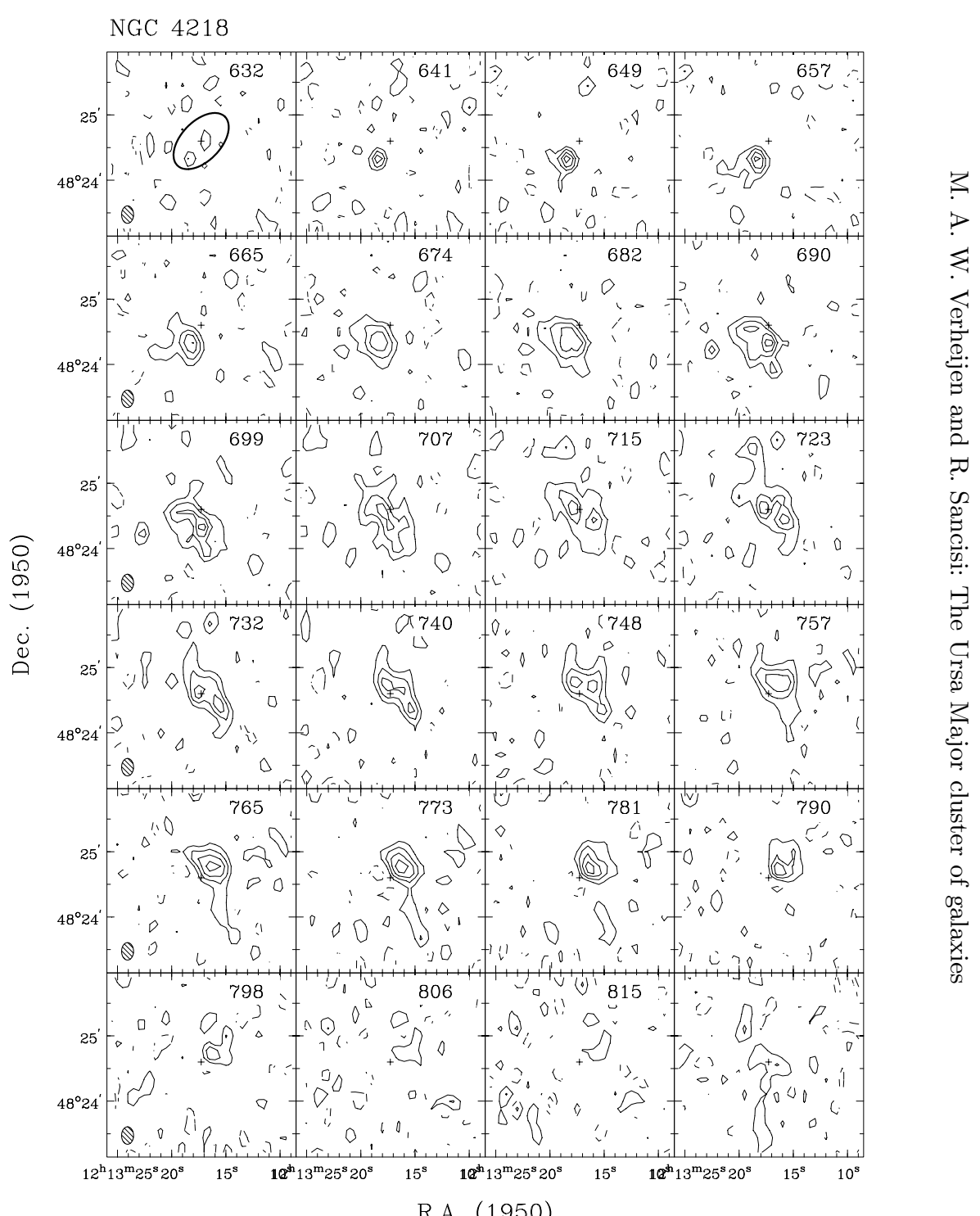

Channel maps at a resolution of $11^{\prime \prime} \times 16^{\prime \prime} \times 19 \mathrm{~km} \mathrm{~s}^{-1}$.

Contour levels at $-3,-1.5$ (dashed), $1.5,3,4.5, \ldots \times \sigma$. 


\section{Observing parameters for NGC 4220}

\begin{tabular}{|c|c|c|}
\hline Length of observation & (hours) & $1 \times 12$ \\
\hline Date of observation & & $\begin{array}{r}\text { 01Jul92 } \\
\text { 14Aug92 }\end{array}$ \\
\hline Field center, $\alpha(1950)$ & & $\begin{array}{l}12: 13: 18 \\
48 \cdot 25 \cdot 00\end{array}$ \\
\hline Central frequency & $(\mathrm{MHz})$ & 1416.82 \\
\hline$V_{\text {hel }}$ of central channel & $\left(\mathrm{km} \mathrm{s}^{-1}\right)$ & 740 \\
\hline Primary beam FWHM & (arcmin) & 37.4 \\
\hline Nr. of interferometers & & 40 \\
\hline Baselines (min-max-incr & (m) & $36-2700-72$ \\
\hline Synthesized beam $(\alpha \times \delta)$ & $(\operatorname{arcsec})$ & $12.3 \times 16.5$ \\
\hline Bandwidth & $(\mathrm{MHz})$ & 5.0 \\
\hline Number of channels & & 63 \\
\hline Channel separation & $\left(\mathrm{km} \mathrm{s}^{-1}\right)$ & 16.57 \\
\hline Velocity resolution & $\left(\mathrm{km} \mathrm{s}^{-1}\right)$ & 33.14 \\
\hline rms noise in one channel & $(\mathrm{K})$ & 2.97 \\
\hline $\begin{array}{l}\text { K-mJy conversion, } \\
\text { equiv. of } 1 \mathrm{mJy} / \text { beam }\end{array}$ & $(\mathrm{K})$ & 2.97 \\
\hline
\end{tabular}

\section{Results from WSRT data}

\begin{tabular}{lr}
\hline \hline $\begin{array}{l}\text { From continuum map: } \\
\text { 21-cm flux density }\end{array}$ & \\
$\begin{array}{r}\text { central point source (mJy) } \\
\text { extended source (mJy) }\end{array}$ & $<0.9(3 \sigma)$ \\
$\begin{array}{l}\text { From global profile: } \\
\left.\text { Integrated HI-flux }(\mathrm{Jy} \mathrm{km} \mathrm{s})^{-1}\right)\end{array}$ & $4.4 \pm 0.3$ \\
Hel. systemic velocity $\left(\mathrm{km} \mathrm{s}^{-1}\right)$ & $914.2 \pm 1.2$ \\
HI profile width, 20\% $\left(\mathrm{km} \mathrm{s}^{-1}\right)$ & $438.1 \pm 1.3$
\end{tabular}

$$
50 \%\left(\mathrm{~km} \mathrm{~s}^{-1}\right) \quad 423.3 \pm 3.3
$$
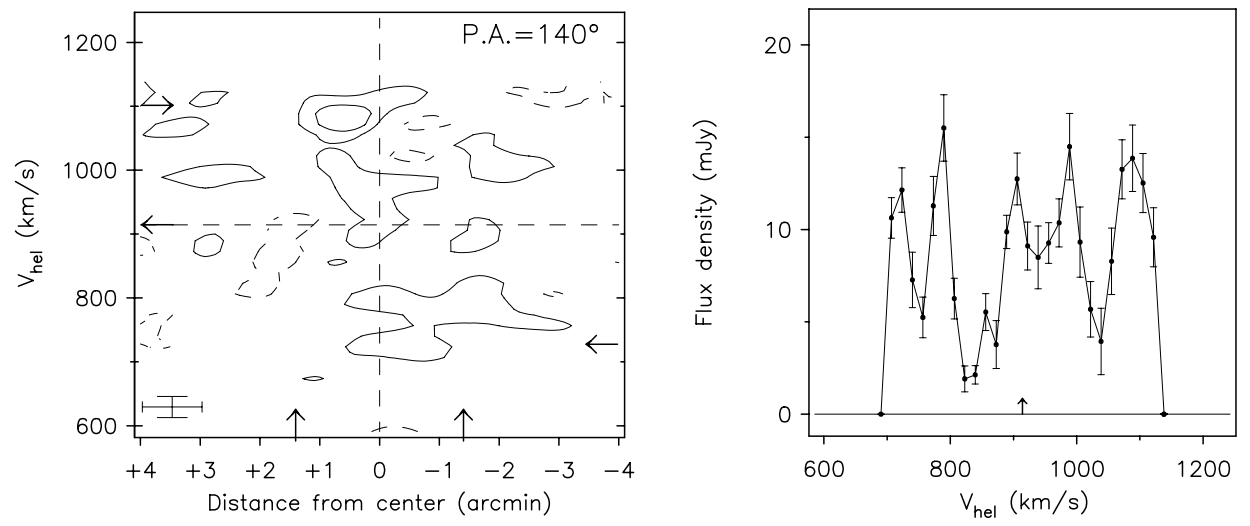

\section{Contour levels for N4220}

Channel maps:

Raw continuum maps:

$\sigma=0.29(\mathrm{~K})$

Position-Velocity diagram

$\sigma=0.28(\mathrm{~K})$

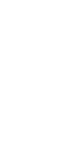

Note: This early type spiral has a very low HI content. The gas is barely detected and occurs only inside the optical region. No meaningful rotation curve could be derived from the positionvelocity diagram.

\section{.}

s.
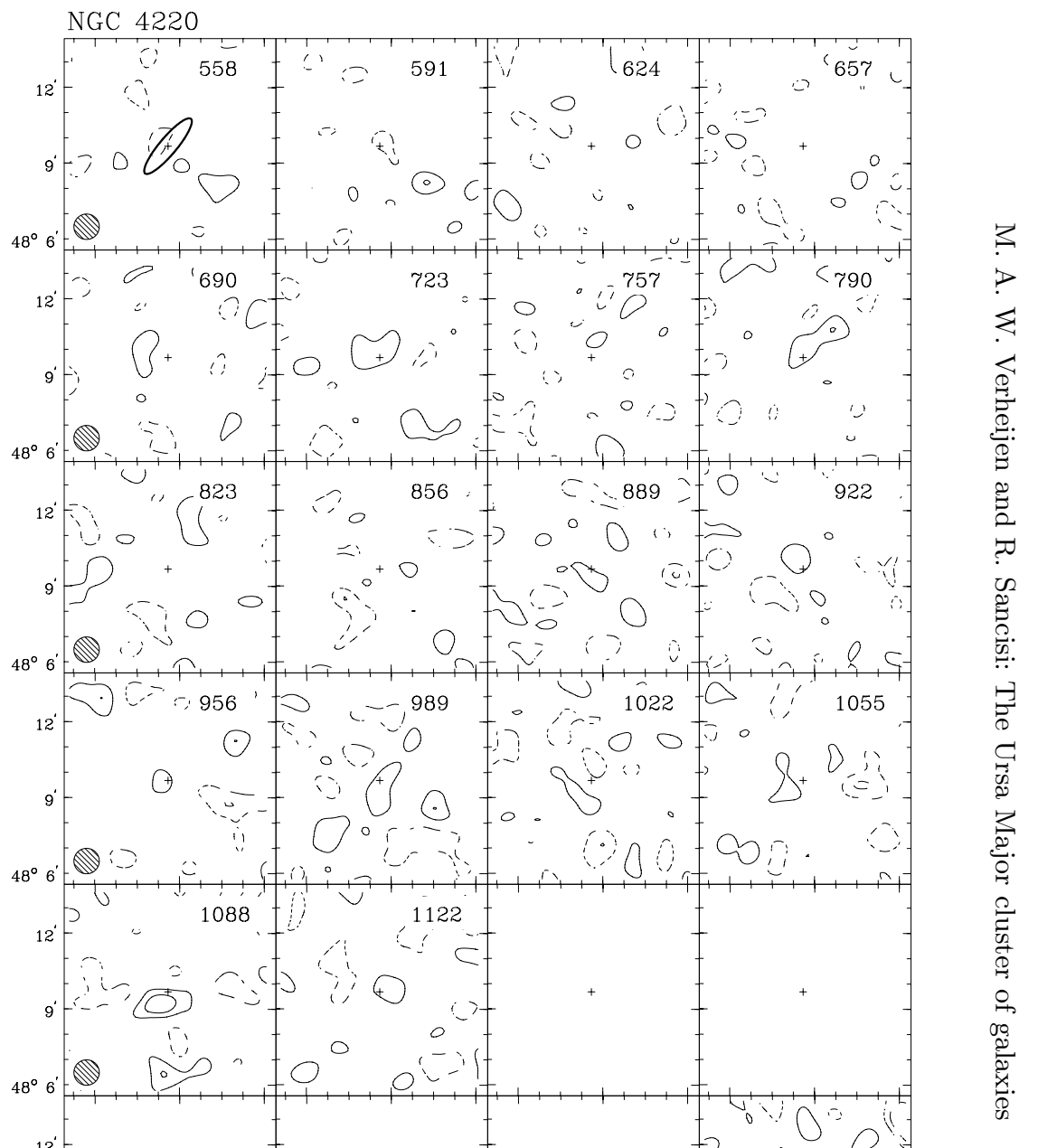

$48^{\circ} 6^{\prime}$

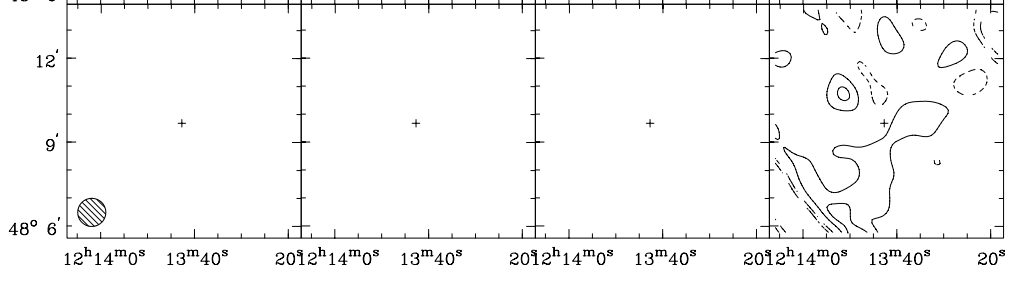

R.A. $(1950)$

Channel maps at a resolution of $60^{\prime \prime} \times 60^{\prime \prime} \times 33 \mathrm{~km} \mathrm{~s}^{-1}$.

Contour levels at $-3,-1.5$ (dashed), $1.5,3,4.5, \ldots \times \sigma$. 